\title{
Healthy Bones for Broiler Chickens
}

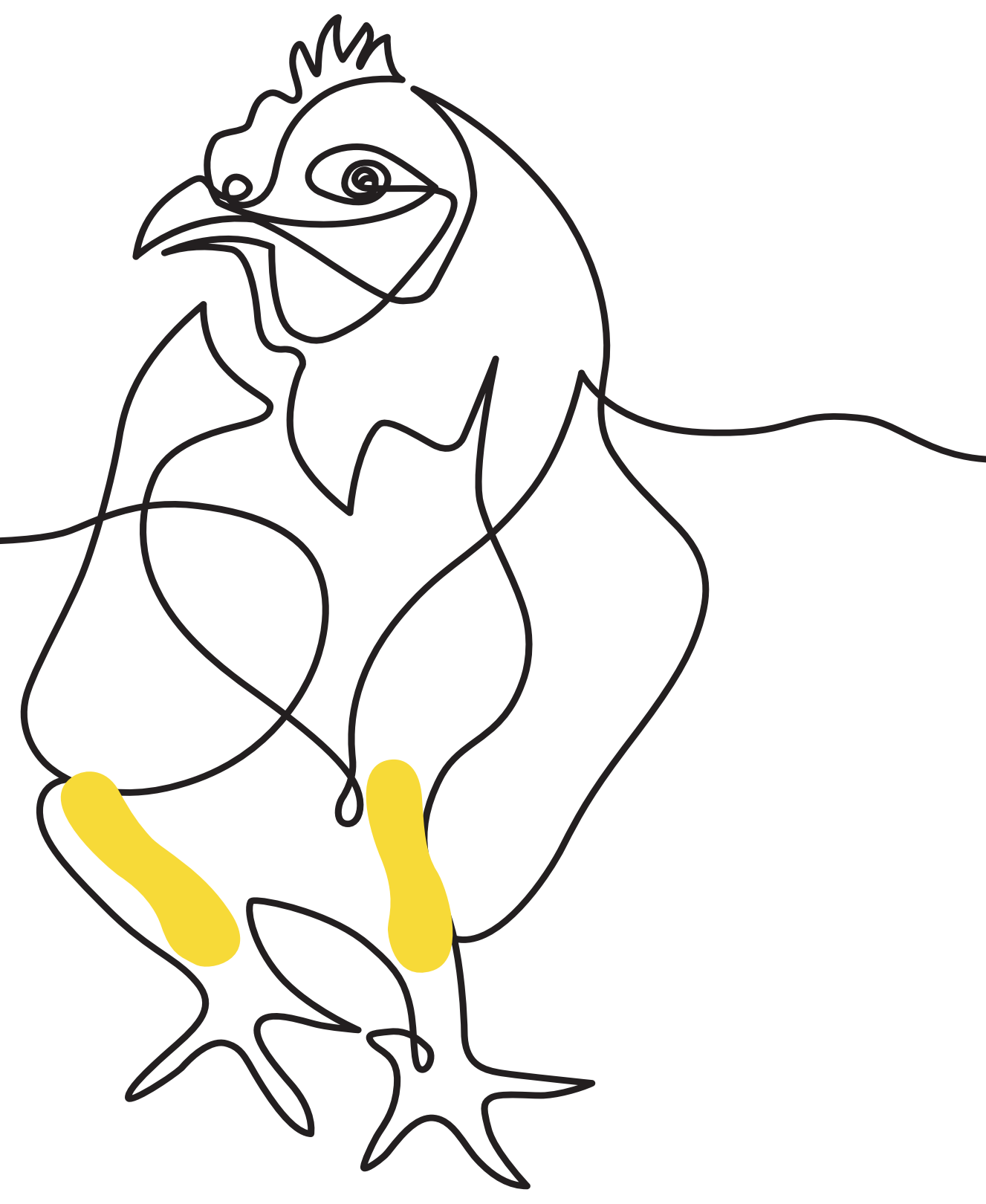

Bahadır Can Güz 
You are cordially invited to attend the public defence of my PhD thesis entitled:

\section{Healthy Bones for Broiler Chickens}

Bahader Can Güz

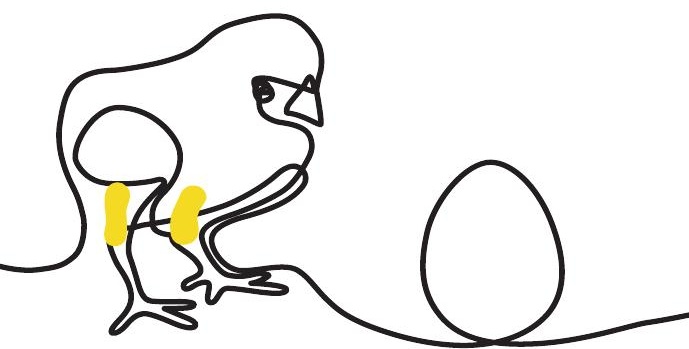

Wednesday, $5^{\text {th }}$ of January 2022 at 1:30 p.m. in the Aula of Wageningen University and Research Generaal Foulkesweg 1, Wageningen

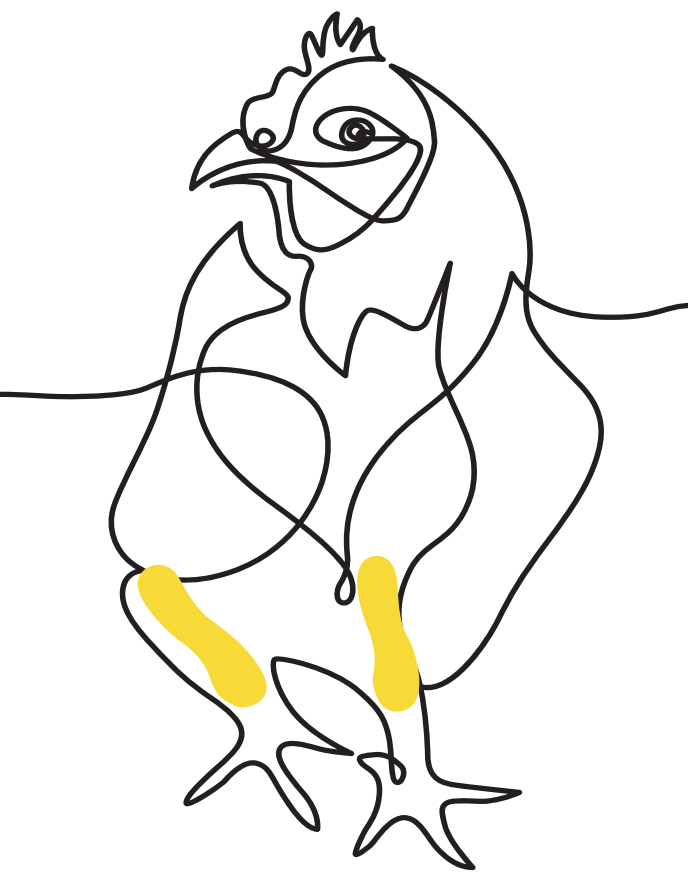

Paranymphs

Jan Wijnen jan.wijnen@wur.nl

Bjorge Laurenssen bjorge.laurenssen@wur.nl 


\section{Propositions}

1. Organic minerals in the diet of broiler chickens result in better bone quality than inorganic minerals. (this thesis)

2. Slower-growing broiler chickens have better bone quality than fast-growing broiler chickens. (this thesis)

3. Research results become valuable when they are implemented.

4. To deny scientific findings that go against superstitious beliefs, hampers the societal debate.

5. Searching on internet for health-related issues is the illness of the 21 st century.

6. Plant-based foods should be tax free.

Propositions belonging to this thesis, entitled

\section{Healthy Bones for Broiler Chickens}

Bahadır Can Güz

Wageningen, 5 January 2022 



\section{Healthy Bones for Broiler Chickens}

Bahadır Can Güz 


\section{Thesis committee}

\section{Promotor}

Prof. Dr B. Kemp

Professor of Adaptation Physiology

Wageningen University \& Research

\section{Co-promotor}

Dr H. van den Brand

Associate professor, Adaptation Physiology Group

Wageningen University \& Research

\section{Other members}

Prof. Dr S. Yalçın, Ege University, Turkey

Prof. Dr E. O. Oviedo-Rondón, North Carolina State University, USA

Prof. Dr L. den Hartog, Wageningen University \& Research

Dr P. Bikker, Wageningen University \& Research

This research was conducted under the auspices of the Graduate School of Wageningen Institute of Animal Sciences (WIAS). 


\title{
Healthy Bones for Broiler Chickens
}

\section{Bahadır Can Güz}

\author{
Thesis
}

submitted in fulfilment of the requirement of the degree of doctor at Wageningen University

by the authority of the Rector Magnificus

Prof. Dr A.P.J. Mol,

in the presence of the

Thesis Committee appointed by the Academic Board

to be defended in public

on January $5^{\text {th }}, 2022$

at 1.30 p.m. in the Aula. 


\section{Bahadır Can Güz}

Healthy Bones for Broiler Chickens

326 pages

\section{PhD Thesis}

Wageningen University, Wageningen, The Netherlands (2022)

With references, with summaries in English and Turkish

ISBN: 978-94-6395-984-1

DOI: https://doi.org/10.18174/553659 


\section{Content of Thesis}

General introduction

Chapter 1 Effects of dietary organic minerals, fish oil, and hydrolysed collagen on growth performance and tibia characteristics of broiler chickens

Chapter 2 Effects of green LED light during incubation and dietary organic macro and trace minerals during rearing on tibia characteristics of broiler chickens at slaughter age

Chapter 3 Effects of organic macro and trace minerals in fast and slower-growing broiler breeders' diet on offspring growth performance and tibia characteristics

Chapter 4 Effects of eggshell temperature pattern during incubation on tibia characteristics of broiler chickens at slaughter age

Chapter 5 Effects of pen enrichment on leg health of fast and slower-growing broiler chickens

General discussion

Summary

Özet (Turkish summary)

Acknowledgements

About the author

Publications

Training and supervision

Colophon 


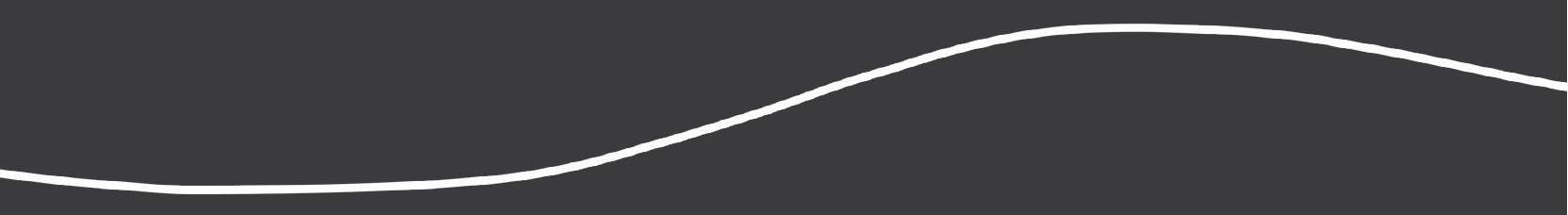




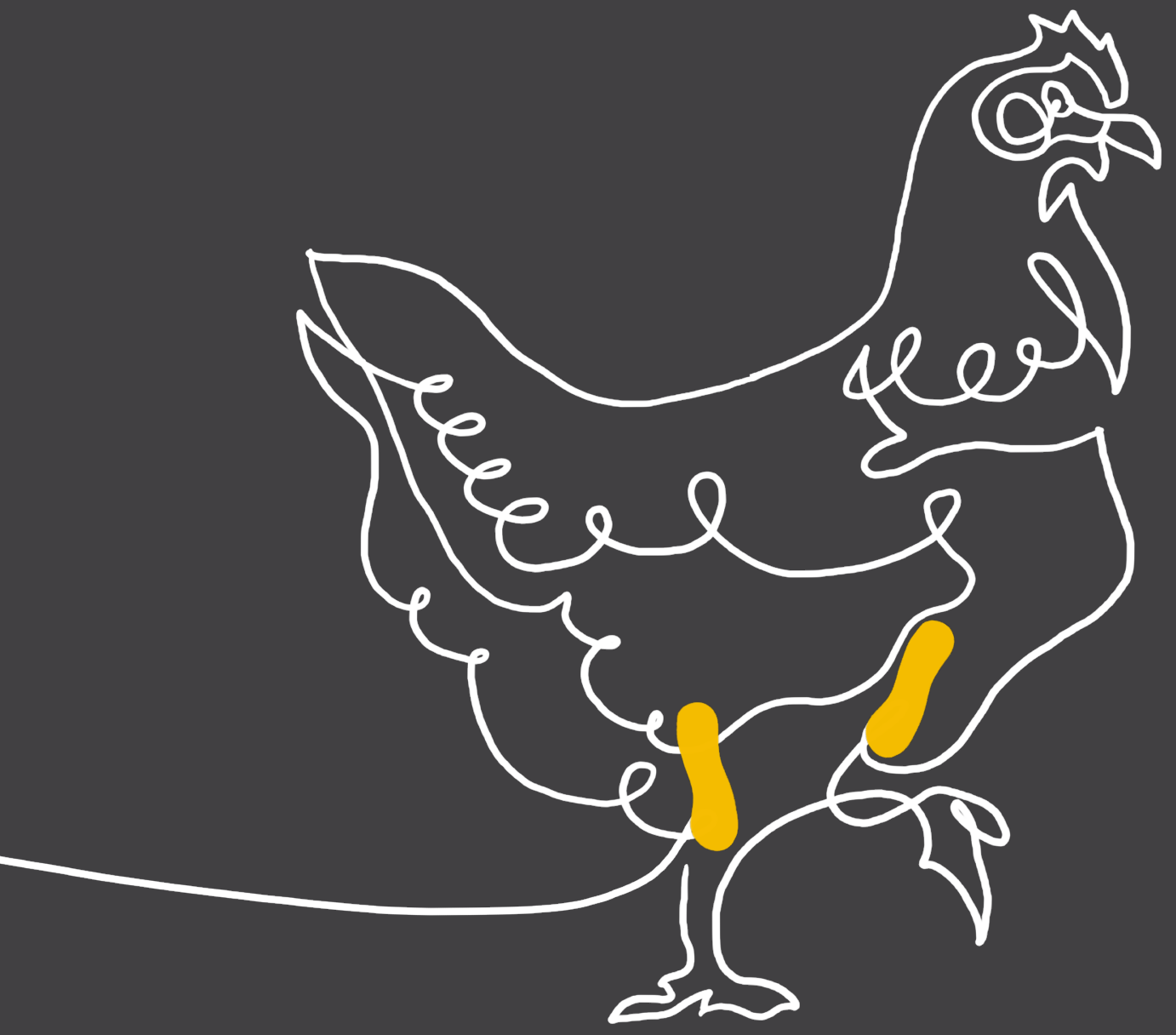

\section{GENERAL \\ INTRODUCTION}


Content of General Introduction 


\section{INTRODUCTION}

\section{SUBOPTIMAL LEG HEALTH IN BROILER CHICKENS}

Leg Bone Abnormalities and Disorders

Welfare Consequences of Suboptimal Leg Health Economic Consequences of Suboptimal Leg Health

\section{BONE QUALITY MEASUREMENTS}

Morphological Characteristics

Biophysical Characteristics

Mechanical Characteristics

\section{POTENTIAL APPROACHES TO IMPROVE LEG HEALTH}

Fast-growing vs. Slower-growing Broiler Chickens

Dietary Macro and Trace Minerals

Incubation Conditions

Environmental Enrichment

$\bullet$

AIM OF THE THESIS

○

OUTLINE OF THE THESIS 


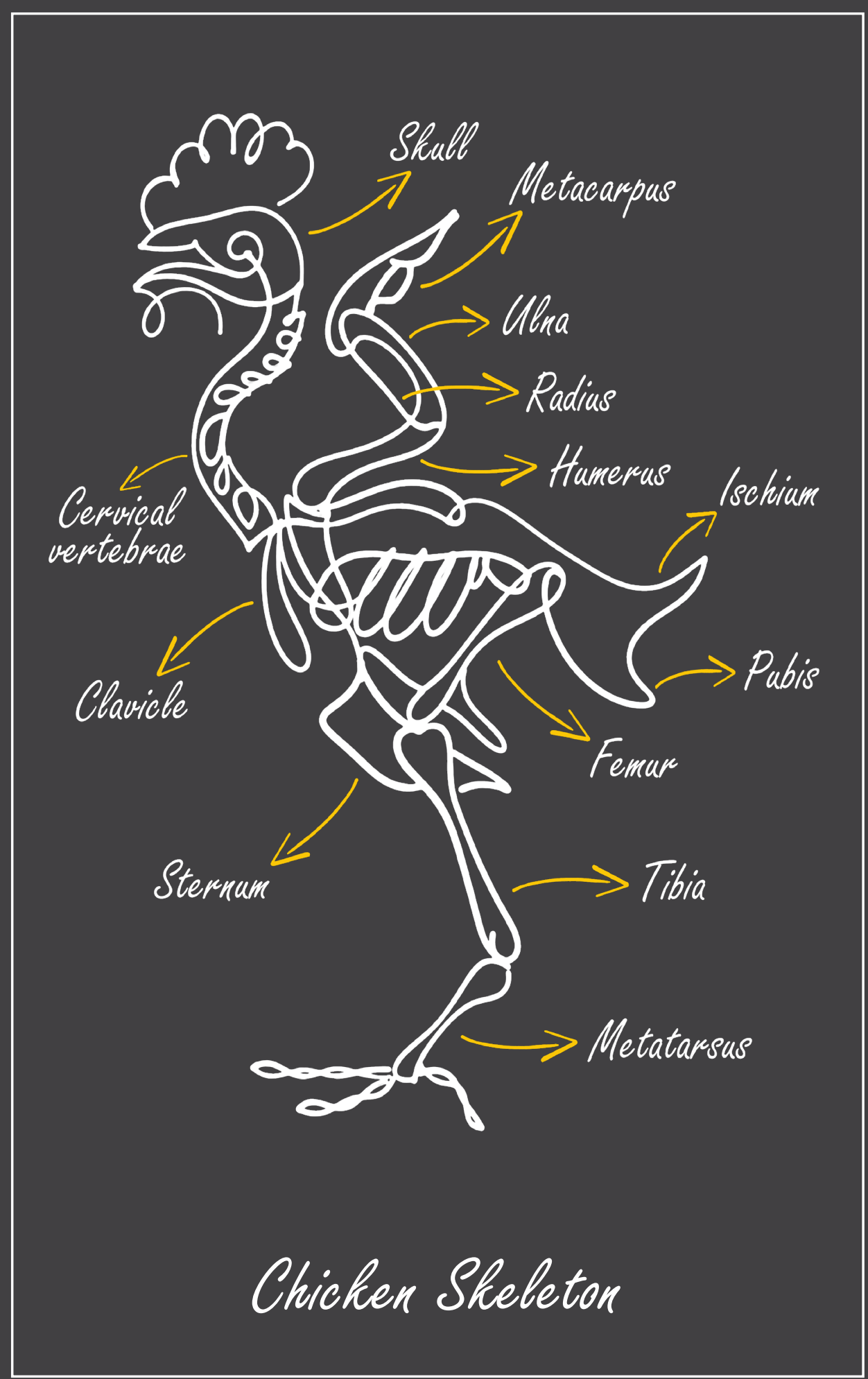




\section{INTRODUCTION}

Chicken meat is one of the main animal protein sources for humans and is nowadays the second most produced and consumed meat in the world (Ritchie and Roser, 2017; FAO, 2021). Due to the rapidly increasing human population, there is a corresponding increase in the demand for meat, which has been forced the broiler (meat-type chicken) industry to be more efficient (Decuypere et al., 2010; Dawkins and Layton, 2012; Mottet and Tempio, 2017). Consequently, broiler chickens have undergone radical phenotypic and genotypic changes as a result of an intense genetic selection (Havenstein et al., 2003; Tona et al., 2004; Vieira and Angel, 2012; Zuidhof et al., 2014; Tallentire et al., 2016; Bessei, 2018). Accompanied by better management conditions and better feed quality, this selection has resulted in an increase in body weight gain by approximately $400 \%$ in last several decades, from approximately $700 \mathrm{~g}$ (1950s) to $3100 \mathrm{~g}$ (2010s) in 42 days (McKay et al., 2000; Knowles et al., 2008; Petracci and Cavani, 2012; Zuidhof et al., 2014; Tallentire et al., 2016).

Despite the fact that fast growth and better feed efficiency have provided numerous advantages e.g., high amount of meat production in a short rearing duration, less environmental pollution and considerable financial benefits for producers, it has also caused some downsides e.g., suboptimal leg health, impaired locomotion and lameness (Bradshaw et al., 2002; Knowles et al., 2008; Tallentire et al., 2016).

One of the major reasons for suboptimal leg health in broiler chickens is an imbalance between high growth rate and immature bones, because the speed of bone development is unable to keep up with this fast growth (Thorp, 1994; Julian, 1998; Angel, 2007; Dibner et al., 2007; Knowles et al., 2008; Sherlock et al., 2010; Shim et al., 2012; Prisby et al., 2014; González-Cerón et al., 2015; Pines and Reshef, 2015), while other reasons are problems related to muscles, joints and tendons, but this thesis focuses on problems related to immature leg bones, specifically the tibia.

Suboptimal leg health leads to difficulties in accessing water and feed (Morris, 1993; Bradshaw et al., 2002; Bessei, 2006; Knowles et al., 2008; González-Cerón et al., 2015), pain (Bessei, 2006; Gocsik et al., 2017), poor welfare (Mckay et al., 2000; Bradshaw et al., 2002; EFSA, 2010; Dawkins and Layton 2012; Gocsik et al., 2017), unwillingness for walking, dustbathing, foraging and preening (Vestergaard and 
Sanotra, 1999; Weeks et al., 2000; Waldenstedt, 2006) and significant economic losses, because of higher mortality, lower slaughter revenues, culling, poor performance and carcass condemnation (Sullivan, 1994; Kestin et al., 1999, 2001: Bradshaw et al., 2002; Mench, 2004; Knowles et al., 2008; EFSA, 2010; Grandin, 2010; Gocsik et al., 2017; Rios et al., 2020).

Due to these welfare and economic consequences, enhancing leg health of broiler chickens has become important. In General Introduction, firstly an overview on suboptimal leg health, leg bone abnormalities and leg disorders in broiler chickens and their welfare and economic consequences will be described. Thereafter, attention will be drawn on bone quality measurements and potential approaches to improve leg health in broiler chickens. The aim and the outline of this thesis are given at the end of General Introduction.

\section{SUBOPTIMAL LEG HEALTH IN BROILER CHICKENS}

Suboptimal leg health is a major welfare issue in broiler chickens and causes considerable economic losses since lame chickens have difficulties accessing feed and water, suffer from dehydration and starvation, or even die, especially in the last weeks of rearing period (Bradshaw et al., 2002; Knowles et al., 2008; Dinev, 2012; Tallentire et al., 2016; Gocsik et al., 2017). Factors affecting leg health in broiler chickens are given in Diagram 1.

In the upcoming sections, firstly leg bone abnormalities and disorders will be described. Thereafter, attention will be drawn on welfare and economic consequences of suboptimal leg health.

\section{Leg Bone Abnormalities and Disorders}

There are several degenerative, developmental and infectious leg bone abnormalities and disorders observed in broiler chickens. Occasionally, it can be difficult to classify these abnormalities and disorders because of their multifactorial causation (Bradshaw et al., 2002). In this thesis, varus valgus deformation (VV), tibia dyschondroplasia (TD), food pad dermatitis (FPD), bacterial chondronecrosis with osteomyelitis (BCO), epiphyseal plate abnormalities (EPA) and epiphysiolysis (EPI) are of particular interest, because (1) these are commonly observed leg bone abnormali- 


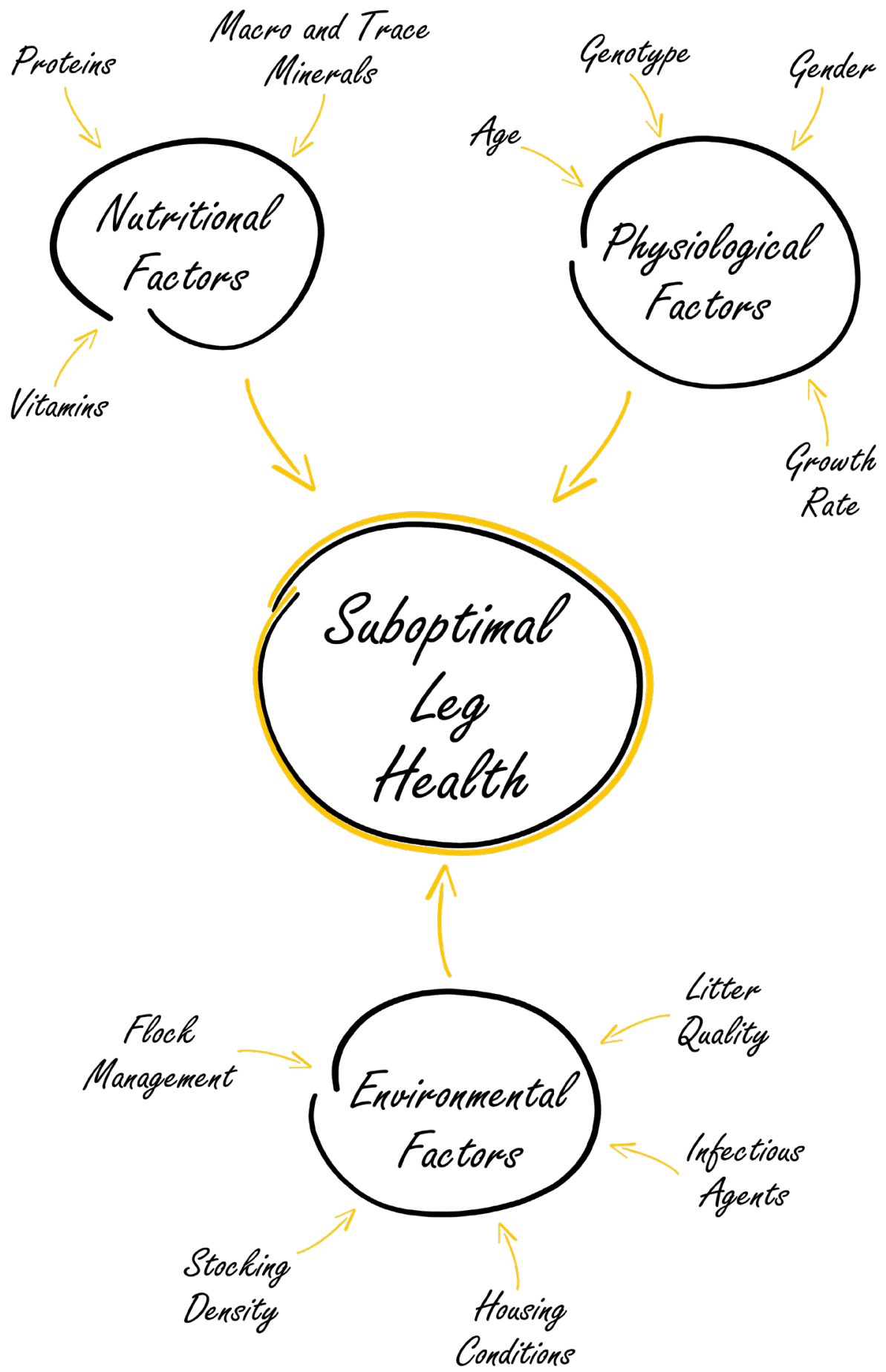

Diagram 1

Factors affecting leg health in broiler chickens

13 
ties and disorders and (2) these can be improved through optimized leg bone development.

\section{Varus valgus deformation}

Varus valgus deformation (VV) is defined as an abnormal angulation of the distal tibia at the intertarsal joint in broiler chickens (Randall and Mills, 1981; Julian, 1984; Riddell and Springer, 1985; Duff and Thorp, 1985; Shim et al., 2012). Varus is defined as inward angulation, whereas valgus is defined as outward angulation of the tibia (Figure 1) (Julian, 1984; Shim et al., 2012). Valgus deformation is more common than varus deformation and legs can differ in VV, e.g., one leg can show varus, whereas the other leg can show valgus (Julian, 1984).

$\mathrm{VV}$ is one of the common bone abnormalities and disorders in broiler chickens which is mostly associated with growth rate (Julian, 1984, 2005; Riddell, 1992; Guo et al, 2019). Despite the prevalence of VV in broiler chickens have been reduced in recent recent years compared to past, it is still ranging from $2 \%$ to $5 \%$ worldwide (Julian, 1984; Cruickshank and Sim, 1986; Newbrey et al., 1988; Bessei, 2006; Guo et al., 2019).

The potential reason of $\mathrm{VV}$ is the heavy body weight related load pressure on immature joints and bones, which causes a disproportionally growing epiphyseal growth plate, finally leading to angulation of the distal tibia at the intertarsal joint (Bradshaw et al., 2002). In severe cases, angulation might lead to fracture of the tibia and separation of the condyle from the tibial shaft, which can be followed, in some cases, by penetration of the skin by the distal tibia shaft (Randall and Mills, 1981) and/or a dislocation of the intertarsal joint (Leterrier and Nys, 1992).

Broiler chickens having $V V$ may suffer from pain that negatively affects their welfare and natural locomotion-related behaviours, such as accessing water and feed, which leads to weight loss or even death, especially in the last weeks of the rearing period (Newbrey et al., 1988; Bessei, 2006). Fast-growing broiler chickens, which reach the slaughter weight from $50 \mathrm{~g}$ to $3100 \mathrm{~g}$ in 42 days, show more developmental bone abnormalities, such as VV, which is mostly seen towards the end of the growth period, compared to slower-growing broiler chickens (Randall and Mills, 1981; 
Julian, 1984; Fleming, 2008). The potential reason is irregular and poor vascular morphology of the epiphyseal growth plate and insufficiently mineralized bones in fast-growing chickens (Thorp, 1994; Shim et al., 2012). Slower-growing chickens, on the contrary, have more time for bone mineralization, which compensates the lack of mineralization in the early growth phase. This may reduce load stress on the skeleton (Shim et al. 2012; Sanchez-Rodriguez et al. 2019) and eventually result in a low incidence of VV.

Regarding gender, the gender distribution of chickens having VV were found as $70 \%$ males and $30 \%$ females (Riddell and Springer, 1985), which can be explained by a higher body weight and growth rate in male broiler chickens compared to females both in fast and slower-growing broiler chickens (Mulder et al., 2009; Demuner et al., 2017; Kryeziv et al., 2018).

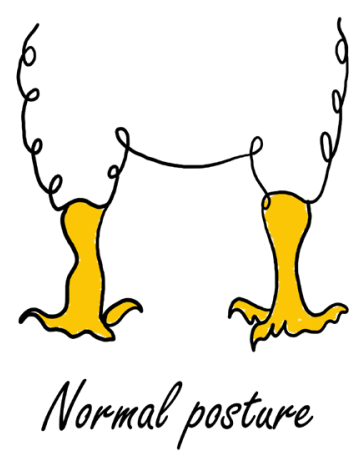

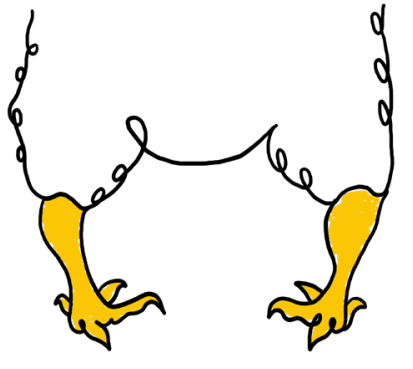

Varus

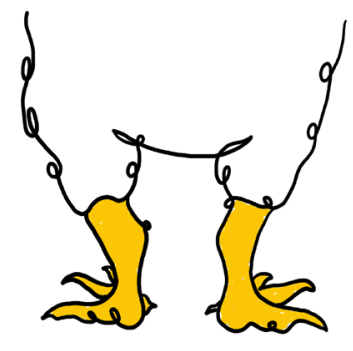

Valgus

Figure 1

Illustration of a broiler chicken with normal posture, varus deformation and valgus deformation in both legs

\section{Tibia Dyschondroplasia}

Tibia dyschondroplasia (TD) is described as an abnormal accumulation of cartilage from the epiphyseal growth plate by maturation of chondrocytes that fail to progress into the next stage. Then, the tissue becomes vascularized and calcified around bone matrix (Hargest et al., 1984; Farquharson and Jefferies, 2000; Leach and Monsonego-Ornan, 2007; Crespo, 2020). Consequently, cartilage mass increases under epiphyseal growth plate (Lowther et al., 1974; Farquharson and Jefferies, 2000; Leach and Monsonego-Ornan, 2007) and finally impair 
bone formation and mineralization (Figure 2) (Sheridan et al., 1978; Farquharson et al., 1992; Lynch et al., 1992; Rath et al., 1994; Ling et al., 2000; Reddi, 2000).

TD causes fractures, which results in abnormal tibia angle, which leads to bone deformities (Capps, 1998; Lynch et al., 1992). It has shown that TD leads to loss of bone strength by causing severe bone weakness, fractures and deformity (Leach Jr and Nesheim, 1972; Lynch et al., 1992; Wong-Valle et al., 1993; Julian 2005) and finally severe pain and unwillingness to walk (Farquharson and Jefferies, 2000; Sanotra et al., 2001).

According to global reports in early 2010 s, approximately $30 \%$ of broiler flocks are affected by TD worldwide, which negatively affects the welfare of the broiler chickens and causes financial losses in global broiler industry (Genin et al., 2012; Pelicia et al., 2012; Shahzad et al., 2015). The high growth rate of broiler chickens is one of the reasons for TD, but not a major factor, only contributes to other reasons e.g., acid/base balance in the epiphyseal growth plate, trace mineral deficiencies in feed and impaired calcium and phosphorus metabolism (Elliot and Edwards, 1994; Praul et al., 2000; Ledwaba and Roberson 2003; Rath et al., 2007; Kapell et al., 2017).

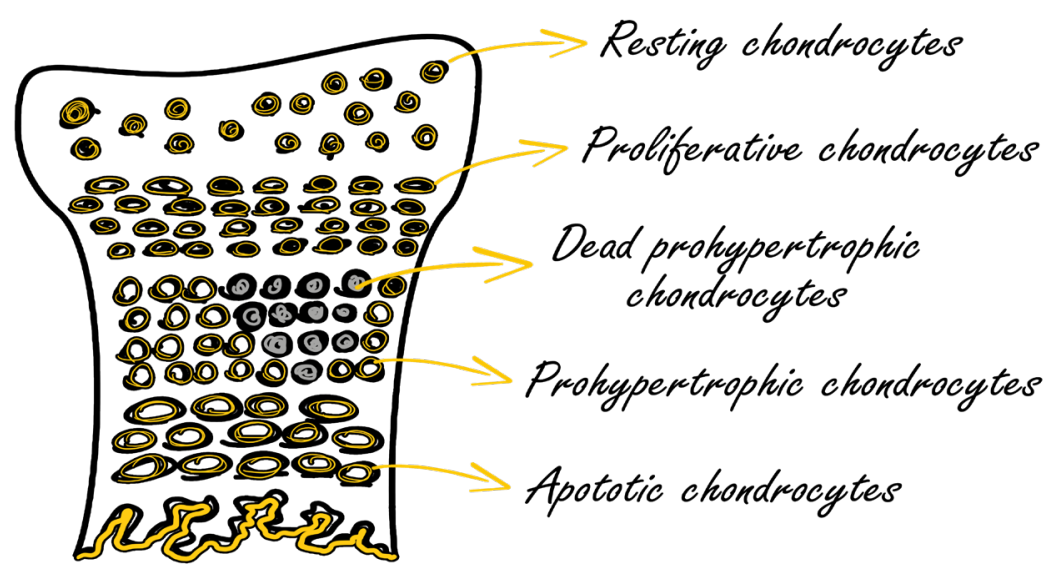

Figure 2

Illustration of bone chondrocytes and tibia dyschondroplasia 


\section{Bacterial chondronecrosis with osteomyelitis}

Bacterial chondronecrosis with osteomyelitis $(\mathrm{BCO})$ is another common leg disorder observed in broiler chickens and is described as necrosis combined with a bacterial infection, mostly occurring in the proximal heads of long bones (McNamee and Smyth, 2000; Wideman and Pevzner, 2012; Wide-

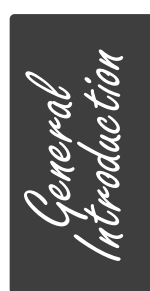
man and Prisby, 2013; Kittelsen et al., 2015; Wideman, 2016). The prevalence has rapidly increased in last few decades and reached over $1 \%$ worldwide (MCNamee et al., 1998; Butterworth, 1999; Bradshaw et al., 2002; Dinev, 2009; Stalker et al., 2010; Wideman and Prisby, 2013).

Despite the fact that $\mathrm{BCO}$ is known as an infectious bone disorder, clinical signs have shown that suboptimal bone development can be a major reason of $\mathrm{BCO}$, because impaired development and vascularization of the epiphyseal growth plate allows more bacteria to enter into the cartilage matrix and bone (Dinev, 2009; Stalker et al., 2010; Wideman and Pevzner, 2012; Wideman and Prisby, 2013; Kittelsen et al., 2015).

\section{Epiphyseal plate abnormalities and epiphysiolysis}

Epiphyseal plate abnormalities are described as malformations in the epiphyseal plate e.g., abnormal length, abnormal thickness and vascularization in cartilage. Epiphysiolysis, which is mostly accompanied with epiphyseal plate abnormalities, is described as separation between the bone head and the articular cartilage that leads to a vulnerable bone. The main reasons of epiphyseal plate abnormalities and epiphysiolysis can be non-infectious e.g., blockage of blood vessels and cartilage degeneration and disorganization, but also infectious e.g., necrotic chondrocytes, or can be both non-infectious and infectious simultaneously (Thorp et al., 1993; Packialakshmi et al., 2015; De Oliveira Peixoto et al., 2019).

\section{Foot pad dermatitis}

Foot pad dermatitis (FPD) is a degenerative and inflammatory disease on the plantarsurface of the footpads and commonly observed in fast-growing broiler chickens (Shepherd and Fairchild, 2010; De Jong etal., 2012, 2015) (Figure 3). FPD presents itself as brown and black coloured lesions on the foot skin with inflammation and necrosis (Greene et al., 1985) and is an important problem 
that negatively affects the welfare, health, locomotion, growth performance and carcass quality of broiler chickens (Algers and Berg, 2001; Allain et al., 2009; Hashimato et al., 2013; Saraiva et al., 2016). The prevalence of FPD and its financial impact show a high variability from $0 \%$ to $90 \%$ worldwide (Kjaer et al., 2006; Kristensen et al., 2006; Pagazaurtundua and Warriss, 2006; Haslam et al., 2007; Zikic et al., 2017) because of its dependency on many factors e.g., leg health, litter type, litter quality, management factors, stocking density, gender, age, genotype, nutritional factors, diseases and lighting program (Mayne, 2005, Meluzzi et al., 2008; De Jong et al., 2012, 2015; Taira et al., 2014). In the Netherlands, 65\% of broiler chickens has been reported to suffer from FPD, while almost $38 \%$ of those chickens have severe footpad lesions (De Jong et al., 2012).

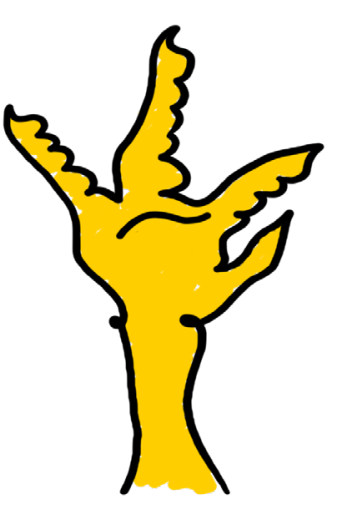

Normal foot pad

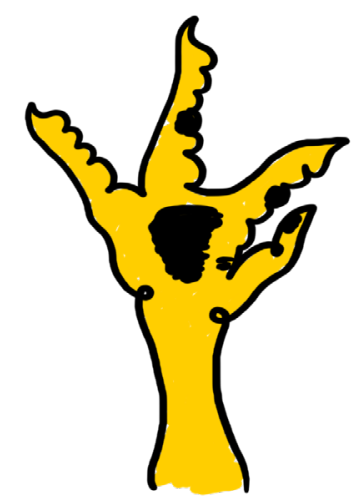

FPD

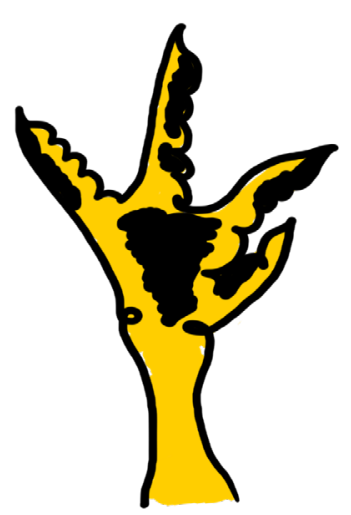

Severe FPD

Figure 3

Illustration of normal food pad, food pad dermatitis and severe food pad dermatitis

In this thesis, varus valgus deformation (VV) (Chapter 1, 3 and 5), tibia dyschondroplasia (TD) (Chapter 3, 4 and 5), food pad dermatitis (FPD), bacterial chondronecrosis with osteomyelitis (BCO), epiphyseal plate abnormalities (EPA) and epiphysiolysis (EPI) (Chapter 2, 3, 4 and 5) will be assessed. Additionally, rotated tibia (RT) and hock burn (HB) will be investigated as well in Chapter 4. Rotated tibia is defined as lateral rotation of the distal tibia on its long axis. Hock burn is defined as burn marks on the leg skin due to sitting and lying on litter containing ammonia. 


\section{Welfare Consequences of Suboptimal Leg Health}

Suboptimal leg health leads to inactivity which negatively affects the welfare of broiler chickens. Because of fast growth and immature bones, they show a tendency to spend most of their time sitting and/or lying, which result in deformation of leg bones (Shim et al., 2012; Sanchez-Rodriguez

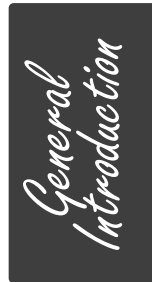
et al. 2019; Hartcher and Lum, 2020), pain (Bessei, 2006; Gocsik et al., 2017), impaired natural behaviours such as dustbathing, foraging and preening (Weeks et al., 2000; Vestergaard and Sanotra, 1999; Waldenstedt, 2006), gait abnormalities (Kestin et al., 1992; Weeks et al., 2000), infectious and/or developmental disorders, as explained in previous section (Wideman and Prisby, 2013; De Jong et al., 2015; Demuner et al., 2017; Kryeziv et al., 2018) and, in extreme cases, broken bones and punctured skin by broken bone (Bradshaw et al., 2002; Bessei, 2006, 2018). Before chickens slaughtered, especially when handling the chickens during depopulation and/or when transporting them to the slaughter plant, immature bones may cause bone breakage (mostly in legs and wings), damaged muscles and punctured skins, which all cause severe pain and poor welfare (Knowles and Wilkins, 1998; Allain et al., 2009; Kittelsen et al., 2015, 2018; Jacobs et al., 2017; Hartcher and Lum, 2020). Improving leg health of broiler chickens may reduce the prevalence of all of these welfare related problems.

\section{Economic Consequences of Suboptimal Leg Health}

Suboptimal leg health, besides its welfare consequences, is also a major economic problem in the broiler industry nowadays. It causes serious financial losses by reducing profit ranging from 10 to $40 \%$ due to high mortality (Sullivian, 1994; Weeks et al., 2000; Wideman et al., 2012), low slaughter revenues (Bradshaw et al., 2002; Jones at al., 2019), downgrading meat quality and increased rejections at slaughter plants, because of bone breakage, damaged muscles, punctured skins and carcass condemnations (Mench, 2004; Venäläinen et al., 2006; Grandin, 2010; Gocsik et al., 2017; Jacobs et al., 2017; Cockram et al., 2018, 2020; Jones at al., 2019; Hartcher and Lum, 2020), and more contamination as a result of contacting wet litter, which threats the food safety (Bradshaw et al., 2002; Bessei, 2006; Knowles et al. 2008). In addition to increased welfare, financial losses might be reduced by improving leg health of broiler chickens. 


\section{BONE QUALITY MEASUREMENTS}

Bone is a rigid tissue which has many functions e.g., providing a rigid skeleton, protecting vital organs, allowing locomotion and storing calcium to regulate mineral homeostasis (Romanoff, 1960; Patt and Maloney, 1975; Cullinane and Einhorn, 2002), which are all vital for health and welfare of broiler chickens. Most of the leg bone health related research in broiler chickens focused on tibia bone (Ruff and Hughes, 1985; Kim et al., 2006), since the tibia is located medially between the femur and metatarsus and is the most loaded and affected leg bone during the gowth period, by e.g., heavy load pressure, infectious and/or non-infectious bone disorders, directly influencing locomotion of the broiler chickens (Nairn and Watson, 1972; Julian, 1998, 2005; Dibner et al., 2007). During the growth period, leg bones of broiler chickens must adapt and modify their morphological, biophysical and mechanical properties to successfully withstand effects of their rapidly increasing body weight (Yair et al., 2012).

In this thesis, tibia morphological characteristics (e.g., weight, proximal length, lateral cortex thickness, femoral and metatarsal side proximal head thicknesses and robusticity index), biophysical characteristics (e.g., osseous volume, pore volume, total volume, volume fraction, mineral content and mineral density) and mechanical characteristics (e.g., ultimate strength, yield strength, stiffness, energy to fracture and elastic modulus) are assessed in all chapters, due to strong relationship between tibia bone quality and leg health.

\section{Morphological Characteristics}

Bone growth occurs in two directions, (1) interstitial (vertical) growth is the growing in length and (2) appositional (horizontal) growth is increasing in thickness (Owen and Friedenstein, 1988; Anderson, 1989; Beresford, 1989; Roach, 1997: Delaissé et al., 2000; Cancedda, 2009). Because of this growth pattern, bone proximal length and lateral cortex thickness as morphological characteristics have been used as indicators of bone quality (Tatara et al., 2011, 2015, Krupski and Tatara, 2007; Charuta et al., 2011,2013; Muszyński et al., 20 18; Harash et al., 2020).

Proximal length is the longitudinal length $(\mathrm{cm})$, cortex thickness is the width of the bone center $(\mathrm{cm})$. Tibia weight, femoral and metatarsal 
side proximal head thicknesses and robusticity index are other common morphological characteristics. Tibia weight is the weight $(\mathrm{g})$ of defleshed tibia bone. Femoral and metatarsal head thicknesses are the widest distances on proximal bone heads (cm). Robusticity index is described as how robust the bone is and calculated by dividing the tibia weight by tibia length ( $\mathrm{g} / \mathrm{cm})$ (Reisenfeld, 1972).

\section{Biophysical Characteristics}

Broiler chickens show fast bone formation, mineralization and remodeling due to the genetic selection for high growth rate (Bain and Watkins, 1993; Yair et al., 2012; Wideman et al., 2012; Johnsson et al., 2015). Throughout the growing phase, a rapid development occurs in tibia (Williams et al., 2000; Yalçın et al., 2001; Applegate and Lilburn, 2002). Bone mineralization is described as a process by which the organic bone matrix is filled with calcium phosphate, which starts with growing and reorganizing small particles and ends with a stable mineralized bone (Litchfield et al., 1998; Vallet-Regi et al., 2004).

Osseous volume, pore volume (Prisby et al., 2014; Muszyński et al., 2018; Harash et al., 2020), mineral content (Akpe et al., 1987; Onyango et al., 2003; Nyman et al., 2005; Kim et al., 2006; Muszyński et al., 2018) and mineral density (Watkins and Southern, 1992; Rath et al., 2000; Onyango et al., 2003; Almeida and Bruno, 2006; Kim et al., 2006; Shim et al., 2012) are the most important biophysical characteristics to assess bone quality in poultry.

Osseous volume is the volume of stiff bone $\left(\mathrm{cm}^{3}\right)$, pore volume is the volume of empty spaces inside bone $\left(\mathrm{cm}^{3}\right)$. Bone mineral content is total bone mineral weight of bone (g) and measured by determining the amount of minerals in the bone. Bone mineral density is a total mineral weight per volume of the bone $\left(\mathrm{g} / \mathrm{cm}^{3}\right.$ ) (Boivin and Meunier 2002; Yair et al., 2017; Harash et al., 2020) and determined by two factors: (1) how many mineral atoms are deposited in the osseous bone and (2) how porous the bone is (Sanchez et al, 1981; Akpe et al, 1987; Clarke, 2008; Muszyński et al., 2018). Volume fraction is another biophysical parameter and described as proportion of total bone volume containing osseus bone and calculated by dividing the osseous volume by the total volume (osseous volume + pore volume). All bone biophysical characteristics can be measured by using dual-energy X-ray absorptiometry or X-ray computed tomography. 


\section{Mechanical Characteristics}

Ultimate strength, yield strength, stiffness and energy to fracture are the most important mechanical characteristics to assess bone quality in poultry (Merkley, 1981; Ruff and Hughes, 1985; Boskey et al., 1999; Park et al., 2003; Kim et al., 2006) and determined by the bone osseous volume, pore volume and the degree of mineralization of the bone matrix (Orban et al., 1993; Boivin and Meunier, 2002; Barber, 2018). Bone mechanical characteristics can be measured by using a three-point bending test (Merkley, 1981; Ruff and Hughes, 1985; Park et al., 2003; Kim et al., 2006; Jungmann et al., 2007). Ultimate strength is the reached maximal load at the breaking point (N). Yield strength is the reached yield load just before the angle changed on slope (inflection point) (N). Stiffness is the angular slope of the selected linear part of the curve $(\mathrm{N} / \mathrm{mm})$. Energy to fracture is the total area under the curve of the selected region (N-mm). Elastic modulus is described as the amount of strain as a result of a particular amount of stress on bone (GPa) (Turner and Burr, 1993; Novitskaya et al., 2011) and calculated by using the following formula (Turner and Burr, 1993):

$$
E=\frac{N S^{3}}{4 \delta T L^{3}}
$$

where $\mathrm{N}$ is the maximal load $(\mathrm{N}), \mathrm{S}$ is the span between the bending fixtures $(\mathrm{mm}), T$ is the tibia thickness $(\mathrm{mm}), L$ is the tibia length $(\mathrm{mm})$ and $\delta$ is the maximum deflection $(\mathrm{mm})$ at the midpoint of the bone. 
Femoral side proximal head thickness

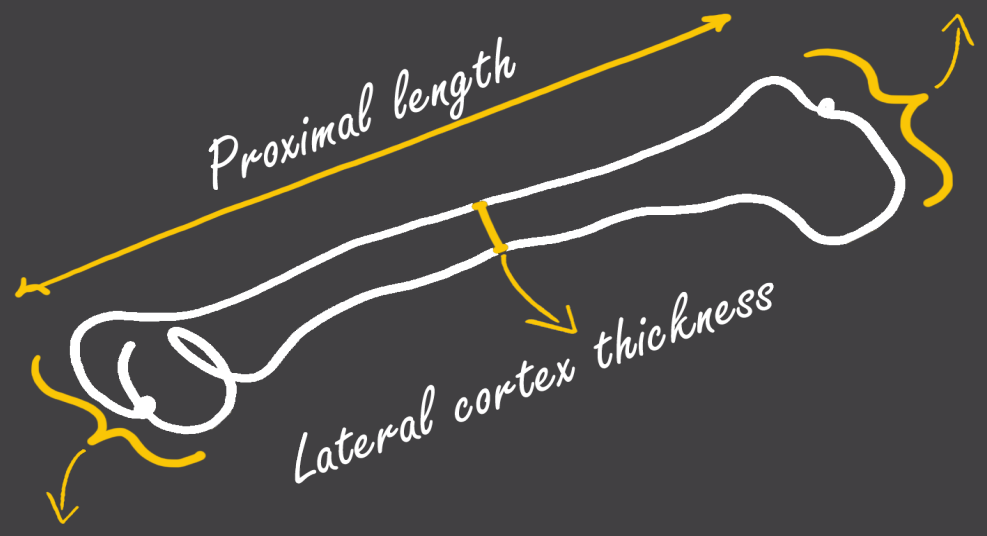

Metatarsal side proximal head thickness

Tibia Morphological Characteristics

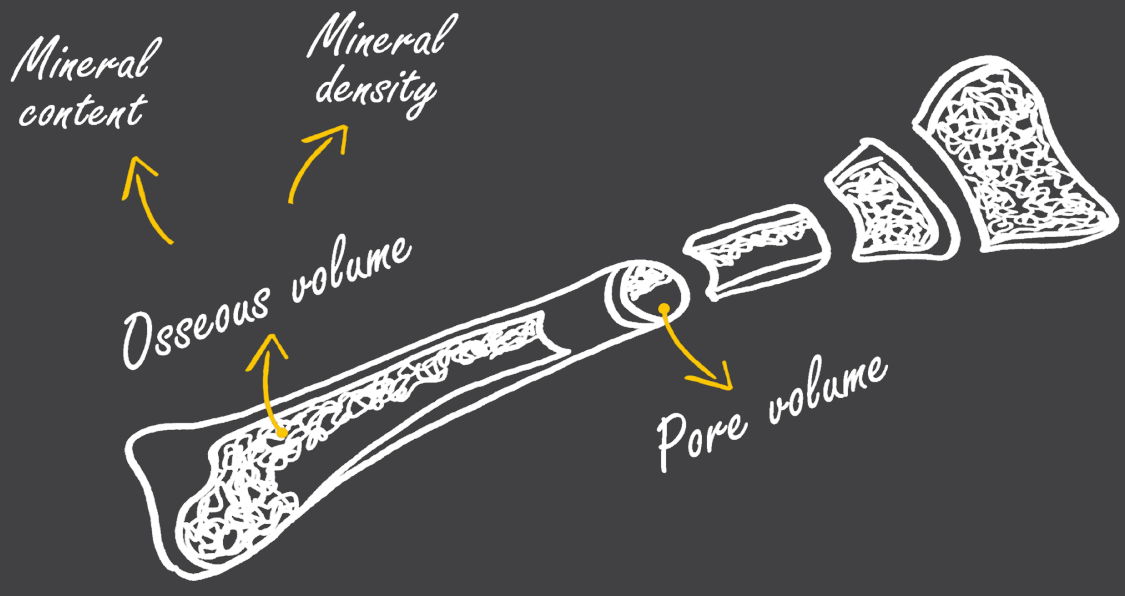

Tibia Biophysical Characteristics 
Three-point bending test
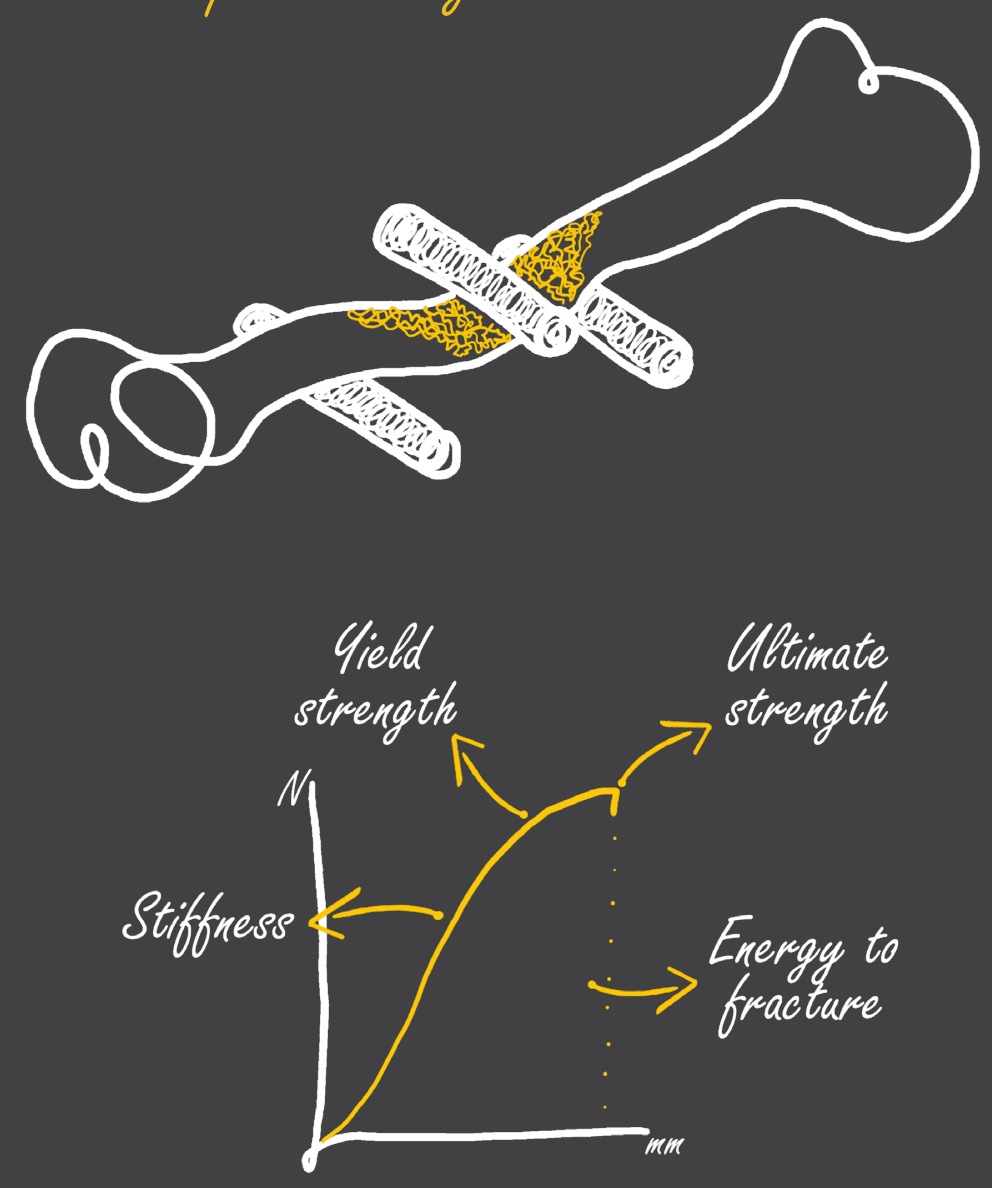

Tibia Mechanical Characteristics 


\section{POTENTIAL APPROACHES \\ TO IMPROVE LEG HEALTH}

There have been many studies aimed to enhance leg health in broiler chickens since suboptimal leg health became a major welfare issue and caused considerable economic losses. In this thesis, the potential approaches to improve leg health of broiler chickens were determined as use of slower-growing broiler chickens, effects of dietary macro and trace minerals, incubation conditions and environmental enrichment.

\section{Fast-growing vs. Slower-growing Broiler Chickens}

The broiler chicken is one of the fastest growing poultry species in the world as a result of an intense genetic selection for high growth rate and feed efficiency (Havenstein et al., 2003; Tona et al., 2004; Siegel et al., 2009; Vieira and Angel, 2012; Zuidh of et al., 2014; Tallentire et al., 2016; Bessei, 2018), which has resulted in an increase in body weight gain by approximately $400 \%$ in the last several decades (Bradshaw et al., 2002; Knowles et al. 2008; Petracci and Cavani, 2012; Zuidhof et al., 2014; Tallentire et al., 2016). The skeleton of the broiler chicken is significantly larger than their wild progenitor. The biggest change has been observed in lower limb bones, particularly the tibia bone, which shows a tripling in thickness and a doubling in length (Bell, 2002; Tickle et al., 2014; Bennett et al., 2018). However, this dimensional improvement in tibia has not been sufficient enough to respond to a rapidly increasing body mass.

It has been well documented that fast-growing broiler chickens have shown more leg and locomotion problems than slower-growing broiler chickens (Williams et al., 2000; Sullivian 1994; Lilburn 1994; Thorp and Waddington, 1997; Torres and Korver, 2018), mainly because fast-growing chickens have more porous and less mineralized leg bones than slower-growing broiler chickens, which results in a higher risk of lameness (Sullivian, 1994; Williams et al., 2000; Williams et al., 2004; Stojcic and Bessei, 2009; Shim et al., 2012; Torres and Korver., 2018), impaired locomotion (Lewis et al., 1997; Reiter and Bessei, 1998; Weeks et al., 2000; Cornetto and Estevez, 2001; Bokkers, 2004; Siegel et al., 2009) and more leg disorders (Bokkers, 2004; Kjaer et al., 2006).

The growth rate also differs between current strains. Fast-growing chickens reach approximately 2500 to $3100 \mathrm{~g}$ slaughter weight in approx- 
imately 38 to 42 days, while slower-growing chickens reach the same body weight in approximately 56 to 60 days (Quentin et al., 2004; Grashorn, 2006; Kokoszyński et al., 2017). Despite many consumers prefer low priced fast-growing chickens, more and more people have concerns regarding animal welfare and product quality, which makes them to pay attention to an additional value when making choices about their purchases (Fanatico ef al., 2005; Castellini et al., 2008; Cooper and Wrathall, 2010; Davies, 2019). Aiming at better welfare and due to sensibilities of consumers, slower-growing chickens rank as nearly $24 \%$ of French and $11 \%$ of British broiler production nowadays (Davies, 2019).

In the Netherlands, the broiler production has been changed in this manner starting from 2014. Slower-growing chickens have been shown a progressive trend since 'Beter Leven' (Better life) project was introduced in 2007, which was followed by a project called 'Kip van Morgen' (Chicken of tomorrow) (saatkamp et al., 2019). In 2019, slower-growing chickens rank as around $35-40 \%$ of broiler production in the Netherlands (Avined, 2019; Davies, 2019). Consequently, the use of slower-growing broiler chickens has become prominent, since they show better compatibility to higher welfare. It is anticipated that this welfare approach will play an important role in future broiler chicken production (Lusk et al., 2019).

In Chapter $\mathbf{3}$ and Chapter $\mathbf{5}$ of this thesis, differences between fast and slower-growing broiler (or broiler breeder) chickens on leg health parameters of broiler chickens (or offspring broilers) are investigated.

\section{Dietary Macro and Trace Minerals}

The most promising nutritional approaches to improve bone health of broiler chickens are macro and trace minerals. Amount and chemical form of macro minerals (calcium and phosphorus) (Driver et al., 2005; Létourneau-Montminy et al., 2008) and trace minerals (iron, copper, manganese, zinc and selenium) (Richards, 1997; Angel, 2007; Dibner et al., 2007; Wang et al., 2019) in the diet play important roles on bone development in broiler chickens, since these minerals are main contributors to bone formation and mineralization. A deficiency of any of these minerals might result in retarded bone growth, strength, morphology and density (Dibner et al., 2007; Létourneau-Montminy et al., 2008; Li et al., 2016, 2020). 
Low or high concentrations of inorganic minerals rather than nutritional requirements in broiler diets have been investigated in several studies. Deficiency of macro and trace minerals in the diet of broiler chickens resulted in lower bone mineral content and lower bone breaking strength (Bar et al., 2003; Sá et al., 2004; McDevitt et al., 2006) and increasing the amount of inorganic minerals in broiler diets have been found to stimulate bone mineralization and resulted in a stronger bone (Driver et al., 2005; L'etourneauMontminy et al., 2008). However, a large amount of inorganic minerals that exceeds the nutritional requirements, may negatively affect each other's bioavailabilities and may cause toxic effects in the chicken and also to the environment (Witkowska et al., 2019). Increasing the amount of inorganic minerals in broiler chickens diet resulted in lower bioavailability and higher mineral levels in manure, which largely exceeds the nutrient requirements of crops (Dozier lii et al., 2003). Consequently, it can be questioned whether or not increasing the amount of inorganic minerals is desired, because of the potential risk of low bioavailability and waste of minerals into the environment (Dozier lii et al., 2003; Bao et al., 2007; Witkowska et al., 2019).

Alternatively, the use of organic minerals might be a more beneficial and sustainable solution (Chang et al., 2016). Compared to inorganic minerals, organic minerals have a higher bioavailability and a higher intestinal absorption capability (Guo et al., 2001; Bao et al., 2007; Huang et al., 2009). This is due to a higher chemical stability and more resistance to changes in $\mathrm{pH}$ throughout the digestive system (Wedekind et al., 1991; Burrell et al., 2004; Selle et al., 2009; Wang et al., 2019). Organic minerals are bound to a carbon complex, often proteins or amino acids, with covalent bonds that have higher binding strength compared to electrovalent bonds observed in inorganic minerals. These strong bonds protect these minerals from binding with other compounds in the chickens' gut (Van der Klis and Kemme, 2002; Bao and Choct, 2009; Gupta et al., 2015) and result in lower antagonistic complex forming with minerals or other diet ingredients (Vieira, 2008; Bao and Choct, 2009). Inorganic minerals form insoluble complexes, which negatively affects gastrointestinal digestion. It has been shown that growth performance, digestibility of minerals and bone mineralization were positively affected when an organic trace mineral premix was used in a diet of broiler chickens compared to an inorganic trace minerals premix (M'sadeq et al., 2018). These 
differences in digestibility can be explained by the fact that organic minerals are easily bound to carbohydrates, fats, proteins and amino acids, which makes them easier to digest (National Research Council, 1994).

The gastro-intestinal $\mathrm{pH}$ has substantial effect on digestion of both macro and trace minerals. Maintaining low pH in the upper gastrointestinal is crucial for better digestion, because a low pH breaks the electrovalent bonds and releases the free ions. Then, soluble ions can be taken up in the small intestine, large intestine and/or caeca (Bao and Choct, 2009).

Regarding macro minerals, it has been observed that limestone, the most used source of inorganic $\mathrm{Ca}$ in broiler and broiler breeder diets, increases the $\mathrm{pH}$ of the small intestine, because of alkaline properties of $\mathrm{CaCO}_{3}$, which negatively affects the digestion and hereby reduces $\mathrm{P}$ and Ca availability (Denbow, 2015; Hamdi et al., 2015; Vieira et al., 2018). Phytate, the source of inorganic $\mathrm{P}$ in broiler and broiler breeder diets, is largely useless for avian species, because phytate binds with phytic acid $\left(\mathrm{C}_{6} \mathrm{H}_{18} \mathrm{O}_{24} \mathrm{P}_{6}\right)$ and form an insoluble and indigestible complex (Morgan et al., 2015; Scholey et al., 2018). Phytic acid contains a high number of protons on the surface, which creates a high electronegative charge in an environment and increases the $\mathrm{pH}$ of the small intestine, which negatively affects the digestion and reduces $\mathrm{P}$ and Ca availability (Denbow, 2015; Vieira et al., 2018).

Regarding trace minerals, broiler and broiler breeder diets are commonly supplemented with inorganic trace minerals, which are bound by weak ionic bonds to a metal-binding agent e.g., oxide and sulphate (Echigo and Kimata, 2010), whereas organic trace minerals consist of strong covalent bonds (Leeson, 2005) and are better absorbed and utilised than inorganic trace minerals, because they are protected from interactions with other components, which hamper each other's bioavailability (scott et al., 1969; Kratzer and Vohra, 1986; Leeson, 2003). It has been shown that serum trace mineral concentrations of broiler chickens were found higher by $22 \%$ for $\mathrm{Mn}, 17 \%$ for $\mathrm{Zn}$ and $20 \%$ for $\mathrm{Cu}$ when minerals were provided in their organic variety instead of inorganic variety (Yenice et al., 2015). In a recent study, digestibility of organic copper in the jejunum was $40.11 \%$ compared to $13.22 \%$ for inorganic copper. For organic $Z n$, the main digestibility difference was found in the ileum, where inorganic Zn digestibility was $9.08 \%$, while organic Zn was digested up to $21.23 \%$ (M'sadeq et al., 2018). 


\section{Macro Minerals}

Calcium ( $\mathrm{Ca}$ ) is the major macro mineral for the chicken skeleton and $99 \%$ of calcium in the body is stored in bones (Shim et al. 2012; Bello et al. 2014). Calcium is also an essential part of enzymes involved in bone development (McDevitt et al., 2006; De Matos, 2008) and also an essential part of bone development related hormones, e.g., GH, IGF-1, T3 and T4 (Rosol and Capen, 1997). Phosphorus (P) is the second major macro mineral for the chicken skeleton and $85 \%$ of phosphorus in the body is stored in bones (Klasing, 1998; Proszkowiec-Weglarz and Angel, 2013). P combines with Ca to form calcium phosphate crystals that builds the bone matrix (Rath et al., 1999; Rubin, 2001; Blake and Fogelman, 2002; Sá et al., 2004; Shim et al, 2012).

Ca and $\mathrm{P}$ are known to be essential for bone mineralization as main components for bone matrix and bone formation (Rath et al., 1999; Blake and Fogelman, 2002; Veum, 2010; Shao et al., 2019). Bone mineralization, as described before, is a process by which the organic bone matrix is filled with calcium phosphate, which starts with growing and reorganizing small particles and ends with a stable mineralized bone (Posner, 1969; Landis, 1996; Vallet-Regi et al., 2004). Ca and P levels in broiler chicken diets directly influence the bone mineralization and determine the strength of bones. Excessive or deficient level of calcium and/or phosphorus led to bone abnormalities (Onyango et al., 2003; Driver et al., 2005; Létourneau-Montminy et al., 2008; Li et al., 2017).

Regarding the source of macro minerals, $\mathrm{Ca}$ is generally sourced from inorganic limestone and supplied as calcium carbonate $\left(\mathrm{CaCO}_{3}\right)$ (Anwar et al., 2017). Inorganic phosphorus is sourced from phosphate rock (Li et al., 2016, 2017, 2020). Ca and P can also be supplied as mono-calcium phosphate or di-calcium phosphate, which is produced by adding sulfuric acid to rock phosphate, creating phosphoric acid, to which calcium carbonate $\left(\mathrm{CaCO}_{3}\right)$ is added after purification (Hamdi et al., 2017, 2018). Other sources of organic $\mathrm{Ca}$ and $\mathrm{P}$ are as follows: processed porcine and bovine bones, oyster shells and eggshells (Scott, 1969; Xu et al., 2020).

Organic sourced $\mathrm{Ca}$ in broiler diet resulted in higher tibia proximal length and lateral cortex thickness compared to inorganic sourced $\mathrm{Ca}$ (Guinotte et al., 1991). Organic sourced P has been found to positively affect the bone strength as a result of its higher bioavailability (Hemme et al., 2005). 


\section{Trace minerals}

Trace minerals play important roles in bone development (Richards, 1997). Copper (Cu), iron (Fe), zinc (Zn) and manganese (Mn) are involved in enzyme activities that are related to embryonic and post-hatch bone development (Dibner et al., 2007). Cu is an element that is part of the crosslinking of collagen and elastin, which gives bones their flexibility (Dibner et al., 2007). Zn is a structural component of carbonic anhydrase, which is essential for the function of osteoclasts (Flis et al., 2019; Richards et al., 2010). Zn is also an important part of alkaline phosphatase, which plays a key function in the formation of new bone (Tomaszewska et al., 2017). Mn is required for formation of extracellular bone matrix (Dibner et al., 2007). Fe is important for cross-linking of collagen, which gives the bone its breaking strength and elasticity (Dibner et al., 2007). Insufficient amount of available Cu and Fe resulted in lower bone strength, even if $\mathrm{Ca}$ and $\mathrm{P}$ levels were adequate (Medeiros et al., 1997). Fe is involved in many enzymes and it is an important component of enzymes that are involved in synthesizing bone collagen (Flis et al., 2019). A deficiency in Fe affects bone morphology, strength, and density (Medeiros et al., 2002). Selenium (Se) is important for the thyroid function, which is important for bone development, as one of the functions of thyroid hormones is to help regulate bone growth (Jianhua et al., 2000).

Regarding the source of trace minerals, organic trace minerals are bound to multiple oligo-peptides rather than to single amino acids, which make them better absorbed and utilized than inorganic trace minerals, because they are protected from interactions with other components (Scott et al., 1982; Kratzer and Vohra, 1986; Leeson, 2003). Organic trace minerals in broiler chickens' diet have been found to be more bioavailable than inorganic forms (Guo et al., 200 1; Bao et al., 2007; Huang et al., 2009), which resulted in higher bone breaking strength and better leg health (McDevitt et al., 2006; Dibner et al., 2007; Ferket et al., 2009; El-Husseiny et al., 2012; Manangi et al., 2012).

Lastly, trans-generational carry-over effect of both macro and trace minerals could be important as well. More available pre-packaged minerals in the egg might positively affect embryonic bone development and later life bone health (Kidd et al., 1992; Dibner et al., 2007). Research has shown that newly hatched chickens with a strong and well-formed skeleton might 
show better locomotion and have a lower prevalence of leg problems in later life (Torres and Korver, 2018).

Organic trace minerals in the broiler breeders' diet have already been shown to result in better embryonic bone mineralization than inorganic trace minerals (Torres and Korver, 2018). However, these carry-over effects from broiler breeders' diet to the offspring embryo are only shown for trace minerals and there is no study on a combination of macro and trace minerals or solely macro minerals. Furthermore, effects of organic minerals in the breeder diet on bone development and leg health of their offspring is hardly studied. Regarding the broiler strain, almost all studies on carry-over effects of minerals in broiler chickens were conducted in fast-growing broiler breeders and not in slower-growing broiler breeders.

Effects of organic macro and trace minerals in the diet of broiler chickens on their leg health related parameters are investigated in Chapter 1 (a combination of organic macro and trace minerals) and Chapter 2 (organic macro and trace minerals separately). In Chapter 1, besides organic macro and trace minerals, fish oil and hydrolyzed collagen in the diet of broiler chickens are investigated as well. Background information regarding these two nutrients is given in that chapter. In Chapter 3, effects of a combination of organic macro and trace minerals in the diet of fast and slower-growing broiler breeders on their offspring' leg health related parameters are investigated.

\section{Incubation Conditions}

The etiology of suboptimal bone development and bone abnormalities observed in broiler chickens seems to have their origin during incubation (Hammond et al., 2007; Yalçın et al., 2007), since the bone development, cartilage formation and bone mineralisation take place during incubation (Applegate and Lilburn, 2002; Hammond et al., 2007; Yalçın et al., 2007; Shim and Pesti, 201 1; Aygün and Narinç, 2016). Several environmental factors, e.g., temperature, light, humidity, ventilation and egg turning, are known to play important roles on the development of the chicken embryos in general (Decuypere et al., 1992, 200 1; Rozenboim et al., 2004; Molenaar et al., 2010; Özkan et al., 2012; Van der Pol et al., 2014; Oviedo-Rondón et al., 2008, 2009; Öznurlu et al., 2016). 


\section{Light during incubation}

In nature, avian embryos are exposed to daylight when the hen leaves her nest to eat and drink (Archer, 2017; Archer and Mench, 2017) and every time she rotates her eggs (Duncan et al., 1978; Archer et al., 2009; Archer and Mench, 2014, 2017). Looking at commercial hatcheries worldwide, eggs are mostly incubated under complete darkness (Archer et al., 2009; Tong et al., 2018), which may negatively affect some physiological mechanisms, including embryonic bone development and bone development-related hormones (Rozenboim et al., 2004; Özkan et al., 2012; Van der Pol et al., 2014, 2019; Huth and Archer, 2015; Archer and Mench, 2017), because chickens have a light-sensitive pineal gland under their skull, at the dorsal surface of their brain. The pineal gland plays a role in seasonal and diurnal rhythmicity by transforming photoperiodic information into output of melatonin (Aige-Gil and Murillo-Ferrol, 1992; Archer et al., 2009), a hormone influencing bone development in chickens (Archer et al., 2009; Huth and Archer, 2015; Archer and Mench, 2017). Light can pass through the eggshell during incubation (Shafey, 2004; Shafey et al., 2005) and it has been found to be an important factor related to embryonic muscular and skeletal development (Aige-Gil and Murillo-Ferrol, 1992; Archer et al., 2009; Van der Pol et al., 2014; Huth and Archer, 2015; Archer and Mench, 2017).

The colour of light during incubation has been found important as well, since chickens have special extraretinal photoreceptors in their eyes and brains (Lewis and Morris, 2000; Rozenboim et al., 2004; Archer, 2018) and they have an ability to see a large spectrum of colours, including ultraviolet light. They also show a high sensitivity to blue, green and red light (Prescott and Wathes, 1999; Archer et al., 2009; Huth and Archer, 2015). Previous studies have shown that white and red light during incubation resulted in improved chick quality compared to complete darkness (Archer, 2017; Yu et al., 2018) or blue light (Hluchý et al., 2012).

In Chapter 2 of this thesis, effects of green LED light during incubation on leg health related parameters of broiler chickens are investigated.

\section{Temperature during incubation}

Alterations in incubation temperature have been demonstrated to influence bone development and later life leg health. The optimum tem- 
perature for embryo development throughout incubation has been determined as $37.8^{\circ} \mathrm{C}$ (Decuypere and Michels, 1992; Lourens et al., 2005). However, a lower or higher temperature than this optimum might influence different physiological mechanisms (Wilson, 1990; French, 2009; Tona et al., 2005; Maatjens et al., 2017; Wijnen et al., 2020), including embryonic bone development (Oviedo-Rondón et al., 2008, 2009; Van der Pol et al., 2014; Öznurlu et al., 2016).

Bone development and growth plate differentiation of broiler chickens begin during incubation (Hammond et al., 2007, Oviedo-Rondón et al., 2008, 2009, Shim and Pesti, 2011, Van der Pol et al., 2014; Aygün and Narinç, 2016). In the first week of incubation, embryonic bone development starts with cartilage formation (Nakane and Tsudzuki, 1999; Atalgin and Kürtül, 2009), which is followed by a rapid increase in mineralization from the second week of incubation onwards (Oviedo-Rondón et al., 2008), which reaches the highest level just before hatch and continues a few days post-hatch (Applegate and Lilburn, 2002; Yalçın et al., 2007; Oviedo-Rondón et al., 2008). Temperature might play an important role in bone development throughout these phases. Consequently, developmental bone abnormalities and disorders might have their origin during incubation (Hammond et al., 2007; Yalçın et al., 2007).

A temperature of $38.0^{\circ} \mathrm{C}$ throughout incubation resulted in longer tibia in broiler chickens at slaughter age than incubation temperatures of $36.0^{\circ} \mathrm{C}$ and $37.0^{\circ} \mathrm{C}$ (Oviedo-Rondón et al., 2008). However, increasing the temperature to $39^{\circ} \mathrm{C}$ during the last 4 days of incubation resulted in impaired bone development compared to incubation temperature of $37.0^{\circ} \mathrm{C}$ (Oviedo-Rondón et al., 2009). Additionally, a high eggshell temperature (EST) of $38.6^{\circ} \mathrm{C}$ throughout incubation resulted in lower tibia, femur and metatarsus bone lengths at hatch than an EST of $36.9^{\circ} \mathrm{C}$ and $37.8^{\circ} \mathrm{C}$ (Van der Pol et al., 2014). An incubation temperature of $38.8^{\circ} \mathrm{C}$ from E10 onwards negatively affected tibia characteristics, including growth plate development, at different days before and at hatch compared to an incubation temperature of $37.8^{\circ} \mathrm{C}$ (Öznurlu et al., 2016). It has been suggested that an incubation temperature of $41.0^{\circ} \mathrm{C}$ from E8 to E14 compared to an incubation temperature of $37.8^{\circ} \mathrm{C}$ can stimulate bone development and particularly the ossification process (Aygün and Narinç, 2016).

In Chapter $\mathbf{4}$ of this thesis, effects of eggshell temperature pattern on leg health related parameters of broiler chickens are investigated. 


\section{Environmental Enrichment}

Another potential approach to stimulate activity and consequently leg health of broiler chickens is environmental enrichment (Reiter and Bessei, 2009; Blatchford et al., 2012; Ohara et al., 2015; Pedersen and Forkman, 2019). In nature, chickens use perches, platforms, ramps and elevated resting areas throughout their history, from wild ancestors to their modern generations (Sandilands et al., 2009; Kaukonen et al., 2016; Riber et al., 2018; Pedersen and Forkman, 2019). However, modern broiler chickens have been found to spend approximately $80 \%$ of their lifespan with passive behaviours (e.g., lying, sitting and resting) (Weeks et al., 2000; Reiter and Bessei, 2009; Zuidhof et al., 2014), which leads to suboptimal leg health (Reiter and Bessei, 1998, 2009; Balog et al., 1997; Bradshaw et al., 2002).

A lower stocking density (Martrenchar et al., 2000; Hall, 2001; Zhao et al., 2013; Pedersen and Forkman 2019), platforms and/or ramps (Kaukonen et al., 2016; Pedersen et al., 2020), well-designed perches (Ventura et al., 2012; Zhao et al., 2013; Kaukonen et al., 2017), large distance between feed and water (Bizeray et al., 2002; Reiter and Bessei 2009; Pedersen et al., 2020), different bedding materials (Toghyani et al., 2010; Riber et al., 2018) and worms/insects in bedding area (Pichova et al., 2016) resulted in lower prevalence of leg disorders and lower mortality rate.

Increasing physical activity and locomotion by pen enrichment may result in lower incidence of leg problems and stimulate tibia morphological, biophysical and mechanical properties (Reiter and Bessei, 2009; Yildiz et al., 2009; Buijs et al., 2012; Kaukonen et al., 2016; Pedersen et al., 20201.

Regarding the relationship between pen enrichment and growth rate, slower-growing broiler chickens spend more time on perches and platforms (Estevez et al., 2002; Bokkers and Koene, 2003; Norring et al., 2016; Wallenbeck et al., 2016), demonstrated better locomotion (Lewis et al., 1997; Reiter and Bessei, 1998; Weeks et al., 2000; Cornetto and Estevez, 2001; Bokkers and Koene, 2004), had less hock and leg problems (Bokkers and Koene, 2004; Kjaer et al., 2006; Zhao et al., 2013), and lower mortality (Havenstein et al., 1994) than fast-growing broiler chickens.

In Chapter $\mathbf{5}$ of this thesis, effects of environmental (pen) enrichment on leg health related parameters of broiler chickens are investigated. 


\section{AIM OF THE THESIS}

The aim of this thesis is to investigate several potential approaches e.g., source of dietary macro and trace minerals, incubation conditions and environmental enrichment in fast and slower-growing broiler chickens to improve leg health by focusing on tibia characteristics and all other leg health related parameters.

\section{OUTLINE OF THE THESIS}

Chapter 1 of this thesis describes effects of a combination of organic macro and trace minerals, fish oil and hydrolyzed collagen in the diet of fast-growing male broiler chickens on (1) growth performance, (2) tibia characteristics, (3) home pen behaviour, (4) leg disorders, (5) locomotion and (6) bone development related blood parameters at 28, 35 and 42 days of age.

Chapter 2 of this thesis describes effects of green light-emitting diode (LED) light during incubation and dietary macro mineral and trace mineral source separately during rearing of fast-growing male broiler chickens on (1) growth performance, (2) tibia characteristics (day 42), (3) home pen behaviour, (4) leg disorders and (5) locomotion. In this experiment, a $2 \times$ $2 \times 2$ factorial arrangement was applied with the following treatments: 1) light during incubation (green LED light or darkness), 2) macro mineral source during rearing (organic or inorganic $\mathrm{Ca}$ and $\mathrm{P}$ ) and 3) trace mineral source during rearing (organic or inorganic Fe, $\mathrm{CU}, \mathrm{Mn}, \mathrm{Zn}$ and $\mathrm{Se}$ ).

Chapter 3 of this thesis describes effects of a combination of organic macro and trace minerals in the diet of fast and slower-growing broiler breeders on (1) the amount of minerals in egg and hatchling, (2) growth performance, (3) tibia characteristics (at similar body weights), (4) home pen behaviour and (5) leg disorders of their male offspring. In this experiment, a $2 \times 2$ factorial arrangement was applied with the following treatments: 1) broiler breeder strain (slower or fast-growing), 2) macro and trace mineral source (organic or inorganic) in the broiler breeder diet.

Chapter 4 of this thesis describes effects of eggshell temperature pattern in week 2 and 3 of incubation of fast-growing male broiler chickens on (1) tibia characteristics (day 41 or 42), (2) leg disorders and (3) lo- 
comotion. In this experiment, a $2 \times 2$ factorial arrangement with 2 eggshell temperatures $\left(37.8^{\circ} \mathrm{C}\right.$ and $\left.38.9^{\circ} \mathrm{C}\right)$ from day 8 to 14 , and 2 eggshell temperatures $\left(36.7^{\circ} \mathrm{C}\right.$ and $\left.37.8^{\circ} \mathrm{C}\right)$ from day 15 till hatch was applied.

Chapter 5 of this thesis describes effects of pen enrichment consisting ramps, platforms, perches, large distance between feed and water and live Black Soldier fly larvae in the moss-peat dust bathing area on (1) growth performance, (2) tibia characteristics (at similar body weights), (3) home pen behaviour, (4) leg disorders and (5) locomotion of fast and slower-growing male broiler chickens. In this experiment, a $2 \times 2$ factorial arrangement was applied with the following treatments: 1) broiler strain (fast or slower-growing), 2) pen enrichment (enriched or non-enriched).

The General Discussion of this thesis summarizes main findings and conclusions from all experiments. How the investigated approaches individually or together give insight in and improve our understanding of leg health of broiler chickens will be discussed. This chapter ends with the main conclusions and future research opportunities. 


\section{REFERENCES}

Aige-Gil, V. and Murillo-Ferrol N. 1992. Effects of white light on the pineal gland of the chick embryo. Histology and histopathology. 7:1-6.

Akpe, M. P., P. E. Waibel, K. Larntz, A. L. Metz, S. L. Noll, and M. M. Walser. 1987. Phosphorus availability bioassay using bone ash and bone densitometry as response criteria. Poultry science. 66:713-720.

Algers, B. and Berg, C., 2001. Monitoring animal welfare on commercial broiler farms in Sweden. Acta Agriculturae Scandinavica, Section A-Animal Science. 51:88-92.

Allain, V., L. Mirabito, C. Arnould, M. Colas, S. Le Bouquin, C. Lupo, and V. Michel. 2009. Skin lesions in broiler chickens measured at the slaughterhouse: relationships between lesions and between their prevalence and rearing factors. British poultry science. 50:407-417.

Almeida Paz, I. C. L. and L. D. G. Bruno. 2006. Bone mineral density. Brazilian journal of poultry science. 8:69-73.

Anderson, H. C. 1989. Mechanism of mineral formation in bone. Laboratory investigation; a journal of technical methods and pathology. 60:320-330.

Angel, R. 2007. Metabolic disorders: limitations to growth of and mineral deposition into the broiler skeleton after hatch and potential implications for leg problems. Journal of applied poultry research. 16:138-149.

Anwar, M. N., V. Ravindran, P. C. H., Morel, G. Ravindran, and A. J. Cowieson. 2017. Effect of calcium source and particle size on the true ileal digestibility and total tract retention of calcium in broiler chickens. Animal feed science and technology. 224:39-45.

Applegate, T. J. and M. S. Lilburn. 2002. Growth of the femur and tibia of a commercial broiler line. Poultry science. 81:1289-1294.

Archer, G. S. 2017. Exposing broiler eggs to green, red and white light during incubation. Animal. 11:1203-1209.

Archer, G. S. and J. A. Mench. 2014. The effects of the duration and onset of light stimulation during incubation on the behavior, plasma melatonin levels, and productivity of broiler chickens. Journal of animal science. 92:1753-1758.

Archer, G. S., H. L. Shivaprasad, and J. A. Mench. 2009. Effect of providing light during incubation on the health, productivity, and behavior of broiler chickens. Poultry science. 88:29-37.

Archer, G.S. and Mench, J.A., 2017. Exposing avian embryos to light affects post-hatch anti-predator fear responses. Applied Animal Behaviour Science. 186, p80-84.

Archer, G.S., 2018. Color temperature of light-emitting diode lighting matters for optimum growth and welfare of broiler chickens. Animal. 12:1015-1021.

Atalgin, S. H. and I. Kürtül. 2009. A morphological study of skeletal development in turkey during the pre-hatching stage. Anatomia, histologia, embryologia. 38:23-30.

Aygün, A. and D. Narinç. 2016. The effects of thermal manipulations during embryogenesis of broiler chicks on growth of embryo and skeletal traits. In AIP conference proceedings. 1726:020015.

Bain, S. D. and B. A. Watkins. 1993. Local modulation of skeletal growth and bone modelling in poultry. Journal of nutrition. 123:317-322.

Balog, J.M., Bayyari, G.R., Rath, N.C., Huff, W.E. and Anthony, N.B., 1997. Effect of intermittent activity on broiler production parameters. Poultry Science. 76:6-12.

Bao, Y. M. and M. Choct. 2009. Trace mineral nutrition for broiler chickens and prospects of application of organically complexed trace minerals: a review. Animal production science. 49:269-282.

Bao, Y. M., M. Choct, P. A. lji, and K. Bruerton. 2007. Effect of organically complexed copper, iron, manganese, and zinc on broiler performance, mineral excretion, and accumulation in tissues. Journal of applied poultry research. 16:448-455.

Bar, A., Razaphkovsky, V., Vax, E. and Plavnik, I., 2003. Performance and bone development in broiler chickens given 25-hydroxycholecalciferol. British poultry science. 44:224-233.

Barber, J. 2018. Anatomy and Biology. In the Chicken. p23-52. 
Bell, D. D. 2002. Anatomy of the chicken. In commercial chicken meat and egg production. p41-58.

Bello, A., P. Y. Hester, P. D. Gerard, W. Zhai, and E. D. Peebles. 2014. Effects of commercial in ovo injection of 25-hydroxycholecalciferol on bone development and mineralization in male and female broilers. Poultry science 93:2734-2739.

Bennett, C. E., R. Thomas, M. Williams, J. Zalasiewicz, M. Edgeworth, H. Miller, B. Coles A. Foster, E. J. Burton, and U. Marume. 2018. The broiler chicken as a signal of a human reconfigured biosphere. Royal society open science. 5.180325:1-11.

Beresford, J. N. 1989. Osteogenic stem cells and the stromal system of bone and marrow. Clinical orthopaedics and related research. 240:270-280.

Bessei, W. 2006. Welfare of broilers: a review. World's poultry science journal. 62:455-466.

Bessei, W. 2018. Impact of animal welfare on worldwide poultry production. World's poultry science journal. 74:211-224.

Bizeray, D., I. Estevez, C. Leterrier, and J. M. Faure. 2002. Effects of increasing environmental complexity on the physical activity of broiler chickens. Applied animal behaviour science. 79:27-41.

Blake, G. M. and I. Fogelman. 2002. Methods and clinical issues in bone densitometry and quantitative ultrasonometry. In Principles of bone biology. p1573-1585.

Blatchford, R. A., G. S. Archer, and J. A. Mench. 2012. Contrast in light intensity, rather than day length, influences the behavior and health of broiler chickens. Poultry science. 91:1768-1774.

Boivin, G. and P. J. Meunier. 2002. The degree of mineralization of bone tissue measured by computerized quantitative contact microradiography. Calcified tissue international. 70:503-511.

Bokkers, E. A. and P. Koene. 2004. Motivation and ability to walk for a food reward in fast-and slow-growing broilers to 12 weeks of age. Behavioural processes. 67:121-130.

Boskey, A. L., T. M. Wright, and R. D. Blank. 1999. Collagen and bone strength. Journal of bone and mineral research. 14:330-335.

Bradshaw, R. H., R. D. Kirkden, and D. M. Broom. 2002. A review of the aetiology and pathology of leg weakness in broilers in relation to welfare. Avian and poultry biology reviews. 13:45-104.

Buijs, S., E. Van Poucke, S. Van Dongen, L. Lens, J. Baert, and F. A. Tuyttens. 2012. The influence of stocking density on broiler chicken bone quality and fluctuating asymmetry. Poultry science. 91:1759-1767.

Burrell, A. L., W. A. Dozier, A. J. Davis, M. M. Compton, M. E. Freeman, P. F. Vendrell, and T. L. Ward. 2004. Responses of broilers to dietary zinc concentrations and sources in relation to environmental implications. British poultry science. 45:225-263.

Burwell, R. G. 1986. The growth of bone. Control and manipulation of animal growth. p53-65.

Butterworth, A. 1999. Infectious components of broiler lameness: a review. World's poultry science journal. 55:327-352.

Cancedda, R. 2009. Cartilage and bone extracellular matrix. Current pharmaceutical design. 15:1334-1348.

Capps, S. G. 1998. Effect of tibial dyschondroplasia on broiler growth and cancellous bone mechanical properties. Avian diseases. p162-167.

Castellini, C., Berri, C., Le Bihan-Duval, E. and Martino, G., 2008. Qualitative attributes and consumer perception of organic and free-range poultry meat. World's Poultry Science Journal. 64:500-512.

Charuta, A., M. Dzierzęcka, M., Komosa, L., Kalinowski, and M. Pierzchała. 2013. Age-and sex-related differences of morphometric, densitometric and geometric parameters of tibiotarsal bone in Ross broiler chickens. Folia biologica. 61:211-220.

Charuta, A., M. Dzierzęcka, T. Majchrzak, E. Czerwiński, and R. G. Cooper. 2011. Computer-generated radiological imagery of the structure of the spongious substance in the postnatal development of the tibiotarsal bones of the Peking domestic duck (Anas platyrhynchos var. domestica). Poultry science. 90:830-835.

Clarke, B. 2008. Normal bone anatomy and physiology. Clinical journal of the american society of nephrology. 3:131-139. 
Cockram, M. S. and K. J. Dulal. 2018. Injury and mortality in broilers during handling and transport to slaughter. Canadian journal of animal science. 98:416-432.

Cockram, M. S., K. J. Dulal, H. Stryhn, and C. W. Revie. 2020. Rearing and handling injuries in broiler chickens and risk factors for wing injuries during loading. Canadian journal of animal science. 100:402-410.

Cornetto, T. and I. Estevez. 2001. Behavior of the domestic fowl in the presence of vertical panels. Poultry Science. 80:1455-1462.

Crespo, R. 2020. Developmental, metabolic, and other noninfectious disorders. Diseases of poultry. pl286-1329.

Cruickshank, J.J. and Sim, J.S., 1986. Morphometric and radiographic characteristics of tibial bone of broiler chickens with twisted leg disorders. Avian diseases. p699-708.

Cullinane, D. M. and T. A. Einhorn. 2002. Biomechanics of bone. In Principles of bone biology. p17-32

Davies, J., 2019. Slow-growing birds are fast becoming mainstream. Poultry World. https:// www. poultryworld. net/Meat/Articles/2019/7/Slow-growing-birds-are-fast-becoming-mainstream-454287E.

Dawkins, M. S. and R. Layton. 2012. Breeding for better welfare: genetic goals for broiler chickens and their parents. Animal welfare. 21:147-166.

De Jong, I. C., A. Lourens, and J. Van Harn. 2015. Effect of hatch location and diet density on footpad dermatitis and growth performance in broiler chickens. Journal of applied poultry research. 24:105-114.

De Jong, I., C. Berg, A. Butterworth, and I. Estevéz. 2012. Scientific report updating the EFSA opinions on the welfare of broilers and broiler breeders. EFSA supporting publications. 9:295E.

De Matos, R. 2008. Calcium metabolism in birds. Veterinary clinics of North America: exotic animal practice. 11:59-82.

De Oliveira Peixoto, J., I. R. Savoldi, A. M. G. Ibelli, M. E. Cantão, F. R. F. Jaenisch, P. F. Giachetto, M. L. Settles, R. Zanella, J. A. P. Marchesi, J. R. Pandolfi, and L. L. Coutinho. 2019. Proximal femoral head transcriptome reveals novel candidate genes related to epiphysiolysis in broiler chickens. BMC genomics. 20:1-17.

Decuypere, E. and H. Michels. 1992. Incubation temperature as a management tool: a review. World's poultry science journal. 48:28-38.

Decuypere, E., V. Bruggeman, N. Everaert, Y. Li, R. Boonen, J. De Tavernier, S. Janssens, and N. Buys. 2010. The broiler breeder paradox: ethical, genetic and physiological perspectives, and suggestions for solutions. British poultry science. 51:569-579.

Delaissé, J. M., M. T. Engsig, V. Everts, M. del Carmen Ovejero, M. Ferreras, L. Lund, T. H. Vu, Z. Werb, B. Winding, A. Lochter, and M. A. Karsdal. 2000. Proteinases in bone resorption: obvious and less obvious roles. Clinica chimica acta. 291:223-234.

Demuner, L. F., D. Suckeveris, J. A. Muñoz, V. C. Caetano, C. G. D. Lima, D. E. D. Faria Filho, and D. E. D. Faria. 2017. Adjustment of growth models in broiler chickens. Pesquisa agropecuária Brasileira. 52:1241-1252.

Denbow, D.M., 2015. Gastrointestinal anatomy and physiology. In Sturkie's avian physiology (pp. 337-366). Academic Press.

Dibner, J. J., J. D. Richards, M. L. Kitchell, and M. A. Quiroz. 2007. Metabolic challenges and early bone development. Journal of applied poultry research. 16:126-137.

Dinev, I. 2009. Clinical and morphological investigations on the prevalence of lameness associated with femoral head necrosis in broilers. British poultry science. 50:284-290.

Dinev, I. 2012. Leg weakness pathology in broiler chickens. Poultry Science. 49:63-67.

Dishon, L., Avital-Cohen, N., Malamud, D., Heiblum, R., Druyan, S., Porter, T.E., Gumułka, M. and Rozenboim, I., 2017. In-ovo monochromatic green light photostimulation enhances embryonic somatotropic axis activity. Poultry science. 96:1884-1890.

Dishon, L., Avital-Cohen, N., Zaguri, S., Bartman, J., Heiblum, R., Druyan, S., Porter, T.E., Gumulka, M. and Rozenboim, I., 2021. In ovo green light photostimulation during the late incubation stage affects somatotropic axis activity. Poultry Science. 100:467-473.

Dozier lii, W.A., Davis, A.J., Freeman, M.E. and Ward, T.L., 2003. Early growth and environmental implications of dietary zinc and copper concentrations and sources of broiler chicks. British Poultry Science. 44:726-731. 
Driver, J. P., G. M. Pesti, R. I. Bakalli, and H. M. Edwards Jr. 2005. Calcium requirements of the modern broiler chicken as influenced by dietary protein and age. Poultry science. 84:1629-1639.

Duff, S. R. I. and B. H. Thorp. 1985. Abnormal angulation/torsion of the pelvic appendicular skeleton in broiler fowl: morphological and radiological findings. Research in veterinary science. 39:313-319.

Duncan, I. J. H., C. J. Savory, and D. G. M. Wood-Gush. 1978. Observations on the reproductive behaviour of domestic fowl in the wild. Applied animal ethology. 4:29-42.

Echigo, T. and M. Kimata. 2010. Crystal chemistry and genesis of organic minerals: a review of oxalate and polycyclic aromatic hydrocarbon minerals. The Canadian mineralogist. 48:1329-1357.

EFSA panel on animal health and welfare. 2010. Scientific opinion on the influence of genetic parameters on the welfare and the resistance to stress of commercial broilers. EFSA journal. 8:1666.

El-Husseiny, O. M., S. M. Hashish, R. A. Ali, S. A. Arafa, L. D. Abd El-Samee, and A. A. Olemy. 2012. Effects of feeding organic zinc, manganese and copper on broiler growth, carcass characteristics, bone quality and mineral content in bone, liver and excreta. International journal of poultry science. 11:368-377.

Elliot, M. A. and H. M. Edwards Jr. 1994. Effect of genetic strain, calcium, and feed withdrawal on growth, tibial dyschondroplasia, plasma 1, 25-dihydroxycholecalciferol, and plasma 25-hydroxycholecalciferol in sixteen-day-old chickens. Poultry science. 73:509-519.

Estevez, I., N. Tablante, R. L. Pettit-Riley, and L. Carr. 2002. Use of cool perches by broiler chickens. Poultry science. 81:62-69.

Fanatico, A.C., Pillai, P.B., Cavitt, L.C., Owens, C.M. and Emmert, J.L., 2005. Evaluation of slower-growing broiler genotypes grown with and without outdoor access: Growth performance and carcass yield. Poultry science. 84:1321-1327.

FAO (Food and Agriculture Organization of the United Nations). 2021. Overview of global meat market developments in 2020. Web link: http://www.fao.org/3/cb3700en/ cb3700en.pdf. Accessed March 27, 2021.

Farquharson, C. and D. Jefferies. 2000. Chondrocytes and longitudinal bone growth: the development of tibial dyschondroplasia. Poultry science. 79:994-1004.

Farquharson, C., C. Whitehead, S. Rennie, B. Thorp, and N. Loveridge. 1992. Cell proliferation and enzyme activities associated with the development of avian tibial dyschondroplasia: an in situ biochemical study. Bone. 13:59-67.

Ferket, P. R., E. O. Oviedo-Rondón, P. L. Mente, D. V. Bohórquez, A. A. Santos Jr, J. L. Grimes, J. D. Richards, J. J. Dibner, and V. Felts. 2009. Organic trace minerals and 25-hydroxycholecalciferol affect performance characteristics, leg abnormalities, and biomechanical properties of leg bones of turkeys. Poultry science. 88:1 18-131.

Fleming, R. H. 2008. Nutritional factors affecting poultry bone health: Symposium on 'Diet and bone health'. In Proceedings of the nutrition society. 67:177-183.

Flis, M., D. Gugała, S. Muszyński, P. Dobrowolski, M. Kwiecień, E. R. Grela, and E. Tomaszewska. 2019. The influence of the partial replacing of inorganic salts of calcium, zinc, iron, and copper with amino acid complexes on bone development in male pheasants from aviary breeding. Animals. 9:237-249.

French, N.A., 2009. The critical importance of incubation temperature. Avian Biology Research. 2:55-59.

Genin, O., A. Hasdai, D. Shinder, and M. Pines. 2012. The effect of inhibition of heat-shock proteins on thiram-induced tibial dyschondroplasia. Poultry science. 91:1619-1626.

Gocsik, É., A. M. Silvera, H. Hansson, H. W. Saatkamp, and H. J. Blokhuis. 2017. Exploring the economic potential of reducing broiler lameness. British poultry science. 58:337-347.

González-Cerón, F., R. Rekaya, and S. E. Aggrey. 2015. Genetic analysis of bone quality traits and growth in a random mating broiler population. Poultry science. 94:883-889.

Grandin, T. 2010. Auditing animal welfare at slaughter plants. Meat science. 86:56-65.

Grashorn, M.A., 2006, September. Fattening performance, carcass and meat quality of slow and fast growing broiler strains under intensive and extensive feeding conditions. In XII Eur. Poult. Conf., Verona, Italy (p. 249). World's Poult. Sci. Assoc. Italian Branch, Bologna, Italy. 
Greene, J. A., R. M. McCracken, and R. T. Evans. 1985. A contact dermatitis of broilers' clinical and pathological findings. Avian pathology. 14:23-38.

Guinotte, F., Y. Nys, and F. De Monredon. 1991. The effects of particle size and origin of calcium carbonate on performance and ossification characteristics in broiler chicks. Poultry science. 70:1908-1920.

Guo, R., P. R. Henry, R. A. Holwerda, J. Cao, R. C. Littell, R. D. Miles, and C. B. Ammerman. 2001. Chemical characteristics and relative bioavailability of supplemental organic copper sources for poultry. Journal of animal science. 79:1132-1141.

Guo, Y., H. Tang, X. Wang, W. Li, Y. Wang, F. Yan, X. Kang, Z. Li, and R. Han. 2019. Clinical assessment of growth performance, bone morphometry, bone quality, and serum indicators in broilers affected by valgus-varus deformity. Poultry science. 98:4433-4440.

Gupta, R. K., S. S. Gangoliya, and N. K. Singh. 2015. Reduction of phytic acid and enhancement of bioavailable micronutrients in food grains. Journal of food science and technology. 52:676-684.

Halevy, O., Piestun, Y., Rozenboim, I. and Yablonka-Reuveni, Z., 2006. In ovo exposure to monochromatic green light promotes skeletal muscle cell proliferation and affects myofiber growth in posthatch chicks. American Journal of Physiology-Regulatory, Integrative and Comparative Physiology. 290(4), p1062-1070.

Hall, A. L. 2001. The effect of stocking density on the welfare and behaviour of broiler chickens reared commercially. Animal welfare. 10:23-40.

Hamdi, M., D. S. Oriol, R. F. Rosselló, R. A. Alemany, and J. F. Pérez. 2017. Comparison of how different feed phosphates affect performance, bone mineralization and phosphorus retention in broilers. Spanish journal of agricultural research. 15:14-24.

Hamdi, M., J. F. Perez, M. P. Létourneau-Montminy, R. Franco-Rosselló, R. Aligue, and D. Solà-Oriol. 2018. The effects ofmicrobial phytases and dietary calcium and phosphorus levels on the productive performance and bone mineralization of broilers. Animal feed science and technology. 243:41-51.

Hamdi, M., López-Vergé, S., Manzanilla, E.G., Barroeta, A.C. and Pérez, J.F., 2015. Effect of different levels of calcium and phosphorus and their interaction on the performance of young broilers. Poultry Science. 94(9), p2144-2151.

Hammond, C. L., B. H. Simbi, and N. C. Stickland. 2007. In ovo temperature manipulation influences embryonic motility and growth of limb tissues in the chick (Gallus gallus). Journal of experimental biology. 210:2667-2675.

Harash, G., K. C. Richardson, Z. Alshamy, H. Hünigen, H. M. Hafez, J. Plendl, and S. Al Masri. 2020. Basic morphometry, microcomputed tomography and mechanical evaluation of the tibiotarsal bone of a dual-purpose and a broiler chicken line. Plos one. 15:0230070.

Hargest, T. E., R. M. Leach, and C. V. Gay. 1985. Avian tibial dyschondroplasia. I. Ultrastructure. The American journal of pathology. 119:175-189.

Hartcher, K. M. and H. K. Lum. 2020. Genetic selection of broilers and welfare consequences: a review. World's poultry science journal. 76:154-167.

Hashimoto, S., K. Yamazaki, T. Obi, and K. Takase. 2013. Relationship between severity of footpad dermatitis and carcass performance in broiler chickens. Journal of veterinary medical science. p13-31.

Haslam, S.M., Knowles, T.G., Brown, S.N., Wilkins, L.J., Kestin, S.C., Warriss, P.D. and Nicol, C.J., 2007. Factors affecting the prevalence of foot pad dermatitis, hock burn and breast burn in broiler chicken. British poultry science. 48:264-275.

Havenstein, G. B., P. R. Ferket, and M. A. Qureshi. 2003. Growth, livability, and feed conversion of 1957 versus 2001 broilers when fed representative 1957 and 2001 broiler diets. Poultry science. 82:1500-1508.

Havenstein, G. B., P. R. Ferket, S. E. Scheideler, and B. T. Larson. 1994. Growth, livability, and feed conversion of 1957 vs 1991 broilers when fed "typical" 1957 and 1991 broiler diets. Poultry science. 73:1785-1794.

Hemme, A., Spark, M., Wolf, P., Paschertz, H. and Kamphues, J., 2005. Effects of different phosphorus sources in the diet on bone composition and stability (breaking strength) in broilers. Journal of Animal Physiology and Animal Nutrition. 89:129-133. 
Hluchý, S., Toman, R., Cabaj, M. and Adamkovičová, M., 2012. The effect of white and monochromatic lights on chicken hatching. Scientific Papers Animal Science and Biotechnologies. 45:408-410.

Huang, Y. L., L. LU, S. F. Li, X. G. Luo, and B. Liu. 2009. Relative bioavailabilities of organic zinc sources with different chelation strengths for broilers fed a conventional corn-soybean meal diet. Journal of animal science. 87:2038-2046.

Huth, J. C. and G. S. Archer. 2015. Effects of LED lighting during incubation on layer and broiler hatchability, chick quality, stress susceptibility and post-hatch growth. Poultry science. 94:3052-3058.

Jacobs, L., E. Delezie, L. Duchateau, K. Goethals, and F. A. Tuyttens, F.A., 2017. Broiler chickens dead on arrival: associated risk factors and welfare indicators. Poultry science. 96:259-265.

Jianhua, H., A. Ohtsuka, and K. Hayashi. 2000. Selenium influences growth via thyroid hormone status in broiler chickens. British journal of nutrition. 84:727-732.

Johnsson, M., K. B. Jonsson, L. Andersson, P. Jensen, and D. Wright. 2015. Genetic regulation of bone metabolism in the chicken: similarities and differences to mammalian systems. PLoS genetics. 11:1005250.

Jones, P. J., J. Niemi, J. P. Christensen, R. B. Tranter, and R. M. Bennett. 2019. A review of the financial impact of production diseases in poultry production systems. Animal production science. 59:1585-1597.

Julian, R. J. 1984. Valgus-varus deformity of the intertarsal joint in broiler chickens. The Canadian veterinary journal. 25:254-258.

Julian, R. J. 1998. Rapid growth problems: ascites and skeletal deformities in broilers. Poultry science. 77:1773-1780.

Julian, R. J. 2005. Production and growth-related disorders and other metabolic diseases of poultry-a review. The veterinary journal. 169:350-369.

Jungmann, R., M. E. Szabo, G. Schitter, R. Y. S. Tang, D. Vashishth, P. K. Hansma, and P. J. Thurner. 2011. Local strain and damage mapping in single trabeculae during threepoint bending tests. Journal of the mechanical behavior of biomedical materials. 4:523-534.

Kapell, D. N. R. G., P. M. Hocking, P. K. Glover, V. D. Kremer, and S. Avendaño. 2017. Genetic basis of leg health and its relationship with body weight in purebred turkey lines. Poultry science. 96:1553-1562.

Karsenty, G. 1999. The genetic transformation of bone biology. Genes \& development. 13:3037-3051.

Kaukonen, E., M. Norring, A. and Valros. 2016. Effect of litter quality on foot pad dermatitis, hock burns and breast blisters in broiler breeders during the production period. Avian pathology. 45:667-673.

Kaukonen, E., M. Norring, and A. Valros. 2017. Perches and elevated platforms in commercial broiler farms: use and effect on walking ability, incidence of tibial dyschondroplasia and bone mineral content. Animal. 11:864-871.

Kestin, S. C., G. Su, and P. Sørensen. 1999. Different commercial broiler crosses have different susceptibilities to leg weakness. Poultry science. 78:1085-1090.

Kestin, S. C., S. Gordon, G. Su, and P. Sørensen. 2001. Relationships in broiler chickens between lameness, liveweight, growth rate and age. Veterinary record. 148:195-197.

Kestin, S. C., T. G. Knowles, A. E. Tinch and N. G. Gregory. 1992. Prevalence of leg weakness in broiler chickens and its relationship with genotype. Veterinary Record. 131:190-194.

Kidd, M.T., Anthony, N.B. and Lee, S.R., 1992. Progeny performance when dams and chicks are fed supplemental zinc. Poultry science. 71:1201-1206.

Kim, W. K., L. M. Donalson, A. D. Mitchell, L. F. Kubena, D. J. Nisbet, and S. C. Ricke. 2006. Effects of alfalfa and fructooligosaccharide on molting parameters and bone qualities using dual energy X-ray absorptiometry and conventional bone assays. Poultry science. 85:15-20.

Kittelsen, K. E., E. G. Granquist, A. L. Aunsmo, R. O. Moe, and E. Tolo. 2018. An evaluation of two different broiler catching methods. Animals. 8:141-150.

Kittelsen, K. E., E. G. Granquist, Ø. Kolbjørnsen, O. Nafstad, and R. O. Moe. 2015. A comparison of post-mortem findings in broilers dead-on-farm and broilers dead-on-arrival at the abattoir. Poultry science. 94:2622-2629. 
Kjaer, J. B., G. Su, B. L. Nielsen, and P. Sørensen. 2006. Foot pad dermatitis and hock burn in broiler chickens and degree of inheritance. Poultry science. 85:1342-1348.

Klasing, K. C. 1998. Comparative avian nutrition (book). Cab International.

Knowles, T. G. and L. J. Wilkins. 1998. The problem of broken bones during the handling of laying hens-A review. Poultry science. 77:1798-1802.

Knowles, T. G., S. C. Kestin, S. M. Haslam, S. N. Brown, L. E. Green, A. Butterworth, S. J. Pope, D. Pfeiffer, and C. J. Nicol. 2008. Leg disorders in broiler chickens: prevalence, risk factors and prevention. Plos one. 3:1545.

Kokoszyński, D., Bernacki, Z., Saleh, M., Stęczny, K. and Binkowska, M., 2017. Body conformation and internal organs characteristics of different commercial broiler lines. Brazilian Journal of Poultry Science, 19:47-52.

Kratzer, F. H. and P. Vohra. 1986. Metal complexes and chelates. In Chelates in Nutrition. p49-61.

Kristensen, H.H., Perry, G.C., Prescott, N.B., Ladewig, J., Ersbøll, A.K. and Wathes, C.M., 2006. Leg health and performance of broiler chickens reared in different light environments. British poultry science, 47:257-263.

Krupski, W. and M. R. Tatara. 2007. Interrelationships between densitometric, morphometric, and mechanical properties of the tibia in turkeys. Bulletin of the veterinary institute in pulawy. 51:621-626.

Kryeziu, A. J., N. Mestani, S. Berisha, and M. A. Kamberi, M. A. 2018. The European performance indicators of broiler chickens as influenced by stocking density and sex. Agronomy research. 16:483-491.

Landis, W. J., K. J. Hodgens, J. Arena, M. J. Song, and B. F. McEwen. 1996. Structural relations between collagen and mineral in bone as determined by high voltage electron microscopic tomography. Microscopy research and technique. 33:192-202.

Leach Jr, R. M. and E. Monsonego-Ornan. 2007. Tibial dyschondroplasia 40 years later. Poultry science. 86:2053-2058.

Leach Jr, R. M. and M. C. Nesheim. 1972. Further studies on tibial dyschondroplasia (cartilage abnormality) in young chicks. The journal of nutrition. 102:1673-1680.

Leblanc, B., M. Wyers, F. Cohn-Bendit, J. M. Legall, E. Thibault, and J. M. Florent. 1986. Histology and histomorphometry of the tibia growth in two turkey strains. Poultry science. 65:1787-1795.

Ledwaba, M. F. and K. D. Roberson. 2003. Effectiveness of twenty-five-hydroxycholecalciferol in the prevention of tibial dyschondroplasia in Ross cockerels depends on dietary calcium level. Poultry science. 82:1769-1777.

Leeson, S. 2003. A new look at the trace mineral nutrition of poultry: Can we reduced environmental burden of poultry manure? In Nutritional biotechnology in the feed and food industries. p125-129.

Leeson, S. 2005. Trace mineral requirements of poultry-validity of the NRC recommendations. In Re-defining mineral nutrition. p107-118.

Leterrier, C. and Y. Nys. 1992. Composition, cortical structure and mechanical properties of chicken tibiotarsi: effect of growth rate. British poultry science. 33:925-939.

Letourneau-Montminy, M. P., P. Lescoat, A. Narcy, D. Sauvant, J. F. Bernier, M. Magnin, C. Pomar, Y. Nys, and C. Jondreville. 2008. Effects of reduced dietary calcium and phytase supplementation on calcium and phosphorus utilisation in broilers with modified mineral status. British poultry science. 49:705-715.

Lewis, P. D. and T. R. Morris, T.R., 2000. Poultry and coloured light. World's poultry science journal, 56:189-207.

Lewis, P. D., G. C. Perry, L. J. Farmer, and R. L. S. Patterson. 1997. Responses of two genotypes of chicken to the diets and stocking densities typical of UK and 'Label Rouge'production systems: I. Performance, behaviour and carcass composition. Meat Science. 45:501-516.

Li, T., G. Xing, Y. Shao, L. Zhang, S. Li, L. Lu, Z. Liu, X. Liao, and X. Luo. 2020. Dietary calcium or phosphorus deficiency impairs the bone development by regulating related calcium or phosphorus metabolic utilization parameters of broilers. Poultry science. 99:32073214. 
Li, W., R. Angel, S. W. Kim, K. Brady, S. Yu, and P. W. Plumstead. 2016. Impacts of dietary calcium, phytate, and nonphytate phosphorus concentrations in the presence or absence of phytase on inositol hexakisphosphate (IP6) degradation in different segments of broilers digestive tract. Poultry science. 95:581-589.

Li, X., Zhang, D. and Bryden, W.L., 2017. Calcium and phosphorus metabolism and nutrition of poultry: are current diets formulated in excess?. Animal production science. 57:23042310.

Lilburn, M.S., 1994. Skeletal growth of commercial poultry species. Poultry science, 73:897-903.

Ling, J., S. A. Kincaid, G. R. McDaniel, and W. Waegell. 2000. Immunolocalization analysis of transforming growth factor- $\beta 1$ in the growth plates of broiler chickens with high and low incidences of tibial dyschondroplasia. Poultry science. 79:1172-1178.

Litchfield, T. M., Y. Ishikawa, L. N. Y. Wu, R. E. Wuthier, and G. R. Saver. 1998. Effect of metal ions on calcifying growth plate cartilage chondrocytes. Calcified tissue international. 62:341-349.

Lourens, A., H. van den Brand, R. Meijerhof, and B. Kemp. 2005. Effect of eggshell temperature during incubation on embryo development, hatchability, and posthatch development. Poultry science. 84:914-920.

Lowther, D. A., H. C. Robinson, J. W. Dolman, and K. W. Thomas. 1974. Cartilage matrix components in chickens with tibial dyschondroplasia. The journal of nutrition. 104:922-929.

Lynch, M., B. H. Thorp, and C. C. Whitehead. 1992. Avian tibial dyschondroplasia as a cause of bone deformity. Avian pathology. 21:275-285.

M'Sadeq, S.A., Wu, S.B., Choct, M. and Swick, R.A., 2018. Influence of trace mineral sources on broiler performance, lymphoid organ weights, apparent digestibility, and bone mineralization. Poultry science. 97:3176-3182.

Maatjens, C. M., I. A. M. van Roovert-Reijrink, B. Engel, C. W. van der Pol, B. Kemp, and H. van den Brand. 2017. Temperature during the last week of incubation. III. Effects on chicken embryo physiology. Poultry science. 96:1451-1458.

Manangi, M. K., M. Vazquez-Anon, J. D. Richards, S. Carter, R. E. Buresh, and K. D. Christensen. 2012. Impact of feeding lower levels of chelated trace minerals versus industry levels of inorganic trace minerals on broiler performance, yield, footpad health, and litter mineral concentration. Journal of applied poultry research. 21:881-890.

Martrenchar, A., D. Huonnic, J. P. Cotte, E. Boilletot, and J. P. Morisse. 2000. Influence of stocking density, artificial dusk and group size on the perching behaviour of broilers. British Poultry Science. 41:125-130.

National Research Council, 1994. Nutrient requirements of poultry: 1994. National Academies Press.

Mayne, R. K. 2005. A review of the aetiology and possible causative factors of foot pad dermatitis in growing turkeys and broilers. World's poultry science journal. 61:256-267.

McDevitt, R. M., G. M. McEntee, and K. A. Rance. 2006. Bone breaking strength and apparent metabolisability of calcium and phosphorus in selected and unselected broiler chicken genotypes. British poultry science. 47:613-621.

McKay, J. C., N. F. Barton, A. N. M. Koerhuis, and J. McAdam. 2000. The challenge of genetic change in the broiler chicken. BSAP occasional publication. 27:1-7.

MCNamee, P. T. and J. A. Smyth. 2000. Bacterial chondronecrosis with osteomyelitis ('femoral head necrosis') of broiler chickens: a review. Avian pathology. 29:477-495.

McNamee, P. T., J. L. McCullagh, B. H. Thorp, H. J. Ball, D. Graham, S. J. McCullough, D. McConaghy, and J. A. Smyth. 1998. Study of leg weakness in two commercial broiler flocks. Veterinary record. 143:131-135.

Medeiros, D. M., J. llich, J. Ireton, V. Matkovic, L. Shiry, and R. Wildman. 1997. Femurs from rats fed diets deficient in copper or iron have decreased mechanical strength and altered mineral composition. The journal of trace elements in experimental medicine: the official publication of the international society for trace element research in humans. 10:197-203.

Meluzzi, A., C. Fabbri, E. Folegatti, and F. Sirri. 2008. Survey of chicken rearing con ditions in Italy: effects of litter quality and stocking density on productivity, foot dermatitis and carcase injuries. British poultry science. 49:257-264. 
Mench, J. 2004. Lameness. In Measuring and auditing broiler welfare. p3-17.

Merkley, J. W. 1981. The effect of sodium fluoride on egg production, egg quality, and bone strength of caged layers. Poultry science. 60:771-776.

Morgan, N.K., Walk, C.L., Bedford, M.R. and Burton, E.J., 2015. Contribution of intestinal-and cereal-derived phytase activity on phytate degradation in young broilers. Poultry science. 94:1577-1583.

Morris, M.P. 1993. National survey of leg problems. Pigs and poultry. 6:016.

Mottet, A. and G. Tempio. 2017. Global poultry production: current state and future outlook and challenges. World's poultry science journal. 73:245-256.

Mulder, H. A., W. G. Hill, A. Vereijken, and R. F. Veerkamp. 2009. Estimation of genetic variation in residual variance in female and male broiler chickens. Animal. 3:1673-1680.

Muszyński, S., E. Tomaszewska, P. Dobrowolski, M. Kwiecień, D. Wiacek, I. Świetlicka, M. Skibińska, M., Szymańska-Chargot, J. Orzeł, M. Świetlicki, and M. Arczewska. 2018. Analysis of bone osteometry, mineralization, mechanical and histomorphometrical properties of tibiotarsus in broiler chickens demonstrates an influence of dietary chickpea seeds (Cicer arietinum L.) inclusion as a primary protein source. PLoS One. 13:0208921.

Nairn, M. E. and A. R. A. Watson. 1972. Leg weakness of poultry-A clinical and pathological characterisation. Australian veterinary journal. 48:645-656.

Nakane, Y. and M. Tsudzuki. 1999. Development of the skeleton in Japanese quail embryos. Development, growth \& differentiation. 41:523-534.

Newbrey, J. W., S. N. Baksi, A. S. Dhillon, N. G. Zimmerman, S. G. Truitt, and R. Riedinger. 1988. Histomorphometry and vitamin D metabolism of valgus-varus deformity in broiler chickens. Avian diseases. p704-712.

Norring, M., E. Kaukonen, and A. Valros. 2016. The use of perches and platforms by broiler chickens. Applied animal behaviour science. 184:91-96.

Novitskaya, E., Chen, P.Y., Hamed, E., Jun, L., Lubarda, V.A., Jasiuk, I. and McKittrick, J., 2011. Recent advances on the measurement and calculation of the elastic moduli of cortical and trabecular bone: a review. Theoretical and applied mechanics, 38:09-297.

Nyman, J. S., M. Reyes, and X. Wang. 2005. Effect of ultrastructural changes on the toughness of bone. Micron. 36:566-582.

Ohara, A., C. Oyakawa, Y. Yoshihara, S. Ninomiya, and S. Sato. 2015. Effect of environmental enrichment on the behavior and welfare of Japanese broilers at a commercial farm. The journal of poultry science. p0150034.

Onyango, E. M., P. Y. Hester, R. Stroshine, and O. Adeola. 2003. Bone densitometry as an indicator of percentage tibia ash in broiler chicks fed varying dietary calcium and phosphorus levels. Poultry science. 82:1787-1791.

Orban, J. I., D. A. Roland Sr, M. M. Bryant, and J. C. Williams. 1993. Factors influencing bone mineral content, density, breaking strength, and ash as response criteria for assessing bone quality in chickens. Poultry science. 72:437-446.

Oviedo-Rondón, E. O., J. Small, M. J. Wineland, V. L. Christensen, P. S. Mozdziak, M. D. Koci, S. V. L. Funderburk, D. T. Ort, and K. M. Mann. 2008. Broiler embryo bone development is influenced by incubator temperature, oxygen concentration and eggshell conductance at the plateau stage in oxygen consumption. British poultry science. 49:666-676.

Oviedo-Rondón, E. O., M. J. Wineland, S. Funderburk, J. Small, H. Cutchin, and M. Mann. 2009. Incubation conditions affect leg health in large, high-yield broilers. Journal of applied poultry research. 18:640-646.

Owen, M. A. and A. J. Friedenstein.1988. Stromal stem cells: marrow-derived osteogenic precursors. In Ciba Found Symposium. p42-60.

Özkan, S., S. Yalçın, E. Babacanoğlu, S. Uysal, F. Karadaş, and H. Kozanoğlu. 2012. Photoperiodic lighting ( 16 hours of light: 8 hours of dark) programs during incubation: 2. Effects on early posthatching growth, blood physiology, and production performance in broiler chickens in relation to posthatching lighting programs. Poultry science. 91:2922-2930. 
Öznurlu, Y., E. Sur, T. Özaydin, I. Celik, and D. Uluisik. 2016. Histological and histochemical evalvations on the effects of high incubation temperature on the embryonic development of tibial growth plate in broiler chickens. Microscopy research and technique. 79:106-110.

Packialakshmi, B., N. C. Rath, W. E. Huff, and G. R. Huff. 2015. Poultry femoral head separation and necrosis: a review. Avian diseases. 59:349-354.

Pagazaurtundua, A. and Warriss, P.D., 2006. Levels of foot pad dermatitis in broiler chickens reared in 5 different systems. British poultry science. 47:529-532.

Park, S. Y., S. G. Birkhold, L. F. Kubena, D. J. Nisbet, and S. C. Ricke. 2003. Effect of storage condition on bone breaking strength and bone ash in laying hens at different stages in production cycles. Poultry science. 82:1688-1691.

Patt, H. M. and M. A. Maloney. 1975. Bone marrow regeneration after local injury: a review. Experimental hematology. 3:135-148.

Pedersen, I. J. and B. Forkman. 2019. Improving leg health in broiler chickens: a systematic review of the effect of environmental enrichment. Animal welfare. 28:215-230.

Pedersen, I.J., Tahamtani, F.M., Forkman, B., Young, J.F., Poulsen, H.D. and Riber, A.B., 2020. Effects of environmental enrichment on health and bone characteristics of fast growing broiler chickens. Poultry science. 99:1946-1955.

Pelicia, K., I. M. Aparecido Jr, E. A. Garcia, A. B. Molino, G. C. Santos, D. A. Berto, J. A. Vieira Filho, E. S. M. Murakami, A. T. Montenegro, and A. M. Silva. 2012. Evaluation of a radiographic method to detect tibial dyschondroplasia lesions in broilers. Brazilian journal of poultry science. 14:129-135.

Petracci, M. and C. Cavani. 2012. Muscle growth and poultry meat quality issues. Nutrients. $4: 1-12$.

Pichova, K., J. Nordgreen, C. Leterrier, L. Kostal, and R. O. Moe. 2016. The effects of food-related environmental complexity on litter directed behaviour, fear and exploration of novel stimuli in young broiler chickens. Applied animal behaviour science. 174:83-89.

Pines, M. and R. Reshef, R. 2015. Poultry bone development and bone disorders. In Sturkie's avian physiology. p367-377.

Posner, A. S. 1969. Crystal chemistry of bone mineral. Physiological reviews. 49:760-792.

Praul, C. A., B. C. Ford, C. V. Gay, M. Pines, and R. M. Leach. 2000. Gene expression and tibial dyschondroplasia. Poultry science. 79:1009-1013.

Prisby, R., T. Menezes, J. Campbell, T. Benson, E. Samraj, I. Pevzner, and R. F. Wideman Jr. 2014. Kinetic examination of femoral bone modeling in broilers. Poultry science. 93:1122-1129.

Proszkowiec-Weglarz, M. and R. Angel. 2013. Calcium and phosphorus metabolism in broilers: effect of homeostatic mechanism on calcium and phosphorus digestibility. Journal of applied poultry research. 22:609-627.

Quentin, M., Bouvarel, I. and Picard, M., 2004. Short-and long-term effects of feed form on fast-and slow-growing broilers. Journal of Applied Poultry Research, 13:540-548.

Randall, C. J. and C. P. J. Mills. 1981. Observations on leg deformity in broilers with particular reference to the intertarsal joint. Avian pathology. 10:407-431.

Rath, N. C., G. R. Bayyari, J. N. Beasley, W. E. Huff, and J. M. Balog. 1994. Age-related changes in the incidence of tibial dyschondroplasia in turkeys. Poultry science. 73:1254-1259.

Rath, N. C., G. R. Huff, W. E. Huff, and J. M. Balog. 2000. Factors regulating bone maturity and strength in poultry. Poultry science, 79:1024-1032.

Rath, N. C., W. E. Huff and G. R. Huff. 2007. Thiram-induced changes in the expression of genes relating to vascularization and tibial dyschondroplasia. Poultry science. 86:2390-2395.

Reddi, A. H. 2000. Initiation and promotion of endochrondral bone formation by bone morphogenetic proteins: potential implications for avian tibial dyschondroplasia. Poultry science. 79:978-981.

Reiter, K. and W. Bessei. 1998. Possibilities to reduce leg disorders in broilers and turkeys. Archiv Für Geflügelkunde. 62:145-149.

Reiter, K. and W. Bessei. 2009. Effect of locomotor activity on leg disorder in fattening chicken. Berliner und Munchener Tierarztliche Wochenschrift. 122:264-270. 
Riber, A. B., H. A. van de Weerd, I. C. De Jong, and S. Steenfeldt. 2018. Review of environmental enrichment for broiler chickens. Poultry science. 97:378-396.

Richards, J. D., J. Zhao, R. J. Harrell, C. A. Atwell, and J. J. Dibner. 2010. Trace mineral nutrition in poultry and swine. Asian-Australasian journal of animal sciences. 23:1527-1534.

Richards, M. P. 1997. Trace mineral metabolism in the avian embryo. Poultry science. 76:152164.

Riddell, C. and R. Springer. 1985. An epizootiological study of acute death syndrome and leg weakness in broiler chickens in western Canada. Avian diseases (book). p90-102.

Riddell, C., 1992. Non-infectious skeleton disorders of poultry-an overview. Bone biology and skeletal disorders in poultry. pl19-145.

Riesenfeld, A., 1972. Metatarsal robusticity in bipedal rats. American journal of physical anthropology. 36:229-233.

Rios, H. V., P. D. Waquil, P. S. de Carvalho, and T. Norton. 2020. How are Information Technologies Addressing Broiler Welfare? A Systematic Review Based on the Welfare Quality ${ }^{\circledR}$ Assessment. Sustainability. 12:1-31.

Ritchie, H. and M. Roser. 2017. Meat and dairy production. Our World in Data. Web link: https://ourworldindata.org/meat-production. Accessed January 20, 2021.

Roach, H. I. 1997. New aspects of endochondral ossification in the chick: chondrocyte apoptosis, bone formation by former chondrocytes, and acid phosphatase activity in the endochondral bone matrix. Journal of bone and mineral research. 12:795-805.

Romanoff, A. L., 1960. The avian embryo. Structural and functional development. The avian embryo. Structural and functional development.

Rosol, T. J. and C. C. Capen. 1997. Calcium-regulating hormones and diseases of abnormal mineral (calcium, phosphorus, magnesium) metabolism. In Clinical biochemistry of domestic animals. p619-702.

Rozenboim, I., Y. Piestun, N. Mobarkey, M. Barak, A. Hoyzman, and O. Halevy. 2004. Monochromatic light stimuli during embryogenesis enhance embryo development and posthatch growth. Poultry science. 83:1413-1419.

Rubin, C., G. Xu, and S. Judex. 2001. The anabolic activity of bone tissue, suppressed by disuse, is normalized by brief exposure to extremely low magnitude mechanical stimuli. Federation of American societies for experimental biology. 15:2225-2229.

Ruff, C. R. and B. L. Hughes. 1985. Bone strength of height-restricted broilers as affected by levels of calcium, phosphorus, and manganese. Poultry science. 64:1628-1636.

Sá, L. M., P. C. Gomes, H. S. Rostagno, L. F. T. Albino, P. R. Cecon, and P. D'Agostini. 2004. Calcium requirement for broiler chicks from 22 to 42 and 43 to 53 days old. Revista Brasileira de Zootecnia. 33:397-406.

Sanchez, T. V., R. C. Myers, J. T. Bond, and G. H. Mayor. 1981. Bone mineral analysis of the rat femur by direct photon absorptiometry. Calcified tissue international. 33:587-590.

Sanchez-Rodriguez, E., C. Benavides-Reyes, C. Torres, N. Dominguez-Gasca, A. I. Garcia-Ruiz, S. Gonzalez-Lopez, and A. B. Rodriguez-Navarro. 2019. Changes with age (from 0 to $37 \mathrm{D}$ ) in tibiae bone mineralization, chemical composition and structural organization in broiler chickens. Poultry science. 98:5215-5225.

Sandilands, V., C. Moinard, and N. H. C. 2009. Providing laying hens with perches: fulfilling behavioural needs but causing injury?. British poultry science. 50:395-406.

Sanotra, G. S., L. G. Lawson, K. S. Vestergaard, and M. G. Thomsen. 2001. Influence of stocking density on tonic immobility, lameness, and tibial dyschondroplasia in broilers. Journal of applied animal welfare science. 4:71-87.

Saraiva, S., Saraiva, C. and Stilwell, G., 2016. Feather conditions and clinical scores as indicators of broilers welfare at the slaughterhouse. Research in veterinary science. 10:75-79.

Scholey, D.V., Morgan, N.K., Riemensperger, A., Hardy, R. and Burton, E.J., 2018. Effect of supplementation of phytase to diets low in inorganic phosphorus on growth performance and mineralization of broilers. Poultry science. 97:2435-2440.

Scott, M. L., M. C. Nesheim, and R. J. Young. 1969. Nutrition of the chicken (book). 
Shao, Y., Q. Wen, S. Zhang, L. Lu, L. Zhang, X. Liao, and X. Luo. 2019. Dietary supplemental vitamin D3 enhances phosphorus absorption and utilisation by regulating gene expression of related phosphate transporters in the small intestine of broilers. British journal of nutrition. 121:9-21.

Shepherd, E. M. and B. D. Fairchild. 2010. Footpad dermatitis in poultry. Poultry science. 89:2043-2051.

Sheridan, A. K., C. R. Howlett, and R. W. Burton. 1978. The inheritance of tibial dyschondroplasia in broilers. British poultry science. 19:491-499.

Sherlock, L., T. G. M. Demmers, A. E. Goodship, I. D. McCarthy, and C. M. Wathes. 2010. The relationship between physical activity and leg health in the broiler chicken. British poultry science. 51:22-30.

Shim, M. Y. and G. M. Pesti. 2011 . Effects of incubation temperature on the bone development of broilers. Poultry science, 90:1867-1877.

Shim, M. Y., A. B. Karnuah, A. D. Mitchell, N. B. Anthony, G. M. Pesti, and S. E. Aggrey. 2012. The effects of growth rate on leg morphology and tibia breaking strength, mineral density, mineral content, and bone ash in broilers. Poultry science. 91:1790-1795.

Siegel, P. B., C. F. Honaker, and W. M. Rauw. 2009. Selection for high production in poultry. p230-242.

Stalker, M. J., M. L. Brash, A. Weisz, R. M. Ouckama, and D. Slavic. 2010. Arthritis and osteomyelitis associated with Enterococcus cecorum infection in broiler and broiler breeder chickens in Ontario, Canada. Journal of veterinary diagnostic investigation. 22:643645.

Stojcic, M. D. and W. Bessei. 2009. The effect of locomotor activity and weight load on bone problems in fast and slow growing chickens. Archiv für Geflügelkunde. 73:242-249.

Sullivan, T. W. 1994. Skeletal problems in poultry: estimated annual cost and descriptions. Poultry science. 73:879-882.

Taira, K., T. Nagai, T. Obi, and K. Takase. 2013. Effect of litter moisture on the development of footpad dermatitis in broiler chickens. Journal of veterinary medical science. p1-20.

Tallentire, C. W., I. Leinonen, and I. Kyriazakis. 2016. Breeding for efficiency in the broiler chicken: A review. Agronomy for sustainable development. 36:1-16.

Tatara, M. R., A. Charuta, W. Krupski, I. Łuszczewska-Sierakowska, A. Korwin-Kossakowska, K. Sartowska, M. Szpetnar, and J. O. Horbańczuk. 2015. Interrelationships between morphological, densitometric and mechanical properties of eggs in Japanese quails (Coturnix Japonica). The journal of poultry science. 53:51-57.

Tatara, M. R., W. Krupski, M. Jankowski, Z. Zduńczyk, J. Jankowski, and T. Studziński. 2011 . Effects of dietary calcium content and vitamin D source on skeletal properties in growing turkeys. British poultry science. 52:718-729.

Thorp, B. H. 1994. Skeletal disorders in the fowl: a review. Avian pathology. 23:203-236

Thorp, B. H., C. C. Whitehead, L. Dick, J. M. Bradbury, R. C. Jones, and A. Wood. 1993. Proximal femoral degeneration in growing broiler fowl. Avian pathology. 22:325-342.

Thorp, B.H. and Waddington, D., 1997. Relationships between the bone pathologies, ash and mineral content of long bones in 35-day-old broiler chickens. Research in veterinary science. 62:67-73.

Tickle, P. G., H. Paxton, J. W. Rankin, J. R. Hutchinson, and J. R. Codd. 2014. Anatomical and biomechanical traits of broiler chickens across ontogeny. Part I. Anatomy of the musculoskeletal respiratory apparatus and changes in organ size. PeerJ. 2:e432.

Toghyani, M., A. Gheisari, M. Modaresi, S. A. Tabeidian, and M. Toghyani. 2010. Effect of different litter material on performance and behavior of broiler chickens. Applied animal behaviour science. 122:48-52.

Tomaszewska, E., S. Muszyński, P. Dobrowolski, M. Kwiecień, A. Winiarska-Mieczan, I. Świetlic$\mathrm{ka}$, and A. Wawrzyniak. 2017. Effect of zinc level and source (zinc oxide vs. zinc glycine) on bone mechanical and geometric parameters, and histomorphology in male Ross 308 broiler chicken. Brazilian journal of poultry science. 19:159-170.

Tona, K., O. M. Onagbesan, Y. Jego, B. Kamers, E. Decuypere, and V. Bruggeman. 2004. Comparison of embryo physiological parameters during incubation, chick quality, and growth performance of three lines of broiler breeders dif fering in genetic composition and growth rate. Poultry science. 83:507-513. 
Tong, Q., I. M. McGonnell, T. G. M. Demmers, N. Roulston, H. Bergoug, C. E. Romanini, R. Verhelst, M. Guinebretière, N. Eterradossi, D. Berckmans, and V. Exadaktylos. 2018. Effect of a photoperiodic green light programme during incubation on embryo development and hatch process. Animal. 12:765-773.

Torres, C. A. and D. R. Korver. 2018. Influences of trace mineral nutrition and maternal flock age on broiler embryo bone development. Poultry science. 97:2996-3003.

Turner, C.H. and Burr, D.B., 1993. Basic biomechanical measurements of bone: a tutorial. Bone. 14:595-608.

Vallet-Regi, M. and J. M. González-Calbet. 2004. Calcium phosphates as substitution of bone tissues. Progress in solid state chemistry. 32:1-31.

Van der Klis, J. D. and P. A. Kemme. 2002. An appraisal of trace elements: inorganic and organic. Poultry feedstuffs: supply, composition and nutritive value. p99-108.

Van der Pol, C. W., I. A. M. van Roovert-Reijink, C. M. Maatjens, I. van den Anker, B. Kemp, and $\mathrm{H}$. van den Brand. 2014. Effect of eggshell temperature throughout incubation on broiler hatchling leg bone development. Poultry science. 93:2878-2883.

Van der Pol, C. W., R. Molenaar, C. J. Buitink, I. A. M. van Roovert-Reijink, C. M. Maatjens, $\mathrm{H}$. van den Brand, and B. Kemp. 2015. Lighting schedule and dimming period in early life: consequences for broiler chicken leg bone development. Poultry science. 94:2980-2988.

Venäläinen, E., J. Valaja, and T. Jalava. 2006. Effects of dietary metabolisable energy, calcium and phosphorus on bone mineralisation, leg weakness and performance of broiler chickens. British poultry science. 47:301-310.

Ventura, B. A., F. Siewerdt, and I. Estevez. 2012. Access to barrier perches improves behavior repertoire in broilers. Plos one. 7:29826.

Vestergaard, S. and G. S. Sanotra.1999. Relationships between leg disorders and changes in the behaviour of broiler chickens. Veterinary record. 144:205-209.

Veum, T. L. 2010. Phosphorus and calcium nutrition and metabolism. Phosphorus and calcium utilization and requirements in farm animals. p94-111.

Vieira, B.S., Caramori, J.G., Oliveira, C.F.S. and Correa, G.S.S., 2018. Combination of phytase and organic acid for broilers: role in mineral digestibility and phytic acid degradation. World's poultry science journal. 74:711-726.

Vieira, S. L. and C. R. Angel. 2012. Optimizing broiler performance using different amino acid density diets: what are the limits?. Journal of applied poultry research. 21:149-155.

Waldenstedt, L. 2006. Nutritional factors of importance for optimal leg health in broilers: A review. Animal feed science and technology. 126:291-307.

Wallenbeck, A., S. Wilhelmsson, L. Jönsson, S. Gunnarsson, and J. Yngvesson. 2016. Behaviour in one fast-growing and one slower-growing broiler (Gallus gallus domesticus) hybrid fed a high-or low-protein diet during a 10-week rearing period. Acta Agriculturae Scandinavica: Section A-Animal science. 66:168-176.

Wang, G., L. Liu, Z. Wang, X. Pei, W. Tao, Z. Xiao, B. Liu, M. Wang, G. Lin, and T. Ao. 2019. Comparison of inorganic and organically bound trace minerals on tissue mineral deposition and fecal excretion in broiler breeders. Biological trace element research. 189:224-232.

Watkins, K.L. and Southern, L.L., 1992. Effect of dietary sodium zeolite A and graded levels of calcium and phosphorus on growth, plasma, and tibia characteristics of chicks. Poultry science. 71:1048-1058.

Wedekind, K. J., E. C. Titgemeyer, A. R. Twardock, and D. H. Baker. 1991. Phosphorus, but not calcium, affects manganese absorption and turnover in chicks. The journal of nutrition. 121:1776-1786.

Weeks, C. A., T. D. Danbury, H. C. Davies, P. Hunt, and S. C. Kestin. 2000. The behaviour of broiler chickens and its modification by lameness. Applied animal behaviour science. 67:111-125.

Wideman Jr, R. F. 2016. Bacterial chondronecrosis with osteomyelitis and lameness in broilers: a review. Poultry science. 95:325-344.

Wideman Jr, R. F. and I. Pevzner. 2012. Dexamethasone triggers lameness associated with necrosis of the proximal tibial head and proximal femoral head in broilers. Poultry science. 91:2464-2474. 
Wideman Jr, R. F. and R. D. Prisby. 2013. Bone circulatory disturbances in the development of spontaneous bacterial chondronecrosis with osteomyelitis: a translational model for the pathogenesis of femoral head necrosis. Frontiers in endocrinology. 3:183.

Wideman Jr, R. F., K. R. Hamal, J. M. Stark, J. Blankenship, H. Lester, K. N. Mitchell, G. Lorenzoni, and I. Pevzner. 2012. A wire-flooring model for inducing lameness in broilers: evaluation of probiotics as a prophylactic treatment. Poultry science. 91:870-883.

Wijnen, H. J., H. van den Brand, A. Lammers, I. A. M. van Roovert-Reijrink, C. W. van der Pol, B. Kemp, and R. Molenaar. 2020. Effects of eggshell temperature pattern during incubation on primary immune organ development and broiler immune response in later life. Poultry science. 99:6619-6629.

Williams, B., D. Waddington, D. H. Murray, and C. Farquharson. 2004. Bone strength during growth: influence of growth rate on cortical porosity and mineralization. Calcified tissue international. 74:236-245.

Williams, B., S. Solomon, D. Waddington, B. Thorp, and C. Farquharson. 2000. Skeletal development in the meat-type chicken. British poultry science. 41:141-149.

Wilson, H. R. 1990. Physiological requirements of the developing embryo: temperature and turning. In Avian incubation. pl45-156.

Witkowska, Z., Świniarska, M., Korczyński, M., Opaliński, S., Konkol, D., Michalak, I., Saeid, A., Mironiuk, M. and Chojnacka, K., 2019. Biofortification of hens' eggs with microelements by innovative bio-based dietary supplement. Journal of animal physiology and animal nutrition. 103:485-492.

Wong-Valle, J., G. R. McDaniel, D. L. Kuhlers, and J. E. Bartels. 1993. Divergent gen etic selection for incidence of tibial dyschondroplasia in broilers at seven weeks of age. Poultry science. 72:421-428.

Xu, Y., Ye, J., Zhou, D. and Su, L., 2020. Research progress on applications of calcium derived from marine organisms. Scientific Reports. 10:1-8.

Yair, R., Z. Uni, and R. Shahar. 2012. Bone characteristics of late-term embryonic and hatchling broilers: Bone development under extreme growth rate. Poultry science. 91:26142620.

Yalçın, S., H. B. Malayoğlu, M. Baka, O. Genin, and M. Pines. 2007. Effect of temperature during the incubation period on tibial growth plate chondrocyte differentiation and the incidence of tibial dyschondroplasia. Poultry science. 86:1772-1783.

Yalçın, S., S. Özkan, E. Coşkuner, G. Bilgen, Y. Delen, Y. Kurtulmus, and T. Tanyalçın. 2001. Effects of strain, maternal age and sex on morphological characteristics and composition of tibial bone in broilers. British poultry science. 42:184-190.

Yıldız, H., M. Petek, G. Sönmez, I. Arıcan, and B. Yılmaz. 2009. Effects of lighting schedule and ascorbic acid on performance and tibiotarsus bone characteristics in broilers. Turkish journal of veterinary and animal sciences. 33:469-476.

YU, Y., Z. Li, Z. Zhong, S. Jin, J. Pan, X. Rao, and Y. Yu. 2018. Effect of monochromatic green LED light stimuli during incubation on embryo growth, hatching performance, and hormone levels. Transactions of the ASABE. 61:661-669.

Zhang, L., Wu, S., Wang, J., Qiao, X., Yue, H., Yao, J., Zhang, H. and Qi, G., 2014. Changes of plasma growth hormone, insulin-like growth factors-l, thyroid hormones, and testosterone concentrations in embryos and broiler chickens incubated under monochromatic green light. Italian Journal of Animal Science, 13:530-535.

Zhang, L., X. D. Zhu, X. F. Wang, J. L. Li, F. Gao, and G. H. Zhou. 2016. Green light-emitting diodes light stimuli during incubation enhances posthatch growth without disrupting normal eye development of broiler embryos and hatchlings. Asian-Australasian journal of animal sciences. 29:1562-1568.

Zhao, J. P., H. C. Jiao, Y. B. Jiang, Z. G. Song, X. J. Wang, and H. Lin. 2013. Cool perches improve the growth performance and welfare status of broiler chickens reared at different stocking densities and high temperatures. Poultry science. 92:1962-1971.

Zikic, D., Djukic-Stojcic, M., Bjedov, S., Peric, L., Stojanovic, S. and Uscebrka, G., 2017. Effect of litter on development and severity of foot-pad dermatitis and behavior of broiler chickens. Brazilian Journal of Poultry science. 19:247-254.

Zuidhof, M. J., B. L. Schneider, V. L. Carney, D. R. Korver, and F. E. Robinson. 2014. Growth, efficiency, and yield of commercial broilers from 1957, 1978, and 2005. Poultry science. 93:2970-2982. 



\author{
B. C. Güz' \\ R. Molenaar ${ }^{1}$ \\ I. C. de Jong ${ }^{2}$ \\ B. Kemp' \\ H. van den Brand \\ M. van Krimpen ${ }^{2}$
}

Poultry Science 2019

98:6552-6563

doi.org/10.3382/ps/pez427

1 Adaptation Physiology Group,

Wageningen University and Research, 6708 PB

Wageningen,

Gelderland,

The Netherlands

2 Wageningen Livestock Research,

Wageningen University and Research, 6708 WD

Wageningen,

Gelderland,

The Netherlands 



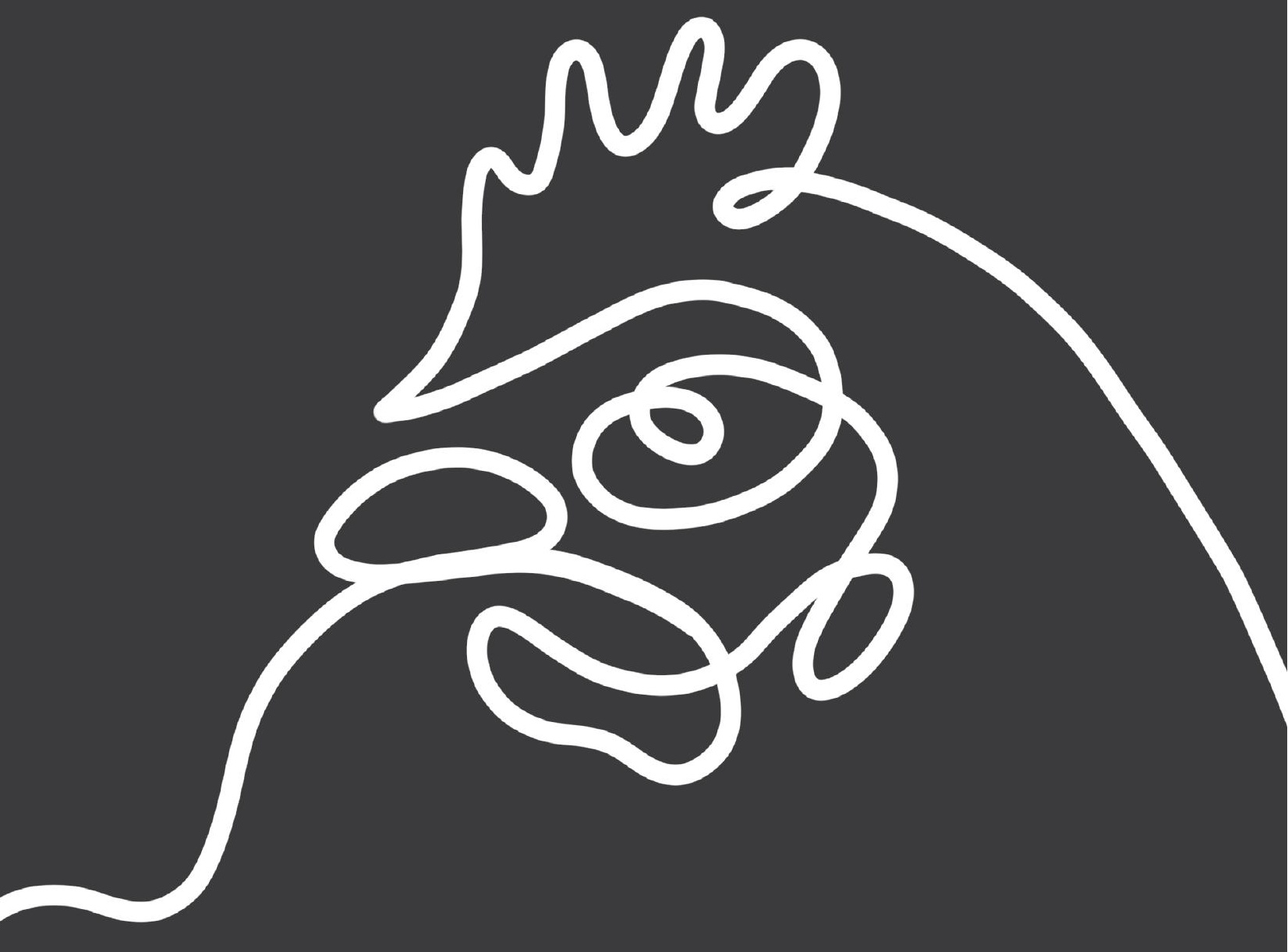




\section{Abstract}

Nutrition is a crucial factor for growth and bone development in broiler chickens. Adjustments in dietary ingredients might affect bone development and consequently locomotion related problems. This study was designed to evaluate effects of dietary organic minerals (ORM), fish oil (FISH) and hydrolyzed collagen (COL) on growth performance and tibia characteristics of broiler chickens. A total of three hundred eighty four 1-day-old Ross 308 male broiler chickens were used in a complete randomized block design with 4 diet groups and 8 replicates per diet group. In the ORM diet, the inorganic macro and trace minerals were replaced by their organic varieties. In the FISH diet, palm oil and soybean oil were partly replaced by FISH. In the COL diet, soybean meal was partly replaced by COL. Results showed that the ORM and $\mathrm{COL}$ diet groups reached a higher body weight (BW) at $42 \mathrm{D}$ of age than the FISH diet group, whereas the control group was in between. The feed conversion ratio between day 1 and 42 was lower in the ORM and $\mathrm{COL}$ diet groups than in both other diet groups. On day 28, 35, and 42, gait score (GS), Varus Valgus, tibia length (TL), thickness, femoral and metatarsal head thickness (THT), mineral content (TMC), mineral density (TMD), breaking strength (TBS), stiffness (TSF) and energy to fracture (TEF) were measured ( $n=3 /$ replicate). The ORM diet group had higher TL at day 42, higher THT at day 28, higher TMC at day 42 , higher TMD at day 28,35 and 42 , higher TBS at day 42 , higher TSF at day 35 and 42 , and higher TEF at day 42 compared to the FISH diet group, with the $\mathrm{COL}$ and control diet groups in between. It can be concluded that replacing dietary inorganic macro and trace minerals by their organic varieties seems to stimulate tibia dimensions, strength, and mineral content of broiler chickens. On the contrary, FISH appears to negatively affect tibia characteristics.

\section{Key words}

Organic minerals, fish oil, collagen, broiler chicken, tibia 


\section{INTRODUCTION}

Leg problems is one of the most important factors affecting health and welfare of broiler chickens nowadays (EFSA, 2010). It has been demonstrated that over $27.6 \%$ of the broiler chickens in the UK suffer from poor locomotion and 3.3\% were unable to walk, especially in the last 2 week of the growing period (Knowles et al., 2008) and this resulted in pain and inability to reach feed and water (Bessei, 2006; Gocsik et al., 2017). It has been shown that approximately $50 \%$ of broiler chickens in Dutch flocks suffered from locomotion related problems at slaughter age (De Jong and Guémené, 2017 ). Locomotion related problems, i.e., weak bones or joints may result in broken bones, punctured skins and/or damaged muscles, especially when handling the chickens during depopulation or when processing in the slaughter plant. Next to welfare problems, carcass quality and slaughter revenues can also be negatively affected by leg problems (Yalçın et al., 1998; Kestin et al., 1999; Mench, 2004).

Most of the locomotion-related research in broiler chickens focused on improving tibia bone development and its strength (Ruff and Hughes, 1985; Kim et al., 2006), because the tibia is the most loaded and affected (by, e.g., bone pathologies) leg bone during the growth period, probably influencing the locomotion of the broiler chickens (Julian, 1998; Dibner et al., 2007).

Various factors to reduce locomotion-related problems in broiler chickens have been studied and nutritional approaches seem to be very promising (Kidd, 2003; Calini and Sirri, 2007). Specific ingredients or nutrients in broiler chicken diets might positively influence bone development, thereby reducing locomotion related problems in later life (Yalçın et al., 1998; Oviedo-Rondon et al., 2006). Among others, organic macro and trace minerals, lipid composition and collagen has been shown or suggested to affect leg bone development. Replacing inorganic by organic macro and trace minerals in broiler diets has shown to improve intestinal absorption of those minerals (Wedekind et al., 1991; Burrell et al., 2004; Wang and XU, 2008), resulting in greater bio-availability and higher bone mineralization in broiler chickens.

Fish oil (FISH) is containing n-6 polyunsaturated fatty acids (PUFA), which differently affect osteoblast function and bone mineralization than 
the common fat sources of broiler diets containing n-3 PUFA, sUch as palm oil, maize oil and soy bean oil (Watkins et al., 1996, 2003), but effects on broiler chickens bone development is largely unknown.

Finally, supplementation with hydrolyzed collagen $(\mathrm{COL})$ in mice diets resulted in a higher bone mineral content and bone mineral density, and a higher concentration of type I collagen and proteoglycans in the bone matrix (Wu et al., 2004; Nomura et al., 2005; Guillerminet et al., 2010, 2012). It can be speculated that $\mathrm{COL}$ in broiler chicken diets might stimulate bone development, but evidence for that is very limited.

The main objectives of this study were to investigate effects of dietary organic macro and trace minerals, fish oil and hydrolyzed collagen on: 1) growth performance; 2) tibia characteristics; 3) locomotion; 4) leg disorders and 5) bone development related blood parameters of broiler chickens.

\section{MATERIALS AND METHODS Experimental Design}

A total of 4 dietary groups, including 1 control diet (CON) and 3 modified diets (FISH, COL and ORM) were compared. Each diet group was replicated 8 times. A total of 32 experimental pens within a complete randomized block design were used. Within each block of 4 pens, diet groups were randomly distributed. Pen was used as the experimental unit and each pen contained 12 male broiler chickens.

\section{Animals and Experimental Procedures}

The experiment was conducted at the Animal Sciences Department of Wageningen University and Research, Wageningen, The Netherlands. All procedures in this study were approved by the Central Commission Animal Experiments, The Hague, The Netherlands (Approval number: 2016.D0138.001).

A total of 384 one-day-old Ross 308 male broiler chickens from a 38-week-old breeder flock were obtained from a commercial hatchery (Lagerwey, Lunteren, The Netherlands). Chickens were vaccinated against infectious bronchitis (eye drop; MSD Animal Health, Boxmeer, The 
Netherlands) upon arrival at the research facility and against Newcastle disease (Nobilis ND Clone 30; eye drop; MSD Animal Health, Boxmeer, The Netherlands) at day 11 of age. Upon arrival at day 0 , all chickens were individually weighed, wing tagged and randomly assigned to 32 pens in a climate-controlled room. Temperature was maintained at $32^{\circ} \mathrm{C}$ until day 3 and thereafter gradually reduced to $22^{\circ} \mathrm{C}$ at day 42 . A continuous light program from arrival to day 3 and a 16L:8D light program from day 4 to 42 was applied. Chickens were raised from arrival to day 42 with ad libitum access to feed and water.

\section{Experimental Diets}

A 3-phase feeding program was applied; starter diets were provided from day 0 to 10, grower diets from day 11 to 28 and finisher diets from day 29 to 42. Dietary treatments were applied throughout all 3 phases. Four experimental diets were used in this experiment.

They are as follows:

\section{1) control (CON);}

2) partly replacement of inorganic by organic macro (Ca, P) and complete replacement of trace minerals ( $\mathrm{Fe}, \mathrm{CU}, \mathrm{Mn}, \mathrm{Zn}$ and $\mathrm{Se}$ )(ORM);

3) partly replacement of palm oil and soybean oil by fish oil (FISH);

4) partly replacement of soybean meal by hydrolyzed collagen (COL).

In the ORM diet, the inorganic macro minerals $\mathrm{Ca}$ and $\mathrm{P}$, provided by limestone and monocalcium phosphate were partly replaced by Calfos (Darling Ingredients Inc., Eindhoven, The Netherlands), an organic $\mathrm{Ca}$ and $\mathrm{P}$ source originating from processed bones. This was done for $70.9 \%$ in the starter diet, for $59.6 \%$ in the grower diet and for $44.7 \%$ in the finisher diet. The trace mineral premix with in ORM was completely replaced by a complete organic sourced trace mineral premix (Optimin, Trouw Nutrition, Tilburg, The Netherlands).

In the FISH diet, palm oil and soybean oil were partly replaced by fish oil $100 \%$ in starter diet, $86.5 \%$ in grower diet, $98.4 \%$ in finisher diet; Trouw Nutrition, Tilburg, The Netherlands). Fish oil content was $39.4 \mathrm{~g} / \mathrm{kg}$ in the starter diet and $50 \mathrm{~g} / \mathrm{kg}$ in the grower and finisher diet. 
In the COL diet, soybean meal was partly $10 \%$ in starter diet, $9.2 \%$ in grower diet, $11 \%$ in finisher diet) replaced by hydrolyzed collagen Hydro-P (Darling Ingredients Inc., Eindhoven, The Netherlands), originating from pigs. Collagen content was $25 \mathrm{~g} / \mathrm{kg}$ in starter, grower and finisher diet.

All diets were produced and pelleted by Research Diet Services (Wijk bij Duurstede, The Netherlands) and analyzed for ash (ISO5984), dry matter (ISO6496), crude fibre (ISO6865), crude fat (ISO6492), crude protein (ISO5983), P (ISO6941) and Ca (ISO 6869). CON and ORM diets were analyzed for Fe, CU, Mn, Zn and Se. CON and FISH diet were analyzed for fatty acid composition, using the method described by khan et al. (2009). Diet compositions, calculated and analyzed nutrient values are shown in Table 1.

\section{Data Collection, Sampling and Measurements}

All chickens were individually weighed on d 0, 10, 21, 28, 35 and 42. Feed intake (FI) was measured per pen for the starter, grower and finisher period. Feed conversion ratios were calculated for all the three phases and over the whole growth period.

Mortality was determined per pen on daily basis. Gait score was evaluated in 2 randomly chosen chickens per pen, using the method of Kestin et al., 1992 on d 27, 34 and 41 and scored within a range of 0 (normal locomotion) to 5 (unable to stand).

To investigate whether the used diets might affect home pen behaviour, observations were performed on d 6, 13, 20, 27, 34 and 41 with morning and afternoon sessions using the scan sampling technique. During 6 to 8 min per day per pen, the number of chickens performing the following activities was scored: eating, drinking, walking, standing, resting, foraging, comfort behaviour, dust bathing and perching.

On d 28, 35 and 42, three chickens per pen were randomly selected and sacrificed by cervical dislocation. At d 42, immediately after sacrificing, chickens were decapitated and a blood sample (10 $\mathrm{ml}$ ) was obtained. Blood samples were centrifuged at $750 \mathrm{~g}$ for $10 \mathrm{~min}$ to obtain serum. Serum of the three samples per pen were pooled $(1.5 \mathrm{ml})$ and frozen at $-20^{\circ} \mathrm{C}$ until further analysis. Serum contents of $\mathrm{Ca}, \mathrm{P}$ and alkaline 


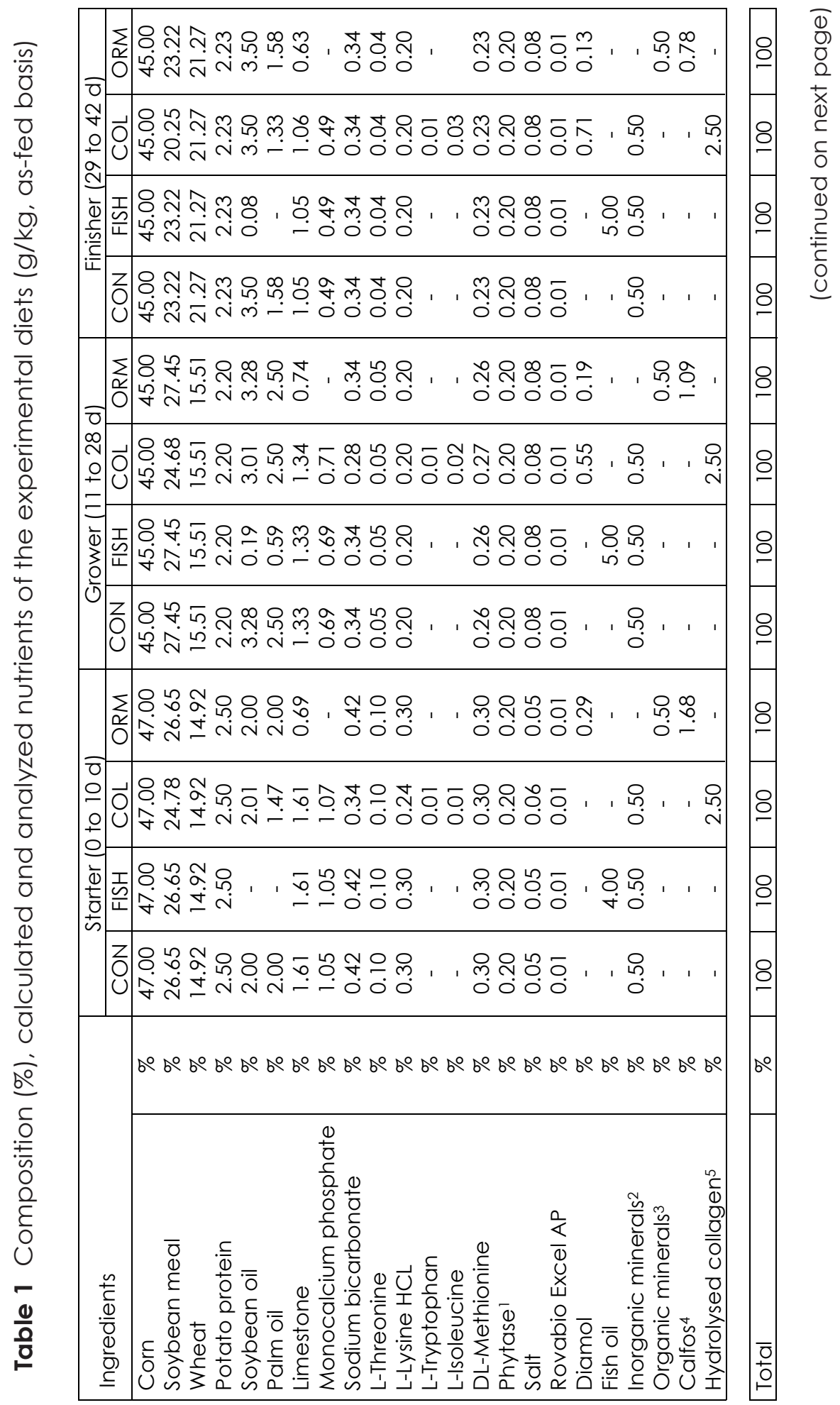




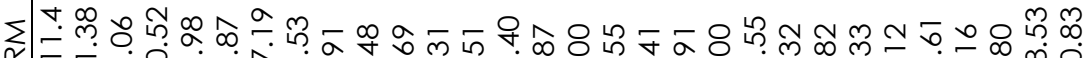
少 $\widetilde{\nabla}$

ข. 니요

ప I

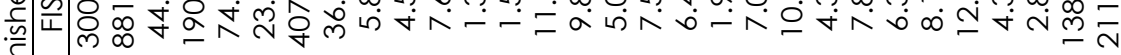

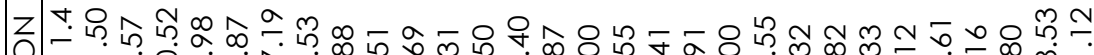

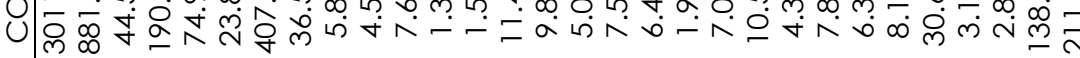

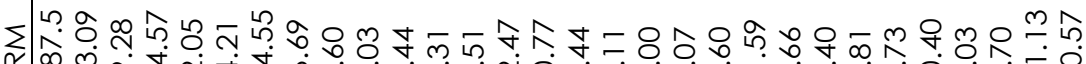

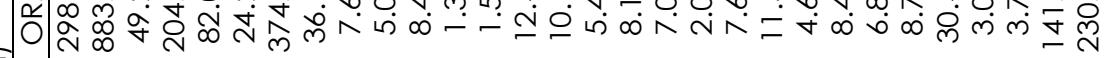
бे

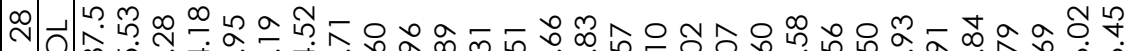

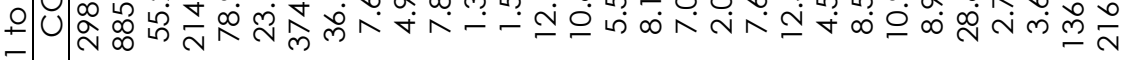

=

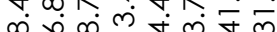

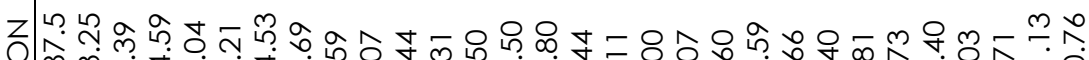

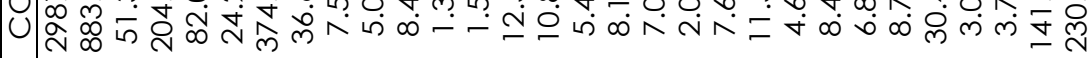

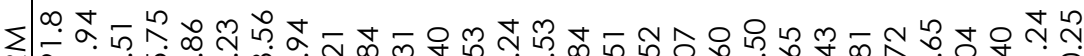

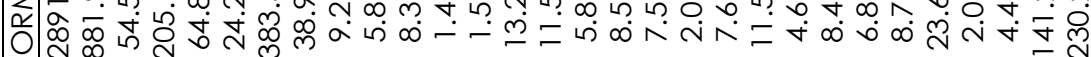
ठ

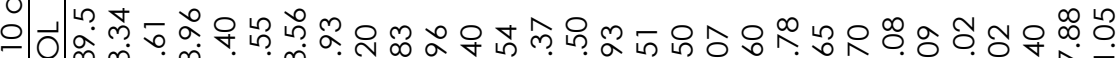

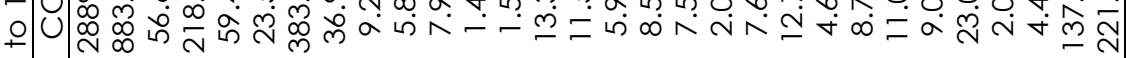
으

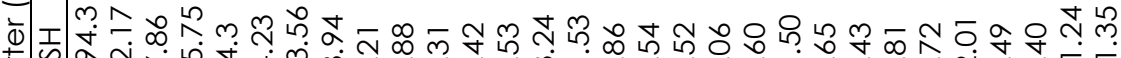

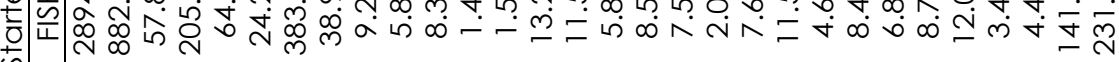

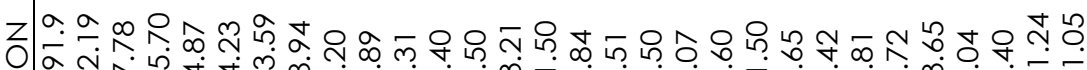
U

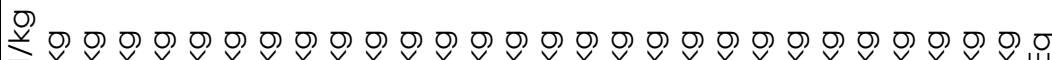

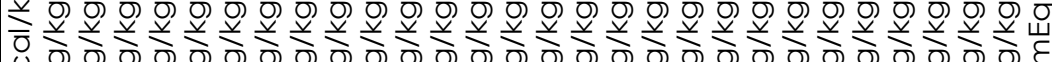
U

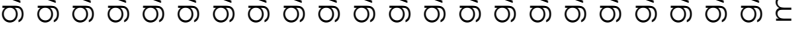




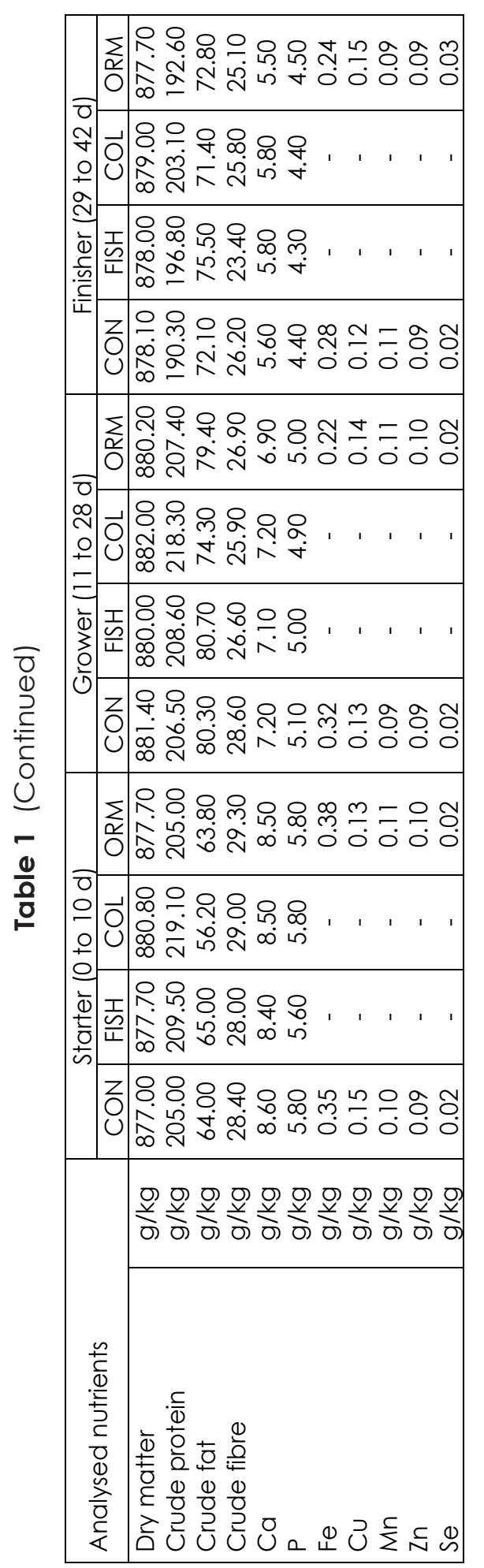

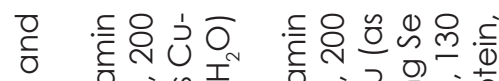
․

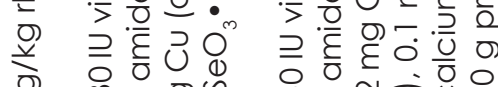
के के o

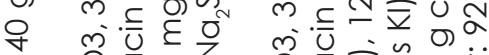

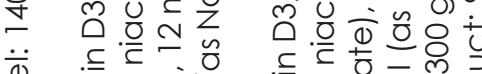

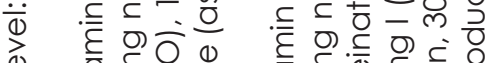

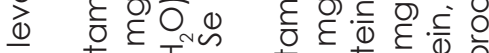

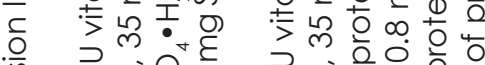

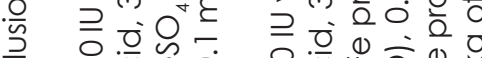

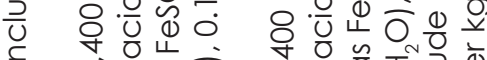
$\cong \quad$ i

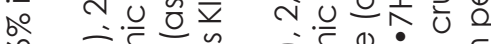
¿

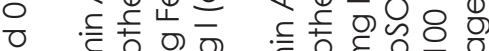

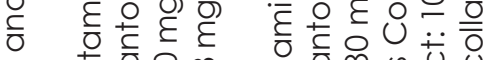

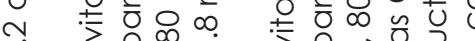
$0.4 \frac{1}{0}$ ᄃ 0 O

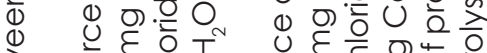

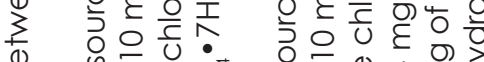

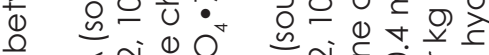

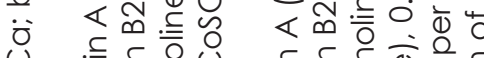

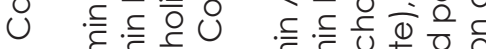

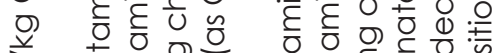

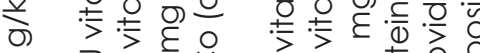

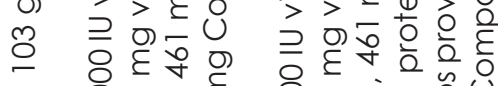

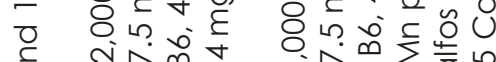

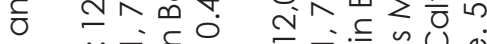

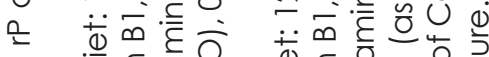

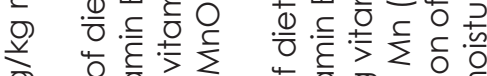
क) ᄂ) त. एँ ब O

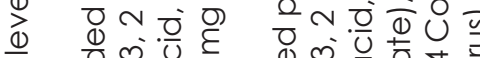
(1) $\frac{0}{2} \dot{\frac{m}{2}}$

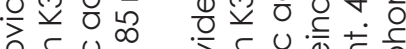
这.

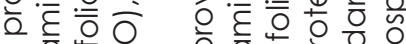

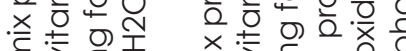
है ब

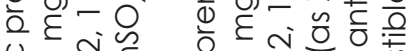

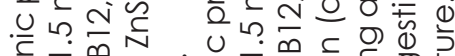
을 क⿺辶一兀

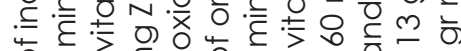

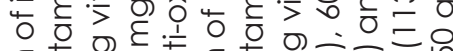

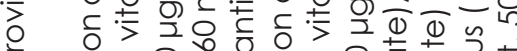

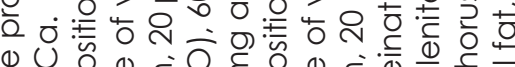

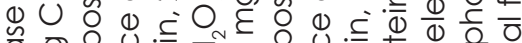

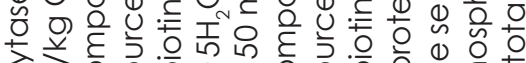

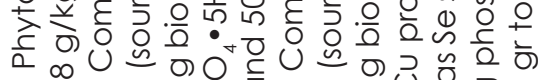


phosphatase (ALP; related to bone mineralisation; Orimo, 2010) were analysed photo-metrically, using a Cobas c701 analyser (Roche ${ }^{\circledR}$, Basel, Switzerland). Serum vitamin D3 (a major Ca and P regulator; Van Leeuwen et al., 2001) was analysed using a 1,25-dihydroxyvitamin D3 ELISA Kit (Elabscience ${ }^{\circledR}$, Houston, Texas, United States) and serum parathyroid hormone (PTH a developmental regulator in cartilage and bone; Martin, 2016) was

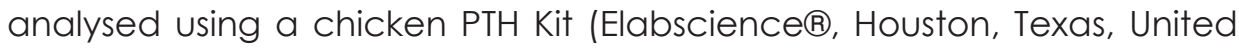
States).

Three slaughtered chickens per pen were checked on Varus Valgus by a veterinarian at each of three slaughtering days (d 28, 35, 42). Varus valgus was scored as present or not and each (small) deviation from normal was scored as present. Both tibia bones were collected, deboned and frozen at $-20^{\circ} \mathrm{C}$. After thawing, proximal length $(\mathrm{cm})$, lateral cortex thickness $(\mathrm{cm})$ and proximal head thickness $(\mathrm{cm})$ at both the femoral and metatarsal side were measured using a digital calliper (Figure 1). Tibia mineral content $(\mathrm{g})$ and mineral density $\left(\mathrm{g} / \mathrm{cm}^{2}\right)$ were analysed using a dual-energy X-ray absorptiometry machine (Horizon® DEXA System by Hologic, Tromp Medical, Castricum, The Netherlands) using pooled samples (2 pens or 6 tibia of the same group per scan).

Tibia bones were subjected to a three-point bending test (method described by Jungmann et al., 2007) using an Instron® electromechanical universal testing machine (Instron® ${ }^{\circledR}$, Norwood, Massachusetts, United States). Maximum load to break the tibia $(\mathrm{N})$, tibia stiffness $(\mathrm{N} / \mathrm{mm})$, and total energy to fracture ( $\mathrm{N}-\mathrm{mm}$ ) were calculated, where;

Stiffness (the slope of the linear part of the curve,

$$
\mathrm{N} / \mathrm{mm})=d x / d y,
$$

where $d x=$ the initial point (a) from $x$-axis $(N), d y=$ the ultimate point (b) from y-axis (mm);

Energy to fracture (the area under the curve, $\mathrm{N}-\mathrm{mm}$ ) $=\int_{\min }^{\max } f(x) \cdot d x$

where $f(x)=$ the curve of $y$-axis, $d x$ is linear integral equation (Figure 2). 
Femoral side proximal head thickness

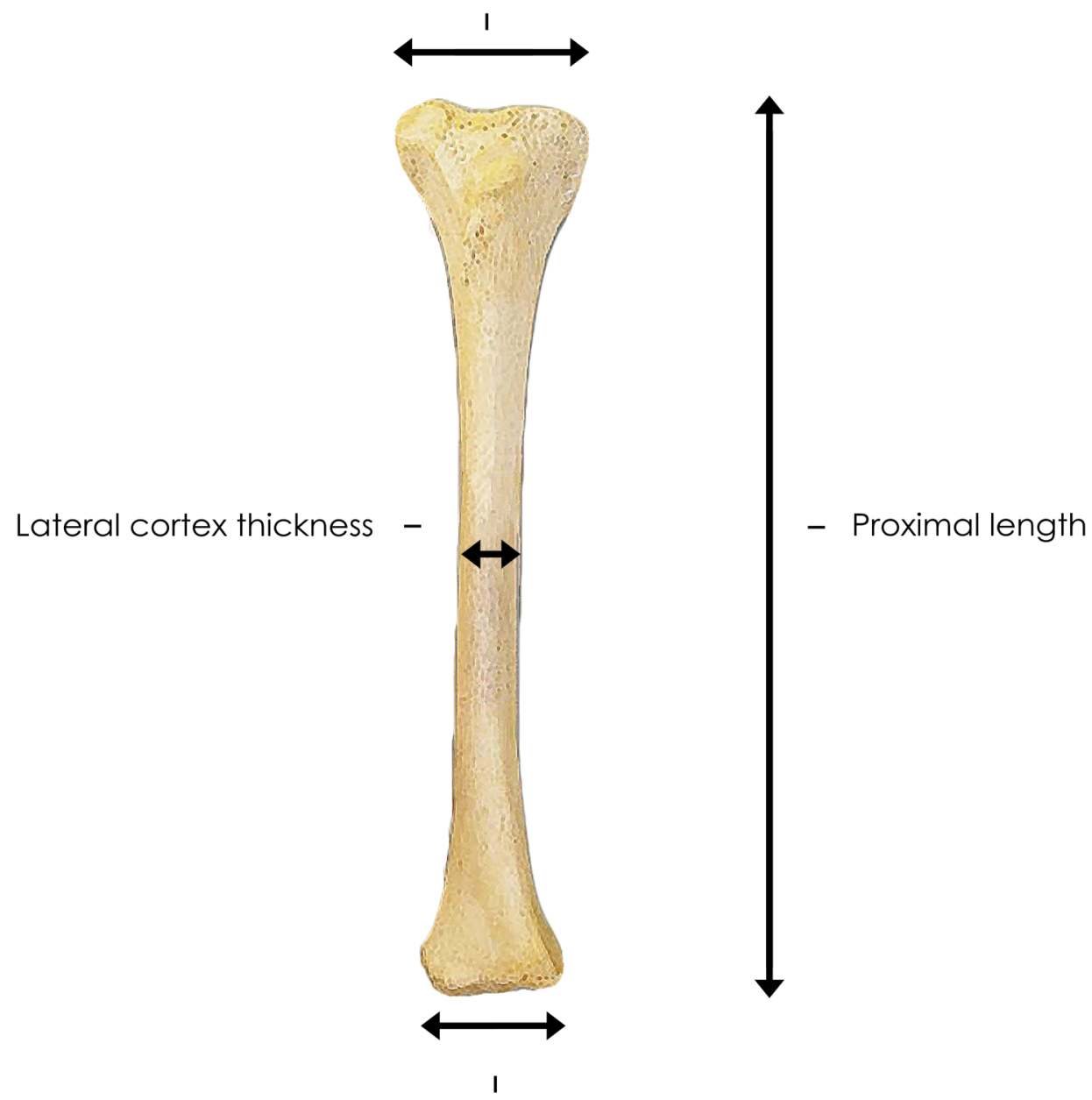

Metatarsal side proximal head thickness

\section{Figure 1}

Morphological measurements of proximal length, lateral cortex thickness, femoral, and metatarsal side bone head thickness on tibia $(\mathrm{cm})$ 


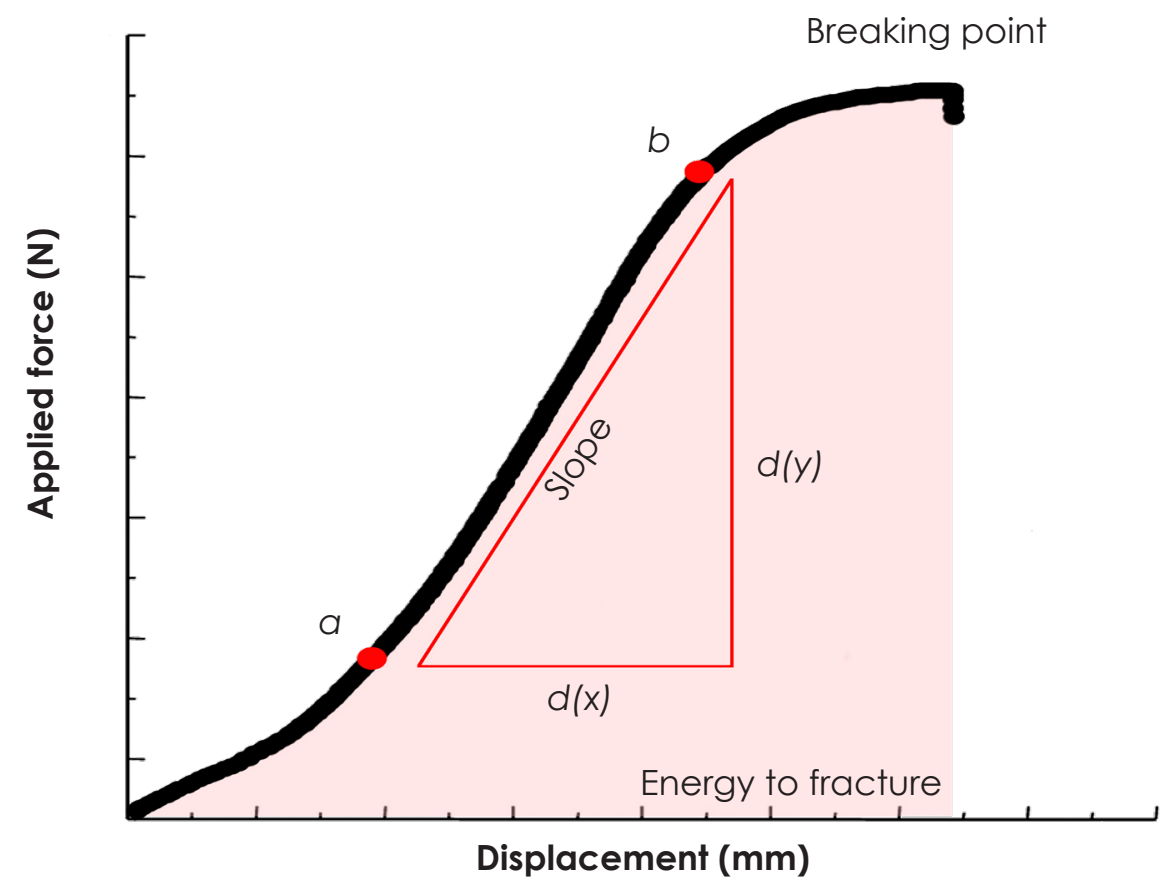

Figure 2

Demonstration of breaking strength (breaking point, N), stiffness (the slope of the linear part of the curve, $\mathrm{N} / \mathrm{mm}$ ), and energy to fracture (the area under the curve, $\mathrm{N}-\mathrm{mm}$ ) during applied force on tibia

\section{Statistical Analysis}

All growth performance data (body weight, feed intake, feed conversion ratio, mortality), tibia characteristics (proximal length, lateral cortex thickness, proximal head thickness, mineral content, mineral density, breaking strength, stiffness and energy to fracture), blood parameters (Ca, P, PTH, ALP and 1.25-dihydroxy vitamin D3) and locomotion-related observations (home pen behaviour and gait score) were subjected to mixed model analysis using the PROC MIXED procedure. In one pen of the CON diet, 3 female chickens were present and this pen was removed from the experiment for all analyses. Pen was used as the experimental unit, except for tibia mineral content and mineral density, where the combination of two pens was used as the experimental unit. 
The overall statistical model used was:

$$
Y i=\mu+\text { Diet group } i+\varepsilon i
$$

where $\mathrm{Yi}=$ the dependent variable, $\mu$ is overall mean, Diet group = Dietary group $(\mathrm{i}$ $=$ control diet, organic minerals diet, fish oil diet, collagen diet) and $\varepsilon=$ the residual error term.

Varus valgus was subjected to generalized linear mixed model analysis, using the PROC GLIMMIX procedure in SAS using same statistical model (Version 9.4, July 2013, SAS Institute Inc., Cary, North Carolina, United States). Block was used as a random effect. BW was added to the model as a covariable for tibia characteristics. Distribution of the means and residuals were examined to verify model assumptions. Results are presented as LSmeans \pm SEM. When multiple comparisons were performed, the level of significance was corrected, using Bonferroni. Effects were considered to be significant when $P \leq 0.05$.

\section{RESULTS \\ Growth Performance}

All growth performance parameters are presented in Table 2. At d 10, the FISH diet group had a lower BW compared to the other diet groups $(\Delta=12-18 \mathrm{~g}, \mathrm{P}=0.001)$. At $\mathrm{d} 42$, the $\mathrm{COL}$ and ORM diet groups had a higher BW than the FISH diet group ( $\Delta=107-138 \mathrm{~g}$; $\mathrm{P}=0.026$ ), with the CON diet group in between and not different from the other diet groups. At the other measuring days, no difference in BW was observed between diet groups.

ADFI was not influenced by diet group throughout the experiment. Chickens of the COL and ORM diet groups had a lower feed conversion ratio (FCR) during the finisher diet phase (d 29 to $42, \Delta=0.09-0.12 ; \mathrm{P}=0.041$ ) and throughout the experiment ( 0 to $42, \Delta=0.06-0.09 ; P=0.024$ ) compared to the FISH and CON diet groups. No significant differences in FCR were found during the starter and grower phase. Mortality was low and not influenced by diet groups ( $1.30 \%$ on average).

\section{Tibia Characteristics}

Tibia characteristics are presented in Table 3. At d 28, the femoral 
side of the proximal tibia head was thicker in ORM diet group compared to the FISH diet group ( $\Delta=0.11 \mathrm{~cm} ; \mathrm{P}=0.047$ ), with both other diet groups in between and not different from the ORM and FISH diet groups. Additionally, at $d 28$, tibia mineral density was higher in the ORM diet group than the other three diet groups $\left(\Delta=0.09-0.17 \mathrm{~g} / \mathrm{cm}^{2} ; \mathrm{P}=0.039\right)$.

At d35, tibia mineral density was higher in the ORM diet group than in the other three diet groups $\left(\Delta=0.15-0.27 \mathrm{~g} / \mathrm{cm}^{2} ; \mathrm{P}=0.018\right)$. Furthermore, at $\mathrm{d} 35$, tibia stiffness was higher in the ORM diet group than in the FISH and $\mathrm{COL}$ diet groups $(\Delta=11 \mathrm{~N} / \mathrm{mm} ; \mathrm{P}=0.039)$, with the CON diet group in between and not different from the other three diet groups.

At $d 42$, chickens of the $C O L$ and ORM diet groups had longer proximal tibia compared to the FISH diet group $(\Delta=0.5-0.6 \mathrm{~cm}$; $P=0.012)$, while the CON diet group was in between and not different from the other diet groups. Tibia mineral content ( $\Delta=3.47-5.02 \mathrm{~g} ; \mathrm{P}=0.013$ ), tibia mineral density $(\Delta=0.063-0.065 \mathrm{~g} / \mathrm{cm} 2 ; P=0.010)$ and tibia breaking strength $(\Delta=28-32$ $\mathrm{N} ; \mathrm{P}=0.001$ ) were higher in the ORM diet group than in the three other diet groups. Tibia stiffness $(\Delta=21-23 \mathrm{~N} / \mathrm{mm} ; \mathrm{P}=0.011)$ and tibia energy to fracture ( $\Delta=17-20 \mathrm{~N}-\mathrm{mm} ; \mathrm{P}=0.013)$ were higher in the ORM diet group than the $\mathrm{CON}$ and FISH diet groups, with the $\mathrm{COL}$ diet group in between and not different from the other diet groups.

\section{Locomotion-Related Observations and Bone Development Related Blood Parameters}

Home pen behaviour parameters (eating, drinking, walking, standing, resting, foraging, comfort behaviour, dust bathing and perching) for each of the scanning days (d 6, 13,20,27, 34 and 41) are presented in Table Al in the Supplementary Data. Locomotion-related observation parameters (gait score and varus valgus) are presented in Table 4. No significant differences between diet groups were found for any of these parameters throughout the experiment. Age based differences were found in eating $(P=0.04)$, drinking $(P=0.003)$, walking $(P=0.003)$, standing $(P=0.004)$, resting $(P<0.001)$ and foraging $(P=0.007)$ behaviours. Blood parameters (Ca, P, ALP, PTH and 1,25-dihydroxyvitamin D3) are presented in Table 5. No significant differences between diet groups were found for any of these blood parameters. 
Table 2 Body weight, feed intake, feed conversion ratio, and mortality of broiler chickens fed a control, fish oil, collagen and organic minerals diet

\begin{tabular}{|l|c|c|c|c|c|c|}
\hline Measurement & Control & Fish Oil & Collagen & $\begin{array}{c}\text { Organic } \\
\text { Minerals }\end{array}$ & SEM & P value \\
\hline $\mathrm{n}$ (pens) & 7 & 8 & 8 & 8 & & \\
Body weight (g) & & & & & & \\
$0 \mathrm{~d}$ & 48 & 48 & 48 & 48 & 1 & 0.967 \\
$10 \mathrm{~d}$ & $248^{\mathrm{a}}$ & $236^{\mathrm{b}}$ & $254^{\mathrm{a}}$ & $254^{\mathrm{a}}$ & 3 & 0.001 \\
$21 \mathrm{~d}$ & 983 & 957 & 975 & 988 & 15 & 0.445 \\
$28 \mathrm{~d}$ & 1706 & 1655 & 1724 & 1724 & 27 & 0.238 \\
$35 \mathrm{~d}$ & 2618 & 2593 & 2647 & 2683 & 30 & 0.208 \\
$42 \mathrm{~d}$ & $3574 \mathrm{ab}$ & $3497 \mathrm{~b}$ & $3635 \mathrm{a}$ & $3604 \mathrm{a}$ & 33 & 0.026 \\
Feed intake (g) & & & & & & \\
$0-10 \mathrm{~d}$ & 240 & 243 & 241 & 246 & 7 & 0.909 \\
$11-28 \mathrm{~d}$ & 1957 & 1953 & 1956 & 1934 & 24 & 0.853 \\
$29-42 \mathrm{~d}$ & 2901 & 2813 & 2891 & 2845 & 42 & 0.633 \\
$0-42 \mathrm{~d}$ & 5098 & 5009 & 5087 & 5025 & 76 & 0.586 \\
& & & & & & \\
Feed conversion & & & & & & \\
ratio (Fl/BWG) & 1.50 & 1.47 & 1.49 & 1.46 & 0.04 & 0.897 \\
$0-10 \mathrm{~d}$ & 1.54 & 1.55 & 1.54 & 1.48 & 0.03 & 0.683 \\
$11-28 \mathrm{~d}$ & $1.64^{\mathrm{b}}$ & $1.63^{\mathrm{b}}$ & $1.60 \mathrm{~b}$ & $1.55^{\mathrm{a}}$ & 0.03 & 0.041 \\
$29-42 \mathrm{~d}$ & $1.54^{\mathrm{b}}$ & $1.56^{\mathrm{b}}$ & $1.55^{\mathrm{b}}$ & $1.51 \mathrm{a}$ & 0.02 & 0.047 \\
$0-42 \mathrm{~d}$ & 1.04 & 1.04 & 2.08 & 1.04 & 0.01 & 0.887 \\
\hline Mortality (\%) & & & & & & \\
\hline
\end{tabular}

$a-b$ Values within a row and factor lacking a common superscript differ $(P \leq 0.05)$. 
Table 3 Tibia characteristics of broiler chickens at 28, 35 and $42 \mathrm{~d}$ of age fed a control, fish oil, collagen or organic minerals diet

\begin{tabular}{|c|c|c|c|c|c|c|}
\hline Measurement & Control & Fish Oil & Collagen & $\begin{array}{l}\text { Organic } \\
\text { Minerals }\end{array}$ & SEM & $\begin{array}{c}P \\
\text { values }\end{array}$ \\
\hline $\mathrm{n}$ (pens) & 7 & 8 & 8 & 8 & & \\
\hline \multicolumn{7}{|c|}{ Tibia proximal length $(\mathrm{cm})$} \\
\hline $28 d$ & 8.3 & 8.3 & 8.4 & 8.4 & 0.02 & 0.233 \\
\hline $35 \mathrm{~d}$ & 10.5 & 10.5 & 10.6 & 10.6 & 0.04 & 0.263 \\
\hline $42 d$ & $13.7 a b$ & $13.4 \mathrm{~b}$ & $13.9 a$ & $14.0 a$ & 0.09 & 0.012 \\
\hline \multicolumn{7}{|c|}{$\begin{array}{l}\text { Tibia lateral cortex thickness } \\
(\mathrm{cm})\end{array}$} \\
\hline $28 d$ & 0.51 & 0.44 & 0.52 & 0.57 & 0.003 & 0.052 \\
\hline $35 d$ & 0.84 & 0.83 & 0.84 & 0.85 & 0.002 & 0.996 \\
\hline $42 \mathrm{~d}$ & 1.22 & 1.21 & 1.23 & 1.25 & 0.002 & 0.747 \\
\hline \multicolumn{7}{|c|}{$\begin{array}{l}\text { Tibia proximal bone head } \\
\text { thickness - femoral side }(\mathrm{cm})\end{array}$} \\
\hline $28 d$ & $1.58 \mathrm{ab}$ & $1.54^{b}$ & $1.59 \mathrm{ab}$ & $1.65^{a}$ & 0.02 & 0.047 \\
\hline $35 d$ & 2.54 & 2.52 & 2.54 & 2.55 & 0.03 & 0.884 \\
\hline $42 d$ & 3.24 & 3.23 & 3.24 & 3.26 & 0.03 & 0.902 \\
\hline \multicolumn{7}{|c|}{$\begin{array}{l}\text { Tibia proximal bone head } \\
\text { thickness - metatarsal side } \\
(\mathrm{cm})\end{array}$} \\
\hline $28 \mathrm{~d}$ & 1.27 & 1.26 & 1.28 & 1.34 & 0.03 & 0.268 \\
\hline $35 d$ & 2.22 & 2.22 & 2.24 & 2.25 & 0.02 & 0.887 \\
\hline $42 \mathrm{~d}$ & 3.01 & 2.99 & 2.99 & 3.05 & 0.03 & 0.635 \\
\hline \multicolumn{7}{|c|}{ Tibia mineral content ${ }^{1}(\mathrm{~g})$} \\
\hline $28 d$ & 7.58 & 7.57 & 7.70 & 7.89 & 0.17 & 0.469 \\
\hline $35 d$ & 12.98 & 12.68 & 13.53 & 15.48 & 0.83 & 0.168 \\
\hline $42 \mathrm{~d}$ & $17.32^{\mathrm{b}}$ & $17.00^{\mathrm{b}}$ & $15.77 \mathrm{~b}$ & $20.79 a$ & 0.84 & 0.013 \\
\hline \multicolumn{7}{|c|}{ Tibia mineral density ${ }^{1}\left(\mathrm{~g} / \mathrm{cm}^{2}\right)$} \\
\hline $28 d$ & $0.141^{\mathrm{b}}$ & $0.133^{b}$ & $0.140^{b}$ & $0.150^{a}$ & 0.003 & 0.039 \\
\hline $35 d$ & $0.262^{\mathrm{b}}$ & $0.250^{\mathrm{b}}$ & $0.251^{b}$ & $0.277 a$ & 0.006 & 0.018 \\
\hline $42 d$ & $0.275^{b}$ & $0.276^{b}$ & $0.274^{b}$ & $0.339 a$ & 0.010 & 0.010 \\
\hline \multicolumn{7}{|c|}{ Tibia breaking strength (N) } \\
\hline $28 d$ & 287 & 278 & 287 & 295 & 2.77 & 0.070 \\
\hline $35 d$ & 302 & 297 & 302 & 307 & 3.74 & 0.651 \\
\hline $42 \mathrm{~d}$ & $297 b$ & $296 \mathrm{~b}$ & $300^{b}$ & $327 a$ & 3.96 & 0.001 \\
\hline \multicolumn{7}{|c|}{ Tibia stiffness (N/mm) } \\
\hline $28 d$ & 257 & 251 & 257 & 264 & 3.41 & 0.067 \\
\hline $35 d$ & $270^{a b}$ & $263^{b}$ & $264^{b}$ & $275^{a}$ & 3.35 & 0.039 \\
\hline $42 \mathrm{~d}$ & $263^{b}$ & $261^{b}$ & $267 \mathrm{ab}$ & $284^{a}$ & 4.91 & 0.011 \\
\hline \multicolumn{7}{|c|}{$\begin{array}{l}\text { Tibia energy to fracture ( } N \text { - } \\
\mathrm{mm} \text { ) }\end{array}$} \\
\hline $28 \mathrm{~d}$ & 279 & 273 & 280 & 287 & 3.51 & 0.066 \\
\hline $35 d$ & 293 & 283 & 289 & 295 & 3.84 & 0.158 \\
\hline $42 \mathrm{~d}$ & $290^{b}$ & $287 \mathrm{~b}$ & $295^{\mathrm{ab}}$ & $307 a$ & 4.54 & 0.013 \\
\hline
\end{tabular}

a-b Values within a row, lacking a common superscript differ $(P \leq 0.05)$.

1 Two pens were combined and used as the experimental unit for mineral content and mineral density parameters. 
Table 4 Locomotion-related observations (gait score and varus valgus) of broiler chickens fed a control, fish oil, collagen and organic minerals diet

\begin{tabular}{|l|c|c|c|c|c|c|}
\hline Measurement & Control & Fish Oil & Collagen & $\begin{array}{c}\text { Organic } \\
\text { Minerals }\end{array}$ & SEM & $\begin{array}{c}P \\
\text { values }\end{array}$ \\
\hline $\mathrm{n}$ (pens) & 7 & 8 & 8 & 8 & & \\
Gait score (score 1 to 5)' & & & & & & \\
27d & 2.0 & 2.0 & 2.0 & 2.0 & -2 & -2 \\
34d & 2.47 & 2.33 & 2.34 & 2.22 & 0.02 & 0.254 \\
$41 \mathrm{~d}$ & 2.61 & 2.66 & 2.58 & 2.48 & 0.03 & 0.125 \\
Varus valgus (\%) & & & & & & \\
28d & 21 & 17 & 17 & 17 & 0.07 & 0.511 \\
$35 d$ & 58 & 66 & 58 & 45 & 0.09 & 0.083 \\
$42 d$ & 66 & 70 & 66 & 63 & 0.06 & 0.114 \\
\hline
\end{tabular}

$a-b$ Values within a row, lacking a common superscript differ $(P \leq 0.05)$.

1 Method of Kestin et al. (1992), scored within a range of 0 (normal locomotion) to 5 (unable to stand).

2 Due to same scores in each diet group, SEM and $\mathrm{P}$ values were not calculated.

3 The percentage of chickens per diet group.

Table 5 Blood parameters of broiler chickens at 42 D of age fed a control, fish oil, collagen and organic minerals diet.

\begin{tabular}{|l|c|c|c|c|c|c|}
\hline Measurement & Control & Fish Oil & Collagen & $\begin{array}{c}\text { Organic } \\
\text { Minerals }\end{array}$ & SEM & $\begin{array}{c}\mathrm{P} \\
\text { values }\end{array}$ \\
\hline $\mathrm{n}$ (pens) & 7 & 8 & 8 & 8 & & \\
Calcium (mmol/l) & 2.60 & 2.51 & 2.58 & 2.64 & 0.05 & 0.506 \\
Phosphorus (mmol/l) & 1.83 & 1.91 & 1.86 & 1.82 & 0.03 & 0.242 \\
Parathyroid (pg/ml) & 331.3 & 371.3 & 385.8 & 483.9 & 46.5 & 0.900 \\
Alkaline phosphatase (U/l) & 1134.9 & 1115.2 & 1137.2 & 1204.7 & 73.3 & 0.860 \\
Vitamin D3 (pg/ml) & 463.2 & 468.3 & 493.3 & 502.1 & 37.6 & 0.931 \\
\hline
\end{tabular}

\section{DISCUSSION}

\section{Growth Performance}

The results of this study showed that replacement of inorganic sourced macro and trace minerals by their organic varieties and replacement of soybean meal by hydrolysed collagen in broiler chicken diets might stimulate growth performance. At d 42, the chickens of the $\mathrm{COL}$ and ORM diet groups had a higher BW compared to the FISH diet group. The findings of the ORM diet group are in line with previous studies that showed that dietary organic sourced $\mathrm{Ca}$ and $\mathrm{P}$ (Tahir et al., 2012) and CU, Fe, Mn, Zn (Bao et al., 2007; Abdallah et al., 2009; Ao et al., 2017) resulted in higher growth performance than their inorganic varieties, because of 
their functions in numerous biochemical reactions, increased bio-availability and less antagonistic impact among each other. It can be speculated whether or not an increase of inorganic minerals compared to the current guidelines will result in comparable effects as when organic minerals are used.

Since hardly any research has been performed on the effects of dietary hydrolysed collagen and fish oil on growth performance of broiler chickens, it requires further investigation to understand the mechanisms involved. Possible explanations for a higher BW results of the $\mathrm{COL}$ diet group might be the protein quality and digestibility of collagen. In chickens, mice and humans, hydrolysed collagen or gelatin has been demonstrated to improve protein digestion and absorption of peptides, rich in proline, hydroxyproline, and glycine (Oesser et al., 1999; Iwai et al., 2005; Ohara et al., 2007; Beyranvand et al., 2018). In addition, the higher BW of the COL diet group might be explained by the high protein content of the COL diets. The FISH diet group had a lower BW than the COL and ORM diet groups. These findings were opposite as compared to other studies, where the dietary fish oil resulted in higher BW and lower FCR of broiler chickens (Lopez-Ferrer et al, 1999; Schreiner et al., 2005). The lower BW of the FISH diet group might possibly explained by unknown interferences by n-6 PUFA in broiler diet, it requires further investigation to understand the mechanisms involved. Another possible explanation might be the lipid peroxidization of fish oil in feed. Many studies have shown that this peroxidation can negatively affect the growth performance of broiler chickens and some other animal species, such as turkeys, rats and pigs, mostly due to decreased bio-availability (Dibner et al., 1996; Liv et al., 2014; Yang et al., 2015). However, in the current study, lipid peroxidation in feed is not analysed.

Despite a higher BW in the ORM and COL diet groups compared to the FISH diet group, FI was similar for all diet groups. As a result of this, lower FCR values for ORM and COL diet groups throughout the growing period, specifically during the finisher diet phase were found. These results on FCR are in accordance with previous studies, stating that broiler chickens fed organic Ca, P, CU, Fe, Mn, Zn had lower FCR levels (Nollet et al., 2007; Abdallah et al., 2009; Tahir et al., 2012; Oliveira et al., 2015; Ao et al., 2017) than broiler chickens fed inorganic mineral varieties, probably 
again due to the higher bio-availability of the organic mineral varieties.

\section{Locomotion-related Observations and Bone Development Related Blood Parameters}

In general, fast-growing broiler chickens suffering from leg problems demonstrated by low locomotion activities and gait abnormalities (Lewis and Hurnik, 1990; Corr et al., 2003; Bessei, 2006; Kittelsen et al., 2017). Whether this can be influenced by diet composition cannot be concluded from the current study, because no influence of the used diet compositions was found for home pen behaviour observations, gait score and varus valgus. Whether the lack of effects of the used diet composition is indeed true or due to e.g. the non-commercial setup (small pens; low stocking density) and consequently the good litter quality in our study needs further investigations. However, looking more into detail to the results of the gait score and rotated tibia, the ORM diet group showed, not significantly, the best gait scores and the lowest prevalence of varus valgus at $d 34$ and 41 . This might suggest that organic minerals not only affecting tibia characteristics, but also result in better leg quality and locomotion.

Looking to the age based behavioural changes, we expected less walking and more resting toward to slaughter age. However, this was not found in the current study, but more or less the opposite was found, which might be explained by the low stocking density in the pen, particularly after the removal of chickens at d 28 and 34 for slaughtering.

Regarding bone related blood parameters, literature demonstrates ambiguous results. Organic sourced $\mathrm{Zn}$ and $\mathrm{Mn}$ in broiler diets resulted in a lower serum ALP level than inorganic varieties of $\mathrm{Zn}$ and $\mathrm{Mn}$ (Yuan et al., 2017). Higher serum Cu and Fe levels were found in another broiler study applying organic sourced $\mathrm{Zn}$ in diet of broiler chickens compared to inorganic Zn (Yalçinkaya et al., 2012). Organic sourced Zn in broiler chickens diet resulted in higher Ca content in plasma compared to control diet containing inorganic sourced Zn (Salim et al., 2012).

It has been concluded that organic sourced $\mathrm{Cu}$ in laying hen diets resulted in higher Cu levels in blood compared to inorganic sourced Cu in diet (Dobrzanski et al., 2008). To our knowledge, no studies have been 
conducted to determine the relationship between dietary hydrolysed collagen or fish oil on bone related blood parameters in chickens. In the current study, serum Ca, P, ALP, vitamin D3 and PTH levels were not influenced by diet groups. Due to limited comparative studies and their inconsistent results regarding the effects of dietary organic macro and trace minerals, hydrolysed collagen and fish oil on blood parameters in poultry or other species, further research is needed.

\section{Tibia characteristics}

Tibia morphological measurements, such as tibia proximal length, and lateral cortex thickness have been used as indicators of bone quality in poultry (Leblanc et al., 1986; Krupski and Tatara, 2007; Charuta et al., 2013). The results obtained in this study indicated that chickens of the ORM diet group had significantly longer tibia at d 42 and thicker femoral and metatarsal side tibia heads at d 28 compared to the FISH diet group. These findings are in agreement with previous studies, indicating the stimulative effects of organic sourced minerals on tibia morphologic characteristics. It has been reported that organic sourced $\mathrm{Ca}$ in broiler chicken diets resulted in higher tibia proximal length and lateral cortex thickness (Guinotte and Monredon, 1991). It has been reported that lower bio-availability of Zn and Mn led to shorter tibia proximal length, shorter lateral cortex thickness and malformations on tibia (scott et al., 1982). A low concentration of Zn in broiler diets also resulted in shorter tibia length, due to its role in bone development by mechanisms influencing longitudinal bone growth at the growth plate (Starcher et al., 1980; Wang et al., 2002; Oviedo-Rondón et al., 2006). In the current study, morphological tibia characteristics of the ORM diet group were stimulated, which can be explained by higher mineral absorption and greater bio-availability. Dietary fish oil had a negative effect on tibia morphological characteristics, which might be explained by different interferences of n-6 PUFA in the mineralisation and ossification mechanisms, and required further investigation.

Tibia mechanical and biophysical measurements, such as bone mineral density (Watkins and Southern, 1992; Rath et al., 2000; Onyango et al., 2003; Kim et al., 2006; Shim et al., 2012), bone mineral content (Akpe et al., 1987; Onyango et al., 2003; Kim et al., 2006) and bone breaking strength 
(Merkley, 1981; Ruff and Hughes, 1985; Park et al., 2003; Kim et al., 2006) have been the most promising parameters to assess bone health in poultry, because of their relationships with locomotion and leg pathologies. A deficiency of $\mathrm{Ca}, \mathrm{P}$ and trace minerals in the diet result in lower bone mineral content and lower breaking strength (Bar et al., 2003; Sá et al., 2004; McDevitt et al., 2006; Van Krimpen et al., 2013). Ca and P are primarily essential for bone mineralisation (Rath et al., 1999; Blake and Fogelman, 2002) and increasing Ca and P in broiler chicken diets might positively influence bone mineralisation and bone Ca content, leading to a stronger bone (Driver et al., 2005a, b; L'etourneau-Montminy et al., 2008; Van Krimpen et al., 2013). Current diets were optimised based on the CVB (CVB, 2012), but the advices for $\mathrm{Ca}$ and $\mathrm{P}$ in this table are not based on bone quality. It can be speculated that the current advised minimal levels are too limited for optimal bone development and bone strength. Instead of increasing dietary inorganic $\mathrm{Ca}$ and $\mathrm{P}$ content, changing the source of $\mathrm{Ca}$ and $\mathrm{P}$ and thereby increasing the bio-availability might work in the same way. In the current study, the highest tibia mineral content and the highest tibia mineral density at slaughter age were observed in the ORM diet group compared to all other diet groups. These findings are in accordance with El-Husseiny et al. (2012) who evaluated effects of replacement of organic Zn, Mn and Cu on tibia mineralisation.

Bone strength is generally assumed to be suboptimal in broiler chickens (Lilburn, 1994; Williams et al., 2004; Sherlock et al., 2010) and the strength is mostly related to the inorganic part of the bone, which is responsible for the hardness (Turner, 1993; Boivin and Meunier, 2002a,b; Bonser and Casinos, 2003). It has been shown that even if the mineral content of the diet can fulfil the requirements for growth performance, the mineral content level might be insufficient to meet the requirements for maximal bone strength (Bar et al., 2003; sa et al., 2004). Hemme et al. (2005) reported that organic sourced P, because of its higher bio-availability, positively affected bone strength. Cu and Fe are vitally important for crosslinking of collagen, which gives the bone its breaking strength and elasticity (Dibner et al., 2007). Insufficient amount of available $\mathrm{CU}$ and Fe resulted in lower bone strength, even if $\mathrm{Ca}$ and $\mathrm{P}$ levels were adequate (Medeiros et al., 1997). In the present study, tibia breaking strength of the ORM diet group was highest at the slaughter age (d 42). This confirms previous studies that dietary organic $\mathrm{Zn}, \mathrm{Mn}, \mathrm{Cu}, \mathrm{Fe}, \mathrm{Se}$ 
and more available $\mathrm{Ca}$ and $\mathrm{P}$ resulted in higher bone breaking strength (McDevitt et al., 2006; Dibner et al., 2007; Ferket et al., 2009; El-Husseiny et al., 2012).

Tibia stiffness and energy to fracture parameters are mostly related to the organic part of bone, which is responsible for flexibility (Velleman, 2000; Turner, 2006). In the current study, tibia stiffness and energy to fracture reached the highest values in the ORM diet group. It is known that especially essential trace minerals play a role in the linkage between elastin and collagen, which supplies bone its stiffness and flexibility (Starcher et al., 1980; Dibner et al., 2007). These two parameters of COL diet group were also high at the slaughter age compared to CON and FISH diet groups, which might be explained by the increased collagen amount in bone and consequently more flexible bone structure.

In conclusion, the hypothesis of this study was that dietary organic minerals, fish oil and hydrolysed collagen might positively affect tibia development in broiler chickens. The results of this study indeed showed that organic macro and trace minerals in the diet positively affect tibia characteristics, but these effects have not been found for the fish oil and hydrolysed collagen. Because of the best tibia characteristics for the organic mineral diet group and the lowest (not significant) values for gait score and rotated tibia, it can be suggested that organic minerals in the diet of fast growing broiler chickens might improve animal welfare and risk on injuries during depopulation, transport and slaughtering. 


\section{ACKNOWLEDGEMENTS}

This project was financed by the public-private partnership "Healthy Bones" (project number BO-47001-011). The financial support of the Ministry of Agriculture, Nature and Food Quality of The Netherlands, Aviagen EPI, Darling Ingredients Inc., ForFarmers, Hubbard, Marel Stork Poultry Processing BV, Nepluvi and Nutreco is gratefully acknowledged. The authors would like to thank Lagerwey hatchery (Lunteren, The Netherlands) for providing a-day-old chicks; Martijn Nieuwenhout at Tromp Medical B.V. (Castricum, The Netherlands) for allowing us to use their X-ray machine. Stefan Veenstra, Henny Reimert, llona van der Anker-Hensen, Mehmet Can Güçlü and the animal care takers are acknowledged for their help during the experiment. 


\section{SUPPLEMENTARY DATA}

Table A1 Home pen behaviour observation parameters of broiler chickens (\%) fed fish oil, collagen and organic minerals diets

\begin{tabular}{|c|c|c|c|c|c|c|c|c|c|}
\hline Measurement & Control & $\begin{array}{c}\text { Fish } \\
\text { Oil }\end{array}$ & Collagen & $\begin{array}{l}\text { Organic } \\
\text { Minerals }\end{array}$ & Overall & SEM & \multicolumn{3}{|c|}{$P$ values } \\
\hline n (pens) & 7 & 8 & 8 & 8 & & & $\begin{array}{c}\text { Diet } \\
\text { group }\end{array}$ & Age & $\begin{array}{c}\text { Diet } \\
\text { group } \\
\text { x age }\end{array}$ \\
\hline Eating & & & & & & 0.33 & 0.16 & 0.04 & 0.88 \\
\hline $6 d$ & 20.1 & 14.8 & 15.5 & 19.8 & $17.5^{c}$ & & & & \\
\hline $13 d$ & 21.8 & 21.5 & 24.5 & 23.8 & $22.9 \mathrm{~b}$ & & & & \\
\hline $20 \mathrm{~d}$ & 20.8 & 20.0 & 21.0 & 20.0 & $20.4^{\mathrm{bc}}$ & & & & \\
\hline $27 d$ & 34.5 & 35.6 & 39.3 & 37.0 & $36.6 a$ & & & & \\
\hline $34 \mathrm{~d}$ & 22.5 & 19.8 & 18.6 & 20.2 & $20.3^{b c}$ & & & & \\
\hline $41 \mathrm{~d}$ & 20.1 & 20.3 & 18.5 & 23.4 & $20.6 \mathrm{bc}$ & & & & \\
\hline Drinking & & & & & & 0.11 & 0.10 & 0.003 & 0.60 \\
\hline $6 \mathrm{~d}$ & 9.1 & 6.4 & 6.5 & 6.0 & $7.0^{\mathrm{b}}$ & & & & \\
\hline $13 d$ & 13.7 & 12.7 & 12.5 & 14.7 & $13.4^{a}$ & & & & \\
\hline $20 d$ & 13.6 & 11.5 & 12.3 & 11.9 & $12.3^{a b}$ & & & & \\
\hline $27 \mathrm{~d}$ & 11.3 & 9.3 & 9.5 & 7.0 & $9.3 \mathrm{~b}$ & & & & \\
\hline $34 \mathrm{~d}$ & 16.1 & 12.8 & 16.5 & 13.0 & $14.6^{a}$ & & & & \\
\hline $41 \mathrm{~d}$ & 16.0 & 13.4 & 14.8 & 14.4 & $14.6 a$ & & & & \\
\hline Walking & & & & & & 0.15 & 0.50 & 0.003 & 0.79 \\
\hline $6 d$ & 13.0 & 15.5 & 12.7 & 15.9 & $14.3^{b c}$ & & & & \\
\hline $13 d$ & 13.0 & 11.3 & 12.5 & 11.0 & $11.9 c$ & & & & \\
\hline $20 d$ & 14.0 & 17.5 & 13.1 & 12.7 & $14.3^{b c}$ & & & & \\
\hline $27 d$ & 15.5 & 15.0 & 13.5 & 14.6 & $14.6^{\mathrm{bc}}$ & & & & \\
\hline $34 d$ & 15.0 & 17.0 & 15.5 & 15.7 & $15.8 a^{b}$ & & & & \\
\hline $41 d$ & 16.5 & 19.2 & 21.7 & 20.2 & $19.4^{a}$ & & & & \\
\hline Standing & & & & & & 0.31 & 0.13 & 0.004 & 0.62 \\
\hline $6 d$ & 16.1 & 19.1 & 16.6 & 20.5 & $18.0^{a}$ & & & & \\
\hline $13 d$ & 11.6 & 13.1 & 13.0 & 10.0 & $11.9 \mathrm{~b}$ & & & & \\
\hline $20 \mathrm{~d}$ & 14.1 & 12.8 & 12.3 & 15.8 & $13.7 \mathrm{~b}$ & & & & \\
\hline $27 d$ & 16.5 & 18.3 & 16.3 & 20.9 & $18.0^{a}$ & & & & \\
\hline $34 d$ & 19.0 & 19.8 & 20.1 & 22.3 & $20.3^{a}$ & & & & \\
\hline $41 \mathrm{~d}$ & 19.4 & 20.8 & 20.6 & 19.2 & $20.0^{a}$ & & & & \\
\hline Resting & & & & & & 0.55 & 0.18 & $<.0001$ & 0.26 \\
\hline $6 d$ & 40.2 & 41.4 & 46.1 & 35.2 & $40.7^{a}$ & & & & \\
\hline $13 d$ & 36.3 & 38.0 & 34.5 & 37.0 & $36.4^{a}$ & & & & \\
\hline $20 d$ & 33.6 & 36.0 & 39.0 & 35.3 & $35.9 a$ & & & & \\
\hline $27 \mathrm{~d}$ & 34.5 & 35.6 & 39.3 & 37.0 & $36.6^{a}$ & & & & \\
\hline $34 \mathrm{~d}$ & 20.4 & 27.0 & 26.8 & 25.0 & $24.8^{b}$ & & & & \\
\hline $41 \mathrm{~d}$ & 22.0 & 22.5 & 22.3 & 19.2 & $21.5^{b}$ & & & & \\
\hline Forage & & & & & & 0.07 & 0.80 & 0.007 & 0.91 \\
\hline $6 d$ & 0 & 0.7 & 0 & 0.5 & $0.3^{b c}$ & & & & \\
\hline $13 d$ & 1.5 & 0.5 & 2.1 & 1.5 & $1.4 \mathrm{ab}$ & & & & \\
\hline $20 d$ & 1.6 & 0.1 & 0.2 & 1.0 & $0.7^{b c}$ & & & & \\
\hline $27 \mathrm{~d}$ & 0.2 & 0 & 0 & 0 & $0.05^{c}$ & & & & \\
\hline $34 d$ & 0 & 0 & 0 & 0 & $0 c$ & & & & \\
\hline $41 \mathrm{~d}$ & 1.4 & 2.0 & 1.5 & 2.6 & $1.8 a$ & & & & \\
\hline
\end{tabular}


Table A1 (Continued)

\begin{tabular}{|c|c|c|c|c|c|c|c|c|c|}
\hline Measurement & Control & $\begin{array}{l}\text { Fish } \\
\text { Oil }\end{array}$ & Collagen & $\begin{array}{l}\text { Organic } \\
\text { Minerals }\end{array}$ & Overall & SEM & \multicolumn{3}{|c|}{$P$ values } \\
\hline $\begin{array}{l}\text { Comfort } \\
\text { Behaviour }\end{array}$ & & & & & & 0.05 & 0.84 & 0.33 & 0.33 \\
\hline $6 d$ & 1.0 & 1.5 & 2.6 & 1.8 & 1.7 & & & & \\
\hline $13 d$ & 2.0 & 2.3 & 1.0 & 1.0 & 1.6 & & & & \\
\hline $20 d$ & 2.3 & 1.8 & 2.1 & 3.3 & 2.4 & & & & \\
\hline $27 d$ & 2.0 & 2.7 & 2.2 & 2.1 & 2.2 & & & & \\
\hline $34 \mathrm{~d}$ & 3.0 & 3.4 & 2.0 & 3.7 & 3.0 & & & & \\
\hline $41 d$ & 4.5 & 1.5 & 0.5 & 1.0 & 1.9 & & & & \\
\hline Dustbathing & & & & & & 0.01 & 0.67 & 0.19 & 0.71 \\
\hline & 0 & 0.5 & 0 & 0.2 & 0.2 & & & & \\
\hline $13 d$ & 0 & 0.5 & 0 & 0.2 & 0.2 & & & & \\
\hline $20 d$ & 0 & 0.2 & 0 & 0 & 0.05 & & & & \\
\hline $27 d$ & 0 & 0 & 0 & 0 & 0 & & & & \\
\hline $34 \mathrm{~d}$ & 0 & 0 & 0.3 & 0.3 & 0.2 & & & & \\
\hline $41 \mathrm{~d}$ & 0 & 0 & 0 & 0 & 0 & & & & \\
\hline
\end{tabular}

$a-b$ Values within a row, lacking a common superscript differ $(P \leq 0.05)$.

* Due to same scores in each diet group in Perching, SEM and $\mathrm{P}$ value were not shown in the table. 


\section{REFERENCES}

Abdallah, A. G., O. M. El-Husseiny, and K. O. Abdel-Latif. 2009. Influence of some dietary organic mineral supplementations. International journal of poultry science. 8:291-298.

Akpe, M. P., P. E. Waibel, K. Larntz, A. L. Metz, S. L. Noll, and M. M. Walser. 1987. Phosphorus availability bioassay using bone ash and bone densitometry as response criteria. Poultry science. 66:713-720.

Ao, T., L. M. Macalintal, M. A. Paul, A. J. Pescatore, R. M. Delles, A. H. Cantor, M. J. Ford, and K. A. Dawson. 2017. Effects of dietary supplementation of organic minerals on the performance of broiler chicks fed oxidised soybean oil. Journal of applied animal nutrition. 5:1-5.

Bao, Y. M., M. Choct, P. A. Iji, and K. Bruerton. 2007. Effect of organically complexed copper, iron, manganese, and zinc on broiler performance, mineral excretion, and accumulation in tissues. Journal of applied poultry research. 16:448-455.

Bar, A., D. Shinder, S. Yosefi, E. Vax, and I. Plavnik. 2003. Metabolism and requirements for calcium and phosphorus in the fast-growing chicken as affected by age. British journal of nutrition. 89:51-60.

Bessei, W. 2006. Welfare of broilers: a review. World poultry science journal. 62:455-466.

Blake, G. M., and I. Fogelman. 2002. Methods and clinical issues in bone densitometry and quantitative ultrasonometry. Pages 1573-1585 in Principles of Bone Biology (Second Edition). J. P. Bilezikian, L. G. Raisz, and G. A. Rodan, ed. Academic Press, Massachusetts, US.

Boivin, G., and P. J. Meunier. 2002a. The degree of mineralization of bone tissue measured by computerized quantitative contact microradiography. Calcified tissue international. 70:503-511.

Boivin, G., and P. J. Meunier. 2002b. Effects of bisphosphonates on matrix mineralization. Journal of musculoskeletal and neuronal interactions. 2:538-543.

Bonser, R. H. C., and A. Casinos. 2003. Regional variation in cortical bone properties from broiler fowl-a first look. British poultry science. 44:350-354.

Burrell, A. L., W. A. Dozier, A. J. Davis, M. M. Compton, M. E. Freeman, P. F. Vendrell, and T. L. Ward. 2004. Responses of broilers to dietary zinc concentrations and sources in relation to environmental implications. British poultry science. 45:225-263.

Calini, F., and F. Sirri. 2007. Breeder nutrition and offspring performance. Brazilian journal of poultry science. 9:77-83.

Charuta, A., M. Dzierz,ecka, M. Pierzchała, R. G. Cooper, E. Poławska, and J. O. Horba'nczuk. 2013. Sex-related differences of morphometric, densitometric, and geometric parameters of tibia and tarsometatarsal bone in 14-month-old ostriches (Struthio camelus). Poultry science. 92:2965-2976.

Corr, S. A., M. J. Gentle, C. C. McCorquodale, and D. Bennett. 2003. The effect of morphology on walking ability in the modern broiler: a gait analysis study. Animal welfare. 12:159-171.

CVB. 2012. Veevoedertabel 2012 [Feeding table], Central Veevoeder Bureau. Lelystad.

De Jong, I. C., and D. Guémené. 2011. Major welfare issues in broiler breeders. World poultry science journal. 67:73-82.

Dibner, J. J., J. D. Richards, M. L. Kitchell, and M. A. Quiroz. 2007. Metabolic challenges and early bone development. Journal of applied poultry research. 16:126-137.

Dobrzañski, Z., M. Korczyñski, K. Chojnacka, H. Górecki, and S. Opaliñski. 2008. Influence of organic forms of copper, manganese and iron on bioaccumulation of these metals and zinc in laying hens. Journal of elementology. 13:309-319.

Driver, J. P., G. M. Pesti, R. I. Bakalli, and H. M. Edwards, Jr. 2005a. Calcium requirements of the modern broiler chicken as influenced by dietary protein and age. Poultry science. 84:1629-1639.

Driver, J. P., G. M. Pesti, R. I. Bakalli, and H. M. Edwards, Jr. 2005b. Effects of calcium and nonphytate phosphorus concentrations on phytase efficacy in broiler chicks. Poultry science. 84:1406- 1417.

El-Husseiny, O. M., S. M. Hashish, R. A. Ali, S. A. Arafa, L. D. A. El-Samee, and A. A. Olemy. 2012. Effects of feeding organic zinc, manganese and copper on broiler growth, carcass 
characteristics, bone quality and mineral content in bone, liver and excreta. International journal of poultry science. 11:368-377.

European Food Safety Authority. 2010. Standard sample description for food and feed. EFSA J. 8:1457.

Ferket, P. R., E. O. Oviedo-Rondón, P. L. Mente, D. V. Bohórquez, A. A. Santos Jr, J. L. Grimes, J. D. Richards, J. J. Dibner, and V. Felts. 2009. Organic trace minerals and 25-hydroxycholecalciferol affect performance characteristics, leg abnormalities, and biomechanical properties of leg bones of turkeys. Poultry science. 88:1 18-131.

Gocsik, É., A. M. Silvera, H. Hansson, H. W. Saatkamp, and H. J. Blokhuis. 2017. Exploring the economic potential of reducing broiler lameness. British poultry science. 58:337-347.

Guillerminet, F., H. Beaupied, V. Fabien-Soulé, D. Tomé, C. L. Benhamou, C. Roux, and A. Blais. 2010. Hydrolyzed collagen improves bone metabolism and biomechanical parameters in ovariectomized mice: an in vitro and in vivo study. Bone. 46:827-834.

Guillerminet, F., V. Fabien-Soulé, P. Even, D. Tomé, C. L. Benhamou, C. Roux, and A. Blais. 2012. Hydrolyzed collagen improves bone status and prevents bone loss in ovariectomized C3H/HeN mice. Osteoporosis international. 23:1909-1919.

Guinotte, F., Y. Nys, and F. de Monredon. 1991. The effects of particle size and origin of calcium carbonate on performance and ossification characteristics in broiler chicks. Poultry science. 70:1908-1920.

Hemme, A., M. Spark, P. Wolf, H. Paschertz, and J. Kamphues. 2005. Effects of different phosphorus sources in the diet on bone composition and stability (breaking strength) in broilers. Journal of animal physiology and animal nutrition. 89:129-133.

Iwai, K., T. Hasegawa, Y. Taguchi, F. Morimatsu, K. Sato, Y. Nakamura, A. Higashi, Y. Kido, Nakabo, and K. Ohtsuki. 2005. Identification of food-derived collagen peptides in human blood after oral ingestion of gelatin hydrolysates. Journal of agricultural and food chemistry. 53:6531-6536.

Jungmann, R., G. Schitter, G. E. Fantner, M. E. Laver, P. K. Hansma, and P. J. Thurner. 2007. Real-time microdamage and strain detection during micromechanical testing of single trabeculae. In Experimental and Applied Mechanics: SEM Annual Conference and Exposition, Springfield, Massachusetts, US, 3-6 June 2007.

Julian, R. J. 1998. Rapid growth problems: ascites and skeletal deformities in broilers. Poultry science. 77:1773-1780.

Kestin, S. C., G. SU, and P. Sorensen. 1999. Different commercial broiler crosses have different susceptibilities to leg weakness. Poultry science. 78:1085-1090.

Kestin, S. C., T. G. Knowles, A. F. Tinch, and N. G. Gregory. 1992. The prevalence of leg weakness in broiler chickens and its relationship with genotype. Veterinary record. 131:190194.

Khan, N. A., J. W. Cone, and W. H. Hendriks. 2009. Stability of fatty acids in grass and maize silages after exposure to air during the feed out period. Animal feed science and technology. 154:183-192.

Kidd, M. T. 2003. A treatise on chicken dam nutrition that impacts on progeny. World poultry science. J. 59:475-494.

Kim, W. K., L. M. Donalson, A. D. Mitchell, L. F. Kubena, D. J. Nisbet, and S. C. Ricke. 2006. Effects of alfalfa and fructooligosaccharide on molting parameters and bone qualities using dual energy X-ray absorptiometry and conventional bone assays. Poultry science. 85:15-20.

Kittelsen, K. E., B. David, R. O. Moe, H. D. Poulsen, J. F. Young, and E. G. Granquist. 2017. Associations among gait score, production data, abattoir registrations, and postmortem tibia measurements in broiler chickens. Poultry science. 96:1033-1040.

Knowles, T. G., S. C. Kestin, S. M. Haslam, S. N. Brown, L. E. Green, A. Butterworth, S. J. Pope, D. Pfeiffer, and C. J. Nicol. 2008. Leg disorders in broiler chickens: prevalence, risk factors and prevention. PLoS One. 3:e1545.

Krupski, W., and M. R. Tatara. 2007. Interrelationships between densitometric, morphometric, and mechanical properties of the tibia in turkeys. Bulletin of the veterinary institute in Pulawy. 51:621-626.

Leblanc, B., M. Wyers, F. Cohn-Bendit, J. M. Legall, E. Thibault, and J. M. Florent. 1986. Histology and histomorphometry of the tibia growth in two turkey strains. Poultry science. 
65:1787-1795.

Létourneau-Montminy, M. P., P. Lescoat, A. Narcy, D. Sauvant, J. F. Bernier, M. Magnin, C. Pomar, Y. Nys, and C. Jondreville. 2008. Effects of reduced dietary calcium and phytase supplementation on calcium and phosphorus utilisation in broilers with modified mineral status. British poultry science. 49:705-715.

Lewis, N. J., and J. F. Hurnik. 1990. Locomotion of broiler chickens in floor pens. Poultry science. 69:1087-1093.

Lilburn, M. S. 1994. Skeletal growth of commercial poultry species. Poultry science. 73:897-903.

Lopez-Ferrer, S., M. D. Baucells, A. C. Barroeta, and M. A. Grashorn. 1999. n-3 enrichment of chicken meat using fish oil: alternative substitution with rapeseed and linseed oils. Poultry science. 78:356-365.

Martin, T. J. 2016. Parathyroid hormone-related protein, its regulation of cartilage and bone development, and role in treating bone diseases. Physical review. 96:831-871.

McDevitt, R. M., G. M. McEntee, and K. A. Rance. 2006. Bone breaking strength and apparent metabolisability of calcium and phosphorus in selected and unselected broiler chicken genotypes. British poultry science. 47:613-621.

Medeiros, D. M., J. llich, J. Ireton, V. Matkovic, L. Shiry, and R. Wildman. 1997. Femurs from rats fed diets deficient in copper or iron have decreased mechanical strength and altered mineral composition. The journal of trace elements in experimental medicine. 10:197-203.

Mench, J. 2004. Lameness. Pages 3-17 in Measuring and Auditing Broiler Welfare. C. A. Weeks, and A. Butterworth, ed. CABI, Wallingford, UK.

Merkley, J. W. 1981. The effect of sodium fluoride on egg production, egg quality, and bone strength of caged layers. Poultry science. 60:771-776.

Nollet, L., J. D. Van der Klis, M. Lensing, and P. Spring. 2007. The effect of replacing inorganic with organic trace minerals in broiler diets on productive performance and mineral excretion. Journal of applied poultry research. 16:592-597.

Nomura, Y., K. Oohashi, M. Watanabe, and S. Kasugai. 2005. Increase in bone mineral density through oral administration of shark gelatin to ovariectomized rats. Nutrition. 21:11201126.

Oesser, S., M. Adam, W. Babel, and J. Seifert. 1999. Oral administration of 14C labeled gelatin hydrolysate leads to an accumulation of radioactivity in cartilage of mice (C57/BL). Journal of nutrition. 129:1891-1895.

Ohara, H., H. Matsumoto, K. Ito, K. Iwai, and K. Sato. 2007. Comparison of quantity and structures of hydroxyproline-containing peptides in human blood after oral ingestion of gelatin hydrolysates from different sources. Journal of agricultural and food chemistry. 55:1532-1535.

Oliveira, T. F. B., A. G. Bertechini, R. M. Bricka, E. J. Kim, P. D. Gerard, and E. D. Peebles. 2015. Effects of in ovo injection of organic zinc, manganese, and cop per on the hatchability and bone parameters of broiler hatchlings. Poultry science. 94:2488-2494.

Onyango, E. M., P. Y. Hester, R. Stroshine, and O. Adeola. 2003. Bone densitometry as an indicator of percentage tibia ash in broiler chicks fed varying dietary calcium and phosphorus levels. Poultry science. 82:1787-1791.

Orimo, H. 2010. The mechanism of mineralization and the role of alkaline phosphatase in health and disease. Journal of nippon medical school. 77:4-12.

Oviedo-Rondón, E. O., P. R. Ferket, and G. B. Havestein. 2006. Nutritional factors that affect leg problems in broilers and turkeys. Avian and poultry biology reviews. 17:89- 103.

Park, S. Y., S. G. Birkhold, L. F. Kubena, D. J. Nisbet, and S. C. Ricke. 2003. Effect of storage condition on bone breaking strength and bone ash in laying hens at different stages in production cycles. Poultry science. 82:1688-1691.

Rath, N. C., J. M. Balog, W. E. Huff, G. R. Huff, G. B. Kulkarni, and J. F. Tierce. 1999. Comparative differences in the composition and biomechanical properties of tibiae of seven-and seventy-two-week-old male and female broiler breeder chickens. Poultry science. 78:1232-1239.

Rath, N. C., G. R. Huff, W. E. Huff, and J. M. Balog. 2000. Factors regulating bone maturity and strength in poultry. Poultry science. 79:1024-1032. 
Ruff, C. R., and B. L. Hughes. 1985. Bone strength of height restricted broilers as affected by levels of calcium, phosphorus, and manganese. Poultry science. 64:1628-1636.

Sá, L. M., P. C. Gomes, H. S. Rostagno, L. F. T. Albino, P. R. Cecon, and P. D'Agostini. 2004. Calcium requirement for broiler chicks from 22 to 42 and 43 to 53 days old. Brazilian journal of animal science. 33:397-406.

Salim, H. M., C. Jo, and B. D. Lee. 2008. Zinc in broiler feeding and nutrition. Avian biology research. 1:5-18.

Schreiner, M., H. W. Hulan, E. Razzazi-Fazeli, J. Böhm, and R. G. Moreira. 2005. Effect of different sources of dietary omega-3 fatty acids on general performance and fatty acid profiles of thigh, breast, liver and portal blood of broilers. Journal of the science of food and agriculture. 85:219-226.

Scott, M. L., M. C. Nesheim, and R. Yang. 1982. Essential inorganic elements. Pages 258-382 in Nutrition of the Chicken. 3rd ed. M. L. Scott and Associates, New York, US.

Sherlock, L., T. G. M. Demmers, A. E. Goodship, I. D. McCarthy, and C. M. Wathes. 2010. The relationship between physical activity and leg health in the broiler chicken. British poultry science. 51:22-30.

Shim, M. Y, A. B. Karnuah, A. D. Mitchell, N. B. Anthony, G. M.Pesti, and S. A. Aggrey. 2012. The effects of growth rate on leg morphology and tibia breaking strength, mineral density, mineral content, and bone ash in broilers. Poultry science. 91:1790-1795.

Starcher, B. C., C. H. Hill, and J. G. Madaras. 1980. Effect of zinc deficiency on bone collagenase and collagen turnover. Journal of nutrition. 110:2095-2102.

Tahir, M., M. Y. Shim, N. E. Ward, C. Smith, E. Foster, A. C. Guney, and G. M. Pesti. 2012. Phytate and other nutrient components of feed ingredients for poultry. Poultry science. 91:928-935.

Turner, C. H. 2006. Bone strength: current concepts. Annals of the new york academy of sciences. 1068:429-446.

Turner, C. H., and D. B. Burr. 1993. Basic biomechanical measurements of bone: a tutorial. Bone. 14:595-608.

Van Leeuwen, J. P. T. M. ,M. Van Driel, G. J. C. Van Den Bemd, and H. A. P. Pols. 2001. Vitamin $D$ control of osteoblast function and bone extracellular matrix mineralization. Critical reviews in eukaryotic gene expression. 11:1-3.

Velleman, S. G. 2000. The role of the extracellular matrix in skeletal development. Poultry science. 79:985-989.

Wang, X., G. J. Fosmire, C. V. Gay, and R. M. Leach, Jr. 2002. Short-term zinc deficiency inhibits chondrocyte proliferation and induces cell apoptosis in the epiphyseal growth plate of young chickens. Journal of nutrition. 132:665-673.

Wang, Y. B., and B. H. XU. 2008. Effect of different selenium source(sodium selenite and selenium yeast) on broiler chickens. Animal feed science and technology. 144:306-314.

Watkins, B. A., Y. Li, H. E. Lippman, and S. Feng. 2003. Modula-tory effect of omega-3 polyunsaturated fatty acids on osteoblast function and bone metabolism. Prostaglandins, leukotrienes and essential fatty acids. 68:387-398.

Watkins, B. A., C. L. Shen, K. G. Allen, and M. F. Seifert. 1996. Dietary (n-3) and (n-6) polyunsaturates and acetylsalicylic acid alter ex vivo PGE2 biosynthesis, tissue IGF-l levels, and bone morphometry in chicks. Journal of bone and mineral research. 11:1321-1332.

Watkins, K. L., and L. L. Southern. 1992. Effect of dietary sodium zeolite A and graded levels of calcium and phosphorus on growth, plasma, and tibia characteristics of chicks. Poultry science. 71:1048-1058.

Wedekind, K. J., E. C. Titgemeyer, A. R. Twardock, and D.H. Baker. 1991. Phosphorus, but not calcium, affects manganese absorption and turnover in chicks. Journal of nutrition. 121:1776-1786.

Williams, B., D. Waddington, D. H. Murray, and C. Farquharson.2004. Bone strength during growth: influence of growth rate on cortical porosity and mineralization. Calcified tissue international. 74:236-245.

Wu, J., M. Fujioka, K. Sugimoto, G. Mu, and Y. Ishimi. 2004. Assessment of effectiveness of oral administration of collagen peptide on bone metabolism in growing and mature rats. Journal of bone and mineral metabolism. 22:547-553. 
Yalçın, S., P. Settar, and O. Dicle. 1998. Influence of dietary protein and sex on walking ability and bone parameters of broilers. British poultry science. 39:251-256.

Yalçınkaya, I., M. Çınar, E. Yildirim, S. Erat, M. Basalan, and T. Güngör. 2012. The effect of prebiotic and organic zinc alone and in combination in broiler diets on the performance and some blood parameters. Italian journal of animal science. 11:298-302.

Yuan, J., Z. Xu, C. Huang, S. Zhou, and Y. Guo. 2011 . Effect of dietary Mintrex-Zn/Mn on performance, gene expression of $\mathrm{Zn}$ transfer proteins, activities of $\mathrm{Zn} / \mathrm{Mn}$ related enzymes and fecal mineral excretion in broiler chickens. Animal feed science and technology. 168:72-79. 
B. C. Güz

R. Molenaar ${ }^{1}$

I. C. de Jong ${ }^{2}$

B. Kemp'

M. van Krimpen ${ }^{2}$

$H$. van den Brand

Poultry Science 2021

100:707-720

doi.org/10.1016/j.psj.2020.11.042

1 Adaptation Physiology Group,

Wageningen University and Research,

6708 PB

Wageningen,

Gelderland,

The Netherlands

2 Wageningen Livestock Research,

Wageningen University and Research, 6708 WD

Wageningen,

Gelderland,

The Netherlands 


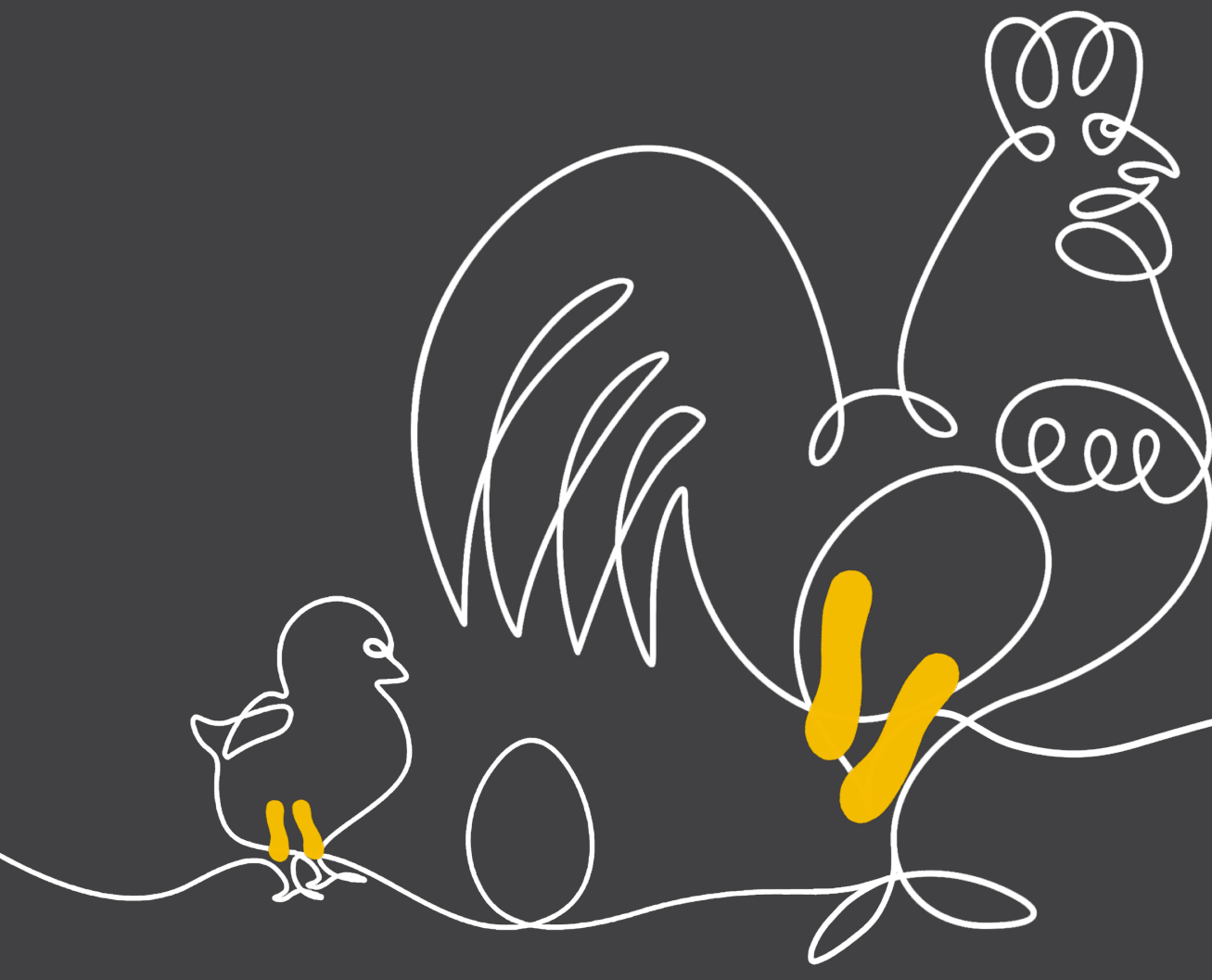

Chapter 2

EFFECTS OF GREEN LED LIGHT DURING INCUBATION AND

DIETARY ORGANIC MACRO AND TRACE MINERALS DURING REARING ON TIBIA CHARACTERISTICS OF BROILER CHICKENS AT SLAUGHTER AGE 


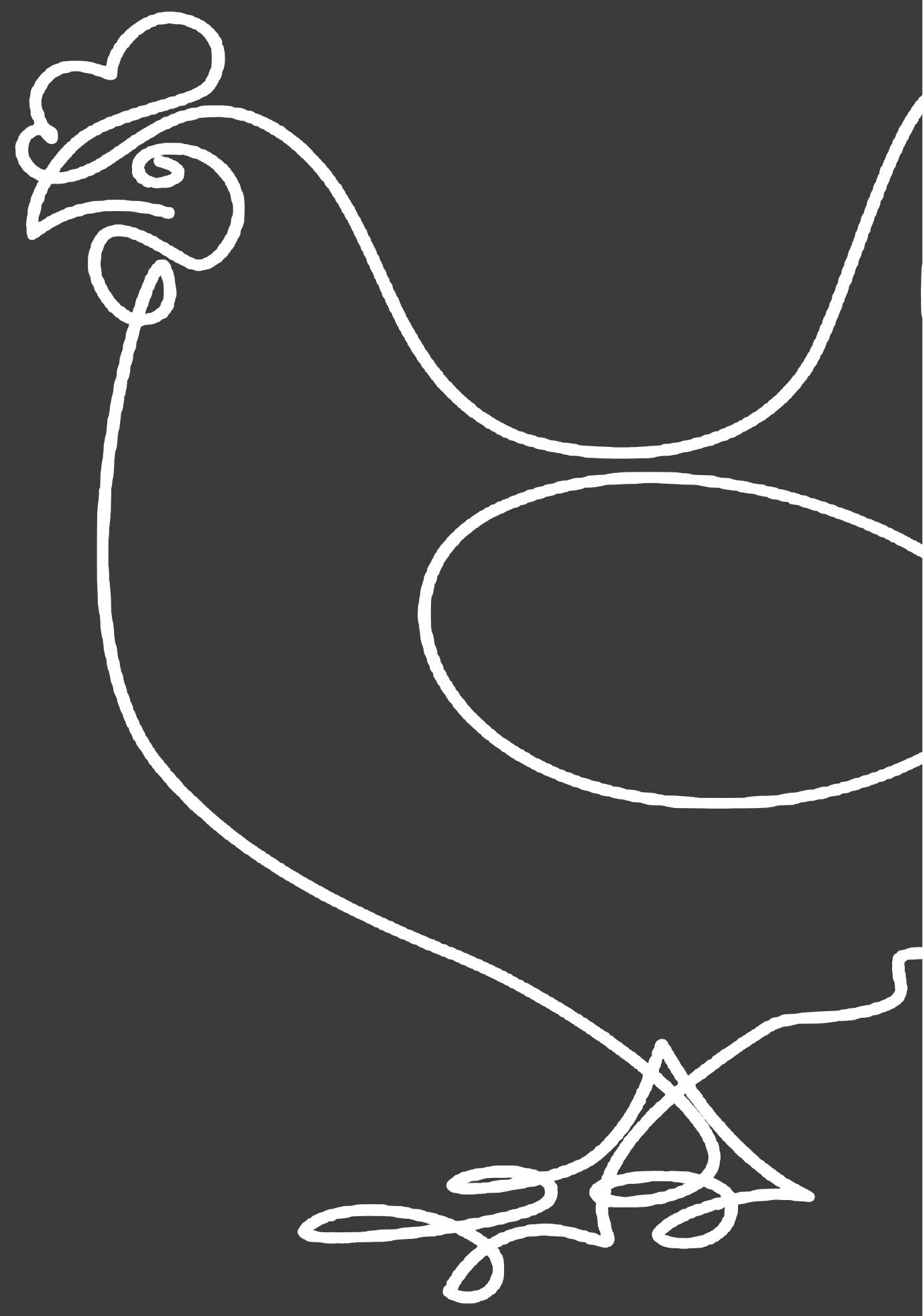




\section{Abstract}

This study was designed to evaluate the effects of green light emitting diode (LED) light during incubation and dietary organic macro and trace minerals during rearing on tibia morphological, biophysical, and mechanical characteristics of broiler chickens at slaughter age. The experiment was setup as a $2 \times 2 \times 2$ factorial arrangement, with the following treatments: 1) light during incubation (green LED light or darkness), 2) macro mineral source during rearing (organic or inorganic $\mathrm{Ca}$ and $\mathrm{P}$ ) and 3 ) trace mineral source during rearing (organic or inorganic $\mathrm{Fe}, \mathrm{Cu}, \mathrm{Mn}, \mathrm{Zn}$, and $\mathrm{Se}$ ). A total of 2,400 eggs (Ross 308) were either incubated under green LED light (16L:8D) or in complete darkness. After hatch, a total of 864 male broiler chickens were reared until slaughter age (day 42) and provided with 1 of 4 diets, differing in macro and/or trace mineral source. During rearing, the experiment had a complete randomized block design with 9 replicate pens per treatment and 12 chickens per pen. At slaughter age (day 42), 2 chickens per replicate were randomly selected and tibia bones were obtained. Tibia weight, length, thickness, osseous volume, pore volume, total volume, mineral content, mineral density, ultimate strength and stiffness were determined. Green LED light during incubation did not affect any of the tibia characteristics. Dietary organic macro minerals positively affected most of the tibia morphological, biophysical, and mechanical characteristics compared to the inorganic macro minerals, whereas trace mineral sources did not affect tibia characteristics. It can be concluded that dietary organic macro minerals $\mathrm{Ca}$ and $\mathrm{P}$ stimulated tibia characteristics, whereas green LED light during incubation and dietary trace minerals during rearing did not affect tibia characteristics, locomotion or leg disorders.

\section{Key words}

green LED, incubation, organic mineral, tibia, broiler chicken 


\section{INTRODUCTION}

Increased attention is being focused on improving leg health of broiler chickens, due to an imbalance between high growth rate and immature bones and joints (Kestin et al., 1992, 2001; Knowles et al., 2008; Sherlock et al., 2010; Güz et al., 2019, 2020). Suboptimal leg health is known to result in pain and thereby negatively affects welfare and natural locomotion-related behaviours, such as accessing water and feed, especially in the last weeks of broilers rearing period (Mckay et al., 2000; Bradshaw et al., 2002; Bessei, 2006; Gocsik et al., 2017). In terms of economy, suboptimal leg health can cause higher mortality, lower slaughter revenues and consequently results in financial losses (Kestin et al., 1999; Mench, 2004).

Two important factors, among others, that seem to affect bone development in broilers are light during incubation (Huth and Archer, 2015a,b; Van der Pol et al., 2017, 2019) and macro and/or trace mineral sources during rearing (Hemme et al., 2005; Ferket et al., 2009; El- Husseiny et al., 2012; Güz et al., 2019).

In commercial hatcheries, eggs are mostly incubated under complete darkness (Archer et al., 2009; Tong et al., 2018). However, in nature, most developing avian embryos are regularly exposed to daylight for short periods of time when the hen leaves her nest to eat and drink (Archer, 2017; Archer and Mench, 2017) and when she rotates her eggs several times in a day (Duncan et al., 1978; Archer et al., 2009; Archer and Mench, 2014, 2017). It is known that light exposure during incubation affects chicken embryo development (Rozenboim et al., 2004; Özkan et al., 2012; Van der Pol et al., 2015, 2017), because broilers have a lightsensitive pineal gland, which produces melatonin (Aige-Gil and Murit10-Ferrol, 1992; Archer et al., 2009). Melatonin influences skeletal development in chickens (Archer et al., 2009; Huth and Archer, 2015a,b; Archer and Mench, 2017).

In addition to light itself, the colour of light might be an important factor, since chickens have special extraretinal photoreceptors in their eyes and brains (Lewis and Morris, 2000; Rozenboim et al., 2004; Sobolewska et al., 2019). Green is a common colour in the natural habitat of avian species and eggs are exposed to green light when the nest is located beneath green leaves. Several studies evaluating green light emitting diode (LED) light during incubation in broiler chickens showed higher body weight and post-hatch pectoral muscle growth compared to complete 
darkness during incubation (Rozenboim et al., 2004; Halevy et al., 2006; Zhang et al., 2014, 2016), as well as higher liver weight, higher antioxidant activity and higher melatonin levels (zhang et al., 2014). Because of the effect of green light on melatonin levels, an effect of incubation with green light on bone development can be expected, but, to our knowledge, this has not been investigated yet.

Once embryonic bone development is stimulated by green light, it is necessary to provide chickens with sufficient minerals for further bone development during rearing as well. This means that bioavailability of minerals is important for optimal bone development during the rearing period (Bao and Choct, 2009; Yenice et al., 2015). Organic macro (Ca and P) and trace (Fe, $\mathrm{CU}, \mathrm{Mn}, \mathrm{Zn}$ and $\mathrm{Se}$ ) minerals have been shown to have a higher bioavailability than inorganic minerals in broilers' diet, due to higher intestinal absorption of those minerals (Wedekind et al., 1991; Bao and Choct, 2009). This higher bioavailability resulted in higher bone mineralization in broiler chickens and better bone quality (Oviedo-Rondon et al., 2006; Ferket et al., 2009; El-Husseiny et al., 2012; Güz et al., 2019). Most studies related to organic minerals in broilers' diets have focused on trace minerals only (Ferket et al., 2009; Zhao et al., 2010; El-Husseiny et al., 2012), or on combinations with organic macro minerals (Ca and P) (Güz et al., 2019), but effects of organic macro minerals only are less clear.

The main objective of this study was to investigate the effect of green LED light during incubation and dietary organic macro and/or trace minerals during rearing on: 1) tibia characteristics, 2) locomotion and 3) leg disorders at slaughter age in fast-growing broiler chickens.

\section{MATERIALS AND METHODS Experimental Design}

The incubation phase of the experiment was conducted at Wageningen University and Research (Wageningen, The Netherlands). The rearing phase was conducted at the experimental facility of a commercial company (ForFarmers N.V., Lochem, The Netherlands). All procedures in this study were approved by the Central Commission for Animal Experiments, The Hague, The Netherlands (Approval number: AVD401002016686). 
The experiment was setup as a $2 \times 2 \times 2$ factorial arrangement with two phases (incubation and rearing). Factors included were 1) light during incubation (green LED or complete darkness), 2) macro mineral source (inorganic vs organic) and 3) trace mineral source (inorganic vs organic) during rearing. In total, there were eight treatments and each treatment was replicated nine times during the rearing phase. As a result, a total of 72 experimental pens within a complete randomized block design were used. Within each block of eight pens, treatment groups were randomly distributed. Pen was used as the experimental unit and each pen contained 12 male broiler chickens.

\section{Incubation Phase}

A total of 2,400 Ross 308 eggs from a 44-week-old breeder flock were obtained from a commercial hatchery (Lagerwey, Lunteren, The Netherlands). All eggs were selected on weight between 61.0 - $65.0 \mathrm{~g}$, transported from the hatchery to the research facility of Wageningen University and Research (Wageningen, The Netherlands) and stored at $18.0^{\circ} \mathrm{C}$ for $48 \mathrm{~h}$. After storage, eggs were randomly distributed over 4 incubators; two large incubators, containing 960 eggs each and two small incubators, containing 240 eggs each. In the large incubators, trays contained 88 eggs in a honey-structure. In the small incubator, trays contained 150 eggs in a row structure (see Figure 1). Per incubator, 4 or 5 eggshell temperature sensors (Pt-100, Sensor Data BV, Rijswijk, The Netherlands) were attached to 4 or 5 individual eggs. All sensors were placed at the equator of the chosen eggs by using heat conducting paste (Dow Corning 340 Heat Sink Compound, Dow Corning GmbH, Wiesbaden, Germany) and a small piece of tape $(2 \times 2 \mathrm{~cm})$. The incubator temperature was continuously adjusted based on the median egg shell temperature to maintain an egg shell temperature of $37.8^{\circ} \mathrm{C}$ throughout incubation. Eggs were turned every $30 \mathrm{~min}$ at an angle of $90^{\circ}$. At embryonic day 8 (E8), all eggs were candled and infertile eggs were removed. At E18, all eggs were candled again and eggs containing a vital embryo were transferred from the trays into hatching baskets, which were placed back in the same incubator. At E21, chicks were taken from the hatching baskets and feather-sexed. Chick quality parameters (red hock, red beak, and navel score) of all hatched chicks 
(both males and females; $n=1,810$ ) were assessed. Red hock and red beak were scored as 0 (absent) or 1 (present). Navel score was assessed as 1 (good), 2 (moderate) or 3 (poor). Thereafter, 30 female chicks per treatment (darkness and green LED light) were randomly selected and body weight, residual yolk weight, yolk free body mass, heart weight, liver weight, stomach weight and intestinal weight were measured. A total of 864 male chicks were individually weighed and tagged with a neck-label, vaccinated against infectious bronchitis (eye drop; MSD Animal Health, Boxmeer, The Netherlands) and transported for half an hour in a climate-controlled van to the rearing facility (Nijkerk, The Netherlands).

\section{Green LED Setup}

Two incubators ( 1 large and 1 small) were equipped with plastic covered and water resistant green LED strips (Josef Barthelme Green LED strips, Barthelme GmbH \& Co., Nürnberg, Germany). These strips were attached underneath hatching trays, meaning that eggs underneath that hatching tray were lighted (Figure 1). Light intensities at egg shell level (approximately $7 \mathrm{~cm}$ distant from the LED strip), measured by a Lux meter (Testo Model 540 Lux meter, Testo BV, Almere, The Netherlands), were on average 288 lux. Green light was applied with a 16L:8D light schedule throughout incubation.

At E18, after transfer of eggs to the hatching baskets, LED strips were attached to metal frames holding the hatching baskets to maintain the green lighting during the last $3 \mathrm{~d}$ of incubation.

\section{Spectrum Measurement Through Egg Shell}

To determine the light spectrum of the green LED light passing through the eggshell, sixteen Ross 308 broiler breeder eggs were chosen from aforementioned collected eggs and egg contents were removed (shell membranes remained).

The shells were placed individually over a LED spectrum meter sensor (AvaSpec ULS2048 Spectrometer, Avantes, Apeldoorn, The Netherlands). Green LED strips were placed $7 \mathrm{~cm}$ distant from the eggshell (comparable to the distance during incubation). The light spectrum passing through the eggshells and average spectrum was determined. 
The peak in light spectrum without an eggshell was obtained at a wavelength of $522 \mathrm{~nm}$ at approximately 57,000 counts, whereas the peak in light spectrum passing through the eggshell was obtained at a wavelength of $536 \mathrm{~nm}$ at approximately 19,000 counts (Figure 2).
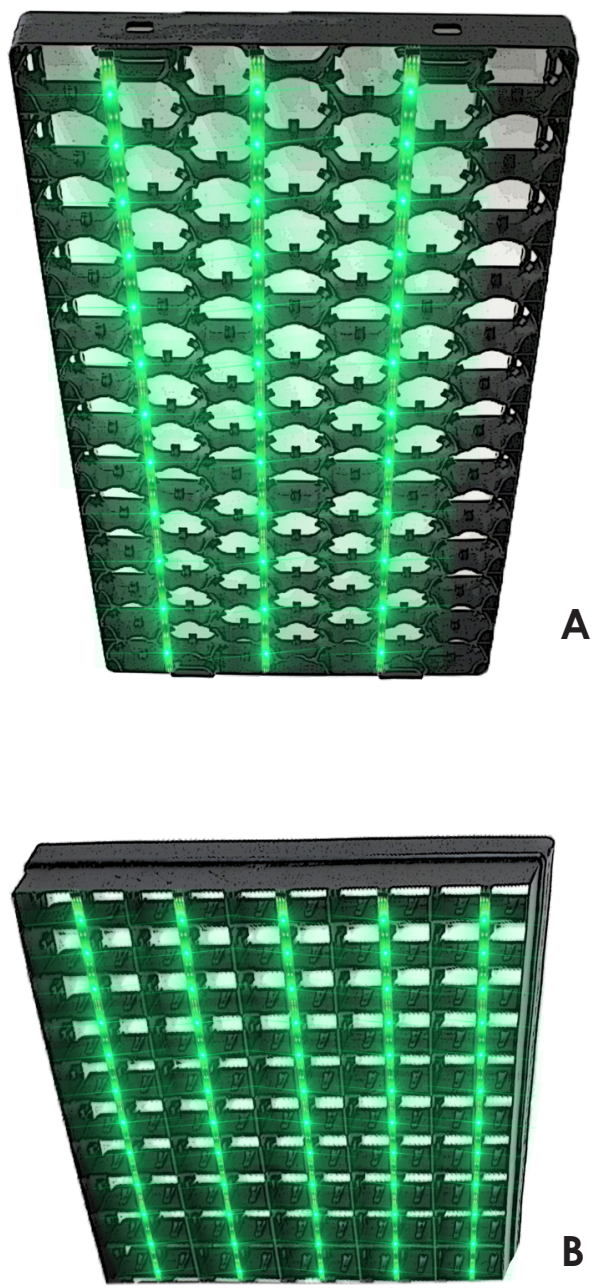

\section{Figure 1}

Assembling of green LED light strips beneath egg trays for the (A) large and (B) small incubator 

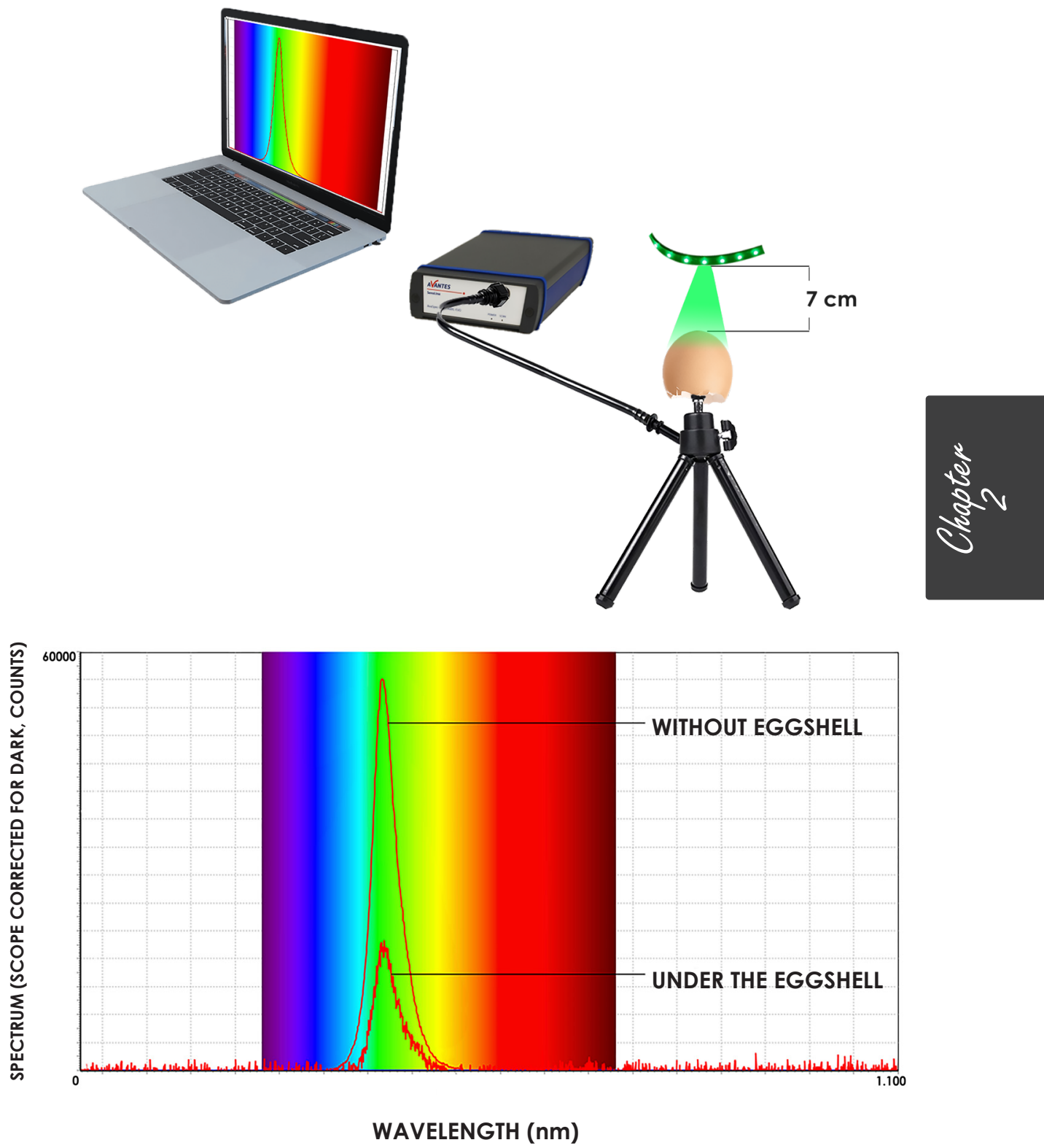

Figure 2

Unfiltered spectrum measurements, using the LED spectrometer without eggshell (direct impose) and through the eggshell of broiler breeder eggs, illuminated by the green LED light strips used in the incubators

Abbreviation: LED, light emitting diode 


\section{Rearing Phase}

Upon arrival at the rearing facility, chickens were randomly assigned to 72 floor pens within 9 blocks of 8 pens in a climate-controlled broiler house. Each pen contained 12 male broiler chickens. Pen size was 0.90 $\mathrm{m} 2(1.2 \times 0.75 \mathrm{~m})$ and the floor was covered with $4-6 \mathrm{~cm}$ wood shavings. Temperature was maintained at $32^{\circ} \mathrm{C}$ until $\mathrm{d} 3$ and thereafter gradually reduced to $22^{\circ} \mathrm{C}$ at $\mathrm{d} 42$. A continuous light program from arrival to $\mathrm{d} 3$ and a 16L:8D light program from d 4 to 42 was applied. Chickens were reared from arrival to $d 42$ with ad libitum access to feed and water.

At d 11, chickens were individually vaccinated against Newcastle disease (Clone 30; eye drop, MSD Animal Health, Boxmeer, The Netherlands).

\section{Experimental Diets}

A 3-phase feeding program was applied; starter diets were provided from d 0 to 10, grower diets from $d 11$ to 28 and finisher diets from $d$ 29 to 42. Dietary treatments were applied throughout all three phases. Four experimental diets were used in this experiment in a $2 \times 2$ factorial arrangement.

They were:

1. inorganic macro and inorganic trace minerals (IMIT);

2. inorganic macro and organic trace minerals (IMOT);

3. organic macro and inorganic trace minerals (OMIT);

4. organic macro and organic trace minerals (OMOT).

In the inorganic macro and organic trace minerals diet, inorganic sourced $\mathrm{Ca}$ and $\mathrm{P}$ were used, but the inorganic trace mineral premix was replaced by a $100 \%$ organic sourced trace mineral premix (Optimin, Trouw Nutrition, Tilburg, The Netherlands). In the organic macro and inorganic trace minerals diet, only the inorganic macro minerals (Ca and P), provided by limestone and monocalcium phosphate, were largely replaced by Calfos (Sonac Vuren B.V., Vuren, The Netherlands), an organic hydroxyapatite form of $\mathrm{Ca}$ and $\mathrm{P}$ source originating from processed porcine bones. 
An inorganic sourced trace mineral premix was used. In the organic macro and organic trace minerals diet, inorganic macro minerals were largely replaced by Calfos and the trace mineral premix with inorganic minerals was completely replaced by a complete organic sourced trace mineral premix (Table 1).

All diets were produced and pelleted by ForFarmers N.V. (Lochem, The Netherlands) and analysed for ash (ISO 5984), dry matter (ISO 6496), crude fibre (ISO 6865), crude fat (ISO 6492), crude protein (ISO 5983), starch (ISO 6493), $\mathrm{Na}$ (ISO 6869), P (ISO 6941), Ca (ISO 6869), and $\mathrm{Fe}, \mathrm{Cu}$, $\mathrm{Mn}, \mathrm{Zn}$, and Se. Diet compositions and calculated and analysed nutrient values are shown in Table 1.

\section{Data Collection, Sampling and Measurements}

All chickens were individually weighed on d 0, 10, 21, 27, 34 and 41. Feed intake $(\mathbf{F I})$ was measured per pen for the starter, grower and finisher period. Feed conversion ratios (FCR) were calculated for the three phases and over the whole growth period, taking mortality into account. Mortality was recorded per pen per day. Gait score of 2 randomly selected chickens per pen was evaluated on $\mathrm{d} 27,34$ and 39 by using the method of Kestin et al. (1992) and scored within a range of 0 (normal locomotion) to 5 (unable to walk). To determine whether the light and diet factors might affect home pen behaviour, observations were performed in morning and afternoon sessions on $\mathrm{d} 6,13,20,27,34$ and 41, using the scan sampling technique (De Jong and Gunnink, 2019). During 3 to 4 min per session per day per pen, the number of chickens performing the following activities was scored: eating, drinking, walking, standing, resting, foraging, comfort behaviour, dust bathing and perching.

On d 42, two chickens per pen were randomly selected. Food pad dermatitis (FPD) was scored on both legs as 0 (no lesions), 1 (mild lesion) or 2 (severe lesion) (Ekstrand et al., 1998). Thereafter, chickens were subjected to electrical stunning, after which they were cut and bled. Breast weight, heart weight, and carcass weight were determined. Breast weight is described as the breast meat which was completely detached from sternum. Carcass weight is defined as the weight of body of the chicken without breast, legs and wings. 


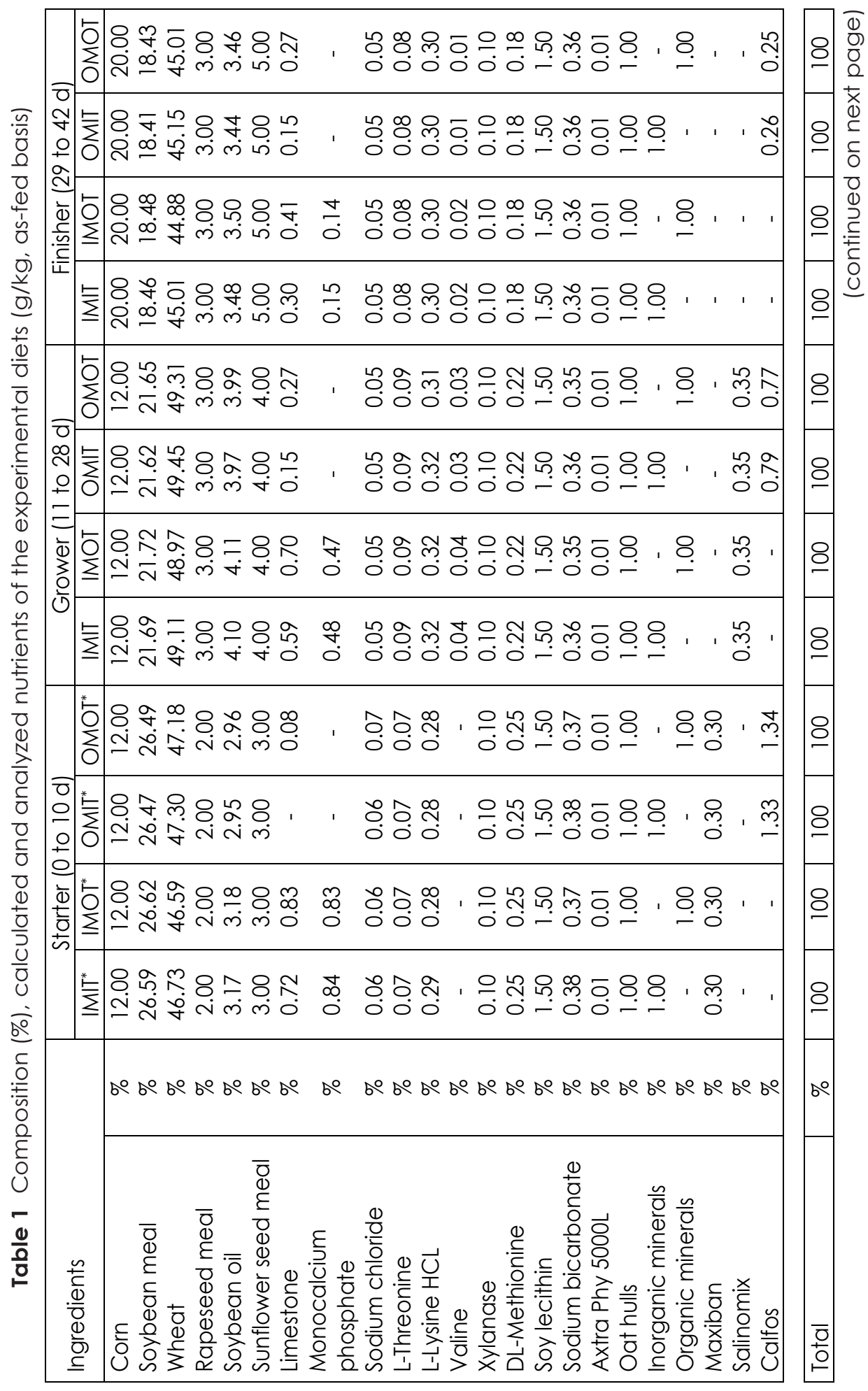



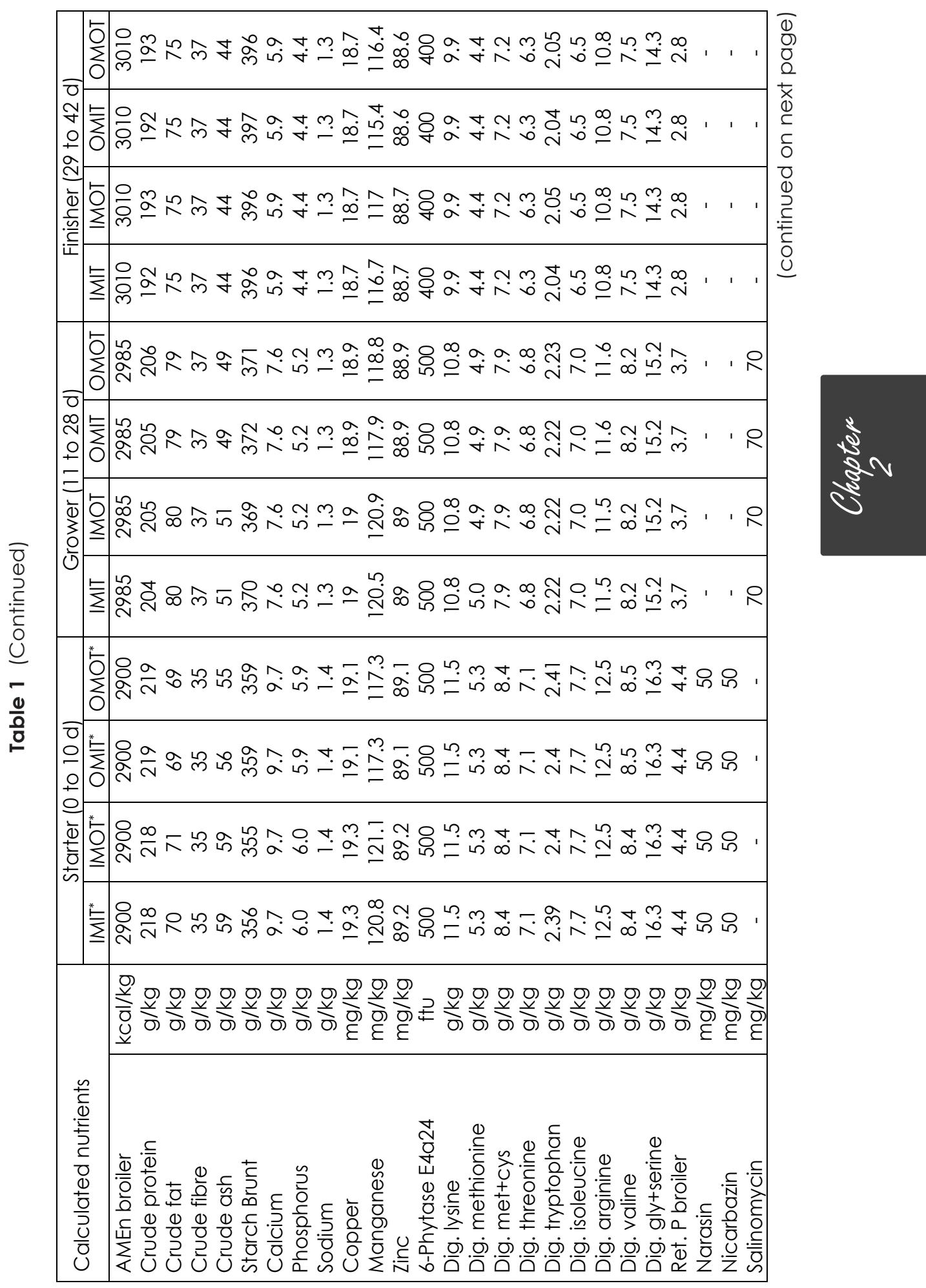


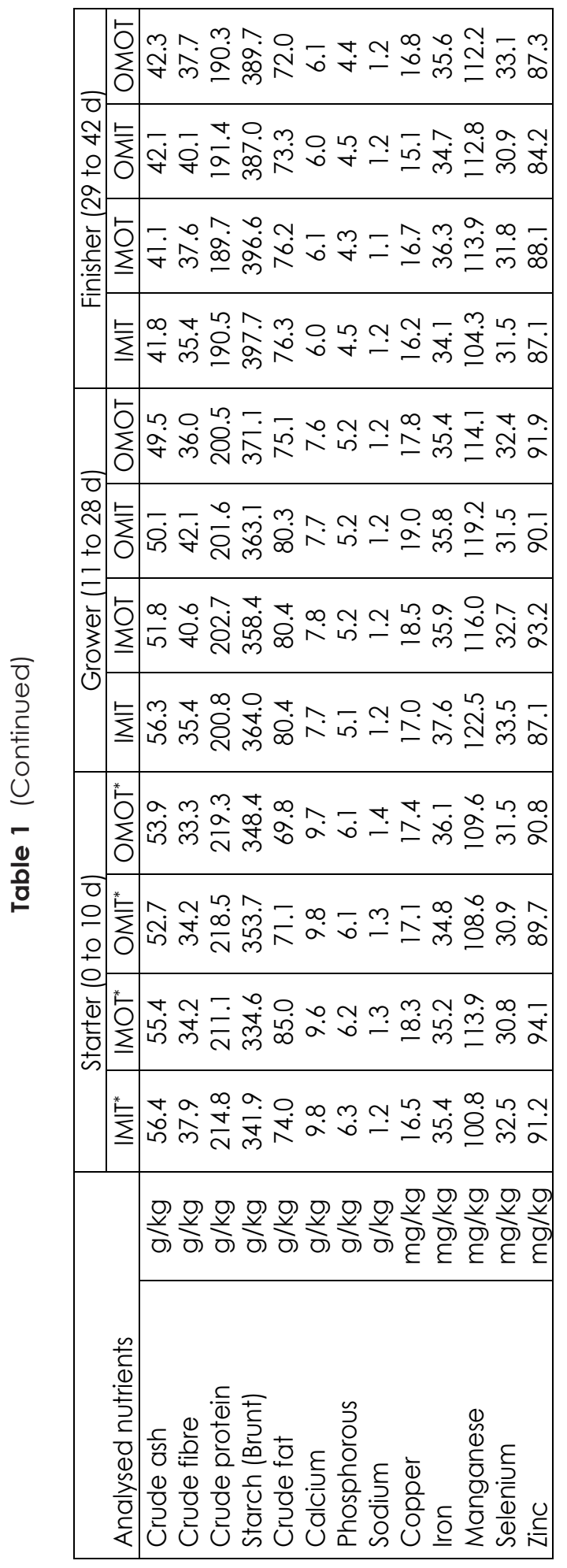

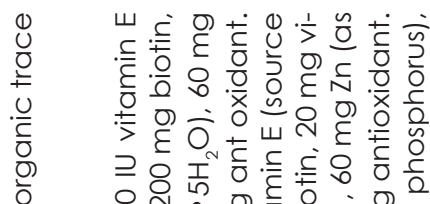
ㅅ․ बी

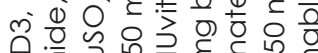

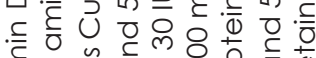
ठิ

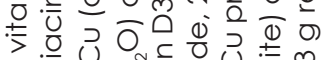
每 ठ ह ह

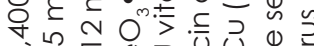

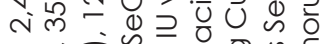

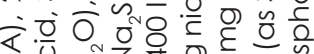
. O U U है

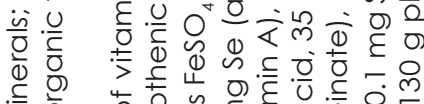
है ठ०

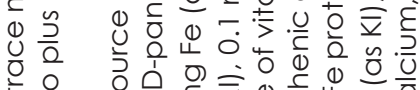

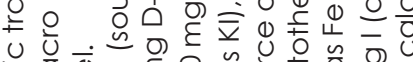

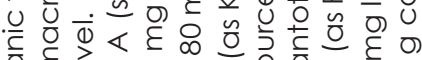

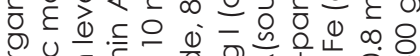
ठำ 든 . ว

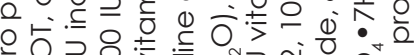

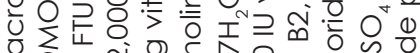

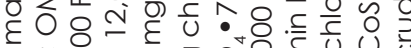
U î

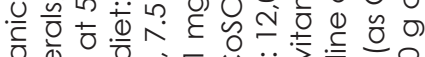
O)

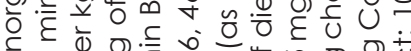
$\therefore$ ○

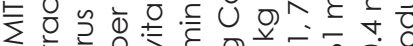
i) 0 ○ 0 O

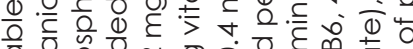

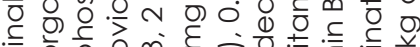

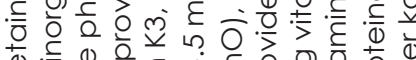

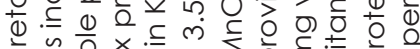

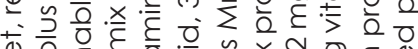

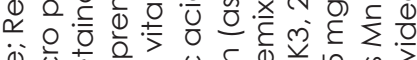

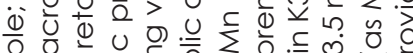
王 ฮิ บำ 方

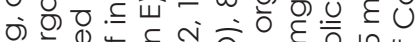
宁

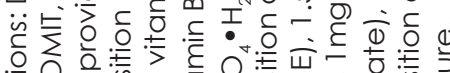

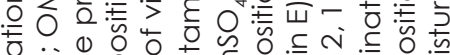

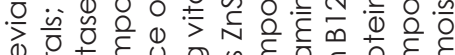
đ

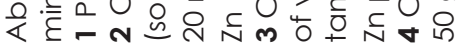


The left leg of each chicken was assessed by a veterinarian on bacterial chondronecrosis with osteomyelitis (BCO), epiphyseal plate abnormalities (EPA) and epiphysiolysis (EPI). All these leg abnormalities were scored in the range of 0 (no abnormalities), 1 (minor abnormality) or 2 (severe abnormality). Right legs were deboned and tibias were obtained, packed, and frozen at $-20^{\circ} \mathrm{C}$. After thawing, tibia weight was determined. Tibia proximal length, lateral cortex thickness, femoral and metatarsal side proximal head thickness, osseous volume, pore volume, total volume (osseous volume + pore volume), volume fraction (osseous volume / total volume), mineral content and mineral density were analysed on individual tibia, using a GE Phoenix 3D X-ray microfocus CT scanner (General Electric Company ${ }^{\circledR}$, Boston, Massachusetts, US) (method described by Bouxsein et al., 2010) (Figure 3). Robusticity index was calculated, using the formula of Reisenfeld (1972):

Robusticity index $(\mathrm{cm} / \mathrm{g})=$ bone proximal length $(\mathrm{cm}) /$ bone weight $(\mathrm{g})$

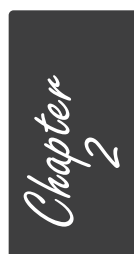

The same tibias, scanned in 3D X-ray measures, were subjected to a three-point bending test (method described by Jungmann et al., 2007), using an Instron ${ }^{\circledR}$ electromechanical universal testing machine (Instron ${ }^{\circledast}$, Norwood, Massachusetts, United States). Ultimate stress (maximal load of breaking point) data was used as the tibia ultimate strength; yield point (reached yield load just before the angle has changed on slope, inflection point) data was used as the tibia yield strength; the slope of the selected linear part of the curve data was used as the tibia stiffness; the area under the curve of selected region data was used as the tibia energy to fracture. Elastic modulus (GPa), which is the amount of strain as a result of a particular amount of stress and directly related to the density of bone (Novitskaya et al., 2017), was calculated using the following formula of Turner and Burr (1993):

$$
E=\frac{N S^{3}}{4 \delta T L^{3}}
$$

where $E$ = the elastic modulus (GPa), $N=$ the maximal load $(N), S=$ the span between bending fixtures $(\mathrm{mm}), T=$ tibia thickness $(\mathrm{mm}), \mathrm{L}=$ tibia length $(\mathrm{mm})$, and $\delta=$ the maximum deflection $(\mathrm{mm})$ at the midpoint of the bone. For more details about X-ray and Instron related bone variables, see Güz et al. (2019, 2020). 


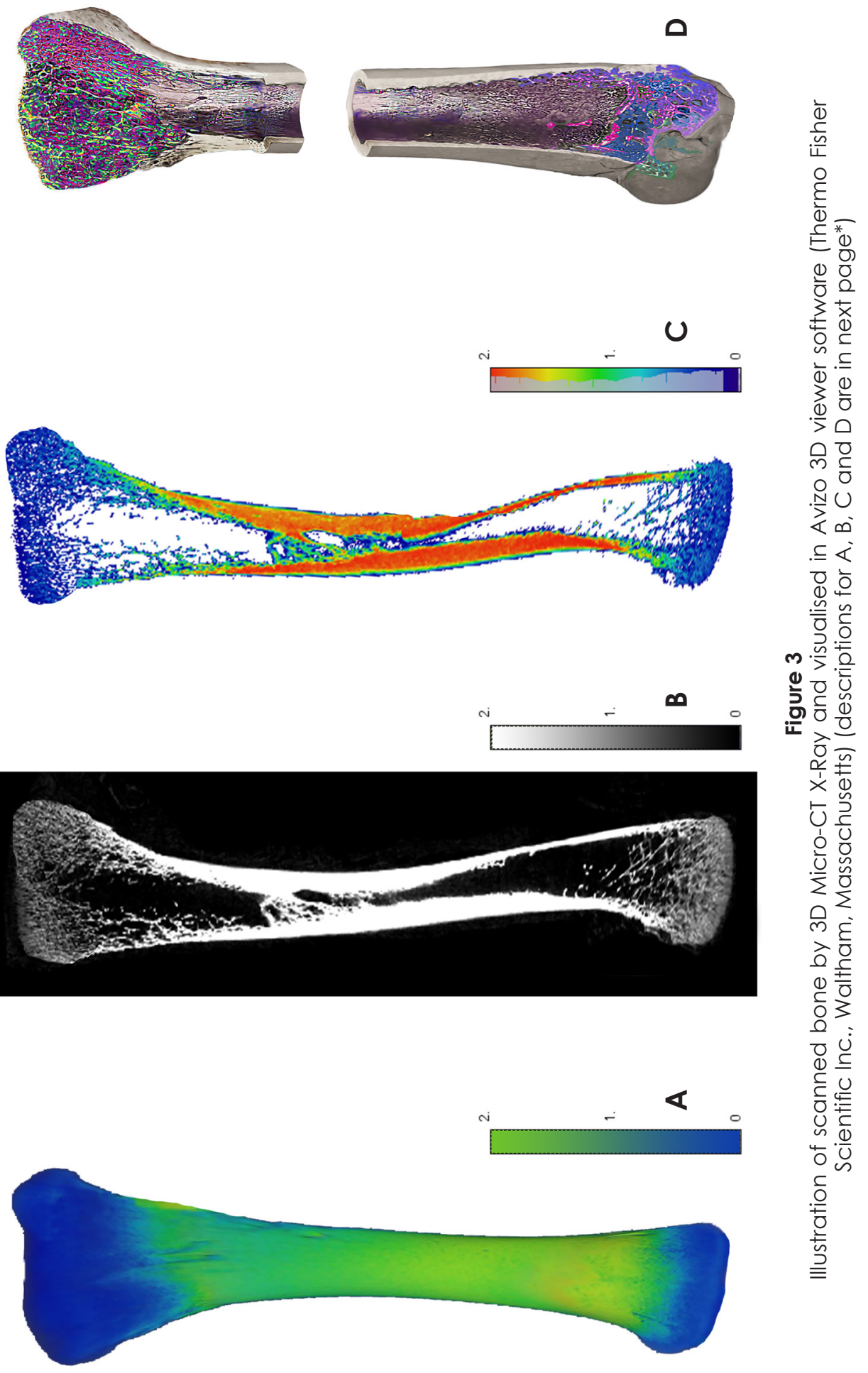


*(A) Three-dimensional tibia outer view. Color scale represents the mineralization areas of bone from blue (less mineralization, 0) to green (more mineralization, 2). (B) Two-dimensional black and white (gray scale) tibia outer layer view. Shades of gray represent the mineralization areas of bone from dark gray (less mineralization) to white (more mineralization). (C) Two-dimensional colored tibia outer layer view. Color scale represents the mineralization areas of bone from blue (less mineralization, 0 ) to red (more mineralization, 2). (D) Three-dimensional tibia inner view scanned by 3D Micro-CT X-Ray scanner. Different colors represent different densities of bone materials and pores.

\section{Statistical Analysis}

All statistical analyses were performed in SAS (Version 9.4, 2013, SAS Institute Inc., Cary, North Carolina, United States).

Hatch data (red hock, red beak, navel score, residual yolk weight, yolk free body mass, heart weight, liver weight, stomach weight and intestines weight) were subjected to mixed model analysis, using the PROC MIXED procedure. Hatchling was used as the experimental unit and incubator was added to the model as a random effect. The statistical model used was:

$$
Y=\mu+\text { Light during incubation }+\varepsilon,[1]
$$

where $Y=$ dependent variable, $\mu=$ overall mean, Light = green LED light or complete darkness during incubation, $\varepsilon=$ residual error.

All growth performance data (BW, Fl, FCR, mortality), tibia morphological, biophysical, and mechanical characteristics, slaughter characteristics, and home pen behaviour were subjected to mixed model analysis, using the PROC MIXED procedure. The statistical model used was:

$Y=\mu+$ Light during incubation + Macro minerals + Trace minerals

$$
+ \text { Interactions }+\varepsilon,[2]
$$

where $Y=$ dependent variable, $\mu=$ overall mean, Light during incubation = green LED light or complete darkness, Macro minerals = Inorganic or organic $\mathrm{Ca}$ and $\mathrm{P}$ during rearing, Trace minerals = Inorganic or organic trace minerals during rearing, Interactions = 2-way and 3-way interactions between light during incubation, macro minerals, and trace minerals, $\varepsilon=$ residual error. 
Pen was used as the experimental unit for all analyses $(n=72)$. The different blocks within the broiler house $(n=8)$ were used as a random effect. Body weight at slaughter age was added to the model as a covariable for tibia characteristics. Model assumptions were approved at both means and residuals. Non-normal distributed data were log-transformed before analyses. Results are provided as LSmeans \pm SEM. When multiple comparisons were performed, the level of significance was corrected, using Bonferroni correction.

Gait score and leg disorders (HB, FPD, EPA, BCO and EPI) were subjected to generalized linear mixed model analysis, using the PROC GLIMMIX procedure, using model 2. Gait score, FPD, EPA, BCO and EPI were analysed at multinomial level. Body weight at slaughter age was added to the model as a covariable for gait score and leg disorders. Effects were considered to be significant at $\mathrm{P} \leq 0.05$.

\section{RESULTS}

Only main effects are shown in all the tables (Table 2, 3 and 4) because only 1 three-way interaction and a few two way interactions were found and these will be discussed in the text.

\section{General Hatch Data}

Hatchability of fertile eggs was on average $87.8 \%$ and was not affected by green LED light or darkness during incubation $(P=0.11)$. Chick quality characteristics (red hock, red beak and navel score of all chickens; and residual yolk weight, yolk free body mass, heart weight, liver weight, stomach weight and intestines weight of 30 female chickens per treatment) are shown in Table A1 in the Supplementary Data. No differences were found in any of those parameters between green LED light incubated chickens and dark incubated chickens ( $P>0.06)$.

\section{Growth performance}

No two or three-way interactions between incubation light, macro mineral, and trace mineral source were found for performance characteristics from $\mathrm{d} 0$ to $\mathrm{d} 42$. Mortality was not affected by any of the treatments (Table 2). 


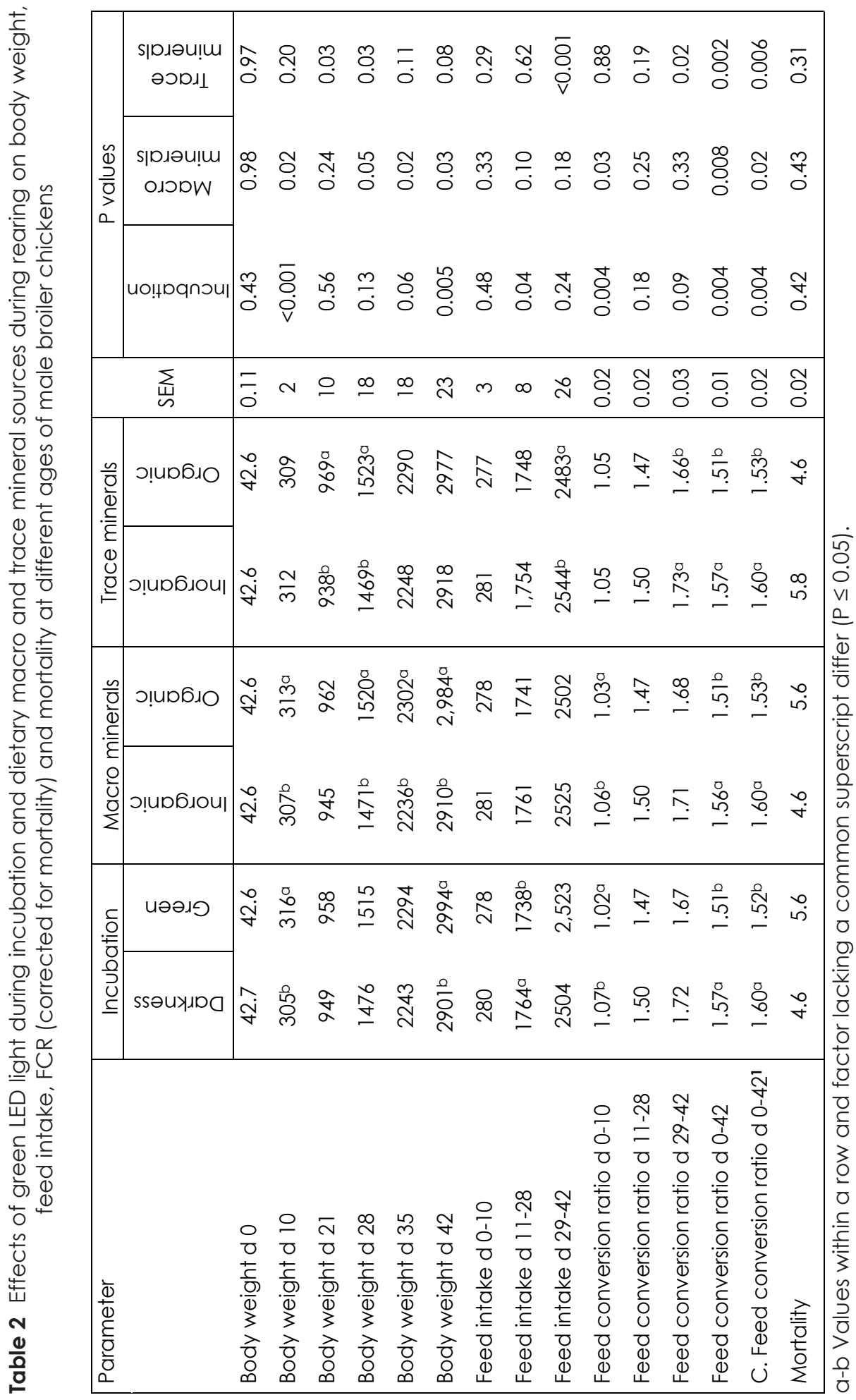


At d $10(\Delta=11 \mathrm{~g} ; \mathrm{P}<0.001)$, d $35(\Delta=51 \mathrm{~g} ; \mathrm{P}=0.06)$ and d 42 ( $\Delta=93 \mathrm{~g}$; $\mathrm{P}=0.005$ ) of age chickens incubated under green LED light had or tended to have a higher body weight compared to chickens incubated under complete darkness (Table 2). Fl between $\mathrm{d} 11$ and 28 was lower in the green light incubated chickens than in the complete darkness incubated chickens ( $\Delta=26 \mathrm{~g} ; \mathrm{P}=0.001$ ). FCR between $\mathrm{d} 0$ and 10 was lower in the darkness incubated chickens $(\Delta=0.05 ; P=0.004)$ than in green light incubated chickens. FCR $(\Delta=0.06 ; \mathrm{P}=0.004)$ and corrected FCR $(\Delta=0.08 ; \mathrm{P}=0.004)$ between $\mathrm{d} 0$ and 42 were lower in green light incubated chickens than in darkness incubated chickens (Table 2).

At day 10 ( $\Delta=6 \mathrm{~g} ; \mathrm{P}=0.02)$, day 28 ( $\Delta=49 \mathrm{~g} ; \mathrm{P}=0.05)$, day 35 ( $\Delta 66 \mathrm{~g}$; $\mathrm{P}=0.02)$ and day $42(\Delta=74 \mathrm{~g} ; \mathrm{P}=0.03)$, chickens fed with organic macro minerals had a higher BW compared to chickens fed with inorganic macro minerals (Table 2). FCR between day 0 and 10 was lower in the chickens fed with inorganic macro minerals $(\Delta=0.03 ; P=0.03$ ) than chickens fed with inorganic macro minerals. FCR between day 0 and 42 ( $\Delta=0.05 ; P=0.008$ ) and corrected FCR $(\Delta=0.07 ; \mathrm{P}=0.02)$ were lower in chickens fed with organic macro minerals than in chickens fed with inorganic macro minerals (Table 2).

At d 21 ( $\Delta=31 \mathrm{~g} ; \mathrm{P}=0.03$ ) and d 28 ( $\Delta=54 \mathrm{~g} ; \mathrm{P}=0.03$ ) chickens fed with organic trace minerals had a higher BW compared to chickens fed with inorganic trace minerals (Table 2). Fl between d 29 and 42 was higher in the chickens fed with inorganic trace minerals $(\Delta=61 \mathrm{~g} ; \mathrm{P}<0.001)$ than in the chickens fed with organic trace minerals. FCR between $\mathrm{d} 0$ and 42 was lower in chickens fed with organic trace minerals $(\Delta=0.03 ; \mathrm{P}=0.03)$ than chickens fed with inorganic trace minerals.

\section{Tibia Morphological Characteristics}

A three-way interaction between light during incubation, macro mineral source and trace mineral source was found on tibia length. However, after correction for Bonferroni, this interaction disappeared. No two-way interaction effects among light during incubation, macro mineral source, and trace mineral source were found for tibia morphological characteristics. No main effects of green LED light were found on tibia morphological characteristics. Main effects of macro and trace mineral 
source were found on tibia length. Chickens fed with organic macro minerals $(\Delta=0.27 \mathrm{~cm} ; \mathrm{P}=0.04)$ or trace minerals $(\Delta=0.21 \mathrm{~cm} ; \mathrm{P}=0.01)$ had a higher tibia length than chickens fed with inorganic varieties (Table 3 ).

\section{Tibia Biophysical Characteristics}

No three-way or two-way interactions between light during incubation, macro mineral source, and trace mineral source were found on tibia biophysical characteristics. Additionally, no main effects of green LED light and dietary trace mineral source were found on tibia biophysical characteristics. Chickens fed with organic macro minerals had a higher osseous volume $\left(\Delta=1.6 \mathrm{~cm}^{3} ; \mathrm{P}=0.02\right)$, higher mineral content $(\Delta=2.2 \mathrm{~g} ; \mathrm{P}=0.001)$ and higher mineral density $\left(\Delta=0.10 \mathrm{~g} / \mathrm{cm}^{2} ; \mathrm{P}=0.001\right)$ compared to chickens fed with inorganic macro minerals (Table 3 ).

\section{Tibia Mechanical Characteristics}

No two-way or three-way interactions between light during incubation, macro mineral source and trace mineral source were found on tibia mechanical characteristics. No main effects of green LED light and dietary trace mineral source were found on tibia mechanical characteristics. Chickens fed organic macro minerals had a higher ultimate strength ( $\Delta=11 \mathrm{~N} ; \mathrm{P}=0.006$ ), higher yield strength $(\Delta=8 \mathrm{~N} ; \mathrm{P}=0.04)$, higher stiffness $(\Delta=12 \mathrm{~N} / \mathrm{mm} ; \mathrm{P}=0.002)$ and higher energy to fracture ( $\Delta=10 \mathrm{~N}-\mathrm{mm}$; $\mathrm{P}=0.002$ ) compared to chickens fed inorganic macro minerals (Table 3 ).

\section{Locomotion Related Observations (Leg Disorders and Gait Score), Slaughter Characteristics and Home Pen Behaviour}

No two-way or three-way interactions between light during incubation, macro mineral source, and trace mineral source were found on gait scores and leg disorders. Chickens incubated under green LED light had higher gait scores on d $34(\Delta=0.19 ; \mathrm{P}=0.02)$ and $\mathrm{d} 39(\Delta=0.31 ; \mathrm{P}=0.001)$ compared to chickens incubated under complete darkness (Table 4). The incidence of leg disorders (FPD, BCO, EPA and EPI) in all treatment groups was very low. EPA was not scored at all in any of the treatment groups. FPD, $\mathrm{BCO}$, and EPI had an average score of $0.28,0.10$ and 0.20 , respectively, 


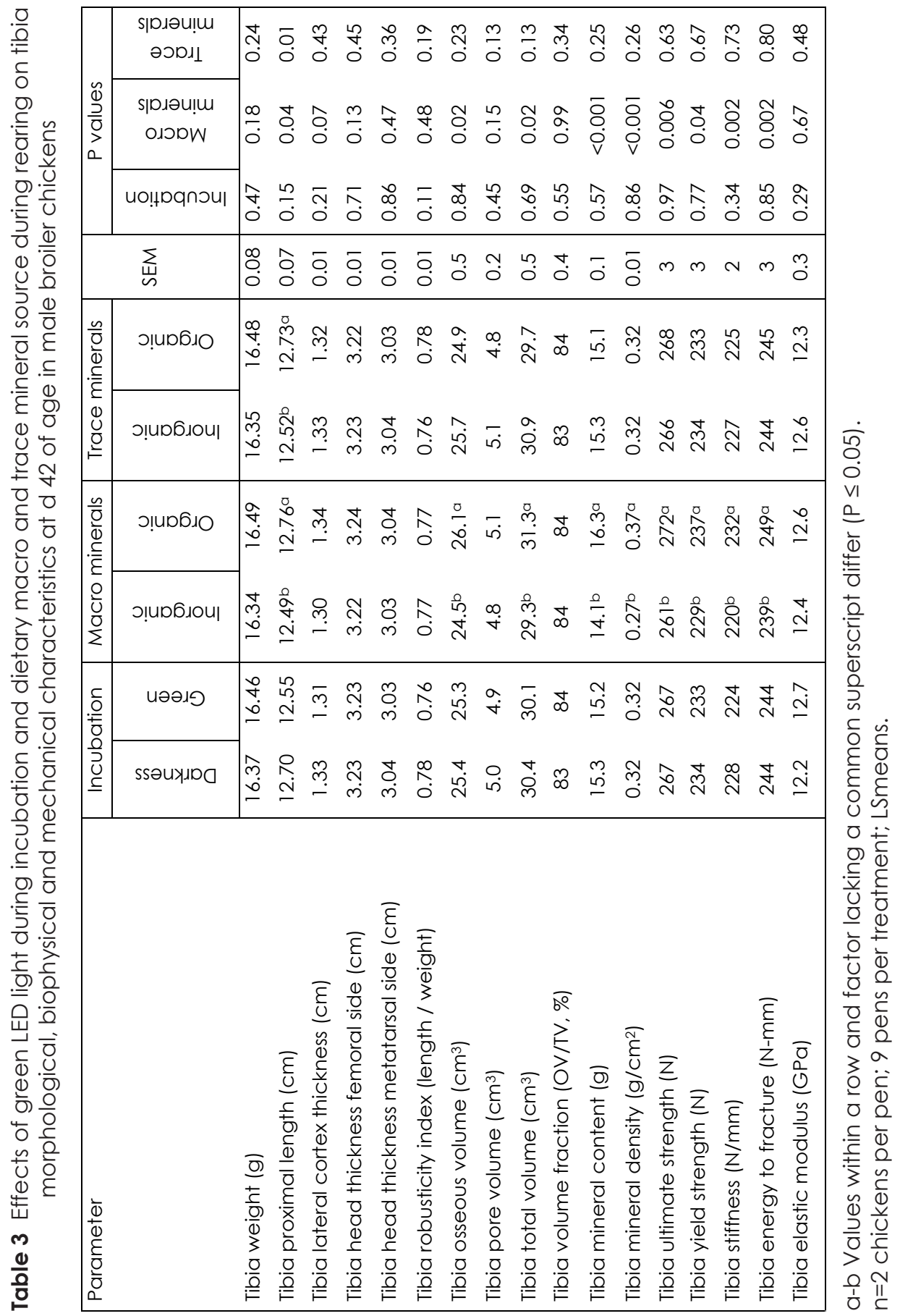




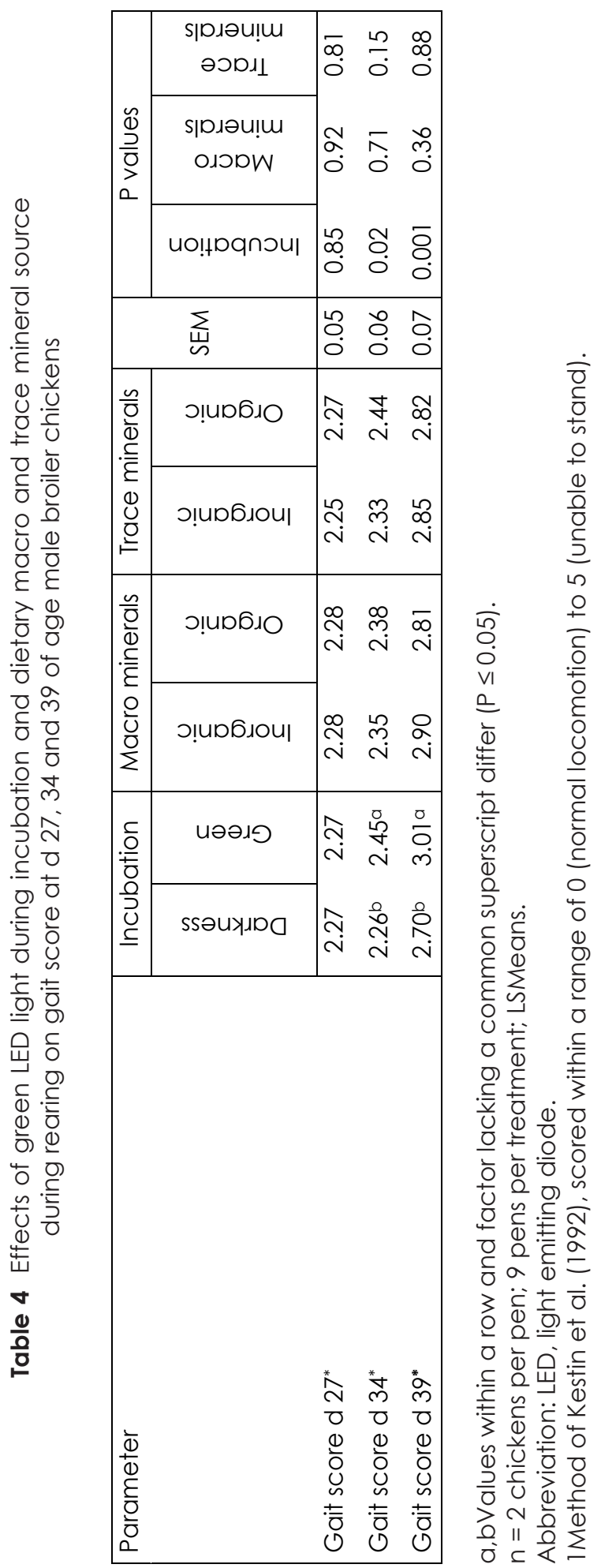


and no significant differences were observed between treatment groups.

Slaughter characteristics (breast weight, heart weight and carcass weight) are presented in Table A2 in the Supplementary Data. Home pen behaviour parameters (eating, drinking, walking, standing, resting, foraging, comfort behaviour, dust bathing and perching) for each of the scanning days (day 6, 13,20,27, 34 and 41) are presented in Table A3 in the Supplementary Data. No difference was found in any of slaughter characteristics and behaviour parameters among treatment groups.

\section{DISCUSSION Green LED Light}

Several environmental factors, such as temperature, humidity, ventilation, and egg turning, are known to play important roles on the development of the chicken embryos (Decuypere et al., 2001; Molenaar et al., 2010).

Light during incubation is another important factor related to embryonic muscular development (Aige-Gil and Murillo-Ferrol, 1992; Archer et al., 2009; Huth and Archer, 2015; Archer and Mench, 2017). Light can pass through the eggshell, as demonstrated in the current study, and by Shafey (2004) and shafey et al. (2005) with light of several wave lengths, meaning that embryos were indeed exposed to light during incubation. The current study showed higher BW at several ages of chickens incubated under green LED light compared to chickens incubated under darkness. This is in agreement with studies of Zhang et al. $(2014,2016)$ and Rozenboim et al. (2004), where green LED light during incubation resulted in higher body weights and especially post-hatch pectoral muscle growth by enhancing proliferation and differentiation of satellite cells during embryonic development and post hatch period, compared to chickens incubated under complete darkness. Zhang et al. (2014) also demonstrated higher liver weight, higher antioxidant activity, and higher melatonin levels in broiler chickens exposed to green LED light during incubation compared to darkness incubated chickens.

Regarding bone development, green LED light during incubation has been found to increase bone development related hormones, such as plasma growth hormone (GH), prolactin (PRL), melatonin (Archer et al., 2009; Huth and Archer, 2015; Archer and Mench, 2017) and hypothalamic growth 
hormone releasing hormone (GHRH), growth hormone receptor (GHR), and insulin-like growth factor-1 (IGF-1) of broiler chickens (Dishon et al., 2017). However, in the current study, no effect of green LED light during incubation was found on tibia morphological, biophysical or mechanical characteristics at slaughter age. Results of the current study suggest that although green light during incubation has been found to stimulate abovementioned hormones, receptors and growth factors (Archer and Mench, 2017; Dishon et al., 2017), and to accelerate body weight gain (Rozenboim et al., 2004; Halevy et al., 2006; Zhang et al., 2014, 2016), its effect on bone development appears limited. It can be speculated that despite green LED light during incubation having a positive influence on growth factors and pectoral muscle growth, by enhancing abovementioned hormones and receptors, these influences seem not sufficient to stimulate bone development.

Although no effect of green LED light during incubation was found on bone characteristics at slaughter age, gait score was worse in chickens incubated in green LED light compared to chickens incubated in darkness, even after correction for differences in BW. This suggests that other factors than bone characteristics (muscles, joints, tendons) may play a role as well in locomotion of broiler chickens, but how these different leg parts interact with locomotion is still unclear. That other factors play a role in locomotion is also demonstrated by the almost complete lack of leg bone pathologies in the current study, whereas the gait score differed between treatments.

\section{Macro Minerals}

This study showed that replacement of inorganic sourced macro minerals ( $\mathrm{Ca}$ and $\mathrm{P}$ ) by their organic varieties resulted in higher body weight in most phases of the rearing period, while preserving similar feed intake, which resulted in lower FCR. These findings are in line with previous studies in which was found that supplying organic macro minerals to broiler chickens stimulated growth performance (Bradbury et al., 2017, 2018). Organic sourced macro minerals have covalent bonds that provide a better binding strength compared to electrovalent bonds in inorganic minerals. This provides these minerals to bind with other compounds more efficiently (Vieira, 2008; Bao and Choct, 2009), resulting in a better chemical stability and in 
more resistance to $\mathrm{pH}$ changes throughout the digestive system (selle at al., 2009; Wang et al. 2019). Finally, this results in lower antagonistic complex forming with minerals or other diet ingredients, resulting in a higher bio-availability and an overall lower FCR.

Thus, replacement of inorganic macro minerals by a similar amount of organic macro minerals in the diet appears to result in comparable or even higher growth performance. Results of the current study suggest that the current broiler diets can be improved related to macro mineral availability for fast growing male broiler chickens, leading to enhanced performance. It can be questioned whether or not a further increase in inorganic macro minerals is desired, because of the potential risk of mineral complex forming and waste of minerals in the environment (Dozier III ef al., 2003; Bao et al., 2007). Alternatively, the use of organic varieties of macro minerals might be a more sustainable solution.

The replacement of inorganic by organic macro minerals (Ca, P) did not only result in enhanced growth, but also in better bone characteristics. Almost all tibia morphological, biophysical, and mechanical characteristics were positively affected by dietary organic macro minerals. Ca and $\mathrm{P}$ are known to be essential for bone development as a main component of the bone matrix (Rath et al., 1999; Blake and Fogelman, 2002), as an essential part of enzymes involved in bone development (McDevitt et al., 2006) and also as an essential part of bone development related hormones, such as GH, IGF-1, T3 and T4 (Parmer at al., 1987; Rosol and Capen, 1997). Increasing Ca and $\mathrm{P}$ levels in broiler chicken diets has been shown to positively influence bone mineralization, leading to a stronger bone (Driver et al., 2005a,b; Létourneau-Montminy et al., 2008). A deficiency of Ca or P may impair bone growth, mineral density, and strength (Bar et al., 2003; Sá et al., 2004; McDevitt et al., 2006; Dibner et al. 2007). A fast increase in bone mineralisation and bone growth during the rearing period of fast growing broilers, expressed in high tibia $\mathrm{Ca}$ and $\mathrm{P}$ level, is crucial for leg bone strength (shao et al., 2019). Onyango et al. (2003) reported that increasing dietary $\mathrm{Ca}$ and $\mathrm{P}$ amount resulted in increased tibia mineral content of broiler chickens. Guinotte et al. (1991) reported that organic sourced $\mathrm{Ca}$ in broiler chicken diets resulted in higher tibia proximal length and lateral cortex thickness compared to inorganic sourced $\mathrm{Ca}$. This is in accordance with results of the current study and 
overall, it can be suggested that organic dietary $\mathrm{Ca}$ and $\mathrm{P}$ may provide better bone health to broiler chickens than the inorganic $\mathrm{Ca}$ and $\mathrm{P}$ sources.

\section{Trace Minerals}

The current study showed that replacement of inorganic trace minerals (Fe, $\mathrm{Cu}, \mathrm{Mn}, \mathrm{Zn}$ and Se) by their organic varieties resulted in (tendencies to a) higher body weight during the rearing period. These findings are in accordance with previous studies that showed that dietary organic sourced CU, Fe, Mn, Zn (Bao et al., 2009; Abdallah et al., 2009; Ao et al., 2009; Huang et al., 2009) resulted in higher growth performance than their inorganic varieties. This is probably because of an increased bio-availability and less antagonistic complex forming with each other, as explained above. Yenice et al. (2015) showed that serum mineral concentrations of broiler chickens were found higher by $22 \%$ for $\mathrm{Mn}, 17 \%$ for $\mathrm{Zn}$ and $20 \%$ for Cu when minerals were provided in their organic variety instead of inorganic variety, which indicates that organic minerals have a higher bio-availability for chickens than inorganic minerals. This higher bio-availability is supported by the findings that the difference in BW gain is particularly due to a higher feed efficiency, rather than due to an increase in Fl. It appears that levels of inorganic trace minerals used in the current experiment might be suboptimal to reach the genetic potential in weight gain of fast growing male broiler chickens. However, a further increase of dietary trace minerals may result in higher wastage of minerals in the environment and even toxic effects (Dozier III et al. 2003). Switching to organic trace minerals may reduce mineral losses in the environment (Nollet et al., 2007; Leeson and Caston, 2008).

Trace minerals are known to play major roles in metabolism, particularly in hormone and enzyme systems (Richards, 1997), from which some are related to embryonic and post-hatch bone development (Angel, 2007: Dibner et al. 2007). Cu has an essential role in bone strength and flexibility (Rucker et al., 1998; Dibner et al. 2007). Zn is essential for the functioning of bone osteoclast cells (Richards et al. 2010). Mn is required for the synthesis of bone cartilage (Eyre, 2004; Dibner et al. 2007). Iron (Fe), iodine (I) and selenium (Se) are also essential trace minerals for bone development by regulating 
thyroid functions, which is known to regulate bone growth (Jianhua et al. 2000; Medeiros et al. 2002; Röttger et al. 2011). Based on these functions in bone related metabolism, it can be suggested that a higher bio-availability of trace minerals, due to the use of organic trace mineral varieties, might improve bone characteristics. Although some previous studies, using organic trace minerals did find a positive effect on bone development (Dibner et al. 2007; El-Husseiny et al., 2012), in the current study, tibia morphological, biophysical and mechanical characteristics were not affected by trace mineral source. These results are in agreement with other previous studies (Abdallah et al., 2009; Zhao et al., 2010). As a result of no effects of trace mineral sources on bone characteristics in this study, it can be speculated that the current guidelines for trace minerals are sufficient for bone development, whereas this does not appear to be the case for growth performance.

In conclusion, using green LED light during incubation, dietary organic macro minerals, and organic trace minerals resulted in higher BW gain of broiler chickens compared to darkness during incubation, inorganic macro minerals, and inorganic trace minerals, respectively. However, only dietary organic macro minerals positively affected most of the tibia morphological, biophysical, and mechanical characteristics. This suggests, on one hand, that macro mineral source rather than trace mineral source appears to be more important for leg bone development in current broiler chicken strains. However, on the other hand, effects of macro or trace mineral source on locomotion and leg pathologies appear to be marginal. 


\section{ACKNOWLEDGEMENTS}

This experiment was the part of the "Healthy Bones" project, financed by a public-private partnership (project number BO-47001-011). The financial support of the Ministry of Agriculture, Nature, and Food Quality of The Netherlands, Aviagen EPI, Darling Ingredients Inc., ForFarmers N.V., Hubbard, Marel Stork Poultry Processing BV, Nepluvi and Nutreco (The Netherlands) is gratefully acknowledged. The authors would like to thank Lagerwey hatchery (Lunteren, The Netherlands) for providing eggs; Remco Hamoen for his assistance and valuable contributions during the 3D micro CT X-ray scanning. Stefan Veenstra, Bert van Nijhuis, Henny Reimert, Marcel Heetkamp, llona van der Anker - Hensen, Monique Ooms, Bjorge Laurenssen, Lotte Stokvis, Jan Wijnen, Olivia Shaw, Anne Steenstra and the animal caretakers are acknowledged for their assistance during the experiment. 


\section{SUPPLEMENTARY DATA}

Table A1 Effects of green LED light during incubation on hatchling characteristics (red hock, red beak, navel score, residual yolk weight, yolk free body mass, heart weight, liver weight, stomach weight and intestines weight) of female broiler chickens

\begin{tabular}{|c|c|c|c|c|}
\hline \multirow[t]{2}{*}{ Parameter ${ }^{1}$} & \multicolumn{2}{|c|}{ Incubation light condition } & \multirow{2}{*}{ SEM } & \multirow{2}{*}{$P$ values } \\
\hline & Darkness & Green LED light & & \\
\hline Chick weight (g) & 42.2 & 42.4 & 0.31 & 0.79 \\
\hline Residual yolk weight (g) & 3.96 & 3.88 & 0.16 & 0.71 \\
\hline Yolk-free body mass (g) & 38.3 & 38.5 & 0.28 & 0.60 \\
\hline Heart weight (\% of YFBM) & 0.81 & 0.84 & 0.02 & 0.18 \\
\hline Liver weight (\% of YFBM) & 2.94 & 2.98 & 0.05 & 0.60 \\
\hline Intestines weight (\% of YFBM) & 6.07 & 5.99 & 0.41 & 0.68 \\
\hline Stomach weight (\% of YFBM) & 6.00 & 6.28 & 0.14 & 0.06 \\
\hline $\operatorname{Red~hock}^{2}(\%)$ & 1.8 & 2.4 & 0.01 & 0.36 \\
\hline Red beak $^{2}(\%)$ & 6.3 & 2.9 & 0.02 & 0.07 \\
\hline Navel score ${ }^{2}$ & 1.26 & 1.29 & 0.03 & 0.37 \\
\hline
\end{tabular}

130 chicks per treatment were assessed for all chick quality parameters, except for red hocks, red beaks, and navel score $(n=864)$.

2 Red hock and red beak of all chicks were scored as 0 (absent) or 1 (present) and expressed as percentage chickens with score 1; navel score was assessed as 1 (good), 2 (moderate), or 3 (poor) and expressed as average score.

Table A2 Effects of green LED light during incubation and dietary macro and trace mineral source during rearing on average body weight and percentages of breast, carcass and heart of male broiler chickens at d 42 of age

\begin{tabular}{|c|c|c|c|c|c|c|c|c|c|c|}
\hline \multirow[t]{2}{*}{ Parameter } & \multicolumn{2}{|c|}{ Incubation } & \multicolumn{2}{|c|}{$\begin{array}{c}\text { Macro } \\
\text { minerals }\end{array}$} & \multicolumn{2}{|c|}{$\begin{array}{c}\text { Trace } \\
\text { minerals }\end{array}$} & \multirow[b]{2}{*}{ SEM } & \multicolumn{3}{|c|}{$P$ values } \\
\hline & 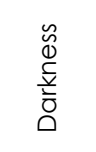 & $\begin{array}{l}\frac{\nwarrow}{\Phi} \\
\frac{0}{\bar{U}}\end{array}$ & 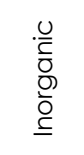 & $\begin{array}{l}\frac{U}{\bar{C}} \\
\overline{0} \\
\overline{0} \\
\overline{0}\end{array}$ & $\begin{array}{l}\frac{U}{\bar{C}} \\
\bar{D} \\
\bar{D} \\
\underline{0} \\
\underline{\underline{O}}\end{array}$ & $\begin{array}{l}\frac{U}{\bar{C}} \\
\overline{0} \\
\overline{0} \\
\overline{0}\end{array}$ & & 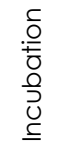 & 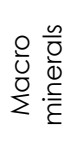 & 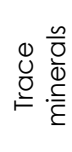 \\
\hline Body weight' $(g)$ & $3,054^{b}$ & $3,130^{a}$ & 3,117 & 3,066 & 3,058 & 3,125 & 26 & 0.05 & 0.18 & 0.08 \\
\hline Breast weight $^{2}(\%)$ & 21.8 & 21.7 & 21.8 & 21.8 & 21.7 & 21.8 & 0.7 & 0.39 & 0.50 & 0.21 \\
\hline Carcass weight 2,3 (\%) & 53.2 & 52.8 & 53.1 & 53.0 & 53.3 & 52.8 & 1.3 & 0.30 & 0.26 & 0.52 \\
\hline Heart weight ${ }^{2}(\%)$ & 0.5 & 0.5 & 0.5 & 0.5 & 0.5 & 0.5 & 0.001 & 0.65 & 0.73 & 0.50 \\
\hline
\end{tabular}

1 Average body weight of slaughtered chickens at d 42.

2 Percentages of pectoralis major plus pectoralis minor and hearth weight of average body weight.

3 Carcass weight (feathers and skin are attached) without both wings (detached from humerus head) and legs (detached from femur head).

$a-b$ Values within a row and factor lacking a common superscript differ $(P \leq 0.05)$.

$\mathrm{n}=2$ chickens per pen; 9 pens per treatment; LSMeans. 
Table A3 Effects of green LED light during incubation and dietary macro and trace mineral source during rearing on home pen behaviour of male broilers (eating, drinking, walking, standing, resting, foraging, comfort behaviour, dustbathing and perching, expressed as percentage of chickens performing each behaviour) for each of the scanning days (day 6 , $13,20,27,34$ and 41 )

\begin{tabular}{|c|c|c|c|c|c|c|c|c|c|c|}
\hline \multirow{2}{*}{ 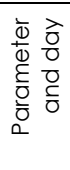 } & \multicolumn{2}{|c|}{ Incubation } & \multicolumn{2}{|c|}{ Macro minerals } & \multicolumn{2}{|c|}{ Trace minerals } & \multirow[b]{2}{*}{ SEM } & \multicolumn{3}{|c|}{$P$ values } \\
\hline & $\begin{array}{l}\tilde{\widetilde{U}} \\
\stackrel{\tilde{D}}{\bar{v}} \\
\overline{\bar{D}} \\
0\end{array}$ & $\begin{array}{l}\frac{\ulcorner}{0} \\
\frac{\bigotimes}{0}\end{array}$ & 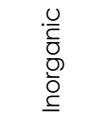 & 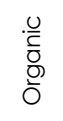 & 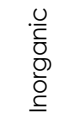 & $\begin{array}{l}\cdot \frac{u}{c} \\
\bar{D} \\
\overline{0} \\
0\end{array}$ & & 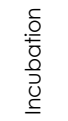 & 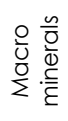 & 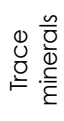 \\
\hline \multicolumn{11}{|c|}{ Eating } \\
\hline $6 d$ & 21.8 & 19.8 & 22.3 & 19.4 & $18.1^{\mathrm{b}}$ & $23.5^{a}$ & 1.7 & 0.40 & 0.23 & 0.03 \\
\hline $13 d$ & 19.8 & 22.5 & 21.2 & 21.1 & 22.3 & 19.9 & 1.2 & 0.11 & 0.95 & 0.17 \\
\hline $20 d$ & 18.1 & 22.0 & 21.3 & 18.9 & 19.6 & 20.6 & 2.5 & 0.28 & 0.51 & 0.78 \\
\hline $27 d$ & 19.9 & 18.5 & 17.6 & 20.8 & 20.3 & 18.1 & 2.5 & 0.71 & 0.39 & 0.54 \\
\hline $34 \mathrm{~d}$ & 19.1 & 20.1 & 20.1 & 19.1 & 19.9 & 20.0 & 1.7 & 0.45 & 0.45 & 0.98 \\
\hline $41 \mathrm{~d}$ & 18.7 & 23.4 & 22.1 & 20.0 & 20.3 & 21.8 & 1.9 & 0.09 & 0.43 & 0.57 \\
\hline \multicolumn{11}{|c|}{ Drinking } \\
\hline $6 d$ & 12.2 & 10.7 & 11.8 & 11.2 & 11.0 & 12.0 & 1.3 & 0.42 & 0.77 & 0.59 \\
\hline $13 d$ & 10.6 & 12.8 & 10.2 & 13.3 & 12.3 & 11.1 & 1.5 & 0.30 & 0.15 & 0.58 \\
\hline $20 d$ & 13.1 & 16.1 & 12.7 & 16.5 & 15.2 & 13.9 & 2.1 & 0.33 & 0.22 & 0.68 \\
\hline $27 d$ & 15.7 & 14.3 & 13.7 & 16.3 & 15.5 & 14.5 & 2.3 & 0.67 & 0.42 & 0.77 \\
\hline $34 \mathrm{~d}$ & 12.9 & 13.4 & 15.8 & 12.7 & 12.1 & 14.3 & 1.6 & 0.84 & 0.08 & 0.32 \\
\hline $41 \mathrm{~d}$ & 13.8 & 13.8 & 13.5 & 14.0 & 14.2 & 13.4 & 1.7 & 0.99 & 0.84 & 0.73 \\
\hline \multicolumn{11}{|c|}{ Walking } \\
\hline $6 d$ & 16.3 & 11.4 & 14.1 & 13.7 & 13.9 & 13.8 & 1.7 & 0.06 & 0.87 & 0.96 \\
\hline $13 d$ & 17.1 & 15.1 & 16.4 & 15.8 & 16.7 & 15.5 & 1.7 & 0.42 & 0.78 & 0.62 \\
\hline $20 \mathrm{~d}$ & 20.7 & 17.0 & 20.3 & 17.3 & 18.2 & 19.5 & 2.1 & 0.22 & 0.31 & 0.67 \\
\hline $27 d$ & 21.2 & 18.2 & 20.0 & 19.4 & 19.7 & 19.6 & 2.2 & 0.35 & 0.85 & 0.98 \\
\hline $34 \mathrm{~d}$ & 15.5 & 14.5 & 15.8 & 14.2 & 15.9 & 14.1 & 1.8 & 0.72 & 0.55 & 0.49 \\
\hline $41 \mathrm{~d}$ & 20.3 & 18.5 & 17.4 & 17.3 & 18.4 & 16.4 & 1.8 & 0.21 & 0.98 & 0.44 \\
\hline \multicolumn{11}{|c|}{ Standing } \\
\hline $6 d$ & 16.4 & 18.7 & 17.7 & 17.4 & 17.7 & 17.4 & 1.9 & 0.41 & 0.93 & 0.93 \\
\hline $13 d$ & 16.1 & 16.9 & 18.1 & 14.9 & 15.2 & 17.9 & 1.8 & 0.72 & 0.21 & 0.28 \\
\hline $20 \mathrm{~d}$ & 18.4 & 20.1 & 19.8 & 18.7 & 22.3 & 16.2 & 2.2 & 0.59 & 0.73 & 0.06 \\
\hline $27 d$ & 17.2 & 19.4 & 19.2 & 17.5 & 17.2 & 19.5 & 2.3 & 0.49 & 0.62 & 0.47 \\
\hline $34 \mathrm{~d}$ & 15.5 & 14.6 & 14.1 & 16.0 & 14.8 & 15.4 & 1.9 & 0.74 & 0.50 & 0.83 \\
\hline $41 \mathrm{~d}$ & 18.7 & 22.9 & 20.8 & 20.8 & 20.9 & 20.6 & 2.0 & 0.15 & 0.99 & 0.90 \\
\hline \multicolumn{11}{|c|}{ Resting } \\
\hline $6 d$ & 34.3 & 31.0 & 30.6 & 34.8 & 35.7 & 29.7 & 2.6 & 0.36 & 0.25 & 0.10 \\
\hline $13 d$ & 33.7 & 28.8 & 31.3 & 31.3 & 29.9 & 32.6 & 2.7 & 0.19 & 0.99 & 0.49 \\
\hline $20 \mathrm{~d}$ & 24.5 & 22.3 & 23.2 & 23.5 & 21.0 & 25.7 & 2.2 & 0.49 & 0.94 & 0.15 \\
\hline $27 d$ & 20.9 & 26.7 & 24.6 & 23.0 & 23.0 & 24.5 & 2.2 & 0.07 & 0.63 & 0.65 \\
\hline $34 \mathrm{~d}$ & 33.5 & 32.8 & 30.3 & 35.9 & 33.5 & 32.8 & 2.7 & 0.88 & 0.14 & 0.86 \\
\hline $41 \mathrm{~d}$ & 27.5 & 22.4 & 24.5 & 25.7 & 24.3 & 25.9 & 2.3 & 0.14 & 0.70 & 0.62 \\
\hline \multicolumn{11}{|c|}{ Forage } \\
\hline $6 d$ & 0.9 & 0.7 & 0.9 & 0.7 & 1.2 & 0.5 & 0.04 & 0.69 & 0.72 & 0.24 \\
\hline $13 d$ & 0.3 & 0.6 & 0.3 & 0.6 & 0.7 & 0.08 & 0.03 & 0.50 & 0.50 & 0.09 \\
\hline $20 d$ & 3.7 & 0.9 & 1.4 & 3.3 & 2.8 & 1.9 & 0.09 & 0.05 & 0.17 & 0.52 \\
\hline $27 d$ & 3.7 & 1.4 & 2.8 & 2.4 & 2.8 & 2.3 & 0.1 & 0.11 & 0.79 & 0.75 \\
\hline $34 \mathrm{~d}$ & 0.7 & 1.7 & 0.9 & 1.5 & 1.0 & 1.5 & 0.06 & 0.25 & 0.47 & 0.54 \\
\hline $41 \mathrm{~d}$ & 0.4 & 0.4 & 0.5 & 0.1 & 0.1 & 0.4 & 0.03 & 0.34 & 0.37 & 0.33 \\
\hline
\end{tabular}


Table A3 (Continued)

\begin{tabular}{|c|c|c|c|c|c|c|c|c|c|c|}
\hline \multicolumn{11}{|c|}{$\begin{array}{l}\text { Comfort } \\
\text { behaviour }\end{array}$} \\
\hline $6 \mathrm{~d}$ & 2.1 & 2.2 & 2.5 & 1.8 & 0.7 & 2.6 & 0.07 & 0.91 & 0.53 & 0.37 \\
\hline $13 d$ & 2.7 & 3.3 & 2.8 & 3.2 & 2.8 & 3.2 & 0.08 & 0.65 & 0.77 & 0.77 \\
\hline $20 d$ & 1.7 & 1.8 & 1.4 & 2.1 & 1.2 & 2.4 & 0.07 & 0.96 & 0.57 & 0.28 \\
\hline $27 d$ & 1.6 & 1.6 & 2.3 & 0.8 & 1.59 & 1.54 & 0.07 & 0.97 & 0.17 & 0.97 \\
\hline $34 \mathrm{~d}$ & 2.2 & 2.2 & 2.4 & 1.9 & 2.6 & 1.7 & 0.06 & 0.96 & 0.65 & 0.35 \\
\hline $41 \mathrm{~d}$ & 1.2 & 2.6 & 1.5 & 2.3 & 2.2 & 1.7 & 0.07 & 0.18 & 0.40 & 0.66 \\
\hline \multicolumn{11}{|c|}{$\begin{array}{l}\text { Dust } \\
\text { bathing }\end{array}$} \\
\hline $6 \mathrm{~d}$ & 0.9 & 0.7 & 0.5 & 1.2 & 0.9 & 0.7 & 0.05 & 0.77 & 0.29 & 0.80 \\
\hline $13 d$ & 0 & 0.3 & 0 & 0.3 & 0.3 & 0.1 & 0.01 & 0.31 & 0.31 & 0.34 \\
\hline $20 \mathrm{~d}$ & 0 & 0 & 0 & 0 & 0 & 0 & 0 & 0 & 0 & 0 \\
\hline $27 d$ & 0 & 0 & 0 & 0 & 0 & 0 & 0 & 0 & 0 & 0 \\
\hline $34 \mathrm{~d}$ & 1.0 & 0.2 & 0.1 & 0.9 & 0.5 & 0.5 & 0.03 & 0.11 & 0.10 & 0.99 \\
\hline $41 \mathrm{~d}$ & 0 & 0 & 0 & 0 & 0 & 0 & 0 & 0 & 0 & 0 \\
\hline
\end{tabular}

a-b Values within a row, lacking a common superscript differ $(P \leq 0.05)$.

Due to same scores in each treatment group for perching, values are not shown. 


\section{REFERENCES}

Abdallah, A. G., O. M. El-Husseiny, and K. O. Abdel-Latif. 2009. Influence of some dietary organic mineral supplementations. International journal of poultry science. 8:291-298.

Aige-Gil, V. and N. Murillo-Ferrol. 1992. Effects of white light on the pineal gland of the chick embryo. Histology and histopathology. 7:1-6.

Angel, R. 2007. Metabolic disorders: limitations to growth of and mineral deposition into the broiler skeleton after hatch and potential implications for leg problems. Journal of applied poultry research. 16:138-149.

Ao, T., J. L. Pierce, R. Power, A. J. Pescatore, A. H. Cantor, K. A. Dawson, and M. J. Ford. 2009. Effects of feeding different forms of zinc and copper on the performance and tissue mineral content of chick. Poultry science. 88:2171-2175.

Archer, G. S. and J. A. Mench. 2017. Exposing avian embryos to light affects post-hatch anti-predator fear responses. Applied animal behaviour science. 186:80-84.

Archer, G. S. 2017. Exposing broiler eggs to green, red and white light during incubation. Animal. 11:1203-1209.

Archer, G. S., H. L. Shivaprasad, and J. A. Mench. 2009. Effect of providing light during incubation on the health, productivity, and behavior of broiler chickens. Poultry science. 88:29-37.

Archer, G. S. and J. A. Mench. 2014. The effects of the duration and onset of light stimulation during incubation on the behavior, plasma melatonin levels, and productivity of broiler chickens. Journal of animal science. 92:1753-1758.

Bao, Y. M. and M. Choct. 2009. Trace mineral nutrition for broiler chickens and prospects of application of organically complexed trace minerals: a review. Animal production science. 49:269-282.

Bao, Y. M., M. Choct, P. A. lji, and K. Bruerton. 2007. Effect of organically complexed copper, iron, manganese, and zinc on broiler performance, mineral excretion, and accumulation in tissues. Journal of applied poultry research. 16:448-455.

Bar, A., D. Shinder, S. Yosefi, E. Vax, and I. Plavnik. 2003. Metabolism and requirements for calcium and phosphorus in the fast-growing chicken as affected by age. British journal of nutrition. 89:51-60.

Bessei, W. 2006. Welfare of broilers: a review. World poultry science. J. 62:455-466.

Blake, G. M. and I. Fogelman. 2002. Methods and clinical issues in bone densitometry and quantitative ultrasonometry. In: Principles of bone biology (1573-1585). Academic Press.

Bradbury, E. J., S. J. Wilkinson, G. M. Cronin, P. C. Thomson, M. R. Bedford, and A. J. Cowieson. 2014. Nutritional geometry of calcium and phosphorus nutrition in broiler chicks. Growth performance, skeletal health and intake arrays. Animal. 8:1071-1079.

Bouxsein, M. L., S. K. Boyd, B. A. Christiansen, R. E. Guldberg, K. J. Jepsen, and R. Müller. 2010. Guidelines for assessment of bone microstructure in rodents using micro-computed tomography. Journal of bone and mineral research. 25:1468-1486.

Bradbury, E. J., S. J. Wilkinson, G. M. Cronin, P. Thomson, C. L. Walk, and A. J. Cowieson. 2017. Evaluation of the effect of a highly soluble calcium source in broiler diets supplemented with phytase on performance, nutrient digestibility, foot ash, mobility and leg weakness. Animal production science. 57:2016-2026.

Bradbury, E. J., S. J. Wilkinson, G. M. Cronin, C. L. Walk, and A. J. Cowieson. 2018. Effects of phytase, calcium source, calcium concentration and particle size on broiler performance, nutrient digestibility and skeletal integrity. Animal production science. 58:271283.

Bradshaw, R. H., R. D Kirkden, and D.M. Broom. 2002. A review of the aetiology and pathology of leg weakness in broilers in relation to welfare. Avian and poultry biology reviews. 13:45-103.

De Jong, I. C. and H. Gunnink. 2019. Effects of a commercial broiler enrichment programme with or without natural light on behaviour and other welfare indicators. Animal. 13:384-391.

Decuypere, E., K. Tona, V. Bruggeman, and F. Bamelis. 2001. The day-old chick: a crucial hinge between breeders and broilers. World's poultry science journal. 57:127-138. 
Dibner, J. J., J. D. Richards, M. L. Kitchell, and M. A. Quiroz. 2007. Metabolic challenges and early bone development. Journal of applied poultry research. 16:126-137.

Dishon, L., N. Avital-Cohen, D. Malamud, R. Heiblum, S. Druyan, T. E. Porter, M. Gumułka and I. Rozenboim. 2017. In-ovo monochromatic green light photostimulation enhances embryonic somatotropic axis activity. Poultry science. 96:1884-1890.

Driver, J. P., G. M. Pesti, R. I. Bakalli, and H. M. Edwards, Jr. 2005a. Calcium requirements of the modern broiler chicken as influenced by dietary protein and age. Poultry science. 84:1629-1639.

Driver, J. P., G. M. Pesti, R. I. Bakalli, and H. M. Edwards, Jr. 2005b. Effects of calcium and nonphytate phosphorus concentrations on phytase efficacy in broiler chicks. Poultry science. 84:1406-1417.

Dozier III, W. A., A. J. Davis, M. E. Freeman, and T. L. Ward. 2003. Early growth and environmental implications of dietary zinc and copper concentrations and sources of broiler chicks. British poultry science. 44:726-731.

Duncan, I. J. H., C. J. Savory, and D. G. M. Wood-Gush. 1978. Observations on the reproductive behaviour of domestic fowl in the wild. Applied animal ethology. 4:29-42.

Ekstrand, C., T. E. Carpenter, I. Andersson, and B. Algers. 1998. Prevalence and control of foot-pad dermatitis in broilers in Sweden. British poultry science. 39:318-324.

El-Husseiny, O. M., S. M. Hashish, R. A. Ali, S. A. Arafa, L. D. A. El-Samee, and A. A. Olemy. 2012. Effects of feeding organic zinc, manganese and copper on broiler growth, carcass characteristics, bone quality and mineral content in bone, liver and excreta. International journal of poultry science. 11:368-377.

Eyre, D. R. 2004. Collagens and cartilage matrix homeostasis. Clinical orthopaedics and related research. 427:1 18-122.

Ferket, P. R., E. O. Oviedo-Rondon, P. L. Mente, D. V. Bohorquez, A. A. Santos, Jr, J. L. Grimes, J. D. Richards, J. J. Dibner, and V. Fe lts. 2009. Organic trace minerals and 25- hydroxycholecalciferol affect performance characteristics, leg abnormalities, and biomechanical properties of leg bones of turkeys. Poultry science. 88:118-131.

Gocsik, É., A. M. Silvera, H. Hansson, H. W. Saatkamp, and H. J. Blokhuis. 2017. Exploring the economic potential of reducing broiler lameness. British poultry science. 58:337-347.

Guinotte, F., Y. Nys, and F. De Monredon. 1991. The effects of particle size and origin of calcium carbonate on performance and ossification characteristics in broiler chicks. Poultry science. 70:1908-1920.

Güz, B. C., R. Molenaar, I. C. de Jong, B. Kemp, H. van den Brand, and M. van Krimpen. 2019 Effects of dietary organic minerals, fish oil, and hydrolyzed collagen on growth performance and tibia characteristics of broiler chickens. Poultry science. 98:6552-6563.

Güz, B. C., R. Molenaar, I. C. de Jong, B. Kemp, M. van Krimpen, and H. van den Brand. 2020. Effects of eggshell temperature pattern during incubation on tibia characteristics of broiler chickens at slaughter age. Poultry science. 99:3020-3029.

Halevy, O., Y. Piestun, I. Rozenboim, and Z. Yablonka-Reuveni. 2006. In ovo exposure to monochromatic green light promotes skeletal muscle cell proliferation and affects myofiber growth in posthatch chicks. American journal of physiology - regulatory integrative and comparative physiology. 290:1062-1070.

Hemme, A., M. Spark, P. Wolf, H. Paschertz, and J. Kamphues. 2005. Effects of different phosphorus sources in the diet on bone composition and stability (breaking strength) in broilers. Journal of animal physiology and animal nutrition. 89:129-133.

Huang, Y. L., L. LU, S. F. Li, X. G. Luo, and B. Liu. 2009. Relative bio availabilities of organic zinc sources with different chelation strengths for broilers fed a conventional corn-soybean meal diet. Journal of animal science. 87:2038-2046.

Huth, J. C. and G. S. Archer. 2015. Comparison of two LED light bulbs to a dimmable CFL and their effects on broiler chicken growth, stress, and fear. Poultry science. 94:2027-2036.

Huth, J. C. and G. S. Archer. 2015. Effects of LED lighting during incubation on layer and broiler hatchability, chick quality, stress susceptibility and post-hatch growth. Poultry science. 94:3052-3058.

Jianhua, H., A. Ohtsuka, and K. Hayashi. 2000. Selenium influences growth via thyroid hormone status in broiler chickens. British journal of nutrition. 84:727-732.

Jungmann, R., G. Schitter, G. E. Fantner, M. E. Laver, P. K. Hansma, and P. J. Thurner. 2007. Real-time microdamage and strain detection during micromechanical testing of 
single trabeculae. In Experimental and Applied Mechanics: SEM Annual Conference and Exposition.

Kestin, S. C., S. Gordon, G. Su, and P. Sørensen. 2001. Relationships in broiler chickens between lameness, liveweight, growth rate and age. Veterinary record. 148:195-197.

Kestin, S. C., T. G. Knowles, A. F. Tinch, and N. G. Gregory. 1992. The prevalence of leg weakness in broiler chickens and its relationship with genotype. Veterinary record. 131:190194.

Kestin, S. C., G. Su, and P. Sørensen. 1999. Different commercial broiler crosses have different susceptibilities to leg weakness. Poultry science. 78:1085-1090.

Knowles, T. G., S. C. Kestin, S. M. Haslam, S. N. Brown, L. E. Green, A. Butterworth, S. J. Pope, D. Pfeiffer, and C. J. Nicol. 2008. Leg disorders in broiler chickens: prevalence, risk factors and prevention. PLoS One. 3:1545-1549.

Leeson, S., and L. Caston. 2008. Using minimal supplements of trace minerals as a method of reducing trace mineral content of poultry manure. Animal feed science and technology. 142:339-347.

Letourneau-Montminy, M. P., P. Lescoat, A. Narcy, D. Sauvant, J. F. Bernier, M. Magnin, C. Pomar, Y. Nys, and C. Jondreville. 2008. Effects of reduced dietary calcium and phytase supplementation on calcium and phosphorus utilisation in broilers with modified mineral status. British poultry science. 49:705-715.

Lewis, P. D., and T. R. Morris. 2000. Poultry and coloured light. World poultry science. J. 56:189207.

McDevitt, R. M., G. M. McEntee, and K. A. Rance. 2006. Bone breaking strength and apparent metabolisability of calcium and phosphorus in selected and unselected broiler chicken genotypes. British poultry science. 47:613-621.

McKay, J. C., N. F. Barton, A. N. M. Koerhuis, and J. McAdam. 2000. The challenge of genetic change in the broiler chicken. BSAP Occasional Publication. 27:1-7.

Medeiros, D. M., A. Plattner, D. Jennings, and B. Stoecker. 2002. Bone morphology, strength and density are compromised in iron-deficient rats and exacerbated by calcium restriction. Journal of nutrition. 132:3135-3141.

Medeiros, D. M., J. llich, J. Ireton, V. Matkovic, L. Shiry, and R. Wildman. 1997. Femurs from rats fed diets deficient in copper or iron have decreased mechanical strength and altered mineral composition. The journal of trace elements in experimental medicine. 10:197-203.

Mench, J. 2004. Lameness. Pages 3-17 in Measuring and auditing broiler welfare. C. A. Weeks, and A. Butterworth, ed. CABI, Wallingford, UK.

Molenaar, R., I. A. M. Reijrink, R. Meijerhof, and H. van den Brand. 2010. Meeting embryonic requirements of broilers throughout incubation: a review. Brazilian journal of poultry science. 12:137-148.

Nollet, L., J. D. van der Klis, M. Lensing, and P. Spring. 2007. The effect of replacing inorganic with organic trace minerals in broiler diets on productive performance and mineral excretion. Journal of applied poultry research. 16:592-597.

Novitskaya, E., P. Y. Chen, E. Hamed, L. Jun, V. A. Lubarda, I. Jasiuk, and J. McKittrick. 2011. Recent advances on the measurement and calculation of the elastic moduli of cortical and trabecular bone: a review. Theoretical and applied mechanics. 38:209-297.

Onyango, E. M., P. Y. Hester, R. Stroshine, and O. Adeola. 2003. Bone densitometry as an indicator of percentage tibia ash in broiler chicks fed varying dietary calcium and phosphorus levels. Poultry science. 82:1787-1791.

Oviedo-Rondón, E. O., P. R. Ferket, and G. B. Havestein. 2006. Nutritional factors that affect leg problems in broilers and turkeys. Avian and poultry biology reviews. 17:89-103.

Özkan, S., S. Yalçın, E. Babacanoğlu, S. Uysal, F. Karadaş, and H. Kozanoğlu. 2012. Photoperiodic lighting (16 hours of light: 8 hours of dark) programs during incubation: 2. Effects on early posthatching growth, blood physiology, and production performance in broiler chickens in relation to posthatching lighting programs. Poultry science. 91:2922-2930.

Parmer, T. G., L. B. Carew, F. A. Alster, and C. G. Scanes. 1987. Thyroid function, growth hormone, and organ growth in broilers deficient in phosphorus. Poultry science. 66:19952004. 
Rao, S. V. R., B. Prakash, K. Kumari, M. V. L. N. Raju, and A. K. Panda. 2013. Effect of supplementing different concentrations of organic trace minerals on performance, antioxidant activity, and bone mineralization in Vanaraja chickens developed for free range farming. Tropical animal health and production. 45:1447-1451.

Rath, N. C., J. M. Balog, W. E. Huff, G. R. Huff, G. B. Kulkarni, and J. F. Tierce. 1999. Comparative differences in the composition and biomechanical properties of tibiae of seven-and seventy-two-week-old male and female broiler breeder chickens. Poultry science. 78:1232-1239.

Riesenfeld, A. 1972. Metatarsal robusticity in bipedal rats. American Journal of Physical Anthropology. 36:229-233.

Richards, J. D., J. Zhao, R. J. Harrell, C. A. Atwell, and J. J. Dibner. 2010. Trace mineral nutrition in poultry and swine. Asian-Australas. Journal of animal science. 23:1527-1534.

Richards, M. P. 1997. Trace mineral metabolism in the avian embryo. Poult. Sci. 76:152-164.

Rosol, T. J. and C. C. Capen. 1997. Calcium-regulating hormones and diseases of abnormal mineral (calcium, phosphorus, magnesium) metabolism. Clinical biochemistry of domestic animals. 23:619-702.

Röttger, A. S., I. Halle, H. Wagner, G. Breves, and G. Flachowsky. 2011. The effect of various iodine supplementations and two different iodine sources on performance and iodine concentrations in different tissues of broilers. British poultry science. 52:115-123.

Rozenboim, I., Y. Piestun, N. Mobarkey, M. Barak, A. Hoyzman, and O. Halevy. 2004. Monochromatic light stimuli during embryogenesis enhance embryo development and posthatch growth. Poultry science. 83:1413-1419.

Rucker, R. B., T. Kosonen, M. S. Clegg, A. E. Mitchell, B. R. Rucker, J. Y. Uriu-Hare, and C. L. Keen. 1998. Copper, lysyl oxidase, and extracellular matrix protein cross-linking. The american journal of clinical nutrition. 67:996-1002.

Sá, L. M., P. C. Gomes, H. S. Rostagno, L. F. T. Albino, P. R. Cecon, and P. D'Agostini. 2004. Calcium requirement for broiler chicks from 22 to 42 and 43 to 53 days old. Brazilian journal of animal science. 33:397-406.

Selle, P. H., A. J. Cowieson, and V. Ravindran. 2009. Consequences of calcium interactions with phytate and phytase for poultry and pigs. Livestock science. 124:126-141.

Shafey, T. M. 2004. Effect of lighted incubation on embryonic growth and hatchability performance of two strains of layer breeder eggs. British poultry science. 45:223-229.

Shafey, T. M., H. A. Al-Batshan, M. M. Ghannam, and M. S. Al-Ayed. 2005. Effect of intensity of eggshell pigment and illuminated incubation on hatchability of brown eggs. British poultry science. 46:190-198.

Shao, Y., G. Sun, S. Cao, L. Lu, L. Zhang, X. Liao, and X. Luo. 2019. Bone phosphorus retention and bone development of broilers at different ages. Poultry science. 98:21 14-2121.

Sherlock, L., T. G. M. Demmers, A. E. Goodship, I. D. McCarthy, and C. M. Wathes. 2010. The relationship between physical activity and leg health in the broiler chicken. British poultry science. 51:22-30.

Sobolewska, A., G. Elminowska-Wenda, J. Bogucka, M. Szpinda, K. Walasik, A. Dankowiakowska, W. Jóźwicki., H. Wiśniewska, and M. Bednarczyk. 2019. The effect of two different green lighting schedules during embryogenesis on myogenesis in broiler chickens. European poultry science. 83.

Tong, Q., I. M. McGonnell, T. G. M. Demmers, N. Roulston, H. Bergoug, C. E. Romanini, R. Verhelst, M. Guinebretière, N. Eterradossi, D. Berckmans, and V. Exadaktylos. 2018. Effect of a photoperiodic green light programme during incubation on embryo development and hatch process. Animal. 12:765-773.

Turner, C. H. and Burr, D. B. 1993. Basic biomechanical measurements of bone: a tutorial. Bone. 14:595-608.

Van der Pol, C. W., van I. A. M. Roovert-Reijink, G. Aalbers, B. Kemp, and H. van den Brand. 2017. Incubation lighting schedules and their interaction with matched or mismatched post hatch lighting schedules: Effects on broiler bone development and leg health at slaughter age. Research in Veterinary Science. 114:416-422.

Van der Pol, C. W., I. A. M. van Roovert-Reijink, C. M. Maatjens, S. W. Gussekloo, S. Kranenbarg, J. Wijnen, R. P. Pieters, H. Schipper, B. Kemp, and H. van den Brand. 2019. Lightdark rhythms during incubation of broiler chicken embryos and their effects on embryonic and post hatch leg bone development. Plos One. 14:0210886. 
Vieira, S. L. 2008. Chelated minerals for poultry. Brazilian journal of poultry science. 10:73-79.

Wang, G., L. J. Liu, W. J. Tao, Z. P. Xiao, X. Pei, B. J. Liu, M. Q. Wang, G. Lin, and T. Y. Ao. 2019. Effects of replacing inorganic trace minerals with organic trace minerals on the production performance, blood profiles, and antioxidant status of broiler breeders. Poultry science. 98:2888-2895.

Wedekind, K. J., E. C. Titgemeyer, A. R. Twardock, and D.H. Baker. 1991. Phosphorus, but not calcium, affects manganese absorption and turnover in chicks. Journal of nutrition. 121:1776-1786.

Yenice, E., C. Mızrak, M. Gültekin, Z. Atik, and M. Tunca. 2015. Effects of organic and inorganic forms of manganese, zinc, copper, and chromium on bioavailability of these minerals and calcium in late-phase laying hens. Biological trace element research. 167:300-307.

Zhang, L., H. J. Zhang, J. Wang, S. G. Wu, X. Qiao, H. Y. Yue, J. H. Yao, and G. H. Qi. 2014. Stimulation with monochromatic green light during incubation alters satellite cell mitotic activity and gene expression in relation to embryonic and posthatch muscle growth of broiler chickens. Animal. 8:86-93.

Zhang, L., X. D. Zhu, X. F. Wang, J. L. Li, F. Gao, and G. H. Zhou. 2016. Green light-emitting diodes light stimuli during incubation enhances posthatch growth without disrupting normal eye development of broiler embryos and hatchlings. Asian-Australasian journal of animal sciences. 29:1562.

Zhao, J., R. B. Shirley, M. Vazquez-Anon, J. J. Dibner, J. D. Richards, P. Fisher, T. Hampton, K. D. Christensen, J. P. Allard, and A. F. Giesen. 2010. Effects of chelated trace minerals on growth performance, breast meat yield, and footpad health in commercial meat broilers. Journal of applied poultry research.19:365-372. 

B. C. GüZ
I. C. de Jong ${ }^{2}$
U. Bol'
B. Kemp'
M. van Krimpen ${ }^{2}$
R. Molenaar
H. van den Brand'

Submitted

1 Adaptation Physiology Group,

Wageningen University and Research, 6708 PB

Wageningen,

Gelderland,

The Netherlands

2 Wageningen Livestock Research,

Wageningen University and Research, 6708 WD

Wageningen,

Gelderland,

The Netherlands 


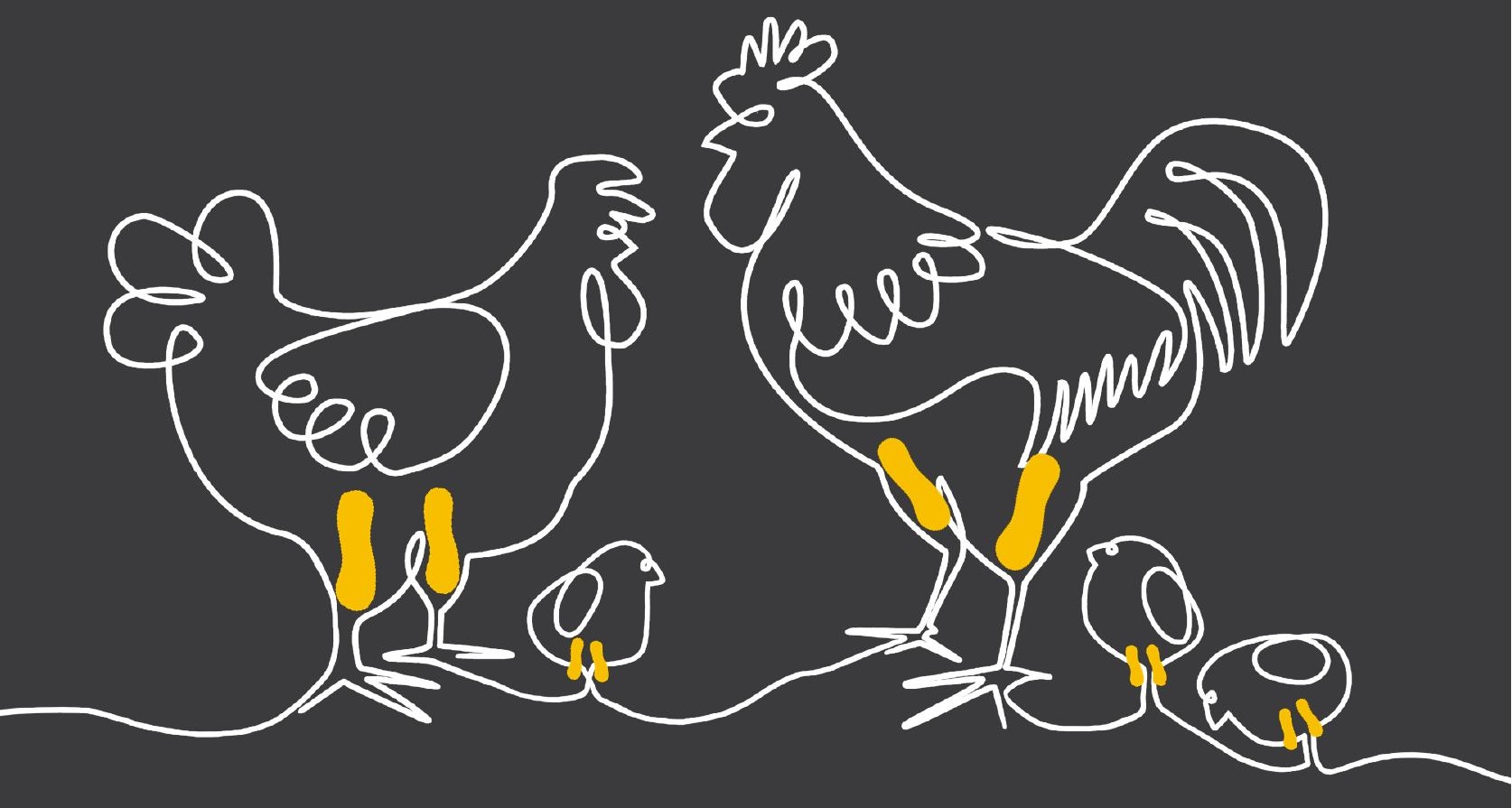

Chapter 3

EFFECTS OF ORGANIC MACRO AND TRACE MINERALS IN FAST AND SLOWER-GROWING BROILER BREEDERS' DIET ON OFFSPRING GROWTH PERFORMANCE AND TIBIA CHARACTERISTICS 


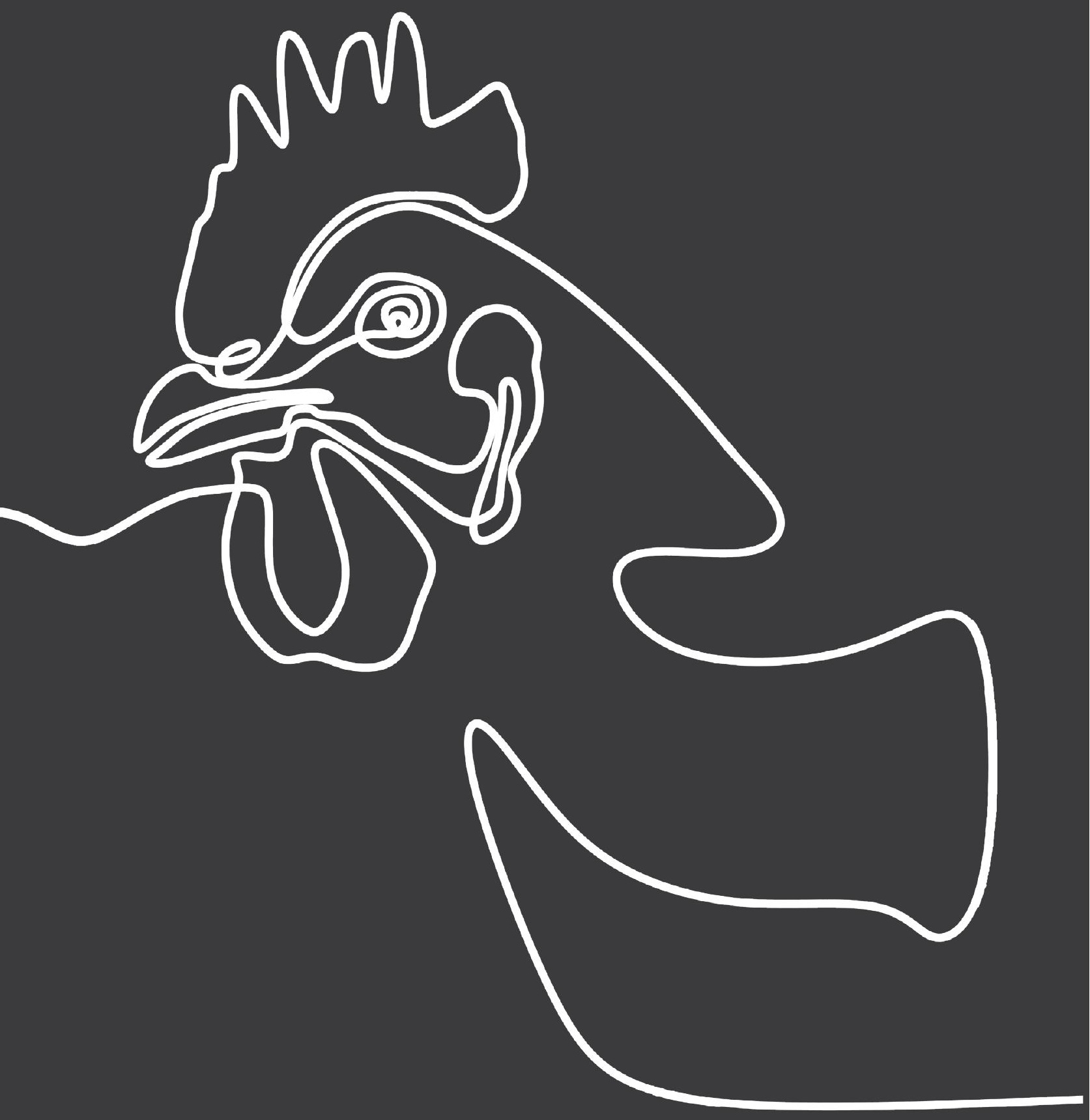




\section{Abstract}

This study was designed to evaluate effects of source of macro and trace minerals (inorganic vs organic) in fast and slower-growing broiler breeders' diets on egg and hatchling mineral content and on offspring tibia morphological, biophysical and mechanical characteristics. After 10 weeks feeding the breeders, eggs were collected and incubated. Eggs and hatchlings were analysed on mineral content. Male chickens were assigned to 32 pens with 12 chickens per pen. At approximately 1700 and 2600 gram BW, three chickens per pen were slaughtered. Tibia characteristics were determined. Organic minerals in the broiler breeder diet resulted in higher Fe and Se concentration in the egg and in higher Se concentration in the hatchling. Despite effects of mineral source on mineral concentration in the eggs and hatchlings were limited, organic minerals in the slower-growing broiler breeder diet resulted in higher offspring $B W$ (day $42, \Delta=115 \mathrm{~g} ; \mathrm{P}=0.03$ ) and advanced tibia development (higher thickness $(\Delta=0.38 \mathrm{~cm} ; \mathrm{P}<0.001$ ), osseous volume $\left(\Delta=5.1 \mathrm{~cm}^{3} ; \mathrm{P}=0.01\right)$ and mineral density $\left(\Delta=0.13 \mathrm{~g} / \mathrm{cm}^{2}\right.$; $\mathrm{P}=0.03$ ) at 2600 gram BW), but this was not observed in fast-growing chickens. This suggests that (1) the difference in feed intake between strains might affect offspring performance, which might indicate that current slower-growing breeder diets might be suboptimal in minerals or that trans-generational mineral availability in slower-growing chickens appears to be more effective on bone development, which might be related to time available for bone development. (2) trans-generational mineral availability in offspring appears to play a role via other mechanisms than via absolute mineral concentrations.

\section{Key words}

organic minerals, tibia characteristics, broiler breeder, broiler chicken 


\section{INTRODUCTION}

In modern broiler chickens, the prevalence of suboptimal leg health is considerable (Bessei, 2006; Knowles et al., 2008; Gocsik et al., 2017). A potential reason is an imbalance between a high growth rate and insufficiently developed leg bones (Williams et al., 2000; Sherlock et al., 2010; Gonzalez-Ceron et al., 2015). Suboptimal leg health negatively affects broiler chickens' locomotion (Bessei, 2006; Gocsik et al., 2017), welfare (Mench, 2004; Bradshaw et al., 2002) and slaughter revenues as a consequence of higher mortality and increased rejections at slaughter plants (sullivian, 1994; Kestin et al., 1999; Mench, 2004; Grandin, 2010).

Fast-growing broiler chickens reach an average slaughter weight of 2500 to 3000 gram in approximately 38 to 42 days, while slower-growing broiler chickens reach the same weight in approximately 48 to 54 days (Quentin et al., 2004; Grashorn, 2006; Kokoszyński et al., 2017). Genetic selection for fast growth in broiler chickens has resulted in high feed efficiency and shorter rearing period, but also in more porous and less mineralised leg bones than slower-growing broiler chickens (Lilburn, 1994; Dixon, 2020; Rayner et al., 2020). Consequently, fast-growing broilers have more leg and locomotion problems than slower-growing broilers (sullivian, 1994; Thorp and Waddington, 1997; Williams et al., 2000; Kokoszyński et al., 2017; Torres and Korver, 2018), expressed a higher risk of lameness and bone breakage (Williams et al., 2004; Stojcic and Bessei, 2009; Shim et al., 2012).

One of the most important factors for bone development is the macro (Ca, P) and trace (Fe, Cu, Mn, Zn, Se) mineral availability (Bao et al., 2007; Yenice et al., 2015). Macro minerals are main components of the bone matrix (Rath et al., 1999; Blake and Fogelman, 2002), whereas both macro (Rosol and Capen, 1997; McDevitt et al., 2006) and trace (Richards, 1997; Angel, 2007; Dibner et al., 2007) minerals are essential parts of bone development related hormones and enzymes. Mineral availability is not only determined by the amount of minerals provided in the diet, but also by the source or origin of the minerals. Minerals used in poultry diets mostly consist of an inorganic form (Van der Klis and Kemme, 2002; Vieira, 2008). However, minerals can also be processed and bound to e.g., an amino acid or a protein (Wang et al., 2019; Khatun et al., 2019) and are then called organic minerals. Organic macro and 
trace minerals in broiler's diet (Huang et al., 2009; zhao et al., 2010) and organic trace minerals in broiler breeders' diet (Torres and Korver, 2018; Bao and Choct, 2009; Swiatkiewicz et al., 2014) have been shown to have a higher bioavailability than inorganic minerals, due to a higher chemical stability and consequently a better intestinal absorption (Wedeking et al., 1991; Wang et al., 2019; Bao and Choct, 2009).

Pre-packaged minerals in the egg are important for embryonic bone development and later life bone health (Kidd et al., 1992; Dibner et al., 2007). Newly hatched chickens with a strong and well-formed skeletal frame might be able to move better and have lower prevalence of leg problems in later life (Torres and Korver, 2018). Organic trace minerals in the breeders' diet have already been shown to result in better embryonic bone mineralization than inorganic trace minerals (Torres and Korver, 2018). However, these carry-over effects from breeders' diet to the offspring embryo are only studied for trace minerals and not for macro minerals. Additionally, effects of mineral source in the breeder diet on offspring bone development and leg health at slaughter age are also hardly studied. Moreover, almost all studies on trans-generational effects of minerals in broiler chickens were conducted in fast-growing broiler breeders and not in slower-growing broiler breeders.

The aim of this study was therefore to investigate effects of mineral source (inorganic vs. organic) in fast and slower-growing broiler breeders' diet on egg and hatchling mineral content and on offspring tibia morphological, biophysical and mechanical characteristics.

\section{MATERIALS AND METHODS Experimental Design}

The experiment was setup as a $2 \times 2$ factorial arrangement with two broiler breeder strains (fast and slower-growing) and two macro and trace mineral sources in the broiler breeder diets (organic and inorganic). Effects of the broiler breeder diet were investigated in the eggs and offspring (hatchlings and later life). During offspring rearing, no treatments were applied. The experiment was conducted at the experimental facility of Wageningen University and Research (Wageningen, The Netherlands). 
All procedures in this study were approved by the Central Commission on Animal Experiments (The Hague, The Netherlands; approval number: 2016.D-0138.005).

\section{Breeder Feeding Phase and Experimental Diets}

At an age of 20 weeks, 132 fast-growing Ross 308 breeders (120 females and 12 males) and 132 slower-growing Hubbard JA57 breeders (120 females and 12 males) were obtained from two commercial rearing farms. Breeders were allocated to 8 pens ( 4 pens per strain with 30 females and 3 males per pen) and provided with a pre-layer diet (different for fast and slower-growing breeders; see Table 1). After 5 weeks of adaptation, they were provided with one of two different layer diets ( 2 pens per strain per diet), containing either inorganic or organic macro (Ca, P) and trace (Fe, Cu, Mn, Zn, Se) minerals for a period of 10 weeks (25 to 34 weeks of age). Composition of these layer diets differed between fast and slowergrowing broiler breeders (Table 1). Consequently, four treatment groups were used. They are as follows:

1) fast-growing broiler breeders fed with inorganic macro and trace minerals (IF);

2) fast-growing broiler breeders fed with organic macro and trace minerals (OF);

3) slower-growing broiler breeders fed with inorganic macro and trace minerals (IS);

4) slower-growing broiler breeders fed with organic macro and trace minerals (OS).

Inorganic macro minerals (Ca, P) were provided by limestone and monocalcium phosphate. In the organic diets, $\mathrm{Ca}$ and $\mathrm{P}$ were largely (see Table 1) replaced by Calfos ${ }^{\circledR}$ (Sonac Vuren B.V., Vuren, The Netherlands), a hydroxyapatite form of $\mathrm{Ca}$ and $\mathrm{P}$, originating from processed porcine bones. Inorganic trace minerals (Fe, Cu, Mn, Zn, Se) were completely replaced by an organic sourced trace mineral premix (Optimin, Trouw Nutrition, Tilburg, The Netherlands). 


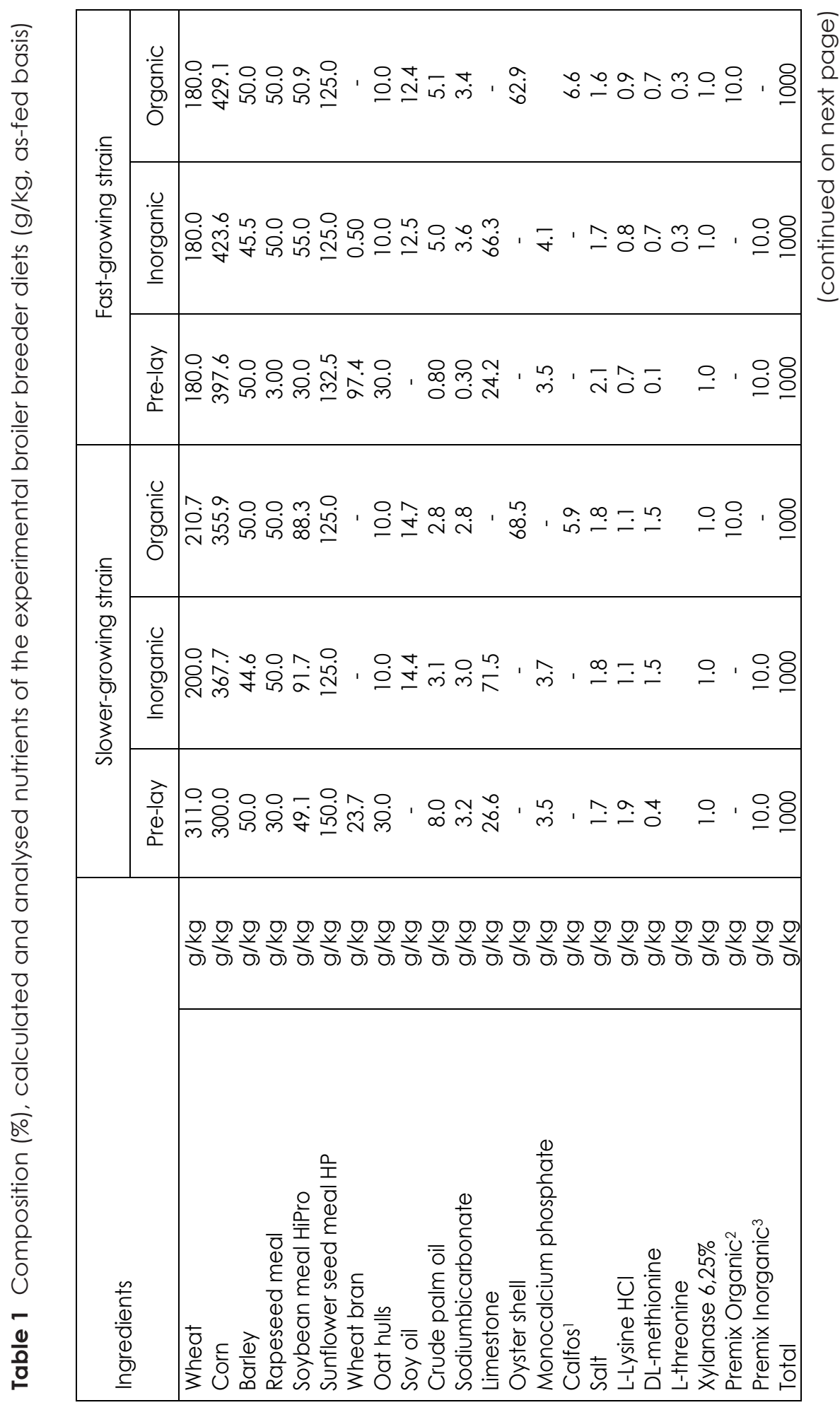




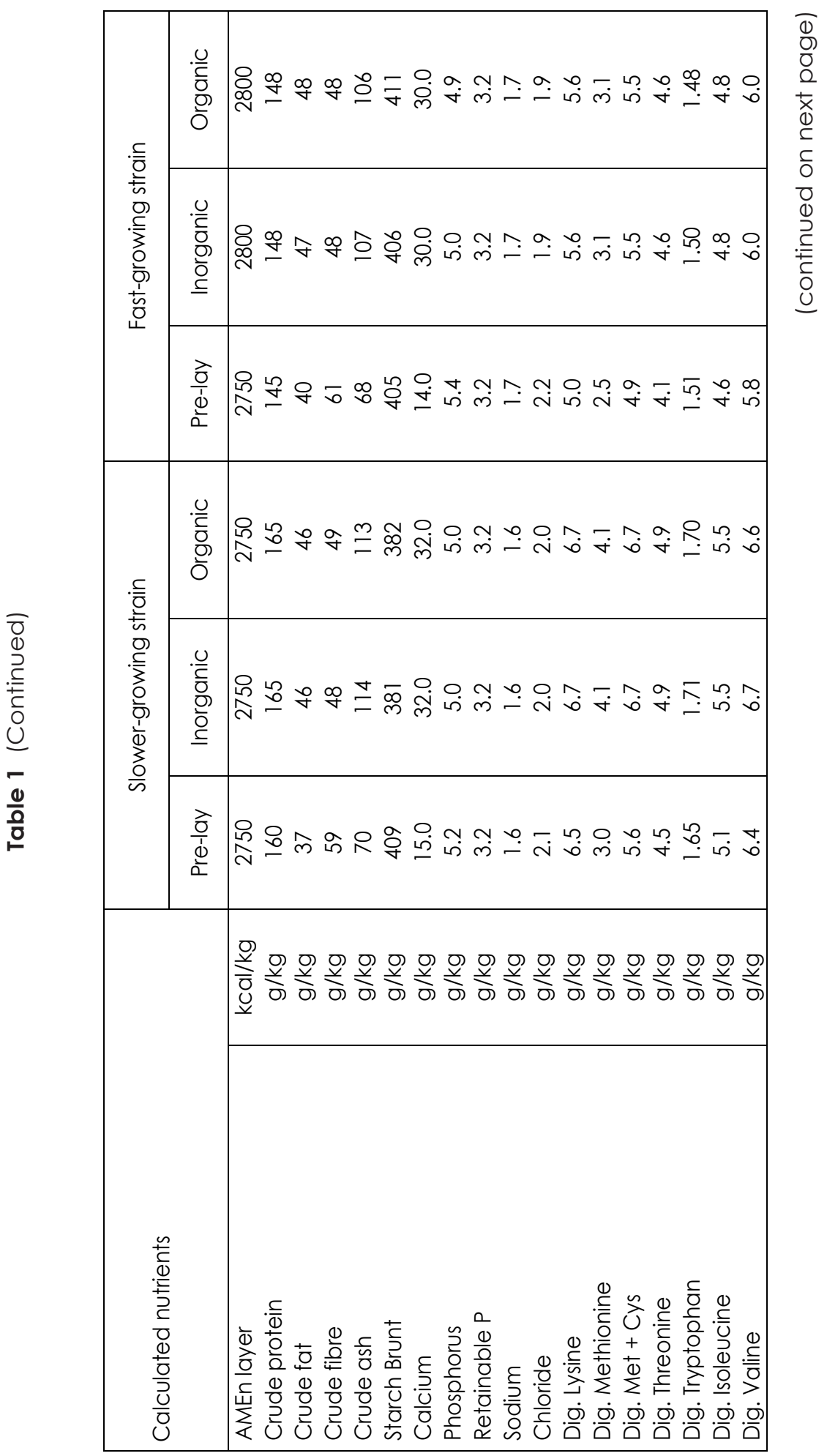



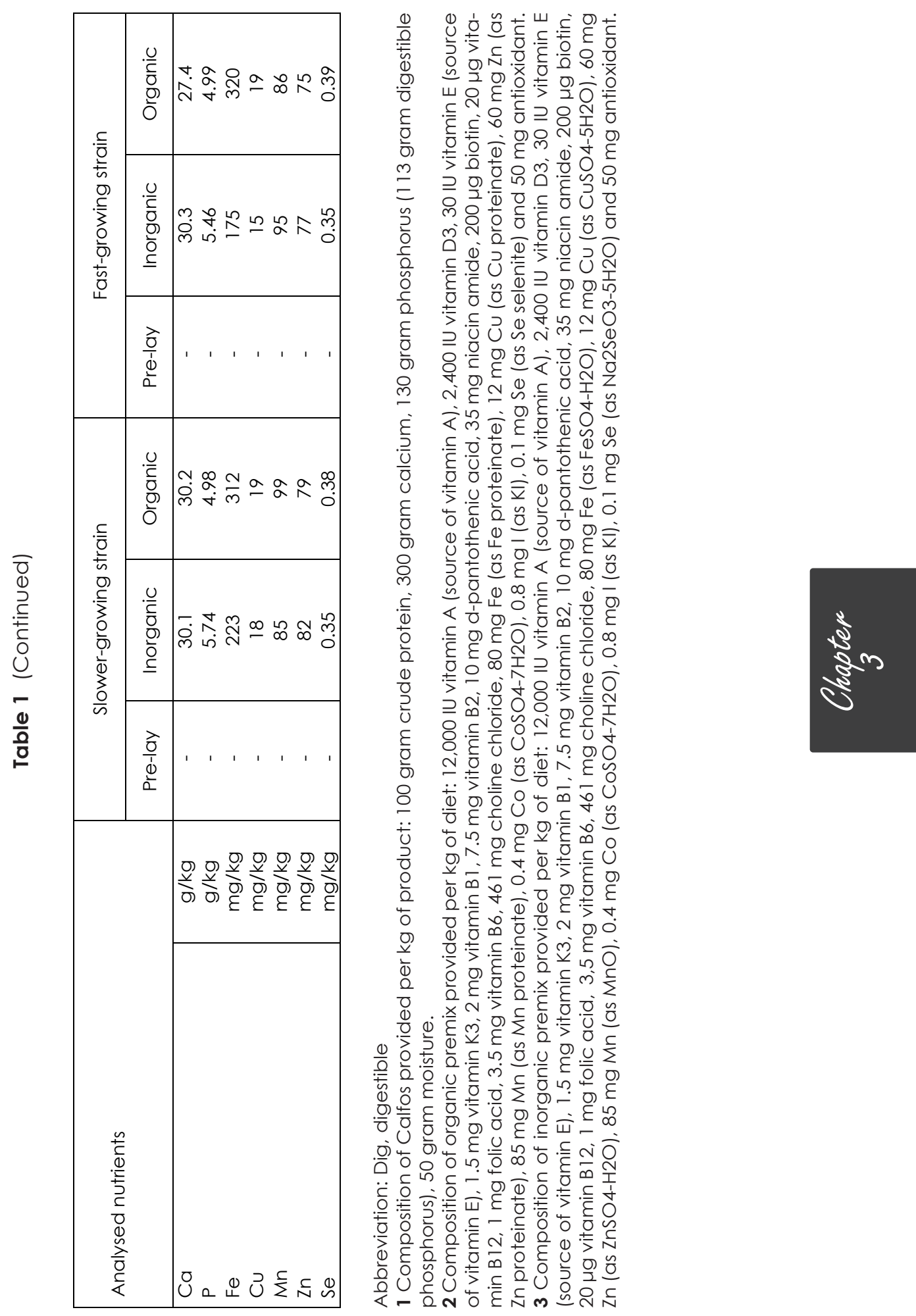
All other raw materials remained the same in both diets, although inclusion levels were different. Breeder diets were produced by ForFarmers N.V. (Heijen, The Netherlands) and analysed for Ca, P, Fe, Cu, Mn, Zn and Se (NutriControl, Veghel, The Netherlands). Diet compositions and calculated and analysed nutrient values are shown in Table 1.

\section{Egg Collection, Storage and Mineral Analysis}

In week 34 of breeders' age, first grade eggs were collected for 5 days, transferred to a storage room and stored at 15 to $16^{\circ} \mathrm{C}$ and a relative humidity of 65 to 70\%. In the same week, 5 eggs per treatment (1 egg per day for 5 days) were collected and analysed for macro (Ca, P) and trace (Fe, Cu, Mn, Zn, Se) minerals by a commercial laboratory (NutriControl, Veghel, The Netherlands), using inductively coupled plasma - optical emission spectrometry (ICP-OES).

\section{Incubation and Hatching Phase}

After a storage duration of 4 to 8 days, 60 eggs per treatment per collection day (300 eggs per treatment, 1200 eggs in total) were randomly distributed over 4 incubators; two large incubators with 440 eggs each (8 trays of 55 eggs per tray) and two small incubators with 160 eggs each (4 trays of 40 eggs per tray). Each incubator contained eggs from all four treatments and each tray contained eggs of one treatment. Per incubator, 4 or 5 eggshell temperature (EST) sensors (Pt-100, Sensor Data BV, Rijswijk, The Netherlands) were attached to 4 or 5 individual eggs. All sensors were placed at the equator of the chosen eggs, using heat conducting paste (Dow Corning 340 Heat Sink Compound, Dow Corning $\mathrm{GmbH}$, Wiesbaden, Germany) and a small piece of tape $(2 \times 2 \mathrm{~cm})$. The incubator temperature was continuously adjusted based on the median temperature of the EST sensors to maintain an EST of $37.8^{\circ} \mathrm{C}$ throughout incubation. Relative humidity of incubators was maintained between 50 and $65 \%$ throughout incubation. Eggs were turned every 30 min at an angle of $90^{\circ}$ and not exposed to light during incubation. At embryonic day 8 (E8), all eggs were candled and infertile eggs were removed. At E18, all eggs were candled again and eggs containing a vital embryo were transferred from trays to hatching baskets, which were placed back 
in the same incubator.

After all chickens had hatched, they were taken from the hatching baskets (day E21.5). Chicken quality parameters (red hock, red beak and navel score) of all hatched chickens (both males and females, $n=1032$ ) were assessed. Red hock and red beak were scored as 0 or 1 (absent or present). Navel score was assessed as 1 (good), 2 (moderate) or 3 (poor) as described by Molenaar et al., 2010. After chicken quality assessment, all chickens were feather-sexed. Male chickens were individually weighed and numbered by using a neck-label, vaccinated against infectious bronchitis (eye drop; MSD Animal Health, Boxmeer, The Netherlands) and transported to one of two adjacent rearing rooms at the same location.

After hatch, 5 randomly chosen male chickens per treatment were killed by cervical dislocation and residual yolks were removed. Yolkfree bodies and residual yolks were analysed separately for macro (Ca, P) and trace (Fe, Cu, Mn, Zn, Se) minerals by a commercial laboratory (NutriControl, Veghel, The Netherlands), using inductively coupled plasma - optical emission spectrometry (ICP-OES).

\section{Broiler Rearing Phase}

Upon arrival at the rearing rooms, male chickens per broiler breeder strain and diet were randomly assigned to 32 pens ( 16 pens per room) within 8 blocks of 4 pens, 12 chickens per pen. Pens $(1.25 \times 2.00 \mathrm{~m})$ were covered with $4-6 \mathrm{~cm}$ wood shavings. Temperature was maintained at $32{ }^{\circ} \mathrm{C}$ until day 3 of age and thereafter gradually reduced to $24{ }^{\circ} \mathrm{C}$ at day 21 and to $20 \cdot \mathrm{C}$ from day 28 onward. A continuous light program from arrival to day 3 of age and a 16L:8D light program from day 4 to 49 was applied. Chickens were raised from arrival to day 42 (fast-growing) or day 49 (slower-growing) with ad libitum access to a similar commercially available diet (ForFarmers, Lochem, The Netherlands). A 3-phase feeding program was applied; a starter diet (ME=2925 kcal $/ \mathrm{kg}, C P=203 \mathrm{~g} / \mathrm{kg}$, dLys $=11.1 \mathrm{~g} / \mathrm{kg}$ ) was provided from day 0 to 14 , a grower diet (ME=2975 $\mathrm{kcal} / \mathrm{kg}, \mathrm{CP}=173 \mathrm{~g} / \mathrm{kg}$, dLys=9.1 g/ $/ \mathrm{kg}$ ) from day 14 to 35 and a finisher diet (ME=3025 kcal $/ \mathrm{kg}, C P=172 \mathrm{~g} / \mathrm{kg}, \mathrm{dLys}=8.6 \mathrm{~g} / \mathrm{kg}$ ) from day 35 to 42 (fastgrowing) or 49 (slower-growing). 
Water was available ad libitum throughout the rearing phase via drinking nipples. At day 11, chickens were vaccinated against Newcastle disease (Clone 30; eye drop, MSD Animal Health, Boxmeer, The Netherlands).

\section{Data Collection, Sampling and Measurements}

All chickens were individually weighed on day $0,10,14,21,28,35$, 42 and 49 of age. Feed intake (FI) was measured per pen for the starter, grower and finisher period and over the whole rearing period. Feed conversion ratios (FCR) were calculated for the same periods, taking mortality into account. Mortality was recorded per pen per day and dead chickens were weighed.

Home pen behaviour was scored on day 16, 23, 30, 37 and 44 with one morning and one afternoon session, using the scan sampling technique(44). During 3 to 4 min per session per pen per day, the number of chickens performing the following activities was scored: eating, drinking, walking, standing, resting, foraging, sitting, dust bathing or perching.

At a body weight (BW) of approximately 1700 gram (day 29 and 38 of age for fast and slower-growing chickens, respectively) and at a BW of approximately 2600 gram (day 38 and 49 of age for fast and slowergrowing chickens, respectively) three chickens per pen were selected for slaughtering. Chickens with a BW close to 1700 or 2600 gram were selected. Chickens were stunned by electrocution, cut and bled. Varus Valgus (VV; each angulation was scored as present, 1; or no angulation, 0) was scored on both legs by visual appraisal. The left leg of all chickens was assessed by a veterinarian on tibia dyschondroplasia (TD), bacterial chondronecrosis with osteomyelitis (BCO), epiphyseal plate abnormalities (EPA) and epiphysiolysis (EPI). These abnormalities were scored in the range of 0 (no abnormalities), 1 (minor abnormality), or 2 (severe abnormality), but analyzed as 0 (no abnormality) or 1 (abnormality), because no score 2 was observed.

The tibia of the right leg was obtained from each chicken and frozen at $-20^{\circ} \mathrm{C}$. After thawing, tibia of two chickens per pen were selected for further analyses. Tibia weight was determined. Tibia proximal length, 
lateral cortex thickness, femoral and metatarsal side proximal head thickness, osseous volume, pore volume, total volume (osseous volume + pore volume), volume fraction (osseous volume / total volume), mineral content and mineral density were analysed on each tibia, using a GE Phoenix 3D X-ray microfocus CT scanner (General Electric Company ${ }^{\circledR}$, Boston, Massachusetts, US) (Bouxsein et al., 2010; Güz et al., 2019, 2021). Robusticity index was calculated, using the following formula (Riesenfeld, 1972):

Robusticity index $(\mathrm{cm} / \mathrm{g})=$ bone proximal length $(\mathrm{cm}) /$ bone weight $(\mathrm{g})$.

The same tibias were then subjected to a three-point bending test (Jungmann et al., 2017), using an Instron ${ }^{\circledR}$ electromechanical universal testing machine (Instron $\AA^{\circledR}$, Norwood, Massachusetts, United States). Ultimate strength (maximal load at breaking point); yield strength (reached yield load the angulation point on slope); tibia stiffness (the slope of the selected linear part of the curve) and energy to fracture (the area under the curve) were measured (Güz et al., 2019, 2021). Elastic modulus (GPa), which is the amount of strain caused by stress (Novitskaya et al., 2017), was calculated using the following formula (Turner and Burr, 1993):

$$
E=\frac{N S^{3}}{4 \delta T L^{3}}
$$

where $E$ is the elastic modulus (GPa), $N$ is the maximal load (N), $S$ is the span between bending fixtures ( $\mathrm{mm}), T$ is the tibia thickness $(\mathrm{mm}), L$ is the tibia length $(\mathrm{mm})$ and $\delta$ is the maximum deflection $(\mathrm{mm})$ at the midpoint of the bone.

\section{Statistical Analysis}

All statistical analyses were performed in SAS (Version 9.4, 2013, SAS Institute Inc., Cary, North Carolina, US).

Hatch data (red hock, red beak and navel score) was subjected to generalized linear mixed model analysis, using PROC GLIMMIX with model 1:

$$
Y=\mu+\text { Mineral }+ \text { Strain + Interaction }+\varepsilon,[1]
$$

where $Y=$ the dependent variable, $\mu$ is the overall mean, Mineral $=$ mineral source in the broiler breeder diet (organic or inorganic), Strain = broiler breeder strain (fast-growing Ross 308 or slower-growing Hubbard JA757), Interaction = interaction between mineral source and strain, $\varepsilon=$ residual error. Incubator was 
added to the model as a random effect.

Red hock and red beak were analysed at binary level (present or not) with a logit link; navel score was analysed at multinomial level (good, moderate or poor) with a cumlogit link. Hatchling was used as the experimental unit.

All growth performance data from day 0 to 42 (BW, Fl, FCR, mortality) was subjected to general linear mixed model analysis, using PROC MIXED with model 1. Pen was used as the experimental unit. Block was used as a random factor.

From day 42 onward, only chickens from the slower-growing strain were present. Consequently, all growth performance data (BW, Fl, FCR, mortality) was subjected to general linear mixed model analysis, using PROC MIXED with model 2. Pen was used as the experimental unit. The statistical model used was:

$$
Y=\mu+\text { Mineral }+\varepsilon,[2]
$$

where $Y=$ the dependent variable, $\mu$ is the overall mean, Mineral $=$ mineral source in the broiler breeder diet (organic or inorganic), $\varepsilon=$ residual error. Block was used as a random factor.

Tibia morphological, biophysical and mechanical characteristics, at two BW classes (1700 and 2600 gram), were subjected to general linear mixed model analysis, using PROC MIXED with model 1, added with BW (actual BW of the individual chickens at slaughter age) as a covariate. Pen was used as the experimental unit. Block was used as a random factor. Chicken nested within pen was added as a random factor to use pen as the experimental unit in the analyses.

Home pen behaviour (eating, drinking, walking, standing, resting, sitting, dust bathing and perching) was subjected to general linear mixed model analysis, using PROC MIXED with model 1. Pen was used as the experimental unit. Block was used as a random factor. Only main effects were analysed and presented, because preliminary analyses demonstrated a lack of interaction effects between mineral source and breeder strain. 
Leg disorders (VV, TD, EPA, BCO and EPI) were subjected to generalized linear mixed model analysis, at two BW classes (1700 and 2600 gram), using PROC GLIMMIX with model 1. VV was scored as present or not (each angulation was scored as 0 (no angulation) or 1 (angulation present). TD was scored in the range of 0 (no abnormalities), 1 (minor abnormality), or 2 (severe abnormality), but analyzed as 0 (no abnormalities) or 1 (abnormalities present), because no score 2 was found. For all these analyses, a logit link was used. Pen was used as the experimental unit. EPA, BCO and EPI were not statistically analysed, because there was only one BCO disorder in the OF group at 1700 gram BW class and no observations were recorded for EPA and EPI.

For continuous data, model assumptions were checked for both means and residuals. Non-normal distributed data were log-transformed before analyses. Results are provided as LSmeans \pm SEM, unless indicated otherwise. When multiple comparisons were performed, the level of significance was corrected, using Bonferroni. Effects were considered to be significant at $\mathrm{P} \leq 0.05$.

\section{RESULTS}

\section{Mineral Analysis}

By incidence, eggs selected for mineral analyses differed in weight between mineral source in the breeder diet and did not represent the average egg weight of the treatments. Consequently, egg mineral composition is expressed as concentration. No interaction effects between mineral source and breeder strain were found in mineral concentrations of albumen + yolk, eggshell, residual yolk or yolk free body (Table 2 and 3). Iron ( $\Delta=3.2 \mathrm{mg} / \mathrm{kg}, \mathrm{P}=0.008)$ and selenium ( $\Delta=0.073 \mathrm{mg} / \mathrm{kg}, \mathrm{P}<0.001$ ) were higher in albumen + yolk of eggs originating from organic minerals fed broiler breeders compared to eggs of inorganic minerals fed broiler breeders, whereas the opposite was found for copper $(\Delta=0.1 \mathrm{mg} / \mathrm{kg}$, $\mathrm{P}=0.05$ ) (Table 2). No strain effect was found for albumen + yolk mineral concentrations.

Selenium concentration was higher $(\Delta=0.11 \mathrm{mg} / \mathrm{kg}, \mathrm{P}=0.05)$ in residual yolk of hatchlings originating from organic minerals fed broiler 
breeders compared to inorganic minerals fed broiler breeders (Table 3 ). In the residual yolk, calcium $(\Delta=0.65 \mathrm{~g} / \mathrm{kg}, P<0.001)$, copper $(\Delta=0.2 \mathrm{mg} /$ $\mathrm{kg}, \mathrm{P}=0.05)$ and manganese $(\Delta=0.4 \mathrm{mg} / \mathrm{kg}, \mathrm{P}=0.003)$ concentrations were lower in fast-growing chickens compared to slower-growing chickens, whereas the opposite was found for phosphorus ( $\Delta=0.04 \mathrm{~g} / \mathrm{kg}, P=0.009)$, iron $(\Delta=7.7 \mathrm{mg} / \mathrm{kg}, P=0.002)$ and selenium $(\Delta=0.19 \mathrm{mg} / \mathrm{kg}, P=0.003)$ concentrations.

Selenium concentration was higher $(\Delta=0.04 \mathrm{mg} / \mathrm{kg}, \mathrm{P}=0.009$ ) in yolk free bodies of hatchlings originating from organic minerals fed broiler breeders compared to inorganic minerals fed broiler breeders. Iron $(\Delta=4.4$ $\mathrm{mg} / \mathrm{kg}, \mathrm{P}<0.001$ ) and selenium concentration ( $\Delta=0.4 \mathrm{mg} / \mathrm{kg}, \mathrm{P}=0.03$ ) were lower in yolk free bodies of slower-growing hatchlings compared to fastgrowing hatchlings (Table 3).

\section{General Hatch Data}

Hatchability of fertile eggs was on average $86 \%$. Hatch characteristics (red hock, red beak and navel score of all chickens) are shown in Table Al in Supplementary Data. No interaction effects between mineral source and breeder strain nor mineral source effects were found for red hock, red beak and navel score. Slower-growing chickens had a higher incidence of red hocks ( $\Delta=3.73 \%, \mathrm{P}=0.02)$ and red beaks $(\Delta=3.06 \%, \mathrm{P}=0.05)$ than fastgrowing chickens. Navel score was not affected by breeder strain.

\section{Growth Performance}

BW at day 10, 14,21, 28, 35 and 42 of the broiler rearing phase showed a significant interaction between mineral source and breeder strain (Table 4). In fast-growing chickens, no effect of mineral source was found on BW at any of the weighing days, but slower-growing chickens originating from organic minerals fed broiler breeders had a higher BW at day 10 ( $\Delta=20 \mathrm{~g}$; $\mathrm{P}=0.04)$, day $14(\Delta=20 \mathrm{~g} ; \mathrm{P}=0.04)$, day $21(\Delta=39 \mathrm{~g} ; \mathrm{P}=0.04)$, day $28(\Delta=65$

g; $P=0.04)$, day $35(\Delta=78 \mathrm{~g} ; \mathrm{P}=0.03)$ and day $42(\Delta=115 \mathrm{~g} ; \mathrm{P}=0.03)$ than the ones originating from inorganic mineral fed broiler breeders. Slowergrowing chickens originating from organic mineral fed broiler breeders had a higher BW at day 49 ( $\Delta=132 \mathrm{~g}, \mathrm{P}=0.004)$ than chickens originating from inorganic minerals fed broiler breeders. 


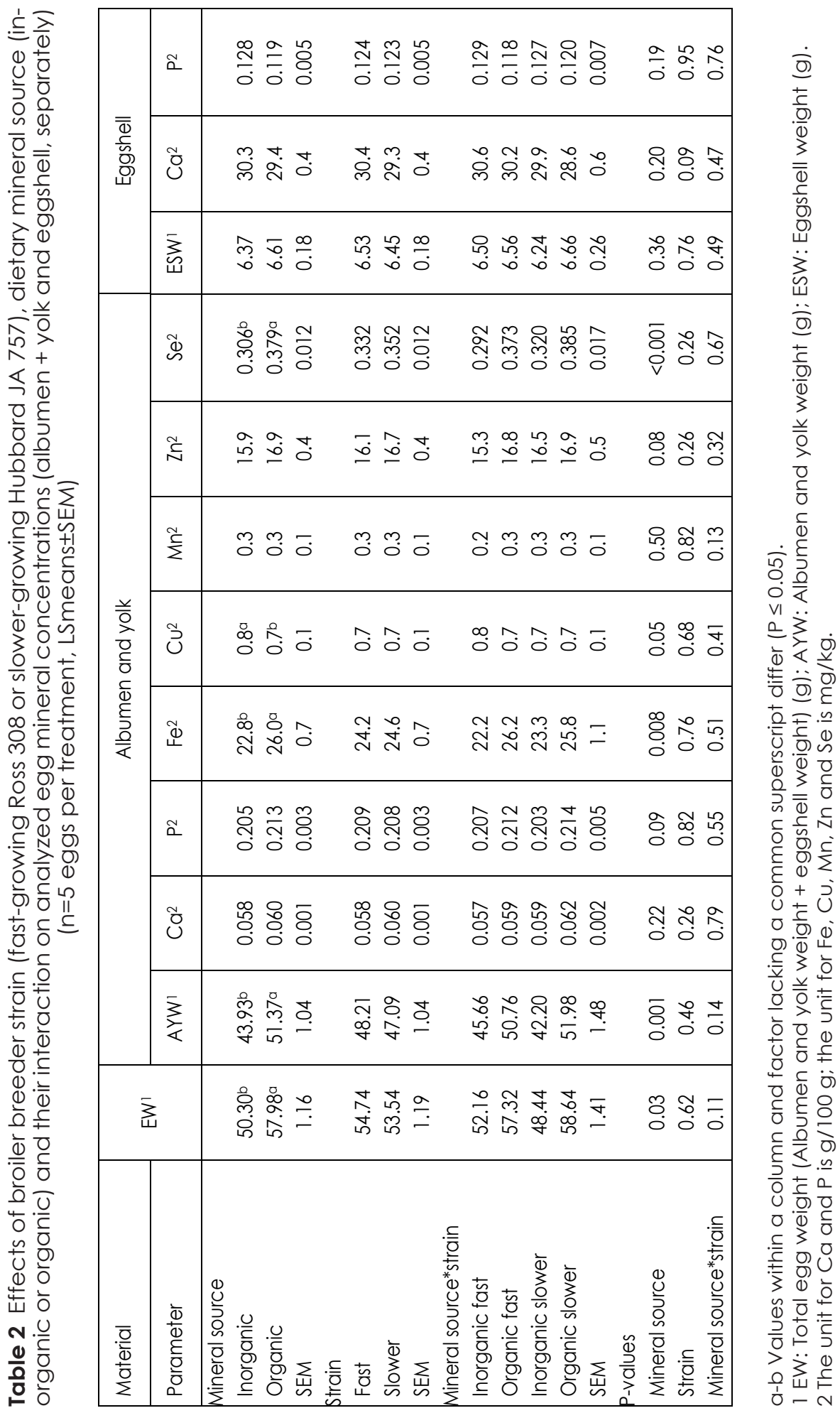




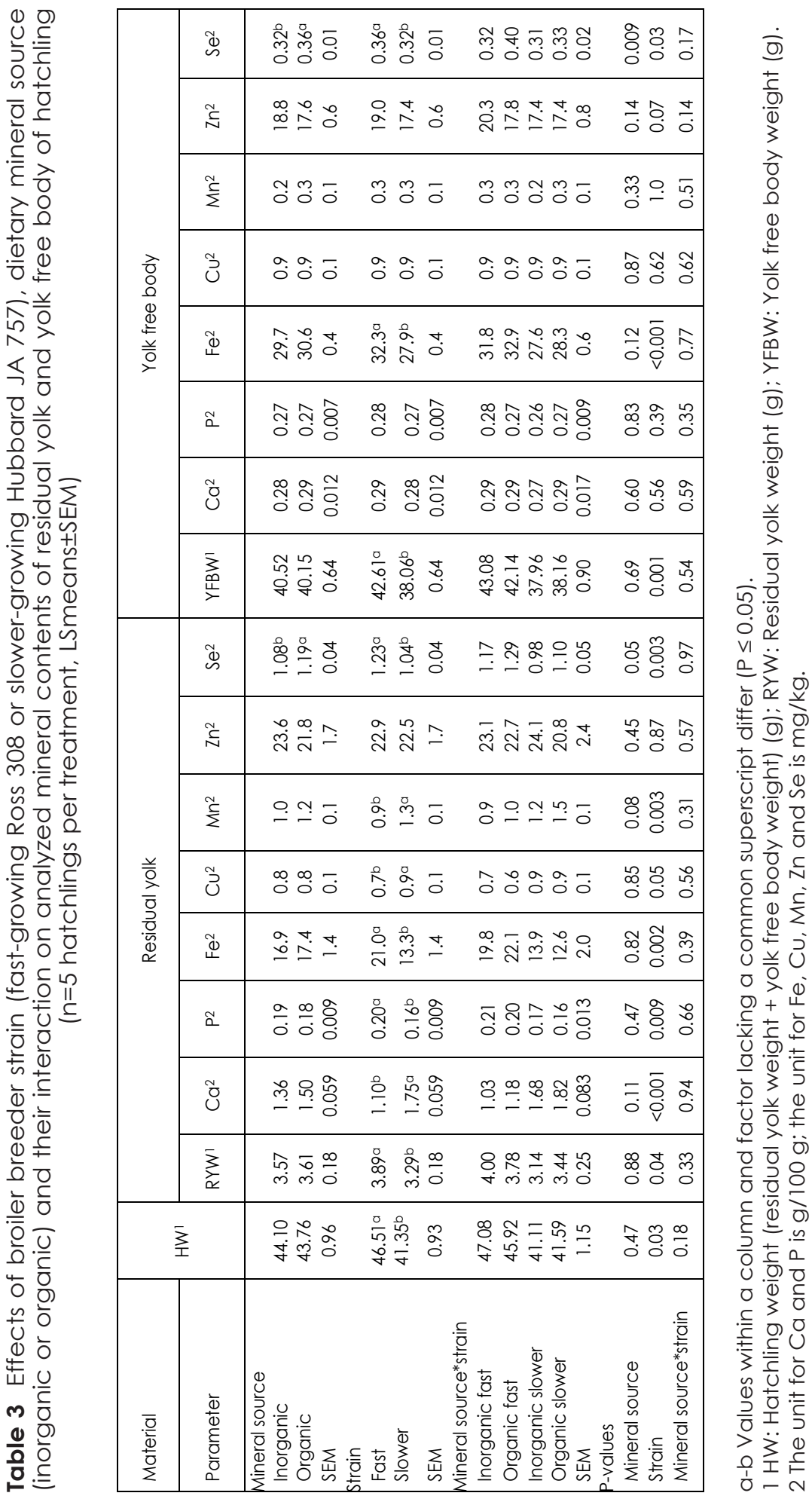




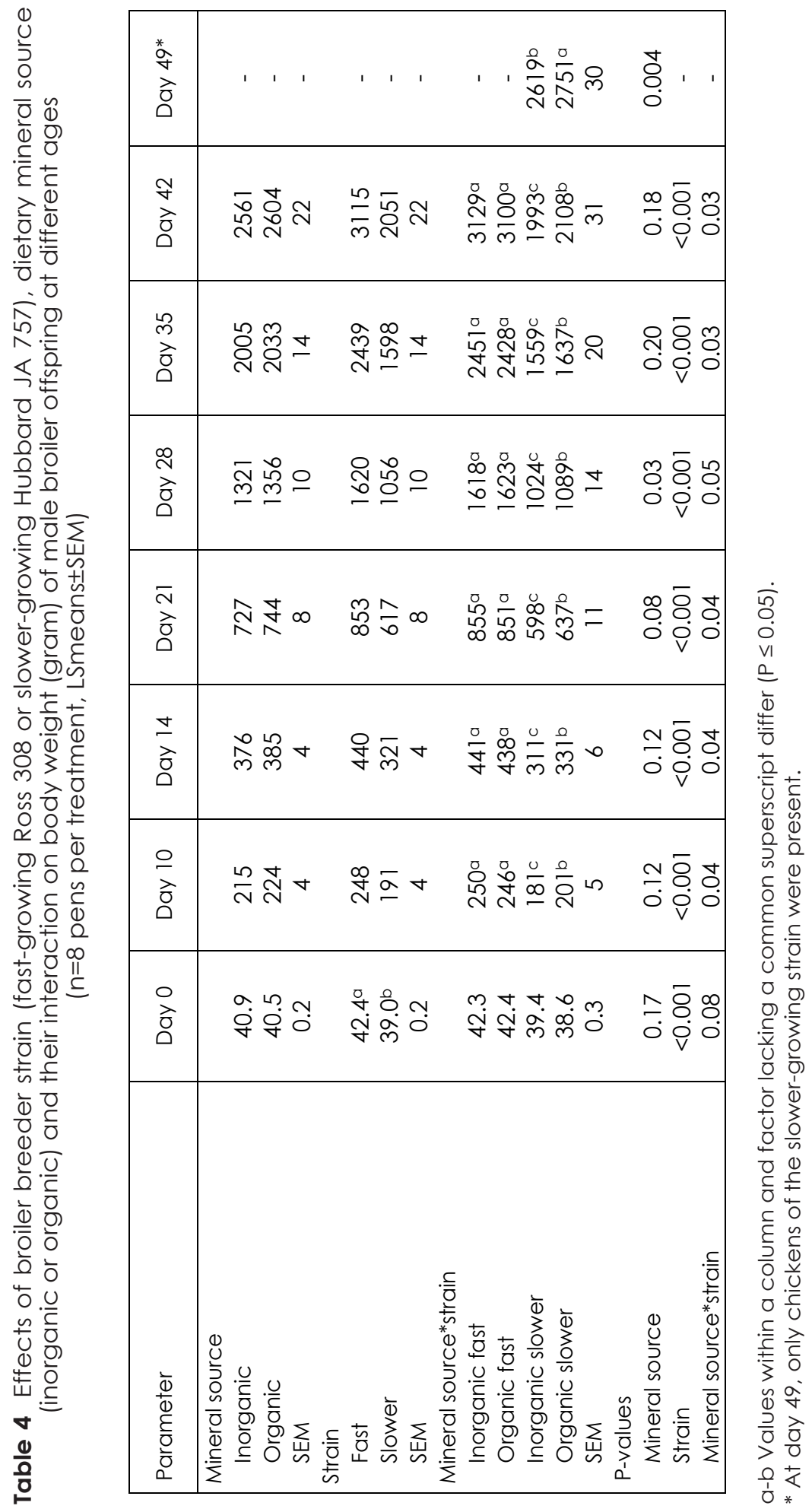




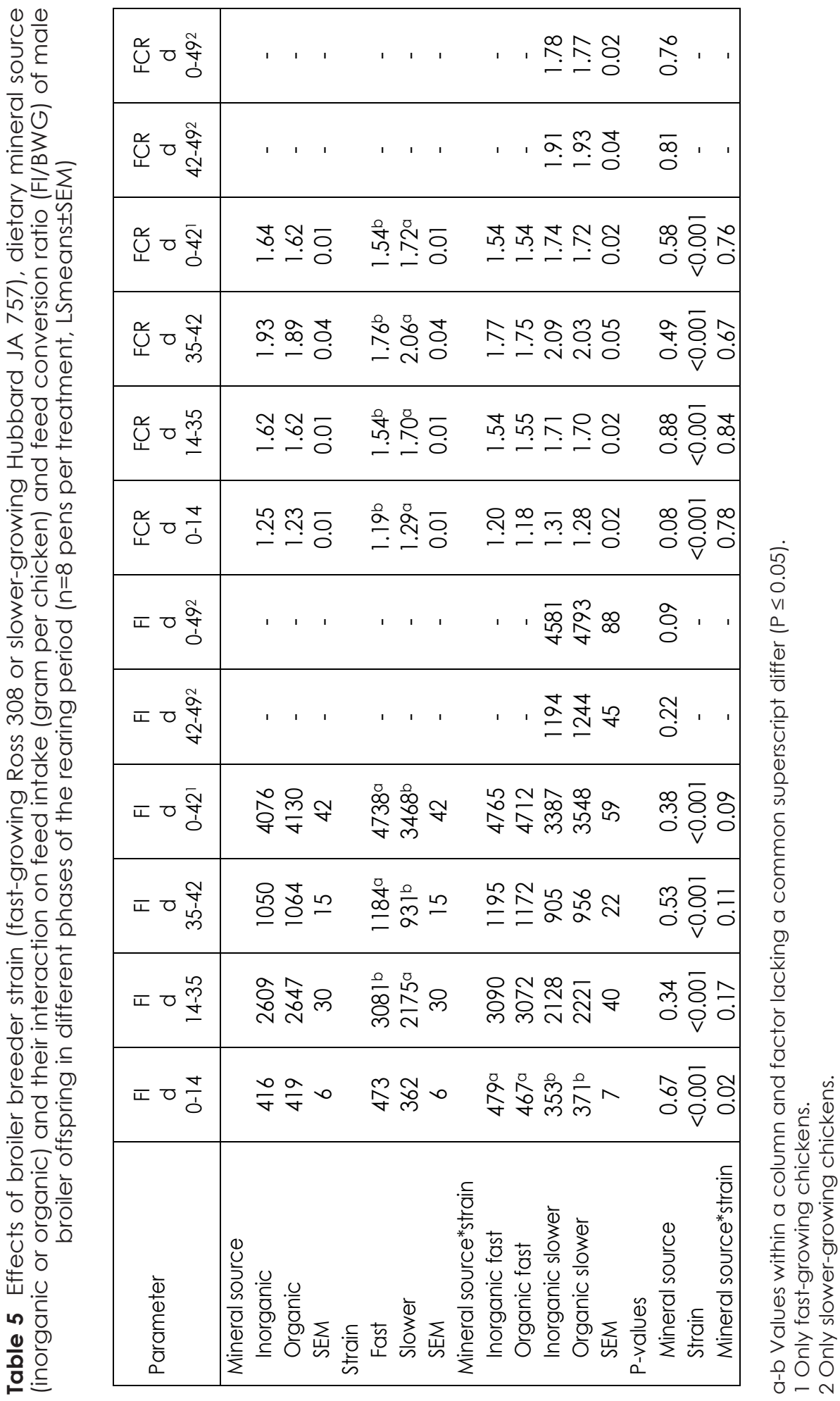


Chickens of the slower- growing strain had a lower BW than fastgrowing strain at day $0(\Delta=3.4 \mathrm{~g}, \mathrm{P}<0.001)$.

Feed intake between days 0-14 showed a significant interaction between mineral source and breeder strain (Table 5), but this effect disappeared after correction for Bonferroni. No further interactions between mineral source and breeder strain were found for FI or FCR and neither mineral source effects were found. Slower-growing chickens had a lower feed intake between days 14-35 ( $\Delta=906 \mathrm{~g}, \mathrm{P}<0.001$ ), 35-42 ( $\Delta=253 \mathrm{~g}$, $\mathrm{P}<0.001)$ and 0-42 ( $\Delta=1270 \mathrm{~g}, \mathrm{P}<0.001)$ than fast-growing chickens. Slowergrowing chickens had a higher FCR than fast-growing chickens between days $0-14(\Delta=0.10, \mathrm{P}<0.001), 14-35(\Delta=0.16, \mathrm{P}<0.001), 35-42(\Delta=0.30, \mathrm{P}<0.001)$ and $0-42(\Delta=0.18, \mathrm{P}<0.001)$.

A total of 12 (3.1\%) dead chickens were recorded during the rearing period. No interaction between mineral source and breeder strain or main effects were found on mortality.

\section{Tibia Morphological Characteristics}

At the 1700 gram BW class, interaction effects between mineral source and broiler breeder strain were found on all tibia morphological characteristics, except for tibia lateral cortex thickness (Table 6). The OS group had a higher tibia weight compared to the other treatment groups ( $\Delta=0.86 \mathrm{~g}$ on average; $\mathrm{P}=0.006$ ), which were similar. For proximal tibia length, tibia femoral side head thickness, tibia metatarsal side head thickness and tibia robusticity index, no effects of mineral source were found in the slower-growing broilers. However, in fast-growing broilers, chickens originating from organic minerals fed broiler breeders had higher proximal tibia length ( $\Delta=0.88 \mathrm{~cm}$; $P=0.02$ ), tibia femoral side head thickness $(\Delta=0.21 \mathrm{~cm} ; P=0.03)$, tibia metatarsal side head thickness $(\Delta=0.23 \mathrm{~cm}$; $\mathrm{P}=0.04)$ and tibia robusticity index $(\Delta=0.07 \mathrm{~cm} / \mathrm{g} ; \mathrm{P}<0.001)$ than chickens originating from inorganic minerals fed broiler breeders. For the 1700 gram BW class, the lateral tibia cortex thickness was higher in slower-growing chickens than in fast-growing chickens $(\Delta=0.17 \mathrm{~cm} ; \mathrm{P}<0.001)$.

At 2600 gram BW class, interaction effects between mineral source and broiler breeder strain were found on lateral tibia cortex thickness, 
femoral side proximal tibia head thickness and tibia metatarsal side proximal tibia head thickness (Table 6). In fast-growing chickens, no effects of mineral source in the breeder diet were found, but slower-growing chickens, originating from organic minerals fed broiler breeders had a higher tibia lateral cortex thickness $(\Delta=0.38 \mathrm{~cm} ; P<0.001)$, tibia femoral side proximal head thickness $(\Delta=0.26 \mathrm{~cm} ; \mathrm{P}=0.009)$ and tibia metatarsal side proximal head thickness $(\Delta=0.15 \mathrm{~cm} ; \mathrm{P}=0.02)$ than chickens originating from inorganic minerals fed broiler breeders. Slower-growing chickens showed a higher tibia weight $(\Delta=1.65 \mathrm{~g} ; \mathrm{P}<0.001)$, proximal tibia length $(\Delta=0.30 \mathrm{~cm} ; \mathrm{P}=0.02)$ and a lower tibia robusticity index $(\Delta=0.07 \mathrm{~cm} / \mathrm{g}$; $\mathrm{P}<0.001$ ) than fast-growing chickens.

\section{Tibia Biophysical Characteristics}

At 1700 gram BW class, no interaction effects between mineral source and broiler breeder strain were found on tibia biophysical characteristics (Table 7). Chickens originating from organic minerals fed broiler breeders had a higher tibia osseous volume $\left(\Delta=1.7 \mathrm{~cm}^{3} ; \mathrm{P}=0.03\right)$, tibia mineral content $(\Delta=1.1 \mathrm{~g} ; \mathrm{P}=0.009)$ and tibia mineral density $(\Delta=0.07 \mathrm{~g} /$ $\mathrm{cm}^{2} ; \mathrm{P}=0.003$ ) than chickens originating from inorganic minerals fed broiler breeders. Slower-growing chickens showed a higher tibia osseous volume $\left(\Delta=7.1 \mathrm{~cm}^{3} ; \mathrm{P}<0.001\right)$, tibia pore volume $\left(\Delta=1.2 \mathrm{~cm}^{3} ; \mathrm{P}<0.001\right)$, tibia total volume $\left(\Delta=8.4 \mathrm{~cm}^{3} ; \mathrm{P}<0.001\right)$ and tibia mineral content $(\Delta=1.9 \mathrm{~g} ; \mathrm{P}<0.001)$ than fast-growing chickens.

At 2600 gram BW class, interaction effects between mineral source and broiler breeder strain were found on tibia osseous volume, tibia total volume and tibia mineral density (Table 7). In fast-growing chickens, no effect of mineral source in the breeder diet was found, but slower-growing chickens originating from organic minerals fed breeders showed a higher tibia osseous volume $\left(\Delta=5.1 \mathrm{~cm}^{3} ; P=0.01\right)$, tibia total volume $\left(\Delta=5.6 \mathrm{~cm}^{3}\right.$; $\mathrm{P}=0.005)$ and tibia mineral density $\left(\Delta=0.13 \mathrm{~g} / \mathrm{cm}^{2} ; \mathrm{P}=0.03\right)$ than chickens originating from inorganic minerals fed breeders. Chickens originating from organic minerals fed broiler breeders had a lower tibia volume fraction $(\Delta=1.8 \% ; \mathrm{P}=0.03)$ and a higher tibia mineral content ( $\Delta=1.4 \mathrm{~g} ; \mathrm{P}<0.001)$ than chickens originating from inorganic minerals fed broiler breeders. 
Slower-growing chickens showed a higher tibia pore volume $\left(\Delta=2.6 \mathrm{~cm}^{3}\right.$; $\mathrm{P}<0.001$ ), tibia mineral content $(\Delta=2.8 \mathrm{~g} ; \mathrm{P}<0.001)$ and lower tibia volume fraction $(\Delta=3.4 \% ; \mathrm{P}<0.001)$ than fast-growing chickens.

\section{Tibia Mechanical Characteristics}

At 1700 gram BW class, no interaction effects between mineral source and broiler breeder strain were found on tibia mechanical characteristics (Table 8). Chickens originating from organic minerals fed broiler breeders had a higher tibia ultimate strength ( $\Delta=22.1 \mathrm{~N} ; \mathrm{P}<0.001$ ), tibia yield strength $(\Delta=20.8 \mathrm{~N} ; \mathrm{P}<0.001)$, tibia stiffness $(\Delta=19.2 \mathrm{~N} / \mathrm{mm} ; \mathrm{P}<0.001)$ and tibia energy to fracture $(\Delta=18.8 \mathrm{~N}-\mathrm{mm} ; \mathrm{P}<0.001)$ than chickens originating from inorganic minerals fed broiler breeders. Slower-growing chickens showed a higher tibia ultimate strength $(\Delta=37.9 \mathrm{~N} ; \mathrm{P}<0.001)$, tibia yield strength $(\Delta=39.1 \mathrm{~N} ; \mathrm{P}<0.001)$, tibia stiffness $(\Delta=39.1 \mathrm{~N} / \mathrm{mm} ; \mathrm{P}<0.001)$ and tibia energy to fracture $(\Delta=35.7 \mathrm{~N}-\mathrm{mm} ; \mathrm{P}<0.001)$ than fast-growing chickens.

At 2600 gram BW class, no interaction effects between mineral source and broiler breeder strain were found on tibia mechanical characteristics (Table 8). Chickens originating from organic minerals fed broiler breeders had a higher tibia ultimate strength $(\Delta=14.1 \mathrm{~N} ; \mathrm{P}=0.04)$, tibia yield strength $(\Delta=14.8 \mathrm{~N} ; P=0.03)$, tibia stiffness $(\Delta=16.0 \mathrm{~N} / \mathrm{mm} ; P=0.04)$ and tibia energy to fracture ( $\Delta=14.2 \mathrm{~N}-\mathrm{mm} ; \mathrm{P}=0.03$ ) than chickens originating from inorganic minerals fed broiler breeders. Slower-growing chickens showed a higher tibia ultimate strength $(\Delta=18.1 \mathrm{~N} ; \mathrm{P}=0.008)$, tibia yield strength $(\Delta=22.6 \mathrm{~N}$; $\mathrm{P}<0.002)$, tibia stiffness ( $\Delta=24.6 \mathrm{~N} / \mathrm{mm} ; \mathrm{P}=0.003$ ) and tibia energy to fracture $(\Delta=20.0 \mathrm{~N}-\mathrm{mm} ; \mathrm{P}=0.004)$ than fast-growing chickens.

\section{Home Pen Behaviour}

Home pen behaviour parameters (eating, drinking, walking, standing, resting, sitting, dust bathing and perching) for each of the observation days (day 16, 23, 30, 37 and 44) are presented in Table A2 in Supplementary Data. Hardly any effect of mineral source in the breeder diet was found on behaviour parameters. Fast-growing chickens showed less walking (day 30 and 37), less standing (all days), less perching (all days) and more resting behaviour (day 30 ) than slower-growing chickens. 


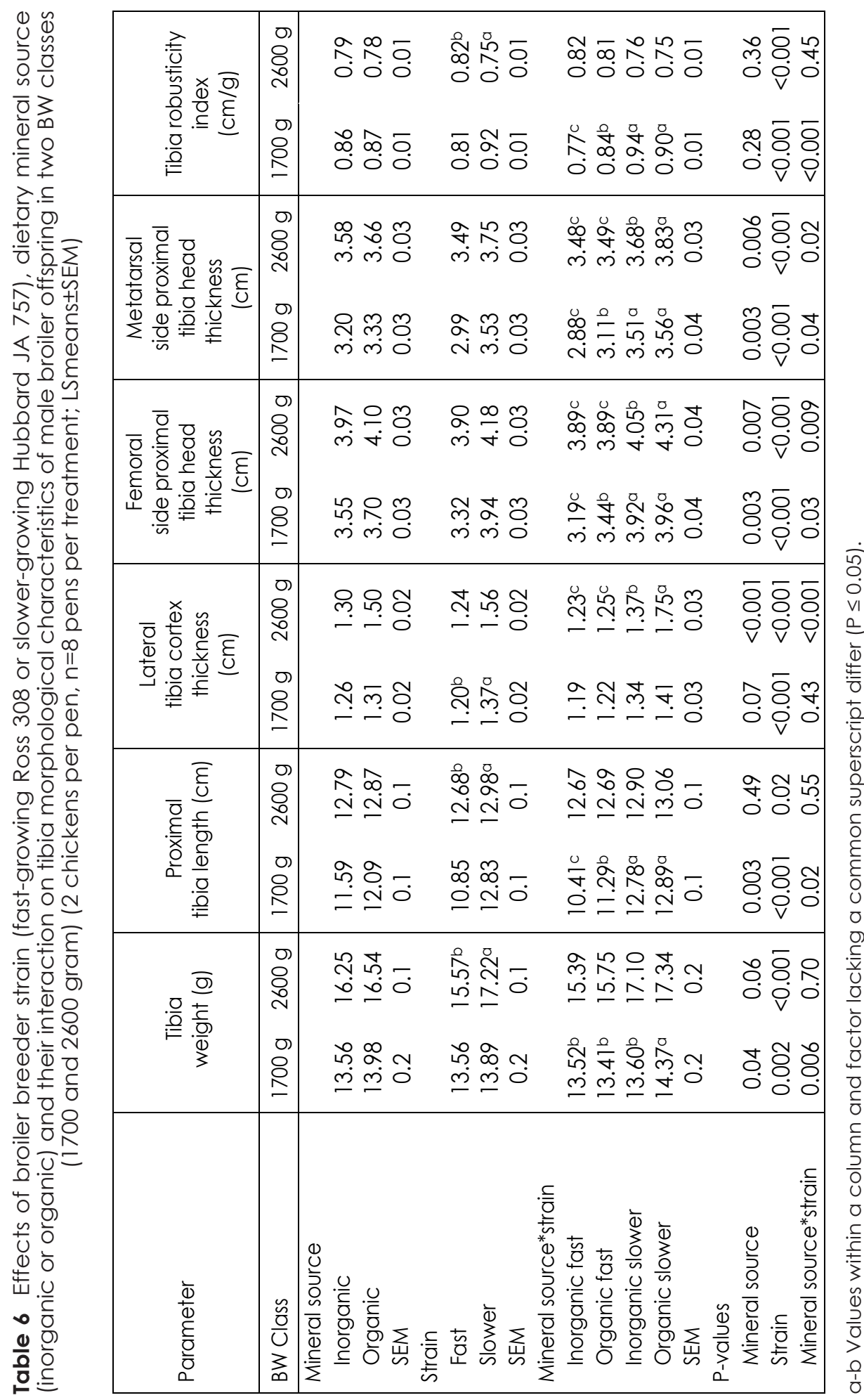




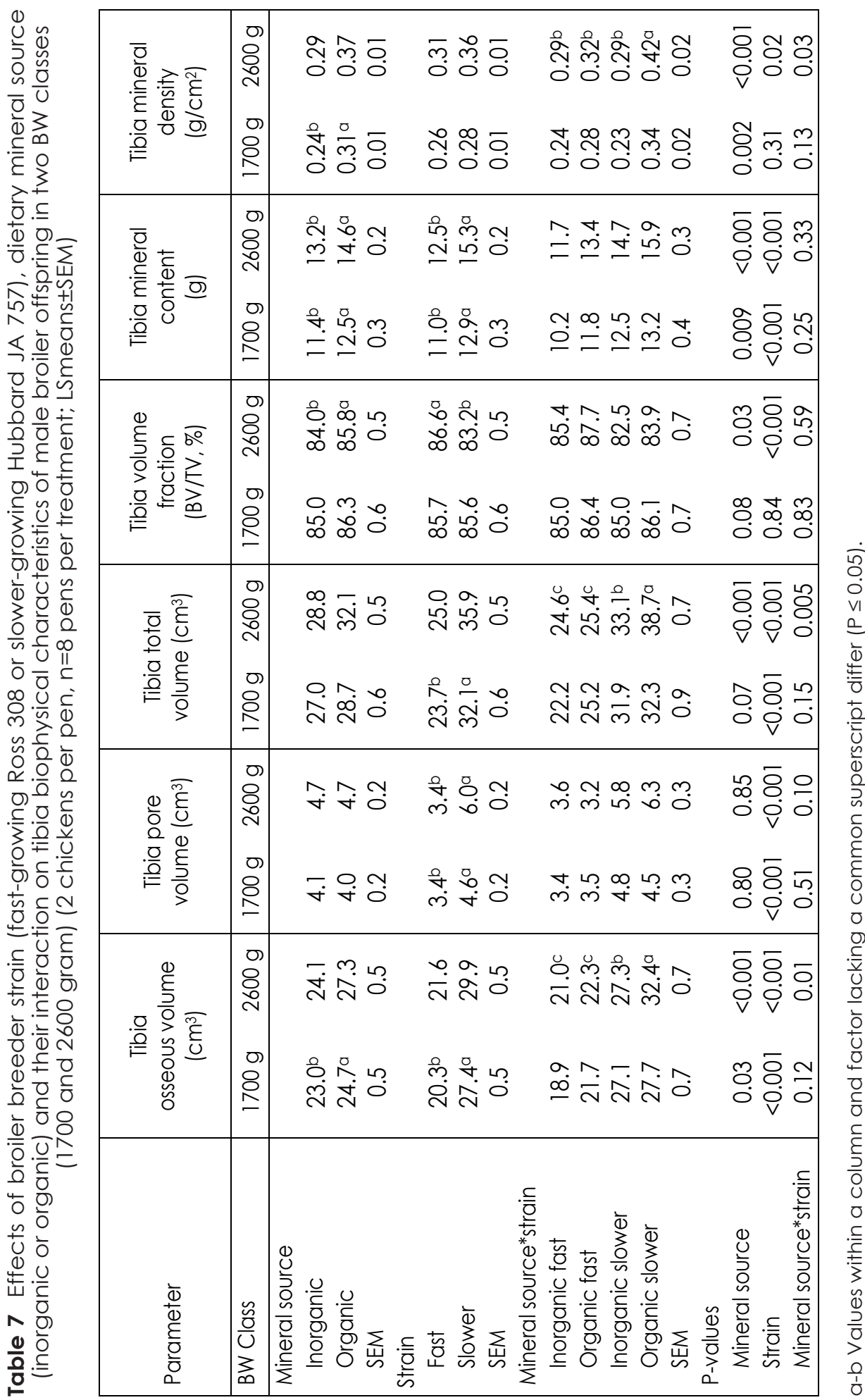




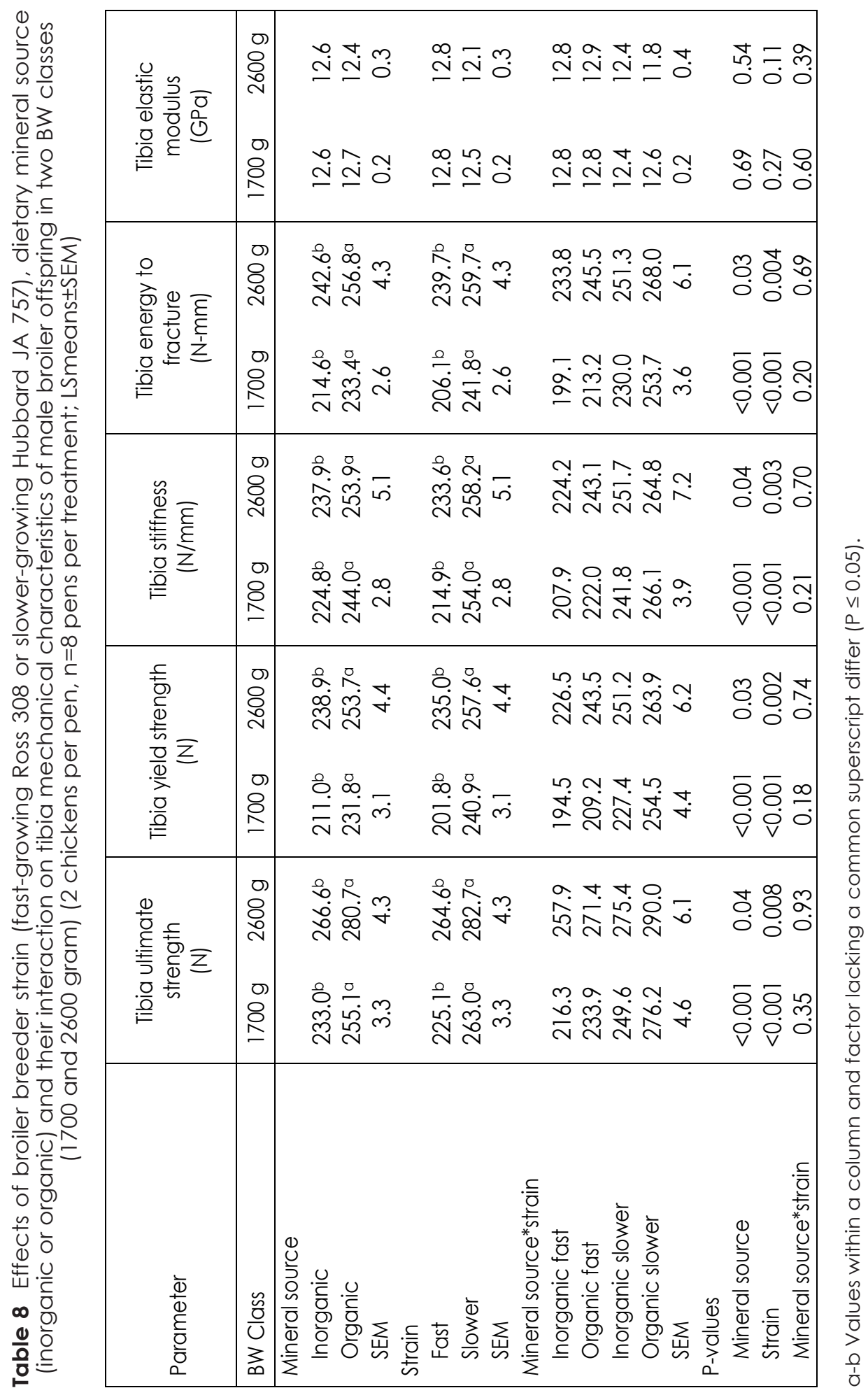




\section{Leg Disorders}

No interaction effects between mineral source and broiler breeder strain were found on VV and TD at both 1700 and 2600 gram BW classes and furthermore, no main effects were found on TD. At 1700 gram and 2600 gram BW classes, fast-growing chickens showed a higher VV incidence than slower-growing chickens ( 1700 gr: 25.0 vs $4.2 \%$, respectively; $P=0.02$; 2600 gr: 37.5 vs $23.0 \%$, respectively; $P=0.04$ ).

\section{DISCUSSION}

\section{Minerals in Eggs and Hatchlings}

Results of mineral analyses in the current study demonstrated that source of the macro and trace minerals in the broiler breeder diet affected the concentrations of some minerals in eggs of both fast and slower-growing broiler breeders. Fe and Se concentrations were found higher in the mixture of yolk and albumen, whereas $\mathrm{Cu}$ was found lower after feeding organic minerals to the breeders compared to their inorganic forms. The other minerals (Ca, P, Mn, Zn) were not influenced by mineral source in the breeder diet nor by broiler breeder strain. The yolk is the main source of nutrients for the embryo and supplies phosphorus and trace minerals, while the eggshell is the main calcium source (Torres and Korver, 2018). Studies have shown that increasing the concentration of macro and trace minerals in the diet of broiler breeder or laying hens hardly influenced their concentrations in the egg, since the amounts of minerals in the egg have certain limits, which is mainly determined by the genetic background of the breeder (Naber, 1979; Angel, 2007).

Changing the mineral source in the maternal diet might be an alternative way to influence mineral concentrations in the egg and consequently in the offspring. Effects of trace mineral source in broiler breeder diets on mineral concentrations in their eggs are hardly investigated, whereas studies on macro mineral source in broiler breeder diets are completely lacking. Furthermore, the exact pathways of macro and trace mineral transfer from the hen to the egg yolk, albumen and egg shell are still unclear (Dacke et al., 2015) and even more interesting, it is unclear whether or not pathways of inorganic and organic mineral transfer from 
breeder to egg differ. Results of the current study about iron and selenium concentration in the egg are in line with previous studies, indicating that organic selenium was found to be transferred more efficiently from broiler breeders (Pappas et al., 2005) and laying hens (Sirichakwal et al., 1984; Miles, 2001; Kidd, 2003) to the egg than inorganic selenium. Organic iron in laying hen diet resulted in higher amount of iron in eggs (Park et al., 2004; Buckiuniene et al., 2017) than inorganic iron in the laying diet. These and our findings suggest that organic selenium and iron in the broiler breeder diet affect the physiological transfer and deposition pathways and finally ends up with increased concentrations in the egg. Regarding manganese, copper and zinc, several studies with laying hens have shown that an organic form of those minerals in the diet also resulted in higher concentrations in eggs than an inorganic form (Dobrzanski et al., 2008; Venglovska et al., 2014; Yenice et al., 2015; saleh et al., 2019), but this increase was not found in the current study. This may be explained by different genetic backgrounds between broiler breeders and laying hens, but further investigation is needed to understand the exact pathways.

Looking at the results of residual yolk and yolk free bodies of hatchlings, selenium appeared to be the only mineral which was affected by the mineral source in the breeder diet in both residual yolk and yolk free bodies. It can be speculated that the higher concentration of selenium in eggs was effectively absorbed and retained in the yolk free body during embryonic development and also more selenium was still left in the residual yolk. The fact that only selenium was affected in the hatchling body and not the other minerals might indicate that selective absorption of minerals through the yolk sac membrane into the blood stream is occurring (Yair and Uni, 2013; Bokkers and Koene, 2003). However, the exact pathways and factors affecting these pathways remain unclear and need to be investigated.

Despite the fact that breeder strain did not show any effect on mineral concentration in the mixture of yolk and albumen nor the eggshell, differences in mineral concentration were found in the residual yolk and yolk free body, but effects were ambiguous. Calcium, copper and manganese were higher in residual yolk of slower-growing hatchlings compared to fast-growing hatchlings, whereas the opposite was found for phosphorus, iron and selenium. This again suggests that selective 
absorption of minerals takes place as indicated above.

Based on the mineral concentrations in the egg, residual yolk and yolk free body, it can be concluded that effects of mineral sources in the breeders' diet on concentrations in the eggs and hatchlings are marginal. However, effects on body weight and tibia characteristics are found (see below), suggesting that other mechanisms than only mineral concentration appears to play a role in trans-generational mineral transfer in broiler chickens.

\section{Growth Performance}

Although effects of mineral source in the broiler breeder diet were limited in relation to mineral concentrations in both eggs and hatchlings, effects on offspring BW were evident, particularly in the slower-growing chickens. From day 10 of age onward, in the slower-growing strain, chickens originating from organic minerals fed broiler breeders had higher BW than chickens originating from inorganic minerals fed broiler breeders, while this effect was not seen in fast-growing chickens. Earlier studies demonstrated that organic trace minerals had a higher bioavailability in fast-growing breeders and finally resulted in higher post-hatch growth performance (Chang et al., 2016; M'sadeq et al., 2018; Araújo et al., 2019) than inorganic trace minerals. It can be speculated why offspring growth rate in the current study was positively affected by organic minerals in the breeder diet of the only slower-growing strain and not in the fast-growing strain. The first reason, as difference in feed intake, might be due to different absorption, mineral deposition or mineral transferring physiologies between slower and fast-growing chickens, because of their different genetic backgrounds (Torres and Korver, 2018; Yair et al., 2013). Secondly, related to first reason and differences in feed intake, because fast and slower-growing breeders received different diets, it can be speculated that the inorganic diet of the slower-growing breeders might have been suboptimal in minerals and consequently they benefit from organic minerals, whereas this was not the case for the fast-growing breeder diet. Thirdly, it might be that slower-growing chickens with better developed bones are more active and better able to reach the feed and water. This is supported by the numerically higher $\mathrm{Fl}$ in the slower-growing chickens originating from 
organic minerals fed breeders compared to the ones originating from inorganic minerals fed breeders. It appears that organic minerals in broiler breeder diets and sufficiently developed and mineralised bones could work together to reach a better growth performance.

Regarding the strain, in the current study, fast-growing broiler chickens, as expected, showed higher body weight and feed efficiency compared to the slower-growing broiler chickens on the same ages. This is in accordance with previous studies showing that fast-growing chickens have been specifically selected for these two parameters (Bokkers and Koene, 2003; Quentin et al., 2004; Benyi et al., 2010, 2015).

\section{Tibia Characteristics}

Calcium mobilization, bone mineralization and growth plate differentiation in the chicken embryo start during the first week of incubation (Blom and Lilja, 2005; Oviedo-Rondón et al., 2009; Van der pol et al., 2019). During this first week, the yolk is the main source of calcium for embryonic bone development. During the second embryonic week, almost all macro and trace minerals are absorbed by the embryo, while calcium from the shell and phosphorus from the yolk sac membrane are released (Richards and Steele, 1987; Ono and Tuan, 1991). Towards the last days of incubation and first days after hatch, phosphorus and trace minerals are suggested to be too low in the residual yolk (Parmer et al., 1987; Ono and Tuan, 1991; Duan et al., 2013), which might limit bone development of chickens. This suggests that prepackaged mineral concentrations in the egg are very important for bone development and later life bone health (Kienholz et al., 1961; Richards and Steele, 1987; Kidd et al., 1992; Dibner et al., 2007).

Macro minerals that are involved in bone development are Ca and P (Angel, 2007), which work together to form calcium-phosphate crystals that build the bone matrix (Rath et al., 1999; Blake and Fogelman, 2002; Shim et al., 2012). They also work as an essential part of enzymes and hormones involved in bone development, such as GH, IGF-1, T3 and T4 (simpson et al., 1967; Rosol and Capen, 1997; McDevitt et al., 2006). Trace minerals also play an important role in bone development. Copper, zinc and manganese are involved in enzyme activities that are related to embryonic and post-hatch bone development (Dibner et al., 2007). Copper, zinc and manganese are stored 
mainly in the egg yolk (Ono and Tuan, 1991; Richards, 1997) and are mobilized throughout incubation to support development of the growth plate of the bone (Medeiros et al., 2002; Flis et al., 2019). Other trace minerals that are essential for bone development are iron and selenium. Iron is involved in many enzymes and protein systems and an important component of synthesising collagen (Medeiros et al., 2002). A deficiency in iron has been reported to negatively affect bone morphology, strength and density in rats (Medeiros et al., 2002). Iron, manganese and selenium are also important for the thyroid function, which helps to regulate bone growth (Caskey et al., 1944; Jianhua et al., 2000).

Changing the mineral sources from inorganic to organic has been found to positively affect embryonic bone development and later life leg health, due to their higher mineral mobilization and bioavailability (Kidd et al., 1992; Park et al., 2004; Echigo and Kimata, 2010; Favero et al., 2013; Oviedo-Rondón et al., 2013). The main reason of the higher mobilization and bioavailability of organic minerals compared to inorganic minerals is related to their bonds. Organic minerals contain covalent bonds that provide a better binding strength with other compounds and result in better chemical stability compared to inorganic minerals, which are bound by weak electrovalent and/or ionic bonds (Vieira, 2008; Bao and Choct, 2009; Chigo and Kimata, 2010; Wang et al., 2019). Results of the current study provided evidence that organic macro and trace minerals in the maternal diet of slower-growing chickens resulted in better offspring tibia characteristics compared to inorganic form of those minerals in the maternal diet. These findings are in line with previous research, indicating that a higher trace mineral availability in the diet of broiler breeder leads to more advanced bone development of their offspring from embryonic phase till slaughter age (Sirichakwal et al., 1984; Dibner et al., 2007; Ferket et al., 2009; Torres and Korver, 2018; Saleh et al., 2019). Despite the fact that concentrations of minerals in the eggs and hatchlings originated from organic and inorganic minerals fed broiler breeders hardly differed, it appears that other mechanisms have played a role on post-hatch body weight gain and bone development, but these mechanisms are currently unclear.

Regarding the strain, slower-growing chickens showed better tibia morphological, biophysical and mechanical characteristics compared 
to fast-growing chickens at both 1700 and 2600 gram BW classes. This difference can probably be explained by the negative correlation between growth rate and bone development. Fast-growing broiler chickens have been shown to have poorer mineralised bones compared to slower-growing broilers (Lilburn, 1994; Velleman, 2000; Bonser and Casinos, 2003). Fast growth is known to result in poorer mineralised bones, due to the fact that mechanisms involved in bone development cannot keep up with fast growth of the broiler, particularly during the first two weeks of the growth phase. Slower growth ensures that there is more time for bone mineralisation, which compensates for the lack of mineralisation in the early growth phase (Shim et al., 2012; Sanchez-Rodriguez et al., 2019).

In conclusion, despite the fact that effects on mineral concentration in eggs and hatchlings were limited, organic macro and trace minerals in the broiler breeder diet showed positive effects on both offspring BW and tibia characteristics in slower-growing chickens, whereas this effect was hardly seen in fast-growing chickens. This suggests that (1) the difference in feed intake between fast and slower-growing broiler breeders might affect offspring performance, which might indicate that current slowergrowing broiler breeder diets might be suboptimal in minerals or that transgenerational mineral availability in slower-growing chickens appears to be more effective on bone development than in fast-growing chickens, which might be related to time available for bone development. (2) transgenerational mineral availability in offspring appears to play a role via other mechanisms than via absolute mineral concentrations in the egg. 


\section{ACKNOWLEDGEMENTS}

This experiment was the part of the "Healthy Bones" project, financed by a public-private partnership (TKI-AF-15203; BO-63-001-004). The financial support of the Ministry of Agriculture, Nature and Food Quality of The Netherlands, Aviagen EPI, Darling Ingredients Inc., ForFarmers N.V., Hubbard, Marel Stork Poultry Processing BV, Nepluvi and Nutreco (The Netherlands) is gratefully acknowledged. The authors would like to thank Remco Hamoen, Bert van Nijhuis, Henny Reimert, Marcel Heetkamp, llona van der Anker-Hensen, Monique Ooms, Bjorge Laurenssen and the animal caretakers for their help during the experiment. 


\section{SUPPLEMENTARY DATA}

Table A1 Effects of broiler breeder strain (fast-growing Ross 308 or slower-growing Hubbard JA 757), dietary mineral source (inorganic or organic) and their interaction on hatchling characteristics (LSmeans \pm SEM)

\begin{tabular}{|c|c|c|c|c|c|}
\hline Parameter & $\mathrm{n}$ & $\begin{array}{c}\text { Egg } \\
\text { weight }{ }^{1} \\
\text { (g) }\end{array}$ & $\begin{array}{c}\text { Red } \\
\text { hock }^{2} \\
(\%)\end{array}$ & $\begin{array}{c}\text { Red } \\
\text { beak }^{2} \\
(\%)\end{array}$ & $\begin{array}{l}\text { Navel } \\
\text { score }\end{array}$ \\
\hline \multicolumn{6}{|l|}{ Mineral source } \\
\hline Inorganic & 517 & $53.5^{a}$ & 5.06 & 6.19 & 1.21 \\
\hline Organic & 515 & $52.7^{b}$ & 6.62 & 6.80 & 1.20 \\
\hline SEM & - & 0.22 & 1.03 & 1.10 & 0.02 \\
\hline \multicolumn{6}{|l|}{ Strain } \\
\hline Fast & 498 & $55.5^{a}$ & $3.97 b$ & $4.97 b$ & 1.19 \\
\hline Slower & 534 & $50.6^{b}$ & $7.70^{a}$ & $8.03 a$ & 1.22 \\
\hline SEM & - & 0.22 & 1.03 & 1.10 & 0.02 \\
\hline \multicolumn{6}{|l|}{ Mineral source*strain } \\
\hline Inorganic fast & 238 & 56.1 & 2.94 & 3.78 & 1.19 \\
\hline Organic fast & 259 & 55.0 & 5.00 & 6.15 & 1.20 \\
\hline Inorganic slower & 278 & 50.9 & 7.17 & 8.60 & 1.23 \\
\hline Organic slower & 257 & 50.4 & 8.24 & 7.45 & 1.21 \\
\hline SEM & - & 0.31 & 1.47 & 1.53 & 0.03 \\
\hline \multicolumn{6}{|l|}{ P-values } \\
\hline Mineral source & - & 0.03 & 0.29 & 0.70 & 0.83 \\
\hline Strain & - & $<0.001$ & 0.02 & 0.05 & 0.35 \\
\hline Mineral source*strain & - & 0.44 & 0.74 & 0.26 & 0.74 \\
\hline
\end{tabular}

a-b Values within a column and factor, lacking a common superscript differ ( $P \leq 0.05$ ).

1 Average egg weight per treatment before incubation by weighing egg trays.

2 Red hock and red beak of all hatchlings were scored as 0 (absent) or 1 (present) and expressed as percentage of chickens with red hocks or red beaks.

3 Navel score was assessed as 1 (good), 2 (moderate) or 3 (poor), analysed binary (good vs moderate plus poor) and expressed as LSmeans.

Table A2 Effects of broiler breeder strain (fast-growing Ross 308 or slower-growing Hubbard JA 757) and dietary mineral source (inorganic or organic) on percentage of male offspring chickens showing the following behaviours in their home pen (eating, drinking, walking, standing, resting, sitting, dustbathing and perching) at day $16,23,30,37$ and 44 of age (all chickens in the pen, $\mathrm{n}=8$ pens per treatment).

\begin{tabular}{|c|c|c|c|c|c|c|c|}
\hline \multirow{2}{*}{$\begin{array}{l}\text { Parameter } \\
\text { and day }\end{array}$} & \multicolumn{2}{|c|}{ Mineral source } & \multicolumn{2}{|c|}{ Strain } & \multirow{2}{*}{ SEM } & \multicolumn{2}{|c|}{$P$-values ${ }^{2}$} \\
\hline & Inorganic & Organic & Fast & Slower & & $\begin{array}{l}\text { Mineral } \\
\text { source }\end{array}$ & Strain \\
\hline Eating & & & & & & & \\
\hline $16 d$ & 3.46 & 2.60 & 4.17 & 1.90 & 1.13 & 0.60 & 0.17 \\
\hline $23 d$ & 2.65 & 2.33 & $4.47 a$ & $0.51^{b}$ & 0.83 & 0.79 & 0.003 \\
\hline $30 \mathrm{~d}$ & 5.70 & 3.06 & 6.57 & 2.19 & 1.59 & 0.25 & 0.06 \\
\hline $37 d$ & 4.84 & 3.83 & 6.56 & 2.11 & 1.64 & 0.67 & 0.06 \\
\hline $44 d^{1}$ & 2.78 & 0.00 & - & - & 1.52 & 0.21 & - \\
\hline
\end{tabular}


Table A2 (Continued)

\begin{tabular}{|c|c|c|c|c|c|c|c|}
\hline \multirow{2}{*}{$\begin{array}{l}\text { Parameter } \\
\text { and day }\end{array}$} & \multicolumn{2}{|c|}{ Mineral source } & \multicolumn{2}{|c|}{ Strain } & \multirow{2}{*}{ SEM } & \multicolumn{2}{|c|}{$P$-values ${ }^{2}$} \\
\hline & Inorganic & Organic & Fast & Slower & & $\begin{array}{l}\text { Mineral } \\
\text { source }\end{array}$ & Strain \\
\hline Drinking & & & & & & & \\
\hline $16 d$ & 4.50 & 2.86 & 4.69 & 2.68 & 1.32 & 0.39 & 0.29 \\
\hline $23 d$ & 4.05 & 7.22 & 6.84 & 4.42 & 1.35 & 0.11 & 0.22 \\
\hline $30 d$ & 3.59 & 2.42 & 2.78 & 3.23 & 1.20 & 0.50 & 0.79 \\
\hline $37 \mathrm{~d}$ & 3.58 & 3.41 & 4.25 & 2.75 & 1.50 & 0.94 & 0.49 \\
\hline $\begin{array}{l}44 \mathrm{~d}^{1} \\
\text { Walking }\end{array}$ & 0.70 & 0.00 & - & - & 0.49 & 0.33 & - \\
\hline $16 d$ & 7.60 & 7.77 & 6.25 & 9.12 & 1.73 & 0.95 & 0.25 \\
\hline $23 d$ & 8.74 & 10.81 & 8.40 & 11.14 & 2.02 & 0.48 & 0.35 \\
\hline $30 \mathrm{~d}$ & 6.22 & 7.58 & $3.86^{b}$ & $9.94^{a}$ & 1.92 & 0.62 & 0.03 \\
\hline $37 d$ & 4.42 & 2.40 & $0.35^{b}$ & $6.47 a$ & 1.07 & 0.19 & $<0.001$ \\
\hline $\begin{array}{l}44 \mathrm{~d}^{1} \\
\text { Standing }\end{array}$ & 8.12 & 6.61 & - & - & 3.10 & 0.74 & - \\
\hline $16 d$ & 9.50 & 7.29 & $3.91^{\mathrm{b}}$ & $12.88^{a}$ & 1.77 & 0.39 & 0.002 \\
\hline $23 d$ & 3.70 & 7.46 & $3.41^{b}$ & $7.75^{a}$ & 1.50 & 0.09 & 0.05 \\
\hline $30 \mathrm{~d}$ & 3.80 & 5.51 & $1.82^{\mathrm{b}}$ & $7.49 a$ & 1.69 & 0.48 & 0.03 \\
\hline $37 d$ & 5.27 & 3.68 & $2.47^{b}$ & $6.47 a$ & 1.31 & 0.40 & 0.04 \\
\hline $\begin{array}{l}44 \mathrm{~d}^{1} \\
\text { Resting }\end{array}$ & 2.37 & 5.48 & - & - & 1.83 & 0.24 & - \\
\hline $16 d$ & 12.98 & 10.16 & 13.92 & 9.21 & 1.99 & 0.33 & 0.11 \\
\hline $23 d$ & $18.82^{a}$ & $9.00^{b}$ & 14.42 & 13.40 & 2.62 & 0.02 & 0.79 \\
\hline $30 \mathrm{~d}$ & 11.75 & 15.29 & $19.36^{a}$ & $7.67 \mathrm{~b}$ & 2.69 & 0.36 & 0.004 \\
\hline $37 \mathrm{~d}$ & 9.08 & 10.56 & 11.33 & 8.31 & 2.53 & 0.68 & 0.41 \\
\hline $\begin{array}{l}44 d^{1} \\
\text { Sitting }\end{array}$ & 7.81 & 8.86 & - & - & 3.65 & 0.85 & - \\
\hline $16 \mathrm{~d}$ & 57.19 & 63.20 & 63.79 & 56.60 & 3.41 & 0.23 & 0.15 \\
\hline $23 d$ & 55.45 & 58.24 & 55.45 & 55.24 & 3.56 & 0.59 & 0.52 \\
\hline $30 \mathrm{~d}$ & 64.73 & 62.96 & 65.61 & 62.08 & 3.26 & 0.71 & 0.45 \\
\hline $37 \mathrm{~d}$ & 69.54 & 73.33 & 74.00 & 68.88 & 3.46 & 0.30 & 0.45 \\
\hline $44 d^{1}$ & 75.97 & 77.57 & - & - & 5.3 & 0.84 & - \\
\hline $\begin{array}{l}\text { Dust } \\
\text { bathing }\end{array}$ & & & & & & & \\
\hline $16 d$ & 1.59 & 2.49 & 2.75 & 1.33 & 1.30 & 0.63 & 0.45 \\
\hline $23 d$ & 2.37 & 2.60 & 2.89 & 2.08 & 0.81 & 0.84 & 0.49 \\
\hline $30 \mathrm{~d}$ & 0.26 & 0.26 & 0.00 & 0.52 & 0.26 & 1.00 & 0.16 \\
\hline $37 \mathrm{~d}$ & 1.39 & 0.69 & 1.04 & 1.04 & 0.85 & 0.57 & 1.00 \\
\hline $\begin{array}{l}44 \mathrm{~d}^{1} \\
\text { Perching }\end{array}$ & 0.69 & 0.69 & - & - & 0.69 & 1.00 & - \\
\hline $16 d$ & 3.20 & 3.62 & $0.52^{b}$ & $6.30^{a}$ & 1.40 & 0.84 & 0.007 \\
\hline $23 d$ & 4.24 & 2.34 & 1.07 & 5.51 & 1.56 & 0.40 & 0.06 \\
\hline $30 \mathrm{~d}$ & 3.97 & 2.92 & $0.00^{b}$ & $6.88^{a}$ & 1.18 & 0.54 & $<0.001$ \\
\hline $37 \mathrm{~d}$ & 1.89 & 2.08 & $0.00^{b}$ & $3.98^{a}$ & 0.90 & 0.89 & 0.003 \\
\hline $44 \mathrm{~d}^{1}$ & 1.56 & 0.78 & - & - & 1.24 & 0.66 & - \\
\hline
\end{tabular}

a-b Values within a row and factor, lacking a common superscript differ ( $\mathrm{P} \leq 0.05)$.

1 Only slower-growing chickens.

2 No interactions between broiler breeder strain and mineral source were observed for any of the behaviours and any of the sampling days. 


\section{REFERENCES}

Angel, R., 2007. Metabolic disorders: limitations to growth of and mineral deposition into the broiler skeleton after hatch and potential implications for leg problems. Journal of Applied Poultry Research. 16:138-149.

Araújo, C.S.S., Hermes, R.G., Bittencourt, L.C., Silva, C.C., Araújo, L.F., Granghelli, C.A., Pelissari, P.H., Roque, F.A. and Leite, B.G.S., 2019. Different dietary trace mineral sources for broiler breeders and their progenies. Poultry science. 98:4716-4721.

Bao, Y.M. and Choct, M., 2009. Trace mineral nutrition for broiler chickens and prospects of application of organically complexed trace minerals: a review. Animal Production Science, 49(4), pp.269-282.

Bao, Y.M., Choct, M., lji, P.A. and Bruerton, K., 2007. Effect of organically complexed copper, iron, manganese, and zinc on broiler performance, mineral excretion, and accumulation in tissues. Journal of Applied Poultry Research. 16:448-455.

Benyi, K., Acheampong-Boateng, O., Norris, D. and Ligaraba, T.J., 2010. Response of Ross 308 and Hubbard broiler chickens to feed removal for different durations during the day. Tropical animal health and production. 42:1421-1426.

Benyi, K., Netshipale, A.J., Mahlako, K.T. and Gwata, E.T., 2015. Effect of genotype and stocking density on broiler performance during two subtropical seasons. Tropical animal health and production. 47:969-974.

Bessei, W., 2006. Welfare of broilers: a review. World's Poultry Science Journal, 62(3), pp.455466.

Blake, G.M. and Fogelman, I., 2002. Methods and clinical issues in bone densitometry and quantitative ultrasonometry. In Principles of bone biology (pp. 1573-1585). Academic Press.

Blom, J. and Lilja, C., 2005. A comparative study of embryonic development of some bird species with different patterns of postnatal growth. Zoology. 108:81-95.

Bokkers, E.A. and Koene, P., 2003. Behaviour of fast-and slow growing broilers to 12 weeks of age and the physical consequences. Applied animalbehaviourscience. 81:59-72.

Bonser, R.H.C. and Casinos, A., 2003. Regional variation in cortical bone properties from broiler fowl--a first look. British poultry science. 44:350-354.

Bouxsein, M.L., Boyd, S.K., Christiansen, B.A., Guldberg, R.E., Jepsen, K.J. and Müller, R., 2010. Guidelines for assessment of bone microstructure in rodents using microcomputed tomography. Journal of bone and mineral research. 25:1468-1486.

Bradshaw, R.H., Kirkden, R.D. and Broom, D.M., 2002. A review of the aetiology and pathology of leg weakness in broilers in relation to welfare. Avian and poultry biology reviews. 13:45-104.

Buckiuniene, V., Gruzauskas, R. and Stanyte, G., 2017. Influence of organic and inorganic iron on laying hens productivity and bloods morphological composition and parameters in blood. In VIII International Scientific Agriculture Symposium," Agrosym 2017", Jahorina, Bosnia and Herzegovina, October 2017. Book of Proceedings (pp. 2261-2266). Faculty of Agriculture, University of East Sarajevo.

Caskey, C.D., Norris, L.C. and Heuser, G.F., 1944. A chronic congenital ataxia in chicks due to manganese deficiency in the maternal diet. Poultry Science. 23:516-520.

Chang, A., Halley, J. and Silva, M., 2016. Can feeding the broiler breeder improve chick quality and offspring performance?. Animal production science. 56:1254-1262.

Dacke, C.G., Sugiyama, T. and Gay, C.V., 2015. The role of hormones in the regulation of bone turnover and eggshell calcification. Sturkie's avian physiology, p549-575.

De Jong, I.C. and Gunnink, H., 2019. Effects of a commercial broiler enrichment programme with or without natural light on behaviour and other welfare indicators. Animal. 13:384-391. 
Dibner, J.J., Richards, J.D., Kitchell, M.L. and Quiroz, M.A., 2007. Metabolic challenges and early bone development. Journal of applied poultry research. 16:126-137.

Dixon, L.M., 2020. Slow and steady wins the race: The behaviour and welfare of commercial faster growing broiler breeds compared to a commercial slower growing breed. Plos one. 15:e0231006.

Dobrzañski, Z., Korczyñski, M., Chojnacka, K., Górecki, H. and Opaliñski, S., 2008. Influence of organic forms of copper, manganese and iron on bioaccumulation of these metals and zinc in laying hens. Journal of elementology. 13:309-319.

Duan, X., Li, M., Wu, F., Yang, N., Nikoo, M., Jin, Z. and Xu, X., 2013. Postfertilization changes in nutritional composition and protein conformation of hen egg. Journal of agricultural and food chemistry. 61:12092-12100.

Echigo, T. and Kimata, M., 2010. Crystal chemistry and genesis of organic minerals: a review of oxalate and polycyclic aromatic hydrocarbon minerals. The canadian mineralogist. 48:1329-1357.

Favero, A., Vieira, S.L., Angel, C.R., Bos-Mikich, A., Lothhammer, N., Taschetto, D. Cruz, R.F.A. and Ward, T.L., 2013. Development of bone in chick embryos from Cobb 500 breeder hens fed diets supplemented with zinc, manganese, and copper from inorganic and amino acid-complexed sources. Poultry science. 92:402-411.

Ferket, P.R., Oviedo-Rondón, E.O., Mente, P.L., Bohórquez, D.V., Santos Jr, A.A., Grimes, J.L., Richards, J.D., Dibner, J.J. and Felts, V., 2009. Organic trace minerals and 25-hydroxycholecalciferol affect performance characteristics, leg abnormalities, and biomechanical properties of leg bones of turkeys. Poultry science. 88:1 18-131.

Flis, M., Gugała, D., Muszyński, S., Dobrowolski, P., Kwiecień, M., Grela, E.R. and Tomaszewska, E., 2019. The influence of the partial replacing of inorganic salts of calcium, zinc, iron, and copper with amino acid complexes on bone development in male pheasants from aviary breeding. Animals. 9:237-249.

Gocsik, É., Silvera, A.M., Hansson, H., Saatkamp, H.W. and Blokhuis, H.J., 2017. Exploring the economic potential of reducing broiler lameness. British poultry science. 58:337-347.

González-Cerón, F., Rekaya, R. and Aggrey, S.E., 2015. Genetic analysis of bone quality traits and growth in a random mating broiler population. Poultry Science. 94:883-889.

Grandin, T., 2010. Auditing animal welfare at slaughter plants. Meat science. 86:56-65.

Grashorn, M.A., 2006, September. Fattening performance, carcass and meat quality of slow and fast-growing broiler strains under intensive and extensive feeding conditions. In XII Eur. Poult. Conf., Verona, Italy (p. 249). World's poultry science association italian branch, Bologna, Italy.

Güz, B.C., Molenaar, R., de Jong, I.C., Kemp, B., van den Brand, H. and van Krimpen, M., 2019. Effects of dietary organic minerals, fish oil, and hydrolyzed collagen on growth performance and tibia characteristics of broiler chickens. Poultry science. 98:65526563.

Güz, B.C., Molenaar, R., de Jong, I.C., Kemp, B., van Krimpen, M. and van den Brand, H., 2021. Effects of green light emitting diode light during incubation and dietary organic macro and trace minerals during rearing on tibia characteristics of broiler chickens at slaughter age. Poultry science. 100:707-720.

Huang, Y.L., LU, L., Li, S.F., LUO, X.G. and Liu, B., 2009. Relative bioavailabilities of organic zinc sources with different chelation strengths for broilers fed a conventional corn-soybean meal diet. Journal of Animal science. 87:2038-2046.

Jianhua, H., Ohtsuka, A. and Hayashi, K., 2000. Selenium influences growth via thyroid hormone status in broiler chickens. British journal of nutrition. 84:727-732.

Jungmann, R., Szabo, M.E., Schitter, G., Tang, R.Y.S., Vashishth, D., Hansma, P.K. and Thurner, P.J., 2011. Local strain and damage mapping in single trabeculae during three-point bending tests. Journal of the mechanical behavior of biomedical materials. 4:523534. 
Kestin, S.C., Su, G. and Sorensen, P., 1999. Different commercial broiler crosses have different susceptibilities to leg weakness. Poultry Science. 78:1085-1090.

Khatun, A., Chowdhury, S.D., Roy, B.C., Dey, B., Haque, A. and Chandran, B., 2019. Comparative effects of inorganic and three forms of organic trace minerals on growth performance, carcass traits, immunity, and profitability of broilers. Journal of advanced veterinary and animal research. 6:66-73.

Kidd, M.T., 2003. A treatise on chicken dam nutrition that impacts on progeny 1, 2. World's poultry science journal. 59:475-494.

Kidd, M.T., Anthony, N.B. and Lee, S.R., 1992. Progeny performance when dams and chicks are fed supplemental zinc. Poultry science. 71:1201-1206.

Kienholz, E.W., Turk, D.E., Sunde, M.L. and Hoekstra, W.G., 1961. Effects of zinc deficiency in the diets of hens. Journal of nutrition. 75:211-221.

Knowles, T.G., Kestin, S.C., Haslam, S.M., Brown, S.N., Green, L.E., Butterworth, A., Pope, S.J., Pfeiffer, D. and Nicol, C.J., 2008. Leg disorders in broiler chickens: prevalence, risk factors and prevention. PloS one. 3:e1545.

Kokoszyński, D., Bernacki, Z., Saleh, M., Stęczny, K. and Binkowska, M., 2017. Body conformation and internal organs characteristics of different commercial broiler lines. Brazilian journal of poultry science. 19:47-52.

Lilburn, M.S., 1994. Skeletal growth of commercial poultry species. Poultry science. 73:897-903.

McDevitt, R.M., McEntee, G.M. and Rance, K.A., 2006. Bone breaking strength and apparent metabolisability of calcium and phosphorus in selected and unselected broiler chicken genotypes. British poultry science. 47:613-621.

Medeiros, D.M., Plattner, A., Jennings, D. and Stoecker, B., 2002. Bone morphology, strength and density are compromised in iron-deficient rats and exacerbated by calcium restriction. The journal of nutrition. 132:3135-3141.

Mench, J., 2004. Lameness. Measuring and auditing broiler welfare. p3-17.

Miles, R.D., 2001. Trace minerals and avian embryo development. Ciência animal brasileira. 2:1-10.

Molenaar, R., Reijink, I.A.M., Meijerhof, R. and Van den Brand, H., 2010. Meeting embryonic requirements of broilers throughout incubation: a review. Brazilian journal of poultry science. 12:137-148.

M'Sadeq, S.A., Wu, S.B., Choct, M. and Swick, R.A., 2018. Influence of trace mineral sources on broiler performance, lymphoid organ weights, apparent digestibility, and bone mineralization. Poultry science. 97:3176-3182.

Naber, E.C., 1979. The effect of nutrition on the composition of eggs. Poultry Science, 58:518528.

Novitskaya, E., Chen, P.Y., Hamed, E., Jun, L., Lubarda, V.A., Jasiuk, I. and McKittrick, J., 2011. Recent advances on the measurement and calculation of the elastic moduli of cortical and trabecular bone: a review. Theoretical and applied mechanics. 38:209-297.

Ono, T. and Tuan, R.S., 1991. Vitamin D and chick embryonic yolk calcium mobilization: Identification and regulation of expression of vitamin D-dependent Ca2+-binding protein, calbindin-D28K, in the yolk sac. Developmental biology. 144:167-176.

Oviedo-Rondon, E.O., Leandro, N.M., Ali, R., Koci, M., Moraes, V. and Brake, J., 2013. Broiler breeder feeding programs and trace minerals on maternal antibody transfer and broiler humoral immune response. Journal of applied poultry research. 22:499-510.

Oviedo-Rondón, E.O., Wineland, M.J., Funderburk, S., Small, J., Cutchin, H. and Mann, M. 2009. Incubation conditions affect leg health in large, high-yield broilers. Journal of applied poultry research. 18:640-646.

Pappas, A.C., Acamovic, T., Sparks, N.H.C., Surai, P.F. and McDevitt, R.M., 2005. Effects of supplementing broiler breeder diets with organic selenium and polyunsaturated fatty acids on egg quality during storage. Poultry science. 84:865-874. 
Park, S.Y., Birkhold, S.G., Kubena, L.F., Nisbet, D.J. and Ricke, S.C., 2004. Review on the role of dietary zinc in poultry nutrition, immunity, and reproduction. Biological trace element research. 101:147-163.

Parmer, T.G., Carew, L.B., Alster, F.A. and Scanes, C.G., 1987. Thyroid function, growth hormone, and organ growth in broilers deficient in phosphorus. Poultry science. 66:19952004.

Quentin, M., Bouvarel, I. and Picard, M., 2004. Short-and long-term effects of feed form on fast-and slow-growing broilers. Journal of applied poultry research. 13:540-548.

Rath, N.C., Balog, J.M., Huff, W.E., Huff, G.R., Kulkarni, G.B. and Tierce, J.F., 1999. Comparative differences in the composition and biomechanical properties of tibiae of seven-and seventy-two-week-old male and female broiler breeder chickens. Poultry science. 78:1232-1239.

Rayner, A.C., Newberry, R.C., Vas, J. and Mullan, S., 2020. Slow-growing broilers are healthier and express more behavioural indicators of positive welfare. Scientific reports, 10:114.

Richards, M.P. and Steele, N.C., 1987. Trace element metabolism in the developing avian embryo: a review. The Journal of experimental zoology. Supplement: published under auspices of the american society of zoologists and the division of comparative physiology and biochemistry. 1:39-51.

Richards, M.P., 1997. Trace mineral metabolism in the avian embryo. Poultry science. 76:152164.

Riesenfeld, A., 1972. Metatarsal robusticity in bipedal rats. American journal of physical anthropology. 36:229-233.

Rosol, T.J. and Capen, C.C., 1997. Calcium-regulating hormones and diseases of abnormal mineral (calcium, phosphorus, magnesium) metabolism. In Clinical biochemistry of domestic animals (p619-702). Academic press.

Saleh, A.A., Eltantawy, M.S., Gawish, E.M., Younis, H.H., Amber, K.A., Abd El, A.E.M.E. and Ebeid, T.A., 2019. Impact of dietary organic mineral supplementation on reproductive performance, egg quality characteristics, lipid oxidation, ovarian follicular development, and immune response in laying hens under high ambient temperature. Biological trace element research. pl-9.

Sanchez-Rodriguez, E., Benavides-Reyes, C., Torres, C., Dominguez-Gasca, N., Garcia-Ruiz, A.I., Gonzalez-Lopez, S. and Rodriguez-Navarro, A.B., 2019. Changes with age (from 0 to $37 \mathrm{D}$ ) in tibiae bone mineralization, chemical composition and structural organization in broiler chickens. Poultry science. 98:5215-5225.

Sherlock, L., Demmers, T.G.M., Goodship, A.E., McCarthy, I.D. and Wathes, C.M., 2010. The relationship between physical activity and leg health in the broiler chicken. British Poultry science. 51:22-30.

Shim, M.Y., Karnuah, A.B., Anthony, N.B., Pesti, G.M. and Aggrey, S.E., 2012. The effects of broiler chicken growth rate on valgus, varus, and tibial dyschondroplasia. Poultry science. 91:62-65.

Shim, M.Y., Karnuah, A.B., Mitchell, A.D., Anthony, N.B., Pesti, G.M. and Aggrey, S.E., 2012. The effects of growth rate on leg morphology and tibia breaking strength, mineral density, mineral content, and bone ash in broilers. Poultry science. 91:1790-1795.

Simpson, C.F., Jones, J.E. and Harms, R.H., 1967. Ultrastructure of aortic tissue in copper-deficient and control chick embryos. The journal of nutrition. 91:283-291.

Sirichakwal, P.P., Newcomer, C.E., Young, V.R. and Janghorbani, M., 1984. Labeling hen's egg with $74 \mathrm{Se}$ for use in human metabolic experiments. The journal of nutrition.114:1159-1168.

Stojcic, M.D. and Bessei, W., 2009. The effect of locomotor activity and weight load on bone problems in fast and slow growing chickens. Archiv für geflügelkunde. 73:242-249. 
Sullivian, T.W., 1994. Skeletal problems in poultry: estimated annual cost and descriptions. Poultry science. 73:879-882.

Świątkiewicz, S., Arczewska-Włosek, A. and Jozefiak, D., 2014. The efficacy of organic minerals in poultry nutrition: review and implications of recent studies. World's poultry science journal. 70:475-486.

Thorp, B.H. and Waddington, D., 1997. Relationships between the bone pathologies, ash and mineral content of long bones in 35-day-old broiler chickens. Research in veterinary science. 62:67-73.

Torres, C.A. and Korver, D.R., 2018. Influences of trace mineral nutrition and maternal flock age on broiler embryo bone development. Poultry science. 97:2996-3003.

Torres, C.A., 2013. Influence of maternal flock age, maternal trace mineral nutrition and incubation temperature on bone development of embryos and chicks. Doctoral dissertation, University of Alberta, Canada.

Turner, C.H. and Burr, D.B., 1993. Basic biomechanical measurements of bone: a tutorial. Bone. 14:595-608.

Van der Klis, J.D. and Kemme, P.A., 2002. An appraisal of trace elements: inorganic and organic. Poultry feedstuffs: supply, composition and nutritive value. p99-108.

van der Pol, C.W., van Roovert-Reijrink, I.A., Maatjens, C.M., Gussekloo, S.W., Kranenbarg, S., Wijnen, J., Pieters, R.P., Schipper, H., Kemp, B. and van den Brand, H., 2019. Light-dark rhythms during incubation of broiler chicken embryos and their effects on embryonic and post hatch leg bone development. PLoS one. 14:e0210886.

Velleman, S.G., 2000. The role of the extracellular matrix in skeletal development. Poultry science. 79:985-989.

Venglovska, K., Gresakova, L., Placha, I., Ryzner, M. and Cobanova, K., 2014. Effects of feed supplementation with manganese from its different sources on performance and egg parameters of laying hens. Czech journal of animal science. 59:147-155.

Vieira, S.L., 2008. Chelated minerals for poultry. Brazilian journal of poultry science. 10:73-79.

Wang, G., Liu, L.J., Tao, W.J., Xiao, Z.P., Pei, X., Liu, B.J., Wang, M.Q., Lin, G. and Ao, T.Y., 2019. Effects of replacing inorganic trace minerals with organic trace minerals on the production performance, blood profiles, and antioxidant status of broiler breeders. Poultry science. 98:2888-2895.

Wedekind, K.J., Titgemeyer, E.C., Twardock, A.R. and Baker, D.H., 1991. Phosphorus, but not calcium, affects manganese absorption and turnover in chicks. The journal of nutrition. 121:1776-1786.

Williams, B., Solomon, S., Waddington, D., Thorp, B. and Farquharson, C., 2000. Skeletal development in the meat-type chicken. British poultry science. 41:141-149.

Williams, B., Waddington, D., Murray, D.H. and Farquharson, C., 2004. Bone strength during growth: influence of growth rate on cortical porosity and mineralization. Calcified tissue international. 74:236-245.

Yair, R. and Uni, Z., 2011. Content and uptake of minerals in the yolk of broiler embryos during incubation and effect of nutrient enrichment. Poultry science. 90:1523-1531.

Yair, R., Shahar, R. and Uni, Z., 2013. Prenatal nutritional manipulation by in ovo enrichment influences bone structure, composition, and mechanical properties. Journal of animal science. 91:2784-2793.

Yenice, E., Mizrak, C., Gültekin, M., Atik, Z. and Tunca, M., 2015. Effects of organic and inorganic forms of manganese, zinc, copper, and chromium on bioavailability of these minerals and calcium in late-phase laying hens. Biological trace element research. 167:300-307.

Zhao, J., Shirley, R.B., Vazquez-Anon, M., Dibner, J.J., Richards, J.D., Fisher, P., Hampton, T., Christensen, K.D., Allard, J.P. and Giesen, A.F., 2010. Effects of chelated trace minerals on growth performance, breast meat yield, and footpad health in commercial meat broilers. Journal of applied poultry research. 19:365-372. 


$$
3
$$



B. C. Güz'
R. Molenaar
I. C. de Jong ${ }^{2}$
B. Kemp'
M. van Krimpen ${ }^{2}$
H. van den Brand'

Poultry Science 2020

99: 3020-3029

doi.org/10.1016/j.psj.2019.12.042

1 Adaptation Physiology Group, Wageningen University and Research, 6708 PB

Wageningen,

Gelderland,

The Netherlands

2 Wageningen Livestock Research, Wageningen University and Research, 6708 WD

Wageningen,

Gelderland,

The Netherlands 


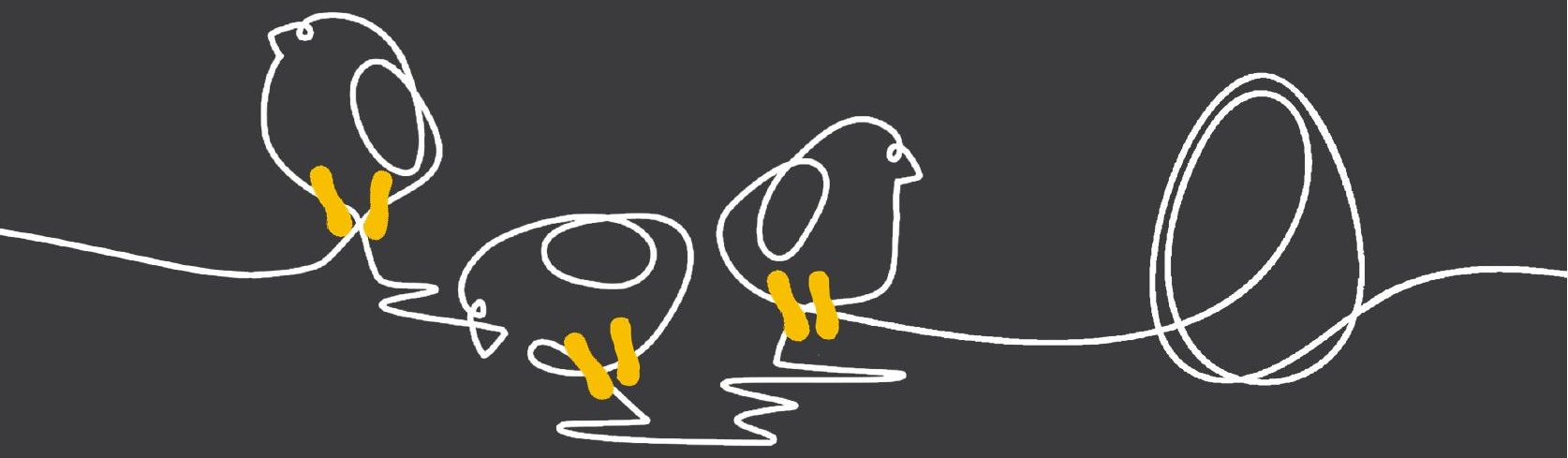

\section{Chapter 4}

EFFECTS OF EGGSHELL

TEMPERATURE PATTERN

DURING INCUBATION ON

TIBIA CHARACTERISTICS

OF BROILER CHICKENS AT

SLAUGHTER AGE 


$$
0
$$




\section{Abstract}

This study was designed to determine effects of eggshell temperature (EST) pattern in week 2 and week 3 of incubation on tibia development of broiler chickens at slaughter age. A total of 468 Ross 308 eggs were incubated at an EST of $37.8^{\circ} \mathrm{C}$ from incubation day (E) 0 to E7. Thereafter, a $2 \times 2$ factorial arrangement with $2 \mathrm{EST}\left(37.8\right.$ and $38.9^{\circ} \mathrm{C}$ ) from E8 to E14 and 2 EST ( 36.7 and $37.8^{\circ} \mathrm{C}$ ) from E15 till hatch was applied. After hatching, chickens were reared until slaughter age with the four EST treatments and 8 replicates per treatment. At $d 41$ and 42 , one male chicken per replicate per day was selected and hock burn and food pad dermatitis were scored. Rotated tibia, tibia dyschondroplasia, epiphyseal plate abnormalities, bacterial chondronecrosis with osteomyelitis and epiphysiolysis were assessed. Tibia weight, length, thickness, head thickness and robusticity index were determined. X-ray analyses (osseous volume, pore volume, total volume, volume fraction, mineral content and mineral density) and a 3-point bending test (ultimate strength, yield strength, stiffness, energy to fracture and elastic modulus) were performed. A high EST $\left(38.9^{\circ} \mathrm{C}\right)$ in week 2 of incubation, followed by a normal EST $\left(37.8^{\circ} \mathrm{C}\right)$ in week 3 resulted in higher mineral content $(P=0.001)$, mineral density $(P=0.002)$, ultimate strength $(P=0.04)$, yield strength $(\mathrm{P}=0.03)$ and stiffness $(\mathrm{P}=0.05)$ compared to the other 3 EST groups (week $2 \times$ week 3 interaction). A high EST $\left(38.9^{\circ} \mathrm{C}\right.$ ) in week 2 of incubation, regardless of the EST in week 3, resulted in a higher tibia weight $(P<0.001)$, thickness $(P=0.05)$, osseous volume $(P<0.001)$, and total volume $(\mathrm{P}<0.001)$ than a normal EST $\left(37.8^{\circ} \mathrm{C}\right)$. It can be concluded that $1.1^{\circ} \mathrm{C}$ higher EST than normal in week 2 of incubation appears to stimulate tibia morphological, biophysical, and mechanical characteristics of broiler chickens at slaughter age. Additionally, a $1.1^{\circ} \mathrm{C}$ lower EST in week 3 of incubation appears to have negative effects on tibia characteristics, particularly in interaction with the EST in week 2 of incubation.

\section{Key words}

eggshell temperature, incubation, tibia, leg health, broiler chickens 


\section{INTRODUCTION}

Leg health has been demonstrated to be suboptimal in fast-growing broiler chickens (Bessei, 2006; Sherlock et al., 2010). The underlying reason might be related to a developmental imbalance between a high growth rate and immaturity of bones and joints (Kestin et al., 1992, 2001). Suboptimal leg health can cause pain and may negatively affect natural locomotion-related behaviours of broiler chickens, such as accessing water and feed, especially in the last weeks of their life (Bessei, 2006; Gocsik et al., 2017). Moreover, leg problems can cause financial losses due to higher mortality, lower slaughter revenues, and increased rejections at slaughter plants (Kestin et al., 1999; Mench, 2004).

One factor that might play a role in bone development is incubation temperature. An optimal temperature for embryo development throughout incubation has been determined as $37.8^{\circ} \mathrm{C}$ (Lourens et al., 2005). A lower or higher temperature than this optimum might influence the quality of hatchlings (Wilson, 1991; French, 1997; Tona et al., 2005) and embryonic bone development as well. Bone development and growth plate differentiation begins during incubation and incubation temperature has been demonstrated to affect bone development and later life leg health of broiler chickens (Hammond et al., 2007; Oviedo-Rondon et al, 2008a,b, 2009; Shim and Pesti, 2017: Van der Pol et al., 2014). Effects of incubation temperature on embryonic bone development and later life leg health might be related to the speed of bone mineralisation during incubation. Embryonic bone development starts with cartilage formation in the first week of incubation (Nakane and Tsudzuki, 1999; Atalgin and Kürtül, 2009), which is followed by a rapid increase in mineralisation from the second week of incubation onward (Oviedo-Rondón et al., 2008b). This is due to growth plate differentiation (Ballock and O'Keefe, 2003; Hammond et al., 2007), which reaches the highest level just before hatch till a few days post-hatch (Applegate and Lillburn, 2002; Yalçin et al., 2007; Oviedo-Rondón et al., 2008b).

A temperature of $38.0^{\circ} \mathrm{C}$ throughout incubation resulted in longer tibia in broiler chickens at slaughter age compared to incubation temperatures of $36.0^{\circ} \mathrm{C}$ and $37.0^{\circ} \mathrm{C}$ (Oviedo-Rondón et al., 2008b). Yalçın et al. (2007) concluded that both low $\left(36.9^{\circ} \mathrm{C}\right)$ or high $\left(39.6^{\circ} \mathrm{C}\right)$ incubation 
temperatures from incubation day (E) 10 until E18 resulted in lower tibia weights at slaughter age compared to a control temperature $\left(37.8^{\circ} \mathrm{C}\right)$. Oviedo-Rondon et al. (2009) found comparable results at slaughter age when applying a high eggshell temperature (EST, $38.9^{\circ} \mathrm{C}$ ) during the last $4 \mathrm{~d}$ of incubation compared to an EST of $36.9^{\circ} \mathrm{C}$. van der Pol et al. (2014) observed that a high EST $\left(38.6^{\circ} \mathrm{C}\right)$ throughout incubation appears to result in lower tibia, femur, and metatarsus lengths at hatch compared to an EST of $36.9^{\circ} \mathrm{C}$ and $37.8^{\circ} \mathrm{C}$. Oznurlu et al. (2016) demonstrated that a $1.0^{\circ} \mathrm{C}$ higher incubation temperature $\left(38.8^{\circ} \mathrm{C}\right.$ vs. $\left.37.8^{\circ} \mathrm{C}\right)$ from E10 onward negatively affected tibia characteristics, including growth plate development, at different sampling days before and at hatch.

These different results among studies might be related to the moment, duration, and level of the incubation temperature that was applied. Moreover, studies differed in using incubation temperature or EST, which might result in differences in actual embryo temperature (Meijerhof and Van Beek, 1993).

It has been suggested that a higher incubation temperature $\left(41.0^{\circ} \mathrm{C}\right)$ than normal $\left(37.8^{\circ} \mathrm{C}\right)$ can stimulate bone development in general and the ossification process (E8 to E14) in particular (Aygun and Narinc, 2016). Contrary to the potential beneficial effects of a higher incubation temperature in the second week of incubation, a higher temperature $\left(39.0^{\circ} \mathrm{C}\right)$ than normal $\left(37.8^{\circ} \mathrm{C}\right)$ in the last week of incubation has been shown to negatively affect chicken development in general and leg bone health in later life in particular (Oviedo-Rondón et al., 2009).

Based on these findings, it can be hypothesised that a combination of a higher temperature than normal in the second week of incubation combined with a lower temperature in the last week of incubation might result in most optimal bone development and later life leg health.

The objectives of this study were to investigate effects of a combination of EST in week $2\left(37.8\right.$ or $\left.38.9^{\circ} \mathrm{C}\right)$ and EST in week $3\left(36.7\right.$ or $\left.37.8^{\circ} \mathrm{C}\right)$ of incubation on 1) tibia characteristics, 2) locomotion during rearing, and 3) leg disorders at slaughter age in fast-growing broiler chickens. 


\section{MATERIALS AND METHODS \\ Experimental Design}

The experiment was setup as a $2 \times 2$ factorial arrangement with 2 EST $\left(37.8\right.$ or $38.9^{\circ} \mathrm{C}$ ) from E8 to El4 and 2 EST (36.7 or $37.8^{\circ} \mathrm{C}$ ) from E15 till hatch. From EO to E7 of incubation, EST was maintained at $37.8^{\circ} \mathrm{C}$ for all eggs. After hatching, chickens were reared in a completely randomized block design until slaughter age and leg bone measurements were performed after slaughtering. All procedures in this study were approved by the Governmental Commission on Animal Experiments, The Hague, The Netherlands; Approval number: 2016.W-0087.001).

\section{Animals, Incubation, Rearing and Housing Management}

A total of 468 Ross 308 eggs from a 44-week-old breeder flock were obtained from a commercial hatchery (Lagerwey, Lunteren, The Netherlands). All eggs were selected on weight within three weight classes: 62.0 - $62.9 \mathrm{~g}(\mathrm{n}=156), 63.0-63.9 \mathrm{~g}(\mathrm{n}=156)$ and 64.0-64.9 $\mathrm{g}(\mathrm{n}=156)$ and stored at $20.0^{\circ} \mathrm{C}$ for $48 \mathrm{~h}$. Thereafter, all eggs were transported from the hatchery to the research facility of Wageningen University and Research (Wageningen, The Netherlands). At the research facility, all eggs were placed in one incubator with a capacity of 4,800 eggs (HatchTech Incubation Technology, B.V., Veenendaal, The Netherlands) for the first $7 \mathrm{~d}$ of incubation. Eggs were equally divided over the four treatment groups ( $n=117$ / treatment group) and placed on eight plastic trays (58 eggs $\times 4$ trays + 59 eggs $\times 4$ trays), in the middle layers of the incubator. Each tray contains Four EST sensors (NTC Thermistors: type DC 95; Thermometrics, Somerset, UK) were attached to four individual eggs, which each egg is on different tray and layer. All sensors were placed at the equator of the chosen eggs by using a heat conducting paste (Dow Corning 340 Heat Sink Compound, Dow Corning, Michigan, USA) and a small piece of tape $(2 \times 2 \mathrm{~cm})$. The incubator temperature was continuously adjusted based on the median temperature of the four EST sensors to maintain an EST of $37.8^{\circ} \mathrm{C}$. Throughout incubation, relative humidity of incubators and CRCs was maintained between $50 \%$ and $65 \%$. Eggs were turned every 30 min at 
an angle of $90^{\circ}$ and not exposed to light during incubation.

At E8, all eggs were candled and fertile eggs were divided over the same two HatchTech incubators and two small climate respiration chambers (CRC). The CRC had a capacity of 550 eggs. Eggs of the different weight classes were divided equally over the two incubators and the two CRCs. One incubator and one CRC were set at an EST of $37.8^{\circ} \mathrm{C}$ and the other ones were set at an EST of $38.9^{\circ} \mathrm{C}$. Within each incubator and CRC, five EST sensors (Pt-100, Sensor Data BV, Rijswijk, The Netherlands) were attached to five randomly chosen eggs, as described above.

At E15, all eggs were candled again and infertile eggs or eggs lacking a vital embryo were removed and a break-out analysis was performed (data not included in this paper). All eggs containing a vital embryo were redistributed over the same two incubators and two CRCs, after which one incubator and one CRC were set at an EST of $36.7^{\circ} \mathrm{C}$ and the other incubator and CRC were set at an EST of $37.8^{\circ} \mathrm{C}$. EST was maintained based on the median temperature of the four EST sensors as described above. The combination of EST in week 2 and week 3 resulted in a $2 \times 2$ factorial arrangement with 4 treatments in total: EST $37.8 \times 36.7^{\circ} \mathrm{C}$; EST $37.8 \times$ $37.8^{\circ} \mathrm{C}$; EST $38.9 \times 36.7^{\circ} \mathrm{C}$; EST $38.9 \times 37.8^{\circ} \mathrm{C}$ (week $2 \times$ week 3 , respectively).

At E18, all eggs were candled again and eggs containing a vital embryo were transferred from the trays to hatching baskets, which were placed back in the same incubator or CRC. From E20 onwards, hatched chicks were counted at every 6 hours of periods and marked as an early (at 486 and $492 \mathrm{~h}$ of incubation), mid (498 and $504 \mathrm{~h}$ of incubation) or late (510 and $516 \mathrm{~h}$ of incubation) hatchers.

At E21, chickens were collected then individually weighed and numbered by using a paw-ring, and transferred to a separate room, where they were placed in specially designed baskets with ad libitum access to feed and water till all chickens had hatched (HatchCare system, Hatchtech BV, Veenendaal, The Netherlands).

Thereafter, chickens were feather-sexed and transferred to two adjacent houses, each having 16 floor pens $(2 \times 1 \mathrm{~m})$, containing saw dust as litter. Chickens were vaccinated against infectious bronchitis (eye drop; MSD Animal Health, Boxmeer, The Netherlands) at $d 0$ and against 
Newcastle disease (Clone 30; eye drop, MSD Animal Health, Boxmeer, The Netherlands) at $d 11$. Each pen contained five male and five female chickens (consisted of early, mid and late hatchers) from the same treatment. Both houses were divided into 4 blocks and the four treatments were randomly divided within each block. Both houses were identical and climate controlled with continuous light from d 0 till $\mathrm{d} 3$ and $16 \mathrm{~h}$ of light and $8 \mathrm{~h}$ of darkness thereafter. The chickens were raised from $d 1$ to 42 with ad libitum access to feed and water. All chickens obtained the same pelleted available starter diet (MEbroiler $=2,850 \mathrm{kcal} / \mathrm{kg} ; \mathrm{CP}=220 \mathrm{~g} / \mathrm{kg}$; apparent $\mathrm{dLys}=11.81 \mathrm{~g} / \mathrm{kg}$ ) from d 0 until 10 of age, a grower diet (MEbroiler = 2,952 $\mathrm{kcal} / \mathrm{kg} ; \mathrm{CP}=209.7 \mathrm{~g} / \mathrm{kg}$; apparent dLys $=12.52 \mathrm{~g} / \mathrm{kg}$ ) from d 10 until 28 of age, and a finisher diet (MEbroiler =2,999 kcal $/ \mathrm{kg} ; \mathrm{CP}=199.8 \mathrm{~g} / \mathrm{kg}$; apparent dLys $=11.14 \mathrm{~g} / \mathrm{kg}$ ) from d 28 until 42 of age (Reseach Diet Service, Wijk bij Duurstede, The Netherlands).

\section{Data Collection, Sampling and Measurements}

At $d 28,35$ and 39 , the gait score of each individual broiler chicken ( $n=320$ ) was measured, using the gait scoring method, described by Kestin et al. (1992) with a range from 0 (normal locomotion) to 5 (unable to stand). At d 41 and 42, one male chicken per pen per day was selected from mid-hatchers randomly to determine leg bone characteristics (8 chickens per treatment per slaughtering day, 64 chickens in total). After weighing the chicken, food pad dermatitis (FPD) and hock burns (HB) were scored. $\mathrm{HB}$ was scored as 0 (not affected), 1 (colour changes), 2 (minor lesions) or 3 (severe lesions). FPD was scored as 0 (no lesions), 1 (mild lesion) or 2 (severe lesion). Thereafter, chickens were stunned, cut and bleeded. The left leg of each chicken was assessed by a veterinarian on rotated tibia (RT), tibia dyschondroplasia (TD), bacterial chondronecrosis with osteomyelitis (BCO), epiphyseal plate abnormalities (EPA) and epiphysiolysis (EPI). All abnormalities were scored in the range of 0 (no abnormalities), 1 (minor abnormality) or 2 (severe abnormality).

Right legs were deboned and tibias were obtained, packed and frozen at $-20^{\circ} \mathrm{C}$. After thawing, tibia weight, proximal length, lateral cortex thickness and femoral and metatarsal side proximal head thickness were measured, using a digital caliper. Robusticity index was calculated using 
the formula of Reisenfeld (1972):

Robusticity index $(\mathrm{cm} / \mathrm{g})=$ bone proximal length $(\mathrm{cm}) /$ bone weight $(\mathrm{g})$

Tibia osseous volume, tibia pore volume, tibia total volume (osseous volume + pore volume), tibia volume fraction (osseous volume / total volume), tibia mineral content and tibia mineral density were analysed on individual tibia, using a GE Phoenix 3D X-ray microfocus CT scanner (General Electric Company ${ }^{\circledR}$, Boston, Massachusetts, United States) (method described by Bouxsein et al., 2010) (Figure 1).

The same tibias, used for X-ray measures, were subjected to a threepoint bending test (method described by Jungmann et al., 2007), using an Instron ${ }^{\circledR}$ electromechanical universal testing machine (Instron ${ }^{\circledR}$, Norwood, Massachusetts, United States). Ultimate stress (maximal load of breaking point) data was used as the tibia ultimate strength; yield point (reached yield load just before the angle has changed on slope) data was used as the tibia yield strength; the slope of the selected linear part of the curvedata was used as the tibia stiffness; the area under the curve of selected region data was used as the tibia energy to fracture.

Elastic modulus (GPa) (Novitskaya et al., 2017), was calculated using the following formula of Turner and Burr (1993):

$$
E=\frac{N S^{3}}{4 \delta T L^{3}}
$$

where $\mathrm{E}=$ the elastic modulus (GPa), $\mathrm{N}$ is the maximal load $(\mathrm{N}), \mathrm{S}=$ the span between bending fixtures $(\mathrm{mm}), T=$ the tibia thickness $(\mathrm{mm}), L=$ the tibia length $(\mathrm{mm})$, and $\delta=$ the maximum deflection $(\mathrm{mm})$ at the midpoint of the bone. For more details about X-ray and Instron related bone variables, see Güz et al. (2019).

\section{Statistical Analysis}

All statistical analyses were performed in SAS (Version 9.4, July 2013, SAS Institute Inc., Cary, North Carolina, United States). Body weight, all tibia morphological, biophysical, and mechanical characteristics, and gait score were subjected to mixed model analysis, using the PROC MIXED procedure. The statistical model used was: 
$Y=\mu+$ ESTweek $2+$ ESTweek $3+$ ESTweek $2 \times$ ESTweek $3+$ house $+\varepsilon$,

where $Y=$ dependent variable, $\mu=$ overall mean, ESTweek $2=$ egg shell temperature of week $2\left(37.8\right.$ or $\left.38.9^{\circ} \mathrm{C}\right)$, ESTweek $3=$ egg shell temperature of week $3\left(36.7\right.$ or $\left.37.8^{\circ} \mathrm{C}\right)$, ESTweek $2 \times$ ESTweek $3=$ interaction between egg shell temperature of week 2 and week 3 , house = rearing house $(1,2), \varepsilon=$ residual error.

Pen was used as the experimental unit for all analyses. Block in the house was used as a random effect. Body weight at slaughter age was added to the model as a covariable for tibia characteristics. Model assumptions were approved at both means and residuals. Non-normal distributed data were transformed using a log-transformation before analyses. Results are provided as LSmeans \pm SEM. When multiple comparisons were performed, the level of significance was corrected, using Bonferroni.

Leg disorders (RT, TD, HB, FPD, EPA, BCO and EPI) were subjected to generalized linear mixed model analysis, using the PROC GLIMMIX procedure, using the same model. Variables of HB, RT and TD were analysed at binary level (present or not); variables of FPD, EPA, BCO and EPI were analysed at multinomial level. Effects were considered to be significant at $P \leq 0.05$.

Tibia morphological, biophysical, and mechanical characteristics were subjected to correlation analysis, using the PROC CORR procedure, to investigate whether or not a relationship among these characteristics exists.

\section{RESULTS Tibia Morphological Characteristics}

No interaction effects between EST in week 2 and week 3 were found on tibia morphological characteristics. Main effects were found of EST in week 2 on tibia weight, lateral cortex thickness, metatarsal tibia head thickness and robusticity index (Table 1). Chickens of the high EST $\left(38.9^{\circ} \mathrm{C}\right)$ in week 2 had a higher tibia weight $(\Delta=0.68 \mathrm{~g} ; \mathrm{P}<0.001)$, lateral cortex thickness $(\Delta=0.04 \mathrm{~cm} ; \mathrm{P}=0.05)$, metatarsal side proximal tibia head thickness $(\Delta=0.05 \mathrm{~cm} ; \mathrm{P}=0.05)$ and lower robusticity index $(\Delta=0.04 \mathrm{~cm} / \mathrm{g}$; $\mathrm{P}<0.001$ ) compared to the normal EST $\left(37.8^{\circ} \mathrm{C}\right)$ in week 2 . No main effects of EST in week 3 were found on tibia morphological parameters. 


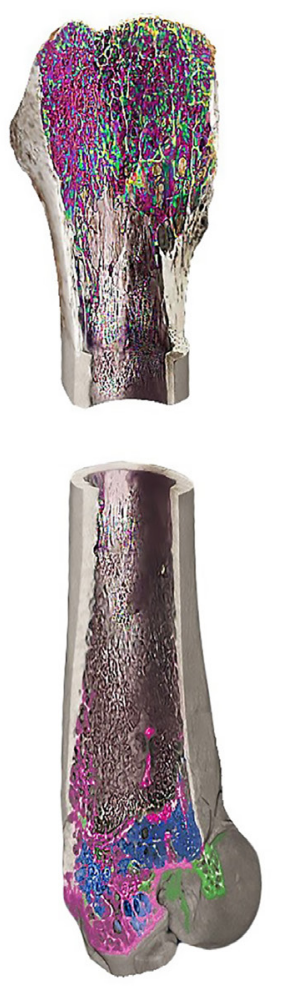

A

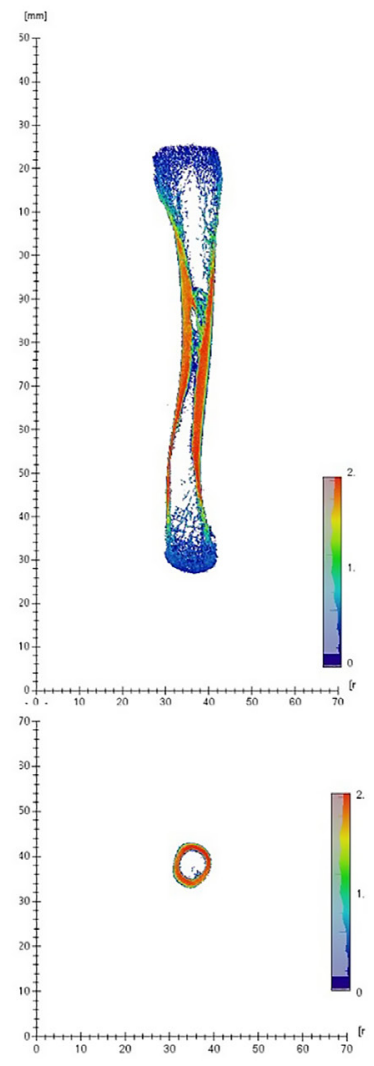

B

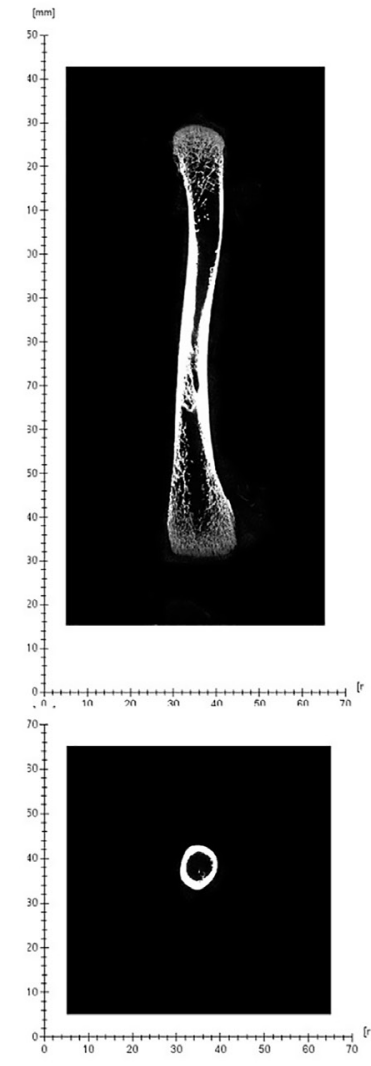

C

Figure 1

Illustration of scanned bone by 3D Micro-CT X-Ray and visualised in Avizo 3D viewer software

A: Three-dimensional tibia inner view scanned by 3D Micro-CT X-Ray scanner. Different colours represent different densities of bone materials and pores

B: Two-dimensional coloured tibia layer view (side) and the layer of middle point of the bone (top). Colour scale represent the mineralisation areas of bone from blue (less mineralisation, 0) to red (more mineralisation, 2)

C: Two dimensional black and white (grey scale) tibia layer view (side) and the layer of middle point of the bone (top). Shades of grey represent the mineralisation areas of bone from dark grey (less mineralisation) to white (more mineralisation). 


\section{Tibia Biophysical Characteristics}

Interaction effects between EST treatments in week 2 and week 3 were found on bone mineral content and tibia mineral density. A normal EST $\left(37.8^{\circ} \mathrm{C}\right)$ in week 2, followed by a normal EST $\left(37.8^{\circ} \mathrm{C}\right)$ in week 3 resulted in a lower tibia mineral content at slaughter age $(\Delta=1.1-1.7 \mathrm{~g}$; $\mathrm{P}=0.001$ ) compared to other three treatment groups. The $37.8 \times 37.8^{\circ} \mathrm{C}$ treatment group (week 2 and 3, respectively) had a lower bone mineral density $\left(\Delta=0.02-0.05 \mathrm{~g} / \mathrm{cm}^{2} ; \mathrm{P}=0.002\right)$ than the $37.8 \times 36.7^{\circ} \mathrm{C}$ and 38.9 $\times 37.8^{\circ} \mathrm{C}$ treatment groups, with the $38.9 \times 36.7^{\circ} \mathrm{C}$ treatment group in between and not different from the other three groups.

Main effects of EST in week 2 were found on osseous volume, pore volume, and total volume. Chickens of the high EST $\left(38.9^{\circ} \mathrm{C}\right)$ treatment group in week 2 had a higher osseous volume $\left(\Delta=5.0 \mathrm{~cm}^{3} ; P<0.001\right)$, pore volume $\left(\Delta=0.7 \mathrm{~cm}^{3} ; \mathrm{P}=0.05\right)$ and total volume $\left(\Delta=5.9 \mathrm{~cm}^{3} ; \mathrm{P}<0.001\right) \mathrm{com}$ pared to the normal EST $\left(37.8^{\circ} \mathrm{C}\right)$ treatment group. Chickens incubated at a normal EST $\left(37.8^{\circ} \mathrm{C}\right)$ in week 3 had a higher volume fraction $(\Delta=2 \%$; $\mathrm{P}=0.008)$ compared to the lower EST $\left(36.7^{\circ} \mathrm{C}\right.$ ) group (Table 2$)$.

\section{Tibia Mechanical Characteristics}

Interaction effects between EST in week 2 and week 3 were found on ultimate strength, yield strength, and stiffness (Table 3). A high EST $\left(38.9^{\circ} \mathrm{C}\right)$ in week 2 followed by a normal EST $\left(37.8^{\circ} \mathrm{C}\right)$ in week 3 resulted in a higher bone ultimate strength $(\Delta=23.2-29.0 \mathrm{~N}$; $P=0.04)$, yield strength $(\Delta=21.3-26.5$ $\mathrm{N} ; \mathrm{P}=0.03)$, and stiffness ( $\Delta=22.9-29.0 \mathrm{~N} / \mathrm{mm} ; \mathrm{P}=0.05)$ at slaughter age than the other three EST treatment groups. Main effects were found for EST in week 2 on energy to fracture and elastic modulus.

Chickens of the high EST $\left(38.9^{\circ} \mathrm{C}\right)$ treatment group had a higher energy to fracture $(\Delta=16.4 \mathrm{~N}-\mathrm{mm} ; \mathrm{P}<0.001$ ) and elastic modulus ( $\triangle=0.4 \mathrm{GPa}$; $\mathrm{P}=0.02)$ compared to the normal EST $\left(37.8^{\circ} \mathrm{C}\right)$ treatment group in week 2. Looking at the main effects in week 3 , chickens incubated at a normal EST $\left(37.8^{\circ} \mathrm{C}\right)$ in week 3 had a higher energy to fracture $(\Delta=12.9 \mathrm{~N}-\mathrm{mm} ; \mathrm{P}=0.03)$ than chickens incubated at a lower EST $\left(36.7^{\circ} \mathrm{C}\right)$ (Table 3). 


\section{Relationship Between Tibia Morphological, Biophysical and Mechanical Characteristics}

Correlation analysis between tibial morphological, biophysical and mechanical characteristics showed the strongest relationships between tibia weight, length, thickness, ultimate strength, stiffness and volume (Table 4). Tibia weight was positively correlated to length ( $r=0.262 ; P=0.03$ ), thickness ( $r=0.306 ; P=0.01)$, ultimate strength ( $r=0.425 ; P=0.005)$, stiffness $(r=0.404 ; P=0.009)$ and osseous volume $(r=0.548 ; P<0.001)$. Tibia length was positively correlated to tibia thickness $(r=0.669 ; P<0.001)$, ultimate strength $(r=0.535 ; P<0.001)$ and stiffness $(r=0.584 ; P<0.001)$. Tibia thickness was positively correlated to ultimate strength $(r=0.658 ; P<0.001)$ and stiffness $(r=0.651 ; P<0.001)$. Ultimate strength was positively correlated to stiffness $(r=0.995 ; P<0.001)$ and osseous volume $(r=0.395 ; P=0.02)$. Stiffness was positively correlated to osseous volume $(r=0.389 ; P=0.005)$.

\section{Body Weight, Locomotion Related Observations and Leg Disorders}

Even if the group of higher EST $\left(38.9^{\circ} \mathrm{C}\right)$ in week 2 and also the same group when interacted with a normal EST $\left(37.8^{\circ} \mathrm{C}\right)$ in week 3 resulted in higher body weight, no interaction between EST in week 2 and week 3 of incubation ( $\mathrm{P}=0.38$ ) or a main effect of EST in week $2(\mathrm{P}=0.23)$ or week 3 $(P=0.90)$ were found on $B W$ of selected chickens at slaughter age (Table 5). Main effects were found of EST in week 2 on gait score at d 39 (Table 5). Chickens of the high EST $\left(38.9^{\circ} \mathrm{C}\right)$ in week 2 of incubation had a lower (better) gait score compared to the normal EST $\left(37.8^{\circ} \mathrm{C}\right)$. No interaction effects between EST in week 2 and week 3, nor a main effect of EST in week 2 or week 3 were found on gait score at d 28 or 35 (Table 5) or FDP, $H B, T D, B C O$, EPA and EPI at slaughter age (Table 6). 


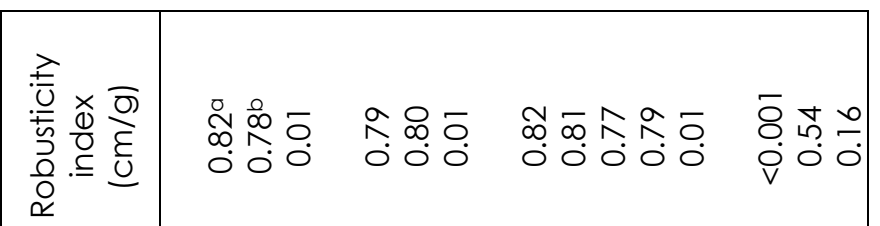




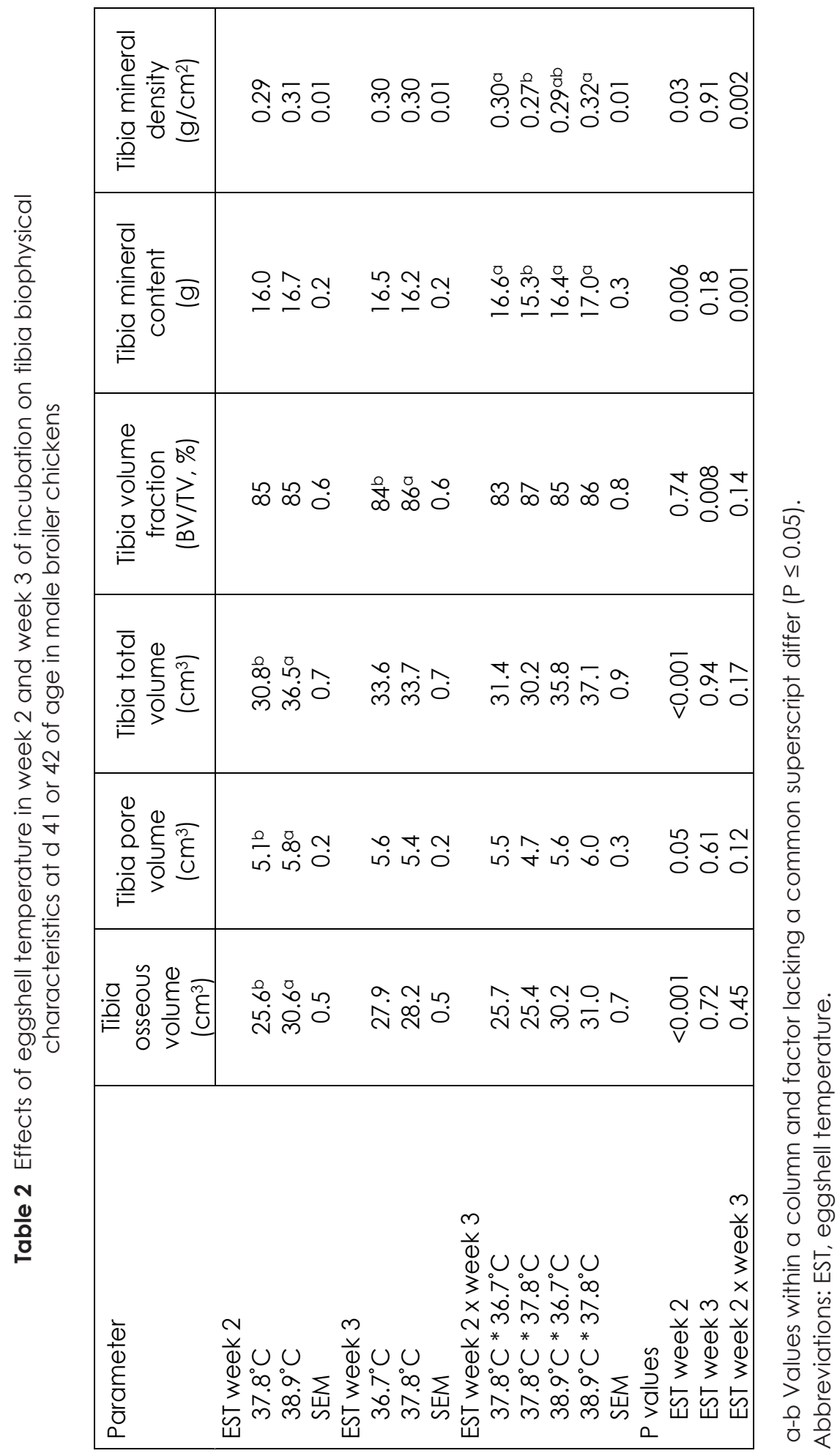




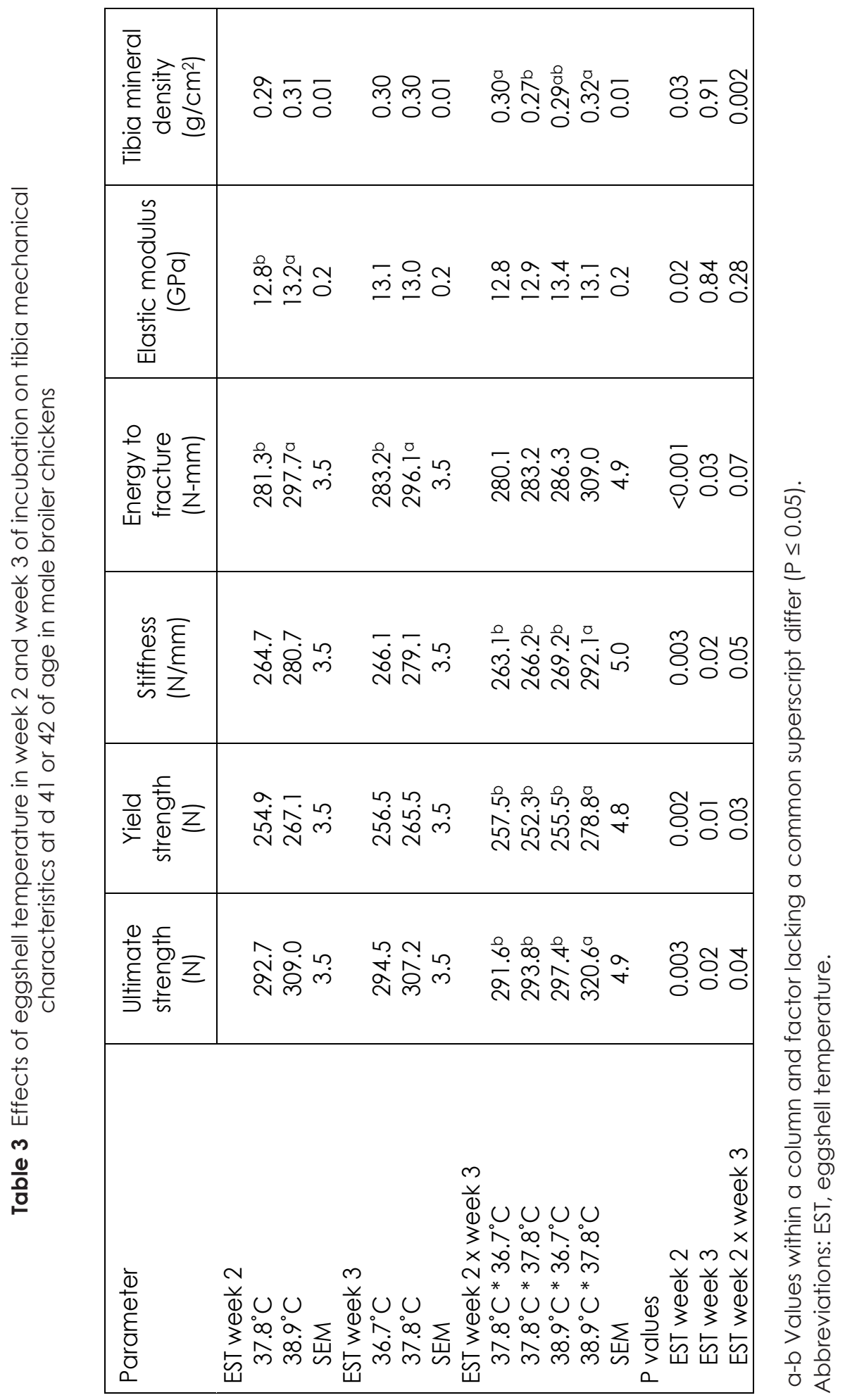


Table 4 Spearman correlation coefficients between tibia morphological, biophysical, and mechanical characteristicslat d 41 or 42 of age in male broiler chickens

\begin{tabular}{|c|c|c|c|c|c|}
\hline Variable\# & $\begin{array}{l}\text { Weight } \\
\text { (g) }\end{array}$ & $\begin{array}{l}\text { Length } \\
\text { (cm) }\end{array}$ & $\begin{array}{l}\text { Thickness } \\
(\mathrm{cm})\end{array}$ & $\begin{array}{c}\text { Ultimate } \\
\text { strength } \\
\text { (N) }\end{array}$ & $\begin{array}{l}\text { Stiffness } \\
(\mathrm{N} / \mathrm{mm})\end{array}$ \\
\hline Weight (g) & - & & & & \\
\hline Length $(\mathrm{cm})$ & $0.262^{* *}$ & - & & & \\
\hline Thickness (cm) & $0.306^{* *}$ & $0.669^{*}$ & - & & \\
\hline $\begin{array}{l}\text { Ultimate } \\
\text { strength (N) }\end{array}$ & $0.425^{*}$ & $0.535^{*}$ & $0.658^{*}$ & - & \\
\hline $\begin{array}{l}\text { Stiffness } \\
(\mathrm{N} / \mathrm{mm})\end{array}$ & $0.404^{*}$ & $0.584^{*}$ & $0.651^{*}$ & $0.995^{*}$ & - \\
\hline $\begin{array}{l}\text { Osseous } \\
\text { volume }\left(\mathrm{cm}^{3}\right)\end{array}$ & $0.548^{*}$ & 0.039 & 0.081 & $0.395^{* *}$ & $0.389^{*}$ \\
\hline
\end{tabular}

1 Significant coefficients are indicated with asterisks, ${ }^{*} P<0.01$ and ${ }^{* *} P<0.05$.

\# A total of 64 bones from 64 chickens were used.

Table 5 Effects of eggshell temperature in week 2 and week 3 of incubation on body weight (BW) at day 41 or 42 of age, gait score 1 at day 28,35 and 39 of age in male broiler chickens

\begin{tabular}{|l|c|c|c|c|}
\hline Parameter & $\begin{array}{c}\text { BW } \\
\text { (g) }\end{array}$ & $\begin{array}{c}\text { Gait score at } \\
\text { d } 28\end{array}$ & $\begin{array}{c}\text { Gait score at } \\
\text { d } 35\end{array}$ & $\begin{array}{c}\text { Gait score at } \\
\text { d } 39\end{array}$ \\
\cline { 2 - 5 } $37.8^{\circ} \mathrm{C}$ & 3469 & 1.9 & 2.1 & $2.4^{\mathrm{b}}$ \\
$38.9^{\circ} \mathrm{C}$ & 3558 & 1.8 & 2.1 & $2.3 \mathrm{a}$ \\
SEM & 51 & 0.02 & 0.03 & 0.04 \\
EST week 3 & 3508 & 1.7 & & \\
$36.7^{\circ} \mathrm{C}$ & 3518 & 1.8 & 2.1 & 2.3 \\
$37.8^{\circ} \mathrm{C}$ & 51 & 0.02 & 0.02 & 2.2 \\
SEM & & & & 0.04 \\
EST week 2x week 3 & 3496 & 1.9 & 2.0 & 2.4 \\
$37.8^{\circ} \mathrm{C} * 36.7^{\circ} \mathrm{C}$ & 3442 & 2.0 & 2.1 & 2.3 \\
$37.8^{\circ} \mathrm{C} * 37.8^{\circ} \mathrm{C}$ & 3521 & 1.8 & 2.1 & 2.3 \\
$38.9^{\circ} \mathrm{C} * 36.7^{\circ} \mathrm{C}$ & 3594 & 1.9 & 2.1 & 2.3 \\
$38.9^{\circ} \mathrm{C} * 37.8^{\circ} \mathrm{C}$ & 72 & 0.03 & 0.02 & 0.04 \\
SEM & & & & \\
P values & 0.23 & 0.12 & 0.10 & 0.04 \\
EST week 2 & 0.90 & 0.63 & 0.12 & 0.81 \\
EST week 3 & 0.38 & 0.30 & 0.32 & 0.31 \\
EST week 2x week 3 & & & & \\
\hline
\end{tabular}

1 Method of Kestin et al. (1992), scored within a range of 0 (normal locomotion) to 5 (unable to stand). Abbreviations: EST, eggshell temperature. 


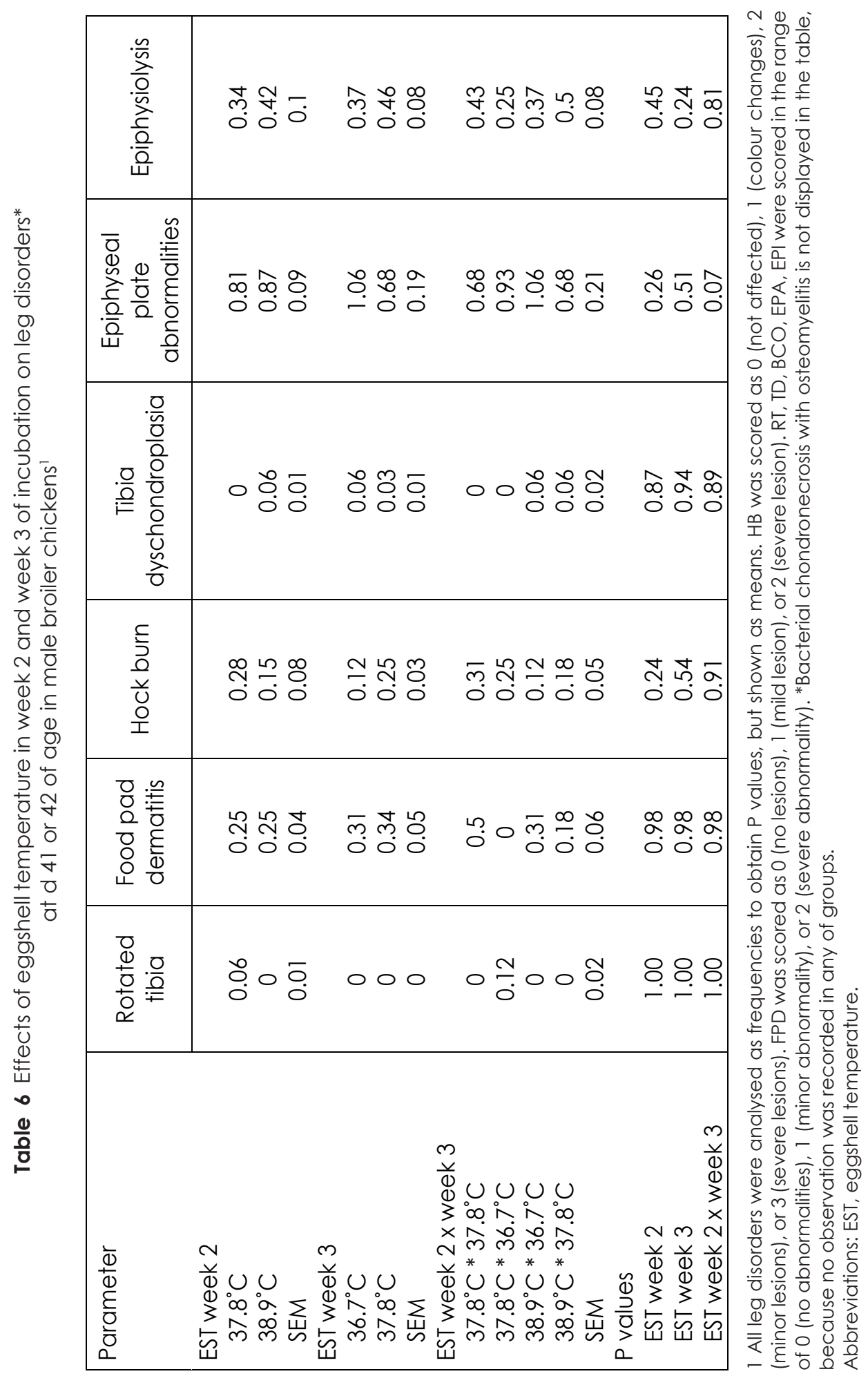




\section{DISCUSSION}

Tibia morphological, biophysical and mechanical characteristics, as assessed in the current experiment, are considered as the most important indicators of bone quality and are interconnected to each other (Leblanc et al., 1986; Rath et al., 2000; Onyango et al., 2003; Krupski and Tatara, 2007; Shim et al., 2012; Charuta et al., 2013). Calcium mobilization, mineralization and ossification of tibia of the chicken embryo begin between E7 and E8 of incubation (Holder, 1978; Blom and Lilja, 2005; Oviedo-Rondón et al., 2008a; Van der Pol et al., 2019). Fast growing broiler chickens have been shown to have lower tibia mineralisation compared to slower growing broilers and other birds and mammals, mostly because of fast growth rate, and consequently they have lower bone volume, mineral content and mineral density (Lilburn, 1994; Velleman, 2000; Bonser and Casinos, 2003). Tibia mineralisation and ossification can be affected by environmental factors (such as incubation temperature) from E7 onward. The rate of mineralisation in the tibia has been shown to be affected by incubation temperature, due to interferences of thyroid hormones, insulin-like growth factor 1 and growth hormone (Rommel et al., 2001; Robson et al., 2002), which all play critical roles in growth plate chondrocyte differentiation (shao et al., 2006). Moreover, several studies have shown that melatonin can promote the osteoblast differentiation and bone formation and thus plays a role in regulating bone growth (Roth et al., 1999; Koyama et al., 2002). Melatonin levels, in turn, can also be affected by the temperature during incubation (Faluhelyi et al., 2009). Rate of osteoblast division in the tibia has been positively affected by higher incubation temperature compared to normal (Robson et al., 2002). Van der Eerden et al. (2003) also indicated that biochemical mechanisms that control endochondral ossification of long bones can be affected by incubation temperatures, mainly during the plateau stage of embryonic development, from the second week of incubation until hatch.

\section{Effects of Incubation Temperature in Week 2}

In the current study, most of the tibia morphological characteristics were positively affected by a higher temperature in the second week of incubation. These results are in agreement with previous research, which indicated that bone mineralisation and ossification are stimulated by a 
higher temperature during the second week of incubation (Rommel et al., 2001; Robson et al., 2002; Shao et al., 2006; Faluhelyi et al., 2009). This is probably due to stimulation of mineralisation, growth plate formation and chondrocyte differentiation by a higher incubation temperature (Rommel et al., 2001; Robson et al., 2002; Shao et al., 2006; Faluhelyi et al., 2009).

One of the first studies on incubation temperature and bone morphological characteristics showed that an increase in incubation temperature from $35^{\circ} \mathrm{C}$ to $40^{\circ} \mathrm{C}$ in steps of $1^{\circ} \mathrm{C}$, during the first $10 \mathrm{~d}$ of incubation resulted in higher tibia weight and length at E10 (Brookes and May, 1972). Particularly, tibia weight appeared to be stimulated by a higher EST in week 2 of incubation, as reflected in a lower robusticity index, which suggests a stronger bone structure (Reisenfeld, 1972). Aygun and Narinc (2016) showed that a high temperature $\left(41^{\circ} \mathrm{C}\right)$ for $3 \mathrm{~h}$ per day during the second week of incubation stimulated tibia morphological development, resulting in longer and thicker tibia.

With respect to leg disorders and gait score, fast-growing broiler chickens in general show low locomotion activities and high (worse) gait scores (Corr et al., 2003; Bessei, 2006; Kittelsen et al., 2017). Chickens reared at higher stocking densities may have poorer gait scores and higher prevalence of leg disorders (Bradshaw et al., 2002) due to less opportunities of locomotion. In the current study, chickens of the high EST $\left(38.9^{\circ} \mathrm{C}\right)$ in week 2 had lower (better) gait scores at d 39 than chicken incubated at the normal EST $\left(37.8^{\circ} \mathrm{C}\right)$ in week 2 , which might be explained by the better tibia characteristics found in the same treatment group. However, no effect was found on the prevalence of leg disorders, which might be due to the non-commercial setup of the experiment with small pens and low stocking density (Oviedo-Rondon et al., 2009) and it is known that the gait score is not always correlated with leg abnormalities (sandilands et al., 2011; Fernandes et al., 2012).

\section{Effects of Incubation Temperature in Week 3}

Regarding the third week of incubation, in the current study, lowering the temperature by $1.1^{\circ} \mathrm{C}$ than normal in week 3 of incubation did not affect morphological tibia characteristics at all. Only a negative effect of a lower EST during week 3 of incubation was found on tibia bone fraction and energy to fracture. In the last week of incubation, embryos become 
very sensitive to incubation temperature due to high metabolism and consequently high heat production (Hulet, 2007; Willemsen et al., 2017). During this week, bone development is also known to reach the highest growth rate compared to previous weeks of incubation. Literature related to temperature in the last week of incubation and bone development demonstrates similar results. Yalçin et al. (2007) concluded that both low $\left(36.9^{\circ} \mathrm{C}\right)$ or high $\left(39.6^{\circ} \mathrm{C}\right)$ incubator temperatures from incubation day E10 until E18 resulted in lower tibia weights at slaughter age compared a control temperature $\left(37.8^{\circ} \mathrm{C}\right)$. Oviedo-Rondon et al. (2009) found comparable results at slaughter age when applying a high EST $\left(38.9^{\circ} \mathrm{C}\right)$ during the last $4 \mathrm{~d}$ of incubation compared to an EST of $36.9^{\circ} \mathrm{C}$.

Based on the results of the current study, it appears that a lower EST $\left(36.7^{\circ} \mathrm{C}\right)$ in the last week of incubation does not have a positive effect on bone development at slaughter age. Maintaining the EST at $37.8^{\circ} \mathrm{C}$ during the last week of incubation may result in a more optimal bone development compared to a lower EST $\left(36.7^{\circ} \mathrm{C}\right)$.

\section{Interactions on Effects of Incubation Temperature Between Week 2 and Week 3}

In the current study, an interaction between EST in week 2 and week 3 of incubation was found for a number of tibia characteristics. However, not all results were in the same direction, which was possibly related to developmental and physiological differences between cortical and trabecular parts of the tibia during the second and third week of incubation (Wineland et al, 2006; Shao et al., 2006). For tibia morphological characteristics, a tendency for an interaction was found for tibia proximal length and femoral side head thickness, indicating that an incubation temperature of $38.9^{\circ} \mathrm{C}$ in week 2 of incubation stimulated tibia dimensions, particularly when EST in week 3 was normal $\left(37.8^{\circ} \mathrm{C}\right)$. This is comparable with the interaction effects for mechanical characteristics, in which ultimate strength, yield strength and stiffness showed the highest values in the $38.9 \times 37.8^{\circ} \mathrm{C}$ group. Regarding tibia biophysical characteristics, an interaction was also found between incubation temperature in week 2 and week 3 in tibia mineral content and tibia mineral density and again the $38.9 \times 37.8^{\circ} \mathrm{C}$ group showed the highest values. 
Interactions as seen in the current study might be explained by the stimulating effect of a higher EST on mineralisation and ossification rate. With a higher EST in week 2 combined with a normal EST in week 3 the highest mineralisation and ossification rate can be expected, resulting in higher mineral content, density, thickness and breaking strength. All these parameters are strongly related to each other (Applegate and Lilburn, 2002; Yair et al., 2012). Based on the lowest values of the $37.8 \times 37.8^{\circ} \mathrm{C}$ treatment group, it can be suggested that higher EST $\left(38.9^{\circ} \mathrm{C}\right)$ in week 2 of incubation is highly important for bone mineralisation and ossification.

In conclusion, a temperature of $38.9^{\circ} \mathrm{C}$ during the second week of incubation stimulates morphological, biophysical and mechanical tibia characteristics of fast-growing broiler chickens at slaughter age compared to a temperature of $37.8^{\circ} \mathrm{C}$. Incubation temperature in the third week appears to interact with the incubation temperature in the second week, resulting in a most advanced tibia development after incubation at $38.9^{\circ} \mathrm{C}$ in week 2 , followed by $37.8^{\circ} \mathrm{C}$ in week 3 of incubation. However, this stimulated bone development due to incubation temperatures did not affect leg disorders in later life.

\section{ACKNOWLEDGEMENTS}

This experiment was the part of the 'Healthy Bones' project, financed by a public-private partnership (project number BO-47001-011). The financial support of the Ministry of Agriculture, Nature, and Food Quality of The Netherlands, Aviagen EPI, Darling Ingredients Inc., ForFarmers, Hubbard, Marel Stork Poultry Processing BV, Nepluvi and Nutreco is gratefully acknowledged. The authors would like to thank Lagerwey hatchery (Lunteren, The Netherlands) for providing eggs; Remco Hamoen for his precious experience in 3D micro CT X-ray scanner. Stefan Veenstra, Henny Reimert, Carla van der Pol, Marcel Heetkamp, Jan Wijnen, Ilona van der Anker-Hensen, Bjorge Laurenssen and the animal caretakers are acknowledged for their help during the experiment. 


\section{REFERENCES}

Applegate, T. J. and M. S. Lilburn. 2002. Growth of the femur and tibia of a commercial broiler line. Poultry science. 81:1289-1294.

Atalgin, S. H. and I. Kürtül. 2009. A morphological study of skeletal development in turkey during the pre-hatching stage. Anatomia, Histologia, Embryologia. 38:23-30.

Aygun, A. and D. Narinc. 2016. The effects of thermal manipulations during embryogenesis of broiler chicks on growth of embryo and skeletal traits. In AIP Conference Proceedings (Vol. 1726, No. 1:020015).

Ballock, R. T. and R. J. O'keefe. 2003. The biology of the growth plate. The journal of bone and joint surgery. 85:715-726.

Bessei, W. 2006. Welfare of broilers: a review. World poultry science journal. 62:455-466.

Blom, J. and C. Lilja. 2005. A comparative study of embryonic development of some bird species with different patterns of postnatal growth. Zoology. 108:81-95.

Bonser, R. H. C. and A. Casinos. 2003. Regional variation in cortical bone properties from broiler fowl-a first look. British poultry science. 44:350-354.

Bradshaw, R. H., R. D Kirkden, and D.M. Broom. 2002. A review of the aetiology and pathology of leg weakness in broilers in relation to welfare. Avian and poultry biology reviews. 13:45-103.

Brookes, M. U. and K. U. May. 1972. The influence of temperature on bone growth in the chick. The journal of anatomy. 111:351-363.

Bouxsein, M. L., S. K. Boyd, B. A. Christiansen, R. E. Guldberg, K. J. Jepsen, and R. Müller. 2010. Guidelines for assessment of bone microstructure in rodents using micro-computed tomography. Journal of bone and mineral research. 25:1468-1486.

Charuta, A., M. Dzierzęcka, M. Komosa, L. Kalinowski, and M. Pierzchala. 2013. Age-and sex-related differences of morphometric, densitometric and geometric parameters of tibiotarsal bone in Ross broiler chickens. Folia Biologica. 61:211-220.

Corr, S. A., M. J. Gentle, C. C. McCorquodale, and D. Bennett. 2003. The effect of morphology on walking ability in the modern broiler: a gait analysis study. Animal welfare. 12:159-171.

Decuypere, E. 1994. Incubation temperature and postnatal development. In Proceedings of 9th European Poultry Conference (Vol. 2:407-410).

Faluhelyi, N., A. Matkovits, A. Párniczky, and V. Csernus. 2009. The in vitro and in ovo effects of environmental illumination and temperature on the melatonin secretion from the embryonic chicken pineal gland. Annals of the new york academy of sciences. 1163:383-385.

Fernandes, B. C. D. S., M. R. F. B Martins, A. A. Mendes, I. C. D. L. A Paz, C. M. Komiyama, E. L. Milbradt, and B. B. Martins. 2012. Locomotion problems of broiler chickens and its relationship with the gait score. Revista brasileira de zootecnia. 41:1951-1955.

French, N. A. 1997. Modelling incubation temperature: the effects of incubator design, embryonic development, and egg size. Poultry science. 76:124-133.

Gocsik, É., A. M. Silvera, H. Hansson, H. W. Saatkamp, and H. J. Blokhuis. 2017. Exploring the economic potential of reducing broiler lameness. British poultry science. 58:337-347.

Jungmann, R., G. Schitter, G. E. Fantner, M. E. Laver, P. K. Hansma, and P. J. Thurner. 2007. Real-time microdamage and strain detection during micromechanical testing of single trabeculae. In Exp. Appl. Mech.: SEM Annual Conference and Exposition 2007 (3 Vols). Curran Associates. p1 1.

Hammond, C. L., B. H. Simbi, and N. C. Stickland. 2007. In ovo temperature manipulation influences embryonic motility and growth of limb tissues in the chick (Gallus gallus). Journal of experimental biology. 210:2667-2675.

Holder, N. 1978. The onset of osteogenesis in the developing chick limb. Development. 44:1529.

Hulet, R. M. 2007. Symposium: Managing the embryo for performance managing incubation: Where are we and why? Poultry science. 86:1017-1019.

Kestin, S. C., T. G. Knowles, A. F. Tinch, and N. G. Gregory. 1992. The prevalence of leg weakness in broiler chickens and its relationship with genotype. Veterinary Record. 131:190-194. 
Kestin, S. C., G. SU, and P. Sørensen. 1999. Different commercial broiler crosses have different susceptibilities to leg weakness. Poultry science. 78:1085-1090.

Kestin, S. C., S. Gordon, G. Su, and P. Sørensen. 2001. Relationships in broiler chickens between lameness, live weight, growth rate and age. Veterinary Record. 148:195-197.

Kittelsen, K. E., B. David, R. O. Moe, H. D. Poulsen, J. F. Young, and E. G. Granquist. 2017. Associations among gait score, production data, abattoir registrations, and postmortem tibia measurements in broiler chickens. Poultry science. 96:1033-1040.

Koyama, H., O. Nakade, Y. Takada, T. Kaku, and K. H. W Lau. 2002. Melatonin at pharmacologic doses increases bone mass by suppressing resorption through down-regulation of the RANKL-mediated osteoclast formation and activation. Journal of bone and mineral research. 17:1219-1229.

Krupski, W. and M. R. Tatara. 2007. Interrelationships between densitometric, morphometric, and mechanical properties of the tibia in turkeys. Bulletin of the veterinary institute in pulawy. 51:621-626.

Leblanc, B., M. Wyers, F. Cohn-Bendit, J. M. Legall, E. Thibault, and J. M. Florent. 1986. Histology and histomorphometry of the tibia growth in two turkey strains. Poultry science. 65:1787-1795.

Lilburn, M. S. 1994. Skeletal growth of commercial poultry species. Poultry science. 73:897-903.

Lourens, A., H. van den Brand, R. Meijerhof, and B. Kemp. 2005. Effect of eggshell temperature during incubation on embryo development, hatchability, and posthatch development. Poultry science. 84(6):914-920.

Meijerhof, R. and G. van Beek. 1993. Mathematical modelling of temperature and moisture loss of hatching eggs. Journal of theoretical biology. 165:27-41.

Mench, J. 2004. Lameness. Pages 3-17 in Measuring and auditing broiler welfare. C. A. Weeks, and A. Butterworth, ed. CABI, Wallingford, UK.

Nakane, Y. and M. Tsudzuki. 1999. Development of the skeleton in Japanese quail embryos. Development, growth \& differentiation. 41:523-534.

Novitskaya, E., P.Y. Chen, E. Hamed, J. Li, V. A. Lubarda, I. Jasiuk, and J. McKittrick. 2011. Recent advances on the measurement and calculation of the elastic moduli of cortical and trabecular bone: a review. Theoretical and applied mechanics. 38: 209-297.

Onyango, E. M., P. Y. Hester, R. Stroshine, and O. Adeola. 2003. Bone densitometry as an indicator of percentage tibia ash in broiler chicks fed varying dietary calcium and phosphorus levels. Poultry science. 82:1787-1791.

Oviedo-Rondón, E.O., J. Small, M. J. Wineland, V. L. Christensen, J. L. Grimes, S. V. L. Funderburk, D. T. Ort, and K. M. Mann. 2008. Effects of incubator temperature and oxygen concentration during the plateau stage of oxygen consumption on turkey embryo long bone development. Poultry science. 87:1484-1492.

Oviedo-Rondón, E.O., J. Small, M. J. Wineland, V. L. Christensen, P. S. Mozdziak, M. D. Koci, S. V. L. Funderburk, D. T. Ort, and K. M. Mann. 2008. Broiler embryo bone development is influenced by incubator temperature, oxygen concentration and eggshell conductance at the plateau stage in oxygen consumption. British poultry science. 49:666-676.

Oviedo-Rondón, E.O., M. J. Wineland, J. Small, H. Cutchin, A. McElroy, A. Barri, and S. Martin. 2009. Effect of incubation temperatures and chick transportation conditions on bone development and leg health. Journal of applied poultry research. 18:671-678.

Oznurlu, Y., E. Sur, T. Ozaydin, I. Celik, and D. Uluisik. 2016. Histological and histochemical evaluations on the effects of high incubation temperature on the embryonic development of tibial growth plate in broiler chickens. Microscopy research and technique. 79:106-110.

Rath, N. C., G. R. Huff, W. E. Huff, and J. M. Balog. 2000. Factors regulating bone maturity and strength in poultry. Poultry science. 79:1024-1032.

Riesenfeld, A. 1972. Metatarsal robusticity in bipedal rats. American journal of physical anthropology. 36:229-233.

Robson, H., T. Siebler, S. M. Shalet, and G. R. Williams. 2002. Interactions between GH, IGF-I, glucocorticoids, and thyroid hormones during skeletal growth. Pediatric research. 52:137-142.

Rommel, C., S. C. Bodine, B. A. Clarke, R. Rossman, L. Nunez, T. N. Stitt, G. D. Yancopoulos, and D. J. Glass. 2001. Mediation of IGF-1-induced skeletal myotube hypertrophy by PI (3) K/Akt/mTOR and PI (3) K/Akt/GSK3 pathways. Nature cell biology. 3:1009-1013. 
Roth, J. A., B. G. Kim, W. L. Lin, and M. I. Cho. 1999. Melatonin promotes osteoblast differentiation and bone formation. Journal of biological chemistry. 274:22041-22047.

Sandilands, V., S. Brocklehurst, N. Sparks, L. Baker, R. McGovern, B. Thorp, and D. Pearson. 2011 . Assessing leg health in chickens using a force plate and gait scoring: how many birds is enough? Veterinary record. 168:77-83.

Shao, Y. Y., L. Wang, and R. T. Ballock. 2006. Thyroid hormone and the growth plate. Reviews in endocrine and metabolic disorders. 7:265-271.

Sherlock, L., T. G. M. Demmers, A. E. Goodship, I. D. McCarthy, and C. M. Wathes. 2010. The relationship between physical activity and leg health in the broiler chicken. British poultry science. 51:22-30.

Shim, M. Y., A. B. Karnuah, A. D. Mitchell, N. B. Anthony, G. M. Pesti, and S. E. Aggrey, S. E. 2012. The effects of growth rate on leg morphology and tibia breaking strength, mineral density, mineral content, and bone ash in broilers. Poultry science. 91:1790-1795.

Shim, M.Y. and G. M. Pesti. 2011. Effects of incubation temperature on the bone development of broilers. Poultry science. 90:1867-1877.

Tona, K., V. Bruggeman, O. Onagbesan, F. Bamelis, M. Gbeassor, K. Mertens, and E. Decuypere. 2005. Day-old chick quality: Relationship to hatching egg quality, adequate incubation practice and prediction of broiler performance. Avian and poultry biology reviews. 16:19.

Turner, C. H., and D. B. Burr. 1993. Basic biomechanical measurements of bone: a tutorial. Bone. 14:595-608.

Van der Eerden, B. C. J., M. Karperien, and J. M. Wit. 2003. Systemic and local regulation of the growth plate. Endocrine reviews. 24:782-801.

Van der Pol, C. W., I. A. M. van Roovert-Reijink, C. M. Maatjens, I. van den Anker, B. Kemp, and $\mathrm{H}$. van den Brand. 2014. Effect of eggshell temperature throughout incubation on broiler hatchling leg bone development. Poultry science. 93:2878-2883.

Van der Pol, C. W., I. A. M. van Roovert-Reijrink, C. M. Maatjens, S. W. Gussekloo, S. Kranenbarg, J. Wijnen, R. P. Pieters, H. Schipper, B. Kemp, and H. van den Brand. 2019. Lightdark rhythms during incubation of broiler chicken embryos and their effects on embryonic and post hatch leg bone development. PloS one. 14:0210886.

Velleman, S. G. 2000. The role of the extracellular matrix in skeletal development. Poultry science. 79:985-989.

Willemsen, H., Y. Li, E. Willems, L. Franssens, Y. Wang, E. Decuypere, and N. Everaert. 2011. Intermittent thermal manipulations of broiler embryos during late incubation and their immediate effect on the embryonic development and hatching process. Poultry science. 90:1302-1312.

Wilson, H. R. 1990. Physiological requirements of the developing embryo: temperature and turning. Avian incubation. 145-156.

Wineland, M. W., V. L. Christensen, I. Yildrum, B. D. Fairchild, K. M. Mann, and D. T. Ort. 2006. Incubator temperature and oxygen concentration at the plateau stage in oxygen consumption affects intestinal maturation of broiler chicks. International journal of poultry science. 5:229-240.

Yair, R., Z. Uni, and R. Shahar. 2012. Bone characteristics of late-term embryonic and hatchling broilers: Bone development under extreme growth rate. Poultry science. 91:2614-2620.

Yalçin, S., H. B. Malayoglu, M. Baka, O. Genin, and M. Pines. 2007. Effect of temperature during the incubation period on tibial growth plate chondrocyte differentiation and the incidence of tibial dyschondroplasia. Poultry science. 86:1772-1783.

Wilson, H. R. 1990. Physiological requirements of the developing embryo: temperature and turning. Avian incubation. 145-156.

Wineland, M. W., V. L. Christensen, I. Yildrum, B. D. Fairchild, K. M. Mann, and D. T. Ort. 2006. Incubator temperature and oxygen concentration at the plateau stage in oxygen consumption affects intestinal maturation of broiler chicks. International journal of poultry science. 5:229-240.

Yair, R., Z. Uni, and R. Shahar. 2012. Bone characteristics of embryonic and hatchling broilers: Bone development under extreme growth rate. Poultry science. 91:2614-2620.

Yalçin, S., H. B. Malayoglu, M. Baka, O. Genin, and M. Pines. 2007. Effect of temperature during the incubation period on tibial growth plate chondrocyte differentiation and the incidence of tibial dyschondroplasia. Poultry science. 86:1772-1783. 
B. C. Güz'

I. C. de Jong ${ }^{2}$

C. S. da Silva ${ }^{2}$

F. Veldkamp'

B. Kemp'

R. Molenaar ${ }^{1}$

H. van den Brand'

Submitted

1 Adaptation Physiology Group,

Wageningen University and Research, 6708 PB

Wageningen,

Gelderland,

The Netherlands

2 Wageningen Livestock Research,

Wageningen University and Research, 6708 WD

Wageningen,

Gelderland,

The Netherlands 


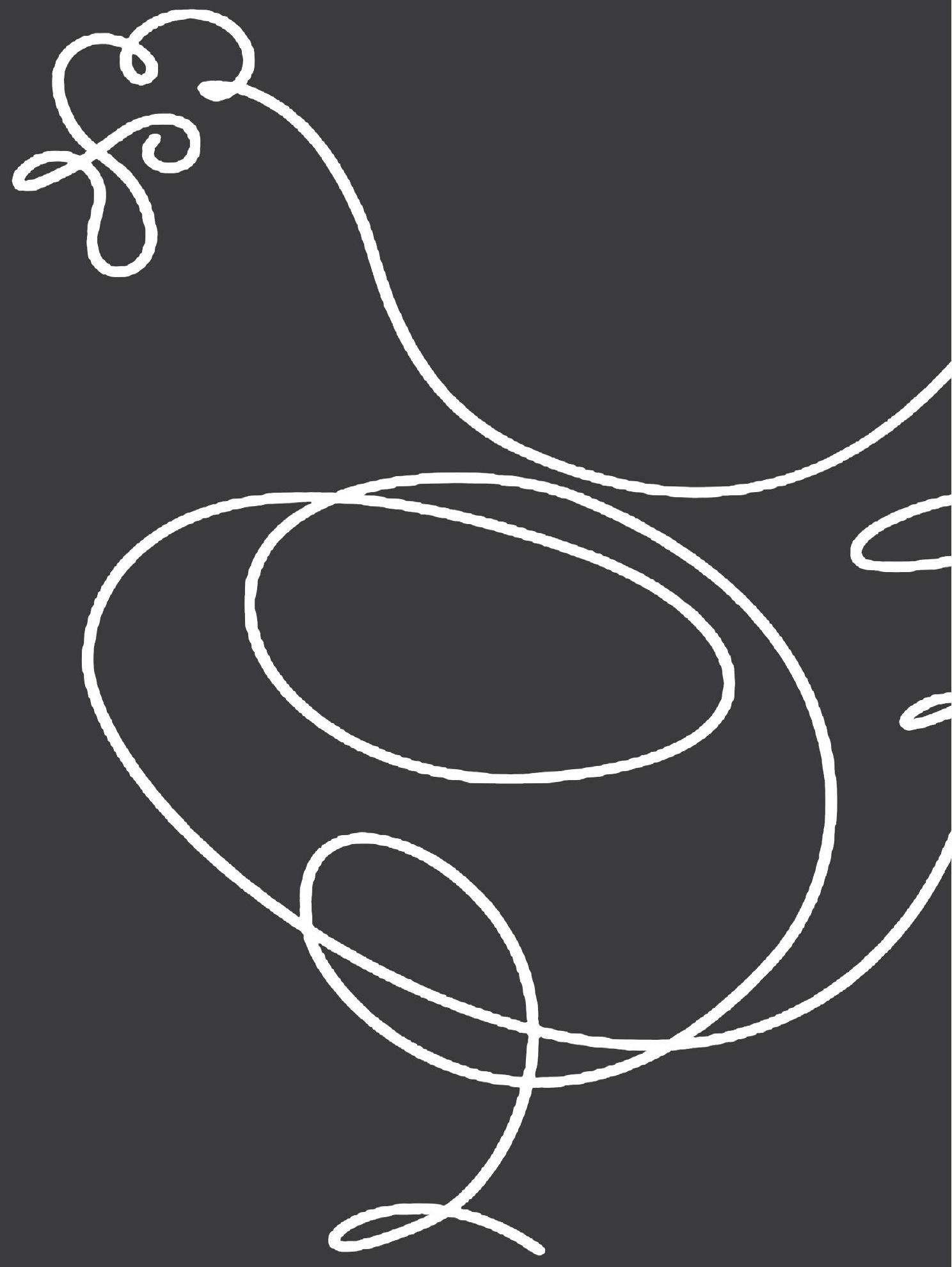




\section{Abstract}

Pen enrichment for broiler (meat-type) chickens is one of the potential strategies to stimulate locomotion and consequently contribute to leg health and welfare. This study was designed to evaluate effects of using a plethora of pen enrichments (barrier perches, angular ramps, horizontal platforms, large distance between feed and water and providing live Black Soldier fly larvae in a dustbathing area) on tibia characteristics, locomotion, leg health and home pen behaviour of fast and slower-growing broiler chickens. The experiment was set up as a $2 \times 2$ factorial arrangement with a total of 840 male broiler chickens in a complete randomized design (7 replicates per treatment and 30 chickens per replicate) with the following treatments: 1) pen enrichment (enriched pen or non-enriched pen); 2) broiler strain (fast-growing Ross 308 or slower-growing Hubbard JA 757). Home pen behaviour and use of enrichment were observed. At approximately 1400 and 2200 gram body weight, two chickens per pen were randomly selected and slaughtered, to investigate tibia morphological, biophysical and mechanical characteristics and leg health. Pen enrichment positively affected tibia biophysical characteristics, e.g., osseous volume $(\Delta=1.8$ $\left.\mathrm{cm}^{3}, \mathrm{P}=0.003\right)$, total volume $\left(\Delta=1.4 \mathrm{~cm}^{3}, \mathrm{P}=0.03\right)$ and volume fraction ( $\Delta=0.02 \%, P=0.002$ ), in both fast and slower-growing chickens, suggesting that pen enrichment particularly affects ossification and mineralization mechanisms. Accordingly, locomotion and active behaviours were positively influenced by pen enrichment. However, pen enrichment resulted in lower body weight gain in both strains, which might be due to higher activity or lower feed intake as a result of difficulties of crossing the barrier perches. Regarding the strain, slower-growing chickens showed consistently more advanced tibia characteristics and more active behaviour than fast-growing chickens. It can be concluded that pen enrichment may lead to more activity and better bone development in both fast and slower-growing chickens.

\section{Key words}

pen enrichment, tibia, slow growing, fast growing, broiler chickens 


\section{INTRODUCTION}

In the last decades, genetic selection on growth rate and feed efficiency in broiler chickens resulted in significant phenotypic and genotypic changes (Havenstein et al., 2003; Knowles et al., 2008; Petracci and Cavani, 2012; Zuidhof et al., 2014). Despite the fact that this selection has provided numerous advantages e.g., high amount of meat production in a short rearing duration, less environmental pollution and considerable financial benefits for producers, it has also caused some downsides e.g., suboptimal leg health. Suboptimal leg health appears to be related to an imbalance between high growth rate and immature bones and joints (Knowles et al., 2008; Sherlock et al., 2010; González-Cerón et al., 2015, Tallentire et al., 2016; Gocsik et al., 2017), which can lead to impaired locomotion (Morris, 1993; Knowles et al., 2008; González-Cerón et al., 2015; Gocsik et al., 2017), pain (Bessei, 2006; Gocsik et al., 2017), poor welfare (McKay et al., 2000; Bradshaw et al., 2002; EFSA, 2012; Gocsik et al., 2017), higher mortality, lower slaughter revenues and significant financial losses (sullivian, 1994; Kestin et al., 1999; Mench, 2004; Knowles et al., 2008; Grandin, 2010).

A potential strategy to promote leg health and welfare of modern broiler chickens might be to stimulate activity and locomotion, e.g., by pen enrichment (Reiter and Bessei, 2009; Blatchford et al., 2012; Ohara et al., 2015; Pedersen et al., 2019). Chickens have been using natural perches, platforms, ramps and elevated resting areas as their natural behaviour throughout their history, from wild ancestors to their modern generations (sandilands ef al., 2009; Kaukonen et al., 2016; Pedersen et al., 2019). This suggests that these types of enrichments are important to fulfil natural behaviours, but current broiler houses mostly lack any form of enrichment. Several studies assessing behaviour showed that broiler chickens spend approximately $80 \%$ of their lifespan with passive behaviours (e.g., lying, sitting and resting) (Weeks et al., 2000; Zuidhof et al., 2014; Reiter and Bessei, 2009). The lack of activity, together with a fast growth rate, may impair bone development, which can result in suboptimal leg health or even lameness (Balog et al., 1997; Bradshaw et al., 2002; Reiter and Bessei, 1998a, 1998b, 2009).

It has been shown that a lower stocking density (Martrenchar et al., 2000; Hall, 2001; Ventura et al., 2012; Zhao et al., 2013; Pedersen et al., 2019), placing platforms and/or ramps (Kaukonen et al., 2016, 2017; Peder sen et al., 2019, 2020), perches 
(Zhao et al., 2013; Kaukonen et al., 2017), large distance between feed and water (Bizeray et al., 2002; Reiter and Bessei, 2009; Pedersen et al., 2020), different dustbathing materials, such as moss-peat (Riber et al., 2018), and worms or insects in a dustbathing area (Pichova et al., 2016; Ipema et al., 2020) resulted in lower prevalence of leg disorders and lower mortality rate. Increasing physical activity and locomotion may thus result in lower incidence of leg problems by stimulating tibia morphological, biophysical and mechanical properties (Reiter and Bessei, 2009; Yildiz et al., 2009; Buijs et al., 2012; Van der Pol et al., 2015; Pedersen et al., 2020).

Another potential strategy to promote leg health and welfare is to reduce growth rate of broiler chickens. Fast-growing broiler chickens demonstrate more leg and locomotion problems than slower-growing broiler chickens (Sulivian, 1994; Williams et al., 2000; Torres and Korver, 2018). The underlying reason is that fast-growing broiler chickens have more porous and less mineralised bones than slower-growing broiler chickens, which are less able to carry the rapidly increased body weight (Williams et al, 2004; Stojcic et al., 2009). It has been found that slower-growing broiler chickens spent more time on perches and platforms (Bokkers and Koene, 2004; Wallenbeck et al., 2016), demonstrated better locomotion (Lewis et al., 1997; Reiter and Bessei, 1998; Weeks et al., 2000; Cornetto et al., 2001; Reiter and Kutritz, 200 1; Bokkers et al., 2004), had less hock and leg problems (Bokkers and Koene, 2004; Kjaer et al., 2006) and lower mortality (Havenstein et al., 1994) than fast-growing broiler chickens.

It can be hypothesized that pen enrichment positively affects bone development and locomotion in both fast and slower-growing broiler chickens, but that effects might be larger in the fast-growing broiler chickens, because they generally show less locomotion. However, effects of pen enrichment on locomotion and leg problems in slower-growing broiler chickens are hardly investigated.

The aim of this study was to investigate effects of a combination of different forms of pen enrichment on (1) tibia characteristics, (2) locomotion, (3) leg health and (4) home pen behaviour of both fast and slow-

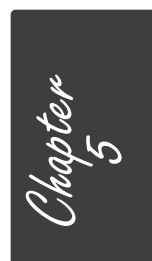
er-growing broiler chickens. 


\section{MATERIALS AND METHODS Experimental Design}

The experiment was setup as a $2 \times 2$ factorial arrangement with two strains of broiler chickens (fast-growing or slower-growing) and two different levels of pen enrichment (enriched or non-enriched). A total of 28 pens (7 pens per treatment, each containing 30 male broiler chickens) within a complete randomized design was used. Fast-growing broiler chickens were reared till day 38 of age, whereas slower-growing broiler chickens were reared till day 49 of age. The experiment was conducted at the research accommodation of Wageningen Bioveterinary Research (Lelystad, The Netherlands). All procedures in this study were approved by the Central Commission on Animal Experiments (The Hague, The Netherlands; approval number: 2016.D-0138.006).

\section{Animals, Rearing and Housing Management}

A total of 420 fast growing (Ross 308, breeder age of 30 weeks) and 420 slower-growing (Hubbard JA 757; breeder age of 28 weeks) day-old male broiler chickens were obtained from a commercial hatchery (Probroed, Groenlo, The Netherlands) and randomly allocated to 28 pens in one broiler house. Half of the chickens per broiler strain were placed in enriched pens, while the other half was placed in non-enriched pens, resulting in the following treatments: enriched fast (EF), non-enriched fast (NF), enriched slower (ES) and non-enriched slower (NS). Pen size of both enriched and non-enriched pens was $3 \times 1 \mathrm{~m}$ and floors in all pens were covered with wood shavings as bedding material. Enriched pens contained two wooden platforms ( $100 \times 20 \times 40 \mathrm{~cm}$, one at each long side of the pen), two wooden ramps $\left(200 \times 20 \mathrm{~cm}\right.$, angle of $\left.11.5^{\circ}\right)$, a dust bathing area in the centre of the pen $(100 \times 100 \mathrm{~cm})$ with peat moss (with a thickness of $2 \mathrm{~cm}$ in week $1,4 \mathrm{~cm}$ in week 2 , and $7.5 \mathrm{~cm}$ from week 3 onwards), two vertical wooden barrier perches $(100 \times 4 \mathrm{~cm}$, adjustable in height from 4 to $16 \mathrm{~cm}$ with steps of $4 \mathrm{~cm}$ at days 7,14 and 21$)$, a maximum distance $(3 \mathrm{~m})$ between feeders and drinkers and provision of live Black Soldier fly larvae (BSFL) in the substrate of the dust bathing area (once daily between 1 1:00 and 11:15 h). The amount of BSFL was determined daily, based on $5 \%$ of 
the expected feed intake, except during the first 7 days, where chickens received a higher level of BSFL ( $10 \%$ on days $0-1,15 \%$ on days $2-4$ and $10 \%$ on days $5-7)$. The reason for using higher percentages in these 7 days is related to the number of larvae available for each chicken. With the low feed intake in this phase, only one or two larvae would have been available per chicken in case only $5 \%$ BSFL was provided. Non-enriched pens included feed and water (at $1 \mathrm{~m}$ distance) and one single long perch (300 $x 4 \mathrm{~cm}$, not adjustable in height). Illustrations of the enriched and non-enriched pens are provided in Figure 1A and 1B.

At day 0 (placement), all broilers were provided with a neck tag for individual identification. House temperature was maintained at $34^{\circ} \mathrm{C}$ at day 0 and gradually decreased to a constant temperature of $18^{\circ} \mathrm{C}$ at 40 days of age. Relative humidity was kept between $60 \%$ and $80 \%$ from 1-7 days of age and between $40 \%$ and $60 \%$ thereafter. The lighting program used was 24L:OD (day 0), 20L:4D (day 1 to 6) and 18L:6D (from day 7 onward, with a continuous dark period during night). At day 0 , chickens were vaccinated against infectious bronchitis (eye drop; MSD Animal Health, Boxmeer, The Netherlands) and at day 11, against Newcastle disease (Clone 30; eye drop, MSD Animal Health, Boxmeer, The Netherlands).

Feed and water were provided ad libitum for all treatments throughout the whole experiment. A 3-phase feeding program was applied; starter diets were provided from day 0 to 14 (ME=2925 kcal/ $/ \mathrm{kg}, C P=203 \mathrm{~g} / \mathrm{kg}$, dLys=11.1 g/kg), grower diets from day 14 to $35(\mathrm{ME}=2975 \mathrm{kcal} / \mathrm{kg}, \mathrm{CP}=171$ $\mathrm{g} / \mathrm{kg}, \mathrm{dLys}=9.1 \mathrm{~g} / \mathrm{kg}$ ) and finisher diets from day 35 to 38 (for fast growing chickens) or 35 to 49 (for slower-growing chickens) (ME=3025 kcal/kg, $\mathrm{CP}=165 \mathrm{~g} / \mathrm{kg}, \mathrm{dLys}=8.6 \mathrm{~g} / \mathrm{kg})$. Coccidiostats $(70 \mathrm{~g} / \mathrm{kg}$ salinomycin) were added to the grower diet. A protein-fat mixture, with a comparable composition as the BSFL, was added to the diet of the non-enriched pens once daily to achieve similar energy and nutrient intake as the broilers in the enriched pens (which received BSFL).

\section{Data Collection, Sampling and Measurements}

All chickens were individually weighed on day $0,7,14,21,28,35,42$, and 49 of age. Feed intake (FI) was determined per pen at the same days. Body weight (BW), Fl and feed conversion ratio (FCR) were calculated for 
the three phases and over the whole growing period, taking mortality into account. FI was calculated without BSFL intake in the enriched pens, but with including the supplement in the non-enriched pens. Mortality was recorded per pen per day.

Home pen behaviour (all chickens per pen) and use of enrichment (all chickens per pen in enriched pens) were scored by direct observation of one observer, using instantaneous scan sampling (De Jong et al., 2019) at day $8,22,29$ and 43 . At these days, broilers were observed in their home pen at four moments $(8: 30,10: 30,13: 00$ and 15:00 h). On day 43 , only slower-growing chickens were present. Per scan, the behaviour of all chickens was scored during 3 to 4 min per scan per day per pen, the number of chickens performing the following activities was scored for home pen behaviour: eating, drinking, walking, standing, sitting, comfort behaviour, foraging, dustbathing, ground pecking, aggression and others. Others was defined as chickens demonstrating a behaviour other than all other behaviours described above. After observing the behaviour in a pen, the number of chickens performing the following activities was scored for use of enrichment: chickens on platforms and ramps, chickens under platforms and ramps, dustbathing chickens and chickens perching on barrier.

Gait score of 4 randomly selected chickens per pen was evaluated on day 27 (fast-growing chickens) and day 35 (slower-growing chickens), to eliminate BW difference. Gait was scored within a range of 0 (normal locomotion) to 5 (unable to walk) (Kestin et al., 1992).

At day 29 and 38, two fast-growing chickens per pen were selected for slaughtering with an average body weight of 1400 and 2200 gram, respectively, whereas at day 38 and 49 , two slower-growing chickens per pen were selected for slaughtering with the same body weights. Chickens were subjected to electrical stunning for euthanizing. Then, Varus-Valgus (VV) was scored, after fixating the legs at the hip joint to stretch the leg, by determining the angle between the tibia and the metatarsus for both the left (VVL) and right leg (VVR), using a goniometer. Thereafter, the left leg of each chicken was dissected and assessed by a veterinarian on tibia dyschondroplasia (TD), bacterial chondronecrosis with osteomyelitis (BCO), epiphyseal plate abnormalities (EPA) and epiphysiolysis (EPI). All these leg 
disorders were scored in the range of 0 (no abnormalities), 1 (minor abnormality) or 2 (severe abnormality).

Right legs were deboned and tibias were packed and frozen at $-20^{\circ} \mathrm{C}$. After thawing, tibia weight was determined. Tibia proximal length, lateral cortex thickness, femoral and metatarsal side proximal head thickness, osseous volume, pore volume, total volume (osseous volume + pore volume), volume fraction (osseous volume / total volume), mineral content and mineral density were analysed on individual tibia, using a GE Phoenix 3D X-ray microfocus CT scanner (General Electric Company ${ }^{\circledR}$, Boston, Massachusetts, US), for details see (Güz et al., 2020, 2021). Illustrations of scanned bones are provided in Figure 2.

Robusticity index was calculated using the following formula (Reisenfeld, 1972):

Robusticity index $(\mathrm{cm} / \mathrm{g})=$ bone proximal length $(\mathrm{cm}) /$ bone weight $(\mathrm{g})$.

The same tibia bones used for 3D X-ray scanning were subjected to a three-point bending test, of which the method is described by (Jungmann et al., 2007), using an Instron ${ }^{\circledR}$ electromechanical universal testing machine (Instron ${ }^{\circledR}$, Norwood, Massachusetts, United States). Maximal load of breaking point was used as the tibia ultimate strength; reached yield load just before the angle has changed on slope data was used as the tibia yield strength; the slope of the selected linear part of the curve data was used as the tibia stiffness; the area under the curve of selected region data was used as the tibia energy to fracture. Elastic modulus (GPa), which is the amount of strain as a result of a particular amount of stress [58], was calculated using the following formula (Turner and Burr, 1993; Novitskaya et al., 2011):

$$
E=\frac{N S^{3}}{4 \delta T L^{3}}
$$

where $\mathrm{E}$ is the elastic modulus (GPa), $\mathrm{N}$ is the maximal load $(\mathrm{N}), \mathrm{S}$ is the span between bending fixtures $(\mathrm{mm}), T$ is the tibia thickness $(\mathrm{mm}), L$ is the tibia length $(\mathrm{mm})$, and $\delta$ is the maximum deflection $(\mathrm{mm})$ at the midpoint of the bone. 


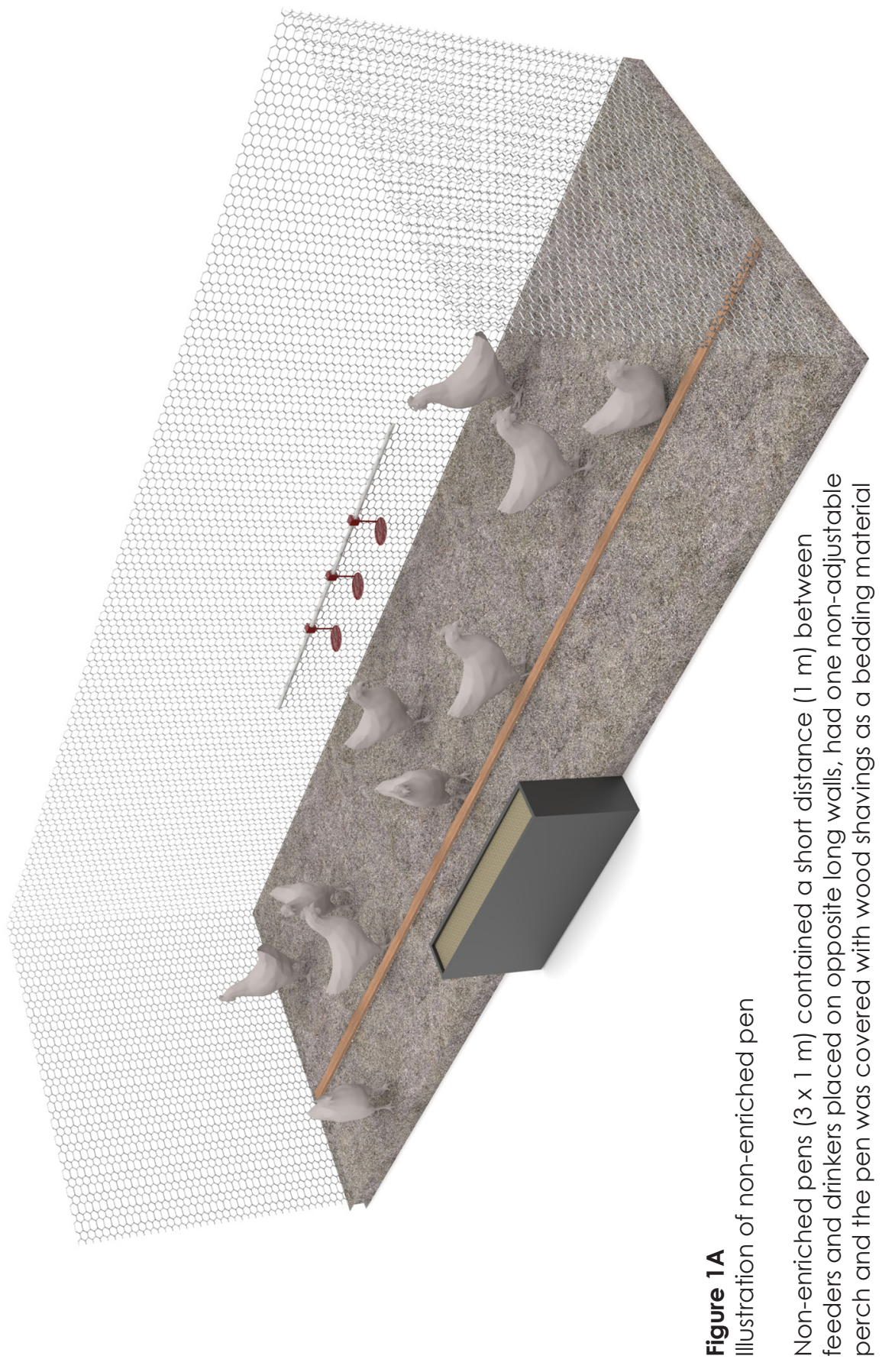



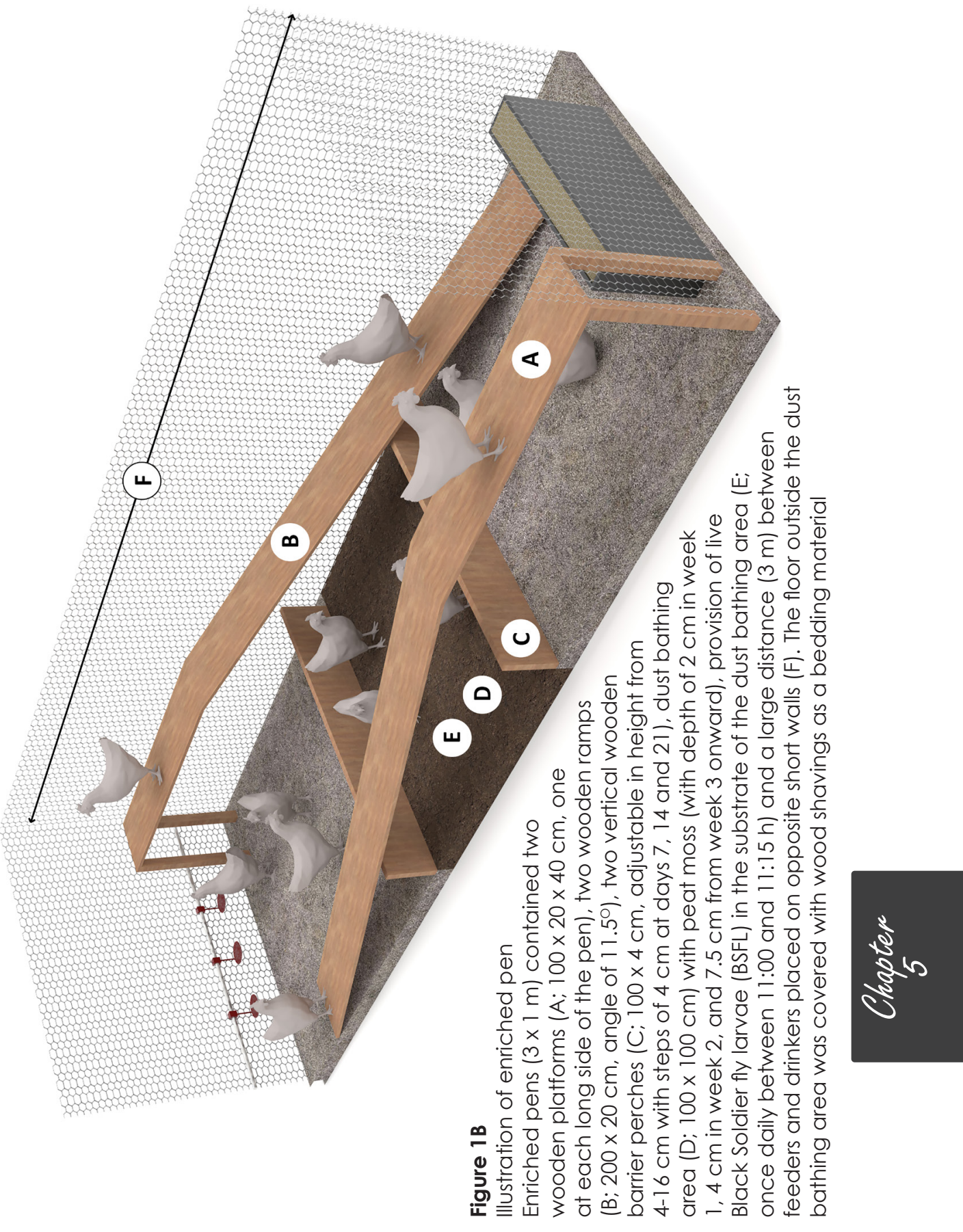


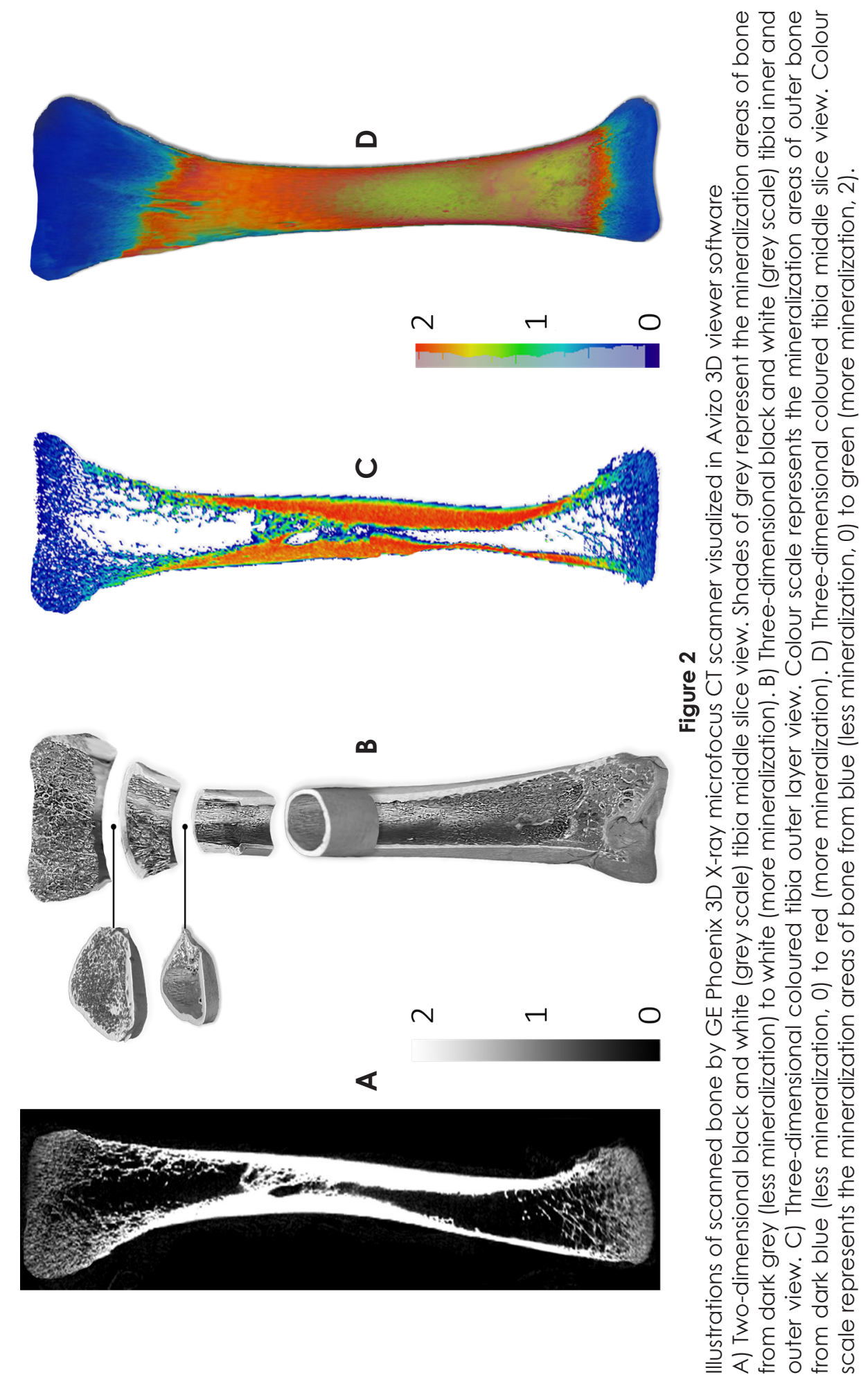




\section{Statistical Analysis}

All statistical analyses were performed in SAS (Version 9.4, 2013, SAS Institute Inc., Cary, North Carolina, US). Model assumptions were approved at both means and residuals for continuous data. Non-normal distributed data were log-transformed before analyses. Pen was used as the experimental unit for all analyses.

All growth performance data from day 0 to 35 (BW, Fl, FCR, mortality) were subjected to general mixed model analysis, using the MIXED procedure with model 1 .

$$
Y=\mu+\text { enrichment + strain +enrichment*strain }+\varepsilon ;[1]
$$

where $Y=$ the dependent variable, $\mu=$ the overall mean, enrichment $=$ whether or not pen enrichment was applied (enriched or non-enriched), strain = broiler strain (fast-growing Ross 308 or slow-growing Hubbard JA757), interaction = 2-way interaction between enrichment and strain, $\varepsilon=$ residual error.

From day 35 onwards, only chickens from the slow-growing strain were present and growth performance data (BW, FI, FCR, mortality) was subjected to general linear mixed model analysis, using the MIXED procedure with model 2.

$$
Y=\mu+\text { enrichment }+\varepsilon \text {; [2] }
$$

where $Y=$ the dependent variable, $\mu$ is the overall mean, enrichment $=$ whether or not pen enrichment was applied (enriched or non-enriched), $\varepsilon=$ residual error.

Tibia morphological, biophysical and mechanical characteristics, at two body weight classes (1400 and $2200 \mathrm{~g}$ ), were subjected to general linear mixed model analysis, using the MIXED procedure with model 1.

Home pen behaviour (eating, drinking, walking, standing, sitting, comfort behaviour, foraging, dustbathing, ground pecking, aggression and others) and gait score were subjected to general linear mixed model analysis, using the MIXED procedure with model 1 (home pen behaviour at day 8,22 and 29) and model 2 (home pen behaviour at day 43). Preliminary analyses demonstrated a lack of interaction between strain and enrichment for home pen behaviour and consequently data is presented for only main effects. 
Gait score at day 27 (fast-growing chickens) and day 35 (slower-growing chickens), when they had similar body weights, were compared, using model 1.

Use of enrichment (chickens on platforms and ramps, chickens under platforms and ramps, dustbathing chickens and chickens perching on barriers) was subjected to general linear mixed model analysis, using the MIXED procedure with model 3.

$$
Y=\mu+\operatorname{strain}+\varepsilon ;[3]
$$

where $Y=$ the dependent variable, $\mu$ is the overall mean, strain $=$ broiler breeder strain (fast-growing Ross 308 or slower-growing Hubbard JA757), $\varepsilon=$ residval error.

To eliminate BW effect between the fast and slower-growing strain, home pen behaviour and enrichment use at day 22 for fast growing chickens and day 29 for slower-growing chickens, when they had similar body weights, were compared, using model 1 (home pen behaviour) or model 3 (enrichment).

VVR and VVL were subjected to general linear mixed model analysis, at two body weight classes (1400 and 2200 gram), using the MIXED procedure with model 1 .

Leg disorders (TD, EPA, BCO and EPI) were subjected to generalized linear mixed model analysis, at two body weight classes (1400 and 2200 gram), using the GLIMMIX procedure with model 1. Leg disorders were scored as 0 (no abnormalities), 1 (minor abnormality), or 2 (severe abnormality), but analyzed as 0 (no abnormalities) or 1 (abnormalities present). EPA, BCO and EPI were not statistically analysed, because there were only three chickens scored with $\mathrm{BCO}$ and no observations at all were recorded for EPA and EPI.

Results are provided as LSmeans \pm SEM, unless indicated otherwise. When multiple comparisons were performed, the level of significance was corrected, using Bonferroni. Effects were considered to be significant at $\mathrm{P}$ $\leq 0.05$. 


\section{RESULTS \\ Growth Performance}

No interaction effects between enrichment and strain were found on BW (Table 1). Chickens in non-enriched pens had a higher BW than chickens in enriched pens at day 21 ( $\Delta=35 \mathrm{~g}, \mathrm{P}=0.02), 28$ ( $\Delta=62 \mathrm{~g}, \mathrm{P}=0.007$ ), 35 ( $\Delta=99 \mathrm{~g}, \mathrm{P}=0.003$ ), 42 (slower-growing chickens only; $\Delta=84 \mathrm{~g}, \mathrm{P}=0.003$ ) and 49 (slower-growing chickens only; $\Delta=93 \mathrm{~g}, \mathrm{P}=0.005$ ). Slower-growing broilers had a lower BW than fast-growing broilers at day $0(\Delta=1.8 \mathrm{~g}), 7$ $(\Delta=29 \mathrm{~g}), 14(\Delta=134 \mathrm{~g}), 21(\Delta=321 \mathrm{~g}), 28(\Delta=540 \mathrm{~g})$ and $35(\Delta=822 \mathrm{~g})$ (all $\mathrm{P}<0.001)$.

No interaction effects between enrichment and strain were found on Fl (Table 2). Chickens in non-enriched pens had a higher Fl than chickens in enriched pens between day 14-35 ( $\Delta=100 \mathrm{~g}, \mathrm{P}=0.004)$, day 0-35 ( $\Delta=109$ g, $\mathrm{P}=0.009$ ), day 35-49 (slower-growing broilers only; $\Delta=56 \mathrm{~g}, \mathrm{P}=0.02$ ) and day 0-49 (slower-growing broilers only; $\Delta=139 \mathrm{~g}, \mathrm{P}=0.002$ ). Slower-growing chickens had a lower FI than fast growing broilers between day 0-14 ( $\Delta=114$ g), day 14-35 ( $\Delta=952 \mathrm{~g}, \mathrm{P}<0.001)$ and day 0-35 ( $\Delta=1067 \mathrm{~g})$ (all $\mathrm{P}<0.001)$.

No interaction effects between enrichment and strain were found on FCR (Table 2). Chickens in non-enriched pens had a lower FCR than chickens in enriched pens between days 0-14 ( $\Delta=0.02, P=0.02)$. Slower-growing chickens had a higher FCR than fast growing chickens between days 0-14 $(\Delta=0.14), 14-35(\Delta=0.13)$ and $0-35(\Delta=0.12)$ (all $P<0.001)$.

\section{Tibia Morphological Characteristics}

At the 1400 gram BW class, no interaction effects between pen enrichment and strain were found on tibia morphological characteristics (Table 3) and neither pen enrichment effects were found. Slower-growing chickens had a higher femoral $(\Delta=0.17 \mathrm{~cm}, P=0.02)$ and metatarsal side proximal tibia head thicknesses $(\Delta=0.12 \mathrm{~cm}, \mathrm{P}=0.04)$ than fast growing chickens.

At the 2200 gram BW class, no interaction effects between enrichment and strain were found on tibia morphological characteristics (Table 3) and neither pen enrichment effects were found. Slower-growing broilers 


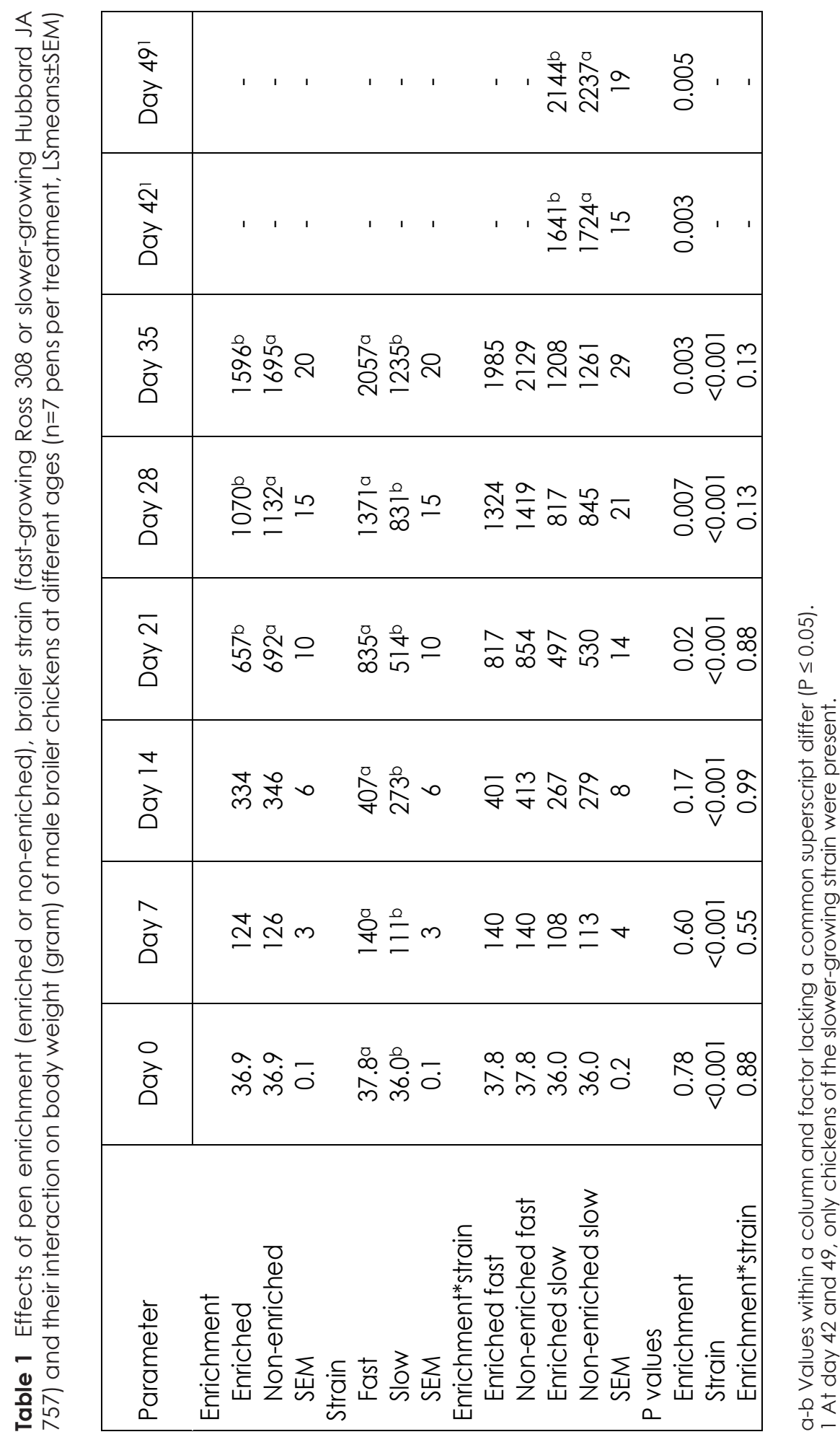




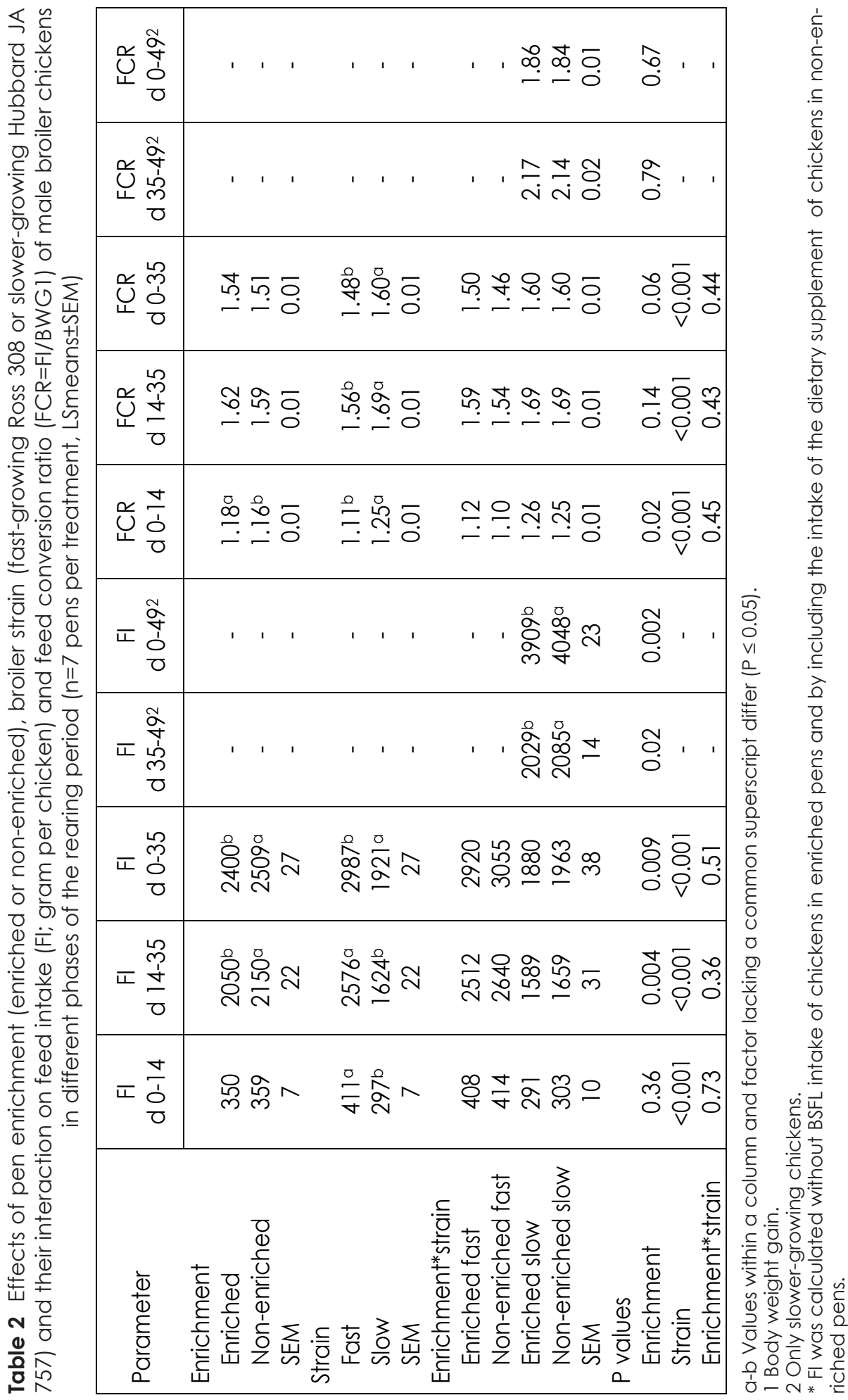


had a higher tibia weight ( $\Delta=0.81 \mathrm{~g}, \mathrm{P}=0.02$ ), proximal tibia length $(\Delta=0.63$ $\mathrm{cm}, \mathrm{P}=0.008)$ and metatarsal side proximal tibia head thicknesses $(\Delta=0.17$ $\mathrm{cm}, \mathrm{P}=0.002$ ) than fast growing broilers.

\section{Tibia Biophysical Characteristics}

At the 1400 gram BW class, no interaction effects between pen enrichment and strain were found on tibia biophysical characteristics (Table 4) and neither pen enrichment effects were found. Slower-growing broilers had a higher tibia osseous volume $\left(\Delta=6.4 \mathrm{~cm}^{3}, P<0.001\right)$, tibia total volume $\left(\Delta=6.6 \mathrm{~cm}^{3}, \mathrm{P}<0.001\right)$, tibia volume fraction $(\Delta=0.04 \%, \mathrm{P}<0.001)$ and tibia mineral content $(\Delta=1.1 \mathrm{~g}, \mathrm{P}<0.001)$ than fast growing broilers.

At the 2200 gram BW class, an interaction between pen enrichment and strain was found on tibia pore volume (Table 4). Enriched slower-growing group resulted in a lower tibia pore volume compared to other groups $\left(\Delta=1.0 \mathrm{~cm}^{3}\right.$ on average; $\left.\mathrm{P}=0.02\right)$. Chickens in non-enriched pens had a lower tibia osseous volume $\left(\Delta=1.8 \mathrm{~cm}^{3}, \mathrm{P}=0.003\right)$, tibia total volume $(\Delta=1.4$ $\left.\mathrm{cm}^{3}, \mathrm{P}=0.03\right)$ and tibia volume fraction $(\Delta=0.02 \%, \mathrm{P}=0.002)$ than chickens in enriched pens. Slower-growing broilers had a higher tibia osseous volume $\left(\Delta=5.9 \mathrm{~cm}^{3}, \mathrm{P}<0.001\right)$, tibia total volume $\left(\Delta=5.4 \mathrm{~cm}^{3}, \mathrm{P}<0.001\right)$, tibia volume fraction $(\Delta=0.05 \%, P<0.001)$, tibia mineral content $(\Delta=0.7 \mathrm{~g}, \mathrm{P}=0.02)$ and tibia mineral density $\left(\Delta=0.05 \mathrm{~g} / \mathrm{cm}^{2}, P<0.001\right)$ than fast-growing broilers.

\section{Tibia Mechanical Characteristics}

At the 1400 gram BW class, no interaction effects between pen enrichment and strain were found on tibia mechanical characteristics and neither pen enrichment effects were found (Table 5). Slower-growing broilers had a higher tibia ultimate strength $(\Delta=21.7 \mathrm{~N}, \mathrm{P}<0.001)$, tibia yield strength $(\Delta=21.0 \mathrm{~N}, \mathrm{P}<0.001)$, tibia stiffness $(\Delta=20.6 \mathrm{~N} / \mathrm{mm}, \mathrm{P}<0.001)$ and tibia energy to fracture $(\Delta=21.9 \mathrm{~N}-\mathrm{mm}, \mathrm{P}<0.001)$ than fast-growing broilers.

At the 2200 gram BW class, no interaction effects between pen enrichment and strain were found on tibia mechanical characteristics (Table 5) and neither pen enrichment effects were found. Slower-growing chickens had a higher tibia ultimate strength $(\Delta=19.4 \mathrm{~N}, \mathrm{P}<0.001)$, tibia yield strength $(\Delta=17.8 \mathrm{~N}, \mathrm{P}<0.001)$, tibia stiffness $(\Delta=21.7 \mathrm{~N} / \mathrm{mm}, \mathrm{P}<0.001)$ and tibia energy to fracture $(\Delta=20.9 \mathrm{~N}-\mathrm{mm}, \mathrm{P}<0.001)$ than fast-growing broilers. 


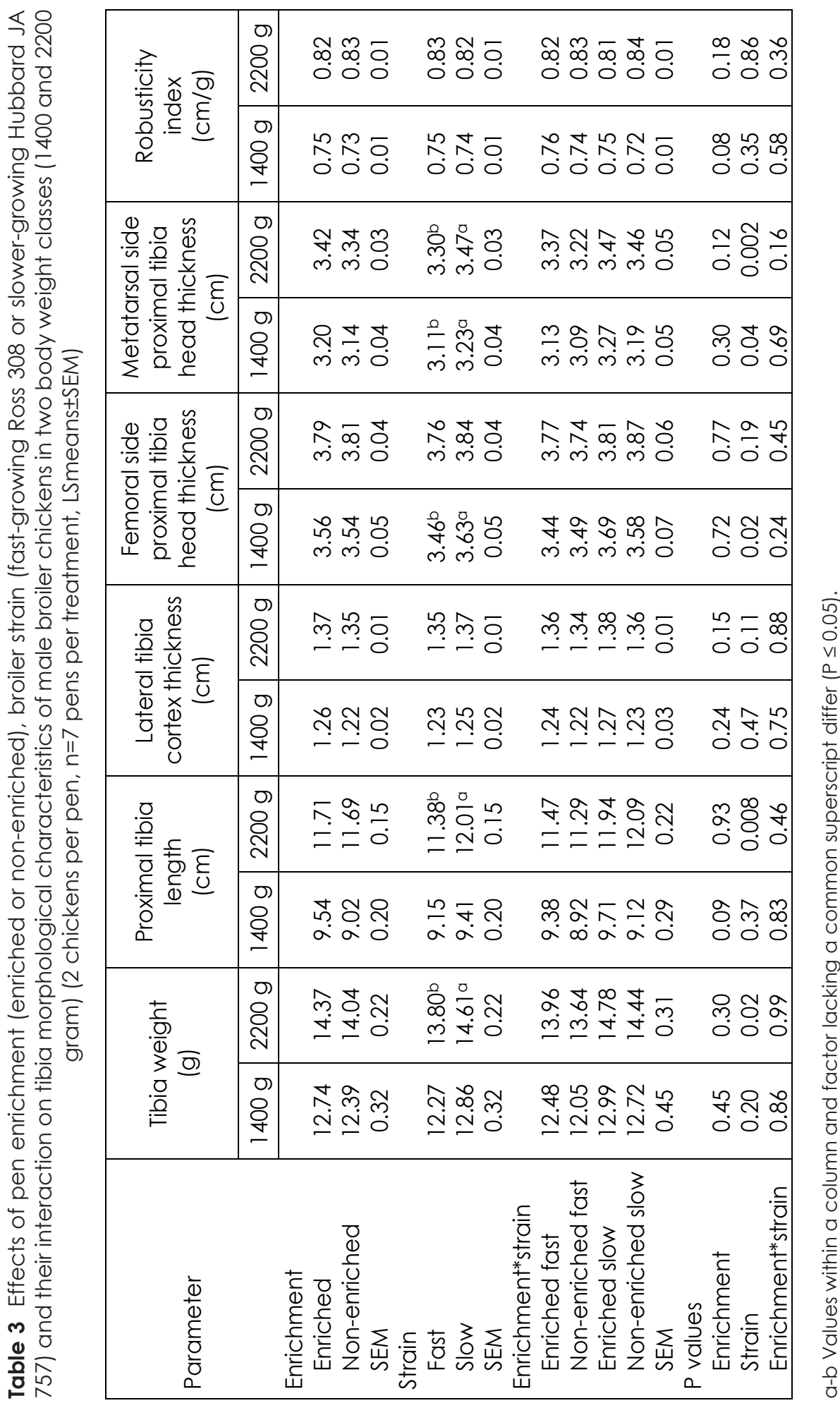

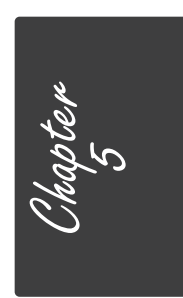




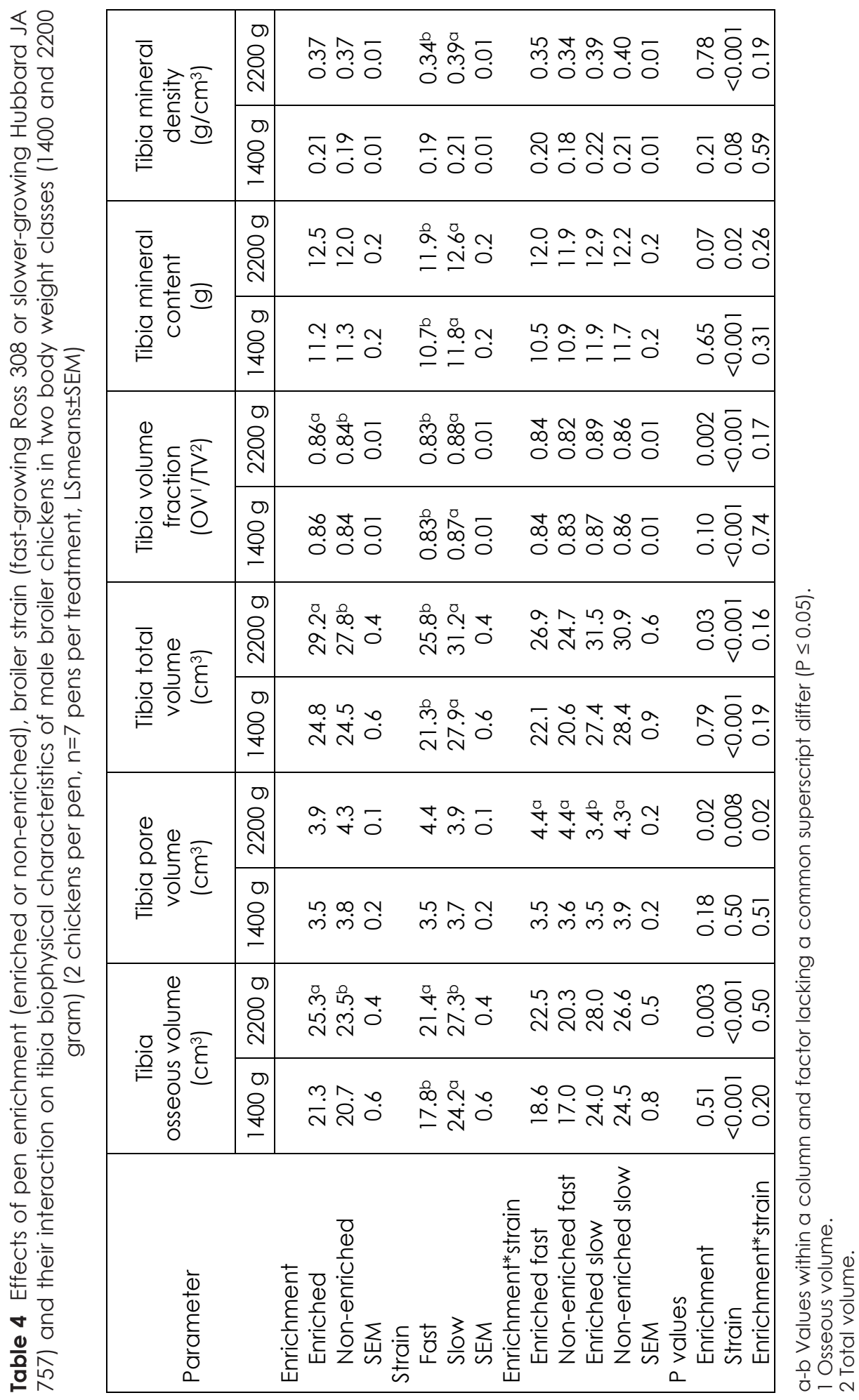




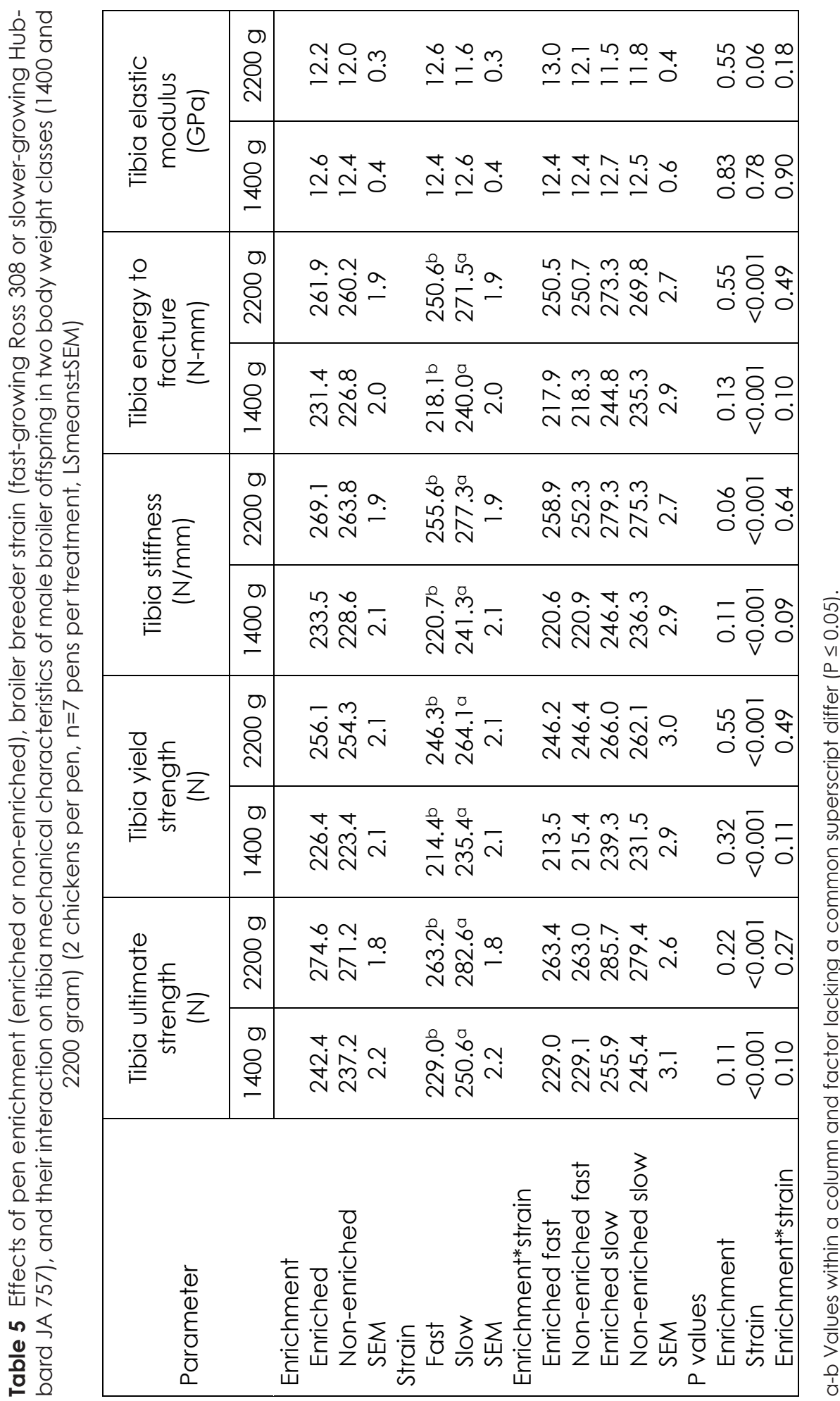

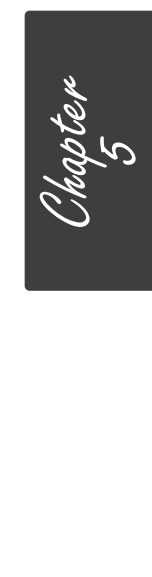




\section{Leg Disorders and Gait score}

No interaction effects between pen enrichment and broiler breeder strain were found on VVR, VVL and TD at both 1400 and 2200 gram BW classes and no main effects of pen enrichment or strain were found on TD. At the 1400 gram BW class, VV angulation was not affected by enrichment or strain (on average $4.90^{\circ}$ ). At the 2200 gram BW class, slower-growing chickens had a lower VVR than fast growing chickens $\left(3.80^{\circ} \mathrm{Vs} 6.04^{\circ}\right.$, respectively, $P=0.003) . V V L$ angulation was not affected by enrichment or strain at this BW class (on average $5.88^{\circ}$ ). No interaction and main effects were found on TD, which was observed in 11 chickens (9.82 \%) at 1400 gram BW class and 10 chickens (8.92 \%) at 2200 BW class.

At similar BW class (day 27 for fast-growing chickens and day 35 for slower-growing chickens), an interaction between pen enrichment and strain was found on gait score. The NS group had a lower gait score compared to NF group (1.66 vs. 2.11, respectively; $P=0.003$ ), while other two groups were in between (both 1.93).

\section{Home Pen Behaviour and Use of Enrichment}

Chickens in non-enriched pens showed less foraging behaviour at day $8(\Delta=8.47 \%, \mathrm{P}<0.001)$, day $22(\Delta=9.19 \%, \mathrm{P}<0.001)$, day $29(\Delta=5.6 \%$, $\mathrm{P}<0.001$ ) and day 43 (slower-growing chickens only, $\Delta=8.86 \%, \mathrm{P}<0.001$ ), less dust bathing behaviour at day $8(\Delta=0.97 \%, P=0.006)$ and less ground pecking behaviour at day 43 (slower-growing chickens only, $\Delta=3.98 \%$, $\mathrm{P}=0.02$ ) than chickens in enriched pens (Table 6). The opposite was found for standing behaviour at day $29(\Delta=4.15 \%, \mathrm{P}=0.007)$, sitting behaviour at day $22(\Delta=8.97 \%, P=0.03)$, ground pecking behaviour at day $8(\Delta=3.71 \%$, $\mathrm{P}=0.002)$ and aggression behaviour at day 29 ( $\Delta=1.05 \%, \mathrm{P}=0.02)$, which behaviours were all higher in non-enriched pens than in enriched pens. Slower-growing chickens showed more walking behaviour at day 8 ( $\Delta=6.99$ $\%, \mathrm{P}=0.001)$, day $22(\Delta=7.6 \%, \mathrm{P}<0.001)$ and day $29(\Delta=5.32 \%, \mathrm{P}<0.001)$, more standing behaviour at day $8(\Delta=2.34 \%, P=0.03)$, day $22(\Delta=3.12 \%$, $\mathrm{P}=0.02)$ and day $29(\Delta=8.91 \%, \mathrm{P}<0.001)$, more foraging behaviour at day $22(\Delta=6.61 \%, P=0.009)$ and day $29(\Delta=3.9 \%, P=0.002)$ and more aggression behaviour at day $22(\Delta=0.94 \%, P=0.03)$ and day $29(\Delta=1.02 \%, P=0.03)$ than 
fast-growing chickens. The opposite was found for eating behaviour at day $8(\Delta=3.04 \%, \mathrm{P}=0.04)$ and day $22(\Delta=1.16 \%, \mathrm{P}=0.03)$ and sitting behaviour at day $22(\Delta=19.28 \%, \mathrm{P}<0.001)$ and day $29(\Delta=8.91 \%, \mathrm{P}<0.001)$ (Table 6).

In enriched pens, a clear strain effect was found on use of different enrichment objects (Table 7). The percentage of chickens on platforms and ramps at day $29(\Delta=14.6 \%, P<0.001)$ and perching on barriers at day $8(\Delta=4.9 \%, \mathrm{P}<0.001)$, day $22(\Delta=14.05 \%, \mathrm{P}<0.001)$ and day $29(\Delta=16.05 \%$, $\mathrm{P}<0.001)$ was higher in slower-growing chickens than in fast growing chickens. The opposite was found for the percentage chickens under platforms and ramps at day $29(\Delta=13.72 \%, P=0.003)$ and dustbathing chickens at day $8(\Delta=1.19 \%, P=0.05)$.

At similar BW, fast growing chickens showed more walking ( $\Delta=5.24$ $\%)$, standing $(\Delta=8.6 \%)$, foraging $(\Delta=2.67 \%)$, dust bathing $(\Delta=0.12 \%)$ and aggression $(\Delta=1.02 \%$ ) behaviour than fast growing chickens, while the opposite was found for eating $(\Delta=0.62 \%)$, drinking $(\Delta=1.54 \%)$, sitting $(\Delta=11.93$ $\%)$ and comfort ( $\Delta=2.32 \%$ ) behaviour. At similar BW, more slower-growing chickens were on platforms and ramps $(\Delta=11.27 \%)$ and perching on barriers $(\Delta=16.1 \%$ ) than fast-growing chickens, whereas the opposite was found for percentage of chickens under platforms and ramps $(\Delta=4.98 \%)$ and dustbathing chickens ( $\Delta=0.03 \%)$. 
Table 6 Effects of pen enrichment (enriched or non-enriched) and broiler strain (fast-growing Ross 308 or slower-growing Hubbard JA 757) on percentage of male chickens showing eating, drinking, walking, standing, sitting, comfort behaviour, foraging, dustbathing, ground pecking, aggression and other behaviours at day

$8,22,29$ and 43 of age ( $n=7$ pens per treatment; LSmeans \pm SEM)

\begin{tabular}{|c|c|c|c|c|c|c|c|}
\hline \multirow{2}{*}{$\begin{array}{l}\text { Parameter } \\
\text { and day (\%) }\end{array}$} & \multicolumn{2}{|c|}{ Enrichment } & \multicolumn{2}{|c|}{ Strain } & \multirow[b]{2}{*}{ SEM } & \multicolumn{2}{|c|}{ P-values ${ }^{2}$} \\
\hline & Enriched & $\begin{array}{l}\text { Non- } \\
\text { enriched }\end{array}$ & Fast & Slow & & Enrichment & Strain \\
\hline \multicolumn{8}{|l|}{ Eating } \\
\hline Day 8 & 6.90 & 6.06 & $8.00^{b}$ & $4.96^{a}$ & 0.94 & 0.54 & 0.04 \\
\hline Day 22 & 1.65 & 1.80 & $2.31^{b}$ & $1.15^{\mathrm{a}}$ & 0.33 & 0.75 & 0.03 \\
\hline Day 29 & 1.78 & 2.78 & 2.86 & 1.69 & 0.46 & 0.14 & 0.09 \\
\hline Day $43^{1}$ & 1.65 & 1.79 & - & - & 0.51 & 0.86 & - \\
\hline \multicolumn{8}{|l|}{ Drinking } \\
\hline Day 8 & 13.17 & 12.01 & 13.76 & 11.42 & 1.58 & 0.61 & 0.31 \\
\hline Day 22 & 5.33 & 5.58 & 5.67 & 5.24 & 0.72 & 0.81 & 0.69 \\
\hline Day 29 & 4.36 & 4.78 & 5.00 & 4.13 & 0.72 & 0.69 & 0.40 \\
\hline Day $43^{1}$ & 3.78 & 3.23 & - & - & 0.58 & 0.51 & - \\
\hline \multicolumn{8}{|l|}{ Walking } \\
\hline Day 8 & 13.42 & 12.27 & $9.35^{b}$ & $16.34^{a}$ & 1.10 & 0.47 & 0.001 \\
\hline Day 22 & 7.92 & 7.09 & $3.70^{b}$ & $11.30^{a}$ & 1.22 & 0.64 & $<0.001$ \\
\hline Day 29 & 5.67 & 6.90 & $3.62^{b}$ & $8.94^{a}$ & 0.82 & 0.31 & $<0.001$ \\
\hline Day $43^{1}$ & 7.99 & 8.56 & - & - & 1.46 & 0.80 & - \\
\hline \multicolumn{8}{|l|}{ Standing } \\
\hline Day 8 & 5.68 & 7.65 & $5.50^{\mathrm{b}}$ & $7.84^{a}$ & 0.72 & 0.07 & 0.03 \\
\hline Day 22 & 4.54 & 5.44 & $3.43^{b}$ & $6.55^{a}$ & 0.81 & 0.44 & 0.02 \\
\hline Day 29 & $5.50^{b}$ & $9.65^{a}$ & $3.12^{b}$ & $12.03^{a}$ & 1.00 & 0.007 & $<0.001$ \\
\hline Day $43^{1}$ & 13.64 & 15.84 & - & - & 1.46 & 0.29 & - \\
\hline \multicolumn{8}{|l|}{ Sitting } \\
\hline Day 8 & 32.57 & 37.36 & 37.61 & 33.31 & 2.44 & 0.18 & 0.14 \\
\hline Day 22 & $45.61^{\mathrm{b}}$ & $54.58^{a}$ & $59.73^{a}$ & $40.45^{b}$ & 2.72 & 0.03 & $<0.001$ \\
\hline Day 29 & 57.36 & 55.23 & $64.78^{a}$ & $47.80^{\mathrm{b}}$ & 2.90 & 0.61 & $<0.001$ \\
\hline Day $43^{1}$ & 50.06 & 58.22 & - & - & 3.97 & 0.18 & - \\
\hline \multicolumn{8}{|c|}{ Comfort behaviour } \\
\hline Day 8 & 7.55 & 9.34 & 7.15 & 9.74 & 1.00 & 0.22 & 0.08 \\
\hline Day 22 & 8.86 & 10.12 & 8.84 & 10.13 & 1.08 & 0.42 & 0.41 \\
\hline Day 29 & 6.19 & 7.60 & 7.27 & 6.52 & 1.12 & 0.39 & 0.64 \\
\hline Day $43^{1}$ & 4.46 & 3.65 & - & - & 0.95 & 0.56 & - \\
\hline \multicolumn{8}{|l|}{ Foraging } \\
\hline Day 8 & $14.45^{a}$ & $5.98^{b}$ & 9.67 & 10.76 & 1.00 & $<0.001$ & 0.45 \\
\hline Day 22 & $12.88^{a}$ & $3.69 b$ & $4.98^{b}$ & $11.59 a$ & 1.64 & $<0.001$ & 0.009 \\
\hline Day 29 & $8.50^{a}$ & $2.90^{b}$ & $3.75^{b}$ & $7.65^{a}$ & 0.79 & $<0.001$ & 0.002 \\
\hline Day $43^{1}$ & $9.78^{a}$ & $0.92^{b}$ & - & - & 1.25 & $<0.001$ & - \\
\hline
\end{tabular}


Table 6 (continued)

\begin{tabular}{|c|c|c|c|c|c|c|c|}
\hline \multirow{2}{*}{$\begin{array}{l}\text { Parameter } \\
\text { and day (\%) }\end{array}$} & \multicolumn{2}{|c|}{ Enrichment } & \multicolumn{2}{|c|}{ Strain } & \multirow[b]{2}{*}{ SEM } & \multicolumn{2}{|c|}{ P-values ${ }^{2}$} \\
\hline & Enriched & $\begin{array}{c}\text { Non- } \\
\text { enriched }\end{array}$ & Fast & Slow & & Enrichment & Strain \\
\hline \multicolumn{8}{|l|}{ Dust bathing } \\
\hline Day 8 & $1.09 a$ & $0.12^{b}$ & 0.90 & 0.30 & 0.23 & 0.006 & 0.07 \\
\hline Day 22 & 0.65 & 0.44 & 0.65 & 0.44 & 0.24 & 0.56 & 0.54 \\
\hline Day 29 & 0.46 & 0.67 & 0.36 & 0.77 & 0.28 & 0.60 & 0.31 \\
\hline Day $43^{1}$ & 0.28 & 0.15 & - & - & 0.17 & 0.59 & - \\
\hline \multicolumn{8}{|c|}{ Ground pecking } \\
\hline Day 8 & $4.83^{b}$ & $8.54^{a}$ & 7.40 & 5.97 & 0.73 & 0.002 & 0.18 \\
\hline Day 22 & 12.02 & 10.76 & 10.60 & 12.17 & 1.17 & 0.46 & 0.36 \\
\hline Day 29 & 10.08 & 8.33 & 9.09 & 9.31 & 1.17 & 0.31 & 0.90 \\
\hline Day $43^{1}$ & $8.10^{a}$ & $4.12^{b}$ & - & - & 0.95 & 0.02 & - \\
\hline \multicolumn{8}{|l|}{ Aggression } \\
\hline Day 8 & 0.35 & 0.22 & 0.22 & 0.35 & 0.17 & 0.60 & 0.60 \\
\hline Day 22 & 0.61 & 0.59 & $0.14^{b}$ & $1.06^{a}$ & 0.29 & 0.97 & 0.04 \\
\hline Day 29 & $0.12^{b}$ & $1.17^{a}$ & $0.14^{b}$ & $1.16^{a}$ & 0.29 & 0.02 & 0.03 \\
\hline Day $43^{1}$ & $0.26^{b}$ & $3.53^{a}$ & - & - & 0.98 & 0.04 & - \\
\hline \multicolumn{8}{|l|}{ Others $^{3}$} \\
\hline Day 8 & - & 0.13 & 0.13 & - & 0.08 & 0.26 & 0.26 \\
\hline Day 22 & - & - & - & - & - & - & - \\
\hline Day 29 & - & - & - & - & - & - & - \\
\hline Day $43^{1}$ & - & - & - & - & - & - & - \\
\hline
\end{tabular}

a-b Values within a factor and row lacking a common superscript differ ( $\mathrm{P} \leq 0.05)$.

1 Only slower-growing chickens.

2 No interactions between broiler breeder strain and pen enrichment were observed for any of the behaviours at any of the sampling days.

3 Chickens demonstrating a behaviour other than eating, drinking, walking, standing, sitting, comfort behaviour, foraging, dustbathing, ground pecking and aggression. 
Table 7 Effects of broiler strain (fast-growing Ross 308 or slower-growing Hubbard JA 757) on percentage of male chickens in enriched pens, using the following enrichment objects (on platforms and ramps, under platforms and ramps, dustbathing, perching on barriers) at day $8,22,29$ and 43 of age ( $n=7$ pens per treatment, LSmeans \pm SEM)

\begin{tabular}{|c|c|c|c|c|}
\hline \multirow{2}{*}{ Parameter and day 1} & \multicolumn{2}{|c|}{ Strain } & \multirow{2}{*}{ SEM } & \multirow{2}{*}{ P-values } \\
\hline & Fast & Slow & & \\
\hline \multicolumn{5}{|c|}{ Chickens on platforms and ramps } \\
\hline Day 8 & 13.69 & 16.18 & 2.49 & 0.50 \\
\hline Day 22 & 17.98 & 24.04 & 2.06 & 0.06 \\
\hline Day 29 & $14.69 \mathrm{~b}$ & $29.25^{a}$ & 1.75 & $<0.001$ \\
\hline Day $43^{2}$ & - & 29.75 & - & - \\
\hline \multicolumn{5}{|c|}{ Chickens under platforms and ramps } \\
\hline Day 8 & 16.95 & 30.35 & 4.51 & 0.06 \\
\hline Day 22 & 22.74 & 16.97 & 2.60 & 0.15 \\
\hline Day 29 & $31.48 a$ & $17.76^{\mathrm{b}}$ & 1.91 & $<0.001$ \\
\hline Day $43^{2}$ & - & 19.55 & - & - \\
\hline \multicolumn{5}{|l|}{ Dustbathing chickens } \\
\hline Day 8 & $1.56^{a}$ & $0.37 b$ & 0.37 & 0.05 \\
\hline Day 22 & 0.4 & 0.63 & 0.26 & 0.54 \\
\hline Day 29 & 0.14 & 0.37 & 0.21 & 0.47 \\
\hline Day $43^{2}$ & - & 0.14 & - & - \\
\hline \multicolumn{5}{|c|}{ Chickens perching on barriers } \\
\hline Day 8 & $2.08^{\mathrm{b}}$ & $6.98 a$ & 0.75 & $<0.001$ \\
\hline Day 22 & $3.92^{\mathrm{b}}$ & $17.97 a$ & 1.53 & $<0.001$ \\
\hline Day 29 & $3.97 \mathrm{~b}$ & $20.02^{a}$ & 0.90 & $<0.001$ \\
\hline Day $43^{2}$ & - & 17.57 & - & - \\
\hline
\end{tabular}

$a-b$ Values within a row lacking a common superscript differ $(P \leq 0.05)$.

1 The percentages of chickens demonstrating no use of enrichment are not demonstrated in the table.

2 Only slower-growing chickens. 


\section{DISCUSSION}

The aim of this study was to investigate effects of a combination of different forms of pen enrichment on tibia characteristics, locomotion, leg health and home pen behaviour of both fast and slower-growing broiler chickens. Hardly any interactions were found between strain and enrichment, indicating that both fast and slower-growing chickens are both able to use several forms of enrichment in a comparable way.

\section{Growth Performance}

Results of the current study showed that pen enrichment resulted in lower body weight and feed intake in both fast and slower-growing chickens. These findings are supported by recent studies (Jordan et al., 2017; Bach et al., 2019), who observed a negative effect of pen enrichment on growth performance in terms of feed intake and body weight gain. However, the results of current study are not in accordance with other studies (Pettit-Riley and Estevez, 200 1; Bizeray et al., 2002; Şimşek et al., 2009; Yıldıım and Taşkın, 2017; Jones et al., 2020), who found no significant effects of pen enrichment on growth performance parameters. This discrepancy among studies might be related to the fact that less complex pen enrichment forms were used in these studies compared to the current study. For example, only barrier perches (Pettit-Riley and Estevez, 2001; Bizeray et al., 2002; Simssek et al., 2009) and mirror, ball, perch and dust (each material in another pen) (Yldirm and Tasskin, 2017) were used in these studies. The lower body weight gain of enriched-housed broilers in the current experiment might be related to 1) the comprehensive enrichment design of the current study, which contains a combination of platforms, angular ramps, barrier perches, large distance between feed and water and live Black Soldier fly larvae in the moss-peat dust bathing area. A plethora of different enrichments might exponentially stimulate physical activity and consequently a higher metabolic energy use, which will result in a lower body weight gain. This higher activity in enriched pens is supported by the higher percentage of chickens showing active behaviours and use of enrichment in both fast and slower-growing chickens. 2) Chickens might have had difficulties to cross the barrier perches to access the feed on one side and water on the other side. This might be due to other chickens perching and consequently blocking the way from one 
side of the pen to the other side. It might also be related to their heavy body weight, particularly in the last week of the rearing period. 3) The intake of BSFL was not taken into account in the enriched pens, which have resulted in lower Fl. Additionally, it can be speculated that the $5 \%$ of BSFL in the diet might have reduced appetite or digestibility of the diet (Ipema et al., 2020). It can be speculated that the level of enrichment, and particularly the height of the barriers was too much in the current study, resulting in a lower Fl and lower BWG. Whether or not a more balanced pen enrichment might have comparable stimulatory effects on activity, while maintaining performance, needs to be investigated.

Regarding the strain, in the current study, body weight and feed intake of fast growing chickens were higher than slower-growing broiler chickens on same ages, which is in accordance with previous studies (Pettit-Riley and Estevez, 2001; Bokkers and Koene, 2004; Benyi et al., 2010, 2015; Dixon, 2020). Due to a use of very young broiler breeders, body weight gain and BW at slaughter was relatively low (Peebles et al., 1999; Nasri et al., 2020), which might have resulted in a low prevalence of leg disorders as well (see below).

\section{Tibia Characteristics}

One of the most important underlying reasons for suboptimal bone development in broiler chickens is high growth rate, while low activity levels is the other one (Weeks et al., 2000; Williams et al., 2000; Bradshaw et al., 2002). The hypothesis of this study was that leg health and bone characteristics in broiler chickens can be improved through pen enrichment, which has previously been confirmed by several studies (Ventura and Siewer, 2012; Zhao et al., 2013; Riber et al., 2018; Pedersen et al., 2019, 2020). Focusing on bone properties, a higher activity has been found to positively affect tibia morphological, biophysical and mechanical characteristics of chickens (Hughes et al., 1993; Reiter and Bessei, 1998; Arnould et al., 2004; Stojcic and Bessei, 2009; Riber et al., 2018; Pedersen et al., 2020). A large distance between feed and water resulted in increased walking activity (Reiter and Bessei, 2009) and better tibia development in broiler chickens (Reiter and Bessei, 1998; Pedersen et al., 2020). Barrier perches resulted in improved tibia characteristics of laying hens (Hughes et al., 1993). Using sand as a dustbathing material and addition of strings for activity stimulation resulted in better bone development in fast growing 
broiler chickens (Arnould et al., 2004).

The results of the current study showed that tibia osseous volume, total volume and volume fraction of both fast and slower-growing broiler chickens and tibia pore volume of slower-growing chickens only were positively affected by pen enrichment, while most of the other tibia characteristics were slightly higher, but not significant. These findings are in agreement with previous studies, indicating the stimulating effects of pen enrichment on bone characteristics (sørensen et al., 2000; Toscano et al., 2013; Pedersen et al., 2020). Tibia characteristics were found correlated with leg disorders and locomotion. Chickens with advanced tibia characteristics showed better locomotion and less leg disorders (sørensen et al., 2000; Toscano et al., 2013). In the current study, it can be suggested that bone mineral deposition is the most stimulated physiological mechanism by pen enrichment, whereas tibia morphological and mechanical characteristics, such as tibia weight, length, strength and stiffness, were not affected. It can be hypothesized that stimulated activity due to pen enrichment particularly affects physiological pathways involved in ossification and mineralization, rather than affecting anatomical and physical tibia characteristics.

Regarding the strain, almost all tibia morphological, biophysical and mechanical characteristics in both body weight classes were higher in slower-growing chickens than in fast-growing chickens. These findings are in line with previous studies, indicating that slower-growing chickens demonstrate better bone characteristics in all ages compared to fast-growing chickens (Lilburn, 1994; Sullivian, 1994; Thorp and Waddington, 1997; Williams et al., 2000; Torres and Corver, 2018). Fast-growing chickens have more porous and less mineralized leg bones than slower-growing broiler chickens, which together with a higher body weight results in a higher risk of lameness (sullivian, 1994; Williams et al., 2000, 2004; Shim et al., 2012; Torres and Corver, 2018), impaired activity and locomotion (Lewis et al., 1997; Reiter and Bessei, 1998; Weeks et al., 2000; Cornetto and Estevez, 2001; Bokkers and Koene, 2004; Siegel et al., 2009) and more leg problems (Bokkers and Koene, 2004; Kjaer et al., 2006).

\section{Leg Disorders and Gait Score}

In the current study, the incidence of TD did not differ between pen enrichment, nor between strains, while other leg disorders (BCO, EPA and 
EPI) were hardly or not observed. These results might be explained by a relatively low stocking density $\left(10\right.$ chickens $\left./ \mathrm{m}^{2}\right)$, which is related to a low prevalence of leg disorders (Lillburn, 1994; Bessei, 2006; Buijs et al., 2009; Buijs et al., 2012). Additionally, BW of the chickens was relatively low, probably related to the use of offspring from young broiler breeders. $V \mathrm{~V}$ angulation in right legs was found to be higher in fast growing chickens than in slower-growing chickens. These results are in line with previous studies, indicating that slower-growing chickens have a relatively lower prevalence of VV compared to fast growing chickens (Haye and Simons, 1978; Hulan et al., 1980; Riddell, 1983). This might be explained by irregular and poor vascular morphology of the epiphyseal growth plate and insufficiently mineralized bones in fast growing, which both are potential causes of VV (Thorp, 1994; Siegel et al., 2009). Slower-growing chickens, on the contrary, have more time for bone mineralization, which compensates the lack of mineralization in the early growth phase, that loads less stress on the skeleton (Arnould et al., 2004; Sanchez-Rodriguez et al., 2019), and eventually result in a low incidence of VV. Despite the fact that $\mathrm{V} V$ angulation in right legs differed between strains in the current study, the maximal average angulation was $6.04^{\circ}$ and it can be disputed whether or not this degree of angulation can be considered as $\mathrm{VV}$ or as a leg disorder.

Better gait was found in slower-growing chickens than in fast growing chickens, both housed in non-enriched pens, while chickens of both strains in enriched pens had a gait score in between. It can be speculated that in fast growing chickens provided with sufficient enrichment, locomotion can be improved, but in case no enrichment is present, fast growing chickens show poorer gait scores than slower-growing chickens. Triggering the activity by pen enrichment might be more beneficial for fast growing chickens, since they have less advanced bone development and poorer leg health than slower-growing chickens (Kestin et al., 2001; Arnould et al., 2004; Toscano et al., 2013; Torres and Korver, 2018; Dixon, 2020; Rayner et al., 2020).

\section{Home Pen Behaviour and Use of Enrichment}

Results of home pen behaviour showed that broiler chickens in enriched pens demonstrated higher or a tendency to higher percentages of active behaviours (e.g., standing, walking, foraging) and lower 
percentages of passive behaviours (e.g., resting, sitting) than chickens in non-enriched pens. This is in accordance with previous studies, indicating that pen enrichment may stimulate physical activity. Placing horizontal platforms (Kaukonen et al., 2016; Pedersen and Forkman, 2019; Pedersen et al., 2020), angular ramps (Birgul et al., 2012) and barrier perches (Ventura et al., 2012; Zhao et al., 2013; Bench et al., 2016; Kaukonen et al., 2017) resulted in stimulated activity in broiler chickens. Using wooden boxes with peat for dust bathing, two platforms with ramps and two bales of peat as a pen enrichment resulted in more wing flapping, wing stretching, body shaking, ground scratching, ground pecking and foraging behaviours in fast growing broiler chickens compared to non-enriched pens (Vasdal et al., 2019). Scattering mealworms (Pichova et al., 2016) and Black Soldier fly larvae (Ipema et al., 2020) on the litter in fast-growing broiler chickens resulted in increased physical activity and locomotion. A large distance between feeder and drinker as a pen enrichment also resulted in a high percentage of active behaviours (Reiter and Bessei, 1996; Jordan et al., 2011; Jones et al., 2020). Different dustbathing materials, such as moss-peat have also been found to contribute to activity of broiler chickens (Riber et al., 2018).

In the current study, slower-growing broiler chickens demonstrated higher or tendency to higher percentages of active behaviours (e.g., standing, walking, foraging) and lower percentages of passive behaviours (e.g., resting, sitting) at all observation days and also on similar body weights (day 22 for fast growing chickens and day 29 for slower-growing chickens) than fast-growing chickens. In addition, use of enrichment objects differed between fast and slower-growing broiler chickens. The most attention-grabbing difference was observed in chickens perching on barriers. A considerably higher percentage of slower-growing chickens were found perching on barriers compared to fast growing chickens at all ages and also at the same body weight. Slower-growing chickens also showed a higher or a tendency to higher preference to go on ramps and platforms, while fast-growing chickens preferred to stay under the platforms and ramps. These findings are in in line with previous studies showing slower-growing chickens demonstrated more active behaviours than fast-growing chickens. Fast-growing broiler chickens showed higher percentages of time sitting idle and lower percentages of time standing and 
walking than slower-growing chickens (Lewis et al., 1997; Reiter and Bessei, 1998; Reiter and Kutritz, 2001; Bokkers and Koene, 2004). Slower-growing chickens have been found to use perches more than fast-growing chickens (Bokkers and Koene, 2004; Jordan et al., 20 1 1; Ventura et al., 2012; Groves and Muir, 2013; Yngvesson et al., 2017). It has been shown that fast-growing broiler chickens showed a preference for lying and sitting on the litter instead of using raised platforms and perches. All these findings might be due to the imbalance between high growth rate and immature bones (Bailie and O'Connell, 2016) of fast-growing chickens than slower-growing chickens, which negatively affects standing, walking and foraging behaviours. This makes that fast-growing chickens have more difficulties with barrier perches to access feed and water, to climb and go down on angular ramps than the slower-growing chickens. Another potential reason for these differences between strains might be related to body weight and heavy breast muscles. However, the current study clearly demonstrates that at the same BW class, still differences in activity related behaviours were present between the fast and slower-growing chickens, which suggests that other aspects than BW appears to play a role as well.

To conclude, in both fast and slower growing chickens, tibia biophysical characteristics were positively influenced by comprehensive pen enrichment, while tibia morphological and mechanical characteristics were not affected, suggesting that pen enrichment particularly affects physiological mechanisms related to ossification and mineralization. Slower-growing chickens showed better tibia characteristics, more locomotion and active behaviours than fast-growing chickens. Pen enrichment resulted in lower body weight gain in both fast and slower-growing chickens, which might be due to higher activity or lower feed intake as a result of difficulties of crossing the barrier perches. The relationship between tibia development and leg health remains unclear, because of the very low incidence of leg disorders in the current study. 


\section{ACKNOWLEDGEMENTS}

This experiment was the part of the "Healthy Bones" project, financed by a public-private partnership (TKI-AF-15203; BO-63-001-004). The financial support of the Ministry of Agriculture, Nature, and Food Quality (The Netherlands), Aviagen EPI, Darling Ingredients Inc., ForFarmers N.V., Hubbard, Marel Stork Poultry Processing BV, Nepluvi and Nutreco (The Netherlands) is gratefully acknowledged. The authors would like to thank Remco Hamoen for his expertise during the 3D micro-CT X-ray scanning. Bert van Nijhuis, Henny Reimert, llona van der Anker-Hensen, Bjorge Laurenssen and the animal caretakers and staff at Wageningen Bioveterinary Research (Lelystad, The Netherlands) are acknowledged for their assistance during the experiment. 


\section{REFERENCES}

Arnould C, Bizeray D, Faure JM, Leterrier C. 2004. Effects of the addition of sand and string to pens on use of space, activity, tarsal angulations and bone composition in broiler chickens. Animal welfare. 13: 87-94.

Bach MH, Tahamtani FM, Pedersen IJ, Riber AB. 2019. Effects of environmental complexity on behaviour in fast-growing broiler chickens. Applied animal behaviour science. 219:104840.

Bailie CL, O'Connell NE. 2016. Perch design preferences of commercial broiler chickens reared in windowed houses. In proceedings of EAAP $67^{\text {th }}$ annual meeting.

Balog JM, Bayyari GR, Rath NC, Huff WE, Anthony NB. 1997. Effect of intermittent activity on broiler production parameters. Poultry science. 76:6-12.

Bench CJ, Oryschak MA, Korver DR, Beltranena E. 2016. Behaviour, growth performance, foot pad quality, bone density, and carcass traits of broiler chickens reared with barrier perches and fed different dietary crude protein levels. Canadian journal of animal science. 97:268-280.

Benyi K, Acheampong-Boateng O, Norris D, Ligaraba TJ. 2010. Response of Ross 308 and Hubbard broiler chickens to feed removal for different durations during the day. Tropical animal health and production. 42:1421-1426.

Benyi K, Netshipale AJ, Mahlako KT, Gwata ET. 2015. Effect of genotype and stocking density on broiler performance during two subtropical seasons. Tropical animal health and production. 47:969-974.

Bessei W. 2006. Welfare of broilers: a review. Worlds poultry science journal. 62: 455-466.

Birgul OB. Mutaf S, Alkan S. 2012. Effects of different angled perches on leg disorders in broilers. Arch für geflügelkunde. 76:44-48.

Bizeray D, Estevez I, Leterrier C, Faure JM. 2002. Effects of increasing environmental complexity on the physical activity of broiler chickens. Applied animal behaviour science. 79:27-41.

Blatchford RA, Archer GS, Mench JA. 2012. Contrast in light intensity, rather than day length, influences the behavior and health of broiler chickens. Poultry science. 91:1768-1774.

Bokkers EA, Koene P. 2004. Motivation and ability to walk for a food reward in fast and slow-growing broilers to 12 weeks of age. Behavioural processes. 67: 21-130.

Bradshaw RH, Kirkden RD, Broom DM. 2002. A review of the aetiology and pathology of leg weakness in broilers in relation to welfare. Avian and poultry biology reviews. 13:45104.

Buijs S, Keeling L, Rettenbacher S, Van Poucke E, Tuyttens FAM. 2009. Stocking density effects on broiler welfare: Identifying sensitive ranges for different indicators. Poultry science. 88:1536-1543.

Buijs S, van Poucke E, Van Dongen S, Lens L, Baert J, Tuyttens FA. 2012. The influence of stocking density on broiler chicken bone quality and fluctuating asymmetry. Poultry science. 91:1759-1767.

Cornetto T, Estevez I. 2001. Behavior of the domestic fowl in the presence of vertical panels. Poultry science. 80:1455-1462.

De Jong IC, Gunnink H. 2019. Effects of a commercial broiler enrichment programme with or without natural light on behaviour and other welfare indicators. Animal. 13:384-391.

Dixon LM. 2020. Slow and steady wins the race: The behaviour and welfare of commercial faster growing broiler breeds compared to a commercial slower growing breed. PLoS one. 15:e0231006.

EFSA panel on animal health and welfare. 2010. Scientific opinion on the influence of genetic parameters on the welfare and the resistance to stress of commercial broilers. EFSA journal. 8:1666.

Gocsik É, Silvera AM, Hansson H, Saatkamp HW, Blokhuis HJ. 2017. Exploring the economic potential of reducing broiler lameness. British poultry science. 58:337-347.

González-Cerón F, Rekaya R, Aggrey SE. 2015. Genetic analysis of bone quality traits and growth in a random mating broiler population. Poultry science. 94:883-889.

Grandin T. 2010.Auditing animal welfare at slaughter plants. Meat science. 86:56-65. 
Groves PJ, Muir WI. 2013. Use of perches by broiler chickens in floor pen experiments. In: Proceedings of the IX European Symposium on Poultry Welfare. p17-20.

Güz BC, Molenaar R, De Jong IC, Kemp B, Van Krimpen M, Van Den Brand H. 2020. Effects of eggshell temperature pattern during incubation on tibia characteristics of broiler chickens at slaughter age. Poultry science. 99:3020-3029.

Güz BC, Molenaar R, De Jong IC, Kemp B, Van Krimpen M, Van den Brand H. 2021. Effects of green light emitting diode light during incubation and dietary organic macro and trace minerals during rearing on tibia characteristics of broiler chickens at slaughter age. Poultry science. 100:707-720.

Hall AL. 2001. The effect of stocking density on the welfare and behaviour of broiler chickens reared commercially. Animal welfare. 10: 23-40.

Havenstein GB, Ferket PR, Qureshi MA. 2003. Growth, livability, and feed conversion of 1957 versus 2001 broilers when fed representative 1957 and 2001 broiler diets. Poultry science. 82:1500-1508.

Havenstein GB, Ferket PR, Scheideler SE, Larson BT. 1994. Growth, livability, and feed conversion of 1957 vs 1991 broilers when fed "typical" 1957 and 1991 broiler diets. Poultry science. 73:1785-1794.

Haye U, Simons PCM. 1978. Twisted legs in broilers. British poultry science. 19:549-557.

Hughes BO, Wilson S, Appleby MC, Smith SF. 1993. Comparison of bone volume and strength as measures of skeletal integrity in caged laying hens with access to perches. Research in veterinary science. 54:202-206.

Hulan HW, Proudfoot F, Ramey D, McRae KB. 1980. Influence of genotype and diet on general performance and incidence of leg abnormalities of commercial broilers reared to roaster weight. Poultry science. 59:748-757.

Ipema AF, Bokkers EA, Gerrits WJ, Kemp B, Bolhuis JE. 2020. Long-term access to live black soldier fly larvae (Hermetia illucens) stimulates activity and reduces fearfulness of broilers, without affecting health. Scientific reports. 10:1-13.

Ipema AF, Gerrits WJ, Bokkers EA, Kemp B, Bolhuis JE. 2020. Provisioning of live black soldier fly larvae (Hermetia illucens) benefits broiler activity and leg health in a frequency-and dose-dependent manner. Applied animal behavioural science. 230: p105082.

Jones PJ, Tahamtani, FM, Pedersen IJ, Niemi JK, Riber AB. 2020. The Productivity and Financial Impacts of Eight Types of Environmental Enrichment for Broiler Chickens. Animals. 10: 378-392.

Jordan D, Stuhec I, Bessei W. 2011 . Effect of whole wheat and feed pellets distribution in the litter on broilers' activity and performance. Arch für geflügelkunde. 75: 98-103.

Jungmann R, Schitter G, Fantner GE, Laver ME, Hansma PK, Thurner PJ. 2007. Real-time microdamage and strain detection during micromechanical testing of single trabeculae. In: Experimental and Applied Mechanics: SEM Annual Conference and Exposition. p0-11.

Kaukonen E, Norring M, Valros A. 2016. Effect of litter quality on foot pad dermatitis, hock burns and breast blisters in broiler breeders during the production period. Avian pathology. 45:667-673.

Kaukonen E, Norring M, Valros A. 2017. Perches and elevated platforms in commercial broiler farms: use and effect on walking ability, incidence of tibial dyschondroplasia and bone mineral content. Animal. 11:864-871.

Kestin SC, Gordon S, SU G, Sørensen P. 2001. Relationships in broiler chickens between lameness, liveweight, growth rate and age. Veterinary record. 148:195-197.

Kestin SC, Knowles TG, Tinch AE, Gregory NG. 1992. Prevalence of leg weakness in broiler chickens and its relationship with genotype. Veterinary record. 131:190-194.

Kestin SC, SU G, Sørensen P. Different commercial broiler crosses have different susceptibilities to leg weakness. Poult Sci. 1999; 78: 1085-1090.

Kjaer JB, Su G, Nielsen BL, Sørensen P. 2006. Foot pad dermatitis and hock burn in broiler chickens and degree of inheritance. Poultry science. 85:1342-1348.

Knowles TG, Kestin SC, Haslam SM, Brown SN, Green LE, Butterworth A. 2008. Leg disorders in broiler chickens: prevalence, risk factors and prevention. PLOS one. 3:1545. 
Lewis PD, Perry GC, Farmer LJ, Patterson RLS. 1997. Responses of two genotypes of chicken to the diets and stocking densities typical of UK and 'Label Rouge production systems: I. Performance, behaviour and carcass composition. Meat science. 45:501-516.

Lilburn MS. 1994. Skeletal growth of commercial poultry species. Poult science. 73:897-903.

Martrenchar A, Huonnic D, Cotte JP, Boilletot E, Morisse JP. 2000. Influence of stocking density, artificial dusk and group size on the perching behaviour of broilers. British poultry science. 41:125-130.

McKay JC, Barton NF, Koerhuis ANM, McAdam J. 2000. The challenge of genetic change in the broiler chicken. BSAP occasional publications. 27:1-7.

Mench J. 2004. Lameness. In: Measuring and auditing broiler welfare. p3-17.

Morris MP. 1993. National survey of leg problems. Pigs and Poultry. 6:16.

Nasri H, Van den Brand H, Najjar T, Bouzouaia M. 2020. Interactions between egg storage duration and broiler breeder age on egg fat content, chicken organ weights, and growth performance. Poultry science. 99:4607-4615.

Novitskaya E, Chen PY, Hamed E, Jun L, Lubarda VA, Jasiuk I. 2011. Recent advances on the measurement and calculation of the elastic moduli of cortical and trabecular bone: a review. Theoretical and applied mechanics. 38:209-297.

Ohara A, Oyakawa C, Yoshihara Y, Ninomiya S, Sato S. 2015. Effect of environmental enrichment on the behavior and welfare of Japanese broilers at a commercial farm. The Journal of poultry science. p.0150034.

Pedersen IJ, Forkman B. 2019. Improving leg health in broiler chickens: a systematic review of the effect of environmental enrichment. Animal welfare. 28:215-230.

Pedersen IJ, Tahamtani FM, Forkman B, Young JF, Poulsen, HD, Riber AB. 2020. Effects of environmental enrichment on health and bone characteristics of fast growing broiler chickens. Poultry science. 99:1946-1955.

Peebles ED, Doyle SM, Pansky T, Gerard PD, Latour MA, Boyle CR. 1999. Effects of breeder age and dietary fat on subsequent broiler performance. 1. Growth, mortality, and feed conversion. Poultry science 78:505-511.

Petracci M, Cavani C. Muscle growth and poultry meat quality issues. Nutrients. 2012; 4: 1-12.

Pettit-Riley R, Estevez I. 2001. Effects of density on perching behavior of broiler chickens. Applied animal behaviour science. 71:127-140.

Pichova K, Nordgreen J, Leterrier C, Kostal L, Moe RO. 2016. The effects of food-related environmental complexity on litter directed behaviour, fear and exploration of novel stimuli in young broiler chickens. Applied animal behaviour science. 174:83-89.

Rayner AC, Newberry RC, Vas J, Mullan S. 2020. Slow-growing broilers are healthier and express more behavioural indicators of positive welfare. Scientific reports. 10:1-14.

Reiter K, Bessei W. 1998. Effect of locomotor activity on bone development and leg disorders in broilers. Archiv für geflügelkunde.

Reiter K, Bessei W. 2009. Effect of locomotor activity on leg disorder in fattening chicken. Berliner und münchener tierärztliche wochenschrift. 122:264-270.

Reiter K, Bessei W. 1996. Effect of the distance between feeder and drinker on behaviour and leg disorders of broilers. In: Proceedings of the $30^{\text {th }}$ international congress of the international society for applied ethology.

Reiter K, Bessei W. 1998. Possibilities to reduce leg disorders in broilers and turkeys. Archiv für geflügelkunde. 62:145-149.

Reiter K, Kutritz B. 2001. Behaviour and leg weakness in different broiler breeds. Archiv für geflügelkunde. 65:137-141.

Riber AB, van de Weerd HA, De Jong IC, Steenfeldt S. 2018. Review of environmental enrichment for broiler chickens. Poultry science. 97:378-396.

Riddell C. Pathology of the skeleton and tendons of broiler chickens reared to roaster weights. I. Crippled chickens. Avian diseases. 1983:950-962.

Riesenfeld A. 1972. Metatarsal robusticity in bipedal rats. American journal of physical anthropology. 36:229-233. 
Sanchez-Rodriguez E, Benavides-Reyes C, Torres C, Dominguez-Gasca N, Garcia-Ruiz Al, Gonzalez-Lopez S. 2019. Changes with age (from 0 to 37 D) in tibiae bone mineralization, chemical composition and structural organization in broiler chickens. Poultry science. 98: 5215-5225.

Sandilands V, Moinard C, Sparks NHC. 2009. Providing laying hens with perches: fulfilling behavioural needs but causing injury? British poultry science. 50:395-406.

Sherlock L, Demmers TGM, Goodship AE, McCarthy ID, Wathes CM. 2010. The relationship between physical activity and leg health in the broiler chicken. British poultry science. $51: 22-30$.

Shim MY, Karnuah AB, Mitchell AD, Anthony NB, Pesti GM, Aggrey SE. 2012. The effects of growth rate on leg morphology and tibia breaking strength, mineral density, mineral content, and bone ash in broilers. Poultry science. 91:1790-1795.

Siegel PB, Honaker CF, Rauw WM. 2009. Selection for high production in poultry. CABI Publishing: Wallingford, UK. p230-242.

Şimşek UG, Dalkilic B, Ciftci M, Cerci IH, Bahsi M. 2009. Effects of enriched housing design on broiler performance, welfare, chicken meat composition and serum cholesterol. Acta veterinaria brno. 78:67-74.

Sørensen P, SU G, Kestin SC. 2000. Effects of age and stocking density on leg weakness in broiler chickens. Poultry science. 79:864-870.

Stojcic MD, Bessei W. 2009. The effect of locomotor activity and weight load on bone problems in fast and slow growing chickens. Arch für geflügelkunde. 73:242-249.

Sullivan TW. 1994. Skeletal problems in poultry: estimated annual cost and descriptions. Poultry science. 73:879-882.

Tallentire CW, Leinonen I, Kyriazakis I. 2016. Breeding for efficiency in the broiler chicken: A review. Agronomy for sustainable development. 36:1-16.

Thorp BH, Waddington D. 1997. Relationships between the bone pathologies, ash and mineral content of long bones in 35-day-old broiler chickens. Research in veterinary science. 62:7-73.

Thorp BH. 1994. Skeletal disorders in the fowl: a review. Avian pathology. 23:203-236.

Torres CA, Korver DR. 2018. Influences of trace mineral nutrition and maternal flock age on broiler embryo bone development. Poultry science. 97:2996-3003.

Toscano MJ, Nasr MAF, Hothersall B. 2013. Correlation between broiler lameness and anatomical measurements of bone using radiographical projections with assessments of consistency across and within radiographs. Poultry science. 92:2251-2258.

Turner CH, Burr DB. 1993. Basic biomechanical measurements of bone: a tutorial. Bone. 14: 595-608.

Van der Pol CW, Molenaar R, Buitink CJ, Van Roovert-Reijink IAM, Maatjens CM, van den Brand H. 2015. Lighting schedule and dimming period in early life: consequences for broiler chicken leg bone development. Poultry science. 94:2980-2988.

Vasdal G, Vas J, Newberry RC, Moe RO. 2019. Effects of environmental enrichment on activity and lameness in commercial broiler production. Journal of applied animal welfare science. 22:197-205.

Ventura, B. A., F. Siewerdt, I. Estevez. 2012. Access to barrier perches improves behavior repertoire in broilers. PloS one. 7:29826.

Wallenbeck A, Wilhelmsson S, Jönsson L, Gunnarsson S, Yngvesson J. 2016. Behaviour in one fast growing and one slower-growing broiler (Gallus gallus domesticus) hybrid fed a high-or low-protein diet during a 10-week rearing period. Acta agriculturae scandinavica, section a - animal science. 66:168-176.

Weeks CA, Danbury TD, Davies HC, Hunt P, Kestin SC. 2000. The behaviour of broiler chickens and its modification by lameness. Applied animal behavioural science. 67:1 11-125.

Williams B, Solomon S, Waddington D, Thorp B, Farquharson C. 2000. Skeletal development in the meat-type chicken. British poultry science. 41:141-149.

Williams B, Waddington D, Murray DH, Farquharson C. 2004. Bone strength during growth: influence of growth rate on cortical porosity and mineralization. Calcified tissue international. 74:236-245. 
Yıldııım M, Taşkın A. The effects of environmental enrichment on some physiological and behavioral parameters of broiler chicks. Braz J Poult Sci. 2017; 19: 355-362.

Yıldız H, Petek M, Sönmez G, Arıcan I, Yılmaz B. 2009. Effects of lighting schedule and ascorbic acid on performance and tibiotarsus bone characteristics in broilers. Turkish journal of veterinary animal science. 33:469-476.

Yngvesson J, Wedin M, Gunnarsson S, Jönsson L, Blokhuis H, Wallenbeck A. 2017. Let me sleep! Welfare of broilers (Gallus gallus domesticus) with disrupted resting behaviour. Acta agriculturae scandinavica, section a - animal science. 67:123-133.

Zhao JP, Jiao HC, Jiang YB, Song ZG, Wang XJ, Lin H. 2013. Cool perches improve the growth performance and welfare status of broiler chickens reared at different stocking densities and high temperatures. Poultry science. 92:1962-1971.

Zuidhof MJ, Schneider BL, Carney VL, Korver DR, Robinson FE. 2014. Growth, efficiency, and yield of commercial broilers from 1957, 1978, and 2005. Poultry science. 93:2970-2982.

Zhao JP, Jiao HC, Jiang YB, Song ZG, Wang XJ, Lin H. 2013. Cool perches improve the growth performance and welfare status of broiler chickens reared at different stocking densities and high temperatures. Poultry science. 92:1962-1971.

Zuidhof MJ, Schneider BL, Carney VL, Korver DR, Robinson FE. 2014. Growth, efficiency, and yield of commercial broilers from 1957, 1978, and 2005. Poultry science. 93:2970-2982. 




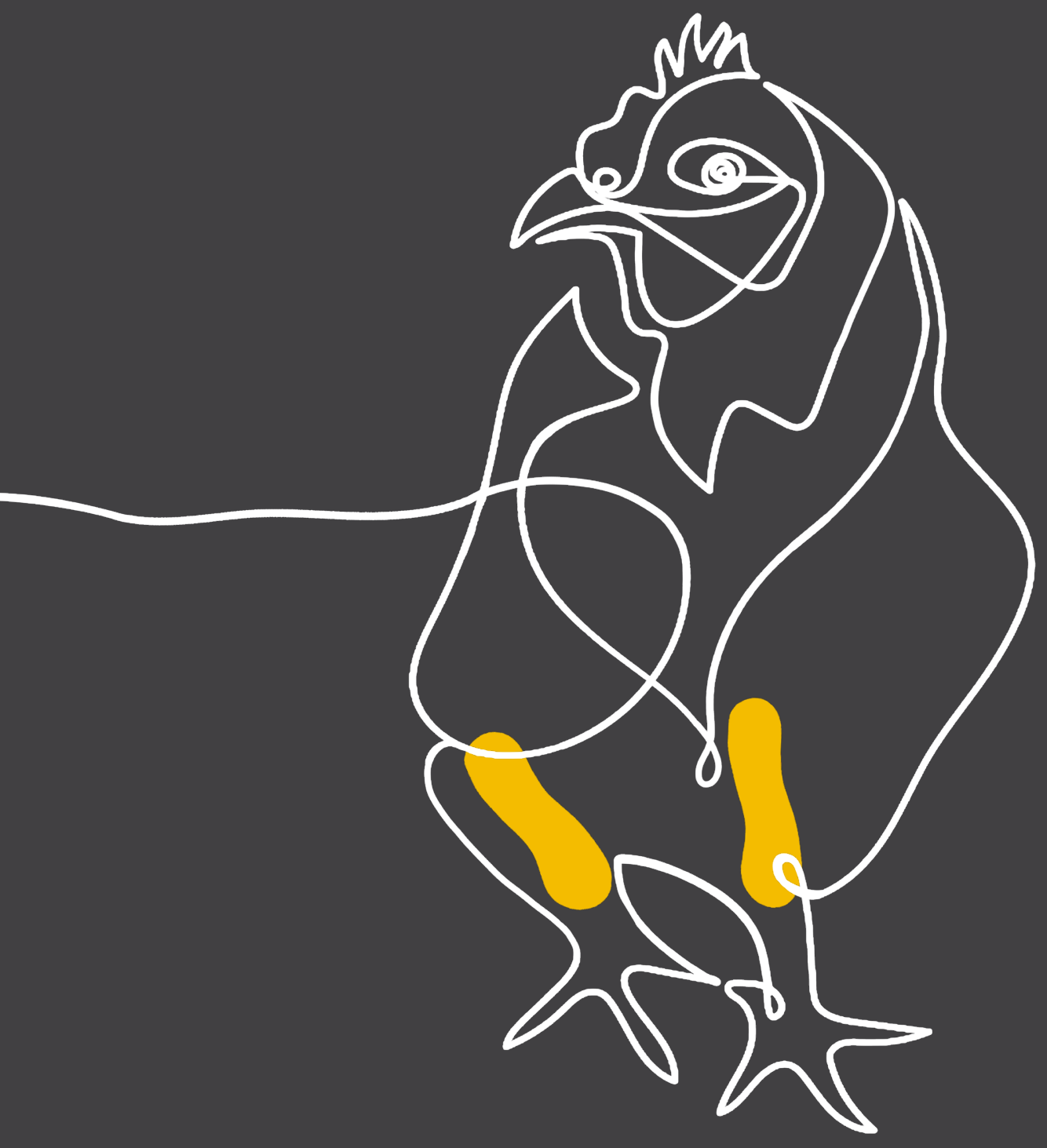

\section{GENERAL \\ DISCUSSION}


Content of General Discussion 


\section{INTRODUCTION}

\section{RECAPITULATION OF FIVE EXPERIMENTS}

\section{INVESTIGATED APPROACHES ON LEG DISORDERS}

\section{INVESTIGATED APPROACHES}

ON TIBIA CHARACTERISTICS

Dietary Organic Macro and Trace Minerals

Light and Temperature During Incubation

Environmental Enrichment

Fast-growing vs. Slower-growing Broiler Chickens

INVESTIGATED APPROACHES

ON LOCOMOTION AND

HOME PEN BEHAVIOUR

\section{INVESTIGATED APPROACHES ON GROWTH PERFORMANCE}

CONCLUSIONS

$\circ$

FUTURE RESEARCH OPPORTUNITIES 


\section{INTRODUCTION}

Suboptimal leg health observed in broiler chickens is nowadays recognized as a global issue (Bessei, 2006, 2018; Rios et al., 2020), since it is a major welfare problem (Kestin et al., 1992; Mckay et al., 2000; Bradshaw et al., 2002; Knowles et al., 2008; Tallentire et al., 2016; Granquist et al., 2019) and causes considerable financial losses (Sullivan, 1994; Kestin et al., 1999; Mench, 2004; Knowles et al., 2008; EFSA, 2010; Gocsik et al., 2017; Rios et al., 2020). Chickens having suboptimal leg health suffer from dehydration and starvation, because of having difficulties accessing feed and water, show less locomotor activity, spend long time sitting or lying and are likely to be distressed and experience pain. Major causes of suboptimal leg health are developmental leg abnormalities and infectious and/or non-infectious leg disorders, which all can result in lameness (Bradshaw et al., 2002; Knowles et al., 2008; Dawkins and Layłon, 2012; González-Cerón et al. 2015; Tallentire et al., 2016; Gocsik et al., 2017).

While there have been some improvements e.g., using high quality feed, healthcare monitoring, environmentally controlled poultry farms and obeying several welfare regulations and laws in a number of countries around the world, welfare problems caused by suboptimal leg health still exist in broiler chickens, which cannot be prevented by only focus on above-mentioned factors (Bradshaw et al., 2002; Bessei, 2006; Oviedo-Rondón et al., 2006; Waldenstedt, 2006).

There are several potential factors related to leg problems in broiler chickens e.g., leg bones, muscles, tendons and joints. The focus of this thesis was on leg bone development, specifically the tibia, since it is the major weight carrying and the most loaded and affected leg bone during the growth period (Nairn and Watson, 1972; Julian, 1998, 2005; Dibner et al., 2007).

The aim of this thesis was to investigate different approaches to improve leg health by focusing on tibia characteristics and other leg health-related parameters of fast and/or slower-growing broiler chickens. Three different approaches were taken into account: (1) dietary factors (source of macro and trace minerals) on broiler chickens (Chapter 1 and Chapter 2) or indirectly via the broiler breeders on offspring broilers (Chapter 3); (2) incubation conditions (light and temperature; Chapter 2 and Chapter 4) and (3) environmental (pen) enrichment (Chapter 5). 
In General Introduction, an overview on suboptimal leg health, bone abnormalities and leg disorders in broiler chickens and their welfare and economic consequences were discussed. Thereafter, bone quality measurements and potential approaches to improve leg bone health in broiler chickens were introduced.

In Chapter 1, effects of different dietary factors (organic macro and trace minerals, fish oil, hydrolyzed collagen) of fast-growing broiler chickens on leg health related parameters were described; in Chapter 2, effects of dietary inorganic and organic macro and trace minerals separately on leg health related parameters of fast-growing broiler chickens were described; in Chapter 3, a study was performed, but in this case inorganic or organic macro and trace minerals in the diet of fast and slower-growing broiler breeders on leg health related parameters of their offspring were described; in Chapter 2, also effects of the use of light (darkness vs. green LED light) during incubation on leg health related parameters of fastgrowing broiler chickens were taken into account; in Chapter 4, effects of eggshell temperature pattern during incubation on leg health of fastgrowing broiler chickens were described; and in Chapter 5, effects of pen enrichment on leg health related parameters of fast and slower-growing broiler chickens were studied.

Male broiler chickens were used in all experiments, since male broiler chickens show more leg health problems due to their higher body weights than females (Mulder et al., 2009; Demuner et al., 2017; Kryeziv et al., 2018).

In the following sections, objectives and main findings from these five experimental chapters are summarized and how the investigated approaches individually or together give insight in and improve our understanding of leg health of broiler chickens are discussed. This chapter ends with main conclusions and future research opportunities. 


\section{RECAPITULATION OF FIVE EXPERIMENTS}

This thesis includes five experiments (Chapter 1, 2, 3, 4 and 5) comprising different approaches and experimental designs with the aim of improving leg health in broiler chickens. The objectives and main findings of five experiment are given in Table 1.

Table 1 Objectives and main findings of five experiments described in this thesis

\begin{tabular}{|c|c|c|}
\hline & Objectives & Main findings \\
\hline $\begin{array}{l}\frac{\bar{d}}{\overline{0}} \\
\frac{\mathrm{d}}{\mathrm{O}}\end{array}$ & $\begin{array}{l}\text { to investigate effects of different } \\
\text { dietary factors (fish oil, hydrolyzed } \\
\text { collagen, a combination of organic } \\
\text { macro and trace minerals) in the diet } \\
\text { of fast-growing broiler chickens on } \\
\text { their; } \\
\text { - tibia morphological, biophysical } \\
\text { and mechanical characteristics } \\
\text { (at day } 28,35 \text { and } 42 \text { ) } \\
\text { - growth performance } \\
\text { - locomotion } \\
\text { - leg disorders } \\
\text { - bone development related blood } \\
\text { parameters } \\
\text { - home pen behaviour }\end{array}$ & $\begin{array}{l}\text { - organic macro and trace minerals in the } \\
\text { diet positively affected tibia } \\
\text { characteristics, while fish oil and } \\
\text { collagen were non-effective } \\
\text { - hydrolyzed collagen and organic macro } \\
\text { and trace minerals in the diet positively } \\
\text { affected growth performance } \\
\text { - better locomotion was achieved by } \\
\text { organic macro and trace minerals fed } \\
\text { broiler chickens }\end{array}$ \\
\hline$\frac{N}{\frac{N}{0}}$ & $\begin{array}{l}\text { to investigate effects of green } \\
\text { LED light (16L:8D) during incubation } \\
\text { and organic macro and trace } \\
\text { minerals separately in the diet of } \\
\text { fast-growing broiler chickens on } \\
\text { their; } \\
\text { - tibia morphological, biophysical } \\
\text { and mechanical characteristics } \\
\text { (at day 42) } \\
\text { - growth performance } \\
\text { - locomotion } \\
\text { - leg disorders } \\
\text { - home pen behaviour }\end{array}$ & $\begin{array}{l}\text { - only dietary organic macro minerals } \\
\text { positively affected most of stimulated } \\
\text { tibia morphological, biophysical and } \\
\text { mechanical characteristics } \\
\text { - green LED light during incubation, } \\
\text { dietary organic macro minerals and } \\
\text { organic trace minerals resulted in better } \\
\text { growth performance compared to } \\
\text { darkness during incubation, inorganic } \\
\text { macro minerals, and inorganic trace } \\
\text { minerals, respectively } \\
\text { - green LED light during incubation } \\
\text { resulted in worse locomotion } \\
\text { - leg disorders were not affected in any } \\
\text { of groups }\end{array}$ \\
\hline
\end{tabular}


Table 1 (continued)

\begin{tabular}{|c|c|c|}
\hline & Objectives & Main findings \\
\hline 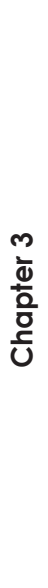 & $\begin{array}{l}\text { to investigate effects of a } \\
\text { combination of organic macro } \\
\text { and trace minerals in the diet of } \\
\text { fast- and slower-growing broiler } \\
\text { breeders' diet on their offspring } \\
\text { broilers'; } \\
\text { - tibia morphological, biophysical } \\
\text { and mechanical characteristics } \\
\text { (at body weight class of } 1700 \text { and } \\
2600 \text { grams) } \\
\text { - mineral concentrations } \\
\text { in egg and hatchling } \\
\text { - leg disorders } \\
\text { - home pen behaviour }\end{array}$ & $\begin{array}{l}\text { - organic macro and trace minerals in } \\
\text { broiler breeder diet positively affected } \\
\text { growth performance and tibia } \\
\text { characteristics of the offspring in } \\
\text { slower-growing chickens. However, this } \\
\text { effect was hardly seen in fast-growing } \\
\text { chickens } \\
\text { - effects on mineral concentrations in } \\
\text { the egg and hatchling were limited, } \\
\text { which suggests mineral availability } \\
\text { appears to play a role in other ways } \\
\text { than via absolute mineral } \\
\text { concentrations }\end{array}$ \\
\hline & $\begin{array}{l}\text { to investigate effects of egg shell } \\
\text { temperature in week } 2\left(37.8^{\circ} \mathrm{C} \text { or }\right. \\
\left.38.9^{\circ} \mathrm{C}\right) \text { and in week } 3\left(36.7^{\circ} \mathrm{C} \text { or }\right. \\
\left.37.8^{\circ} \mathrm{C}\right) \text { of incubation of fast-growing } \\
\text { broiler chickens on their; } \\
\text { - tibia morphological, biophysical } \\
\text { and mechanical characteristics } \\
\text { (at day } 41 \text { and 42) } \\
\text { - body weight } \\
\text { - locomotion } \\
\text { - leg disorders }\end{array}$ & $\begin{array}{l}\text { - } 38.9^{\circ} \mathrm{C} \text { during the second week of } \\
\text { incubation stimulated tibia } \\
\text { morphological, biophysical and } \\
\text { mechanical characteristics } \\
\text { compared to } 37.8^{\circ} \mathrm{C} \\
\text { - incubation temperature of } 38.9^{\circ} \mathrm{C} \text { in } \\
\text { week } 2 \text {, followed by } 37.8^{\circ} \mathrm{C} \text { in week } 3 \\
\text { of incubation resulted in the most } \\
\text { advanced tibia characteristics } \\
\text { - body weight, locomotion and leg } \\
\text { disorders were not affected in any of } \\
\text { groups }\end{array}$ \\
\hline 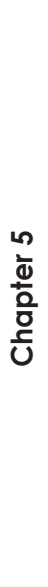 & $\begin{array}{l}\text { to investigate effects of a } \\
\text { combination of different forms of } \\
\text { pen enrichment on in fast- and } \\
\text { slower-growing broiler chickens on } \\
\text { their; } \\
\text { - tibia morphological, biophysical } \\
\text { and mechanical characteristics } \\
\text { (at body weight class of } 1400 \text { and } \\
2200 \text { grams) } \\
\text { - growth performance } \\
\text { - locomotion } \\
\text { - leg disorders } \\
\text { - home pen behaviour }\end{array}$ & $\begin{array}{l}\text { - tibia biophysical characteristics were } \\
\text { positively influenced by } \\
\text { comprehensive pen enrichment, } \\
\text { while tibia morphological and } \\
\text { mechanical characteristics were not } \\
\text { affected } \\
\text { - slow-growing chickens showed } \\
\text { better tibia characteristics, more } \\
\text { locomotion and active life } \\
\text { behaviours than fast-growing } \\
\text { chickens } \\
\text { - pen enrichment resulted in lower } \\
\text { body weight gain in both fast- and } \\
\text { slower-growing chickens }\end{array}$ \\
\hline
\end{tabular}

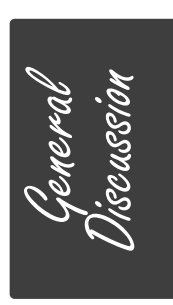


Investigated Approaches on Leg Disorders 


\section{Info box 1}

Because the point of origin of this thesis was suboptimal leg health, leg abnormalities and leg disorders, first effects of different approaches on leg disorders will be discussed.

Effects of investigated approaches on tibia characteristics, locomotion, home pen behaviour and growth performance will be discussed later.

There have been several degenerative, developmental and infectious leg bone abnormalities and disorders reported in broiler chickens (Bradshaw et al., 2002; Shim et al., 2012; Guo et al, 2019; Crespo, 2020), which have been attributed to their one-sided genetic selection towards fast growth and feed efficiency (Lewis and Hurnik, 1990; Kestin et al., 1992; Weeks et al., 2000; Corr et al., 2003; Bessei, 2006, 2018; Kittelsen et al., 2017).

In this thesis, varus valgus deformation (VV) (Chapter 1, 3 and 5), tibia dyschondroplasia (TD) (Chapter 3, 4 and 5), food pad dermatitis (FPD), bacterial chondronecrosis with osteomyelitis (BCO), epiphyseal plate abnormalities (EPA) and epiphysiolysis (EPI) (Chapter 2, 3, 4 and 5) were assessed. Because (1) they are commonly observed leg bone abnormalities and disorders and (2) they can be reduced through optimized leg bone development. Additionally, rotated tibia (RT) and hock burn (HB) were assessed in Chapter 4.

Effects of replacement of a combination of inorganic macro and trace minerals by their organic varieties in the diet of fast-growing broiler chickens and in the diet of fast and slower-growing broiler breeders on $\mathrm{VV}$ at 28, 35 and 42 days of age of fast-growing broiler chickens (Chapter 1) or offspring broilers on 1700 and 2600 gram BW classes (Chapter 3) were investigated. VV was assessed by a veterinarian and scored as present or not and each (small) angulation from straight was scored as present. Results of these two studies showed that no significant differences were observed between treatment groups. However, VV has been found considerably high in all groups in Chapter 1, ranging from 63 to 70 $\%$, while it has been found relatively low, ranging from 14 (1700 gram BW class) to 28 (2600 gram BW class) \% in Chapter 3. Because it is disputable which deviation in angulation from straight can be considered as VV,

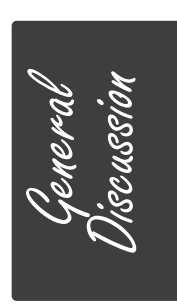


in Chapter $\mathbf{5}$ another method of scoring $\mathrm{VV}$ was used. After fixating the legs at the hip joint to stretch the leg, the angle between the tibia and the metatarsus for both legs were measured, using a digital goniometer.

Effects of a combination of ramps, platforms, barrier perches, large distance between feed and water and live Black Soldier fly larvae in the moss-peat dust bathing area as pen enrichment were investigated on $\mathrm{VV}$ of fast and slower-growing broiler chickens in Chapter 5. At the 1400 gram BW class, VV angulation was not affected by enrichment or strain (on average $\left.4.90^{\circ}\right)$. However, at the 2200 gram BW class, slower-growing chickens had a lower $V V$ on their right legs than fast-growing chickens $\left(3.80^{\circ}\right.$ vs $6.04^{\circ}$, respectively, $\left.P=0.003\right)$. $V V$ on left legs was not affected by enrichment or strain at this BW class (on average $5.88^{\circ}$ ). These results, which are supported by better tibia characteristics of slower-growing chickens, might be explained by high growth rate and immature bones of fast-growing broiler chickens compared to slower-growing chickens.

Considering all studies described in this thesis on the assessment method of VV, several conclusions can be drawn: (1) Despite organic macro and trace minerals in broiler or breeder diet resulted in improvements in tibia characteristics, they had no influence on VV, potentially due to the method used in these studies (Chapter 1 and 3). Further investigation is needed with a novel method used in Chapter $\mathbf{5}$ or another method which needs to be developed in future. (2) VV should not be assessed by subjective observation, but by objectively measuring the angulation between the tibia and metatarsus.

Tibia dyschondroplasia in Chapter 3, 4 and 5; food pad dermatitis, bacterial chondronecrosis with osteomyelitis, epiphyseal plate abnormalities and epiphysiolysis in Chapter 2, 3, 4 and 5; and rotated tibia and hock burn in Chapter $\mathbf{4}$ have been assessed. The incidence of all these leg disorders was found very low in all studies.

Considering all studies described in this thesis on leg disorders, several conclusions can be drawn: (1) Because of marginal results of assessed leg disorders in this thesis, influences of organic macro and trace minerals, incubation conditions and pen enrichment on leg disorders and also relationship among tibia characteristics, locomotion, body weight and leg 
disorders remained inconclusive. (2) Because of relatively small sample size, low stocking density and better management factors in experiments compared to commercial broiler farms, occurrences of above-mentioned leg disorders might have been found marginal. (3) Assessment methods can be quite subjective and may differ between persons. (4) Since these leg disorders have been taken into consideration in genetic selection applications (claimed by current broiler breeding companies e.g., Aviagen and Hubbard) to keep up with welfare schemes and to raise financial gain, the incidence of these leg disorders and diseases may have been reduced in current broiler strains. 
Investigated Approaches on Tibia Characteristics 


\section{Info box 2}

In Chapter 1, palm oil and soybean oil were partly replaced by fish oil in the FISH group. Soybean meal was partly replaced by hydrolyzed collagen in the COL group. Because fish oil and hydrolyzed collagen in the diet did not influence any of tibia characteristics, locomotion, leg disorders and home pen behaviour, results related to these nutrients were only discussed in Chapter 1.

\section{Dietary Organic Macro and Trace Minerals}

Macro minerals (calcium and phosphorus) (Driver et al., 2005; Angel, 2007; Létourneau-Montminy et al., 2008) and trace minerals (iron, copper, manganese, zinc and selenium) (Richards, 1997, 2010; Angel, 2007; Dibner et al., 2007) in the diet of broiler chickens have been demonstrated to play important roles on bone development and consequently leg health, since these minerals are main contributors to bone formation and mineralization as a component of bone matrix, as an essential part of enzymes involved in bone development or as an essential part of hormones or growth factors that are involved in regulating bone development. A deficiency of any of these minerals might result in retarded bone growth, strength, morphology and density (Dibner et al., 2007; Létourneau-Montminy et al., 2008; Li et al., 2016, 2020).

Concentrations of these minerals in broiler chickens and broiler breeders' diet have been addressed in a number of studies by the aim of improving leg health. However, studies on the form of these minerals in the diet of broiler and broiler breeder chickens were limited. In Chapter 1, 2 and $\mathbf{3}$, effects of the source of these minerals (inorganic vs. organic) in broiler or broiler breeder diets were investigated regarding their effects on tibia morphological, biophysical and mechanical characteristics (directly or via the breeder on their offspring).

In Chapter 1, effects of replacement of a combination of inorganic macro and trace minerals by their organic varieties in diet were investigated on tibia morphological, biophysical and mechanical characteristics at 42 days of age of fast-growing broiler chickens. Results of this study showed that a combination of organic macro and trace minerals resulted in improvements in most of the tibia characteristics. 
Figure 1A describes relative differences (\%) between selected tibia characteristics investigated in Chapter 1. In this figure, tibia characteristics at 42 days of age of chickens fed with diet containing a combination of inorganic macro and trace minerals were set at $100 \%$. Chickens fed with a diet containing a combination of organic macro and trace minerals had 2.2\% higher tibia proximal length, $2.5 \%$ higher lateral cortex thickness, $20.0 \%$ higher tibia mineral content, $23.3 \%$ higher mineral density, $10.1 \%$ higher ultimate strength, $8.0 \%$ higher stiffness and $5.9 \%$ higher energy to fracture than chickens fed with diet containing inorganic minerals.

Chapter 1 showed that all tibia biophysical and mechanical characteristics of broiler chickens were significantly affected by a combination of organic macro and trace minerals, while tibia morphological characteristics were slightly higher, but not significant. These results are in line with previous studies, indicating that organic minerals in the diet of broiler chickens stimulated tibia characteristics (Starcher et al., 1980; Wang et al., 2008, 2019; Oviedo-Rondón et al., 2006; Ferket et al., 2009; Zhao et al., 2010; El-Husseiny et al., 2012). However these studies investigated trace minerals only, meaning that it remains unclear which mineral group (macro minerals, trace minerals or their combination) is most effective on these bone characteristics. Therefore, to reveal the more effective mineral group (macro or trace minerals or their combination) on tibia characteristics of broiler chickens, inorganic and organic macro and trace minerals separately were studied in broiler diets in Chapter 2.

Chapter 2 showed that organic macro minerals had a strong effect on bone development compared to inorganic minerals, while trace mineral source hardly affected bone development. Figure 1B describes relative differences (\%) between selected tibia characteristics investigated in Chapter 2. Results of inorganic macro and trace minerals were set at 100\%. Chickens fed with a diet containing organic macro minerals had 2.2 $\%$ higher tibia proximal length, $6.5 \%$ higher osseous volume, $15.6 \%$ higher tibia mineral content, $37.0 \%$ higher mineral density, $4.2 \%$ higher ultimate strength, $5.5 \%$ higher stiffness and $4.2 \%$ higher energy to fracture than chickens fed with diet containing inorganic macro minerals. Chickens fed with a diet containing organic trace minerals had $1.6 \%$ higher tibia 
proximal length, while other tibia characteristics did not differ from chickens fed with a diet containing inorganic trace minerals. Almost all tibia morphological, biophysical and mechanical characteristics of broiler chickens fed with diet containing organic macro minerals were significantly affected, while no significant difference was found on any of tibia characteristics of broiler chickens fed with diet containing organic trace minerals.

Based on the combination of Chapter 1 and Chapter 2, it can be suggested that in the diet of broiler chickens, particularly the source of macro minerals seems to play a major role in bone development, rather than the source of trace minerals. Considering this difference, several conclusions can be drawn: (1) Bone development might need more available $\mathrm{Ca}$ and $\mathrm{P}$ rather than trace minerals since the $\mathrm{Ca}$ and $\mathrm{P}$ are known as major components for bone matrix and bone formation (Rath et al., 1999; Li et al., 2020), while trace minerals are known as supportive components on bone mineralization (Jianhua et al., 2000; Dibner et al., 2007; Flis et al., 2019). (2) Concentrations of macro minerals in current fast-growing broiler chickens' diet might be insufficient for optimal bone development.

As it has been found that organic minerals via the diet of broiler chickens can affect tibia characteristics, it can be speculated that the same might occur when organic minerals are fed to broiler breeders (Bao and Choct, 2009; Świątkiewicz et al., 2014; Torres and Korver, 2018; Wang et al., 2019), since calcium mobilization, bone mineralization and growth plate differentiation in the embryo begin during the first week of incubation (Blom and Lilja, 2005; Oviedo-Rondon et al., 2009). Towards the last days of incubation and first days after hatch, phosphorus and trace minerals have been found to be very low in the residual yolk (Ono and Tuan, 1991; Duan et al., 2013; Yair et al., 2013), which might be considered as a limitation for bone development of chickens after hatch. This suggests that availability of pre-packaged macro and trace minerals in the egg are important for embryonic bone development and later life bone health (Kidd et al., 1992; Dibner et al., 2007). 


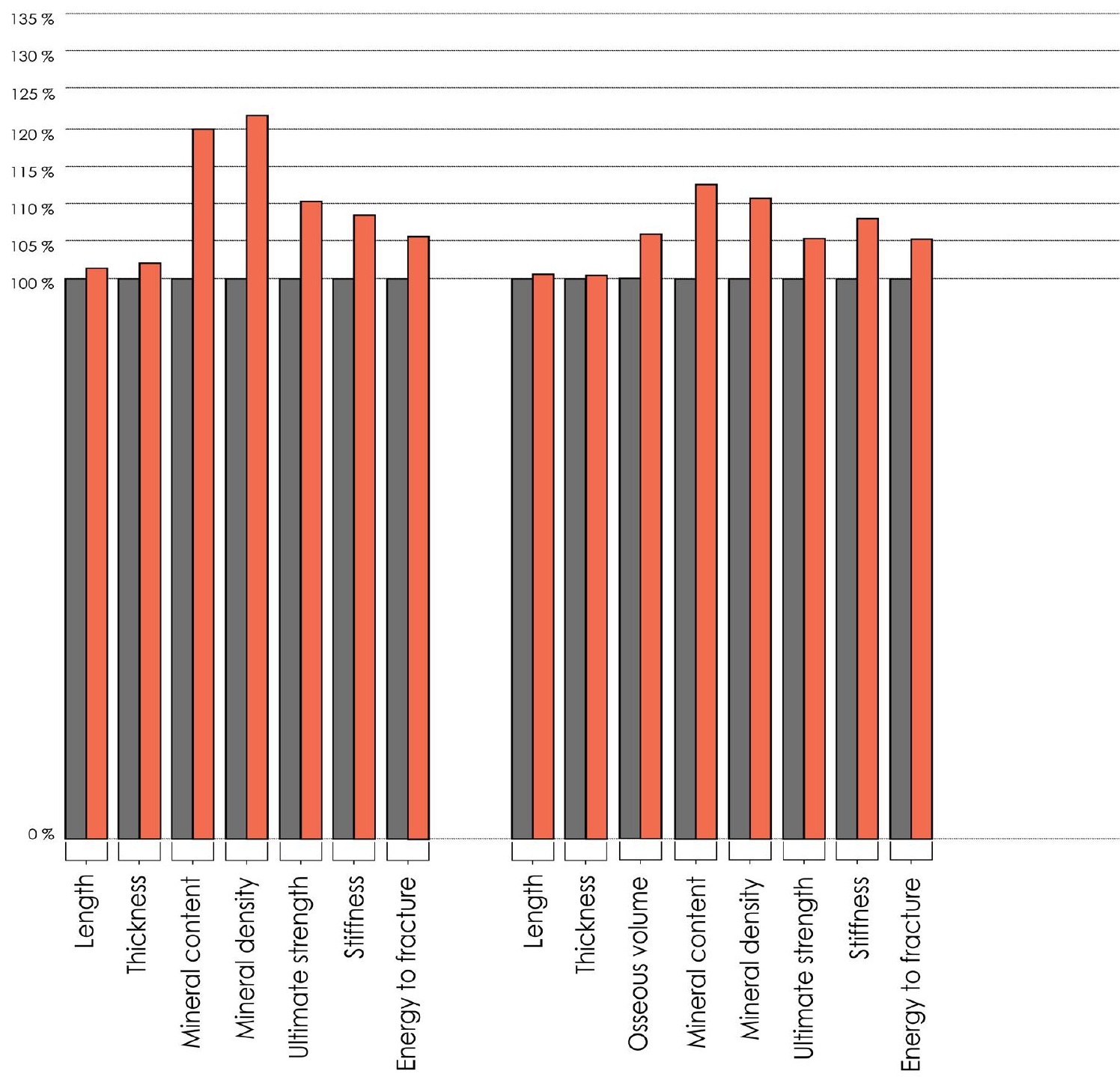

\section{Chapter 1}

\section{Figure 1A}

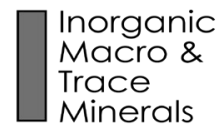

\section{Chapter 3}

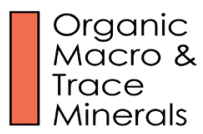

Relative differences (\%) between selected tibia morphological, biophysical and mechanical characteristics of fast-growing chickens fed with a diet containing a combination of organic or inorganic macro and trace minerals (Chapter1) and fast-growing chickens originating from broiler breeders fed with a diet containing a combination of organic or inorganic macro and trace minerals (Chapter 3 ). Chickens fed with diet containing a combination of inorganic macro and trace minerals were set at $100 \%$. 


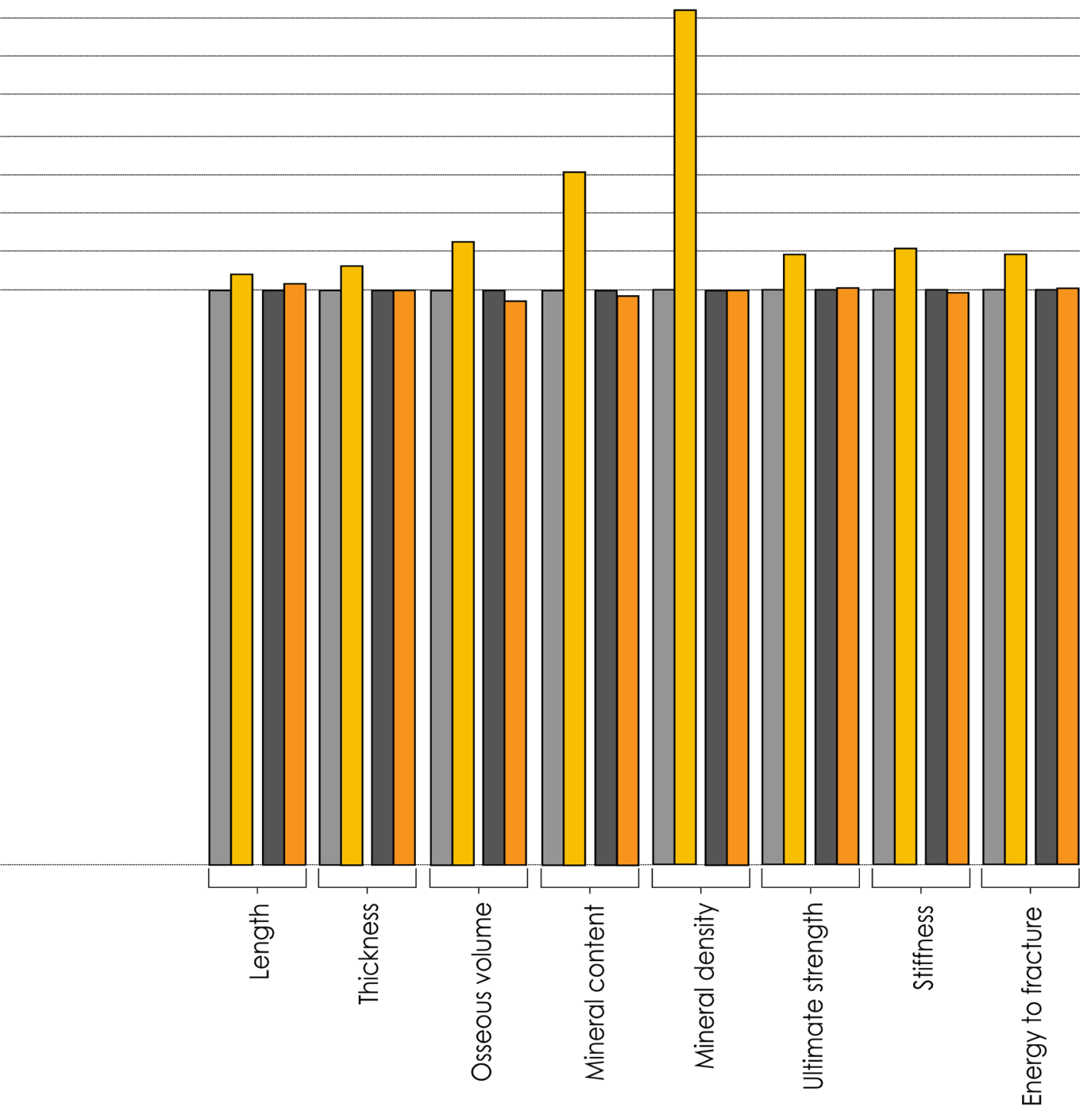

Chapter 2

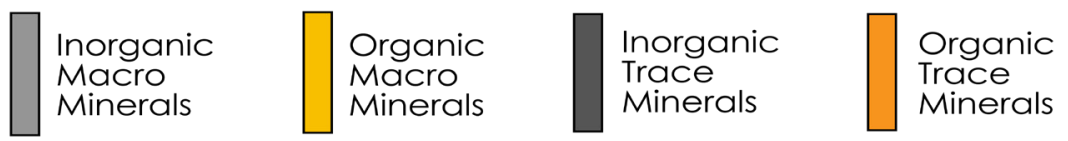

Figure 1B

Relative differences (\%) between selected tibia morphological, biophysical and mechanical characteristics of fast-growing chickens fed with a diet containing organic or inorganic macro and trace minerals separately (Chapter 2). Chickens fed with diet containing inorganic macro and trace minerals separately were set at $100 \%$. 
The mineral concentrations and availabilities in the diet of broiler breeders can influence mineral deposition in the egg. Replacing inorganic trace minerals by their organic varieties in the diet of broiler breeders resulted in improved embryonic tibia and femur characteristics, specifically mineralization and calcification (Favero et al., 2013). Organic trace minerals in the diet of laying hens resulted in higher mineral concentrations in the liver, the medullary bone and blood serum (Yenice et al., 2015). However, previous studies have shown that increasing the concentration of inorganic macro and trace minerals in the diet of broiler breeder or laying hens hardly influenced their concentrations in the egg. It appears that the amounts of minerals in the egg have certain limits, which is mainly determined by the genetic background of the breeder (Naber, 1979; Angel, 2007; Ratriyanto et al., 2018). Replacing inorganic macro and trace minerals by their organic varieties in the diet of broiler breeder might be an alternative way to influence mineral availability in the egg and consequently in the offspring. This has not been investigated until the study described in Chapter 3 .

In Chapter 3, trans-generational carry-over effects of inorganic or organic macro and trace minerals was of particular interest. Replacement of a combination of organic macro and trace minerals by their organic varieties in the diet of fast and slower-growing broiler breeders were investigated on mineral concentrations in egg and hatchling, and offspring broilers' tibia morphological, biophysical and mechanical characteristics at 1700 and 2600 gram BW classes.

\section{Info box 3}

Effects of replacement of a combination of organic macro and trace minerals by their organic varieties in the diet of broiler breeders on their offspring broilers are discussed in this section. Comparison between selected tibia characteristics of fast and slower-growing chickens regardless the mineral source in breeder diet will be discussed later in the "Fast-growing vs. Slower-growing Chickens" section.

Chapter $\mathbf{3}$ showed that only small differences in concentrations of macro and trace minerals in egg and hatchling were found after feeding inorganic or organic macro and trace minerals to the breeders. Fe and Se concentrations were found higher in the mixture of yolk and albumen, whereas Cu was found lower after feeding organic minerals to the 
breeders compared to their inorganic forms. The other minerals ( $\mathrm{Ca}, \mathrm{P}$, $\mathrm{Mn}, \mathrm{Zn}$ ) were not influenced by mineral source in the breeder diet nor by broiler breeder strain. Based on these results, it can be concluded that effects of mineral sources in the breeders' diet on concentrations in the eggs and hatchlings appears to be marginal. Since effects on BW (which will be discussed later) and tibia characteristics have been found (see below), it can be speculated that other mechanisms than only mineral concentration appears to play a role in trans-generational mineral transfer in broiler chickens.

Regarding tibia characteristics, Chapter $\mathbf{3}$ showed that a combination of inorganic macro and trace minerals in the diet of broiler breeders resulted in better tibia characteristics, particularly in slower-growing chickens, but these effects hardly observed in fast-growing chickens. Figure $1 \mathrm{~A}$ shows relative differences (\%) between selected tibia characteristics of only fast-growing chickens investigated in Chapter $\mathbf{3}$ (for comparison with results of Chapter 1 and 2), while Figure 2 shows relative differences (\%) of selected tibia characteristics between fast and slower-growing broiler chickens investigated in Chapter 3.

In Figure 2, fast and slower-growing broiler chickens originating from broiler breeders fed with a diet containing a combination of inorganic macro and trace minerals were set at $100 \%$.

Fast-growing chickens originating from breeders fed with diet containing a combination of organic macro and trace minerals had $6.2 \%$ higher osseous volume, $14.5 \%$ higher tibia mineral content, $10.3 \%$ higher mineral density, $5.2 \%$ higher ultimate strength, $8.4 \%$ higher stiffness and 5.0 $\%$ higher energy to fracture than fast-growing chickens originating from breeder fed with diet containing inorganic minerals.

Slower-growing chickens originating from breeder fed with diet containing a combination of organic macro and trace minerals had $27.7 \%$ higher lateral cortex thickness, $18.7 \%$ higher osseous volume, $8.2 \%$ higher tibia mineral content, $44.8 \%$ higher mineral density, $5.3 \%$ higher ultimate strength, $5.2 \%$ higher stiffness and $6.6 \%$ higher energy to fracture than slower-growing chickens originating from breeder fed with diet containing inorganic minerals. 
Almost all tibia morphological, biophysical and mechanical characteristics of slower-growing offspring broilers were significantly affected, while particularly tibia biophysical and mechanical characteristics of fast-growing offspring broilers were slightly higher, but not significant. However, because only the combination of macro and trace mineral sources was used in Chapter 3, whether or not trans-generational carry-over effect of organic macro minerals, trace minerals or their combination are most effective remained unclear and needs further investigation.

Regarding the results of tibia characteristics in Chapter 3, several conclusions can be drawn: (1) Mineral source in the breeder diet is important for offspring leg bone development. (2) Despite the fact that concentrations of minerals in the eggs and hatchlings originated from organic and inorganic minerals fed broiler breeders hardly differed, it can be speculated that trans-generational mineral availability in offspring appears to play a role via other mechanisms than via absolute mineral concentrations, but these mechanisms are currently unclear. (3) Because organic macro and trace minerals in the broiler breeder diet showed positive effects on tibia characteristics in slower-growing chickens, whereas this effect was hardly seen in fast-growing chickens, this might suggest that the difference in feed intake between fast and slower-growing broiler breeders might affect the mineral intake. (4) Current slower-growing broiler breeder diets might be suboptimal in mineral concentrations. (5) Trans-generational mineral availability in slower-growing chickens might be more effective on bone development than in fast-growing chickens, which might be related to time available for bone development.

Considering effects of minerals in Chapter 1, 2 and $\mathbf{3}$ on tibia morphological, biophysical and mechanical characteristics, several conclusions can be drawn: (1) Dietary organic minerals result in higher bone mineralization in broiler chickens than inorganic minerals. (2) Trans-generational mineral availability in offspring broilers appears to play a role via other mechanisms than via absolute mineral concentrations in the egg. (3) Slower-growing chickens appears to benefit more from organic minerals than fast-growing chickens in the breeder diet, which might be related to the time available for bone development or to the current mineral levels in the breeder diets. (4) It appears that the source of macro minerals 


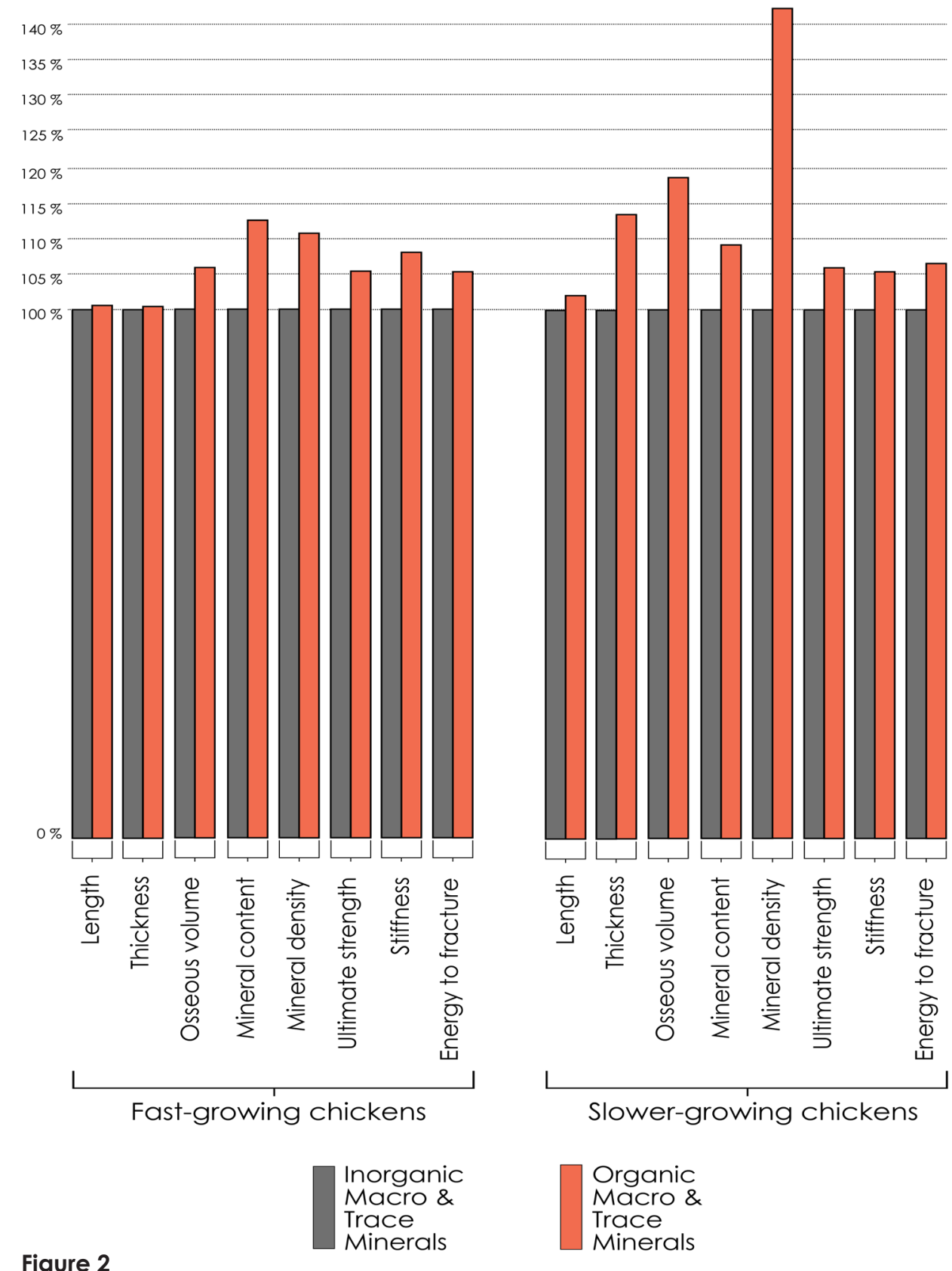

Relative differences (\%) between selected tibia morphological, biophysical and mechanical characteristics of fast and slower-growing chickens at BW class of 2600 gram (38 and 49 days of age for fast and slower-growing chickens, respectively) originating from broiler breeders fed with a diet containing a combination of organic or inorganic macro and trace minerals. Chickens fed with diet containing a combination of inorganic macro and trace minerals were set at $100 \%$. Presented data belongs to Chapter 3.

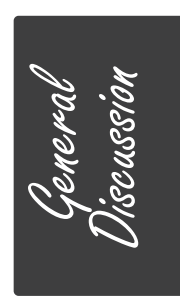


in the diet of fast-growing broiler chickens seems to more effective on tibia characteristics than trace minerals. (5) Tibia biophysical characteristics in broilers seem to respond stronger to the source of minerals both directly in the broiler diet or indirectly via the broiler breeder diet than morphological and mechanical characteristics.

\section{Light and Temperature During Incubation}

In this thesis, effects of green LED light in Chapter 2 and effects of eggshell temperature pattern in Chapter $\mathbf{4}$ during incubation on leg health parameters e.g., tibia morphological, biophysical and mechanical characteristics, leg disorders and locomotion of broiler chickens were investigated.

\section{Green LED light during incubation}

Chickens have an ability to see a large spectrum of colours including ultraviolet light due to having special extraretinal photoreceptors in their eyes and brains (Lewis and Morris, 2000; Rozenboim et al., 2004; Archer, 2017, 2018). They show a high sensitivity to blue, green and red light (Prescott and Wathes, 1999; Archer et al., 2009; Huth and Archer, 2015). Previous studies have shown that white and red light compared to complete darkness during incubation resulted in improved chick quality at hatch and green light during incubation resulted in improved growth performance (Archer, 2017; Yu et al., 2018).

While designing the experiment described in Chapter 2, green LED light during incubation was taken into account, since no study investigated effects of green light during incubation on tibia characteristics of broiler chickens.

Green light during incubation have been shown to positively affect growth performance (comparable results on growth performance have been found in this study as well and will be discussed later) and post-hatch pectoral muscle growth by enhancing proliferation and differentiation of satellite cells during embryonic development and post hatch period (Rozenboim et al., 2004; Halevy et al., 2006; Zhang et al., 2014, 2016), higher liver weight, higher antioxidant activity and higher melatonin levels (Zhang et al., 2014), higher plasma growth hormone, prolactin and melatonin (Archer et al., 2009; 
Huth and Archer, 2015; Archer and Mench, 2017), higher hypothalamic growth hormone releasing hormone, growth hormone receptor and insulin-like growth factor-1 of broiler chickens (Dishon et al., 2017).

Results of Chapter 2 showed that green LED light during incubation has no influence on any of tibia morphological, biophysical and mechanical characteristics at slaughter age, which suggest that despite the fact that stimulating effects of green light on growth performance was found, it does not seem sufficient to stimulate bone development.

\section{Temperature during incubation}

Despite the eggshell temperature for optimal embryonic development of broiler chickens has been determined as $37.8^{\circ} \mathrm{C}$ throughout incubation, a higher or lower eggshell temperatures than this optimal temperature in specific phases of incubation might have a positive effect as well on chick quality (Lourens et al., 2005; Tona et al., 2005; Maatjens et al., 2017; Wijnen et al., 2020), which might also include embryonic bone development and later life leg health of broiler chickens (Hammond et al., 2007; Oviedo-Rondón et al., 2008, 2009; Shim and Pesti, 201 1; Van der Pol et al., 2014). There have been several studies (Yalçın et al., 2007; Oviedo-Rondón et al., 2009; Aygün and Narinç, 2016) conducted on embryonic bone development by making adjustments in temperature (lower or higher) during the different time intervals of incubation, since embryonic bone development starts with cartilage formation in the first week of incubation (Nakane and Tsudzuki, 1999; Atalgin and Kürtül, 2009; Groves and Muir, 2014), which is followed by growth plate differentiation and rapid increase in mineralization from the second week of incubation onward (Ballock and O'keefe, 2003; Hammond et al., 2007; Oviedo-Rondón et al., 2008), which reaches the highest level just before hatch till a few days post-hatch (Applegate and Lilburn, 2002; Yalçın et al., 2007; OviedoRondón et al., 2008).

However, a high temperature during the second week of incubation followed by a lower temperature during the third week of incubation on tibia characteristics of broiler chickens has not been investigated until the study described in Chapter 4. This chapter described effects of eggshell temperature pattern in week $2\left(37.8^{\circ} \mathrm{C}\right.$ or $\left.38.9^{\circ} \mathrm{C}\right)$ and week $3\left(36.7^{\circ} \mathrm{C}\right.$ or

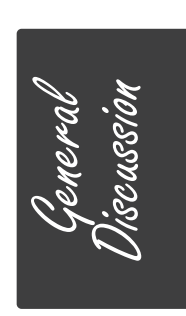


$37.8^{\circ} \mathrm{C}$ ) of incubation on tibia characteristics of fast-growing broiler chickens at 41 and 42 days of age. Figure 3 describes relative differences (\%) between selected tibia characteristics investigated in Chapter 4. In this figure, results of selected tibia characteristics of chickens incubated at eggshell temperature of $37.8^{\circ} \mathrm{C}$ throughout the incubation were set at $100 \%$.

Chickens incubated at an eggshell temperature of $38.9^{\circ} \mathrm{C}$ in the second week of incubation had $3.2 \%$ higher lateral cortex thickness, $19.5 \%$ higher osseous volume, $4.4 \%$ higher mineral content, $6.9 \%$ higher mineral density, $5.6 \%$ higher ultimate strength, $6.0 \%$ higher stiffness and $5.8 \%$ higher energy to fracture than chickens incubated at eggshell temperature of $37.8^{\circ} \mathrm{C}$. Chickens incubated at eggshell temperature of $36.7^{\circ} \mathrm{C}$ in third week of incubation had $1.6 \%$ lower lateral cortex thickness, $1.1 \%$ lower osseous volume, $1.9 \%$ higher mineral content, 4.3 \% lower ultimate strength, $4.9 \%$ lower stiffness and $4.6 \%$ lower energy to fracture than chickens incubated at eggshell temperature of $37.8^{\circ} \mathrm{C}$. Results of the study described in Chapter 4 showed that $1.1^{\circ} \mathrm{C}$ higher eggshell temperature than optimal in the second week of incubation stimulated post-hatch tibia morphological, biophysical and mechanical characteristics of broiler chickens at slaughter age. However, a $1.1^{\circ} \mathrm{C}$ lower eggshell temperature in third week of incubation negatively affected post-hatch tibia characteristics. These results suggest that a lower eggshell temperature $\left(36.7^{\circ} \mathrm{C}\right)$ in the last week of incubation is not effective on bone development, while maintaining the optimal eggshell temperature $\left(37.8^{\circ} \mathrm{C}\right)$ during the last week of incubation may result in better bone development compared to a low eggshell temperature.

Considering effects of incubation factors in Chapter $\mathbf{2}$ and $\mathbf{4}$ on tibia morphological, biophysical and mechanical characteristics, several conclusions can be drawn: (1) Green LED light during incubation has no effect on tibia characteristics. (2) An eggshell temperature of $38.9^{\circ} \mathrm{C}$ compared to $37.8^{\circ} \mathrm{C}$ in the second week of incubation stimulated most of the tibia morphological, biophysical and mechanical characteristics of broiler chickens at slaughter age. (3) An eggshell temperature of $36.7^{\circ} \mathrm{C} \mathrm{com-}$ pared to $37.8^{\circ} \mathrm{C}$ has no effect on tibia characteristics. 


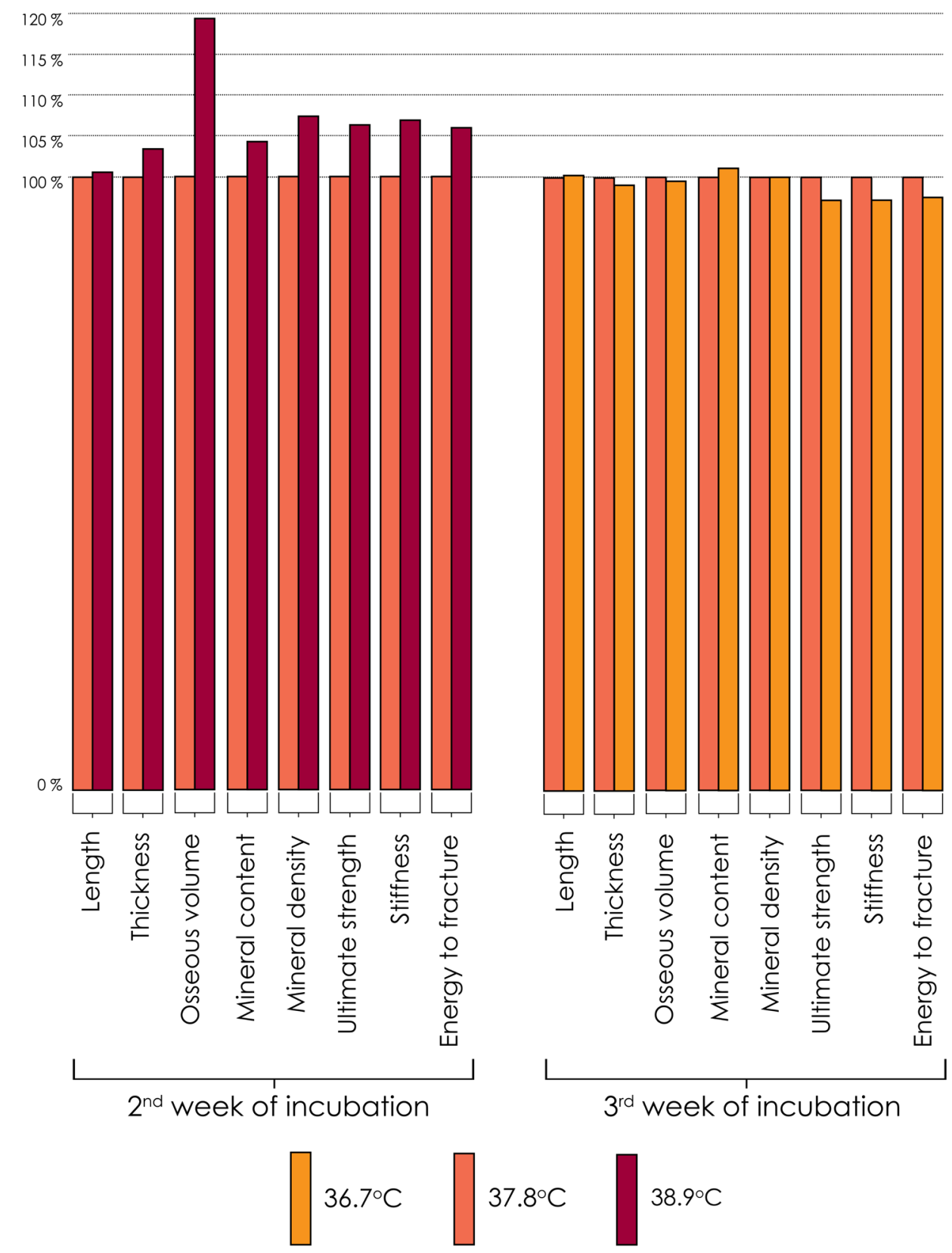

Figure 3

Relative differences (\%) between selected tibia morphological, biophysical and mechanical characteristics of fast-growing broiler chickens incubated with eggshell temperature of $37.8^{\circ} \mathrm{C}$ or $38.9^{\circ} \mathrm{C}$ throughout the second week of incubation followed by $36.7^{\circ} \mathrm{C}$ or $37.8^{\circ} \mathrm{C}$ throughout the third week of incubation. An EST of $37.8^{\circ} \mathrm{C}$ throughout incubation was used as reference and set at $100 \%$. Presented data belongs to Chapter 4. 


\section{Environmental Enrichment}

Broiler chickens have been shown to spend approximately $80 \%$ of their lifespan with passive behaviours e.g., lying, sitting and resting, due to their suboptimal leg health (Lewis and Hurnik, 1990; Kestin et al., 1992; Weeks et al., 2000; Corr et al., 2003; Bessei, 2006; Kittelsen et al., 2017) and impaired locomotion (Kestin et al., 1992; Weeks et al., 2000), which all negatively affects the welfare. Environmental enrichment might be a promising approach to stimulate activity and consequently enhance leg health of broiler chickens (Reiter and Bessei, 2009; Blatchford et al., 2012; Ohara et al., 2015; Kaukonen et al., 2016; Pedersen and Forkman, 2019; Pedersen et al., 2020). There have been several studies conducted by the aim of stimulating the activity of broiler chickens. However, these studies included simple pen enrichment e.g., only barrier perches (Pettit-Riley and Estevez, 2001; Bizeray et al., 2002; simşek et al., 2009), mirror, ball, perch and dust (each material in another pen) (YIldirm and Taşkın, 2017), scattering mealworms (Pichova et al., 2016) and Black Soldier fly larvae on the litter (Ipema et al., 2020). A plethora of different pen enrichment materials in both fast and slower-growing chickens has not been investigated until the study described in Chapter 5.

In Chapter 5, effects of a combination of ramps, platforms, barrier perches, large distance between feed and water and live Black Soldier fly larvae in the moss-peat dust bathing area as a pen enrichment were investigated on tibia morphological, biophysical and mechanical characteristics of fast and slower-growing broiler chickens. Effects of pen enrichment is discussed in this section. Figure 4 describes relative differences (\%) between selected tibia characteristics of fast and slowergrowing chickens investigated in Chapter 5. Fast and slower-growing broiler chickens in non-enriched pens were set at $100 \%$.

\section{Info box 4}

Effects of pen enrichment are discussed in this section. Comparison between selected tibia characteristics of fast and slower-growing chickens regardless the pen enrichment will be discussed later in "Fastgrowing vs. slower-growing chickens" section. 

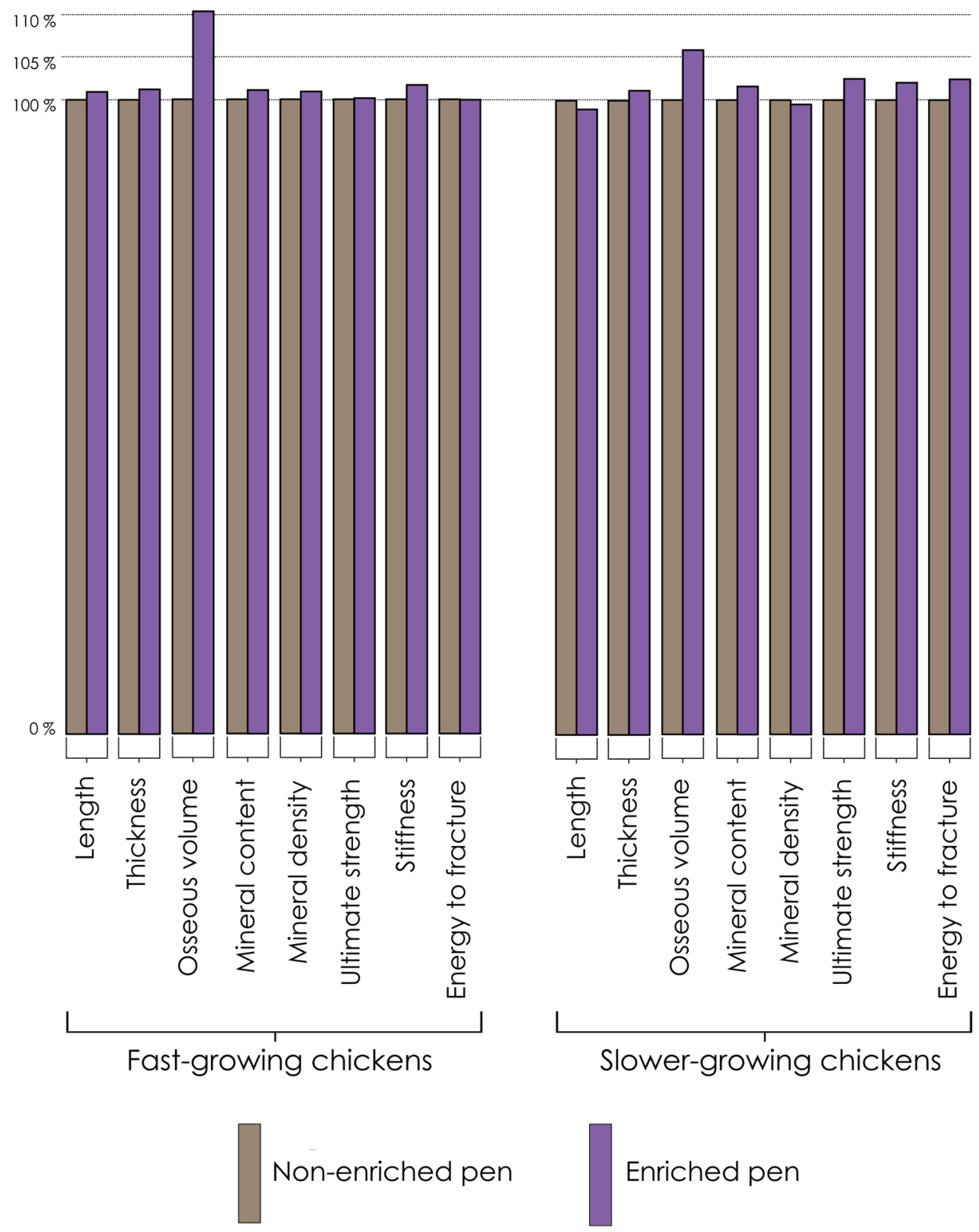

\section{Figure 4}

Relative differences (\%) between selected tibia morphological, biophysical and mechanical characteristics of fast-growing broiler chickens in enriched or nonenriched pens at BW class of 2200 gram (38 and 49 days of age for fast and slowergrowing chickens, respectively). Fast and slower-growing broiler chickens in nonenriched pens were set at $100 \%$. Presented data belongs to Chapter 5. 
Fast-growing chickens in enriched pens had $1.6 \%$ higher tibia proximal length, $1.5 \%$ higher lateral cortex thickness, $10.8 \%$ higher osseous volume, $2.9 \%$ higher mineral density and $2.6 \%$ higher stiffness than fastgrowing chickens in non-enriched pens.

Slower-growing chickens in enriched pens had $1.3 \%$ lower tibia proximal length, $1.5 \%$ higher lateral cortex thickness, $5.3 \%$ higher osseous volume, $5.7 \%$ higher tibia mineral content, $2.6 \%$ lower mineral density, 2.2 $\%$ higher ultimate strength, $1.5 \%$ higher stiffness and $1.3 \%$ higher energy to fracture than slower-growing chickens in non-enriched pens.

Previous research showed that pen enrichment resulted in stimulated tibia characteristics (Reiter and Bessei, 2009; Ylldz et al., 2009; Buijs et al., 2012), probably due to increased physical activity and locomotion. However, these studies investigated basic tibia properties e.g., bone length, weight and breaking strength.

In Chapter 5, in-depth tibia characteristics, including osseous volume, pore volume and total volume, were assessed. Results of the study described in Chapter $\mathbf{5}$ showed that tibia biophysical characteristics, particularly osseous volume, total volume and volume fraction, of both fast and slower-growing broiler chickens were positively influenced by comprehensive pen enrichment, while tibia morphological and mechanical characteristics were not significantly affected in fast-growing chickens and only mechanical characteristics were found slightly higher in slower-growing chickens.

Regarding the results of tibia characteristics in Chapter 5, several conclusions can be drawn: (1) Bone mineral deposition is the most stimulated physiological mechanism by pen enrichment, while most of the other tibia characteristics did not differ. (2) Because growth performance was negatively affected by pen enrichment (will be discussed later), this might reduce the heavy load pressure on leg bones and contribute tibia characteristics compared to heavier chickens in non-enriched pens. 


\section{Fast-growing vs. Slower-growing Broiler Chickens}

Fast growth rate and body weight gain in fast-growing broiler chickens are known to be directly associated with several health and welfare issues e.g., suboptimal leg health, impaired locomotion and lameness (Bradshaw et al., 2002; Bessei, 2006; Knowles et al., 2008; Meluzzi and Sirri, 2009; Tallentire et al., 2016). Slower-growing broiler chickens has come into prominence nowadays, since they have better developed bones, show more active behaviours, lower levels of sitting/lying on the litter, a lower occurrence of leg disorders and leg disorders, a lower percentage of lameness and lower mortality than fast-growing broiler chickens. Potential reasons might be that (1) the imbalance between high growth rate and immature bones is higher in fast-growing broiler chickens compared to slower-growing chickens (Sullivian, 1994; Bokkers et al., 2004; Williams et al., 2004; Kjaer et al., 2006; Stojcic and Bessei, 2009; Shim et al., 2012) and (2) the speed of bone development is unable to keep up with this rapidly increased body weight in fast-growing broiler chickens, which is more balanced in slower-growing broiler chickens (Bokkers and Koene, 2004; Siegel et al., 2009; Shim et al., 2012; Torres and Corver, 2018).

Despite the fact that leg health and welfare of slower-growing broiler chickens have been found better than of fast-growing broiler chickens, there was no research comparing tibia characteristics of fast and slower-growing broilers side by side within the same experimental design. Considering this lack of knowledge in literature, in Chapter 3 and Chapter 5, slower-growing broiler chickens (Hubbard JA 757) and fastgrowing broiler chickens (Ross 308) were compared.

\section{Info box 5}

Comparison between tibia characteristics of fast and slower-growing chickens regardless the other experimental factors (mineral source in broiler breeder diet in Chapter 3; pen enrichment in Chapter 5) is discussed in this section. Effects of mineral source in broiler breeder diet was discussed in the "Dietary Organic Macro and Trace Minerals" and effects of pen enrichment was discussed in "Environmental Enrichment".

Chapter 3 showed that slower-growing chickens demonstrated better tibia morphological, biophysical and mechanical characteristics compared to fast-growing chickens at both 1700 and 2600 gram body

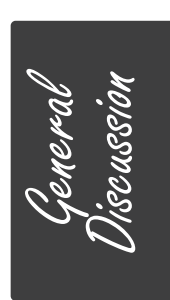


weight classes.

Figure 5 describes relative differences (\%) between selected tibia characteristics of fast and slower-growing chickens investigated in Chapter 3. In this figure, results of selected tibia characteristics of fast-growing broiler chickens (originating from breeders fed with inorganic macro and trace minerals) at BW class of 2600 gram (38 and 49 days of age for fast and slower-growing chickens, respectively) were fixed as constant percentages of $100 \%$. Slower-growing chickens had $1.8 \%$ higher tibia proximal length, $11.4 \%$ higher lateral cortex thickness, $30 \%$ higher osseous volume, $25.6 \%$ higher mineral content, same level of mineral densities, 6.8 \% higher ultimate strength, $12.3 \%$ higher stiffness and $7.5 \%$ higher energy to fracture than fast-growing chickens.

Chapter 5 showed that slower-growing chickens demonstrated better tibia morphological, biophysical and mechanical characteristics compared to fast-growing chickens at both 1400 and 2200 gram body weight classes. Figure 5 describes relative differences (\%) between selected tibia characteristics investigated in Chapter 5. In this figure, results of selected tibia characteristics of fast-growing chickens (in non-enriched pens) at BW class of 2600 gram (38 and 49 days of age for fast and slowergrowing chickens, respectively) were set at $100 \%$. Slower-growing chickens had $7.1 \%$ higher tibia proximal length, $1.5 \%$ higher lateral cortex thickness, $31 \%$ higher osseous volume, $2.5 \%$ higher mineral content, $17.6 \%$ higher mineral density, $6.2 \%$ higher ultimate strength, $9.2 \%$ higher stiffness and 7.6 $\%$ higher energy to fracture than fast-growing chickens.

In both Chapter 3 and Chapter 5, remarkable differences were observed between fast and slower-growing broiler chickens, particularly in biophysical characteristics, which is followed by mechanical characteristics and morphological characteristics, respectively.These results are in line with previous research, indicating that a negative correlation between growth rate and bone development has been found. The main reason is that mechanisms involved in bone development cannot keep up with fast growth speed in fast-growing broiler chickens, while slower growth provides more time for bone mineralization in slower-growing broiler chickens, which might compensate for the lack of mineralization in the early growth phase (Shim et al., 2012; Sanchez-Rodriguez et al., 2019). 


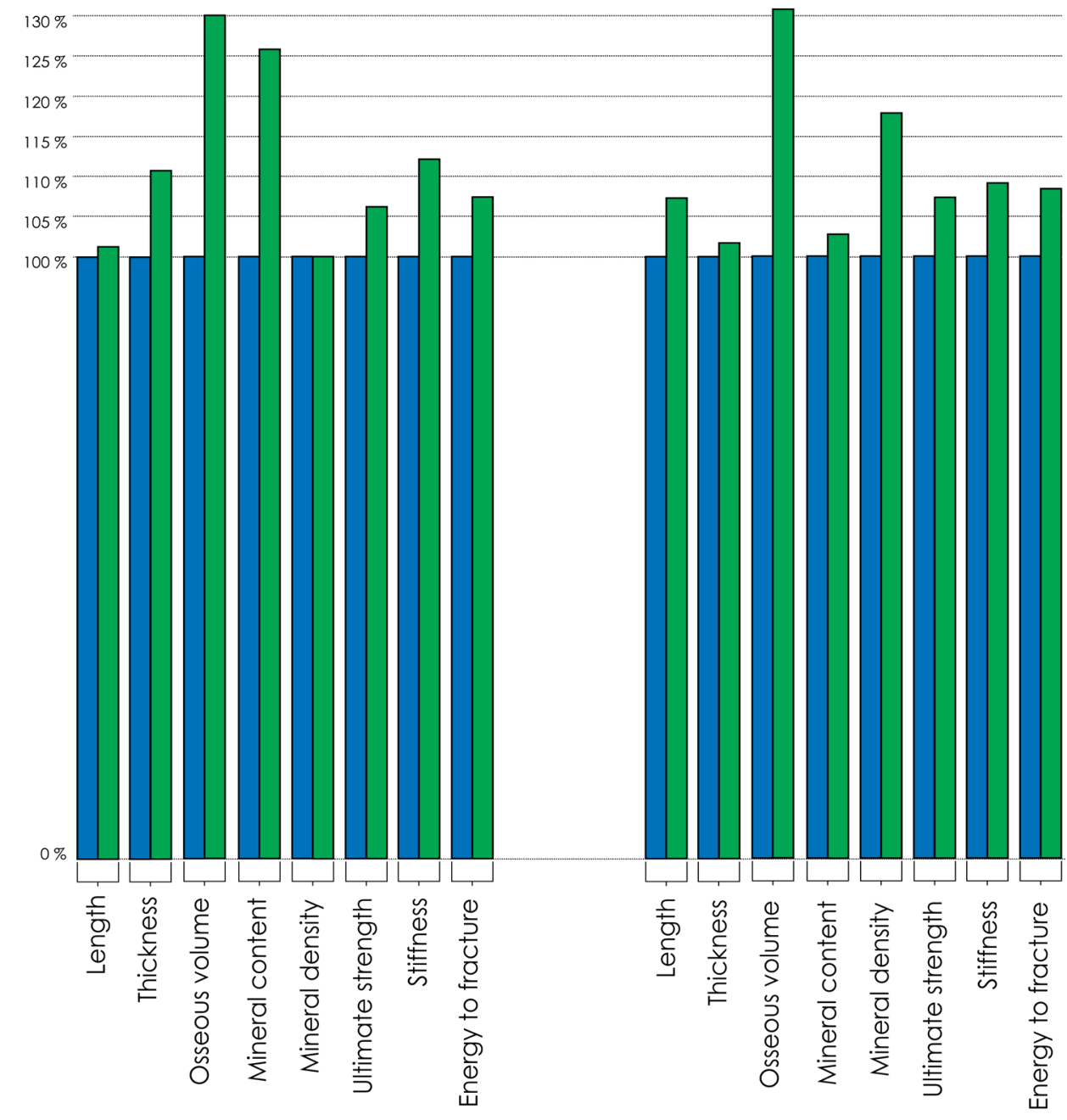

Chapter 3

Chapter 5

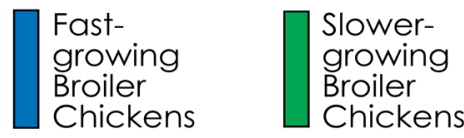

Figure 5

Relative differences (\%) between selected tibia morphological, biophysical and mechanical characteristics of fast and slower-growing chickens at BW class of 2600 gram (38 and 49 days of age for fast and slower-growing chickens, respectively) originating from broiler breeders fed with a diet containing a combination inorganic macro and trace minerals (Chapter 3 ) and fast and slower-growing chickens in non-enriched pens at BW class of 2200 gram (38 and 49 days of age for fast and slower-growing chickens, respectively) (Chapter 5). Results of fast-growing broiler chickens were set at $100 \%$. 
Investigated Approaches on Locomotion and Home Pen Behaviour 
Suboptimal leg health observed in broiler chickens leads to inactivity, poor locomotion (Kestin et al., 1992; Weeks et al., 2000), spending more time sitting and/or lying (Shim et al. 2012; Sanchez-Rodriguez et al. 2019; Hartcher and Lum, 2020) and impaired natural behaviours such as dustbathing, foraging and preening (Vestergaard and Sanotra, 1999; Weeks et al., 2000; Waldenstedt, 2006). One of the major reasons has been specified as an imbalance between high growth rate and immature bones, while the speed of bone development is unable to keep up with this fast growth speed (Angel, 2007; Dibner et al., 2007; Knowles et al., 2008; Sherlock et al., 2010; Shim et al., 2012; Prisby et al., 2014; González-Cerón et al., 2015). In this thesis, gait score and home pen behaviour parameters were determined.

\section{Locomotion}

Gait was scored in Chapter 1, 2, 4 and 5 within a range of 0 (normal locomotion) to 5 (unable to walk) by using the method of (Kestin et al., 1992). In Chapter 1, effects of replacement of a combination of inorganic macro and trace minerals by their organic varieties in the diet of fast-growing broiler chickens on gait score was evaluated. Two chickens per pen were randomly chosen on rearing days 27,34 and 41 , but no significant differences between treatment groups were found. In Chapter 2, green LED light during incubation and dietary organic macro minerals and trace minerals separately during rearing of fast-growing male broiler chickens on gait score was evaluated. Two chickens per pen were randomly chosen on rearing days of 27,34 and 39 , but again no significant differences between inorganic or organic macro and trace minerals were found. However chickens incubated under green LED light had higher gait scores on day 34 and 39 compared to chickens incubated under complete darkness, even after correction for differences in individual body weights of these chickens. In Chapter 4, effects of eggshell temperature pattern in week 2 and 3 of incubation of fast-growing male broiler chickens on gait score was evaluated. All chickens were chosen on rearing days of 27,34 , and 41 . Chickens of the high EST $\left(38.9^{\circ} \mathrm{C}\right)$ in week 2 of incubation had a lower (better) gait score compared to the normal EST $\left(37.8^{\circ} \mathrm{C}\right)$. In Chapter 5, effects of comprehensive pen enrichment on gait score of fast and slower-growing broiler chickens was evaluated. Four chickens per pen were randomly chosen on day 27 (fast-growing chickens)

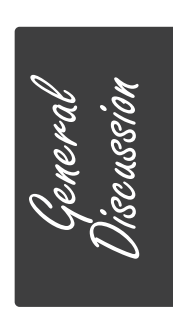


and day 35 (slower-growing chickens), to eliminate body weight differences. At similar BW class (day 27 for fast-growing chickens and day 35 for slower-growing chickens), an interaction between pen enrichment and strain was found. Slower-growing chickens in non-enriched pens had a lower (better) gait score compared to fast-growing chickens in non-enriched pens, while both fast and slower-growing chickens in enriched pens groups were in between.

\section{Home Pen Behaviour}

Home pen behaviour were observed in Chapter 1, 2, 3 and 5 by direct observation, using instantaneous scan sampling (De Jong and Gunnink, 2019; De Jong et al., 2021). The number of chickens performing the following behaviours was scored: eating, drinking, walking, standing, sitting, comfort behaviour, foraging, dustbathing, ground pecking and aggression.

\section{Info box 6}

Observed activities, observation durations and observation moments for home pen behaviour differ between chapters. The reason is that instantaneous scan sampling method was under development (De Jong et al., 2019) during the experiments described in this thesis. The Materials and Methods section of all the chapters contains the relevant information.

In Chapter 1, effects of replacement of a combination of inorganic macro and trace minerals by their organic varieties in the diet of fast-growing broiler chickens on home pen behaviour was evaluated and no differences between treatment groups were found. In Chapter 2, green LED light during incubation and dietary organic macro minerals and trace minerals separately during rearing of fast-growing male broiler chickens on home pen behaviour was evaluated and no differences between treatment groups was found. In Chapter 3, effects of replacement of a combination of organic macro and trace minerals by their organic varieties in the diet of fast and slower-growing broiler breeders on home pen behaviour of their offspring broilers were investigated and no mineral source effect between treatment groups was found, but fast-growing chickens showed less active behaviours e.g., walking standing, perching than slower-growing chickens. In Chapter 5, effects of a combination 
of ramps, platforms, barrier perches, large distance between feed and water and live Black Soldier fly larvae in the moss-peat dust bathing area as a pen enrichment were investigated on home pen behaviour of fast and slower-growing broiler chickens. Chickens in non-enriched pens showed less active behaviours e.g., walking, standing, foraging, dust bathing and ground pecking than chickens in enriched pens. Regarding the strain, slow-growing chickens showed more walking, standing and foraging behaviour than fast-growing chickens.

Considering Chapter 1, 2, 3 and $\mathbf{5}$ on home pen behaviour, several conclusions can be drawn: (1) Despite tibia development of fast-growing broiler chickens was stimulated by dietary organic minerals, no effect was found on home pen behaviour. (2) Investigated approaches do not seem sufficient enough to stimulate activity. (3) Fast-growing broiler chickens showed a preference for lying and sitting on the litter instead of using raised platforms and perches compared to slower-growing chickens (4) Study on pen enrichment demonstrated that at the same BW class, still differences in activity related behaviours were present between the fast and slower-growing chickens, which might suggest that speed of bone development affects activity of broiler chickens. 
Investigated Approaches on Growth Performance 
In all chapters of this thesis, growth performance parameters were investigated in addition to leg health indicators e.g., tibia characteristics, leg disorders, locomotion and home pen behaviour, due to the following reasons: (1) Some of the investigated approaches e.g., organic macro and trace minerals and green LED light during incubation have previously been shown to stimulate growth performance of broiler chickens. (2) Because body weight is known to be related to tibia characteristics, individual body weight of slaughtered chickens has been used as a covariable.

In Figure 6, relative differences (\%) between body weights of all chapters are given. In this figure, results of body weight of control groups at different ages were set at $100 \%$ (details are given in Figure 6).

\section{Dietary Organic Macro and Trace Minerals}

Dietary organic trace minerals in the diet of broiler chickens have been shown to result in higher growth performance than their inorganic varieties. However, most of the studies related to organic minerals in the diet of broiler chickens focused on trace minerals (iron, copper, manganese, zinc and selenium) only (Bao et al., 2007; Abdallah et al., 2009; Ao et al., 2017), while effects of a combination of organic macro and trace minerals or macro minerals (calcium and phosphorus) only in both diet of broiler chickens were lacking.

Considering that lack of knowledge in literature, in Chapter 1, replacement of a combination of inorganic macro and trace minerals by their organic varieties in the diet of fast-growing broiler chickens on their growth performance throughout the rearing period was investigated. Chapter 1 showed that a combination of organic macro and trace minerals in the diet of fast-growing broiler chickens resulted in higher growth performance. At d 42 of age, chickens fed a diet containing a combination of organic macro and trace minerals had 30 gram (0.8\%; Figure 6a) higher body weight, 73 gram lower feed intake and 3 points lower feed conversion ratio compared to chickens fed a diet containing a combination of inorganic macro and trace minerals.

These findings are in line with previous studies indicating that dietary organic trace minerals resulted in higher growth performance compared

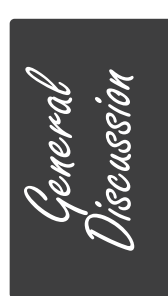


to their inorganic varieties (Bao et al., 2007; Abdallah et al., 2009; Ao et al., 2017). However, which mineral group (macro minerals, trace minerals or their combination) is most effective still remained unclear. Therefore, in Chapter 2, inorganic and organic macro and trace minerals separately were studied in diet of broiler chickens to reveal the most effective mineral group (macro or trace minerals or their combination) on growth performance of broiler chickens.

\section{Info box 7}

In Chapter 1, palm oil and soybean oil were partly replaced by fish oil in FISH group. Soybean meal was partly replaced by hydrolyzed collagen in COL group. Relative differences (\%) in body weight results of these groups can be found in Figure 6a, but all growth performance results related to these nutrients were only discussed in Chapter 1.

Chapter 2 showed that dietary macro mineral source was more effective on growth performance than dietary trace mineral source throughout the rearing period. At d 42 of age, on one hand, chickens fed a diet containing organic macro minerals had 74 gram (2.5\%; Figure 6a) higher body weight, 46 gram lower feed intake and 5 points lower feed conversion ratio compared to chickens fed a diet containing inorganic macro minerals. On the other hand, chickens fed a diet containing organic trace minerals had 59 gram (2.0\%; Figure 6a) higher body weight, 71 gram lower feed intake and 6 points lower feed conversion ratio compared to chickens fed a diet containing inorganic trace minerals. Despite the fact that these differences are close to each other, only dietary organic macro minerals created significance on body weight.

Comparing the growth performance parameters between Chapter 1 and Chapter 2, it appears that: (1) Macro minerals source is of more importance for growth performance of broiler chickens than trace minerals source. (2) Because macro minerals have been found more effective on tibia characteristics as well rather than trace minerals in Chapter 2, it can be speculated that better developed bones might stimulate activity, which makes chicken more able to reach the feed and water. (3) As it was suggested in the tibia characteristics section, the diet of current fast-growing broiler chickens might be suboptimal in $\mathrm{Ca}$ and $\mathrm{P}$, which 
results in adverse effects on tibia characteristics, but also in lower growth performance.

Organic trace minerals in the diet of broiler breeders have also been shown to stimulate post-hatch growth performance of their offspring (BaO and Choct, 2009; Świątkiewicz et al., 2014; Chang et al., 2016; M'sadeq et al., 2018; Torres and Korver, 2018; Araújo et al., 2019; Wang et al., 2019). However, effects of a combination of organic macro and trace minerals in broiler breeder diet on growth performance of their offspring broiler were lacking in literature. Therefore, in Chapter 3, replacement of a combination of inorganic macro and trace minerals by their organic varieties in the diet of fast and slower-growing broiler breeder chickens on growth performance of their offspring broilers were investigated.

Chapter 3 showed that organic macro and trace minerals in the diet of fast and slower-growing broiler breeder chickens resulted in higher growth performance of offspring broilers of slow-growing strain, while this effect was not seen in fast-growing chickens.

In fast-growing chickens, at 42 days of age, chickens originating from broiler breeders fed with diet containing a combination of organic macro and trace minerals had 29 gram (0.9\%; Figure 6a) higher body weight and 53 gram lower feed intake compared to chickens originating from broiler breeders fed with a diet containing a combination of inorganic macro and trace minerals. Because the difference between treatment groups was relatively low, feed conversion ratio did not differ.

In slower-growing chickens at 49 days of age, chickens originating from broiler breeders fed with diet containing a combination of organic macro and trace minerals had 132 gram (4.8\%; Figure 6a) higher body weight, 88 gram lower feed intake and 1 point lower feed conversion ratio compared to chickens originating from broiler breeders fed with a diet containing a combination of inorganic macro and trace minerals.

These results suggest that: (1) Absorption, mineral deposition and mineral transferring physiologies between slower and fast-growing chickens might differ because of their different genetic backgrounds (Yair et al., 2013; Torres and Korver, 2018). (2) The diet of slower-growing broiler breeders might be suboptimal in minerals. 
Considering Chapter 1, 2 and $\mathbf{3}$ on growth performance, several conclusions can be drawn: (1) Increasing mineral availability by using organic minerals in the diet, instead of further increase in the amount of inorganic minerals, may result in stimulated growth performance. (2) Macro minerals appear to play the major role in growth performance compared to trace minerals. (3) Trans-generational mineral availability seems to be more effective on growth performance of slower-growing offspring compared to fast-growing offspring.

\section{Green LED Light During Incubation}

Green LED light during incubation have been shown to stimulate body weight and post-hatch pectoral muscle growth (Halevy et al., 2006; zhang et al., 2014, 2016; Dishon, 2018, 2021), higher liver weight, higher antioxidant activity and higher melatonin levels (zhang et al., 2014) in broiler chickens compared to complete darkness during incubation.

In Chapter 2, effects of green LED light (16L:8D) during incubation on growth performance of broiler chickens throughout the rearing period were investigated. Results of this study showed that green LED light during incubation positively affected the growth performance. Chickens incubated under green LED light had 93 gram (3.2 \%; Figure 6a) higher body weight, 9 gram lower feed intake and 6 points lower feed conversion ratio compared to chickens incubated under complete darkness.

Previous research has underlined the reason for better growth performance of broiler chickens incubated under green light as stimulated growth-related hormones and receptors e.g., plasma growth hormone, prolactin, melatonin (Archer et al., 2009; Huth and Archer, 2015; Archer and Mench, 2017), hypothalamic growth hormone releasing hormone, growth hormone receptor and insulin-like growth factor-1 (Dishon et al., 2017, 2018, 2021).

\section{Info box 8}

In Chapter 4, effects of eggshell temperature pattern in week $2\left(37.8^{\circ} \mathrm{C}\right.$ or $\left.38.9^{\circ} \mathrm{C}\right)$ and week $3\left(36.7^{\circ} \mathrm{C}\right.$ or $\left.37.8^{\circ} \mathrm{C}\right)$ of incubation on body weight of fast-growing broiler chickens at 41 and 42 days of age were investigated. Because no significant differences between groups were found, only relative differences (\%) in body weight results of these groups were given in Figure $6 \mathrm{~b}$, this will not be discussed further. 


\section{Environmental Enrichment}

In Chapter 5, effects of using ramps, platforms, barrier perches, large distance between feed and water and live Black Soldier fly larvae in the moss-peat dust bathing area as a pen enrichment on growth performance of fast and slower-growing broiler chickens throughout the rearing period were investigated. Chapter 5 showed that growth performance of both fast and slower-growing broiler chickens was negatively affected by pen enrichment. In fast-growing chickens, at 35 days of age, chickens in enriched pens had 144 gram (6.8 \%; Figure 6b) lower body weight, 89 gram lower feed intake and 6 points higher feed conversion ratio compared to chickens in non-enriched pens. In slower-growing chickens, at 49 days of age, chickens in enriched pens had 93 gram (4.3\%; Figure 6b) lower body weight, 39 gram lower feed intake and 6 points higher feed conversion ratio compared to chickens in non-enriched pens.

These findings are in line with previous studies indicating that pen enrichment, despite the beneficial effects on tibia characteristics and activity (Reiter and Bessei, 2009; Blatchford et al., 2012; Ohara et al., 2015; Pedersen and Forkman, 2019), may negatively affect growth performance parameters (Bach et al., 2019; Jones et al., 2020; De Jong et al., 2021). Potential reasons for these findings can be: (1) Comprehensive pen enrichment design might exponentially stimulate physical activity and increase the use of metabolic energy, which consequently results in a lower body weight gain. (2) Considering the experimental pen design of the current study only, chickens might have difficulties to cross the barrier perches to access the feed on one side and water on the other side due to perching chickens blocking the way and/or heavy body weight of chickens particularly in the last week of the rearing period. 


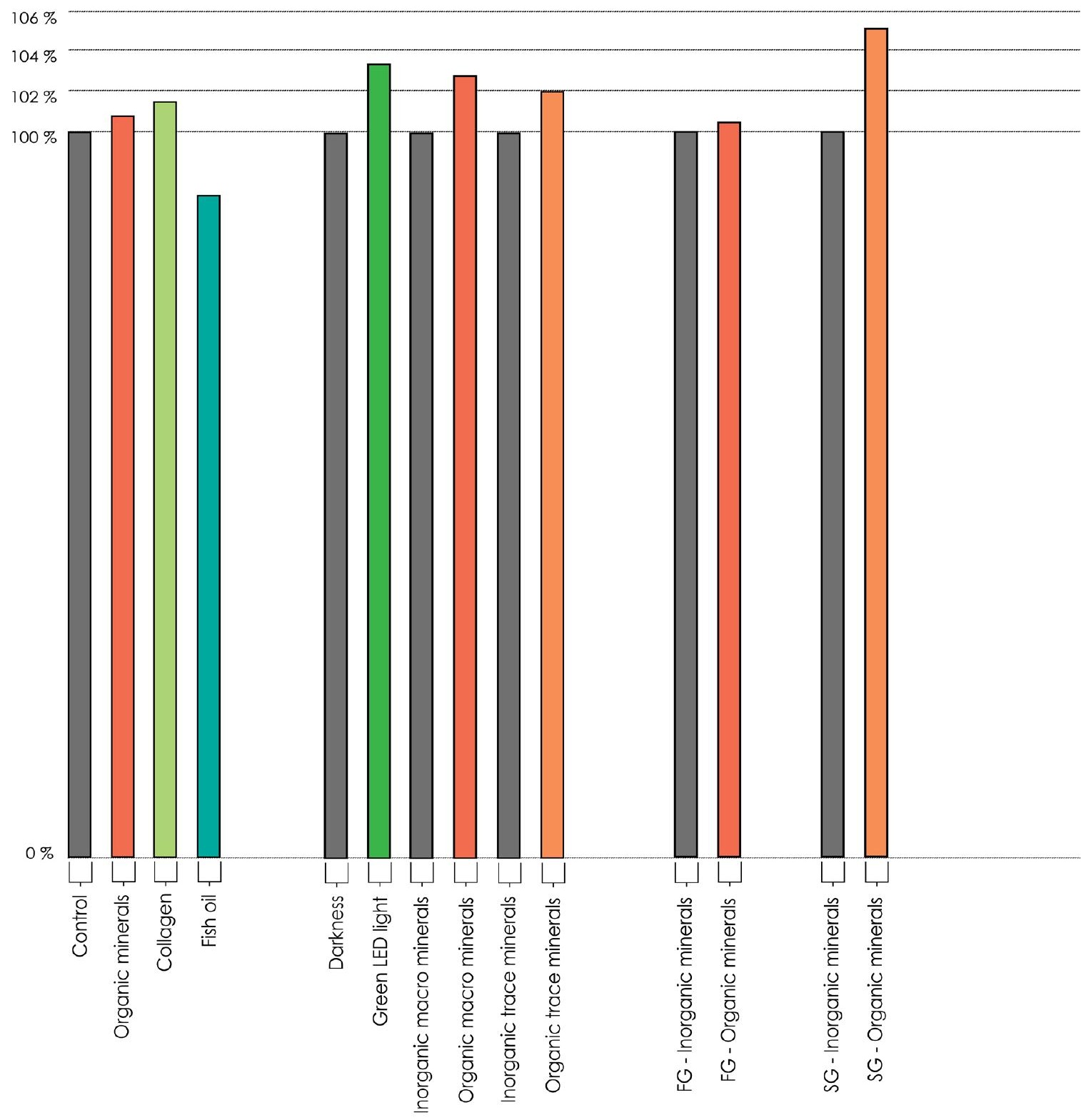

Day 42

Chapter 1
Day 42

Chapter 2
Day 42 Day 49

\section{Chapter 3}

\section{Figure 6a}

Relative differences (\%) between body weights at 42 days of age of fast-growing broiler chickens (Chapter 1, 2 and 3) and at 49 days of age of slower-growing broiler chickens (Chapter 3). Body weights of control groups were set at $100 \%$. 


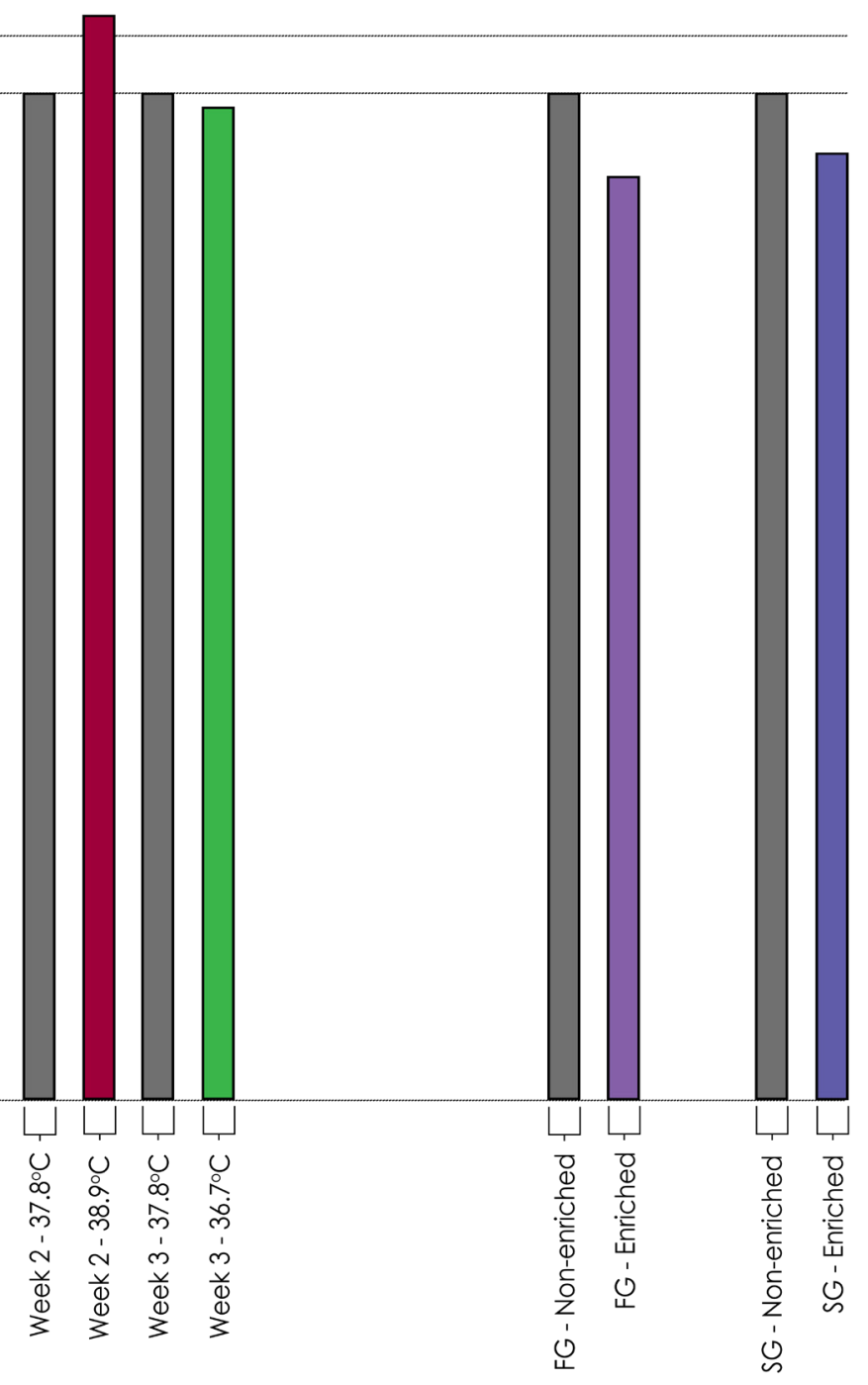

Day 41 or 42

Day 35 Day 49

\section{Chapter 4}

\section{Chapter 5}

Figure $6 \mathrm{~b}$

Relative differences (\%) between body weights at 41 or 42 days of age of fastgrowing broiler chickens (Chapter 4), at 35 days of age of fast-growing broiler chickens (Chapter 5) and at 49 days of age of slower-growing broiler chickens (Chapter 5). Body weights of control groups were set at $100 \%$.

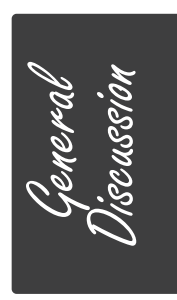




\section{Relationships Between Body Weight and Tibia Characteristics}

A linear regression analysis was performed between individual body weights of slaughtered chickens and selected tibia characteristics (e.g., tibia length, ultimate strength, osseous volume and mineral density) of same chickens in all chapters. Higher body weight showed positive correlation with tibia length and ultimate strength, while it showed negative correlation with tibia osseous volume and mineral density.

Considering these results, several conclusions can be drawn: (1) Higher body weight at slaughter age is in parallel with tibia dimensions and strength. (2) It might be that because mineralization in bone takes places to increase bone dimensions and strength to adapt itself to body dimensions, bone volume and mineral density can be negatively affected with rapidly increasing body weight. (3) Investigated approaches in this thesis (e.g., organic minerals in diet of broiler and broiler breeders, temperature and light during incubation and environmental enrichment) had no effect on relationships between body weight and selected tibia characteristics. This may suggest that despite improved bone characteristics were found in all chapters, this improvement was not sufficient enough to compensate the negative high body weight effect on bone. 


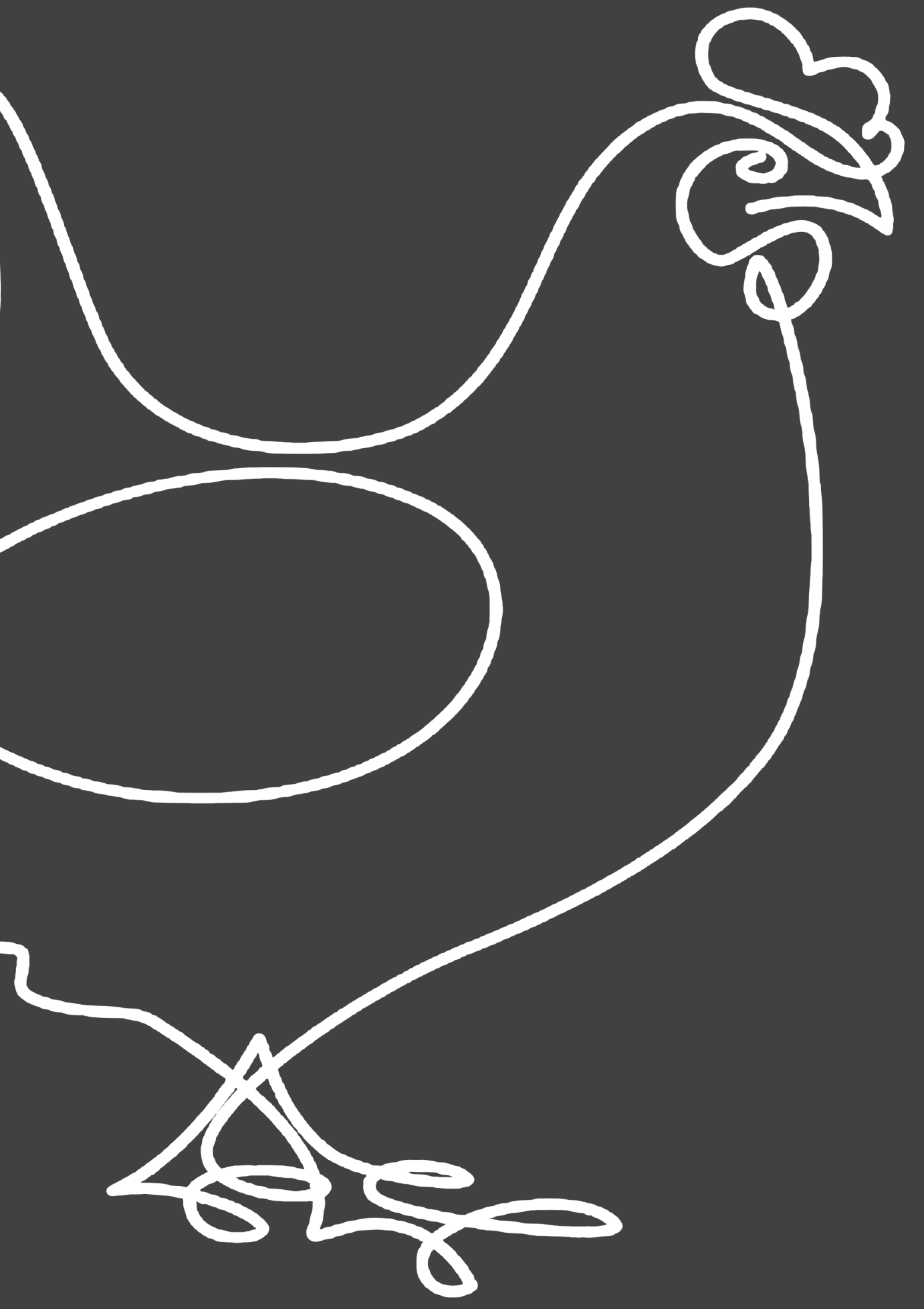


Conclusions 


\section{Dietary Organic Macro and Trace Minerals}

Replacement of a combination of inorganic macro and trace minerals by their organic varieties in the diet of fast-growing broiler chickens has been found to positively affect growth performance and tibia morphological, biophysical and mechanical characteristics.

Replacement of a combination of inorganic macro and trace minerals by their organic varieties in the diet

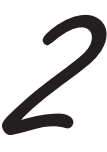
of fast and slower-growing broiler breeders resulted in an increased trans-generational mineral availability, stimulated growth performance and tibia characteristics in slower-growing offspring broilers, while this positive effect was not observed in fast-growing offspring broilers.

In addition to previous conclusion, trans-generational mineral availability in offspring broiler chickens appears to play a role via other mechanisms than via absolute mineral concentrations in the egg, since mineral concentrations in eggs and hatchlings are marginal.

Replacement of inorganic macro minerals only in the diet of fast-growing broiler chickens by their organic varieties seems to more effective than organic trace minerals only on tibia morphological, biophysical and mechanical characteristics.

\section{Light and Temperature During Incubation}

Green LED light during incubation resulted in stimulated body weight gain, but no positive effects were found on any of tibia characteristics of broiler chickens, which may cause worse locomotion and consequently worse welfare because of increased body weight especially towards the slaughter day. 
An eggshell temperature of $38.9^{\circ} \mathrm{C}$ during the second week of incubation is more effective on tibia characteristics of fast-growing broiler chickens at slaughter age compared to an eggshell temperature of $37.8^{\circ} \mathrm{C}$. Furthermore, incubation temperature in the third week appears to interact with the incubation temperature in the second week. An eggshell temperature of $38.9^{\circ} \mathrm{C}$ in second week, followed by $37.8^{\circ} \mathrm{C}$ in third week resulted in the most advanced tibia characteristics and better locomotion. An eggshell temperature of $36.7^{\circ} \mathrm{C}$ in week 3 of incubation appears to have negative effects on tibia characteristics. However, no effect was found on growth performance and leg disorders.

\section{Environmental Enrichment}

Pen enrichment positively affected tibia biophysical characteristics of both slower and fast-growing chickens, while tibia morphological and mechanical characteristics were not affected. Furthermore, slower-growing chickens showed better locomotion compared to fast-growing chickens. Pen enrichment also resulted in lower body weight gain in both fast and slower-growing chickens

\section{Overall Conclusions}

In all experiments described in this thesis, the relationship between tibia development and leg abnormalities and disorders remained unclear due to very low incidence of leg abnormalities and disorders.

Correlation analysis between tibial morphological, biophysical and mechanical characteristics showed strong relationships among tibia weight, length, thickness, ultimate strength, stiffness and osseous volume, which suggests that these selected tibia characteristics are interconnected to each other. 
Tibia biophysical characteristics, compared to morphological and mechanical characteristics, seem to give better response to organic minerals both directly in broiler diet on broiler chickens and via broiler breeder diet on their offspring broilers, higher eggshell temperature during second week of incubation and comprehensive pen enrichment.

Growth performance parameters were directly stimulated by a combination or separate macro and trace minerals in the diet of fast-growing broiler chickens, green LED light during incubation of fast-growing broiler chickens and were indirectly stimulated by a combination of macro minerals in the diet of broiler breeders on only slower-growing offspring broiler chickens. 
Fature Research Opportanaties 
Based on this thesis, the following future research opportunities can be formulated:

Replacement of a combination of inorganic macro and trace minerals by their organic varieties resulted in better growth performance and tibia characteristics. However, organic $\mathrm{Ca}$ and $\mathrm{P}$ were used together in a diet. Future research may investigate $\mathrm{Ca}$ and $\mathrm{P}$ separately to reveal the major role player on growth performance and bone characteristics.

Replacement of inorganic macro minerals and trace minerals separately (and also Ca and P separately)

2 by their organic varieties in the diet of broiler breeders is recommended to investigate the carry-over effect of these minerals in fast and slower-growing broiler breeders to their offspring and to reveal the major role player mineral in trans-generational effect.

Since the most effective mineral group was determined as organic macro minerals, replacement of inorganic macro minerals by their organic varieties in the diet of broiler breeders, followed by replacement of inorganic macro minerals by their organic varieties in the diet of offspring broilers is recommended to investigate both trans-generational carry-over and direct effect of these minerals in both fast and slower-growing broiler chickens.

Regardless other treatment effects, slower-growing broiler chickens consistently showed better leg health parameters and more active behaviours. In this thesis, only a combination of a macro and trace minerals via broiler breeders on offspring broilers and pen enrichment on slower-growing chickens were investigated. Other approaches, e.g., a combination or separate organic macro and trace minerals in the diet, an eggshell temperature pattern during incubation and a green LED light during incubation, were only investigated on fast-growing

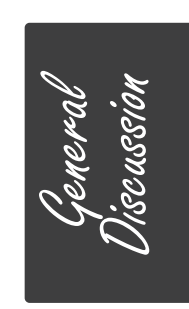


chickens. These approaches are worthwhile to investigate in slower-growing chickens in future research.

Trans-generational mineral availability in offspring broiler chickens appears to play a role via other mechanisms than via absolute mineral concentrations in the egg, since mineral concentrations in eggs and hatchlings are marginal. Future research may investigate the pathways involved in mineral absorption, deposition and transfer in current fast and slower- growing broiler breeders.

No effect of green LED light during incubation was found on leg health parameters, but only higher growth performance of fast-growing chickens. Investigating other colours e.g., white, blue and red light during incubation is recommended for future research on leg health parameters of broiler chickens, since these colours have been shown to positively influence embryonic development compared to complete darkness during incubation.

Temperature pattern during incubation has been found to affect embryonic bone development. Increasing the eggshell temperature from $37.8^{\circ} \mathrm{C}$ to $38.9^{\circ} \mathrm{C}$ throughout the second week of incubation, followed by $37.8^{\circ} \mathrm{C}$ eggshell temperature throughout third week of incubation, resulted in stimulated tibia characteristics. Future research may investigate this increased eggshell temperature in the second week of incubation together with post-hatch tibia characteristics enhancing investigated approaches in this thesis, e.g., organic macro minerals in the diet of broiler chickens, which both approaches exponentially stimulate tibia characteristics. 
Pen enrichment resulted in improved tibia biophysical characteristics in both fast and slower-growing broiler chickens, while tibia morphological and mechanical characteristics were not affected and growth performance was negatively affected. Future research may investigate pen enrichment with a combination of other growth performance enhancing investigated approaches in this thesis, e.g., organic macro and traceminerals and green LED light during incubation, which may stimulate tibia morphological and mechanical characteristics as well as biophysical characteristics, while keeping desirable growth performance.

A very low incidence of leg disorders and marginal effect on locomotion were found in all experiments described in this. To reveal the potentials of investigated approaches used in this thesis, e.g., organic macro and trace minerals in the diet of broiler and broiler breeder chickens, eggshell temperature pattern and environmental enrichment in a large scale field trial is recommended for future research on leg disorders and locomotion of both fast and slower-growing broiler chickens.

As a continuation of previous future research opportunity, in all experiments described in this thesis, male broiler chickens or male offspring broilers were used due to their higher body weights and consequently tendencies towards more leg health problems. To correspond commercial production, a large scale field investigation including both males and females is recommended for future research on leg disorders and locomotion of broiler chickens. 


\section{REFERENCES}

Abdallah, A. G., El-Husseiny, O. M. and Abdel-Latif, K. O., 2009. Influence of some dietary organic mineral supplementations. International journal of poultry science. 8:291-298.

Angel, R. 2007. Metabolic disorders: limitations to growth of and mineral deposition into the broiler skeleton after hatch and potential implications for leg problems. Journal of applied poultry research. 16:138-149.

Ao, T., Macalintal, L.M., Paul, M.A., Pescatore, A.J., Delles, R.M., Cantor, A.H., Ford, M.J. and Dawson, K.A., 2017. Effects of dietary supplementation of organic minerals on the performance of broiler chicks fed oxidised soybean oil. Journal of applied animal nutrition. 5:1-5.

Applegate, T. J. and M. S. Lilburn. 2002. Growth of the femur and tibia of a commercial broiler line. Poultry science. 81:1289-1294.

Araújo, C.S.S., Hermes, R.G., Bittencourt, L.C., Silva, C.C., Araújo, L.F., Granghelli, C.A., Pelissari, P.H., Roque, F.A. and Leite, B.G.S., 2019. Different dietary trace mineral sources for broiler breeders and their progenies. Poultry science. 98:4716-4721.

Archer, G. S. 2017. Exposing broiler eggs to green, red and white light during incubation. Animal. 11:1203-1209.

Archer, G. S., H. L. Shivaprasad, and J. A. Mench. 2009. Effect of providing light during incubation on the health, productivity, and behavior of broiler chickens. Poultry science. 88:29-37.

Archer, G.S., 2018. Color temperature of light-emitting diode lighting matters for optimum growth and welfare of broiler chickens. Animal. 12:1015-1021.

Atalgin, S. H. and I. Kürtül. 2009. A morphological study of skeletal development in turkey during the pre-hatching stage. Anatomia, histologia, embryologia. 38:23-30.

Aygün, A. and D. Narinç. 2016. The effects of thermal manipulations during embryogenesis of broiler chicks on growth of embryo and skeletal traits. In AIP conference proceedings. 1726:020015.

Ballock, R.T. and O'Keefe, R.J., 2003. The biology of the growth plate. JBJS, 85(4), pp.715-726.

Bao, Y. M. and M. Choct. 2009. Trace mineral nutrition for broiler chickens and prospects of application of organically complexed trace minerals: a review. Animal production science. 49:269-282.

Bao, Y. M., M. Choct, P. A. lji, and K. Bruerton. 2007. Effect of organically complexed copper, iron, manganese, and zinc on broiler performance, mineral excretion, and accumulation in tissues. Journal of applied poultry research. 16:448-455.

Bessei, W. 2006. Welfare of broilers: a review. World's poultry science journal. 62:455-466.

Bessei, W. 2018. Impact of animal welfare on worldwide poultry production. World's poultry science journal. 74:211-224.

Bizeray, D., I. Estevez, C. Leterrier, and J. M. Faure. 2002. Effects of increasing environmental complexity on the physi-cal activity of broiler chickens. Applied animal behaviour science. 79:27-41.

Blatchford, R. A., G. S. Archer, and J. A. Mench. 2012. Contrast in light intensity, rather than day length, influences the behavior and health of broiler chickens. Poultry science. 91:1768-1774.

Blom, J. and Lilja, C., 2005. A comparative study of embryonic development of some bird species with different patterns of postnatal growth. Zoology. 108:81-95.

Bokkers, E. A. and P. Koene. 2004. Motivation and ability to walk for a food reward in fast-and slow-growing broilers to 12 weeks of age. Behavioural processes. 67:121-130.

Bradshaw, R. H., R. D. Kirkden, and D. M. Broom. 2002. A review of the aetiology and pathology of leg weakness in broilers in relation to welfare. Avian and poultry biology reviews. 13:45-104.

Buijs, S., E. Van Poucke, S. Van Dongen, L. Lens, J. Baert, and F. A. Tuyttens. 2012. The influence of stocking density on broiler chicken bone quality and fluctuating asymmetry. Poultry science. 91:1759-1767.

Chang, A., Halley, J. and Silva, M., 2016. Can feeding the broiler breeder improve chick quality and offspring performance? . Animal production science. 56:1254-1262. 
Corr, S. A., Gentle, M. J., McCorquodale, C. C. and Bennett, D., 2003. The effect of morphology on walking ability in the modern broiler: a gait analysis study. Animal welfare. 12:159-171.

Crespo, R. 2020. Developmental, metabolic, and other noninfectious disorders. Diseases of poultry. p1286-1329.

Dawkins, M. S. and R. Layton. 2012. Breeding for better welfare: genetic goals for broiler chickens and their parents. Animal welfare. 21:147-166.

De Jong, I.C. and Gunnink, H., 2019. Effects of a commercial broiler enrichment programme with or without natural light on behaviour and other welfare indicators. Animal. 13:384-391.

De Jong, I.C., Blaauw, X.E., van der Eijk, J.A., da Silva, C.S., van Krimpen, M.M., Molenaar, R. and van den Brand, H., 2021. Providing environmental enrichments affects activity and performance, but not leg health in fast-and slower-growing broiler chickens. Applied animal behaviour science. p105375.

Demuner, L.F., Suckeveris, D., Muñoz, J.A., Caetano, V.C., Lima, C.G.D., Faria, D.E.D. and Faria, D.E.D., 2017. Adjustment of growth models in broiler chickens. Pesquisa agropecuária brasileira, 52:1241-1252.

Dibner, J. J., J. D. Richards, M. L. Kitchell, and M. A. Quiroz. 2007. Metabolic challenges and early bone development. Journal of applied poultry research. 16:126-137.

Dishon, L., Avital-Cohen, N., Zaguri, S., Bartman, J., Heiblum, R., Druyan, S., Porter, T.E., Gumulka, M. and Rozenboim, I., 2021. In ovo green light photostimulation during the late incubation stage affects somatotropic axis activity. Poultry science. 100:467-473.

Dishon, L., Avital-Cohen, N., Zaguri, S., Bartman, J., Heiblum, R., Druyan, S., Porter, T.E., Gumulka, M. and Rozenboim, I., 2018. In-ovo green light photostimulation during different embryonic stages affect somatotropic axis. Poultry science. 97:1998-2004.

Dishon, L., Avital-Cohen, N., Zaguri, S., Bartman, J., Heiblum, R., Druyan, S., Porter, T.E., Gumulka, M. and Rozenboim, I., 2021. In ovo green light photostimulation during the late incubation stage affects somatotropic axis activity. Poultry science, 100:467-473.

Driver, J. P., G. M. Pesti, R. I. Bakalli, and H. M. Edwards Jr. 2005. Calcium requirements of the modern broiler chicken as influenced by dietary protein and age. Poultry science. 84:1629-1639.

Duan, X., Li, M., Wu, F., Yang, N., Nikoo, M., Jin, Z. and Xu, X., 2013. Postfertilization changes in nutritional composition and protein conformation of hen egg. Journal of agricultural and food chemistry. 61:12092-12100.

EFSA panel on animal health and welfare. 2010. Scientific opinion on the influence of genetic parameters on the welfare and the resistance to stress of commercial broilers. EFSA journal. 8:1666.

El-Husseiny, O. M., S. M. Hashish, R. A. Ali, S. A. Arafa, L. D. Abd El-Samee, and A. A. Olemy. 2012. Effects of feeding organic zinc, manganese and copper on broiler growth, carcass characteristics, bone quality and mineral content in bone, liver and excreta. International journal of poultry science. 11:368-377.

Favero, A., Vieira, S.L., Angel, C.R., Bos-Mikich, A., Lothhammer, N., Taschetto, D., Cruz, R.F.A. and Ward, T.L., 2013. Development of bone in chick embryos from Cobb 500 breeder hens fed diets supplemented with zinc, manganese, and copper from inorganic and amino acid-complexed sources. Poultry science. 92:402-41 1.

Ferket, P. R., E. O. Oviedo-Rondón, P. L. Mente, D. V. Bohórquez, A. A. Santos Jr, J. L. Grimes, J. D. Richards, J. J. Dibner, and V. Felts. 2009. Organic trace minerals and 25-hydroxycholecalciferol affect performance characteristics, leg abnormalities, and biomechanical properties of leg bones of turkeys. Poultry science. 88:118-131.

Flis, M., Gugała, D., Muszyński, S., Dobrowolski, P., Kwiecień, M., Grela, E.R. and Tomaszewska, E., 2019. The influence of the partial replacing of inorganic salts of calcium, zinc, iron, and copper with amino acid complexes on bone development in male pheasants from aviary breeding. Animals, 9:237-249.

Gocsik, É., A. M. Silvera, H. Hansson, H. W. Saatkamp, and H. J. Blokhuis. 2017. Exploring the economic potential of reducing broiler lameness. British poultry science. 58:337-347.

González-Cerón, F., R. Rekaya, and S. E. Aggrey. 2015. Genetic analysis of bone quality traits and growth in a random mating broiler population. Poultry science. 94:883-889. 
Granquist, E.G., Vasdal, G., De Jong, I.C. and Moe, R.O., 2019. Lameness and its relationship with health and production measures in broiler chickens. Animal. 13:2365-2372.

Groves, P.J. and Muir, W.I., 2014. A meta-analysis of experiments linking incubation conditions with subsequent leg weakness in broiler chickens. PLoS one, 9:e102682.

Guo, Y., H. Tang, X. Wang, W. Li, Y. Wang, F. Yan, X. Kang, Z. Li, and R. Han. 2019. Clinical assessment of growth per-formance, bone morphometry, bone quality, and serum indicators in broilers affected by valgus-varus deformity. Poultry science. 98:4433-4440.

Halevy, O., Piestun, Y., Rozenboim, I. and Yablonka-Reuveni, Z., 2006. In ovo exposure to monochromatic green light promotes skeletal muscle cell proliferation and affects myofiber growth in posthatch chicks. American journal of physiology-regulatory, integrative and comparative physiology. 290:1062-1070.

Hammond, C. L., B. H. Simbi, and N. C. Stickland. 2007. In ovo temperature manipulation influences embryonic motility and growth of limb tissues in the chick (Gallus gallus). Journal of experimental biology. 210:2667-2675.

Hartcher, K.M. and Lum, H.K., 2020. Genetic selection of broilers and welfare consequences: a review. World's poultry science journal. 76:154-167.

Huth, J.C. and Archer, G.S., 2015. Effects of LED lighting during incubation on layer and broiler hatchability, chick quality, stress susceptibility and post-hatch growth. Poultry science. 94:3052-3058.

Ipema, A.F., Bokkers, E.A., Gerrits, W.J., Kemp, B. and Bolhuis, J.E., 2020. Long-term access to live black soldier fly larvae (Hermetia illucens) stimulates activity and reduces fearfulness of broilers, without affecting health. Scientific reports. 10:1-13.

Jianhua, H., Ohtsuka, A. and Hayashi, K., 2000. Selenium influences growth via thyroid hormone status in broiler chickens. British journal of nutrition. 84:727-732.

Julian, R.J., 1998. Rapid growth problems: ascites and skeletal deformities in broilers. Poultry science. 77:1773-1780.

Julian, R.J., 2005. Production and growth-related disorders and other metabolic diseases of poultry-a review. The veterinary journal. 169:350-369.

Kaukonen, E., Norring, M. and Valros, A., 2016. Effect of litter quality on foot pad dermatitis, hock burns and breast blisters in broiler breeders during the production period. Avian pathology. 45:667-673.

Kestin, S. C., G. SU, and P. Sørensen. 1999. Different commercial broiler crosses have different susceptibilities to leg weakness. Poultry science. 78:1085-1090.

Kestin, S. C., T. G. Knowles, A. E. Tinch and N. G. Gregory. 1992. Prevalence of leg weakness in broiler chickens and its relationship with genotype. Veterinary record. 131:190-194.

Kidd, M.T., Anthony, N.B. and Lee, S.R., 1992. Progeny performance when dams and chicks are fed supplemental zinc. Poultry science. 71:1201-1206.

Kittelsen, K.E., David, B., Moe, R.O., Poulsen, H.D., Young, J.F. and Granquist, E.G., 2017. Associations among gait score, production data, abattoir registrations, and postmortem tibia measurements in broiler chickens. Poultry science. 96:1033-1040.

Kjaer, J. B., G. Su, B. L. Nielsen, and P. Sørensen. 2006. Foot pad dermatitis and hock burn in broiler chickens and degree of inheritance. Poultry science. 85:1342-1348.

Knowles, T. G., S. C. Kestin, S. M. Haslam, S. N. Brown, L. E. Green, A. Butterworth, S. J. Pope, D. Pfeiffer, and C. J. Nicol. 2008. Leg disorders in broiler chickens: prevalence, risk factors and prevention. PloS one. 3:e1545.

Kryeziu, A.J., Mestani, N., Berisha, S. and Kamberi, M.A., 2018. The European performance indicators of broiler chickens as influenced by stocking density and sex. Agronomy research. 16:483-491.

Letourneau-Montminy, M. P., P. Lescoat, A. Narcy, D. Sauvant, J. F. Bernier, M. Magnin, C. Pomar, Y. Nys, and C. Jondreville. 2008. Effects of reduced dietary calcium and phytase supplementation on calcium and phosphorus utilisation in broilers with modified mineral status. British poultry science. 49:705-715.

Lewis, N.J. and Hurnik, J.F., 1990. Locomotion of broiler chickens in floor pens. Poultry science. 69:1087-1093.

Lewis, P.D. and Morris, T.R., 2000. Poultry and coloured light. World's poultry science journal, 56:189-207. 
Li, T., G. Xing, Y. Shao, L. Zhang, S. Li, L. Lu, Z. Liu, X. Liao, and X. Luo. 2020. Dietary calcium or phosphorus deficiency impairs the bone development by regulating related calcium or phosphorus metabolic utilization parameters of broilers. Poultry science. 99:32073214.

Li, W., R. Angel, S. W. Kim, K. Brady, S. Yu, and P. W. Plumstead. 2016. Impacts of dietary calcium, phytate, and nonphytate phosphorus concentrations in the presence or absence of phytase on inositol hexakisphosphate (IP6) degradation in different segments of broilers digestive tract. Poultry science. 95:581-589.

Lourens, A., Van den Brand, H., Meijerhof, R. and Kemp, B., 2005. Effect of eggshell temperature during incubation on embryo development, hatchability, and posthatch development. Poultry science. 84:914-920.

Maatjens, C. M., I. A. M. van Roovert-Reijrink, B. Engel, C. W. van der Pol, B. Kemp, and H. van den Brand. 2017. Temperature during the last week of incubation. III. Effects on chicken embryo physiology. Poultry science. 96:1451-1458.

McKay, J. C., N. F. Barton, A. N. M. Koerhuis, and J. McAdam. 2000. The challenge of genetic change in the broiler chicken. BSAP occasional publication. 27:1-7.

Meluzzi, A. and Sirri, F., 2009. Welfare of broiler chickens. Italian journal of animal science. 8:161-173.

Mench, J. 2004. Lameness. In Measuring and auditing broiler welfare. p3-17.

M'Sadeq, S.A., Wu, S.B., Choct, M. and Swick, R.A., 2018. Influence of trace mineral sources on broiler performance, lymphoid organ weights, apparent digestibility, and bone mineralization. Poultry science. 97:3176-3182.

Mulder, H.A., Hill, W.G., Vereijken, A. and Veerkamp, R.F., 2009. Estimation of genetic variation in residual variance in female and male broiler chickens. Animal. 3:1673-1680.

Naber, E.C., 1979. The effect of nutrition on the composition of eggs. Poultry science. 58:518528.

Nairn, M.E. and Watson, A.R.A., 1972. Leg weakness of poultry-A clinical and pathological characterisation. Australian veterinary journal, 48:645-656.

Nakane, Y. and M. Tsudzuki. 1999. Development of the skeleton in Japanese quail embryos. Development, growth \& differentiation. 41:523-534.

Ohara, A., C. Oyakawa, Y. Yoshihara, S. Ninomiya, and S. Sato. 2015. Effect of environmental enrichment on the behavior and welfare of Japanese broilers at a commercial farm. The journal of poultry science. p0150034.

Ono, T. and Tuan, R.S., 1991. Vitamin D and chick embryonic yolk calcium mobilization: Identification and regulation of expression of vitamin D-dependent Ca2+-binding protein, calbindin-D28K, in the yolk sac. Developmental biology. 144:167-176.

Oviedo-Rondón, E. O., J. Small, M. J. Wineland, V. L. Christensen, P. S. Mozdziak, M. D. Koci, S. V. L. Funderburk, D. T. Ort, and K. M. Mann. 2008. Broiler embryo bone development is influenced by incubator temperature, oxygen concentration and eggshell conductance at the plateau stage in oxygen consumption. British poultry science. 49:666-676.

Oviedo-Rondón, E. O., M. J. Wineland, S. Funderburk, J. Small, H. Cutchin, and M. Mann. 2009. Incubation conditions affect leg health in large, high-yield broilers. Journal of Applied poultry research. 18:640-646.

Oviedo-Rondón, E.O., Ferket, P.R. and Havenstein, G.B., 2006. Nutritional factors that affect leg problems in broilers and turkeys. Avian and poultry biology reviews. 17:89-103.

Pedersen, I.J. and Forkman, B., 2019. Improving leg health in broiler chickens: a systematic review of the effect of environmental enrichment. Animal welfare. 28:215-230.

Pedersen, I.J., Tahamtani, F.M., Forkman, B., Young, J.F., Poulsen, H.D. and Riber, A.B., 2020. Effects of environmental enrichment on health and bone characteristics of fast-growing broiler chickens. Poultry science. 99:1946-1955.

Pettit-Riley, R. and Estevez, I., 2001. Effects of density on perching behavior of broiler chickens. Applied animal behaviour science. 71:127-140.

Prisby, R., Menezes, T., Campbell, J., Benson, T., Samraj, E., Pevzner, I. and Wideman Jr, R.F., 2014. Kinetic examination of femoral bone modeling in broilers. Poultry science. 93:1122-1129. 
Rath, N.C., Balog, J.M., Huff, W.E., Huff, G.R., Kulkarni, G.B. and Tierce, J.F., 1999. Comparative differences in the composition and biomechanical properties of tibiae of seven-and seventy-two-week-old male and female broiler breeder chickens. Poultry science. 78:1232-1239.

Ratriyanto, A., Prastowo, S. and Widyas, N., 2018, September. Follicular characteristics of quails fed diets containing different nutrient contents. In AIP conference proceedings (Vol. 2014, No. 1, p. 020006).

Reiter, K. and W. Bessei. 2009. Effect of locomotor activity on leg disorder in fattening chicken. Berliner und munchener tierarztliche wochenschrift. 122:264-270.

Rios, H. V., P. D. Waquil, P. S. de Carvalho, and T. Norton. 2020. How are Information Technologies Addressing Broiler Welfare? A systematic review based on the welfare quality assessment. Sustainability. 12:1-31.

Rozenboim, I., Piestun, Y., Mobarkey, N., Barak, M., Hoyzman, A. and Halevy, O., 2004. Monochromatic light stimuli during embryogenesis enhance embryo development and posthatch growth. Poultry science. 83:1413-1419.

Saleh, A.A., Eltantawy, M.S., Gawish, E.M., Younis, H.H., Amber, K.A., Abd El, A.E.M.E. and Ebeid, T.A., 2019. Impact of dietary organic mineral supplementation on reproductive performance, egg quality characteristics, lipid oxidation, ovarian follicular development, and immune response in laying hens under high ambient temperature. Biological trace element research. 195:506-514.

Sanchez-Rodriguez, E., Benavides-Reyes, C., Torres, C., Dominguez-Gasca, N., Garcia-Ruiz, A.I., Gonzalez-Lopez, S. and Rodriguez-Navarro, A.B., 2019. Changes with age (from 0 to $37 \mathrm{D}$ ) in tibiae bone mineralization, chemical composition and structural organization in broiler chickens. Poultry science. 98:5215-5225.

Sherlock, L., Demmers, T.G.M., Goodship, A.E., McCarthy, I.D. and Wathes, C.M., 2010. The relationship between physical activity and leg health in the broiler chicken. British Poultry Science. 51:22-30.

Shim, M. Y. and G. M. Pesti. 201 1. Effects of incubation temperature on the bone development of broilers. Poultry science, 90:1867-1877.

Shim, M. Y., A. B. Karnuah, A. D. Mitchell, N. B. Anthony, G. M. Pesti, and S. E. Aggrey. 2012. The effects of growth rate on leg morphology and tibia breaking strength, mineral density, mineral content, and bone ash in broilers. Poultry science. 91:1790-1795.

Siegel, P.B., Honaker, C.F. and Rauw, W.M., 2009. Selection for high production in poultry (pp. 230-242). CABI Publishing: Wallingford, UK.

Simsek, U.G., Dalkilic, B., Ciftci, M., Cerci, I.H. and Bahsi, M., 2009. Effects of enriched housing design on broiler performance, welfare, chicken meat composition and serum cholesterol. Acta veterinaria brno. 78:67-74.

Starcher, B.C., Hill, C.H. and Madaras, J.G., 1980. Effect of zinc deficiency on bone collagenase and collagen turnover. The Journal of nutrition. 110:2095-2102.

Stojcic, M. D. and W. Bessei. 2009. The effect of locomotor activity and weight load on bone problems in fast and slow growing chickens. Archiv für geflügelkunde. 73:242-249.

Sullivan, T. W. 1994. Skeletal problems in poultry: estimated annual cost and descriptions. Poultry science. 73:879-882.

Świątkiewicz, S., Arczewska-Włosek, A. and Jozefiak, D., 2014. The efficacy of organic minerals in poultry nutrition: review and implications of recent studies. World's poultry science journal, 70:475-486.

Tallentire, C. W., I. Leinonen, and I. Kyriazakis. 2016. Breeding for efficiency in the broiler chicken: A review. Agronomy for sustainable development. 36:1-16.

Tona, K., Bruggeman, V., Onagbesan, O., Bamelis, F., Gbeassor, M., Mertens, K. and Decuypere, E., 2005. Day-old chick quality: Relationship to hatching egg quality, adequate incubation practice and prediction of broiler performance. Avian and poultry biology reviews. 16:109-119.

Torres, C. A. and D. R. Korver. 2018. Influences of trace mineral nutrition and maternal flock age on broiler embryo bone development. Poultry science. 97:2996-3003.

Van der Pol, C. W., I. A. M. van Roovert-Reijrink, C. M. Maatjens, I. van den Anker, B. Kemp, and $\mathrm{H}$. van den Brand. 2014. Effect of eggshell temperature throughout incubation on broiler hatchling leg bone development. Poultry science. 93:2878-2883. 
Vestergaard, S. and Sanotra, G.S., 1999. Relationships between leg disorders and changes in the behaviour of broiler chickens. Veterinary record. 144:205-209.

Waldenstedt, L. 2006. Nutritional factors of importance for optimal leg health in broilers: A review. Animal feed sci-ence and technology. 126:291-307.

Wang, G., L. Liu, Z. Wang, X. Pei, W. Tao, Z. Xiao, B. Liu, M. Wang, G. Lin, and T. Ao. 2019. Comparison of inorganic and organically bound trace minerals on tissue mineral deposition and fecal excretion in broiler breeders. Biological trace element research. 189:224-232.

Wang, Z., Cerrate, S., Yan, F., Sacakli, P. and Waldroup, P.W., 2008. Comparison of different concentrations of inorganic trace minerals in broiler diets on live performance and mineral excretion. International Journal of Poultry science. 7:625-629.

Weeks, C. A., T. D. Danbury, H. C. Davies, P. Hunt, and S. C. Kestin. 2000. The behaviour of broiler chickens and its modification by lameness. Applied animal behaviour science. 67:111-125.

Wijnen, H. J., H. van den Brand, A. Lammers, I. A. M. van Roovert-Reijink, C. W. van der Pol, B. Kemp, and R. Molenaar. 2020. Effects of eggshell temperature pattern during incubation on primary immune organ develop-ment and broiler immune response in later life. Poultry science. 99:6619-6629.

Williams, B., D. Waddington, D. H. Murray, and C. Farquharson. 2004. Bone strength during growth: influence of growth rate on cortical porosity and mineralization. Calcified tissue international. 74:236-245.

Yair, R., Shahar, R. and Uni, Z., 2013. Prenatal nutritional manipulation by in ovo enrichment influences bone structure, composition, and mechanical properties. Journal of animal science. 91:2784-2793.

Yalçın, S., H. B. Malayoğlu, M. Baka, O. Genin, and M. Pines. 2007. Effect of temperature during the incubation period on tibial growth plate chondrocyte differentiation and the incidence of tibial dyschondroplasia. Poultry science. 86:1772-1783.

Yenice, E., Mızrak, C., Gültekin, M., Atik, Z. and Tunca, M., 2015. Effects of organic and inorganic forms of manganese, zinc, copper, and chromium on bioavailability of these minerals and calcium in late-phase laying hens. Biological trace element research, 167:300-307.

Yildirim, M. and Taskin, A., 2017. The effects of environmental enrichment on some physiological and behavioral parameters of broiler chicks. Brazilian journal of poultry science. 19:355-362.

Yıldız, H., M. Petek, G. Sönmez, İ. Arıcan, and B. Yılmaz. 2009. Effects of lighting schedule and ascorbic acid on performance and tibiotarsus bone characteristics in broilers. Turkish journal of veterinary and animal sciences. 33:469-476.

Yu, Y., Li, Z., Zhong, Z., Jin, S., Pan, J., Rao, X. and Yu, Y., 2018. Effect of monochromatic green LED light stimuli during incubation on embryo growth, hatching performance, and hormone levels. Transactions of the ASABE. 61:661-669.

Zhang, L., Zhang, H.J., Wang, J., Wu, S.G., Qiao, X., Yue, H.Y., Yao, J.H. and Qi, G.H., 2014. Stimulation with monochromatic green light during incubation alters satellite cell mitotic activity and gene expression in relation to embryonic and posthatch muscle growth of broiler chickens. Animal. 8:86-93.

Zhang, L., Zhu, X.D., Wang, X.F., Li, J.L., Gao, F. and Zhou, G.H., 2016. Green light-emitting diodes light stimuli during incubation enhances posthatch growth without disrupting normal eye development of broiler embryos and hatchlings. Asian-Australasian journal of animal sciences. 29:1562-1568.

Zhao, J., Shirley, R.B., Vazquez-Anon, M., Dibner, J.J., Richards, J.D., Fisher, P., Hampton, T., Christensen, K.D., Allard, J.P. and Giesen, A.F., 2010. Effects of chelated trace minerals on growth performance, breast meat yield, and footpad health in commercial meat broilers. Journal of applied poultry research. 19:365-372. 


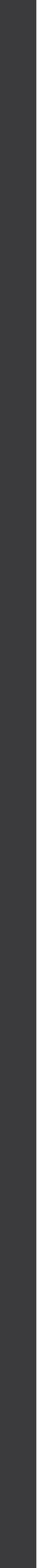


Summary

Oren

(Turkish summary)

Acknowledgements

About the author

Publications

Training and supervision

Colophon 

Chicken meat is one of the main animal protein sources for humans and is nowadays the second most produced and consumed meat in the world. Since 1950s, broiler (meat-type) chickens have undergone radical phenotypic and genotypic changes as a result of an intense genetic selection for fast growth and better feed efficiency. Accompanied by better management conditions and better feed quality, this selection has provided several advantages, such as high amount of meat production in a short time, less environmental pollution and considerable financial benefits for producers. However, it has also caused downsides for broiler chickens, such as poor leg health, impaired locomotion and lameness. One of the main reasons for these downsides is an imbalance between high growth rate and immature bones, because the speed of bone development is unable to keep up with fast growth. Poor leg health causes serious welfare issues and considerable economic losses, since these chickens have difficulties accessing feed and water, suffer from pain, dehydration and starvation as results of high risk of leg bone fractures, developmental bone abnormalities and infectious and/or non-infectious leg disorders.

The main focus of this thesis is on leg bone development, specifically tibia, since it is the major weight carrying and the most loaded and affected leg bone during the growth period. The aim of this thesis was to investigate different approaches to improve leg health of fast and slower-growing broiler chickens by (1) dietary factors on the broiler chickens or indirectly via the broiler breeders, (2) incubation conditions and (3) environmental enrichment.

The General Introduction of this thesis first describes suboptimal leg health, bone abnormalities and leg disorders observed in broiler chickens and their welfare and economic consequences. Then continues with leg bone quality measurements, such as bone morphological, biophysical and mechanical characteristics. Lastly, gives information on potential approaches, which are investigated in this thesis to improve leg health in broiler chickens.

Chapter 1 of this thesis describes effects of a combination of organic macro and trace minerals, fish oil and hydrolyzed 
collagen in the diet of fast-growing male broiler chickens on growth performance, tibia morphological, biophysical and mechanical characteristics (day 28, 35 and 42), leg disorders, locomotion and home pen behaviour. Results of this study showed that organic minerals in the diet positively affected tibia morphological, biophysical and mechanical characteristics and also growth performance, while hydrolyzed collagen in the diet did not affect tibia characteristics and fish oil in the diet negatively affected these characteristics. It can be concluded that bone development might need more available minerals in the diet of current fast-growing broiler chickens.

Chapter 2 of this thesis describes effects of green LED light during incubation, the separate effects of macro minerals source and trace minerals source during rearing on growth performance, tibia morphological, biophysical and mechanical characteristics (day 42), leg disorders, locomotion and home pen behaviour of fast-growing male broiler chickens. Results of this study showed that green LED light during incubation did not influence any of the tibia characteristics. Organic macro minerals positively affected tibia characteristics compared to inorganic minerals and trace mineral source did not affect these characteristics. It can be concluded that despite green light during incubation have been shown to positively affect growth performance in this study, it did not affect tibia characteristics. The source of macro minerals in the diet of broiler chickens seems to play a major role in bone development, rather than the source of trace minerals.

Chapter 3 of this thesis describes effects of a combination of organic macro and trace minerals in the diet of fast and slower-growing broiler breeders on the amount of minerals in egg and hatchling, growth performance, tibia morphological, biophysical and mechanical characteristics (at similar body weights), leg disorders and home pen behaviour of their male offspring broiler chickens. Results of this study showed that mineral concentration in eggs and hatchlings were hardly influenced, but almost all tibia morphological, biophysical and mechanical characteristics of 
slower-growing offspring broilers were positively affected, whereas this effect was hardly seen in fast-growing chickens. It can be concluded that trans-generational mineral availability in offspring appears to play a role via other mechanisms than via absolute mineral concentrations in the egg and the source of minerals in slower-growing breeders' diet appears to be more effective on bone development of their offspring than in fast-growing chickens, which might be related to time available for bone development.

Chapter $\mathbf{4}$ of this thesis describes effects of eggshell temperature pattern in week $2\left(37.8^{\circ} \mathrm{C}\right.$ or $\left.38.9^{\circ} \mathrm{C}\right)$ and week $3\left(36.7^{\circ} \mathrm{C}\right.$ and $37.8^{\circ} \mathrm{C}$ ) of incubation of fast-growing male broiler chickens on tibia morphological, biophysical and mechanical characteristics (day 41 or 42), leg disorders and locomotion. Results of this study showed that a temperature of $38.9^{\circ} \mathrm{C}$ during the second week of incubation improved tibia characteristics of fast-growing broiler chickens. Incubation temperature in the third week appears to interact with the incubation temperature in the second week, resulting in a most advanced tibia development after incubation at $38.9^{\circ} \mathrm{C}$ in week 2 , followed by $37.8^{\circ} \mathrm{C}$ in week 3 of incubation. It can be concluded that a $1.1^{\circ} \mathrm{C}$ higher EST than normal in week 2 of incubation appears to stimulate tibia morphological, biophysical and mechanical characteristics of broiler chickens. However, a $1.1^{\circ} \mathrm{C}$ lower EST in week 3 of incubation appears to have negative effects on tibia characteristics.

Chapter $\mathbf{5}$ of this thesis describes effects of pen enrichment consisting of ramps, platforms, perches, large distance between feed and water and live Black Soldier fly larvae in the moss-peat dust bathing area on growth performance, tibia morphological, biophysical and mechanical characteristics (at similar body weights), leg disorders, locomotion and home pen behaviour of fast and slower-growing male broiler chickens. Results of this study showed that pen enrichment positively affected tibia biophysical characteristics in both fast and slower-growing chickens, while no effect was found on tibia morphological and mechanical characteristics. It can be concluded that pen enrichment can stimulate 
pathways involved in ossification and mineralization, rather than anatomical and physical bone properties.

The General Discussion of this thesis summarizes the objectives and main findings from five experimental chapters and discusses how investigated approaches individually or together give insight in and improve our understanding of leg health of broiler chickens. This chapter ends with main conclusions and future research opportunities.

\section{The main conclusions of this thesis are:}

(1) Replacement of a combination of inorganic macro and trace minerals by their organic varieties in the diet of fast-growing broiler chickens positively affected growth performance and tibia characteristics.

(2) Replacement of a combination of macro and trace minerals by their organic varieties in the diet of fast and slower-growing broiler breeders resulted in stimulated growth performance and tibia characteristics in slower-growing offspring broilers, while no effect was observed in fast-growing offspring broilers.

(3) Trans-generational mineral availability in offspring broilers appears to play a role via other mechanisms than via absolute mineral concentrations in the egg, since mineral concentrations in eggs and hatchlings were not affected by mineral source.

(4) Replacement of inorganic macro minerals in the diet of fast-growing broiler chickens by their organic varieties seems to be more effective than organic trace minerals on tibia characteristics.

(5) Green LED light during incubation resulted in stimulated body weight gain, but no effect was found on bone development of broiler chickens.

(6) An eggshell temperature of $38.9^{\circ} \mathrm{C}$ during the second week of incubation was more effective on tibia characteristics of fast-growing broiler chickens compared to an eggshell temperature of $37.8^{\circ} \mathrm{C}$. An eggshell temperature of $38.9^{\circ} \mathrm{C}$ in second week, followed by $37.8^{\circ} \mathrm{C}$ in third week resulted in the most advanced 
tibia characteristics, while a $1.1^{\circ} \mathrm{C}$ lower EST in week 3 of incubation appears to have negative effects on tibia characteristics.

(7) Pen enrichment positively affected tibia biophysical characteristics of both slower and fast-growing chickens, while no effect was found on tibia morphological and mechanical characteristics. Pen enrichment also resulted in lower body weight gain in both fast and slower-growing chickens, potentially due to higher activity and use of metabolic energy or lower feed intake.

(8) It appears that tibia biophysical characteristics seem to give better response to source of the mineral both in broiler and broiler breeder diets, eggshell temperature during incubation and pen enrichment compared to morphological and mechanical characteristics,

(9) The relationship between tibia development and leg abnormalities and disorders remained unclear due to very low incidence of leg abnormalities and disorders. 

Tavuk eti, insanlar için temel hayvansal protein kaynaklarından biridir ve günümüzde dünyada en çok üretilen ve tüketilen ikinci et konumundadır. 1950'lerden bu yana, broiler (et tipi) tavuklar, hızı büyüme ve daha iyi yem verimliliği için yoğun bir genetik seçilim sonucunda radikal değişikliklere uğramıştır. Daha iyi yetiştirme koşulları ve daha iyi yem kalitesinin de eşlik ettiği bu genetik seçilim, kısa sürede yüksek miktarda et üretimi, daha az çevre kirliliği ve üreticiler için yüksek kazançlar gibi birçok avantaj sağlamıştır. Ancak, bacak ve kemik sağlığı bu durumdan olumsuz etkilenmiş, hareketsizlik ve topallık gibi olumsuzluklara da neden olmuştur. Bunun ana nedenlerinden biri, yüksek büyüme hızı ile olgunlaşmamış kemikler arasındaki dengesizliktir. Çünkü kemik gelişim hızı, bu olağanüstü hızı büyümeye ayak uyduramamaktadır. Sağlıksız bacak kemikleri, etlik tavuklarda ciddi refah sorunlarına, yem ve suya erişimlerinde güçlüklere, kemiklerde yüksek kırılma riskine, gelişimsel kemik anormalliklerine, bulaşıcı ve/veya bulaşıcı olmayan hastalıklara ve sonuç olarak çok ciddi ekonomik kayıplara neden olmaktadır.

Bu tez, tavukların büyüme döneminde en fazla yük taşıyan ve en çok etkilenen bacak kemiği olan tibia (kaval) kemiğine yoğunlaşmaktadır. Bu tezin amacı, etlik tavukların bacak sağlığını ve kemik gelişimini iyileştirmek için farklı yaklaşımları araştırmaktır. Bu yaklaşımlar (1) etlik tavukların veya etlik damızlık tavukların yemlerine yapılan katkılar, (2) kuluçka koşulları ve (3) çevresel zenginleştirmedir.

Tezin Giriş bölümü, öncelikle etlik tavuklarda gözlenen sağlıksız bacakları, kemik anormalliklerini ve bu olumsuz durumların tavukların refahı üzerine etkilerini ve ekonomik sonuçlarını anlatmaktadır. Daha sonra, kemiğin morfolojik, biyofiziksel ve mekanik özellikleri gibi kalite ölçümleri üzerine detaylı bilgi vermektedir. Son olarak, etlik tavuklarda bacak ve kemik sağlığını iyileştirmek için bu tezde araştııılan yaklaşımlar hakkında genel bilgi verilmektedir.

Tezin 1. Bölümünde, hızlı büyüyen etlik tavukların yemlerine organik makro mıneraller (kalsiyum ve fosfor) ve iz mıneraller (demir, bakır, manganez, çinko ve selenyum), balık yağı ve hidrolize kollajen kombinasyonunun büyüme performansı, tibia kemiği morfolojik, 
biyofiziksel ve mekanik özellikleri (28, 35 ve 42. gün), bacak sağlığı bozuklukları, hareket ve davranışları üzerindeki etkileri araştııımıştır. Bu çalışmanın sonuçları, yemdeki organik minerallerin tibia kemiğinin morfolojik, biyofiziksel ve mekanik özelliklerini ve ayrıca büyüme performansını olumlu yönde etkilediğini, yemdeki hidrolize kollajenin kemik özelliklerini etkilemediğini ve yemdeki balık yağının kemik özelliklerini olumsuz etkilediğini göstermiştir. Hızlı büyüyen etlik tavukların yemlerinde kemik gelişimi için daha yarayışlı minerallere intiyaç duyduğu sonucuna ulaşılmıştır.

Tezin 2. Bölümünde, hızı büyüyen etlik tavuklara kuluçkada uygulanan yeşil LED ışığın etkileri, yetiştirme aşamasında organik makro (kalsiyum ve fosfor) ve iz minerallerin (demir, bakır, manganez, çinko ve selenyum) büyüme performansı, tibia kemiği morfolojik, biyofiziksel ve mekanik özellikleri (42. gün), bacak sağlığı bozuklukları, hareket ve davranışları üzerindeki etkileri araştııımıştır. Bu çalışmada, organik makro minerallerin inorganik makro minerallere göre kemik özelliklerini olumlu yönde etkilediği ve organik iz minerallerin kemik özelliklerine etkisi olmadığı saptanmıştır. Bu çalışmada kuluçka sırasında yeşil LED ışığın büyüme performansını olumlu yönde etkilediği bulunmuş olmasına rağmen tibia kemiği özelliklerini etkilemediği tespit edilmiştir. Etlik tavukların yemlerindeki makro minerallerin kaynağının kemik gelişiminde önemli bir rol oynadığı sonucuna varımıştır.

Tezin 3. Bölümünde, hızlı ve daha düşük hızlı büyüyen etlik damızlık tavukların yemlerindeki organik makro (kalsiyum ve fosfor) ve iz minerallerin (demir, bakır, manganez, çinko ve selenyum) kombinasyonunun yumurta ve civciv gövdesindeki mineral miktarı, etlik tavukların büyüme performansı, tibia kemiği morfolojik, biyofiziksel ve mekanik özellikleri (benzer canlı ağırlık düzeylerinde), bacak sağlığı bozuklukları, hareket ve davranışları üzerindeki etkileri araştııımıştır. Bu çalışmada, yumurtalarda ve civcivlerde mineral miktarının etkilenmediği, ancak daha düşük hızlı büyüyen etlik tavukların neredeyse tüm tibia morfolojik, biyofiziksel ve mekanik özelliklerinin olumlu yönde etkilendiği, buna karşın hızlı büyüyen etlik tavuklarda bu etkinin görülmediği saptanmıştır. Civcivlerde 
damızlık tavuktan gelen mineral miktarının yumurtadaki mineral miktarından ziyade başka mekanizmalar yoluyla etki ettiği ve daha düşük hızlı büyüyen damızlıkların yemlerindeki mineral kaynağının bir sonraki jenerasyonda kemik gelişimi üzerinde daha etkili olduğu sonucuna varımıştır.

Tezin 4. Bölümünde, hızlı büyüyen etlik tavuklarda kuluçkanın 2. haftasında normal $\left(37.8^{\circ} \mathrm{C}\right)$ ve yüksek $\left(38.9^{\circ} \mathrm{C}\right)$ ve 3 . haftasında düşük $\left(36.7^{\circ} \mathrm{C}\right)$ ve normal $\left(37.8^{\circ} \mathrm{C}\right)$ yumurta kabuğu sıcaklığının tibia kemiği morfolojik, biyofiziksel ve mekanik özellikleri (41 veya 42. gün), bacak sağlığı bozuklukları ve hareket üzerindeki etkileri araştırımıştır. Bu çalışmada, kuluçkanın ikinci haftasında $38.9^{\circ} \mathrm{C}$ 'lik yüksek sıcakığın hızlı büyüyen etlik tavukların tibia kemiği özelliklerini geliştirdiği saptanmıştır. Ayrıca, üçüncü haftadaki kuluçka sıcaklığının ikinci haftadaki kuluçka sıcaklığı ile bağlantılı olduğu tespit edilmiştir. 2. haftada $38.9^{\circ} \mathrm{C}$ 'lik yüksek sıcaklık ve ardından 3. haftada $37.8^{\circ} \mathrm{C}$ 'lik normal sıcaklıkta kuluçkalanan tavuklarda en gelişmiş tibia özellikleri saptanmıştır. Kuluçkanın 2. haftasında normalden $1.1^{\circ} \mathrm{C}$ daha yüksek yumurta kabuğu sıcaklığının etlik tavukların tibia kemiği morfolojik, biyofiziksel ve mekanik özelliklerini uyardığı sonucuna varılmıştır. Bununla birlikte, kuluçkanın 3. haftasında $1.1^{\circ} \mathrm{C}$ daha düşük yumurta kabuğu sıcaklığının tibia kemiği özellikleri üzerinde olumsuz etkileri olduğu görülmektedir.

Tezin 5. Bölümünde, hızlı ve daha düşük hızlı büyüyen etlik tavukların yaşam alanlarına rampalar, platformlar, tünekler, yem ve su arasındaki büyük mesafe ve turba yosunu ile kaplı kum banyosu alanında canlı siyah asker sineği larvalarından oluşan çevresel zenginleştirme elemanları eklemenin büyüme performansı, tibia kemiği morfolojik, biyofiziksel ve mekanik özellikleri (benzer vücut ağırlıklarında), bacak sağlığı bozuklukları, hareket ve davranışları üzerindeki etkileri araştırımışır. Bu çalışmada, çevresel zenginleştirmenin hem hızlı hem de daha düşük hızı büyüyen tavuklarda tibia kemiği biyofiziksel özelliklerini olumlu yönde etkilediği, tibia morfolojik ve mekanik özellikleri üzerinde ise herhangi bir etki bulunmadığı saptanmıştır. Çevresel zenginleştirmenin anatomik ve fiziksel kemik özelliklerinden ziyade kemikte mineralizasyonla ilgili 
mekanizmaları uyarabileceği sonucuna varılmıştır.

Tezin Tartışma bölümünde, beş deneysel bölümden elde edilen ana bulgular özetlenmiş ve tek tek veya birlikte araştıılan yaklaşımların, etlik tavukların bacak sağlığını geliştirmemize ilişkin anlayışımızı nasıl değiştirdiği ve geliştirdiği tartışılmıştır. Bu bölüm, ana sonuçlar ve gelecekteki araştırma olanakları ile sona ermektedir.

\section{Bu tezin ana sonuçları şunlardır:}

(1) Hızı büyüyen etlik tavukların yemlerinde bulunan inorganik makro ve iz minerallerin organik çeşitleriyle değiştirilmesi, büyüme performansını ve tibia kemiği özelliklerini olumlu yönde etkilemiştir.

(2) Hızlı ve daha düşük hızlı büyüyen etlik damızık tavukların yemlerinde bulunan inorganik makro ve iz minerallerin organik çeşitleriyle değiştirilmesinin, daha düşük hızlı büyüyen yavru etlik tavukların büyüme performansını ve tibia kemiği özelliklerini olumlu etkilediği, hızı büyüyen yavru etlik tavuklarda herhangi bir etki gözlenmediği saptanmıştır.

(3) Etlik tavuklarda nesiller arası mineral aktarımının, yumurtadaki ve kuluçkadaki mineral miktarından ziyade mineral kaynağından etkilendiği ve yumurtadaki mutlak mineral konsantrasyonları dışında başka mekanizmalar yoluyla işlediği saptanmıştır.

(4) Hızlı büyüyen etlik tavukların yemlerindeki inorganik makro minerallerin organik çeşitleriyle değiştirilmesi, tibia kemiği özellikleri üzerinde organik iz minerallerden daha etkili olduğu saptanmıştır.

(5) Kuluçka sırasında uygulanan yeşil LED ışık, vücut ağırlığı artışına neden olmuştur, ancak kemik gelişimi üzerinde herhangi bir etki saptanmamıştır.

(6) Kuluçkanın ikinci haftasında $38.9^{\circ} \mathrm{C}$ 'lik yumurta kabuğu sıcaklığı, $37.8^{\circ} \mathrm{C}$ 'lik bir yumurta kabuğu sıcaklığına kıyasla hızı büyüyen etlik tavukların tibia kemiği özelliklerini geliştirdiği saptanmıştır. İkinci haftada $38.9^{\circ} \mathrm{C}$ 'lik bir yumurta kabuğu sıcaklığı, ardından üçüncü haftada $37.8^{\circ} \mathrm{C}$ ile en gelişmiş tibia özellikleriyle sonuçlanırken, kuluçkanın 3. haftasında $1.1^{\circ} \mathrm{C}$ daha düşük yumurta kabuğu sıcaklığı nın tibia özellikleri üzerinde olumsuz etkileri olduğu bulunmuştur. 
(7) Çevresel zenginleştirme, hem hızlı hem de daha düşük hızIı büyüyen etlik tavukların tibia kemiği biyofiziksel özelliklerini olumlu yönde etkilerken, tibia morfolojik ve mekanik özellikleri üzerinde herhangi bir etkisi bulunmamıştır. Çevresel zenginleştirme ayrıca, potansiyel olarak daha yüksek aktivite ve metabolik enerji kullanımı veya daha düşük yem tüketimi nedeniyle hem hızlı hem de daha düşük hızlı büyüyen tavuklarda daha düşük vücut ağırlığı ile sonuçlanmıştır.

(8) Tibia kemiği biyofiziksel özelliklerinin hem etlik tavuk hem de etlik damızlık tavuk yemlerindeki mineral kaynağına, kuluçka sırasında yumurta kabuğu sıcaklığına ve çevresel zenginleştirmeye, tibia kemiği morfolojik ve mekanik özelliklere kıyasla daha iyi yanıt verdiği saptanmıştır.

(9) Tibia kemiği gelişimi ile bacak anormallikleri ve bacak sağlığı bozuklukları arasındaki ilişki, tüm deneylerde saptanan az miktardaki bacak anormallikleri ve bacak sağlığı bozukluklarından dolayı belirsizliğini korumuştur. 

I know that acknowledgements is the most read part of the thesis. So, I tried to make it enjoyable without forgetting anyone.

My PhD adventure started on first days of 2017 and completed on first days of 2022, after almost 1830 days. It was a great experience but also compelling and challenging both personally and professionally. I would not have been able to make it any of 1830 days without the help and support from many people whom I would like to acknowledge below.

I would like to start to express my gratefulness to dear Marinus van Krimpen (deceased, 31.12.19). Dear Marinus, we have first seen each other during my online PhD interview. I thought that he looks like a harsh and a tough person. Then, you broke down my prejudices when we met faceto-face. Looking back to these 3 years, I remember how kind, considered and gentle you were. I remember how you were pushing yourself to look strict and serious person at work because you, as a project leader, had to be, but you could not succeed because of your friendly and warm-blooded heart. I will always remember your facial changes between laughing and seriousness. We spent 3 years alongside and many hours in our internal and group meetings to make our project better and better. We had nice car travels to collaborate other universities. I remember one of our trips between Eindhoven and Wageningen when you asked me what is the meaning of my name. Then you surprised when I said Bahadir means brave and Can means heart, so it is Braveheart. Then, I said I just realized that meaning today after your question, because this meaning is only in English, not Turkish, then we laughed. I will always remember that day. I remember $\mathrm{my} 30^{\text {th }}$ birthday and your wishful words to me while we shared a delicious apple pie. I remember how proud I was when I invited you and Henry to be keynote speakers in the conference at Antalya. I remember the day you asked me to join a meeting with dean and vice dean of Ankara University, Faculty of Agriculture. I had a chance to meet them through you and continue to work with them after going back to Turkey, thanks to you. And I remember that heartbreaking day, the first day of 2020, when I got the email saying you suddenly passed away. I could not believe it first, then read it again and again... I will always remember you, your voice, your laughs...

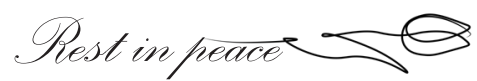


I would like to thank to my promotor Bas Kemp. Dear Bas, I am grateful for everything. You are the most cheerful and pleasant chair holder and promoter I have ever seen. I appreciate your critical remarks and suggestions in all our papers and this thesis thanks to your broad experience. I will always remember how friendly and warm-hearted you were. I wish you a healthy and great life.

The biggest thank of this thesis belongs to my dear co-promotor and daily supervisor Henry van den Brand. Dear Henry, we have gone through a long way together. I will never be able to put into words how impactful your knowledge, research experience, dedication, guidance and actions were. I will always remember your comforting, supportive and sometimes blustery (but necessary) talks at all critical points. These were such powerful tools and milestones that helped me with all difficulties. You have always patiently guided and encouraged me in my adventure to achieve my scientific independence and doctoral degree. I will always remember how you influenced and changed my life, my thoughts, my way of thinking, my decision-making mechanisms and many more things, impossible to fit here. In short, I am getting my PhD thanks to you. I believe we will continue to collaborate and see each other in future. I wish you a healthy and great life with your loved ones.

I would like to thank to the rest of my supervision team. Ingrid de Jong and Roos Molenaar.

Dear Ingrid, I am grateful to know you. You are one of the few people who is the best at what they do. Many thanks for all of your efforts to make our project, experiments and papers better. I appreciate your knowledge and experience, and also your friendly and warm-blooded heart. I wish you a healthy and great life with your loved ones.

Dear Roos, thanks for everything. I have learned a lot from you. I will always remember how friendly, chatty and energetic you were. You energized even the long, exhausting and boring slaughtering days. Many thanks for all of your efforts. I wish you a healthy and great life with your loved ones.

I want to continue with some special thanks. 
First one goes to Tom Berghof. Dear Tom, on that day, somewhere in the summer of 2016, I found you on Linkedln, then my PhD adventure started. I am grateful for your helpfulness, all of your detailed answers and your guidance. You directed me to Henry and I took my first step into my PhD project. I am also grateful for you to be my buddy during my first days in Wageningen. Do you remember your hand-drawn Wageningen map for me? I will keep it in my Wageningen memories box forever. I wish you a healthy and great life with your loved ones.

My second special thanks go to Henny Reimert. Dear Henny, it is a great pleasure to know you. You are super kind, friendly, helpful, good-humored and cheerful person. I will always remember your precious help during slaughtering days, our super nice chat while we travelling to Castricum together, when we went through the Houtribdijk, a $27 \mathrm{~km}$ long dike on the North Sea. You are the first person which I e-mailed in Dutch. I will never forget your happiness when I wrote in Dutch. I wish you a healthy and great life with your loved ones.

My last special thanks go to Lotte Stokvis. Dear Lotte, it is a great pleasure to know you. I owe you a lot. I started my first experiment just after starting my PhD thanks to your protocols and prototypes that you prepared before me. Many thanks for waking up in the middle of the night to come the Nijkerk to help slaughtering and bone collection for all day. Many thanks for our nice chats and laughs during coffee breaks. I will always remember your extraordinary help and friendliness. I hope life brings you lots of happiness and health. I am looking forward to see you on the stage when you defend your thesis.

I would like to thank Olivia Shaw and Anne Steenstra for their exceptional help in green LED light and organic minerals experiment conducted at Nijkerk. I will never forget how much physical work we did, while we also enjoyed. It was really tough to manage, weigh, feed 720 chickens and try to keep them clean, healthy and alive.

I got the chance to supervise MSC and BSC thesis students. I would like to thank Florence Edson, Robert van der Klis, Thijs Versantvoort, Ute Bol and Wisse Zwanenburg. I am very glad to know each of you and work with you guys. You contributed data and nice perspectives in this thesis. 
I would like to thank Fleur Veldkamp for her exceptional help in enrichment experiment conducted at Lelystad. Fleur, I appreciate your effort throughout the experiment. Your co-author place in enrichment paper is well deserved. I wish you a great success in your PhD journey.

I would like to thank Jeroen Bakker. Dear Jeroen, you were a nice road friend. We did a good job in validation experiment at Schothorst Feed Research and also during analyses. I am very glad to know you. I wish you a great success in future and in your PhD journey.

I would like to thank two nice people at Axis building. Remco Hamoen, many thanks for your extraordinary assistance in 3D X-ray CT scanner and your remarkable mind in coding the X-ray software. I could not do anything with that huge machine and its crazy features without you. Jos Sewalt, thanks for your valuable help during bone breaking tests. I could not manage without your expertise.

I would like to thank all super genius people of Technical Development Studio at Innovatron building. I have learned super futuristic things, 3D printing and prototype modelling thanks to all of you. I have a special thanks to Johan Belgraver for his precious help for Instron prototype designing and 3D printing.

I would like to thank all Carus personnel, but especially Arjan van Dolderen, Jacco Klomp, Sherine Gasho-Asaad and Ries Verkerk. You were the best work force at Carus. I could not do anything without your help. Many thanks for your energy and time.

It is time to go back to ADP office.

I would like to start with two best secretaries of WUR, Nanette van Hapert and Lora van der Kleijn. Many thanks to help with all of my paper works, arrangements and difficulties with your always smiley faces, calming talks, helpfulness and friendliness in every situation at every time. I wish you a healthy and great life with your loved ones.

I would like to continue to thank research assistants of Adaptation Physiology Group. I would not have been able to manage all the intensive physical work during hatching and slaughtering days and also analyze days at lab without their help. 
Bjorge Laurenssen, you are the king of knife skills, slaughtering and dissection. Many thanks for being my paranymph and for accompanying me on my defense day. I will always remember your friendliness during our road trips and helpfullness on slaughtering days. I wish you a healthy and great life with your lovely family and all of your loved ones.

Ilona van den Anker-Hensen, many thanks for waking up in the middle of the night to come the Carus for counting hatched chicks, all nice lab talks while we were preparing the bones for analyses, a nice trip between Wageningen and Lelystad and we shocked when we saw a swan family in the middle of the highway and thanks for nice chats and laughs about everything during coffee breaks. I wish you a healthy and great life with your loved ones.

Marcel Heetkamp, thanks for all the work you have done during incubation phases of my three experiments. I have learned many things from you. You are the king of Climate Respiration Lab. I wish you a healthy and great life.

Rudie Koopmanschap, many thanks for all your help in the lab and equipment purchases for my experiments. I will always remember your facial expression when I told you that I need at least 10 meters of green LED strip and you said I cannot buy a party stuff by using project money. I wish it was for a party. I will always remember your always-smiley face and loughs. Enjoy your retirement.

Ger de Vries Reilingh, do you remember the day I put the warm chicken meats and bones into -80 freezer and caused the alarm in the middle of night because of a rapid decrease in temperature which made you come to Zodiac. One day after that day, we have met. You were super helpful all the time. Enjoy your retirement.

It is time to talk about my PhD colleagues.

The first place belongs to Jan Wijnen. Dear Jan, many thanks for being my paranymph. I will always remember your extraordinary help, friendliness and our nice chats. The $4^{\text {th }}$ chapter of this thesis exist because of you and our nice experimental collaboration. I hope life brings you and your lovely family lots of happiness and health. I sincerely want my first visit to the Netherlands to be for your defense. I am looking forward to this day. 
I would like continue with Francesca Marcato. Dear Francesca, we both have gone through a challenging but a great PhD adventure together at the same time at the same room. I will always remember your friendliness, supportive talks and always smiley face, and our gossips and laughs of course. As you said in your acknowledgements, what kind of journey was that between Bern and Wageningen. I forgot how many trains we changed. It was tiring but also fun. I am glad that we have met and became friends. I wish you a healthy and great life with Andrea and your future family.

Maarten Hollemans, what a trip and conference was that in Dubrovnik. It is a great pleasure to know you Maarten. You were the most good-humored and cheerful person in ADP. Thanks all of the days that you came and picked us from tropical room for coffee break. I wish you a healthy and great life with your loved ones.

Jesse Heijmans, it is a great pleasure to know you Jesse. You are super kind, friendly and cheerful person. Thanks for all of our chats and laughs. I am looking forward to see you on the stage when you defend your thesis. I wish you a healthy and great life with your loved ones.

Allyson Ipema, it is a great pleasure to know you Allyson. You are super friendly, helpful and cheerful person. Many thanks for all of your help during slaughtering days of my last experiments. I wish you a great success in your PhD and defense.

I would like to thank all my PhD colleagues at tropical room. Anouschka Middelkoop, Carolina da Silva, Eline Burgers, Francesca Marcato, Junnan Ma, Lisette van der Zande, Lu Luo, Michel Verwoolde, Novi Mayasari, Sofie van Nieuwamerongen, Tom Berghof and Wei Xu. I know tropical room got its name from intercontinental people inside it, but also the temperature. How can we forget how hot our room was during hot summer days? It was a great pleasure to know all of you guys. Comparing to other PhD common rooms of other groups in Zodiac and in other buildings (Yes, I have heard many strange stories and rumors), we were super silent, harmonious, gentle and respectful to each other. So, we were very lucky. I wish all of you a splendid future. 
I would like to thank all other people at Adaptation Physiology Group. Aart Lammers, Akke Kok, Anne van den Oever, Ariette van Knegsel, Bas Rodenburg, Carla van der Pol, Elsbeth Stassen, Henk Parmentier, Inonge Reimert, Jerine van der Eijk, Joop Artz, Kevin Lin, Klaas Frankena, Liesbeth Bolhuis, Lisette Graat, Manon van Marwijk, Monique Ooms, Nicoline Soede, Renny van Hoeij, Shai Barbut and Suresh Neethirajan. Many thanks for your friendliness and kindness. I would also like to thank all pleasant people of Wageningen Livestock Research, especially Jan van Harn, Piet van Wikselaar and Rick van Emous. I am very glad to know each of you.

I would like to thank all dedicated young people at De Veetelers, the study association for Animal Sciences students of Wageningen University and Research. I am grateful that you introduced my project in your webpage and journal.

It was a great pleasure and honor to be part of the history of Wageningen University and Research by designing 100 th years of WUR special logos and sweaters. I will always remember the moments and excitement when my name was announced. I will remember all laughs and applauses while I was talking about my chicken-invaded life. I would like to thank the Rector Magnificus and Executive Board of Wageningen University and Research, all cheerful people working at Atlas building, Merel Arkenbout (project group Gifts \& Gadgets 100 years WUR), Geurt Heimensen (Program manager 100 years WUR), Louise Fresco (Chairman of the Executive Board and Chairman of the Steering Committee 100 years WUR), Marnix de Vries (Unigear) and Dieuwertje de Wagenaar (Circular Economy Student Hub).

I would like to thank all people in WIAS PhD Council 2018-2020. Eline Burgers, Evelien Bos, Francesca Marcato, Hugo Loning, Annemiek Blasweiler, Julia Celis Moreno, Joëlle Janssen, Ibrahim Jibrila and Henri van Kruistum.

It is time to talk about WIAS Annual Conference 2020.

I am grateful to be a chairman of this conference. I would like to thank my organization team, Julia Celis Moreno, Timo Stäudle and Windi al Zahra. We did a great job guys. I will always remember how we perfectly 
handled everything, every detail, every nice touch. If I try to write all things one by one, it would not fit here. It was a great conference and a great experience for me both personally and professionally. It has been almost two years since the conference but people are still talking about how great our conference was. We made it what no one could have imagined. I would also like to thank our keynote speakers. Bernice Bovenkerk, Coen Elemans, Graham Glastow, Laurent Frantz and Rod Wilson. It is a great honor to know all of you. I have also some more thanks to our volunteer helpers, Denge Esentürk, Hakkı Gürkan, Ivy van Kemenade, Olivia Shaw, Paulina Krzysica, Sven Beekman and Thijs Hendrikse. We could not succeed without your help during conference guys, many thanks. I am very glad to know each of you.

I would like to thank two super nice people at WIAS, Janneke van Seters and Marianne Bruining. Dear Janneke, I appreciate your comforting talks at all critical points of my PhD journey and your great help during preparations of WIAS Annual Conference 2020. Dear Marianne, many thanks for everything, your always smiley face, calming talks and keeping track of my PhD training.

I would like to thank all nice people at Ministry of Agriculture, Nature and Food Quality of the Netherlands, Aviagen EPI, Darling Ingredients Inc., ForFarmers N.V., Hubbard, Marel Stork Poultry Processing B.V., Nepluvi and Nutreco, who was involved in the Healthy Bones project.

I would like to thank General Directorate of Agricultural Research and Policies in Turkish Ministry of Agriculture and Forestry for my PhD scholarship including monthly salaries, tuition fees and health expenses. I would like to thank all kind people working at the General Directorate of Higher Education and Foreign Education in Turkish Ministry of National Education for handling all my requests and paperwork. I would like to thank all people working at Education Consultancy in Embassy of Turkey in The Hague.

I was so lucky that Wageningen brought me exceptional Turkish friends who made all my days pleasant with their funny stories, positive energies and optimisms which made my PhD challenges easier. I want to start with my first Turkish men of Wageningen team. First thank goes to Engin Yücel. We spent more than 3 years unitedly in that small village and 
shared many things in our nice containerhood. You showed me how a person would not take the world seriously by going with the flow in everything and everywhere. Samet Sağlam, I will always remember our football watching gatherings accompanied by pizza and beer at your room and your complaints about WUR, The Netherlands and its filthy weather. We will see each other soon and remember all of these stories. Damla Coşkun, thanks for everything, all nice gatherings, your supportive words and our wine-full nights in front of B20, Engin's mansion, an everything is possible place. I will always remember our Panda visiting. It was indeed a great day, full of fun. Hafize Kaya, many thanks for all our laughs, trips and gossips. How can we forget this huge open-air mental asylum and various people inside it? Then we realized, we are two of them, we must be crazy too. I will always remember your support, your funny stories, your chicken-related gifts for me and many more things. Dilek Bolat Erkalaycıoğlu, you were the best cook of Wageningen, thanks to your homeland, Adana, and its great cuisine. Many thanks for all our gatherings at your room (always tea included). You are now a mother of a beautiful baby, Doğa. I wish you a great future with your lovely family.

It is time to talk about my second Turkish men of Wageningen team,

Kadir Demirağ, Murat Yanat, Görkem Şener and Tolga Ayeri. After everyone gone, you came up guys. Kadir, I am grateful for our friendship, laughs, tea talks and lunch breaks. And also great journeys in The Netherlands and Germany. You have a spectacular talent and voice. I want to see you hold on to your dreams and reach great places. Hope to see you in Turkey, buddy. Murat, you are one of the few people who has amazing potential and intelligence. I am very glad to know you. You are also the one that we will leave in Wageningen. Protect and watch our small village. I am looking forward to see you on the stage when you defend your thesis. I wish you a healthy and great life buddy. Görkem and Tolga, thanks for all of our gatherings guys. I know we will continue to do that in Turkey. I am very glad to know each of you.

I would like to thank all of my other Turkish, German, Dutch and Greek friends in Wageningen, too many names to fit here, who made this small village more livable place for me. 
And of course, my great Spanish friends. Nicole Pradas Ferazzoli, David Fuentenebro Navas, Mauro Castello Sanjuan and Arcadio Garcia Perez. I have met you thanks to Deniz and I am super glad to know each of you. You are the most energetic group I have ever seen in Wageningen. Many thanks for all of our nice gatherings, dinners and birthday parties. I hope the next party would be our wedding in Turkey. I am looking forward to see you. I wish all of you a healthy and great life.

It is time to talk about Esra Karaduman. Dear Esra, you are the only witness of my academic life from bechelor to this day. We shared many great, funny and also difficult moments. We fought together against the two-faced, dishonest and fraud people we encountered in our MSc adventure. You are one of those rare people who are always on the side of truth and justice. And you are a great friend. It is wonderful to have you in my life. I wish you a splendid and healthy future.

It is time to talk about my two brothers, the family I choose.

Civan Lezgin Kahraman, you are a four-leaf clover, almost impossible to find and lucky to have. We started this road together from the same place at the same time. I am extraordinarily grateful to the universe for bringing us together. You are the most sensible, warm-hearted, truthful and soulful person I have ever known. I do not believe that no thankfulness sentence in my brain and heart would fit here. We are one of those rare people who understand each other even with just a glance. Thank you for being a remarkably wonderful person, friend, brother and family to me. Thank you for being there and never letting me down. Thank you for your eternal support in my last 2200 days and all upcoming days in future.

Mehmet Can Güçlü, you entered my life as a friend during the first weeks of my PhD adventure in Wageningen, but over the time, day by day, become my best friend, then my eternal brother. The things that bind us together are our similar and unique spirits. We have already a thousand of memories, hundreds of laughs and jokes, which all have one reason: a great brotherhood. If I didn't tell you what I go through every day during my PhD, I would never be able to maintain my mental health and my calmness. We have learned many things from each other. I believe that we make each other a better person. Thank you always stick with me 
even when I am not at my best. Thank you for being my friend, brother and family. It's great to know that you will be in every day of my life.

It is time to talk about love

Wageningen gave me a wonderful gift that will remain in my heart until the last seconds of my life. My love, my beautiful fiancé, my bride to be, Deniz Güngöroğlu. Before you, I was just a person trying to fill the gaps in his world, than you came and became my world. Our destinies worked jointly to brought us together in Wageningen. You became my everything and my biggest purpose of living. You are the one who understands my tears, while the rest of the world know me by my smile. No matter how frustrated I am, you find the words to make me feel better. You have an amazing way on comforting, nurturing and easing everything. You make me feel loved. Many thanks for that. I don't know what I would do without you. You make the world a brighter place for me, for us. Thank you for all of your eternal support during last 2 years and all upcoming days of future until eternity. I love you deeply and forever.

I would like to finish my words with my family.

Semra Güz, Süleyman Güz and Damla Güz. Many thanks for always supporting me in whatever I decide to do and helping me in whatever ways possible.

My lovely mom, thank you for your endless love and for being supportive all the time. You are the one who kept me alive in my hardest moments and difficult times. I am so happy to have you. I feel blessed, cared and loved, all because of you. Thank you for everything.

My dear dad, I know no matter how old I get, I will never stop needing you. Your guidance is all I have ever needed. Since childhood, you have taught me to be a good, nice and kind person before being a man. And I still stand by your advice.

My dear sister, thanks for being my best friend and someone I can share everything with. I am so grateful that we have stayed so close every time. It is such an extraordinary thing knowing there is someone else in the world who gets everything from me and will always be there for me.

I love all of you. 


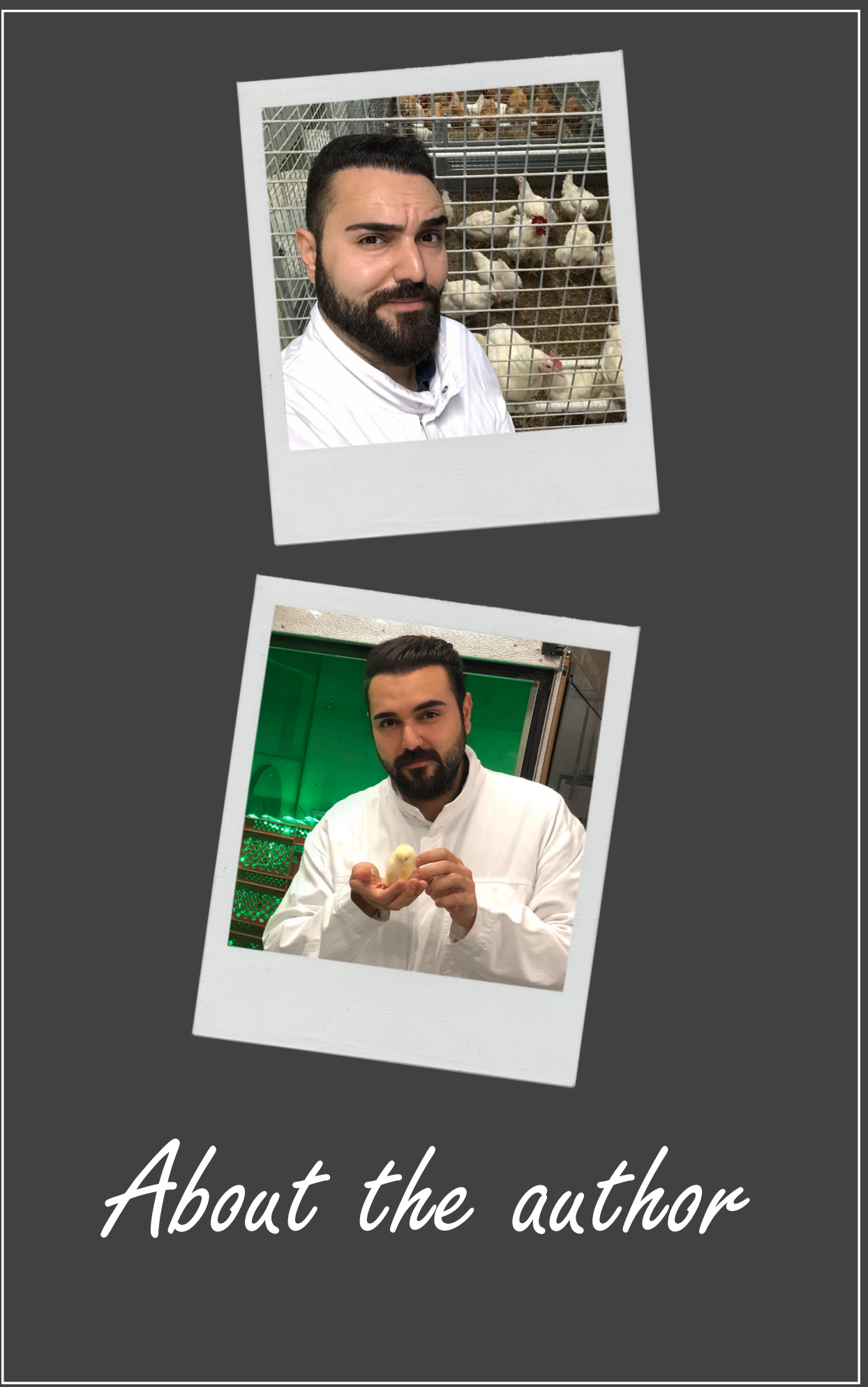


Bahadır Can Güz was born on $17^{\text {th }}$ of January 1989 in Turkey. From his childhood, he had a close relationship with chicken world because his father worked as a manager in an egg production company between 1985 and 2015. Eggs, chicks, chickens, feed ingredients, disease names, vaccine names and many more chicken related terms were a part of his family talk. After exposing this much information with his young and inquiring mind, he decided to take a step forward in poultry science combined with his education. In 2007, he enrolled in the Agricultural Engineering BSC program at Ege University, Izmir, Turkey. After studying engineering part of his BSc degree, he chose Animal Sciences subdivision in 2010. During the last year of his BSC, he took poultry related BSc level courses and wrote a thesis, "The past, present, and future of incubators", to finish his undergraduate study. In 2012, after obtaining his BSc degree, he started his MSC degree in Poultry Management, Breeding and Nutrition group in Animal Sciences Department at Ege University. After taking poultry science related MSc level courses for one year, he involved many poultry-related experiments and one of them was his project called "In-Ovo leptin in broiler chickens". After successfully finishing the experiment, he wrote a thesis, "Effects of in-Ovo leptin injection in broiler breeder eggs on hatchability and growth performance of broiler chickens" and received his master's degree in 2015. He wrote a book together with his supervisor called "The Egg". In the same year, he got a scholarship from "Turkish Ministry of National Education" in behalf of "Turkish Ministry of Agriculture and Forestry" to get his PhD degree abroad. His first aim was to get a position at Wageningen University and Research in the Netherlands, since this university is known as the best university in Agriculture, Forestry and Environmental Sciences in the world for many years. In 2017, during the first days of January, he started his PhD adventure at Adaptation Physiology Group at Wageningen University and Research. He involved in a project called "Healthy Bones" established by Wageningen University and Research in collaboration with Wageningen Livestock Research, Ministry of Agriculture, Nature and Food Quality of the Netherlands, and seven poultry-associated commercial companies, Aviagen EPI, Darling Ingredients Inc., ForFarmers N.V., Hubbard, Marel Stork Poultry Processing B.V., Nepluvi and Trouw Nutrition (Nutreco) (in alphabetical order). The results of his $\mathrm{PhD}$ research are presented in this thesis. 


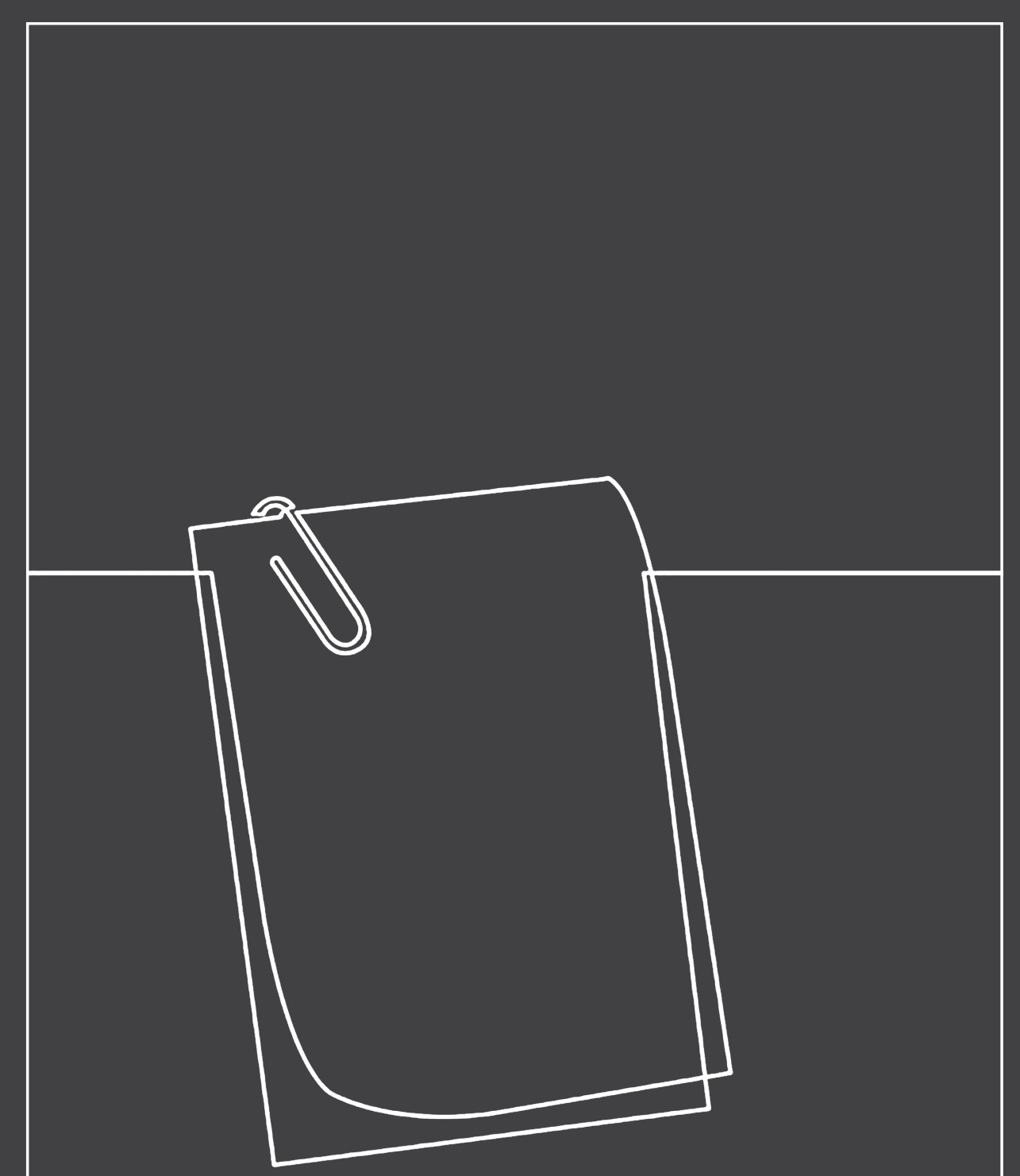

Pablications 
Peer -reviewed scientific papers

Güz, B.C., Molenaar, R., de Jong, I.C., Kemp, B., van den Brand, H. and van Krimpen, M., 2019. Effects of dietary organic minerals, fish oil, and hydrolyzed collagen on growth performance and tibia characteristics of broiler chickens.

Poultry Science, 98(12), pp.6552-6563

DOI: https://doi.org/10.3382/ps/pez427

Güz, B.C., Molenaar, R., De Jong, I.C., Kemp, B., Van Krimpen, M. and Van Den Brand, H., 2020. Effects of eggshell temperature pattern during incubation on tibia characteristics of broiler chickens at slaughter age.

Poultry Science, 99(6), pp.3020-3029

DOI: https://doi.org/10.1016/j.psj.2019.12.042

Güz, B.C., Molenaar, R., de Jong, I.C., Kemp, B., van Krimpen, M. and van den Brand, H., 2021. Effects of green light emitting diode

3 light during incubation and dietary organic macro and trace mineras during rearing on tibia characteristics of broiler chickens at slaughter age.

Poultry Science, 100(2), pp.707-720

DOI: https://doi.org/10.1016/j.psj.2020.11.042

Güz, B.C., de Jong, I.C., Bol, U.E., Kemp, B., van Krimpen, M., Molehaar, R. and van den Brand, H., 2021. Effects of organic macro and trace minerals in fast and slower-growing broiler breeders' diet on offspring tibia characteristics and growth performance. (submitted)

Güz, B.C., de Jong, I.C., da Silva, C.S., Veldkamp, F., Kemp, B., Molenaar, R. and van den Brand, H., 2021. Effects of pen enrichment on leg health of fast and slower-growing broiler chickens. (submitted)

319 


\section{Conference and symposia proceedings}

Güz, B.C., Molenaar, R., van Krimpen, M.M., van den Brand, H., de Jong, I.C. and Kemp, B., 2018. Effects of dietary supplementation of organic minerals, fish oil and hydrolyzed collagen on growth performance and tibia characteristics of broiler chickens. In WIAS Science Day 2018: WIAS: Work on your Impact in Animal Science and Society, pp. 27-27

https://library.wur.nl/WebQuery/wurpubs/533552

Güz, B.C., Molenaar, R., de Jong, I.C., Kemp, B., van den Brand, H. and van Krimpen, M.M., 2018. Effects of dietary replacement of organic minerals, fish oil and hydrolyzed collagen on tibia characteristics of broiler chickens. In International Poultry Science Congress of WPSA Turkish Branch'2018, pp. 479-479

https://library.wur.nl/WebQuery/wurpubs/547371

Güz, B.C., Molenaar, R., de Jong, I.C., Kemp, B., van den Brand, H. and van Krimpen, M.M., 2018. Effects of organic minerals, fish oil and hydrolyzed collagen in broiler diets on growth performance and tibia characteristics. In The 15th European Poultry Conference (EPC), pp. 261-261

https://library.wur.nl/WebQuery/wurpubs/54741 1

Güz, B.C., Molenaar, R., Wijnen, H.J., van Krimpen, M.M., de Jong, I.C. and van den Brand, H., 2018. Eggshell temperature pattern during incubation affects leg bone characteristics of broiler chickens at slaughter age. In Abstracts of the Incubation and Fertility Research Group:(IFRG/WPSA Working Group 6), pp. 19-19. https://library.wur.nl/WebQuery/wurpubs/547687

Güz, B.C., Molenaar, R., de Jong, I., van Krimpen, M. and van den

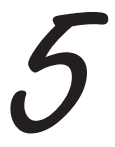
Brand, H., 2019. Eggshell temperature patterns during incubation on tibia characteristics of broiler chickens at slaughter age. In 5th International Poultry Meat Congress, Antalya, Turkey, pp. 18-18 http://beyazetkongresi.com/BESD-BIR-5.UBEK-BILDIRIKITABI-TR.pdf 
Güz, B.C., Molenaar, R., de Jong, I., van Krimpen, M. and van den Brand, H., 2019. Eggshell temperature patterns during incubation on tibia characteristics of broiler chickens at slaughter age. In WIAS Science Day 2019, Lunteren, The Netherlands, pp. 11-11 https://library.wur.nl/WebQuery/wurpubs/549538

Güz, B.C., Molenaar, R., de Jong, I.C., van Krimpen, M.M. and van den Brand, H., 2019. Effects of green LED light during incubation and dietary mineral variety during rearing on tibia characteristics of broiler chickens at slaughter age. In Proceedings of the 17th International Conference on Production Diseases in Farm Animals, pp. 193-193

https://library.wur.nl/WebQuery/wurpubs/552410

\section{Others}

van Krimpen, M.M., Güz, B.C., de Jong, I.C., van den Brand, H. and Molenaar, R., 2019. Organische mineralen goed voor botkwaliteit. De Pluimveehouderij, 49(14), pp.11-15

https://www.forfarmers.nl/bestanden/ForFarmers_NL/PDF-documenten-NL/PDF-Pluimvee/49524-1/Artikel_pluimveehouderij_PPS_ onderzoek_Healthy_Bones.pdf

van Krimpen, M.M., Güz, B.C., de Jong, I.C., van den Brand, H. and

Molenaar, R., 2018. Onderzoek naar sterkere botten. De Pluimveehouderij, (April 2018), pp.28-29

https://library.wur.nl/WebQuery/wurpubs/547477

van den Brand, H., Güz, B.C., Molenaar, R. and de Jong, I.C. 2021. 3 Organic minerals promote broiler leg quality. Poultry World, 8 (September 2021), pp.12-13. 


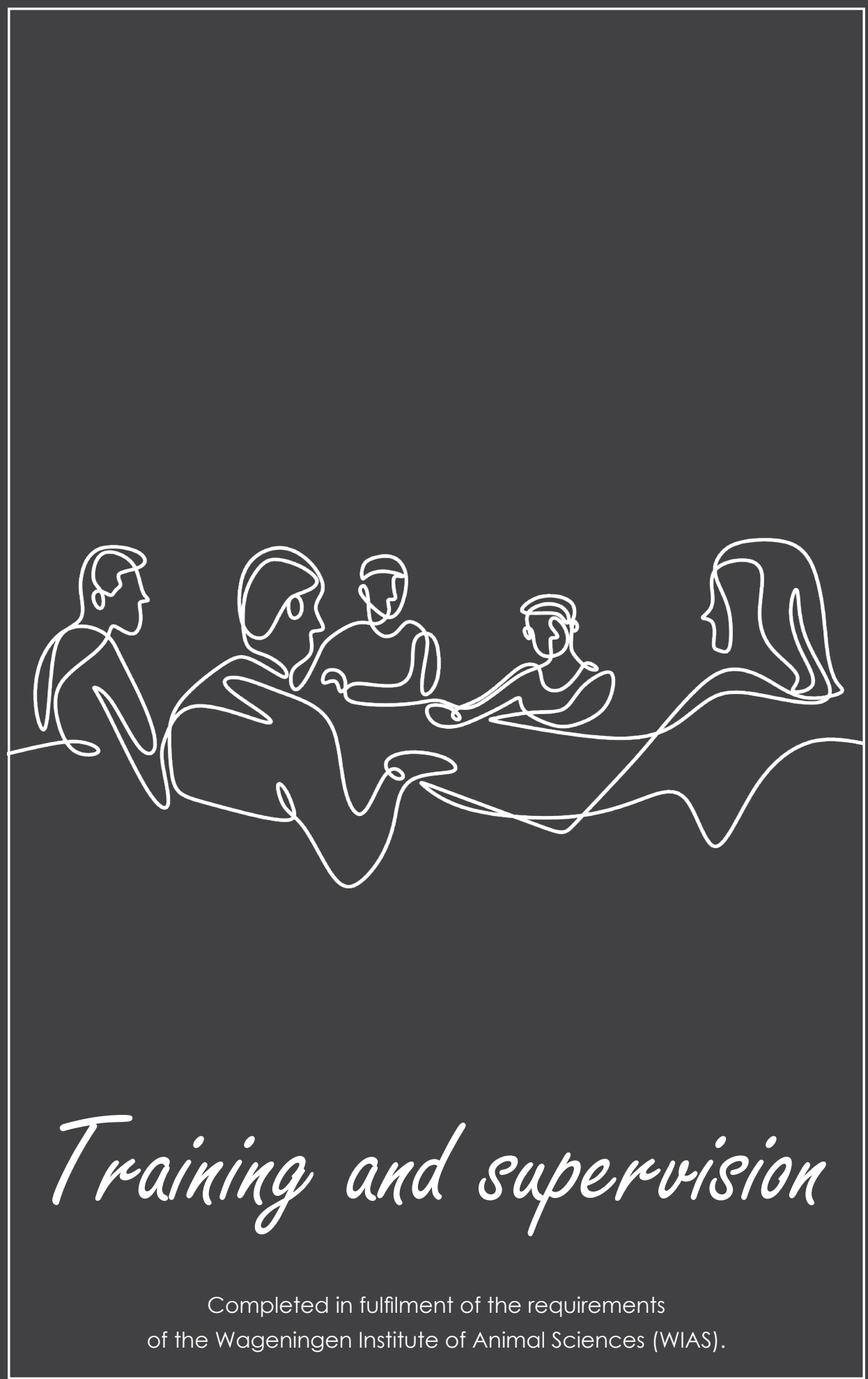




\section{Basic courses}

WIAS Introduction Day

\section{Disciplinary and interdisciplinary courses}

Writing Research Proposal

Advanced Statistics Course on Design of Experiments

Statistics for the Life Sciences

In-Depth Knowledge on Bone (Bologna, Italy)

\section{Professional competences}

Information Literacy Including Endnote Introduction

2017

Project and Time Management

Scientific Writing

Brain Training 2018

WAPS Council Membership 2019

WIAS Annual Conference 2020 - Chairman

\section{Societal relevance}

Societal Impact of Your Research

2019

\section{Presentations}

$15^{\text {th }}$ European Poultry Conference, Dubrovnik, Croatia

2018

WIAS Science Day, Wageningen, The Netherlands

2018

WIAS Science Day, Lunteren, The Netherlands

2019

$5^{\text {th }}$ International Poultry Meat Congress, Antalya, Turkey

2019

$17^{\text {th }}$ International Conference on Production Diseases in Farm 


\title{
Supervising MSc and BSc Theses
}

\author{
Ute Bol
}

Effects of Dietary Mineral Sources in Broiler

Breeders on Leg Health of Their Offspring

\section{Florence Edson}

Effect of Diet Composition and Incubation Temperature on Wing Characteristics of Broiler Chickens

\section{Thijs Versantvoort}

A Comparative Experiment on Measuring Articular Cartilage and Incubation Temperature

\section{Robert van der Klis}

Pen Enrichment and Bone Development

in Broiler Chickens

\section{Wisse Zwanenburg}

Influence of Genetics and Incubation Conditions on Chicken Wing Bone Development 


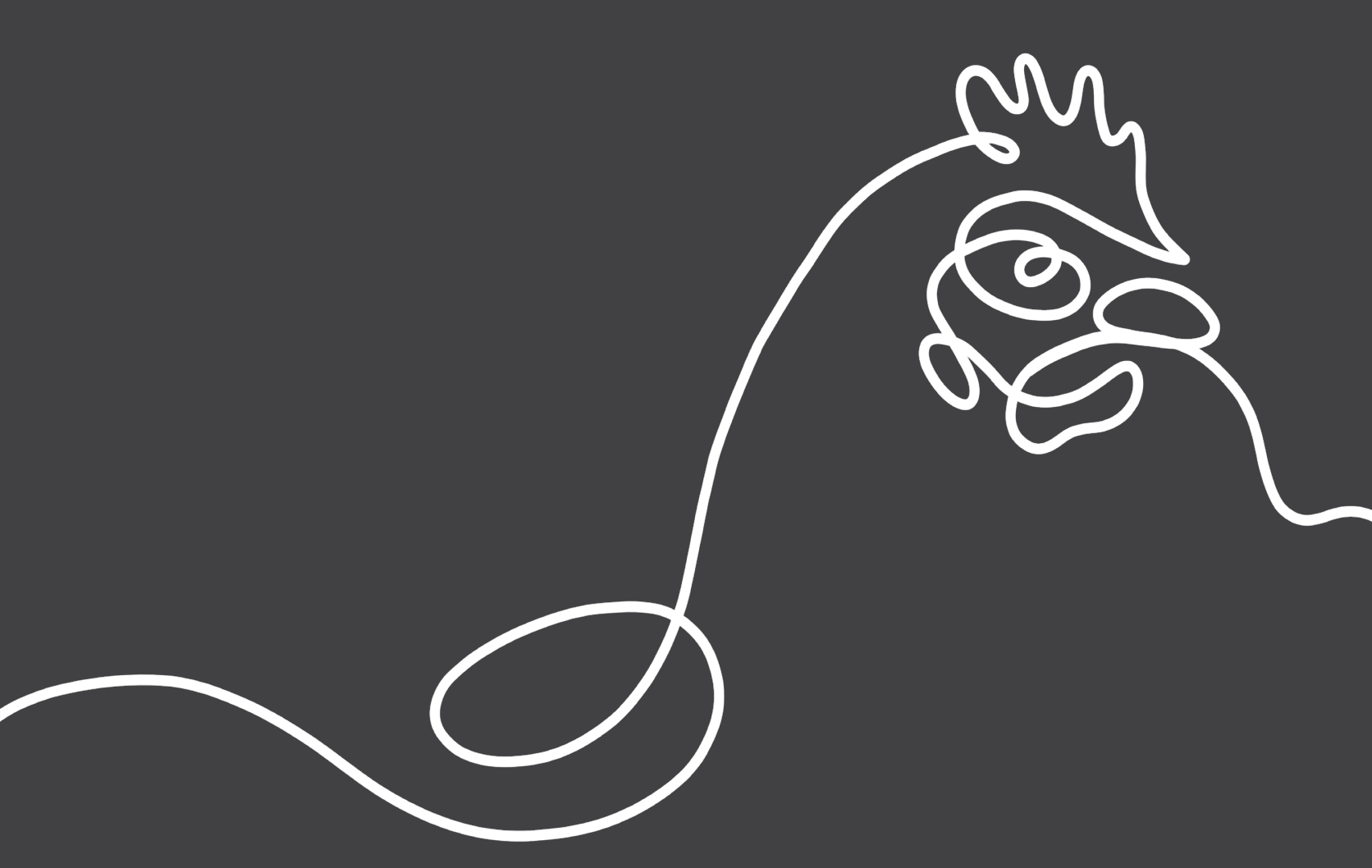




\section{Colophon}

The research described in this thesis was carried out in the project called "Healthy Bones", funded by a public-private partnership, involving Wageningen University and Research, Wageningen Livestock Research, Ministry of Agriculture, Nature and Food Quality of The Netherlands, Aviagen EPI, Darling Ingredients Inc., ForFarmers N.V., Hubbard, Marel Stork Poultry

Processing B.V., Nepluvi and Trouw Nutrition (Nutreco).

The author was financially supported by a PhD scholarship acquired from Turkish Ministry of National Education in behalf of General Directorate of Agricultural Research and Policies in Turkish Ministry of Agriculture and Forestry.

Cover design, illustrations and lay-out: Bahadır Can Güz Printing: ProefschriftMaken.nl

ISBN: 978-94-6395-984-1

DOI: https://doi.org/10.18174/553659

(C) Bahadır Can Güz, 2022

All rights reserved.

No part of this production may be reproduced, stored in a retrieval system, or transmitted in any form or by any means (electronic, mechanical, photocopying, recording or otherwise) without prior written permission by the author. 



$$
\sqrt{2}
$$

